\title{
RADTRAD:
}

\section{A Simplified Model for \\ RADionuclide Transport and \\ Removal And Dose Estimation}

Manuscript Completed: June 1997

Date Published: April 1998

Prepared by

S. L. Humphreys/SNL

T. J. Heames/ITSC

L. A. Miller/SNL

D. K. Monroe/SNL

Sandia National Laboratories

P.O. Box 5800

Albuquerque, NM 87185-0748

ITSC

8015 Mountain Place NE

Suite 210

Albuquerque, NM 87110

DISTRBBUTION OF THS DOCUMENT IS UMLMITED

J.Y. Lee, NRC Project, Manager

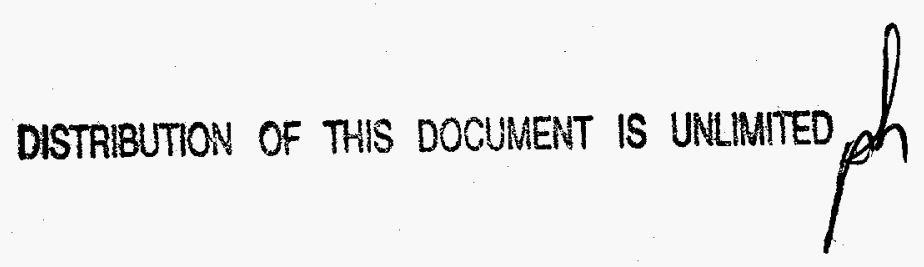

Prepared by

Division of Reactor Program Management

Office of Nuclear Reactor Regulation

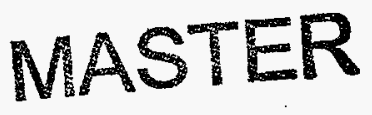

U.S. Nuclear Regulatory Commission

Washington, DC 20555-0001

NRC Job Code J2453 


\section{DISCLAIMER}

This report was prepared as an account of work sponsored by an agency of the United States Government. Neither the United States Government nor any agency thereof, nor any of their employees, makes any warranty, express or implied, or assumes any legal liability or responsibility for the accuracy, completeness, or usefulness of any information, apparatus, product, or process disclosed, or represents that its use would not infringe privately owned rights. Reference herein to any specific commercial product, process, or service by trade name, trademark, manufacturer, or otherwise does not necessarily constitute or imply its endorsement, recom. mendation, or favoring by the United States Government or any agency thereof. The views and opinions of authors expressed herein do not necessarily state or reflect those of the United States Government or any agency thereof. 


\section{DISCLAIMER}

Portions of this document may be illegible electronic image products. Images are produced from the best available original document. 


\begin{abstract}
This report documents the RADTRAD computer code developed for the U.S. Nuclear Regulatory Commission (NRC) Office of Nuclear Reactor Regulation (NRR) to estimate transport and removal of radionuclides and dose at selected receptors. The document includes a users' guide to the code, a description of the technical basis for the code, the quality assurance and code acceptance testing documentation, and a programmers' guide. The RADTRAD code can be used to estimate the containment release using either the NRC TID-14844 or NUREG-1465 source terms and assumptions, or a user-specified table. In addition, the code can account for a reduction in the quantity of radioactive material due to containment sprays, natural deposition, filters, and other natural and engineered safety features. The RADTRAD code uses a combination of tables and/or numerical models of source term reduction phenomena to determine the time-dependent dose at user-specified locations for a given accident scenario. The code system also provides the inventory, decay chain, and dose conversion factor tables needed for the dose calculation. The RADTRAD code can be used to assess occupational radiation exposures, typically in the control room; to estimate site boundary doses; and to estimate dose attenuation due to modification of a facility or accident sequence.
\end{abstract}




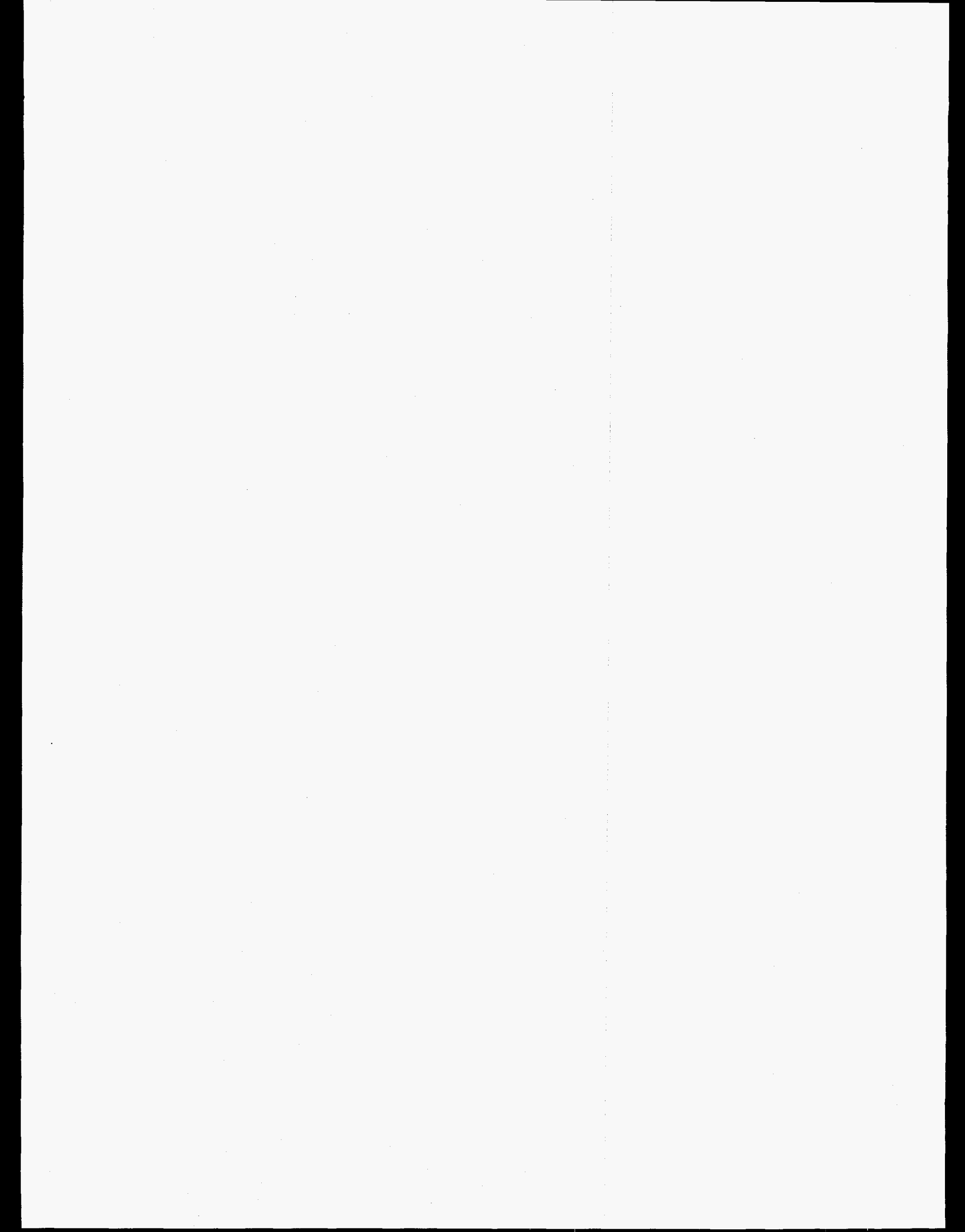




\section{Contents}

Introduction

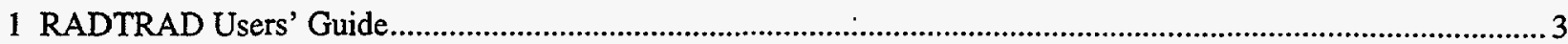

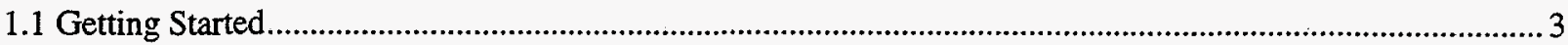

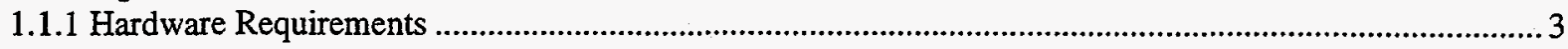

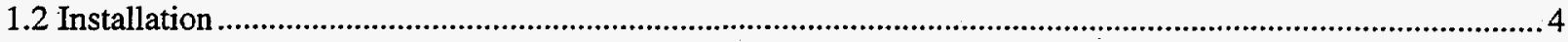

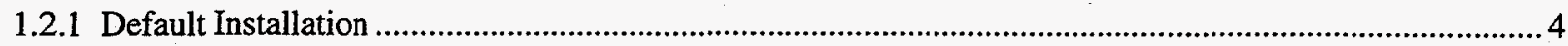

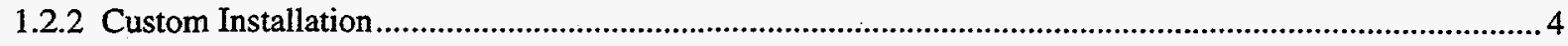

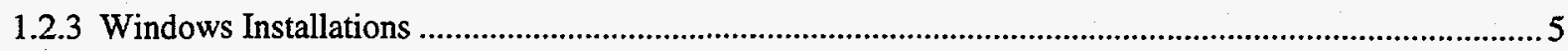

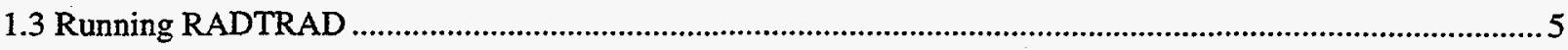

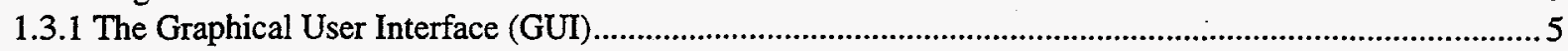

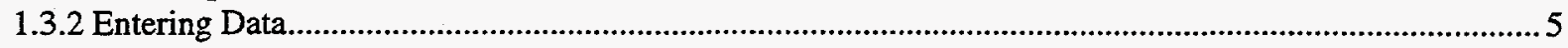

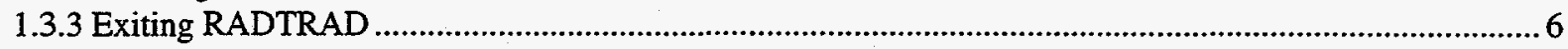

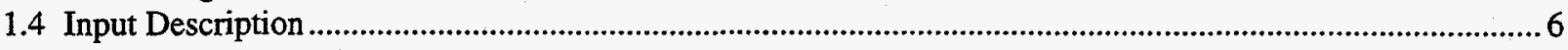

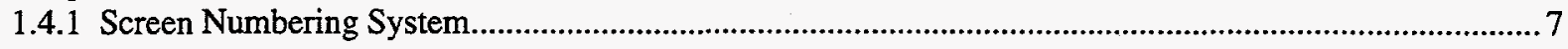

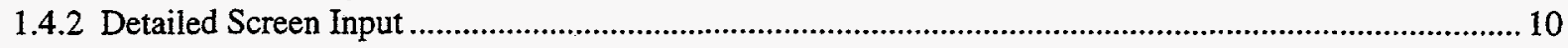

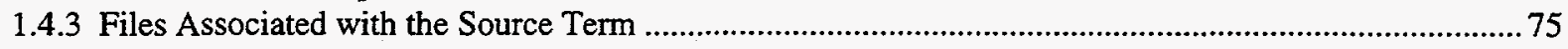

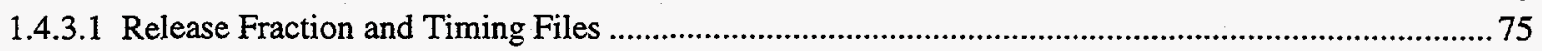

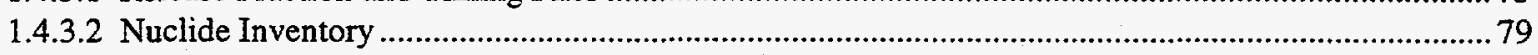

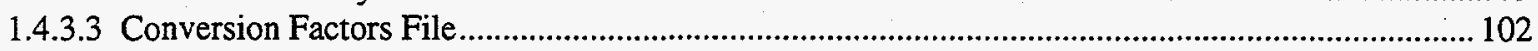

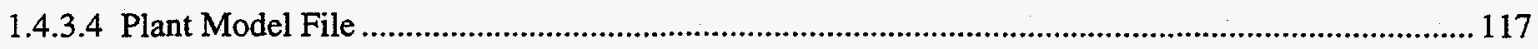

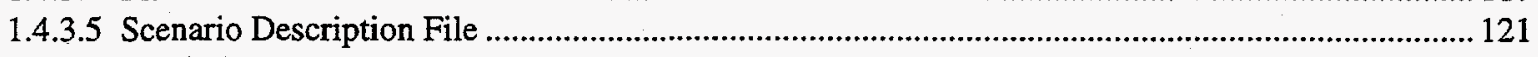

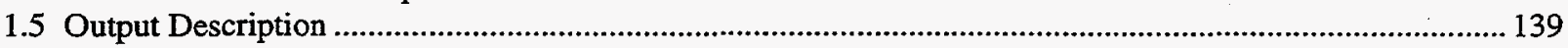

1.5.1 Sample Output ............................................................................................................................. 139

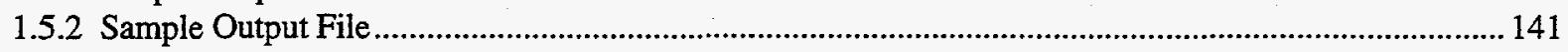

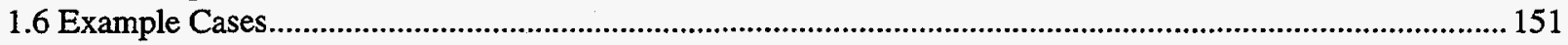

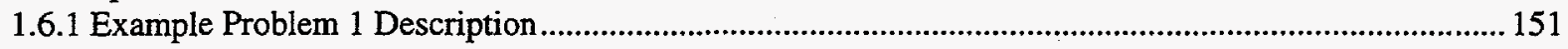

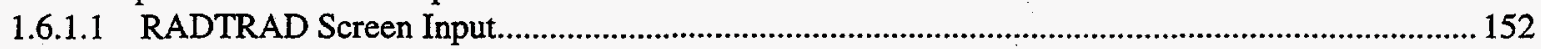

1.6.2 Example Problem 2 Description........................................................................................................... 160

1.6.3 Example Problem 3 Description ....................................................................................................... 170

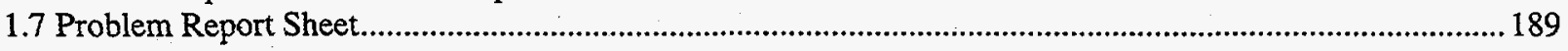

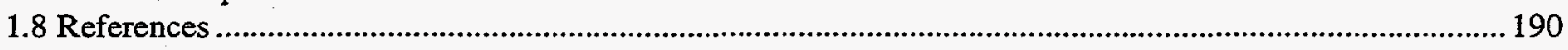

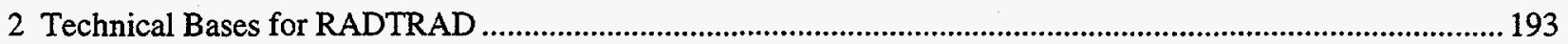

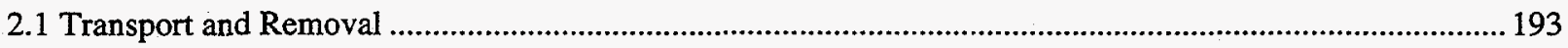

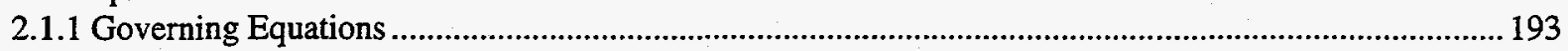

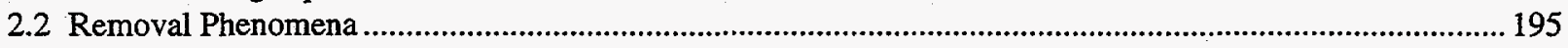

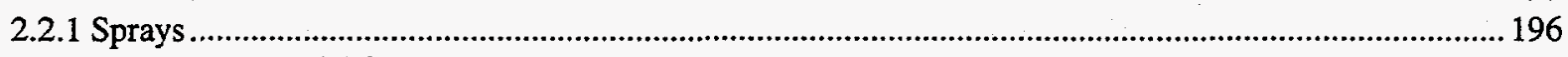

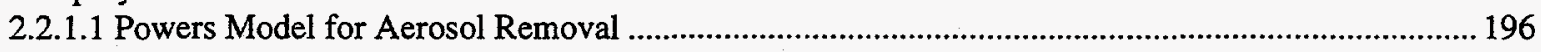

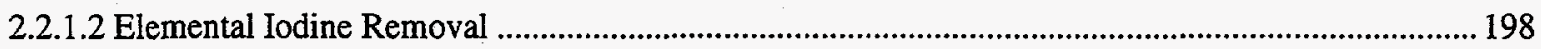

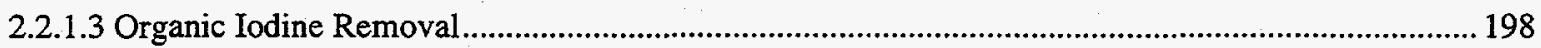

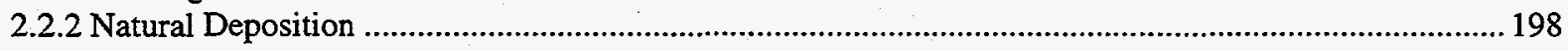

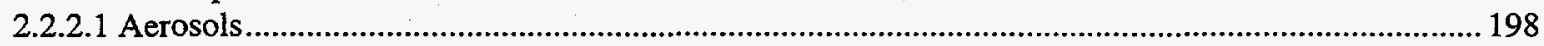

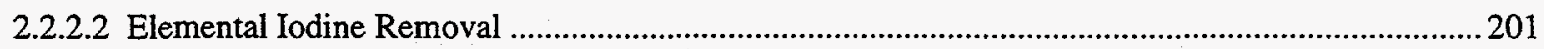

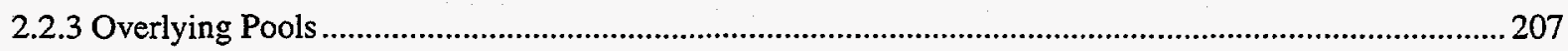

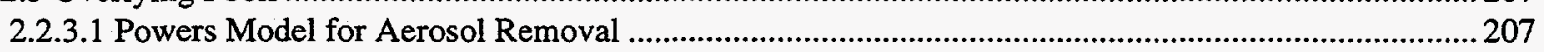

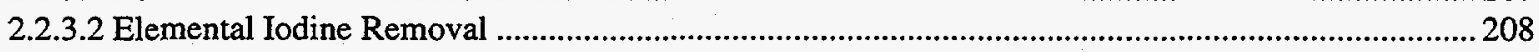

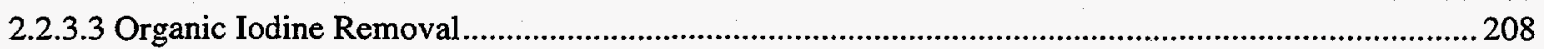

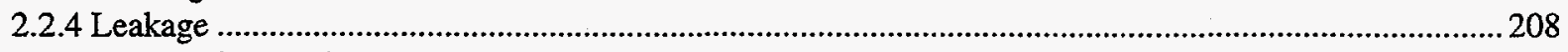

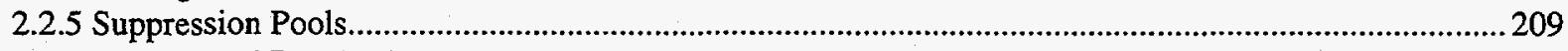

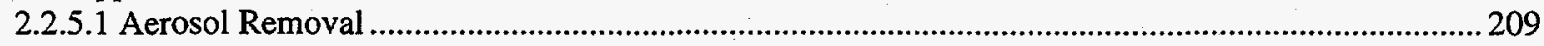

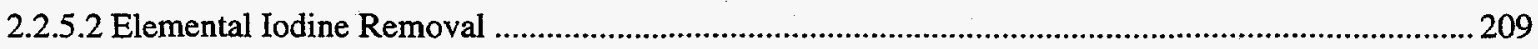

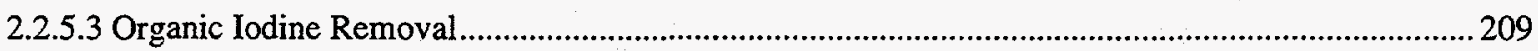

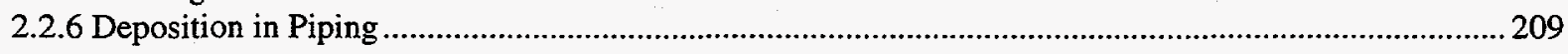




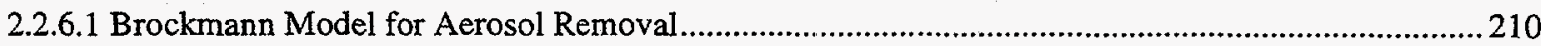

2.2.6.2 Bixler Model for Elemental Iodine Removal.............................................................................. 212

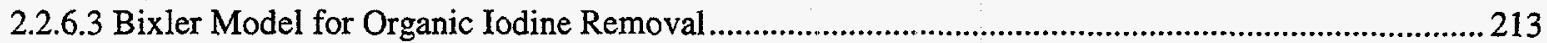

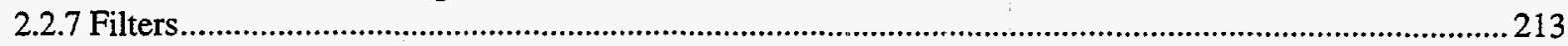

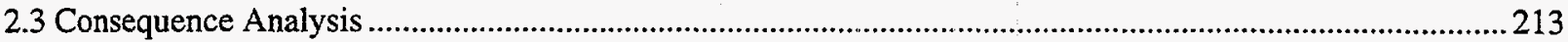

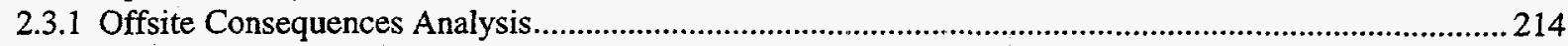

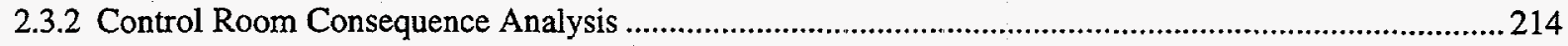

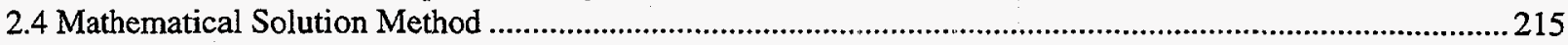

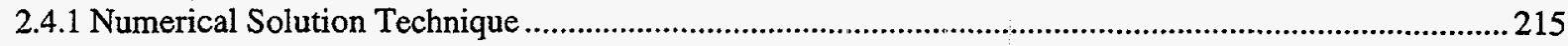

2.4.2 Transport Calculations..........................................................................................................................2 216

2.4.2.1 Calculating the Transport and Removal Coefficients ......................................................................218

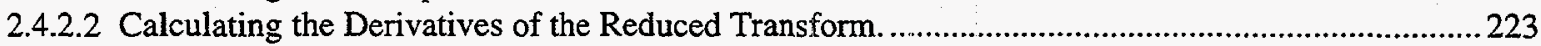

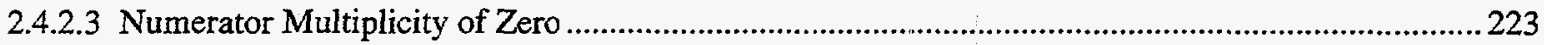

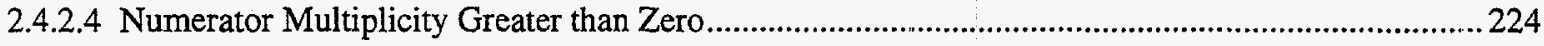

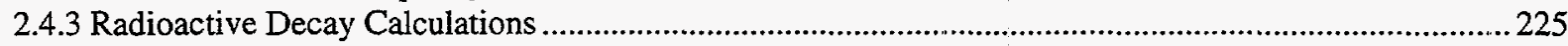

2.4.3.1 Simulation of Decay without Daughter In-growth ..........................................................................225

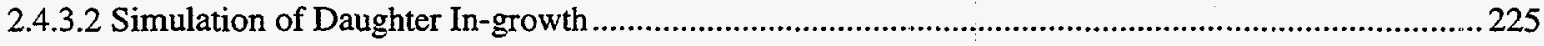

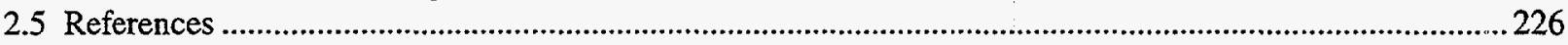

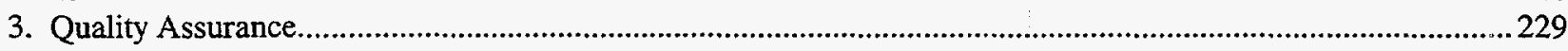

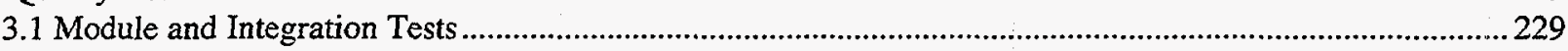

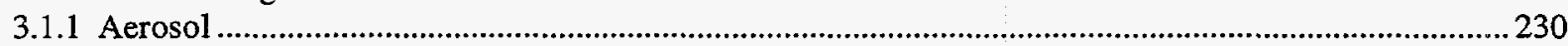

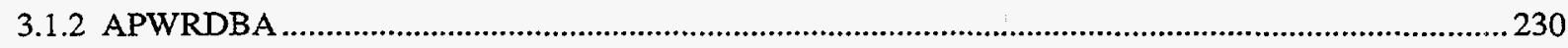

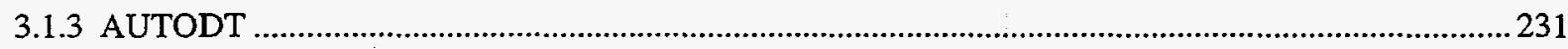

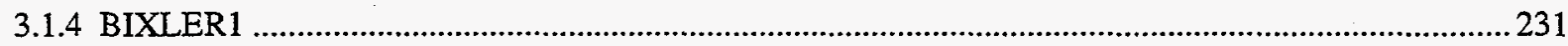

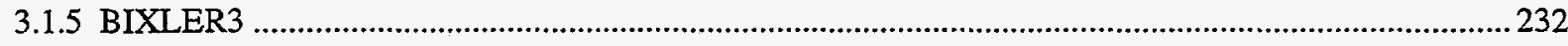

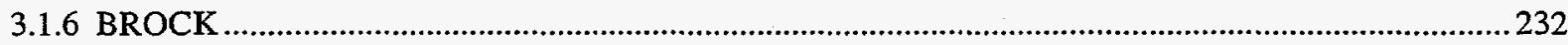

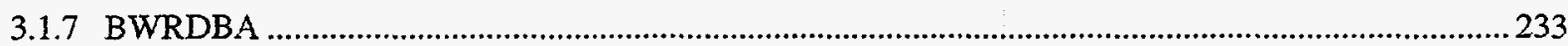

3.1.8 BWRSA

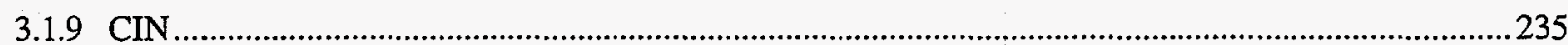

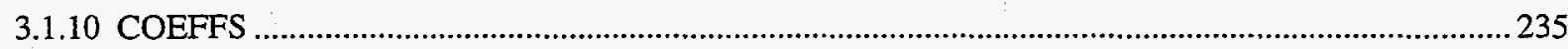

3.1.11 COENUM

3.1.12 COUT .

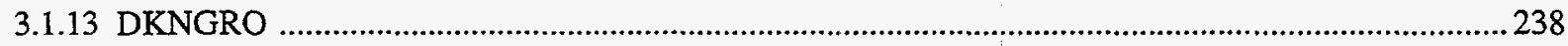

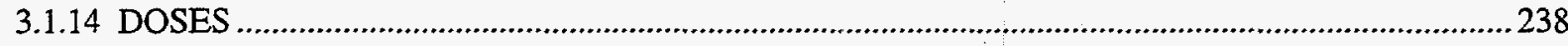

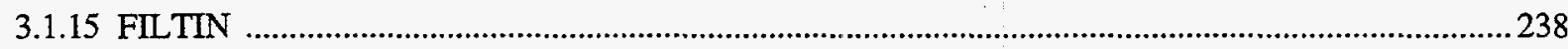

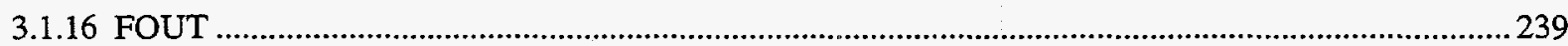

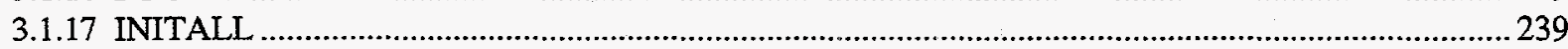

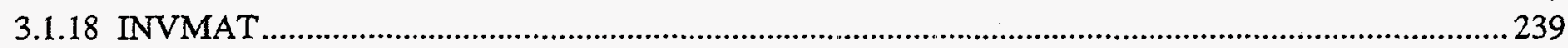

3.1.19 LUPC1

3.1.20 LUPC2

3.1.21 LUPL2

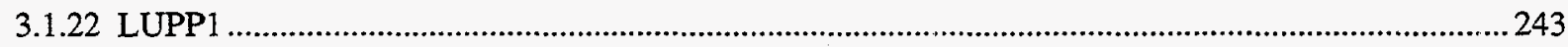

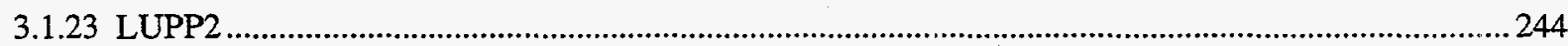

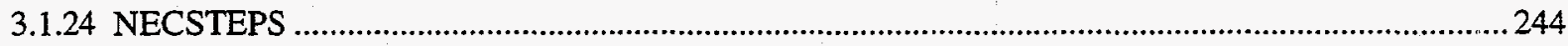

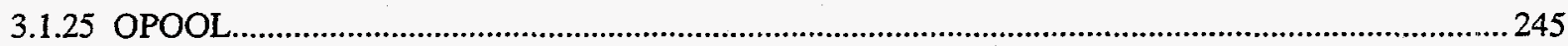

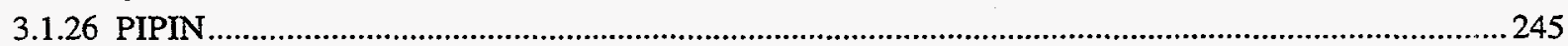

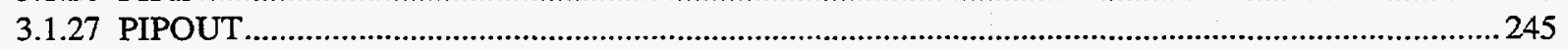

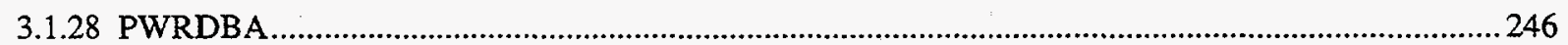

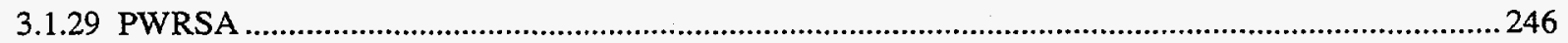

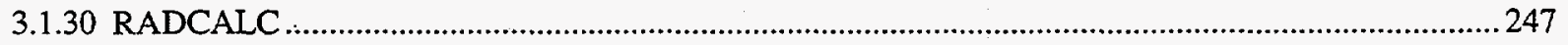

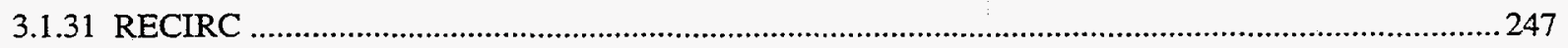

3.1.32 RESADER

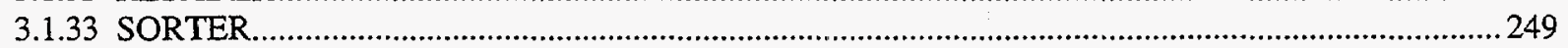

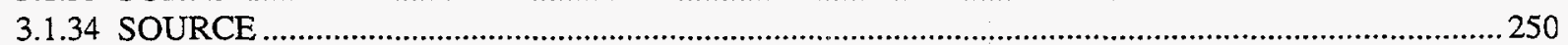

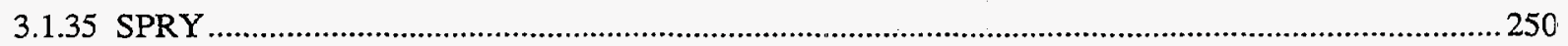




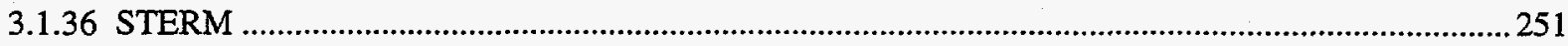

3.1.37 SUMPWR

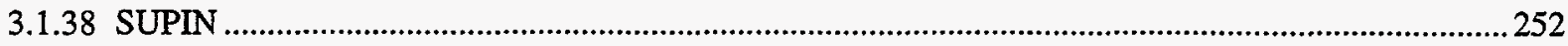

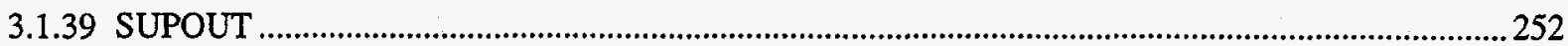

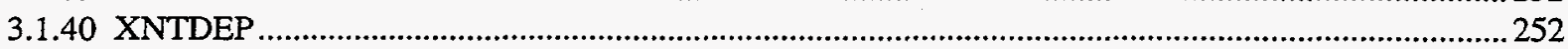

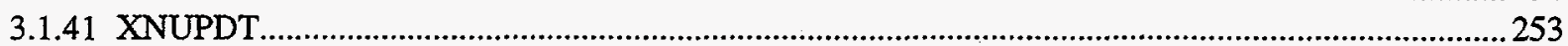

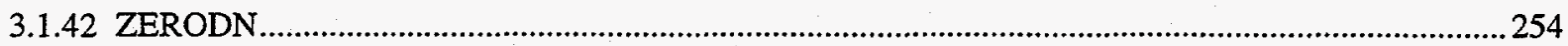

3.1.43 ZPORC

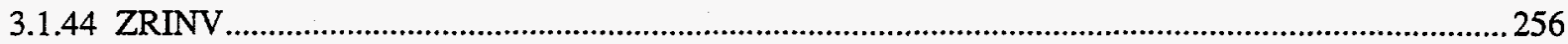

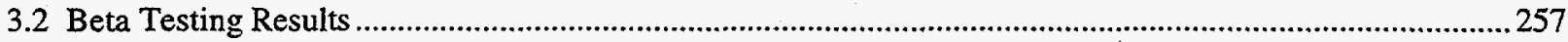

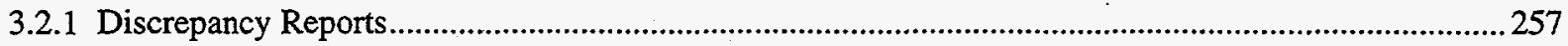

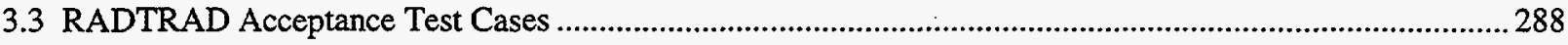

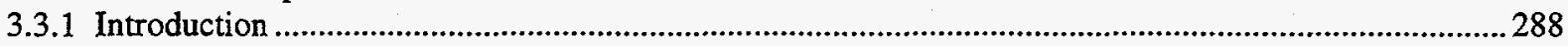

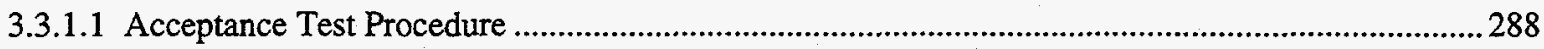

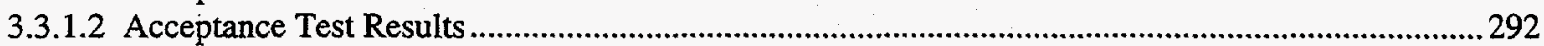

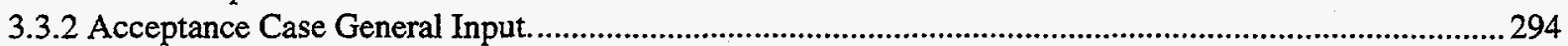

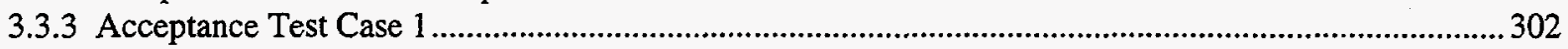

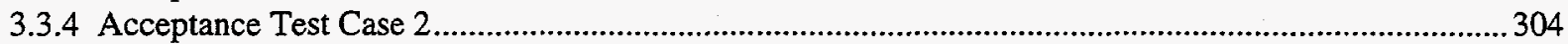

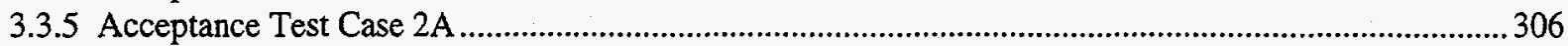

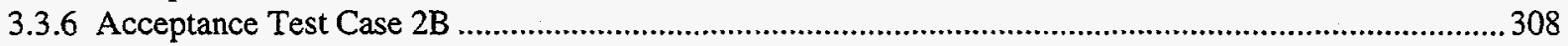

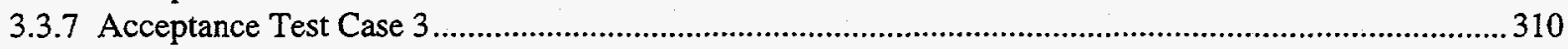

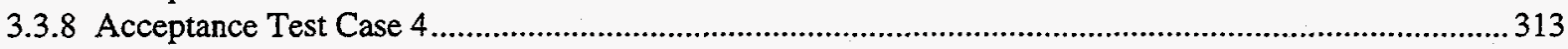

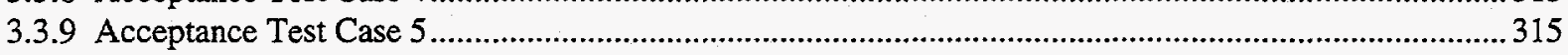

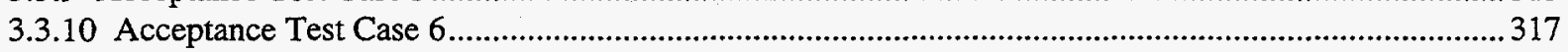

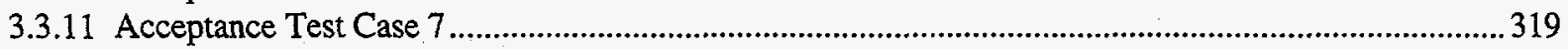

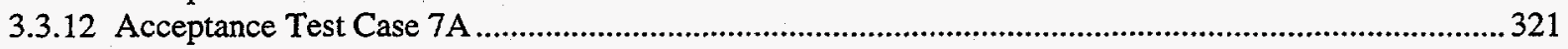

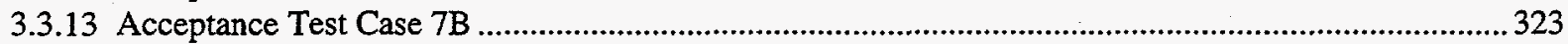

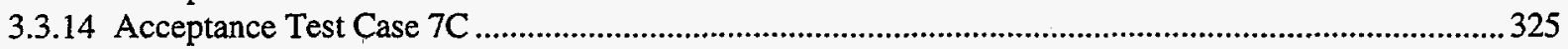

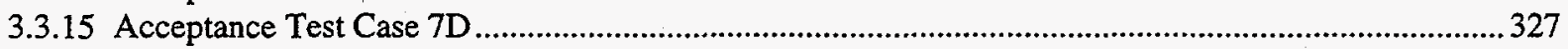

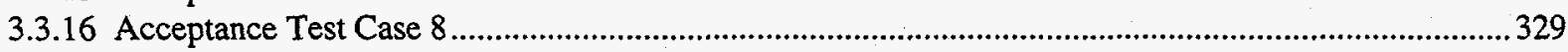

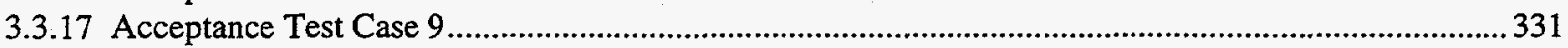

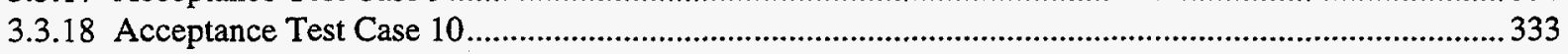

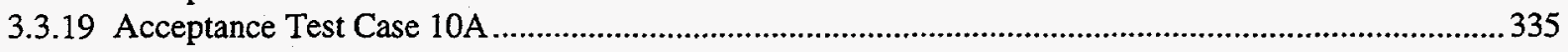

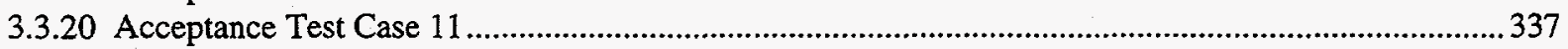

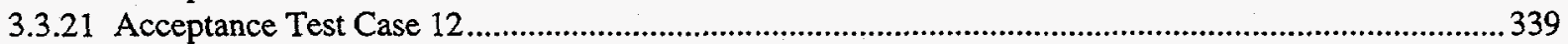

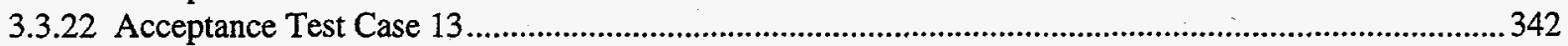

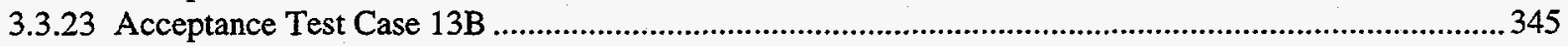

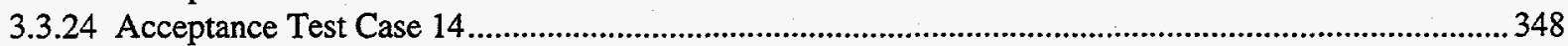

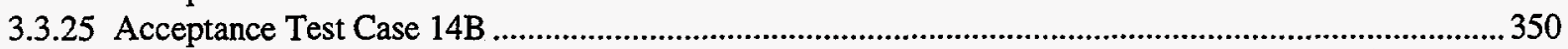

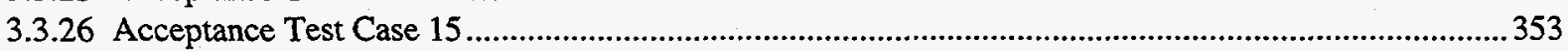

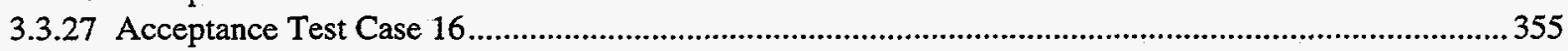

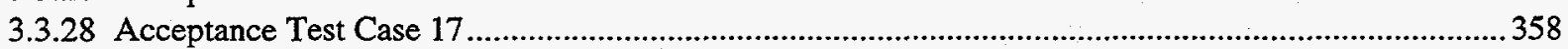

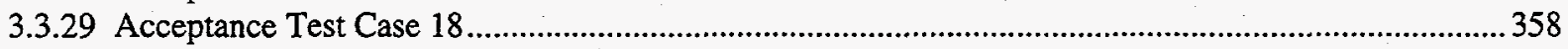

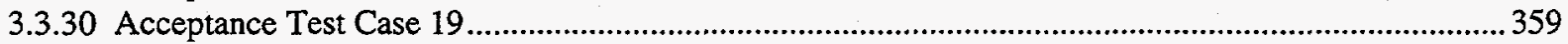

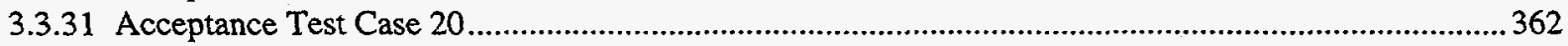

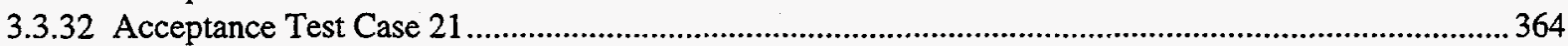

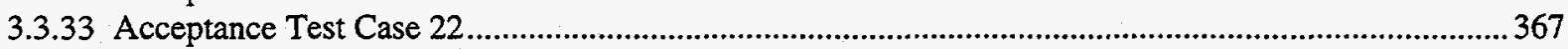

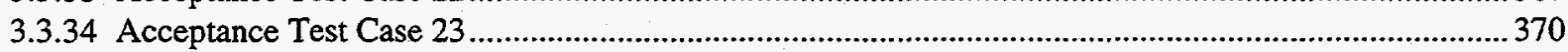

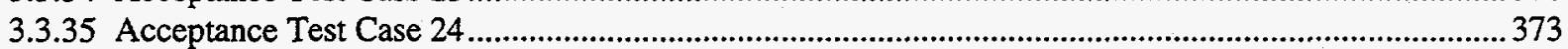

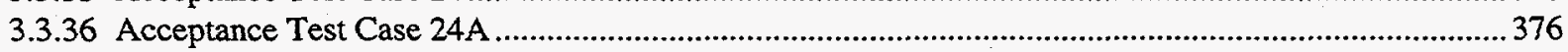

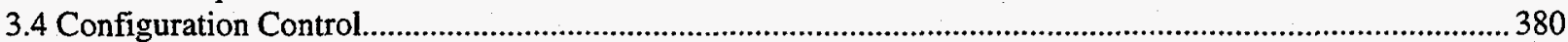

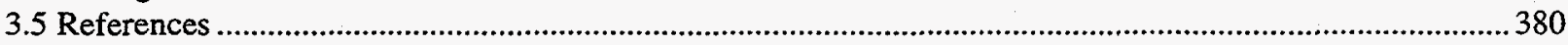

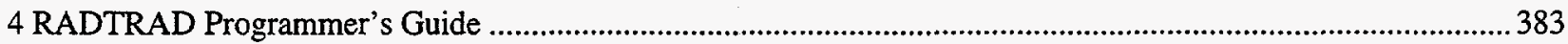

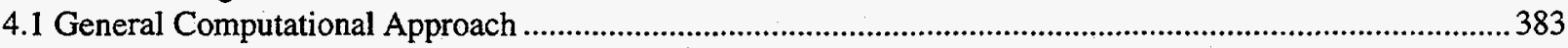

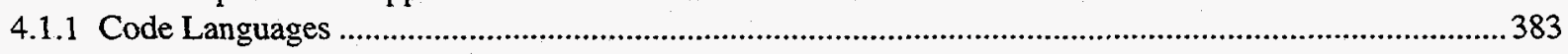




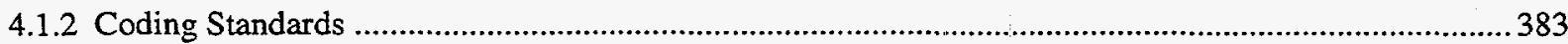

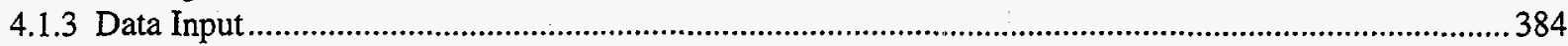

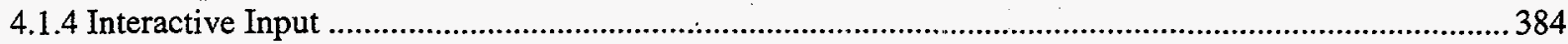

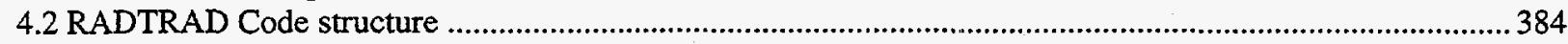

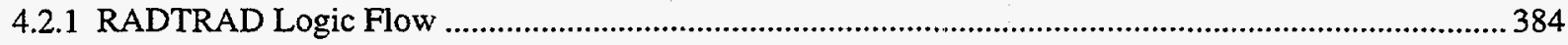

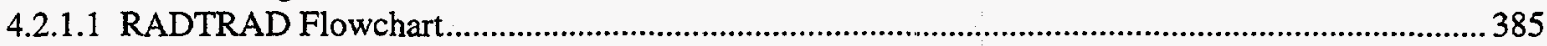

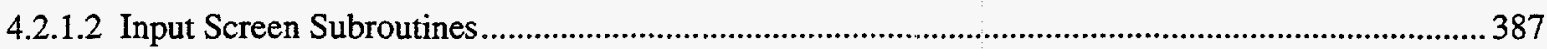

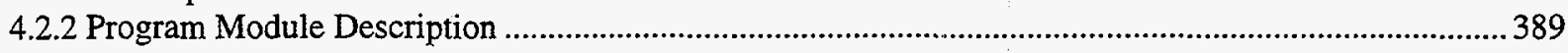

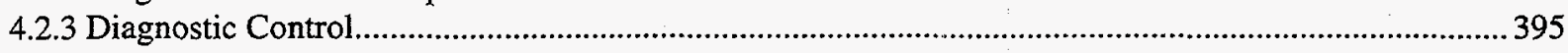

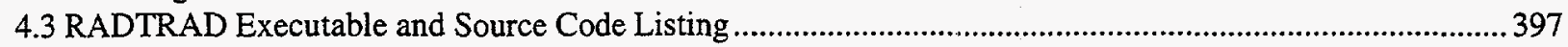




\section{Acknowledgments}

The RADTRAD project particularly recognizes, and is deeply indebted to, Dr. Jay Y. Lee the U.S. Nuclear Regulatory Commission (USNRC) technical monitor. In addition the project wishes to thank Dr. Dana A. Powers for many valuable discussions on modeling of phenomena and J. Van Ramsdell of Pacific Northwest National Laboratory (PNNL) for the interpretation of code results.

The authors also wish to acknowledge the many contributors to the project. In particular, we would like to mention Scott Ashbaugh of Innovative Technology Solutions Corporation (ITSC); Steve Stage (PNNL); Nathan Bixler, Fred Harper, Kelly Hays, Ken Bergeron, and Ken Washington of Sandia National Labs (SNL); David Chanin of Technadyne Engineering Consultants (TEC); and Barry Zalcman (USNRC). The authors are indebted to Ms. Noreen DeHerrera, SNL and Ms. Joanna Leonard, ITSC, for their efforts in preparing this manual. 


\section{Introduction}

The potential radiological consequences of nuclear power reactor accidents depend in part upon the amount, form, and species of the radioactive material released during the postulated accident. The RADionuclide Transport, Removal, and Dose (RADTRAD) model estimates doses at offsite locations; for example, the exclusion area boundary (EAB) or the low population zone (LPZ), and in the control room. The code has two optional source terms to describe fission product release from the reactor coolant system: those specified in "Calculation of Distance Factors for Power and Test Reactor Sites" (TID-14844) along with Regulatory Guides 1.3 and 1.4; and those specified for boiling water reactors (BWRs) and pressurized water reactors (PWRs) in "Accident Source Terms for Light Water Nuclear power Plants" (NUREG-1465). As radioactive material is transported through the containment, the user can account for sprays and natural deposition that may reduce the quantity of radioactive material. Material can flow between buildings, from buildings to the environment, or into control rooms through high-efficiency particulate air (HEPA) filters, piping, or other connectors. An accounting of the amount of radioactive material retained due to these tortuous pathways is maintained. Decay and in-growth of daughters can be calculated over time as the material is transported. The code contains over 25 model and table options to perform these tasks. It is anticipated that the code will be used to estimate attenuation of source terms as a result of modification for a facility or accident sequence.

The RADTRAD code was developed by the Accident Analysis and Consequence Assessment Department at Sandia National Laboratories (SNL) for the U.S. Nuclear Regulatory Commission (NRC), Office of Nuclear Reactor Regulation (NRR), Division of Reactor Program Management.

The code documentation is divided into four parts. The first chapter is a users' guide to the RADTRAD code. It contains the information necessary to install the code, generate an input file, run the code, and examine the output. The second chapter contains the technical basis for the code. It provides the user with the underlying assumptions and the simplifications in the models developed for the code. The third chapter is the quality assurance documentation. It includes the module testing, beta testing, and acceptance testing documentation. Chapter 4 is a programmer's guide to the code. It details the logic flow, the relationships between subprograms, and the purpose of each subprogram. Since this chapter is intended to help those who may be called upon to modify the code, it also includes a code listing. 


\section{RADTRAD Users' Guide}

This users' guide to the RADTRAD code consists of six sections. The first two sections will assist you in getting started. They describe the hardware requirements for and installation of the code. The third section describes how to run the code. It covers the user interface and data entry. The fourth section describes the input and associated files required by RADTRAD. It provides a description of each item and gives acceptable limits for the items. The fifth section shows a sample of a problem report sheet that can be used to report errors, inadequacies, or problems with the code. The sixth section contains two example problems and lists partial RADTRAD output for each problem.

\subsection{Getting Started}

This section of the users' guide covers the installation of the RADTRAD code on your computer. The code is supplied on a single disk. If you have the required hardware and sufficient disk space, installation of the code will be a simple matter.

\subsubsection{Hardware Requirements}

RADTRAD runs on personal computers using an 80386 or better processor (an IBM-compatible computer) with a math coprocessor. To ensure that the program will run correctly, the computer must have at least the following

- a high-density floppy disk drive (3.5-inch disk) and a hard disk with 3.5 megabytes of available space

- 4 megabytes of RAM

- a mouse

Running RADTRAD requires a minimum of 512 kilobytes of conventional free memory. If RADTRAD will not run, check to see if there is sufficient memory available. An error message will be displayed on the monitor. However, the message may flash by so rapidly that it isn't readable.

RADTRAD is designed to run with a mouse and thus a mouse is required. It facilitates moving through the program and selecting program options. However, RADTRAD can be run on computers without a mouse. If a mouse is not available, RADTRAD may be run by bypassing the graphical interface used for data input. An ASCII text editor can be used to create the user input files that control RADTRAD. RADTRAD may then be run by typing

radtrad / $b$ filename.pmf filename.sdf filename.txt

where filename plus the extensions are the plant model file, the scenario description file, and the output file. The contents and format of the specification files are described in Section 1.4.3. 


\subsection{Installation}

RADTRAD is a DOS program and must be installed from the DOS prompt (consult your manual if you are attempting to install RADTRAD from a DOS shell or menu program). To install, exit Windows and insert the RADTRAD floppy disk into the 3.5-inch disk drive. Then follow the directions for either the default or the custom directory installation below.

\subsubsection{Default Installation}

Change to the disk drive on which you inserted the floppy disk. Example:

If a: is the 3.5-inch floppy disk drive, then type:

a:

at the command prompt and press the ENTER key.

Next, type install at the command prompt, press the enter key, and follow the instructions on the screen. This will install RADTRAD in the C:IRADTRAD directory. To run RADTRAD, change to the RADTRAD directory. Example:

Type:

cd c: Iradtrad

at the command prompt and press the ENTER key.

Next, type radtrad at the command prompt and press the ENTER key.

\subsubsection{Custom Installation}

Change to the disk drive in which you inserted the floppy disk. Example:

If $\mathrm{a}:$ is the 3.5-inch floppy disk drive, then type:

a:

at the command prompt and press the ENTER key.

Next type install $n: \mid$ your_path $\mid$ your_directory and press the ENTER key: where $n$ is your driver letter, your_path is the path name of your directory, and your_directory is the name of the directory where you want to install RADTRAD. This will install RADTRAD in the specified directory. To run RADTRAD, change to the specified directory. Example:

Type:

cd n: lyour_pathlyour_directory 
at the command prompt and press the ENTER key; where $\boldsymbol{n}$ is your drive letter, your_path is the path name of your directory and your_directory is the name of the directory specified above.

Next type radtrad at the command prompt and press the ENTER key.

\subsubsection{Windows Installations}

Installation or execution of Windows is neither supported nor recommended. The user interface sometimes conflicts with Windows $3.1,3.11$, and Windows NT. It is completely incompatible with Windows 95. To install RADTRAD, use one of the installation procedures described above.

\subsection{Running RADTRAD}

RADTRAD runs under the DOS operating system. RADTRAD provides a graphical user interface for data entry, initiating execution of dose calculations, examining results, and printing output.

\subsubsection{The Graphical User Interface (GUI)}

RADTRAD consists of a series of screens that provide information about the code and that accept input required for the calculations. This section presents general information about interacting with the computer to run RADTRAD. Online help is available during data entry. Help is obtained by pressing the F1 key on the keyboard

\subsubsection{Entering Data}

In general, the instructions that follow will assume that you are using a mouse. However, the arrow keys may be used as keyboard substitutes for the mouse actions required to run the program. RADTRAD uses several methods to enter input:

- command buttons

- text boxes

- choice lists

Command buttons initiate some sort of action. To activate a command button, place the mouse cursor on the button and click with the left button on the mouse. Command buttons are labeled. The labels include OK, CANCEL, and EDIT. The OK button causes the code to return to the previous input screen and save the data that have been entered or modified. The CANCEL button causes the program to return to the previous input screen without saving any of the data that you have entered or modified. The EDIT button causes the program to advance to a screen where data can be input for the highlighted entry. 
Text boxes are used for entering either text or numerical information. The information is entered from the keyboard. You make a text box active by clicking on the box. Information about the text box (for example, the units required and the range of appropriate values) appears at the bottom of the screen. When you move from a text box after entering data, RADTRAD will check the entry to ensure that it is proper. If an improper entry is detected, a message at the bottom of the screen will appear and you will typically be returned to the text box. where the error is.

Choice lists are used to obtain information when the number of options is small. A choice list is used to specify options such as deposition mechanisms, transfer path, and compartment type. After you activate the choice list, you can move within the list using the mouse.

There is a menu bar at the top of the data input screens that lists three options - File, Review, and Help. You use the menu bar by clicking on a listed item to can gain access to the item. The File option allows you to save the current input data, enter the DOS shell, or exit RADTRAD. The Review option allows you to examine the current input data for either the plant or scenario file. The Help option directs you to RADTRAD Help. If you have questions about entering specific data items, you may be able to find an answer in Help without leaving the program or without having to use this manual. The ESC key is used to return to the data input screens. This key can also be used during the input process to return to the previous screen. In general, with several touches of the ESC key, the user will return to the initial screen and be able to start over again or correct errors. There is a bar at the bottom of the screen that can provide additional information about the current selection.

\subsubsection{Exiting RADTRAD}

The method used to exit RADTRAD depends on where you are in the program. If you are at the main menu screen, you may exit the program by using the Exit RADTRAD selection. While you are elsewhere, you may exit the program by clicking on Exit in the menu bar under the File option. When you exit RADTRAD in any of these ways, your screen will return to its initial operating mode.

\subsection{Input Description}

All input to the code is through a graphical user interface (GUI). Input to the GUI may consist of selecting an option by using the mouse, entering an option number, or entering data. Data are entered in appropriate units. When the user activates a field to enter input data, the units needed automatically appear at the bottom of the GUI. On the following pages a layout is shown that indicates the relationships between screens and subscreens. A number is assigned to every screen so that during the discussion of the individual screens, the user can relate to the layout. There are currently over 60 screens and subscreens that the user could see during problem set-up. It is recommended that the user examine all subscreens related to the screens being used because defaults are limited. Note that there are a few screens that are not available. In future versions of the code they will be activated. After the screen layout, each screen is shown in a black-andwhite representation of the GUI with a brief description of its use. The actual screens are colored; thus highlighting and the highlighted characters are more obvious. 
The underlying scheme of the code is that the user must first specify a nuclear power plant model and then specify a scenario description. The plant model consists of time-independent information and describes the physical structure of the plant. The user will specify the compartments, the transfer pathways that connect the compartments, and information pertaining to them (e.g., compartment volumes, the existence of filters, and suppression pools). In addition, the user will select a BWR or PWR inventory and source term. The inventory is divided into several chemical groups based on the elements in these groups with similar transport properties. The scenario description contains time-dependent information about the state of the plant and allows the user to run several different scenarios with the same plant model. The user may limit the fission products released as part of the source term. The user will be asked to select the fraction of the iodine chemical group that is elemental, organic, and aerosol. For each of the transfer pathways, the user will specify the information necessary to calculate transport and retention through that pathway (e.g., the user will specify filter efficiencies).

The user may also specify the locations of interest for calculating worker dose. Integrated air concentrations at the various locations will be determined by the code, and the user will input the exposure time, breathing rate, and other specifics needed to calculate dose equivalents.

\subsubsection{Screen Numbering System}

The input screens are numbered in a hierarchical manner. The more zeros there are in the screen number, the higher the decision level of the screen. Thus the main menu screen is 0000 , whereas a table input deposition screen could be $2352 \mathrm{~T}$. The addition of a letter to the screen number denotes tabular input when it is the letter $\mathrm{T}$ and model input if it is a letter $\mathrm{M}$. All models have an optional table input to allow the user to compare the results from using the model with results using a more familiar tabular decontamination factor.

To move from one screen to another, the user must follow the lines on the screen layout. Therefore to go from the natural deposition aerosol removal table (SN 2341T) to the spray aerosol removal table (SN 2311T), the user should go from SN 2341T (choose OK), to SN 2341 (choose OK), 2340 (choose OK), SN 2300A (choose Sprays), SN 2310 (choose Aerosol. Removal), SN 2311 (choose User-Specified Removal Coefficients), and thus enter SN 2311T. This may at first be cumbersome, but because it follows the screen layout, the user soon becomes adept. 


\section{RADTRAD Screen Layout}

Main Menu (0000)

Plant Model (1000)

- Load Plant Model (1100)

- Nuclide Inventory (1200)

- Select Compartment (1300)

L Compartment Description (1310)

- Select Pathway (1400)

L Transfer Pathway Description (1410)

L Select Compartment (1411)

- Review Plant Model (1500)

L Save Plant Model (1600)

\section{Scenario Description (2000)}

- Load Scenario Description (2100)

- Source Term Parameters (2200)

L Select Source Term Compartment (2210)

Release Fractions

- Select Compartment (2300)

$L$ Compartment Features (2300A)

L Sprays

- Aeroso

Removal (2311)

Table (2311T)

Model (2311M)

- Elemental I

Removal

Table (2312T)

Lrganic I

Removal

Table (2313T) and Timing (2220) Products (2230)

Recirculating

Filter

Pool (2350)

Table (2330T)
Deposition (2340)

\begin{tabular}{|l} 
Aerosol \\
Removal (2341) \\
Table (234lT) \\
Model (2341M) \\
Model (2341M2) \\
\\
Elemental I \\
$\quad$ Removal \\
$\quad$ Table (2342T) \\
\end{tabular}

- Aerosol

Removal (2352)

Table (2352T)

Model (2352M)

Elemental I

Removal

Table (2353T)

- Organic I

Removal

Table (2354T)

L Initial Water

Volume (2351) 


\section{RADTRAD Screen Layout Continued}

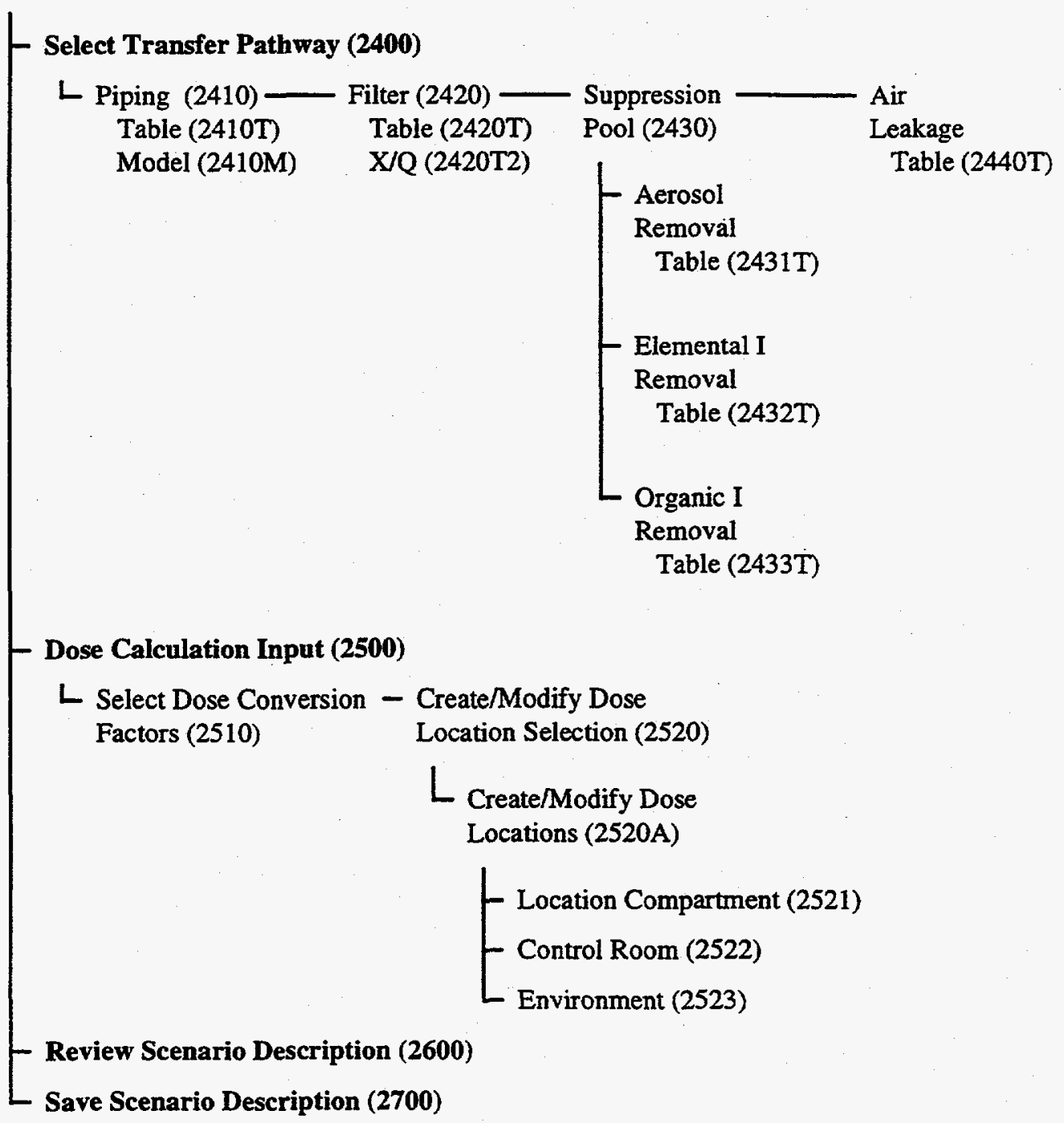

Calculate Results (3000)

Examine Results (4000)

$\llcorner$ Examine Output File (4100)

Exit RADTRAD (5000) 


\subsubsection{Detailed Screen Input}

This section contains all of the screens that are used for the RADTRAD user-interface. The order of the screens presented follows the order that a user would normally encounter them when inputting a new problem. The following conventions will be used when describing the screens:

Bold with Initial Caps

Italics with Initial Caps

SMALL CAPS represents the name of a screen

represents the name of a data field or non-numeric user-

specified data

represents a key on the keyboard. e.g., ESC

The convention used to number the screens is explained in Section 1.4.1.

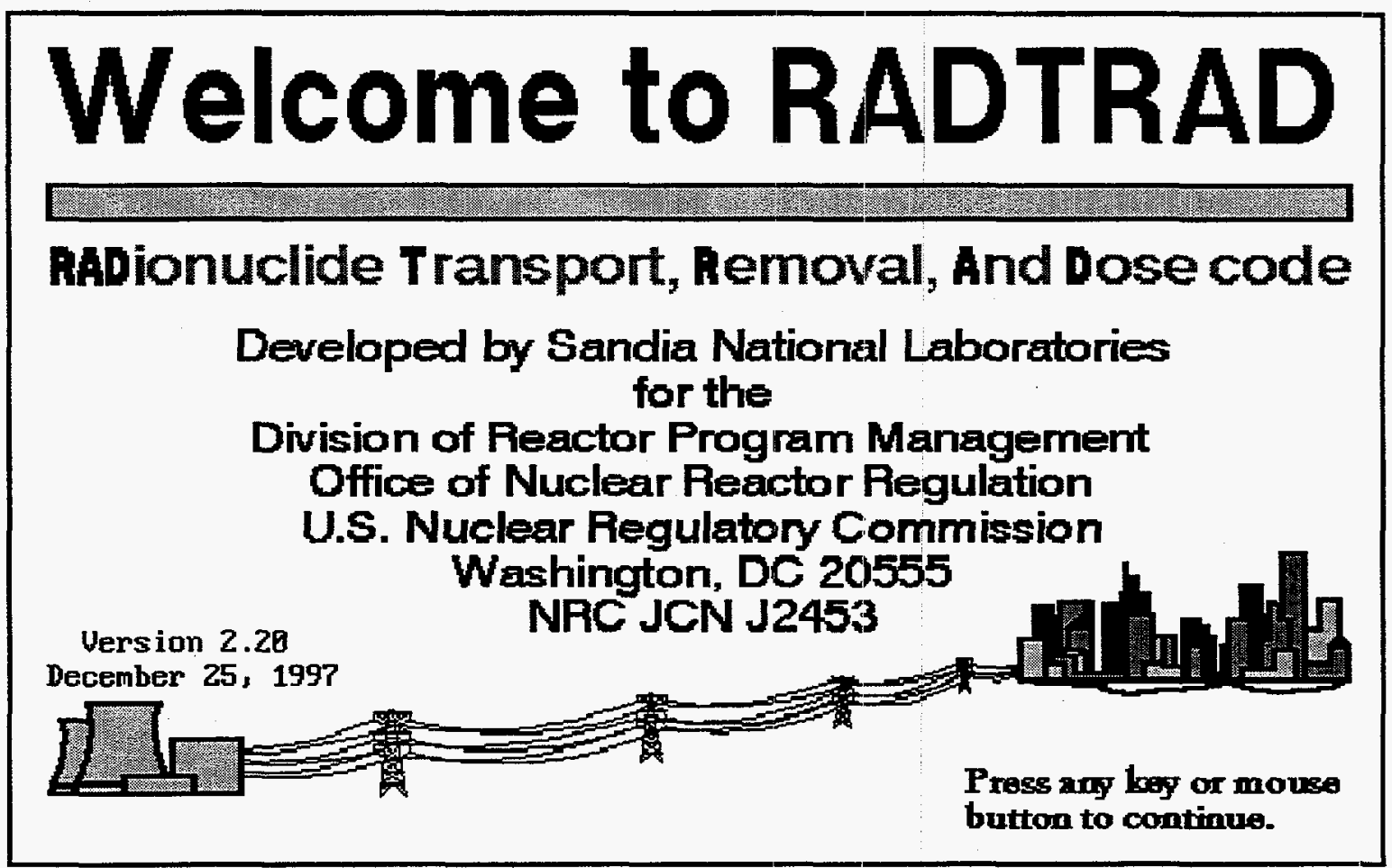

This is the title screen and has no number associated with it. It indicates that a successful loading and initiation of the RADTRAD code has occurred. The user should note the version number and date in the lower left corner. This information allows others, such as the developers, to determine which code version the user has. Press any key or mouse button to continue. 


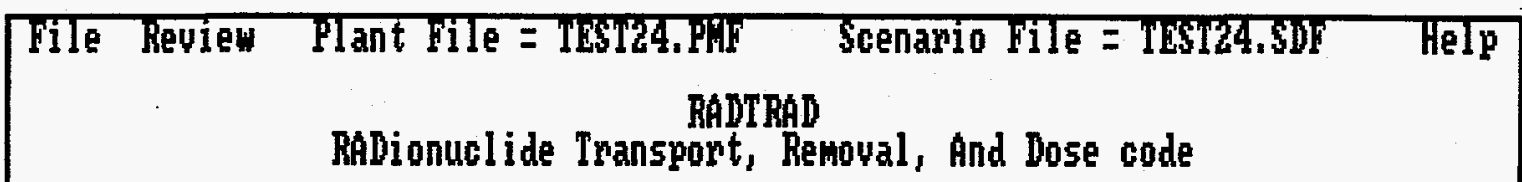

(1) Plant Model

(2) Scenario Description

(3) Calculate Results

(4) Examine Resul ts

Version 2.20

December 25, 1997

(5) Exit RADTRáD

The RADTRAD, RADionuclide Transport, Removal, And Dose code main menu is screen number 0000 . This screen allows the user to develop the input and run RADTRAD. The user should go through the listed options in order. Typically the user will first model the plant, then model the scenario, and finally calculate results. A Scenario Description cannot be defined without a Plant Model to which the scenario can be referenced. Also, a Scenario Description and Plant Model must be available for a calculation to be performed. The user may examine results from previous calculations without running the code by choosing Examine Results and specifying the output file from that calculation. The user may Exit RADTRAD from the main menu by choosing this option at any time. 


File keview Plant File $=$ IEST24.PFF Scenario File $=$ IEST24.SDF Help
Plant Model

(1) Load Plant Model

(2) Nuclide Inventory

(3) Compartment Description
(4) Transfer Pathways

(5) Review Plant Model

(6) Save Plant Model

\section{(7) Return to Main Menu}

The Plant Model menu is screen number. This screen allows the user to describe the timeindependent plant input. The user may select a previously defined plant model for modification or create a new plant model. In most cases a previously defined model will exist; the user then clicks on the first option to retrieve the file into an active status. The user selects the Nuclide Inventory, Compartment Description, or Transfer Pathways options in order to specify or modify information about the plant model in those areas. The Review Plant Model option allows the user to examine the plant model input as it is currently defined. The Save Plant Model option allows the user to save the current plant model in a file of their choice. The name of the current plant file is shown on top of the screen. Selecting Return to Main Menu will return the user to screen number 0000 . 


File Review Plant File = TEST24.PHI Scenario File = TEST24.SDF Help
Plant Model
Load Plant Model

\section{Enter Plant Hodel path and file name}

\section{ACCEPT \EST24, PNF}

ok

CANCEL

The file nawe can be up to 40 characters long,

The Load Plant Model is screen number 1100. This screen allows the user to specify the path and file name of the input data that describe the plant model that the user wishes to modify. Clicking on OK or CANCEL will return the user to the Plant Model menu (screen number 1000). 


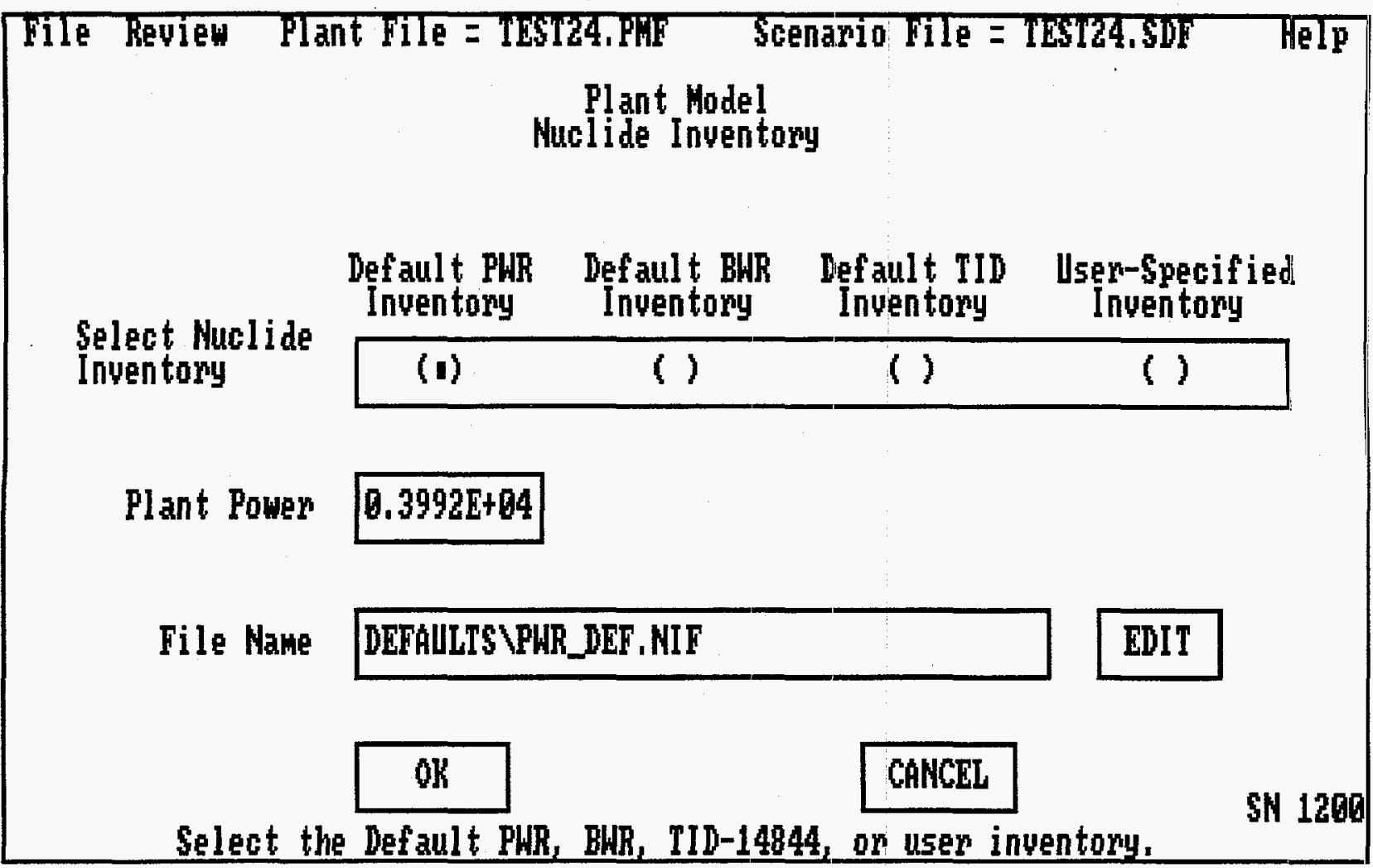

The Nuclide Inventory is screen 1200. This screen allows the user to select from the available options for nuclide inventories. It is invoked when the user selects Nuclide Inventory on the Plant Model menu. Currently the user may select a typical BWR, PWR or the NRC's generic Technical Information Document (TID) inventory as detailed in Section 1.4.3.3. In addition, inventory files for only ${ }^{131}$ I have been supplied in the DEFAULTS subdirectory. Alternatively, the user may supply a file that contains the user-specified inventory. The user must enter the nominal thermal Plant Power (MWt) in the appropriate location. The ratio of the input power to inventory power is used to determine the inventory used for the plant analysis. Only one of the options can be selected (i.e., a PWR and BWR inventory cannot be specified at the same time). If the user specifies a file containing an inventory, the file format will be checked for consistency with the description in Section 1.4.3.3 and an error will result if the format is incorrect. Clicking on OK or CANCEL will return the user to the Plant Model menu (screen number 1000). 


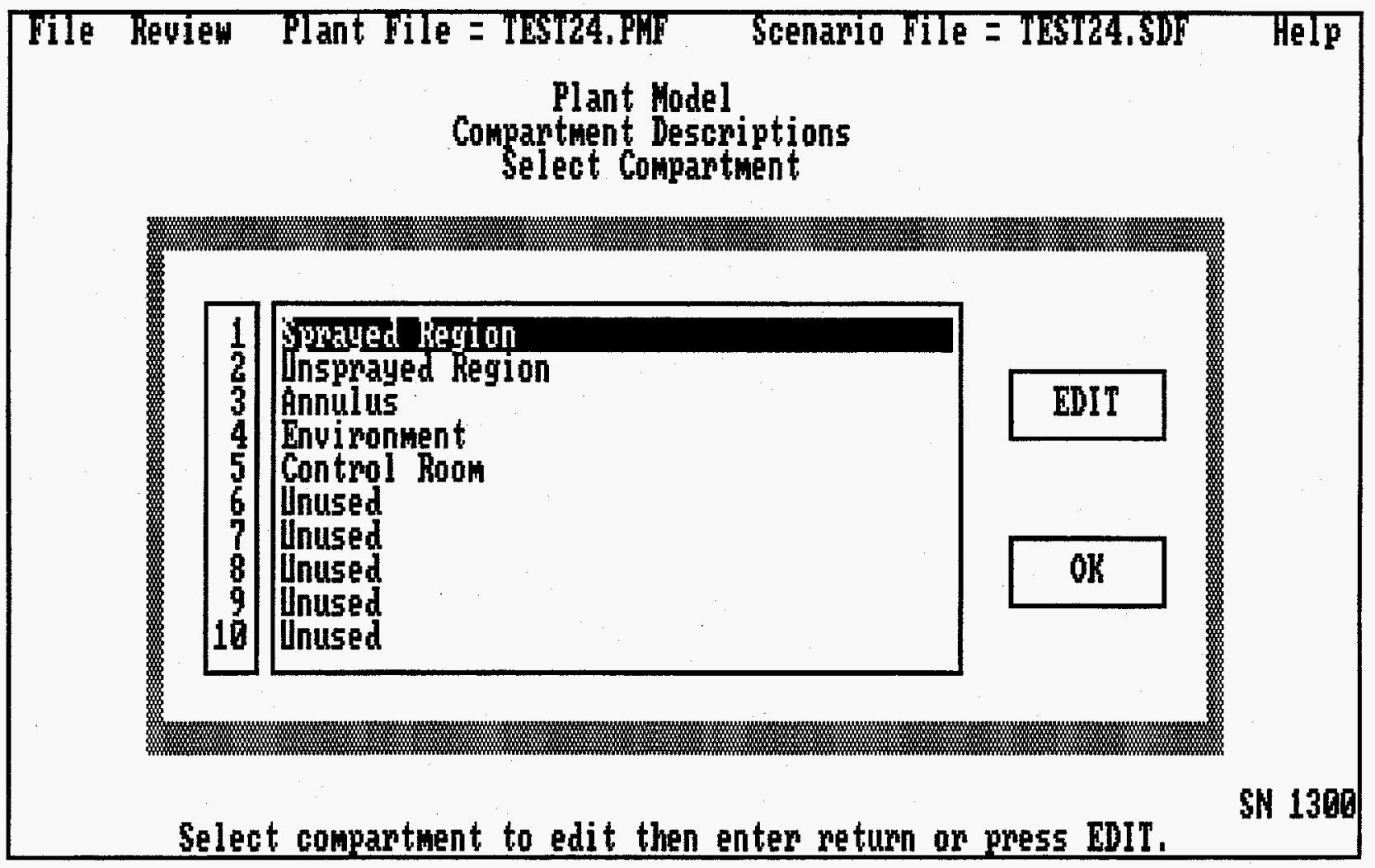

The Select Compartment menu is screen number 1300. It is invoked when the user selects Compartment Description from the Plant Model menu. To add a compartment, the user highlights the first available Unused entry and clicks on EDIT. This will bring up a subscreen that allows data to be input. Upon returning from the subscreen, the new compartment name entered will have replaced the Unused entry and the next Unused entry will be highlighted. This continues until the user has defined all the compartments. A maximum of 10 compartments are allowed in a plant model. The more compartments that there are, the slower the code executes. The speed of execution increases more than linearly for an increase in compartments, but less than the square. Clicking on OK will return the user to the Plant Model menu (screen number 1000). 


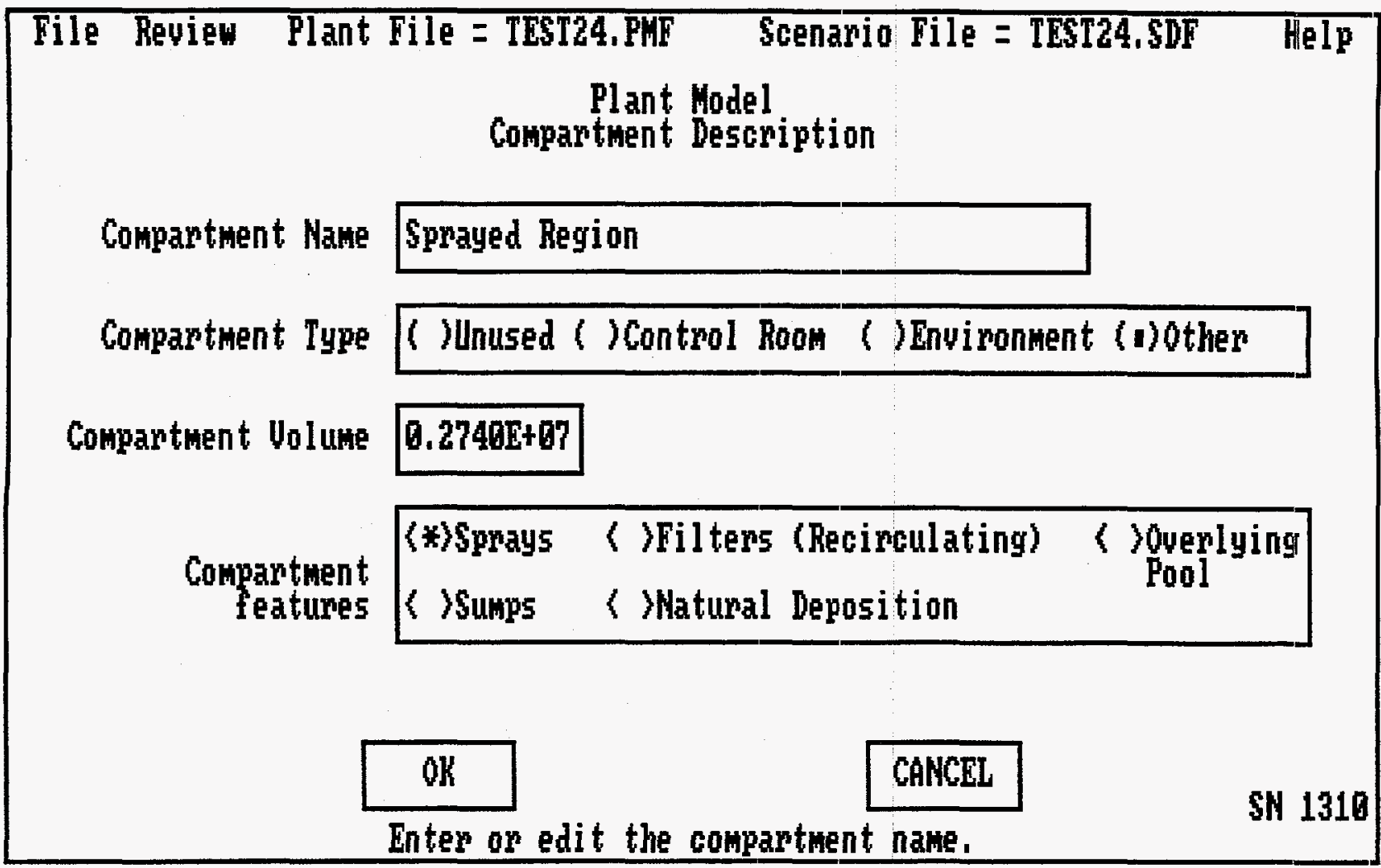

The Compartment Description is screen number 1310. This screen allows the user to enter specific information for a compartment. The name of the compartment is input at the top of the page where the example shows Sprayed Region. This name will be used throughout the input and output as the identifier for this compartment. The user may use up to 40 characters for this identifier. The Compartment Volume is in units of cubic feet and must be input in scientific notation. If the compartment represents a control roorn or the environment, the appropriate box must be checked. Other represents volumes where doses will not be calculated, such as containments, auxiliary buildings, and turbine rooms. Only one control room is allowed in a calculation; hence if a user needs the dose in another room, there must be two separate calculations. If the compartment represents the environment, both the compartment volume and compartment features are removed from the screen when the user clicks on the Environment button, indicating that the user does not use these inputs. Unused is checked only when the user wants to eliminate the volume from the calculation but retain its data in the input deck. The features available in the compartment are also listed. The user must select the appropriate box(es) on this screen to activate the removal mechanism screens (screen numbers 2300-2354T) during the scenario model input. The user should choose only features that are appropriate for the volume being described. For example, an Overlying Pool may exist in a containment but not in a control room because it is associated with postvessel failure scenarios. Clicking on $\mathbf{O K}$ or on CANCEL will return the user to the Select Compartment menu (screen number 1300). 


\begin{tabular}{|c|c|c|}
\hline File Review Plant File = IESI24,PNI Scenario File = TEST24, SDT Help \\
Plant Model \\
Transfer Pathways \\
Select Pathway
\end{tabular}

The Select Pathway menu is screen number 1400. This screen allows the user to identify the flow paths that connect compartments. It is invoked when the user selects Transfer Pathways on the Plant Model screen. To add a pathway, the user must specify information about each transfer pathway by selecting the first available Unused entry, which is then highlighted, and click on EDIT. This brings up a subscreen. The user continues entering transfer paths in this way until the problem is defined. The number of paths does not dramatically affect the execution time. Clicking on OK or on CANCEL will return the user to the Plant Model menu (screen number 1000). 


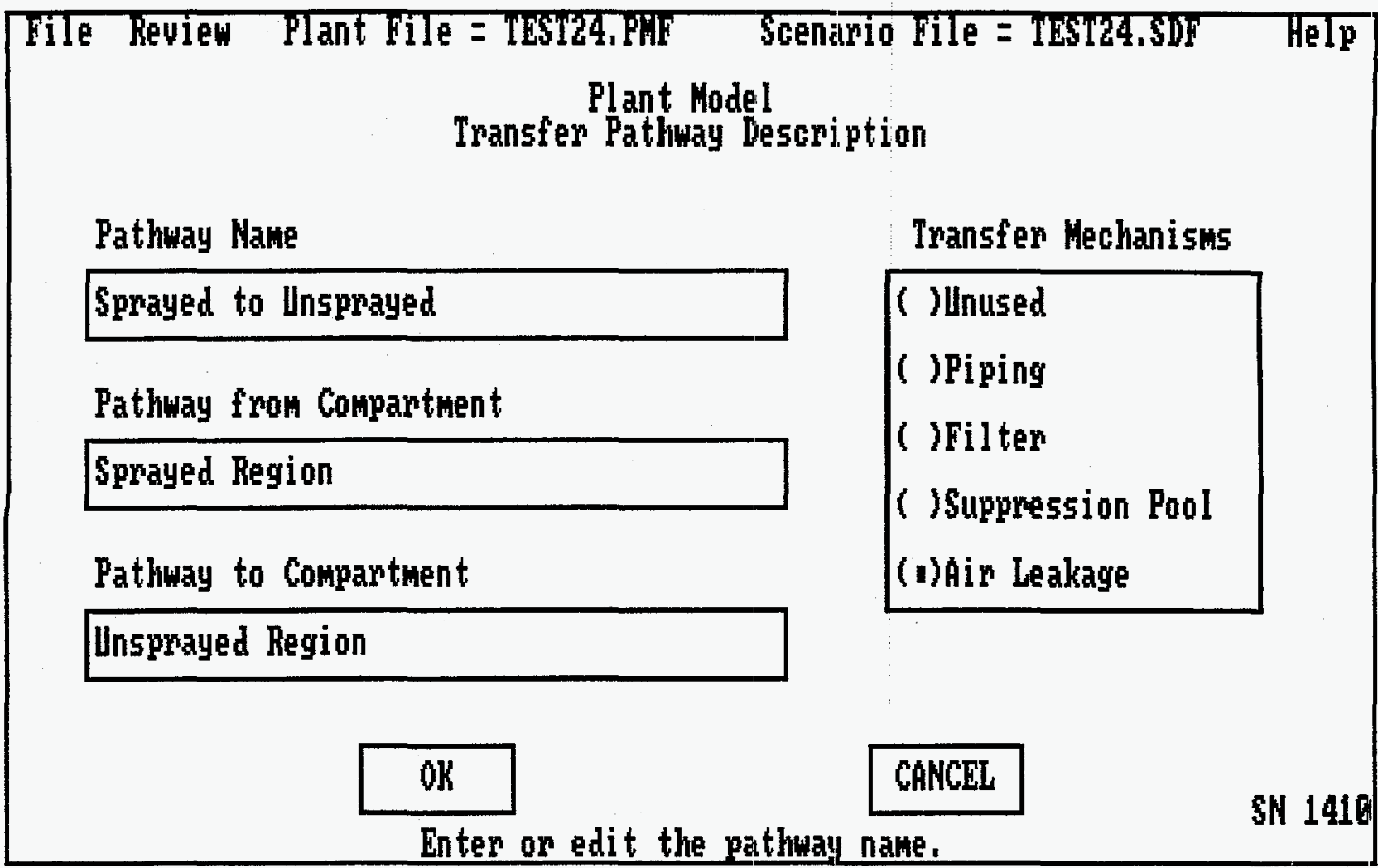

The Transfer Pathway Description is screen 1410. This screen allows the user to enter specific information for the transfer pathway selected in screen 1400. The user may have multiple paths between compartments; for example, filtered and unfiltered air from the environment to the control room. The name of the transfer pathway is input at the top of the screen where the example shows Sprayed to Unsprayed. A double click on the Pathway from Compartment will activate a list of compartment names on screen 1411. The user highlights one and returns, thus identifying the "from" compartment. This method ensures correct linkage with earlier data. The Pathway to Compartment is then double clicked and entered. The user must click on an appropriate transfer mechanism for the pathway to be able to activate the transfer mechanism screens (screen numbers 2400-2440T) during the scenario description (only one is allowed for each pathway). The choice of a pathway Transfer Mechanism is a function of the data available to the user. For example, the leakage option allows for no filtration, but a variation in flow versus time, while filters allow for variation in filtration versus time at constant flow. In addition, the filter option can access the environment as its Pathway from Compartment. Both the Piping and Suppression Pool options allow for a variation of both filtration and flow versus time. In addition, the Piping option has a model to calculate deposition as a function of geometry, flow, and the pressure and temperature. Unused allows the user to remove a transfer path from the calculation but retain it in the input deck. It is recommended that the user be familiar with the acceptance tests detailed in Section 3.3 to specify transfer path mechanisms appropriate for the problem. For example, Air Leakage has been used for containment leaks and Filters for unfiltered in-flow into the control room. Clicking on OK or on CANCEL will return the user to the Select Pathway screen (screen number 1400). 


File Review Plant File = TEST24, PHF Scenanio File = IESL24, SDF Help
Plant Model
Transfer Pathway Compantment
Select Compartment

The Select Compartment menu is screen number 1411. This screen allows the user to select the compartments at the beginning and end of a transfer pathway. The user highlights the desired compartment and clicks $\mathbf{O K}$ to return to the transfer pathway screen and correctly input the connection volume. Clicking on OK or CANCEL will return the user to the Transfer Pathway Description screen (screen number 1410). 


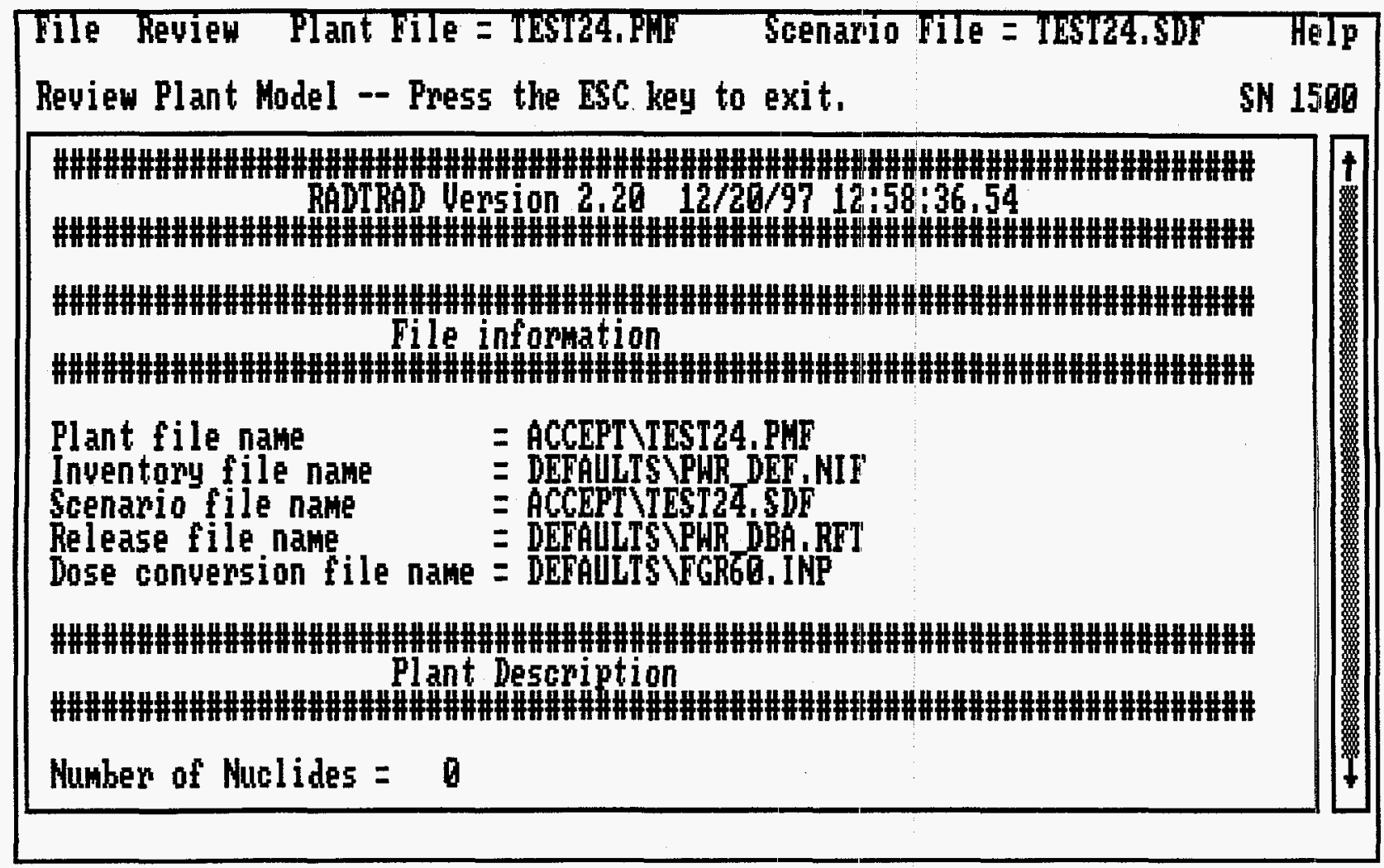

The Review Plant Model is screen number 1500. This screen allows the user to review the input used to define the current plant model by using the scroll bar on the right-hand side of the screen. Return to the Plant Model menu (screen number 1000) by using the escape key (ESC) on the keyboard. 


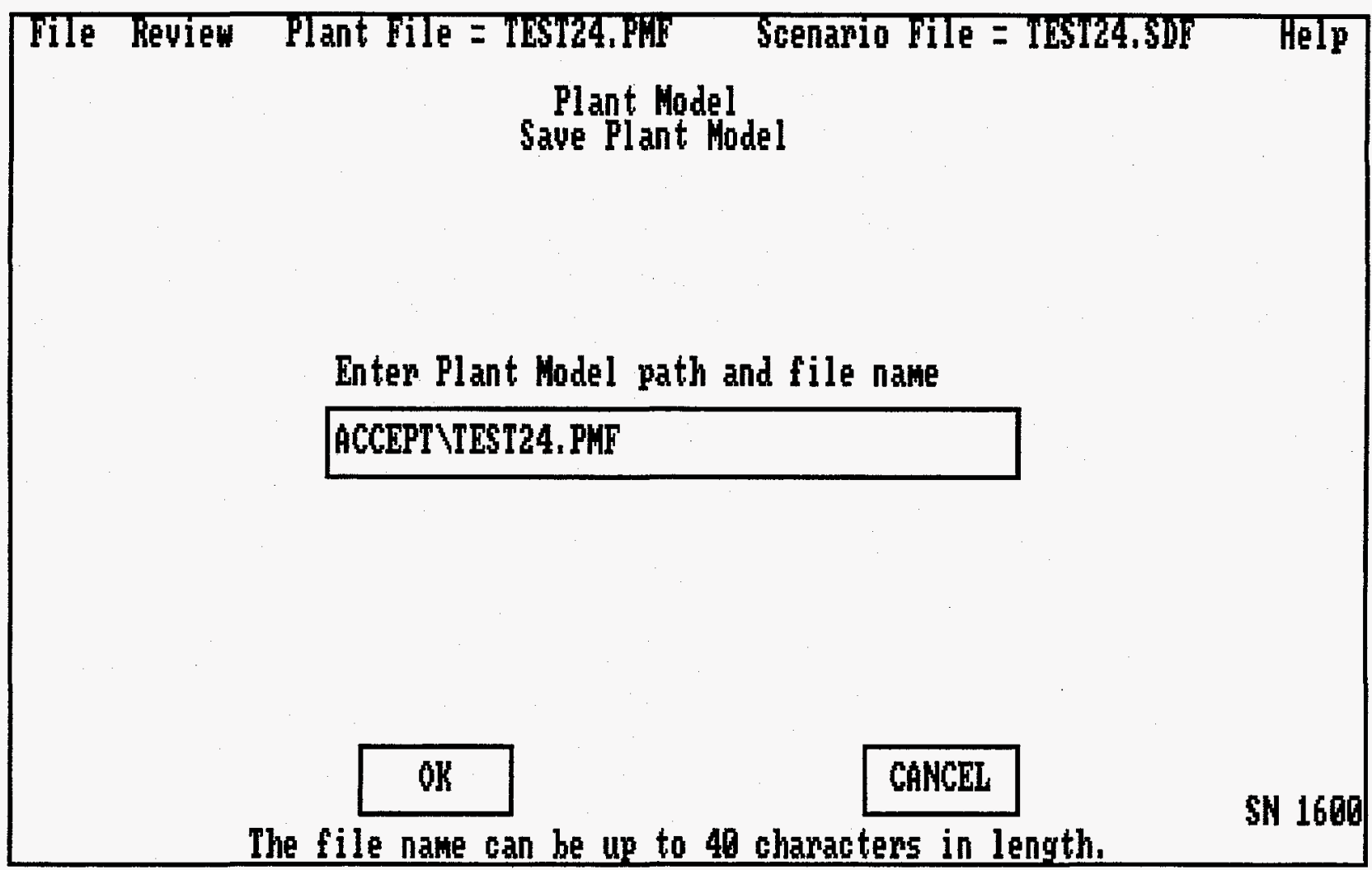

The Save Plant Model is screen number 1600. This screen allows the user to specify a file name for saving the plant model. It is invoked when the user selects Save Plant Model on screen 1000. Note the convention of XXX.PMF where XXX is the user description and PMF stands for plant model file. Typical files names may include subdirectories such as ACCEPT TEST10.PMF or simply PERRYMAN.PMF. Clicking on OK or CANCEL will return the user to the Plant Model menu (screen number 1000). The user should be aware that the PMF files available with the code are read only and that trying to save changes using the original file name will result in a system error. 


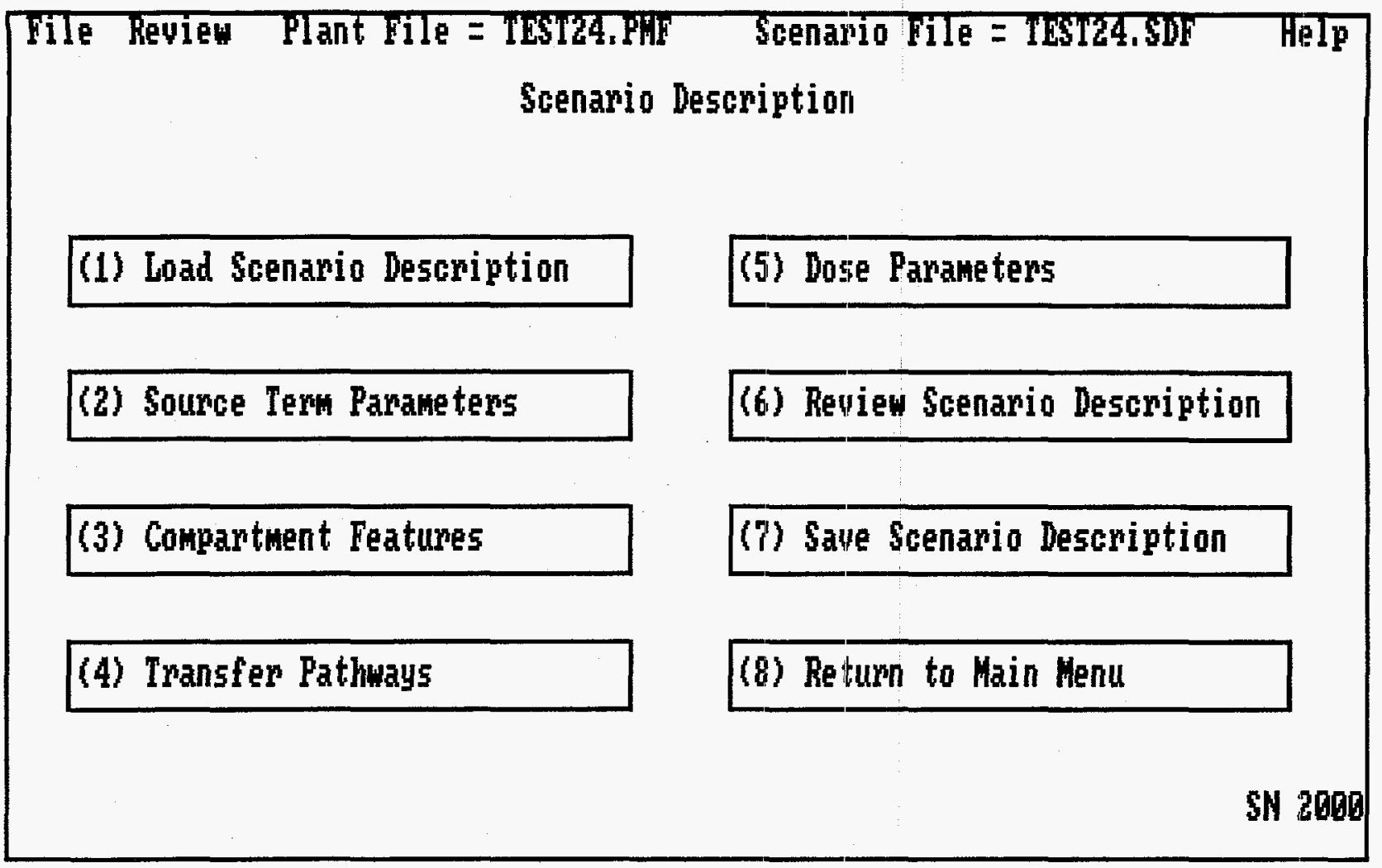

The Scenario Description menu is screen number 2000. It allows the user to input the timedependent aspects of the problem. It is invoked when the user selects Scenario Description on screen 1000. The user must have a plant model file (XXX.PMF) active before starting this file. The user may select a previously defined scenario description or may create a new one. From this screen the user may choose to develop time-dependent information concerning the Source Term, the Compartments, and the Transfer Pathways. View Scenario Description allows the user to examine the scenario input as it is currently defined. The user may view the status of the plant at any selected time after accident initiation. The Save Scenario Description option allows the user to save the current scenario in a file of his or her choice. The name of the current file is shown on the top of the screen. Selecting Return to Main Menu will return the user to screen number 0000 . 


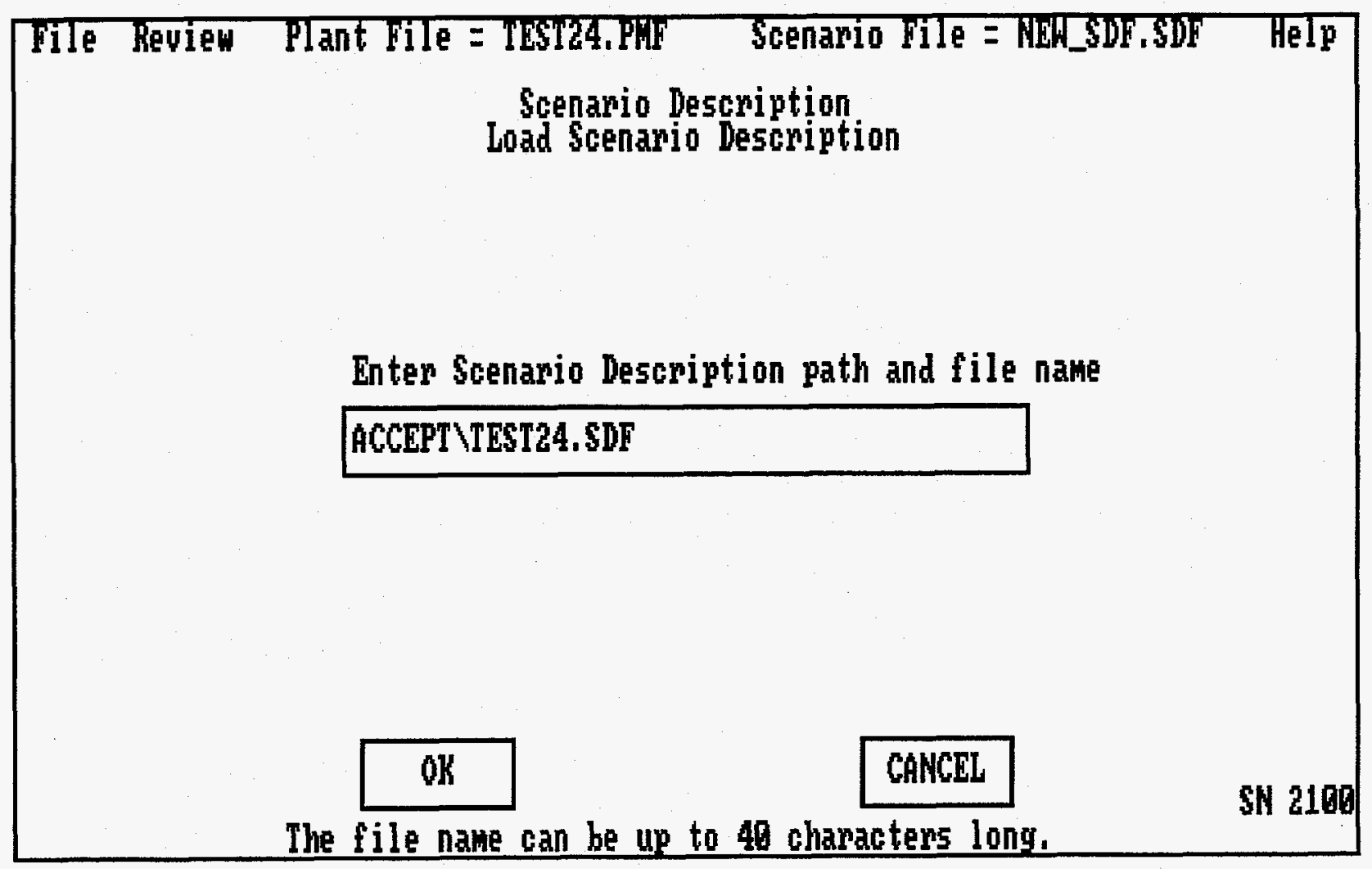

The Load Scenario Description is screen number 2100. This screen allows the user to specify the path and file name of the input data that contain the scenario description the user wishes to modify. Clicking on OK or CANCEL will return the user to the Scenario Description menu (screen number 2000). 


\section{Tile keview Plant File = TEST24,PHF Sgenanio File = TEST24,SDF help \\ Scenario Description \\ Source Term Parameters}

(1) Source Term Compartment

(2) Release Fractions and Timing

(3) Decay \& Daughter Products

(4) Iodine Chenical Form

(5) Return to Scenario Description

The Source Term Parameters menu is screen number 2200. This screen allows the user to enter subscreens and provide information on the source term. It is invoked when the user selects Source Term on screen 1000. The user must go through each subscreen if this is a new scenario, because defaults are limited. The user inputs the Compartment to which radioactive materials are released, the Release Fractions and Timing, the possibility of Decay \& Daughter Products and the Chemical Form of the released iodine. Selecting Return to Scenario Description will return the user to screen number 2000. 


\section{File Review Plant File = TEST4.PHF Scenario File = lWST24.SDP Help \\ Sounce Term Pamaneters \\ Select Source Tern compartment}

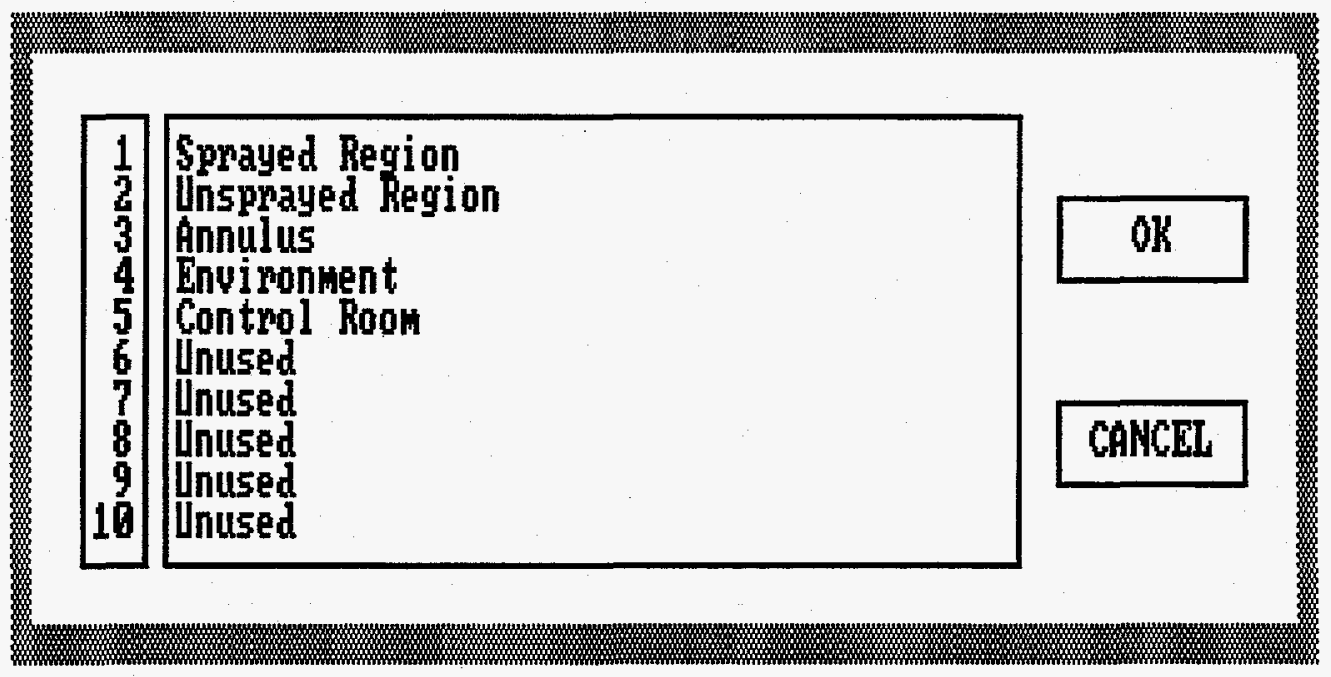

Click on name to edit name - double click to select.

The Select Source Term Compartment menu is screen number 2210. This screen allows the user to specify the compartment in which the source term is released by selecting a compartment from the list in the plant model. Clicking on OK or CANCEL will return the user to the Source Term Parameters menu (screen number 2200). 


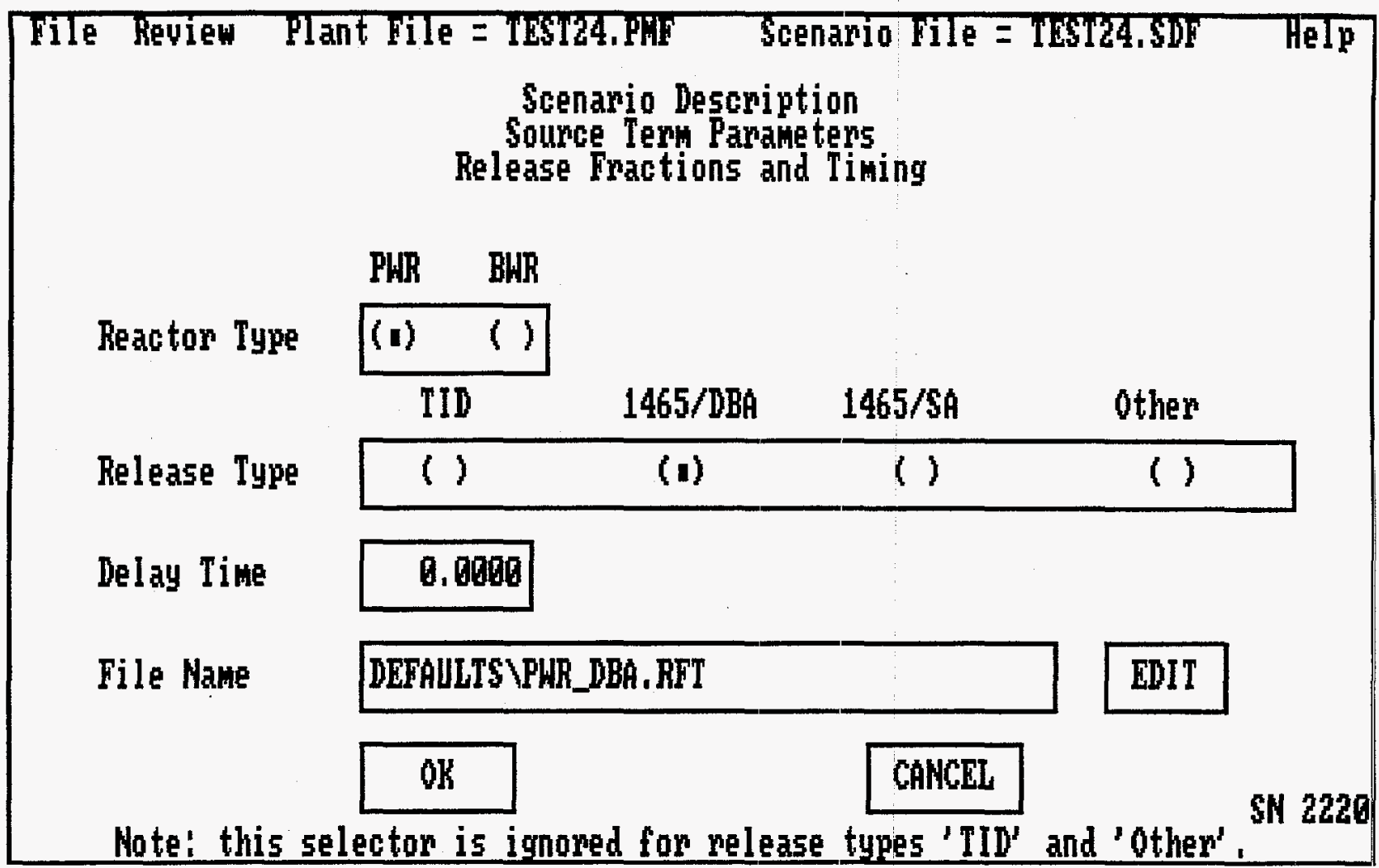

The Release Fractions and Timing screen is screen number 2220. This screen allows the user to select PWR, BWR, TID, or default values for the fractions, timing, and amount of nonradioactive aerosol released. This screen is invoked when the user selects Release Fractions and Timings on screen 2200. The selection for release fractions should be consistent with what was chosen on screen number 1200 for the plant nuclide inventory. The code has files for the TID, design basis, and severe accident release patterns which are described in Section 1.4.3.1. These should be used for most scenarios. If the user chooses to modify the release fractions or timings, this is the screen where it can be done. The user may also modify the time between shutdown and the start of release on this screen by using the Delay Time field. This allows the inventory to decay before its release. Clicking on OK or CANCEL will return the user to the Source Term Parameters menu (screen number 2200). 


\section{File Review Plant File = TEST24.PHI Scenanio file = IUST24,SDr Help \\ Scenario Description \\ Source Tern Paranetens \\ Decay Daughter Products}

\begin{tabular}{|ccc|}
\hline No Decay & Decay & Decay \\
No Daughter Products & No Daughter Products & Daughter Products \\
(1) & $($ ) & $($ ) \\
\hline
\end{tabular}

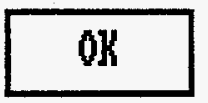

CANCEL

Select the desired option.

The Decay \& Daughter Products menu is screen number 2230. It is invoked by selecting Decay \& Daughter Products on screen number 2200. This screen allows the user to specify whether fission product decay will be included and whether daughter products will also be included in the calculation. Daughter in-growth is not possible without decay. Section 2.4.3 discusses the theory used in these models. Clicking on OK or CANCEL will return the user to the Source Term Parameters menu (screen number 2200). 


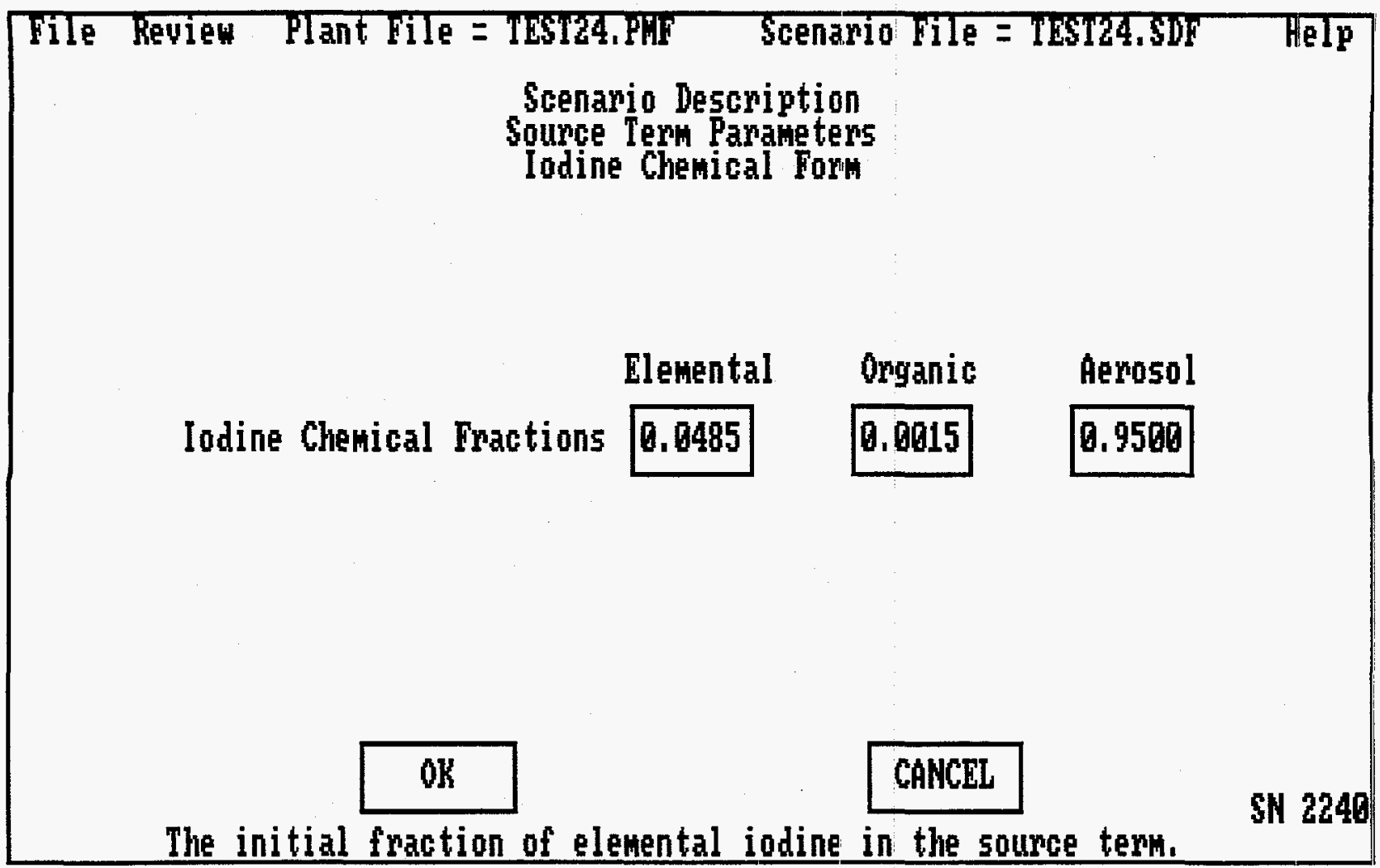

The Iodine Chemical Form menu is screen number 2240. This screen allows the user to specify the chemical form of the iodine as it is released into the compartment chosen in screen number 2210. This screen is invoked when the user specifies lodine Chemical Form on screen 2200. The fractional amounts of each form must sum to 1.0 or an error message will result. The TID release assumes an iodine form that is $\sim 91 \%$ elemental while NUREG/1465 assumes that the iodine is $95 \%$ aerosol. The user should be consistent. Removal processes affect the chemical form differently. Typically, aerosols are the easiest to remove and organics the most difficult. This screen has been used for revolatilization studies in which the organic release is higher at the end than it is at the beginning. For that case, acceptance test 13 , the end-state fractions, were used here and an organic iodine deposition rate versus time was used to get the proper release pattern. Clicking on OK or CANCEL will return the user to the Source Term Parameters menu (screen number 2200). 


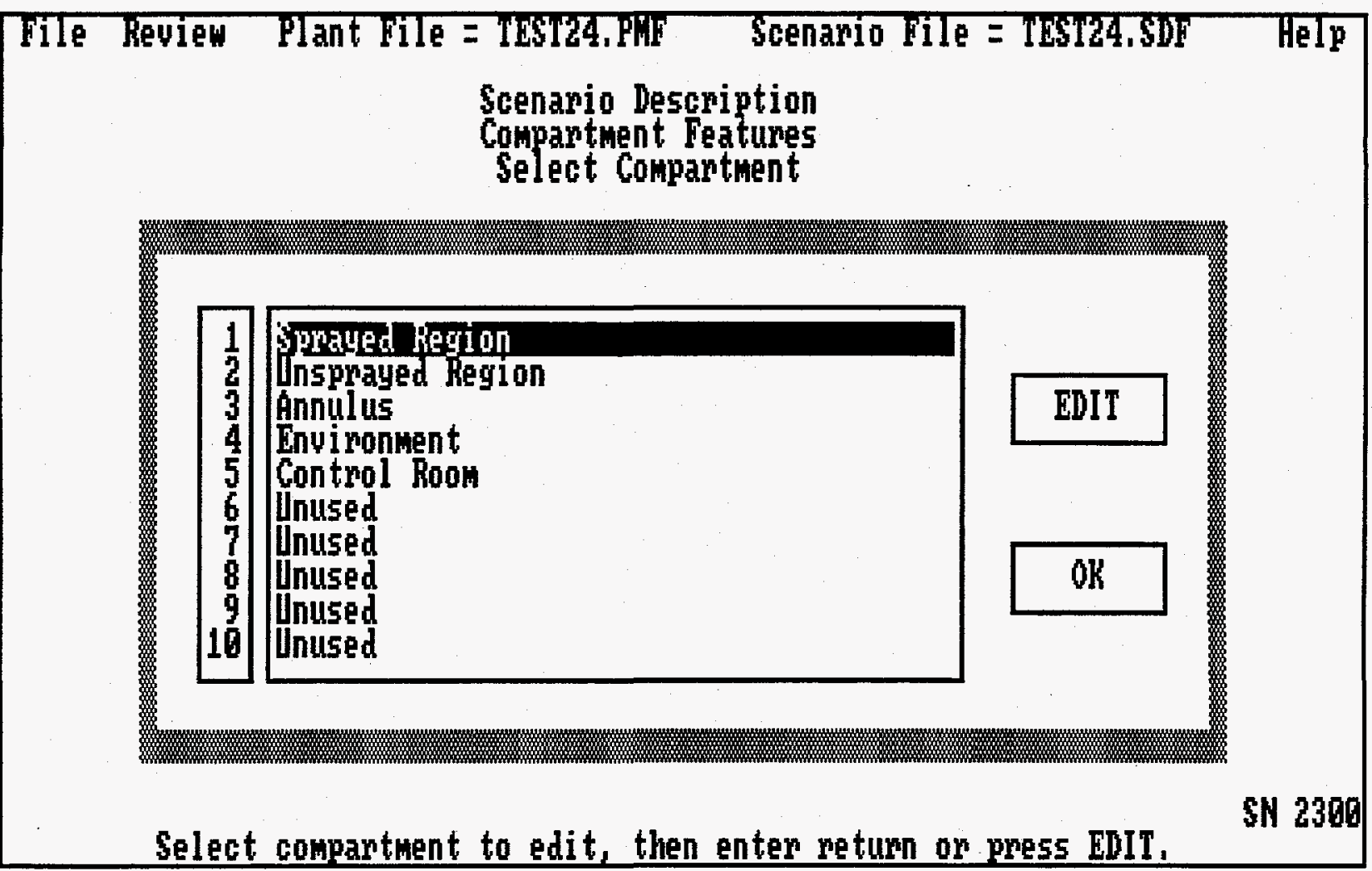

The Select Compartment menu of the scenario file is screen number 2300. It is invoked when the user selects Compartments on the Scenario Description screen. The compartment names are taken from the current plant file. The user must specify information about each compartment by highlighting that compartment and clicking on the EDIT button. If the user clicks on OK, the user is returned to the Scenario Description menu (screen number 2000). 


\begin{tabular}{|c|c|c|}
\hline $\begin{array}{r}\text { File Review Plant File = } \\
\text { Scenario }\end{array}$ & $\begin{array}{l}\text { ST24.PNI Scenario File = } \\
\text { cription: Compartment Featur } \\
\text { Sprayed Region }\end{array}$ & $4.50 \mathrm{r}$ \\
\hline Selected Removal Mechanisms & Edit Renoval Mechanisms & \multirow{2}{*}{$\begin{array}{l}\text { Compantinent } \\
\text { Detailed } \\
\text { Output } \\
\text { Requested }\end{array}$} \\
\hline Sprays $\quad\langle *\rangle$ & (1) Sprays & \\
\hline Sumps & (2) Sunps & $\operatorname{Yes}() \mathrm{No}(11)$ \\
\hline Filters & (3) Fil tens (recirculating) & \\
\hline Matural Deposition \langle\rangle & (4) Natural Deposition & \\
\hline Overlying Pool & (5) Overlying Pool & \\
\hline$\overline{\mathrm{OK}}$ & CANCEL & \\
\hline
\end{tabular}

The Compartment Features menu is screen number 2300A. This screen allows the user to input data about a compartment's features and to enhance the output concerning the compartment. The compartment name is listed at the top of the screen. The user can specify information about the individual compartment features by selecting them only if there is an $\mathrm{X}$ in the box to the left of the option. The $\mathrm{X}$ will appear if the option had been chosen in the plant: model input of screen 1310. If the user wants to add a feature, he or she must first go back to screen 1310 , mark it there, return to this screen, and click on the feature button. If the user desires detailed output regarding the results from the features in this compartment, the box under Compartment Detailed Output Requested must be checked. Detailed output includes the isotopic mass distributions for this volume as well as the group deposition on all surfaces and in all pathways connected to this volume. An example of this output is given in Section 1.5. Clicking on OK will return the user to the Select Compartment screen (screen number 2300). 


\begin{tabular}{|c|c|c|c|}
\hline Tile & $\begin{array}{r}\text { Review Plant File: } \\
\text { C }\end{array}$ & $\begin{array}{l}\text { I24. Pll Scenario File = TEST24.SDF } \\
\text { enario Description } \\
\text { twent Features: Sprays }\end{array}$ & Help \\
\hline & & Sprayed Region & \\
\hline & Selected Models & Edit Model Parametens & \\
\hline & Aerosol $\langle *\rangle$ & (1) Aerosol Renoval & \\
\hline & Elemental Iodine $\langle *\rangle$ & (2) Elemental Iodine Renoval & \\
\hline & Onganic Iodine $\langle *\rangle$ & (3) Onganic Iodine Removal & \\
\hline & & (4) Return to Compartwent Features Henu & \\
\hline & & & SH 2310 \\
\hline
\end{tabular}

The Sprays menu is screen number 2310. This screen allows the user to include information for spray removal of fission products if the spray feature was chosen in screen 1310 and clicked on in screen 2300A. The user may specify information on Aerosol Removal, Elemental lodine Removal, and/or Organic Iodine Removal by selecting these options. All fission products removed by sprays are placed in the sump of the compartment indicated at the top of the screen. Selecting Return to Compartment Features Menu will return the user to screen number 2300A. 


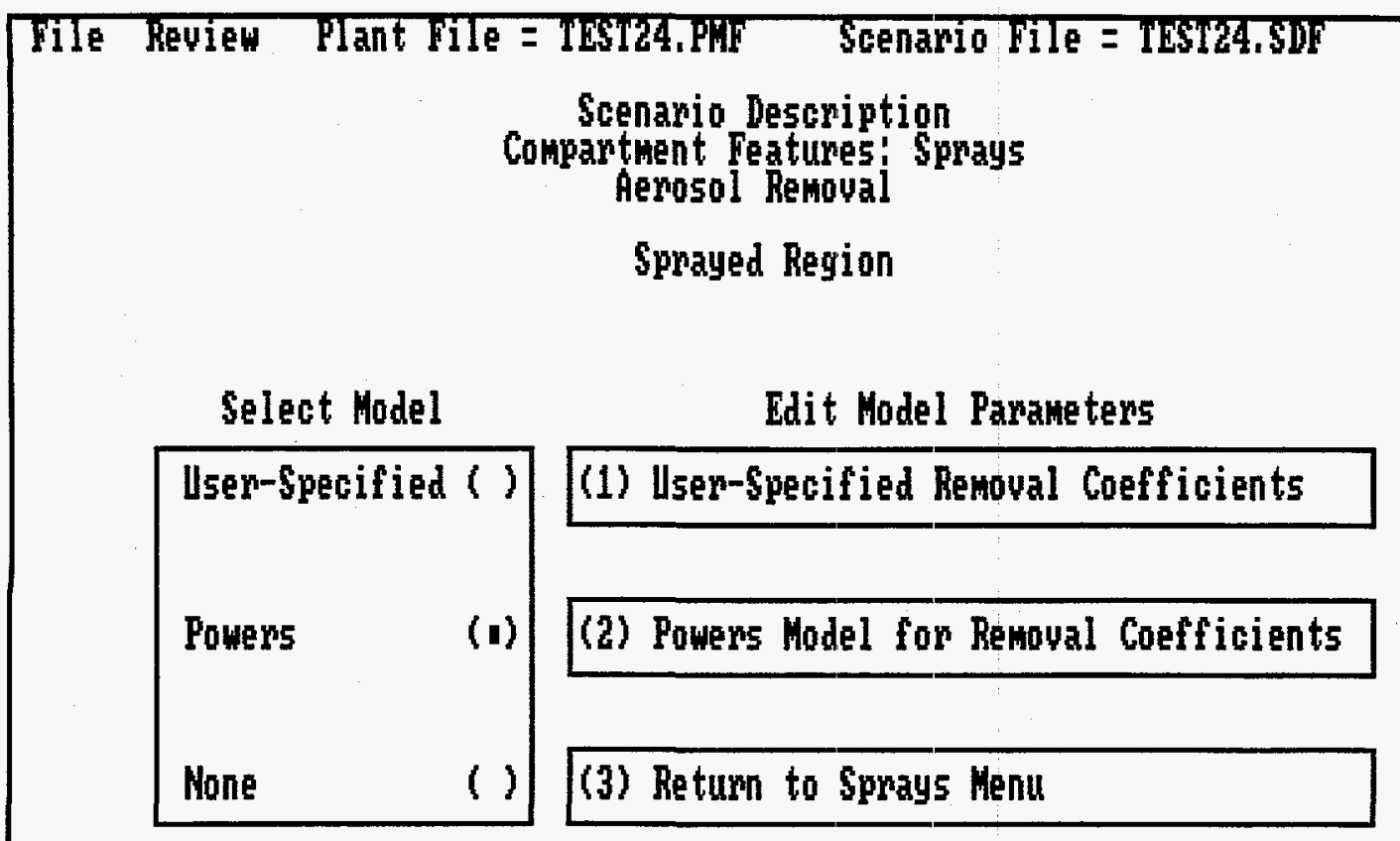

SN 2311

The Aerosol Removal by Sprays menu is screen number 2311. This screen allows the user to choose either the User-Specified Removal Coefficients as a function of time or the Powers Model. This screen is entered by selecting Aerosol Removal on screen 2310. The user may specify only one of these methods for providing the code with removal coefficients. If both are specified, an error message will result and the user will have to clear one of the subscreens. Selecting Return to Sprays Menu returns the user to screen number 2310. 


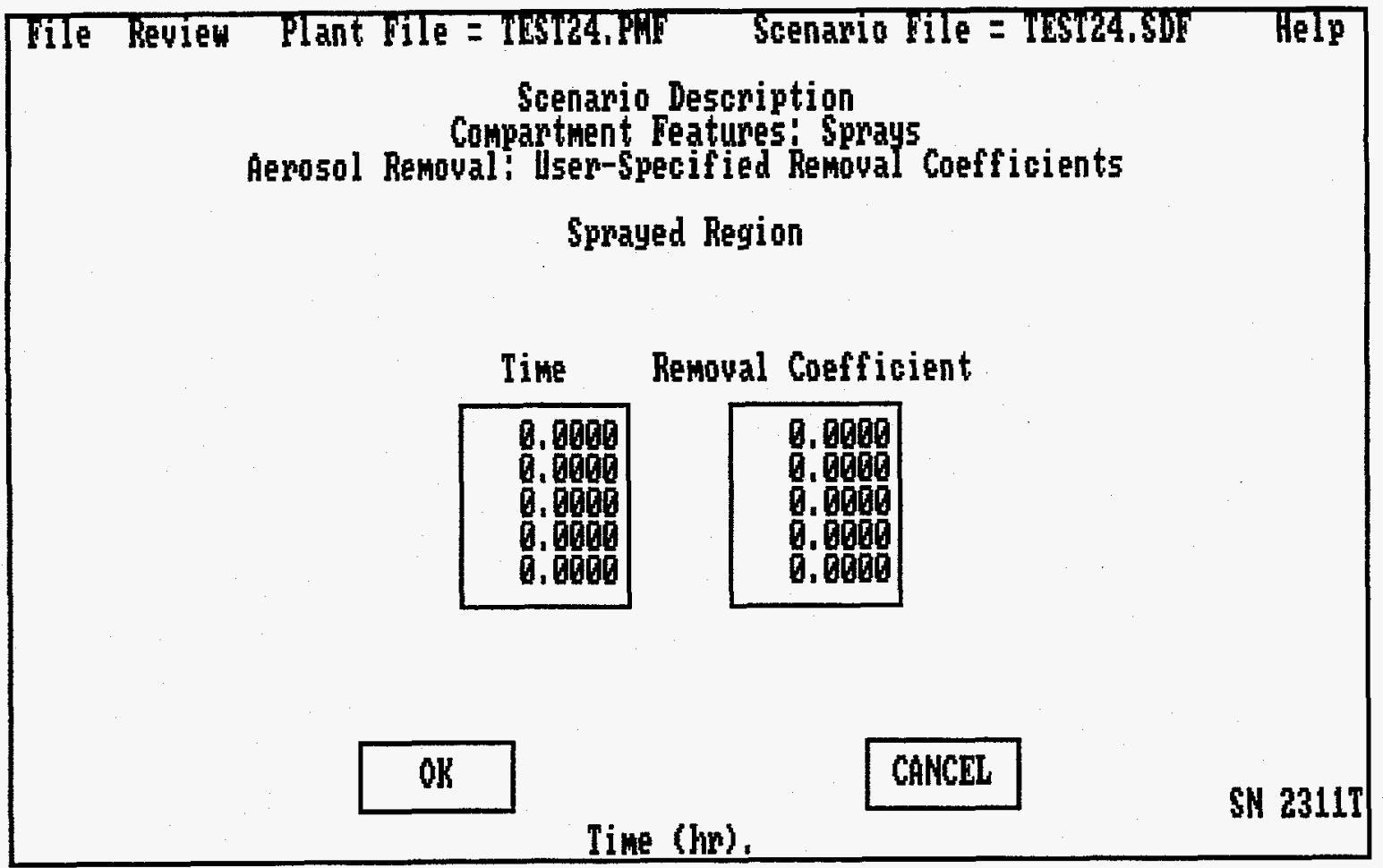

The User-Specified Removal Coefficients for aerosols are screen number 2311T. This screen allows the user to specify the time dependence of the aerosol removal coefficient. When the sprays are not operating, the user should specify a removal coefficient of 0.0 . The Removal Coefficient will change in a stepwise fashion at each Time that it is defined to change. Acceptance test case 23 details typical values for a PWR; the units are fraction removed of the total aerosol mass inserted into the containment per second. Clicking on OK or CANCEL will return the user to the Aerosol Removal by Sprays menu (screen number 2311). 


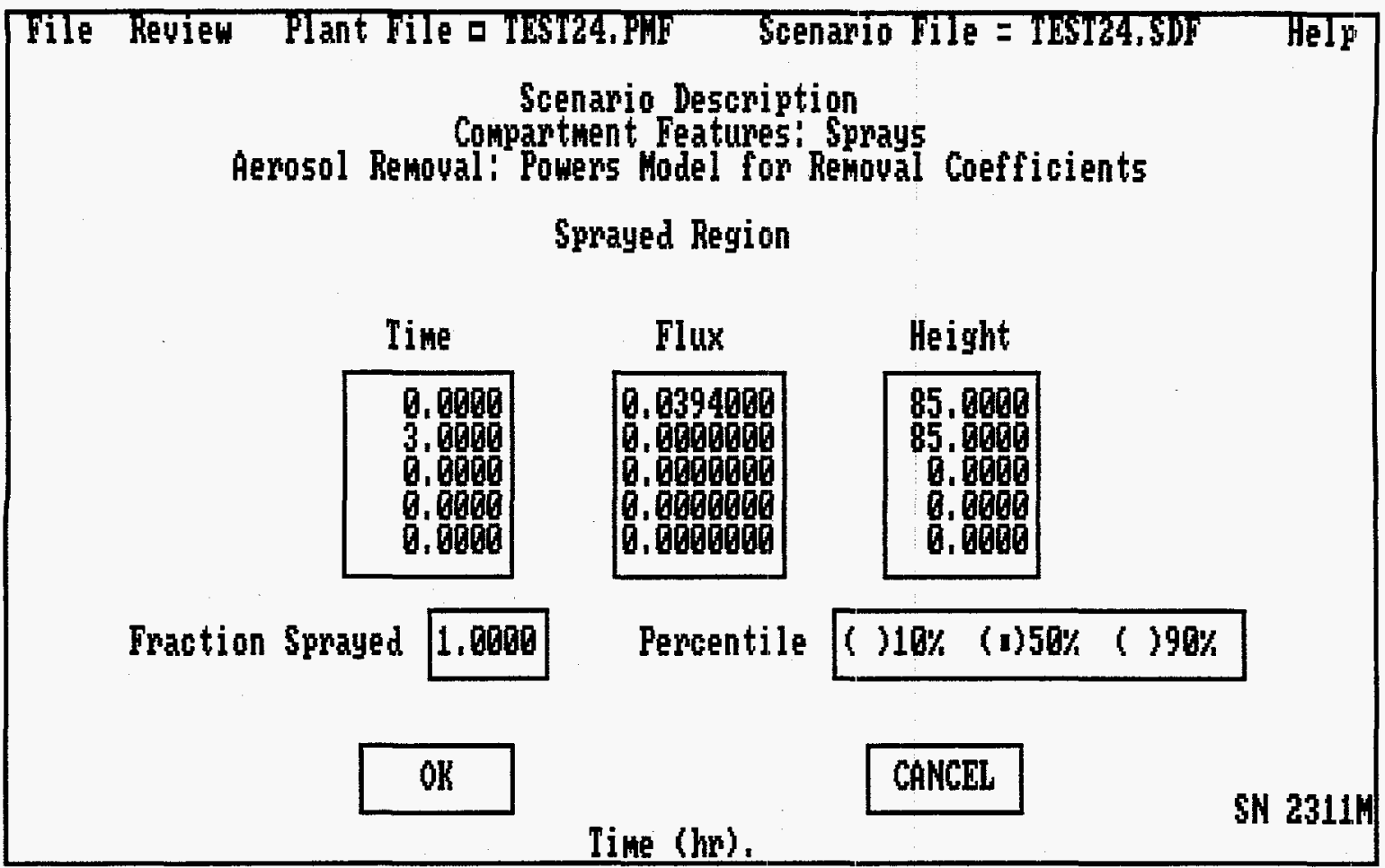

The Powers Model for Removal Coefficients for aerosols is screen number 2311M. This screen allows the user to specify the parameters necessary to calculate the aerosol removal by sprays using a model developed by Powers and Burson [Po93] as a function of time. The Flux is in units of volumetric flow of spray per area of coverage, typically taken as the containment volume over its height $\left(\mathrm{ft}^{3} / \mathrm{min}-\mathrm{ft}^{2}\right)$. The Height of sprays above the floor is in feet. The range of values used during the development of the model was from 0.3 to $80 \mathrm{ft}^{3} / \mathrm{s}-\mathrm{ft}^{2}$ of spray water Flux and from 16 to $160 \mathrm{ft}$ full height. The values will change in a stepwise fashion at each Time that they are defined to change. The user may also specify which Percentile of the model should be used in the calculation. Ten percent represents the lower bound, 50\% represents the median or best estimate, and $90 \%$ represents an upper bound that results in the largest decontamination factor. The user may also specify the Fraction of sprayed volume to total compartment volume. The theory used in developing this model is given in Section 2.1. Note that acceptance test case 24 used this option and separated sprayed and unsprayed zones due to the high removal ratio chosen. Clicking on OK or CANCEL will return the user to the Aerosol Removal by Sprays menu (screen number 2311). 


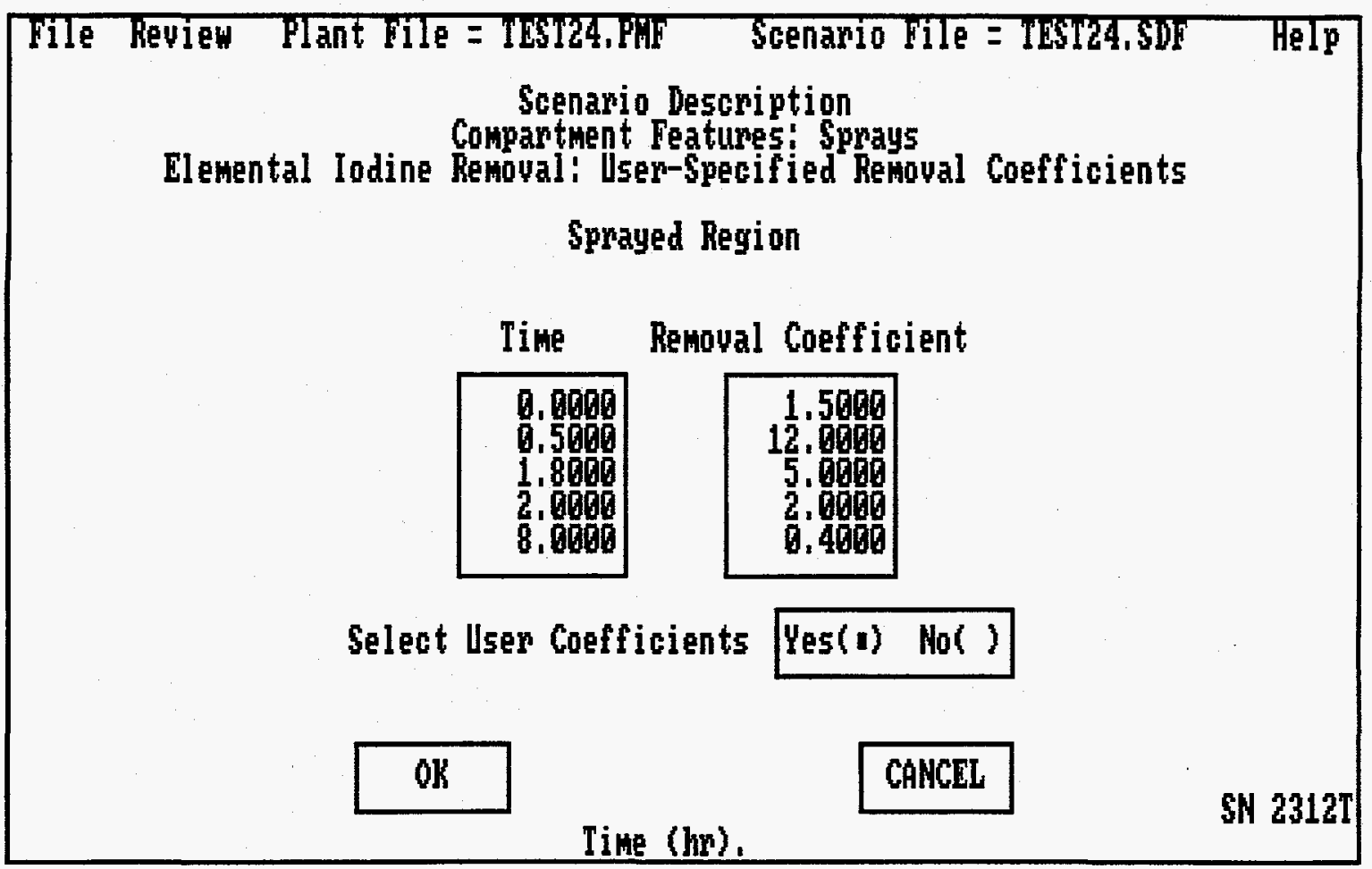

The User-Specified Removal Coefficients for elemental iodine are obtained in screen number $2312 \mathrm{~T}$. This screen allows the user to specify the time dependence of the elemental iodine removal coefficient $\left(\mathrm{hr}^{-1}\right)$. When the sprays are not operating, then the user should specify a removal coefficient of 0.0 . In acceptance test cases 23 and 24, the elemental iodine removal coefficient had the same value as the aerosol removal coefficient for case 23. The Removal Coefficient will change in a stepwise fashion at each Time that it is defined to change. The user must have the Select User Coefficients button on Yes for the code to use the tabular input. When it is set to $\mathrm{No}$, then the removal coefficient is always 0.0. Clicking on OK or CANCEL will return the user to the Sprays menu (screen 2310). 


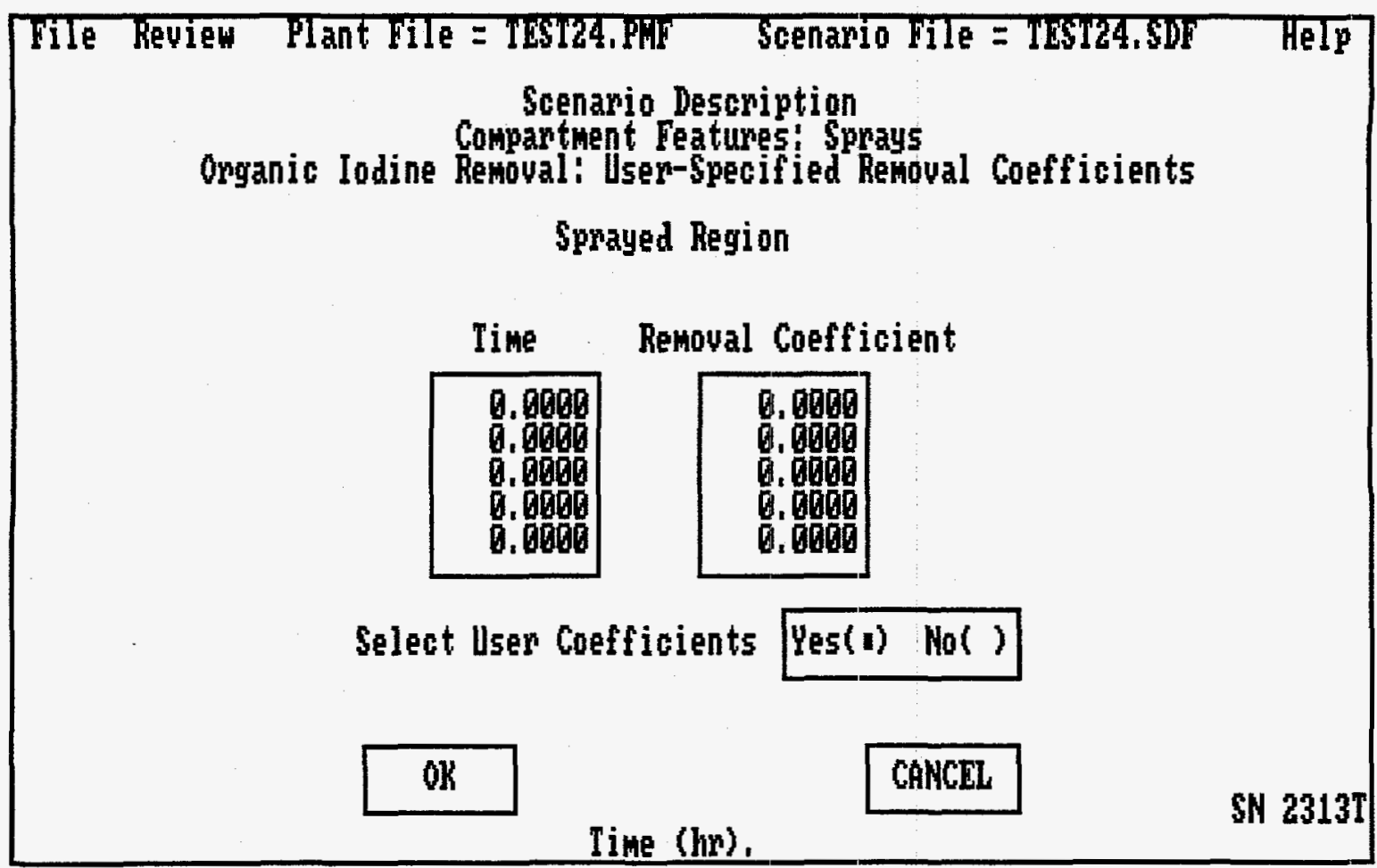

The User-Specified Removal Coefficients for organic iodine are screen number 2313T. This screen allows the user to specify the time dependence of the organic iodine removal coefficient. When the sprays are not operating, then the user should specify a removal coefficient of 0.0 . The Removal Coefficient will change in a stepwise fashion at each Time that it is defined to change. Organics are generally not affected by sprays; hence organic iodine removal coefficients of 0.0 are reasonable. The user must have the Select User Coefficients button on Yes for the code to use the tabular input. When it is set to No, the removal coefficient is always 0.0. Clicking on OK or CANCEL will return the user to the Sprays menu (screen number 2310). 


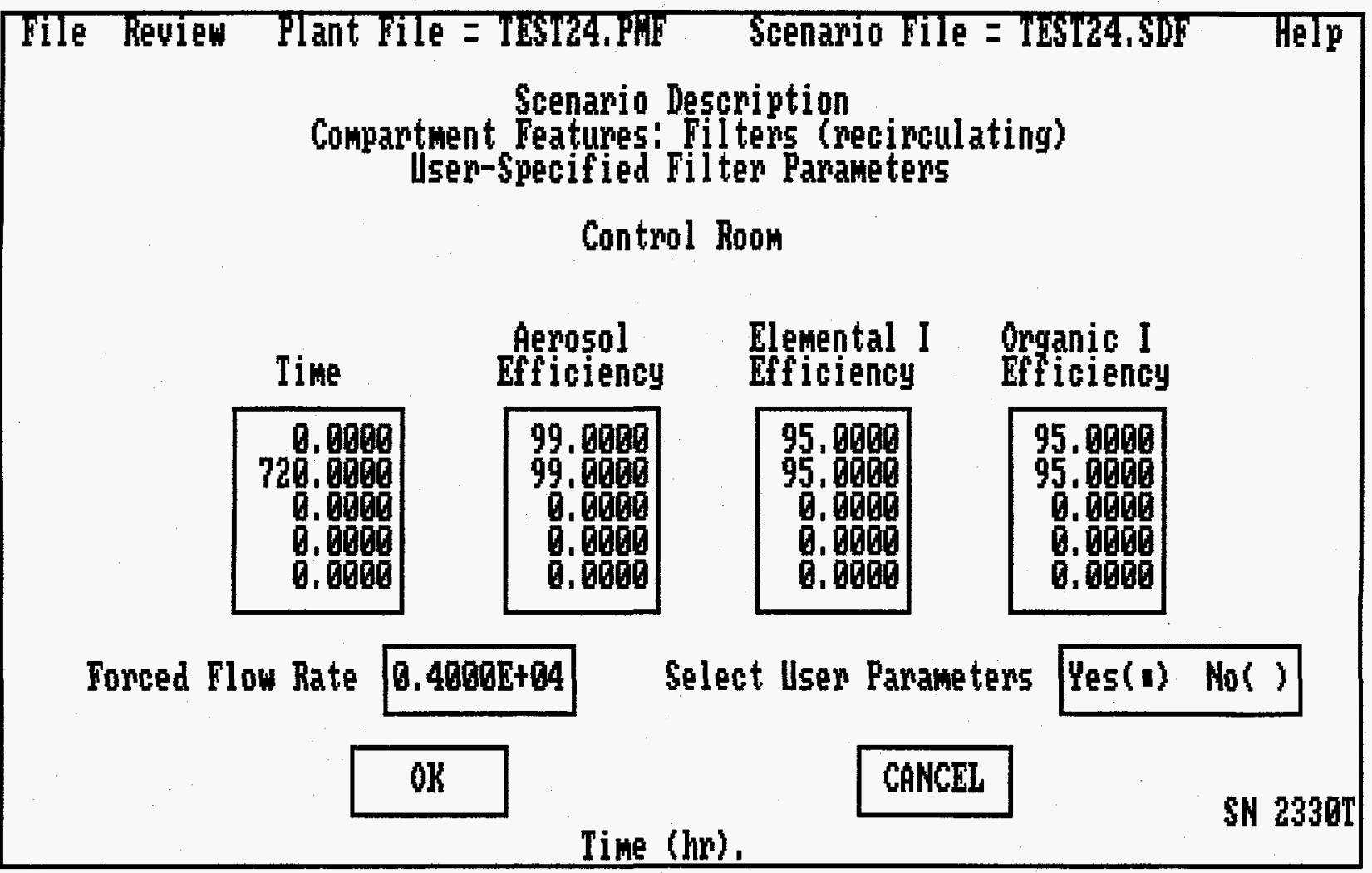

The User-Specified Filter Parameters are screen number 2330T. The screen is reached by initializing Filters (Recirculating) on screen 1310 and then clicking on Filters (Recirculating) on screen 2300A. This screen allows the user to enter information about the effectiveness of a recirculating air filter within a compartment, such as a control room, as a function of time. The capture efficiencies are input separately for the Aerosol, Elemental I, and Organic I groups. It is assumed that the noble gas group cannot be captured. The efficiencies will change in a stepwise fashion at each Time that they are defined to change. The constant Forced Volumetric Flow Rate is also input. If the user requires a variable flow rate, then the user-defined pipe feature is recommended. The user must have the Select User Parameters button on Yes for the code to use the tabular input. When it is set to $\mathrm{No}$, then the efficiencies are all 0.0 . Recirculating filters were used extensively in the acceptance test problems; typical values can be found in Section 3.3.

Clicking on OK or CANCEL will return the user to the Compartment Features menu (2300A). 


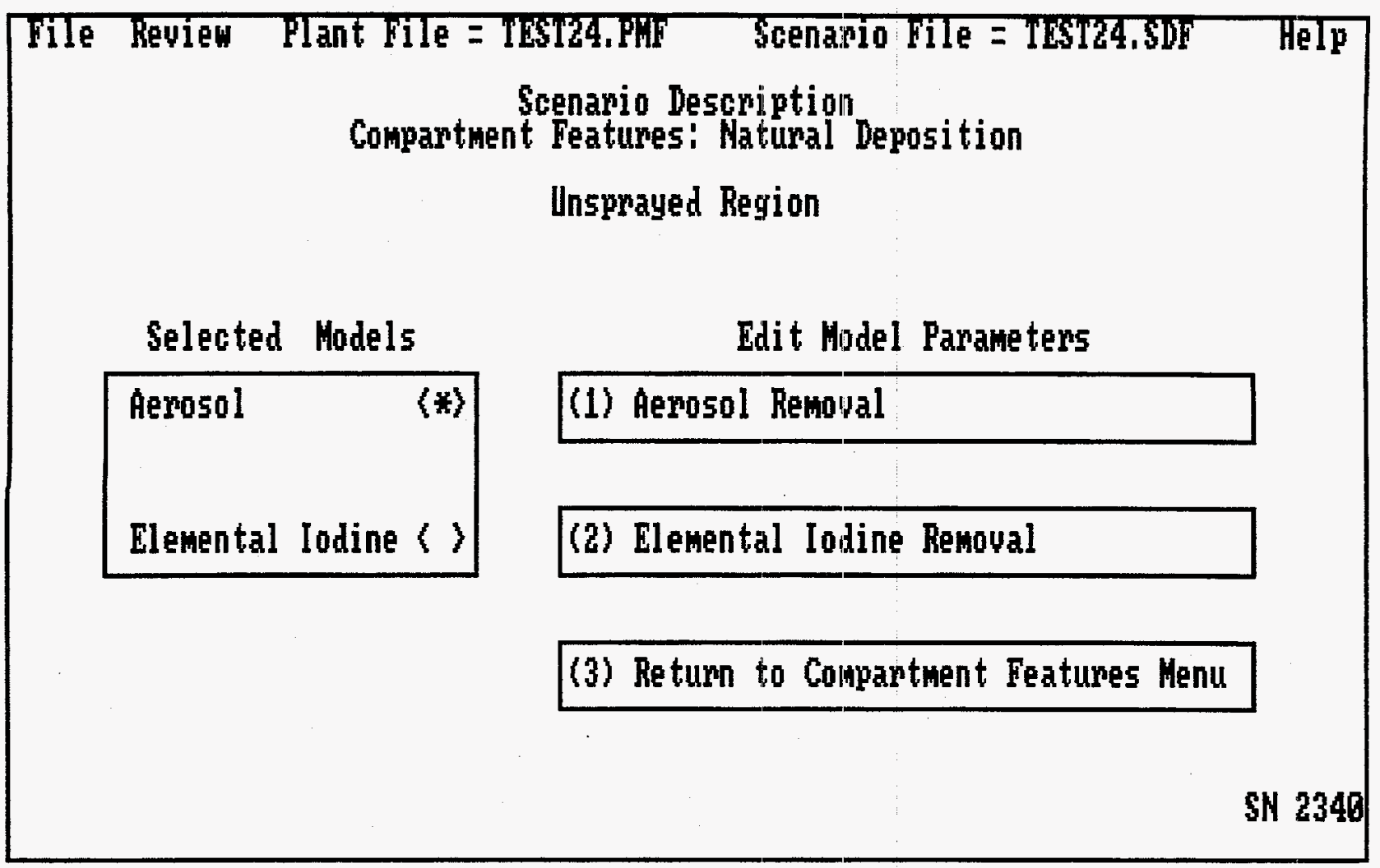

The Natural Deposition menu is screen number 2340. This screen is reached by initializing the Natural Deposition button on screen 1310 and by double clicking on the Natural Deposition pad on screen $2300 \mathrm{~A}$ This screen allows the user to include information on natural deposition. The user may specify information on Aerosol or Elemental Iodine Removal by selecting these options and double clicking. Selecting Return to Compartment Features Menu returns the user to screen number 2300A. 


\begin{tabular}{|c|c|c|c|}
\hline \multirow{2}{*}{\multicolumn{2}{|c|}{ File Review }} & $\begin{array}{l}\text { TEST24.PhP Scenario File = TESL24.SDF } \\
\text { Scenario Description } \\
\text { ent Features: Natural Deposition } \\
\text { Aerosol Renoval } \\
\text { Unsprayed Region }\end{array}$ & \\
\hline & & Edit Model Paraneters & \\
\hline User-sp & (1) & (1) User-Specified Renoval Coefficients & \\
\hline Henry & () & (2) Henry Aerosol Deposition Model & \\
\hline Powers & ( ) & (3) Powers Aerosol Deposition Containment Model & \\
\hline & ( ) & (4) Return to Matural Deposition Menu & \\
\hline
\end{tabular}

The Aerosol Removal by Natural Deposition menu is screen number 2341. This screen allows the user to choose the aerosol Removal Coefficients as a function of time or implement one of the two models for natural deposition of aerosols. Only one of the options may be active. If information is specified for more than one of the options, then the last model selected is used. Selecting Return to Natural Deposition Menu returns the user to screen number 2340. 


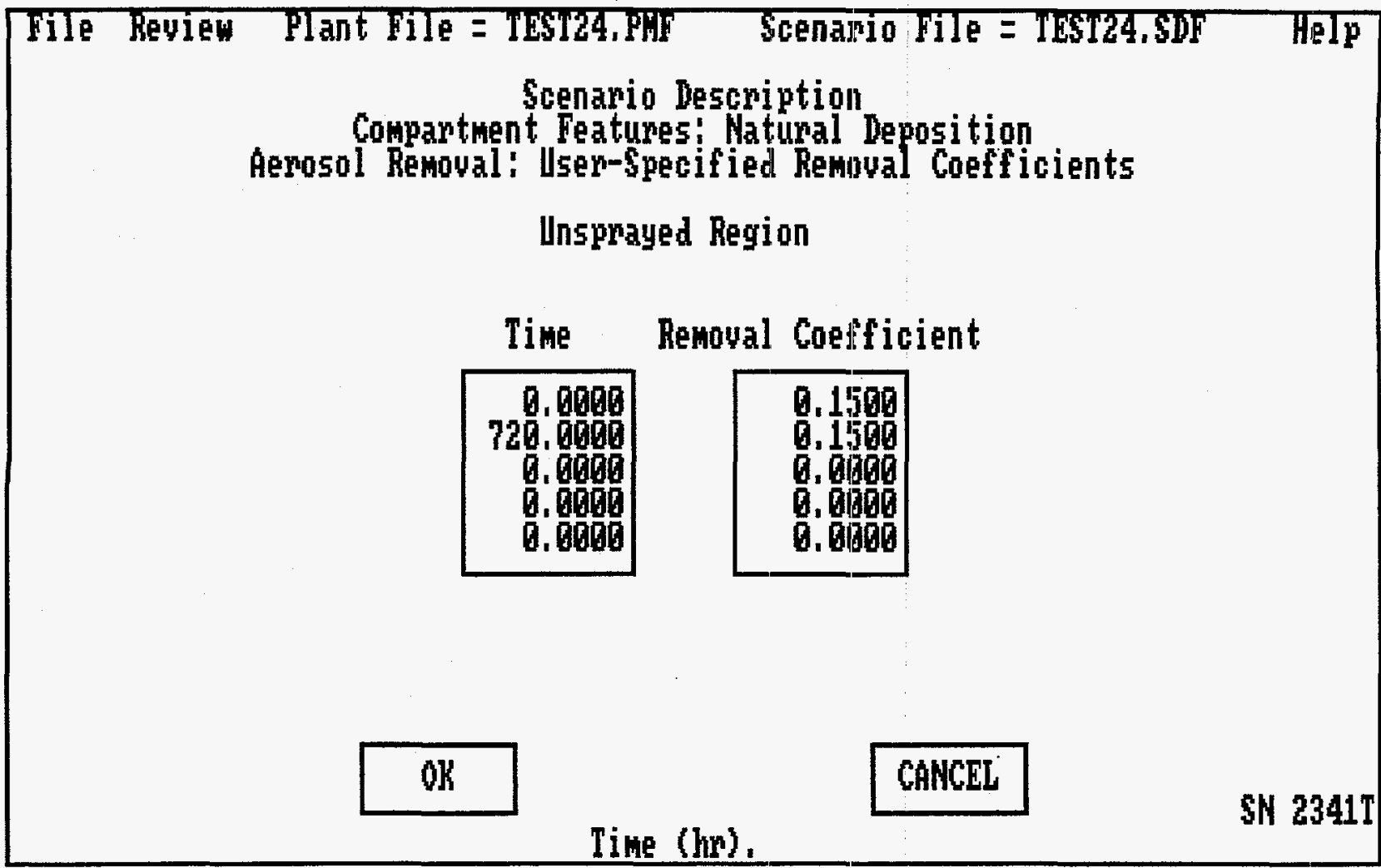

The User-Specified Removal Coefficients for aerosols are screen number 2341T. This screen allows the user to specify the removal coefficient $\left(\mathrm{hr}^{-1}\right)$ as a function of time for the natural deposition of aerosols. The Removal Coefficient will change in a stepwise fashion at each Time it is defined to change. Acceptance test cases 4, 9, 22, 23 and 24 used this option with a value of $0.15 / \mathrm{hr}$ or $4.167 \times 10^{-5} / \mathrm{s}$ for input. Clicking on OK or CANCEL will return the user to the Aerosol Removal by Natural Deposition menu (screen number 2341). 


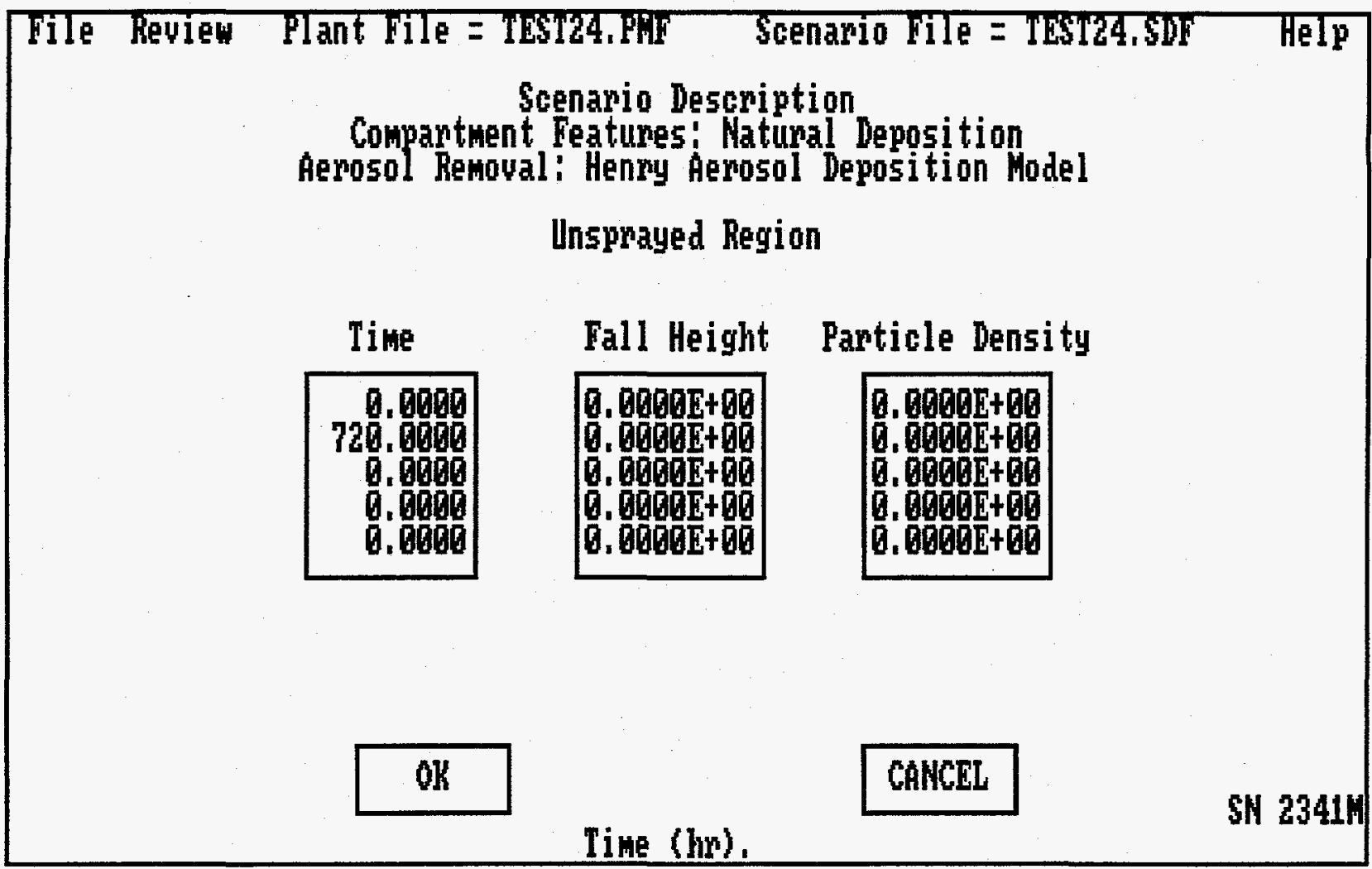

The Henry Aerosol Deposition Model for aerosol removal [Fa84] is screen number 2341M. This screen allows the user to provide input for the Henry correlation for natural deposition as described in Section 2.2.1.1.1. The deposition Fall Height $(\mathrm{ft})$ and Particle Density $\left(\mathrm{g} / \mathrm{cm}^{3}\right)$ will change in a stepwise fashion at each time it is defined to change. Acceptance test cases 5 and $10 \mathrm{~A}$ exercised this option using a fall height of $16.4 \mathrm{ft}$ and the density of water $\left(1 \mathrm{~g} / \mathrm{cm}^{3}\right)$ for the particle density. In many situations the aerosols are not at their theoretical density due to the way they were formed. If the user knows the effective density, it should be used. The range of applicability of this simple model is not well defined. Clicking on OK or CANCEL will return the user to the Aerosol Removal by Natural Deposition menu (screen number 2341). 


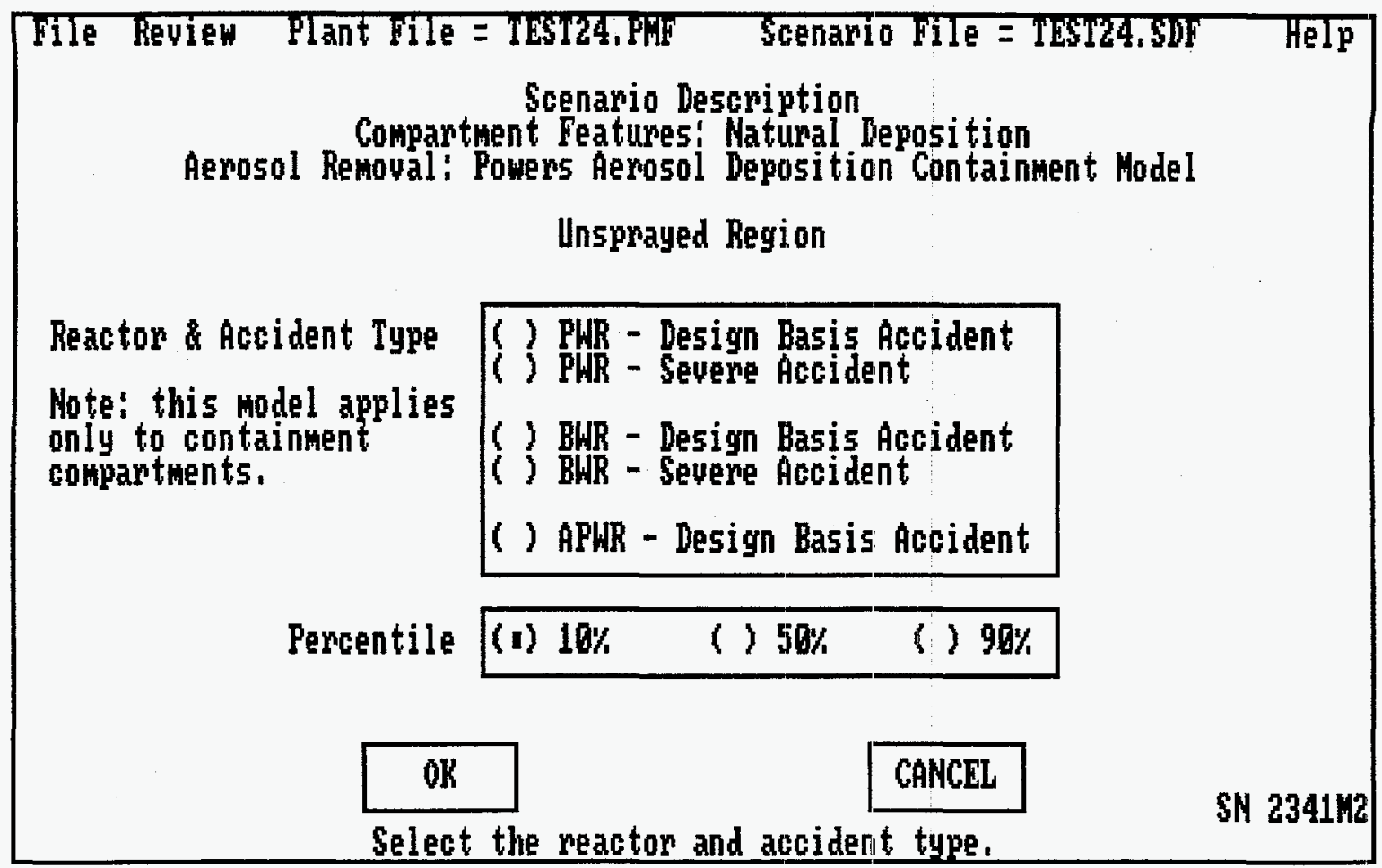

The Powers Aerosol Deposition Containment Model for aerosol removal [Po95], is screen number 2341M2. This screen allows the user to provide input for the Powers model for natural deposition of aerosols. This model is reasonable only for a large building and, according to the author, is not valid for deposition in passages such as pipes. The model is described in Section 2.2.1.1.2. The user should be consistent in the choice of reactor and accident types with the release fractions chosen on screen 2220 . The user must specify an uncertainty percentile; $10 \%$ represents the lower bound, 50\% the best estimate or median, and $90 \%$ the upper bound or largest removal coefficient. Acceptance test case 10 exercised this option. It was a PWR design and looked at the best estimate solution for a design basis accident. Clicking on OK or CANCEL will return the user to the Aerosol Removal by Natural Deposition menu (screen number 2341). 


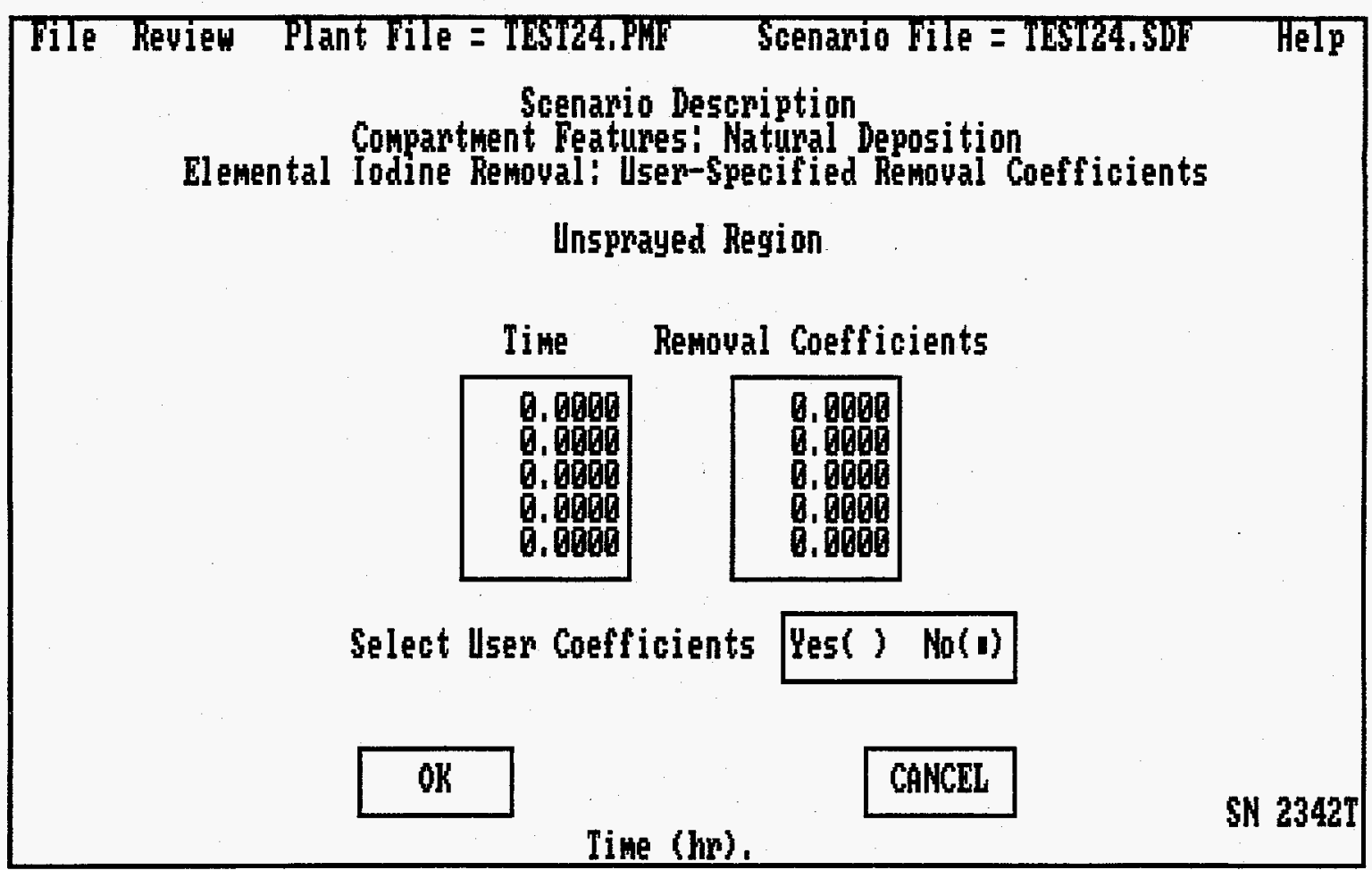

The User-Specified Removal Coefficients for elemental iodine are screen number 2342T. This screen allows the user to specify the removal coefficient as a function of time for the natural deposition of elemental iodine. The Removal Coefficient will change in a stepwise fashion at each Time it is defined to change. Acceptance test cases 4, 5, 9, 10, 23 and 24 all exercised this option by using the above table; however, there was no elemental Iodine Removal Coefficient input. The user must have the Select User Coefficients button on Yes for the code to use the tabular input. When it is set to No the removal coefficient is always 0.0 . Clicking on OK or CANCEL will return the user to the Natural Deposition menu (screen number 2340). 


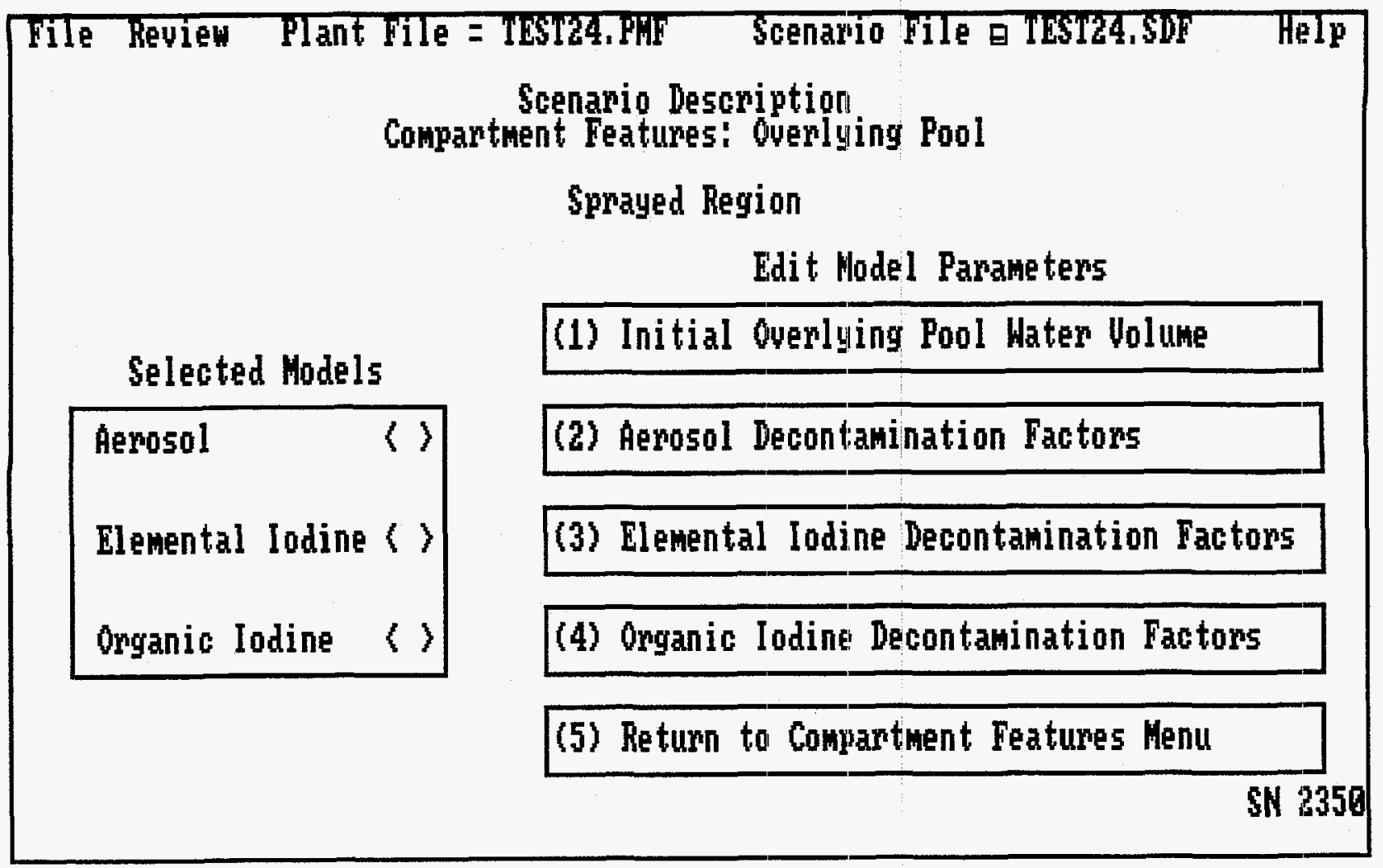

The Overlying Pool is described in screen number 2350. This screen is entered by initializing the Overlying Pool option on screen 1310 and then choosing the Overlying Pool pad on screen $2300 \mathrm{~A}$. This screen allows the user to reduce the source term due to an overlying pool that may be available after the vessel fails and part of the core falls to the containment floor. The user must input an Initial Overlying Pool Water Volume and may choose any or all of the decontamination methods. The default is a decontamination factor of 1.0 , or no decontamination of any group. This option is typically used after vessel failure when the partially ejected core and the releasing fission products are covered with water. It has not been assessed because it deals only with severe accident space. Selecting Return to Compartment Features Menu returns the user to screen number $2300 \mathrm{~A}$. 


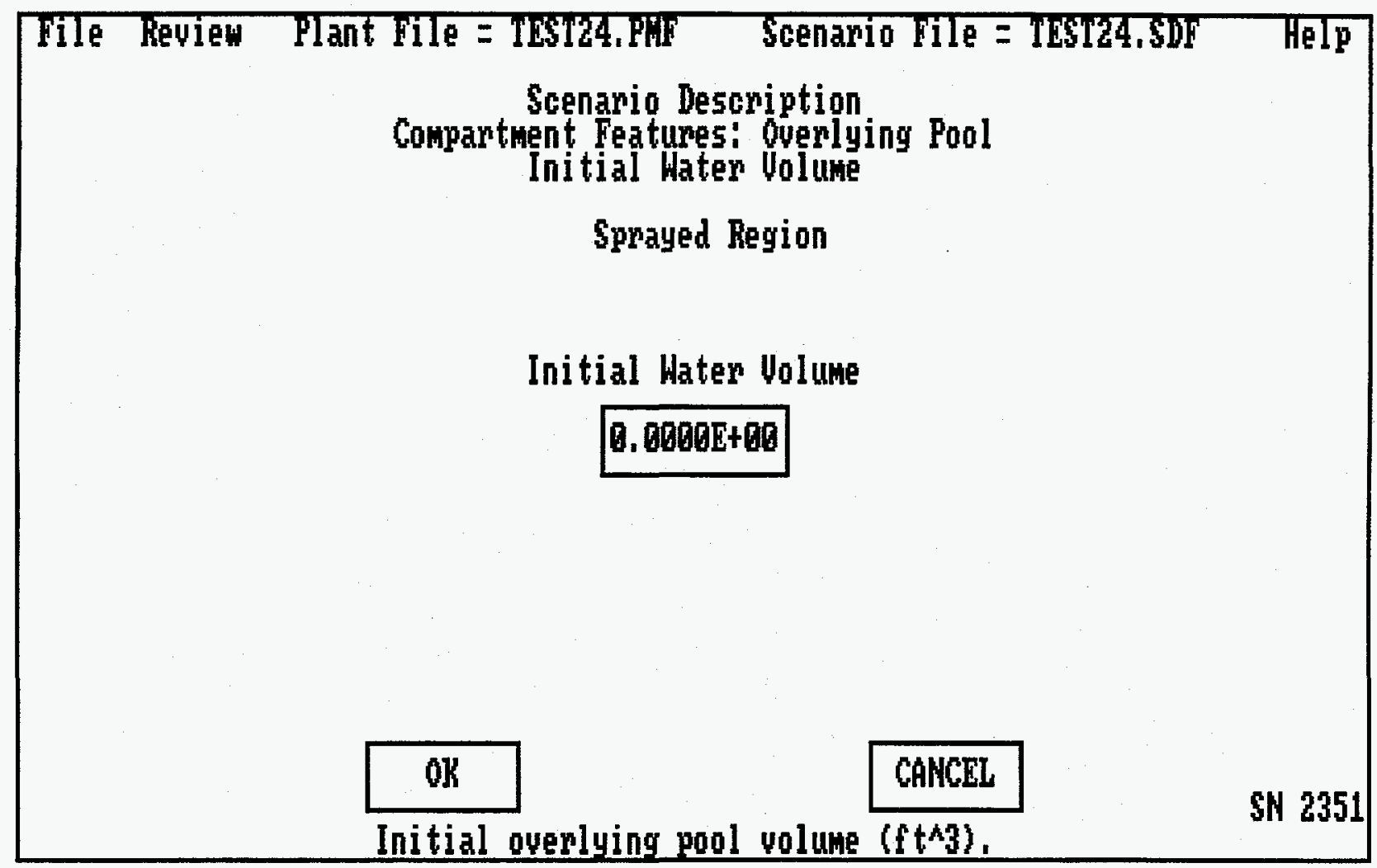

The Overlying Pool Initial Water Volume is screen number 2351. This screen allows the user to input the Initial Water Volume $\left(\mathrm{ft}^{3}\right)$ overlying the molten core. Clicking on OK or CANCEL will return the user to the Overlying Pool menu (screen number 2350). 


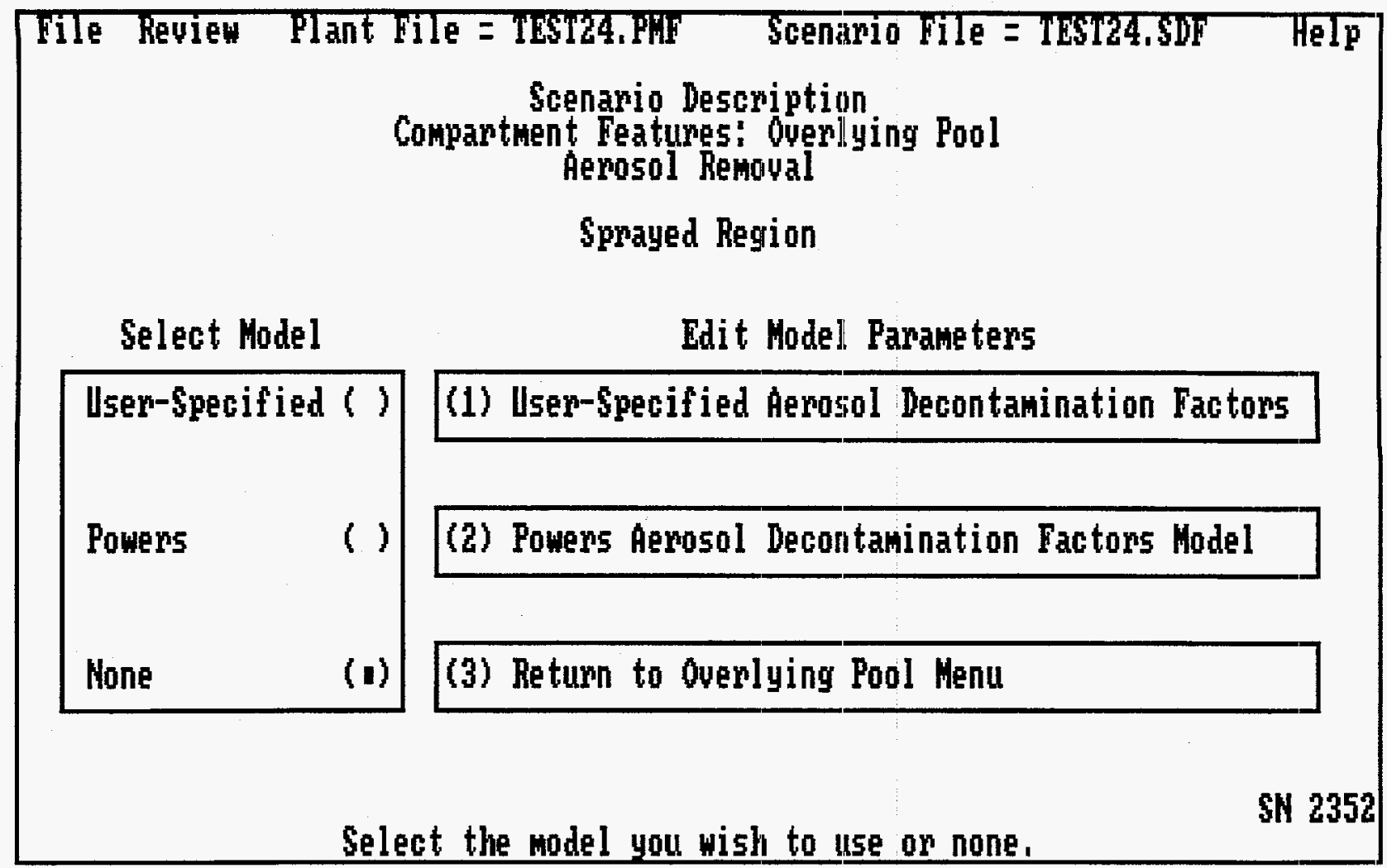

The Overlying Pool Aerosol Removal menu is screen number 2352. This screen allows the user to choose between a user-specified time-dependent decontamination factor and the Powers model [PO92] for aerosol decontamination factor in overlying pools. Only one of these methods may be used. Selecting Return to Overlying Pool Menu will return the user to screen number 2350. 


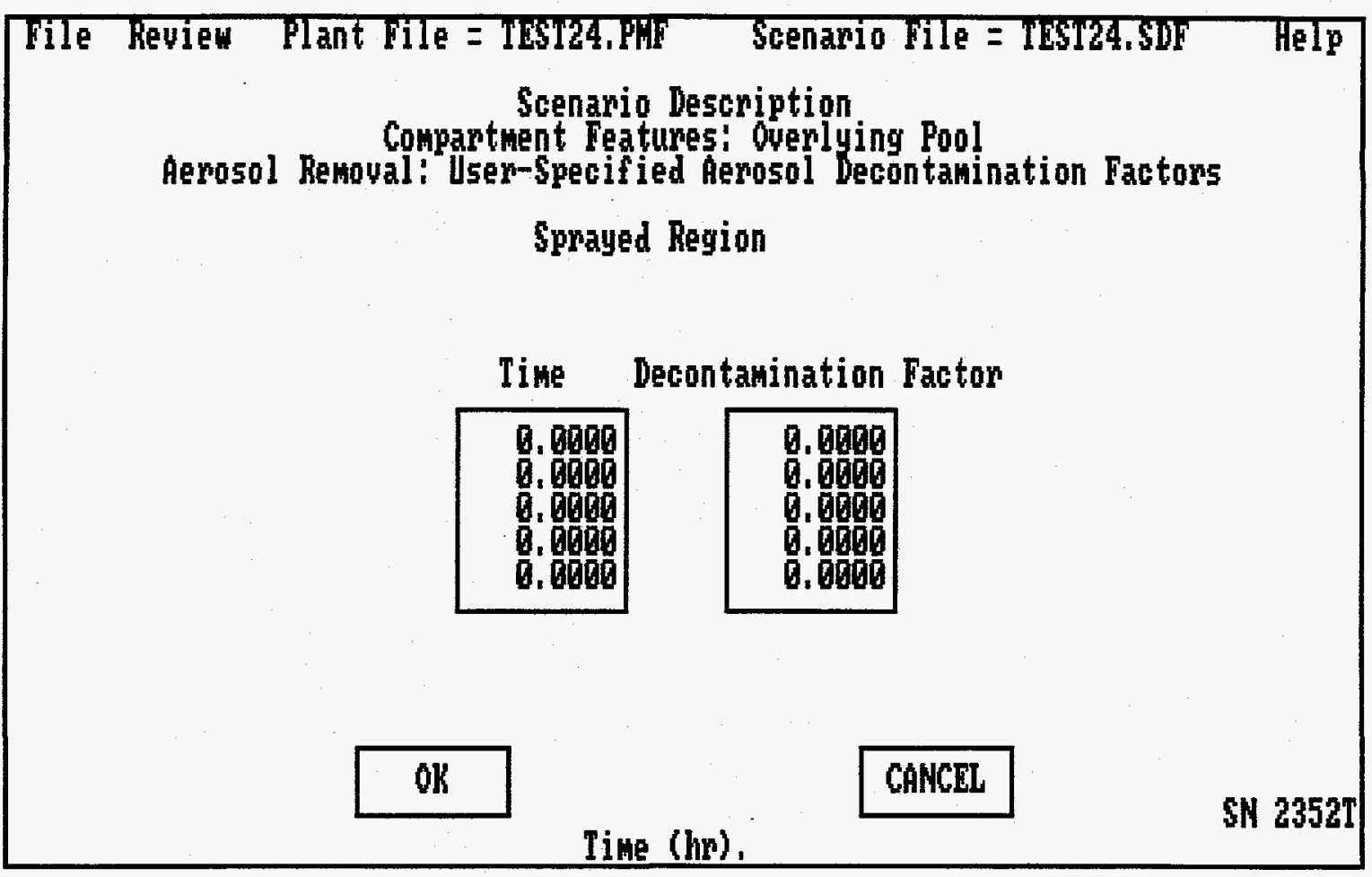

The User-Specified Aerosol Decontamination Factors for aerosol removal due to an overlying pool are screen number $2352 \mathrm{~T}$. This screen allows the user to specify the aerosol

decontamination factor for the overlying pool as a function of time from accident initiation. The Decontamination Factor will change in a stepwise fashion at each Time that it is defined to change. The decontamination factor must be $\geq 1.0$. Clicking on OK or CANCEL will return the user to Overlying Pool Aerosol Removal menu (screen number 2352). 


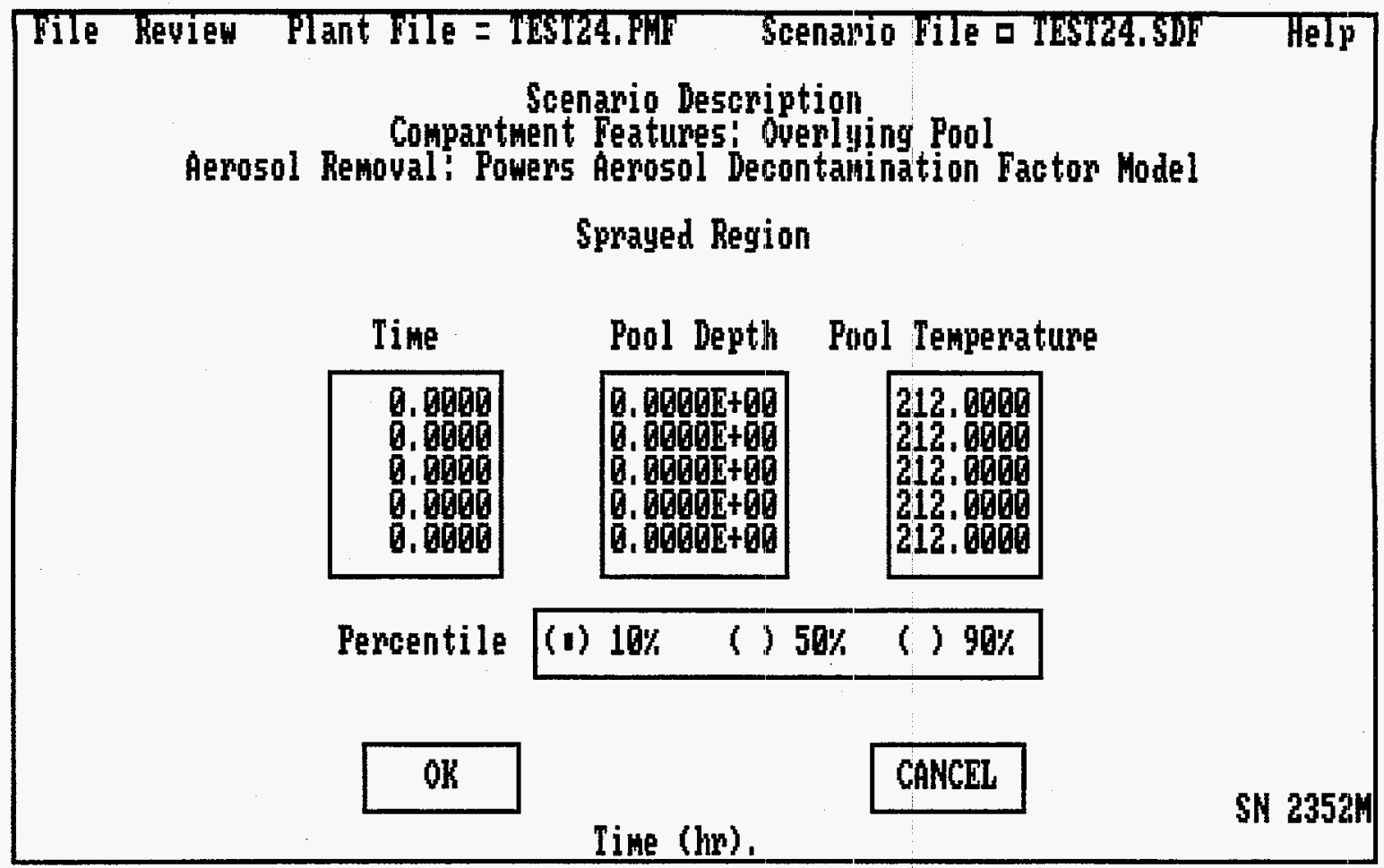

The Powers Aerosol Decontamination Factor Model for aerosol removal is screen number $2352 \mathrm{M}$. This screen allows the user to specify the Pool Depth ( $\mathrm{ft}$ ) and degree of subcooling $\left({ }^{\circ} \mathrm{F}\right)$ as a function of time. The derived model, as described in Section 2.2.3.1, only examined pool depths between 1 and $6 \mathrm{ft}$; it also examined subcooling between 0 and $126^{\circ} \mathrm{F}$ (if the compartment pressure is $1 \mathrm{~atm}$, this corresponds to water temperatures between 86 and $212^{\circ} \mathrm{F}$ ). Its use outside these ranges is not recommended. The pool height and pool subcooling will change in a stepwise fashion at each time that they are defined to change. The lower bound is associated with the $10 \%$ option, the best estimate with the 50\% option, and the upper bound with the $90 \%$ option. Clicking on OK or CANCEL will return the user to the Overlying Pool Aerosol Removal menu (screen number 2352). 


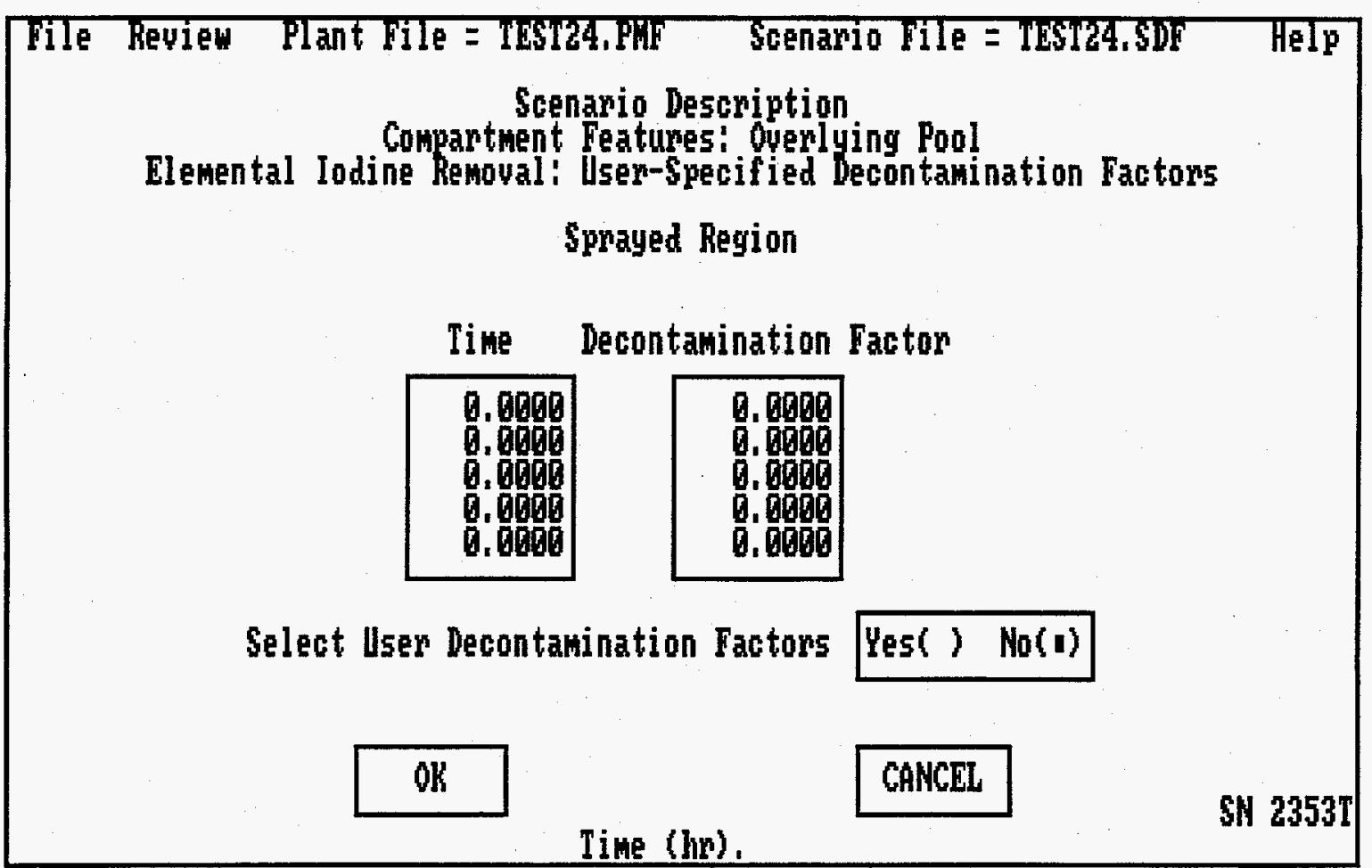

The User-Specified Decontamination Factors for elemental iodine removal are screen number 2353T. This screen allows the user to specify the elemental iodine decontamination factor for the overlying pool as a function of time. Decontamination factors for elemental iodine would probably be large for the range of pool sizes allowed by the aerosol decontamination model. The Decontamination Factor will change in a stepwise fashion at each Time that it is defined to change. The user must have the Select User Decontamination Factors button on Yes for the code to use the tabular input. When it is set to No, the decontamination factor is always 1.0. Clicking on OK or CANCEL will return the user to the Overlying Pool menu (screen number 2350). 


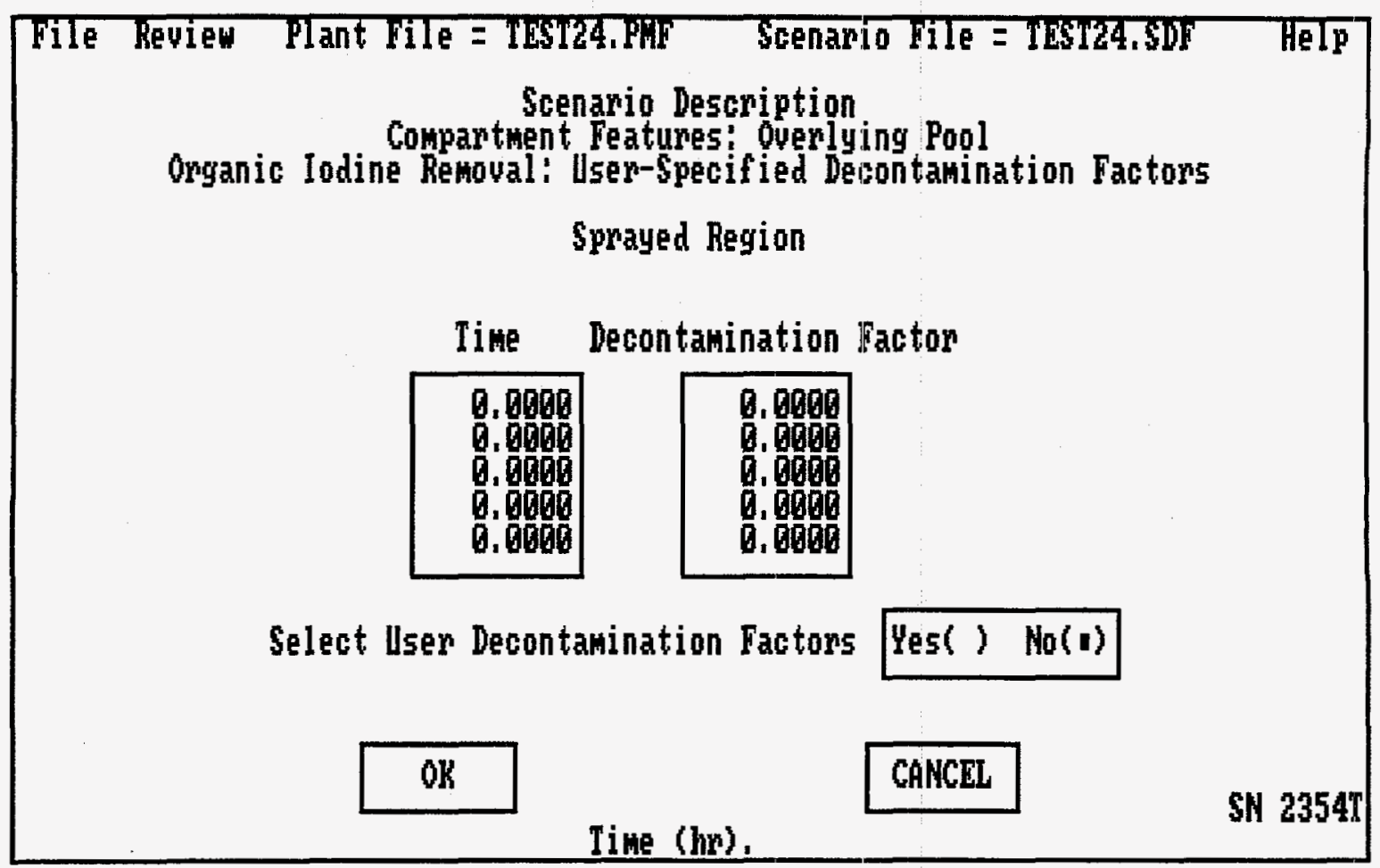

The User-Specified Decontamination Factors for organic iodine removal are screen number $2354 \mathrm{~T}$. This screen allows the user to specify the organic iodine decontamination factor for the overlying pool as a function of time. Decontamination factors for organic iodine would probably be small for the range of pool sizes allowed by the aerosol decontamination model. The Decontamination Factor will change in a stepwise fashion at each Time that it is defined to change. The user must have the Select User Decontamination Factors button on Yes for the code to use the tabular input. When it is set to No, the decontamination factor is always 1.0. Clicking on OK or CANCEL will return the user to the Overlying Pool menu (screen number 2350). 
$\cdot(0002$ แəว.วง $)$

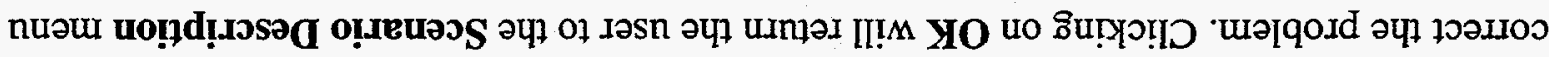

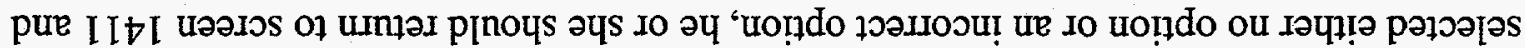

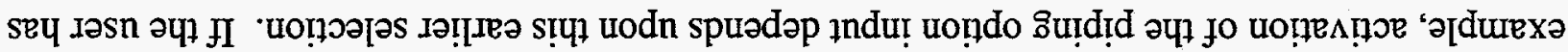

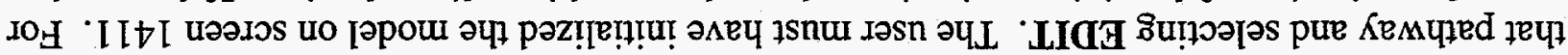

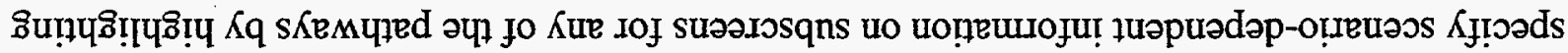

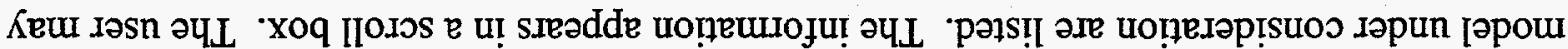

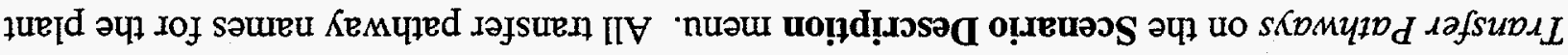

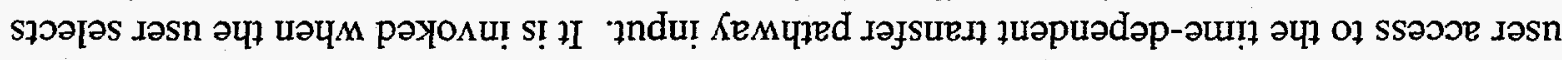

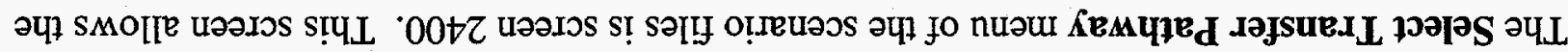

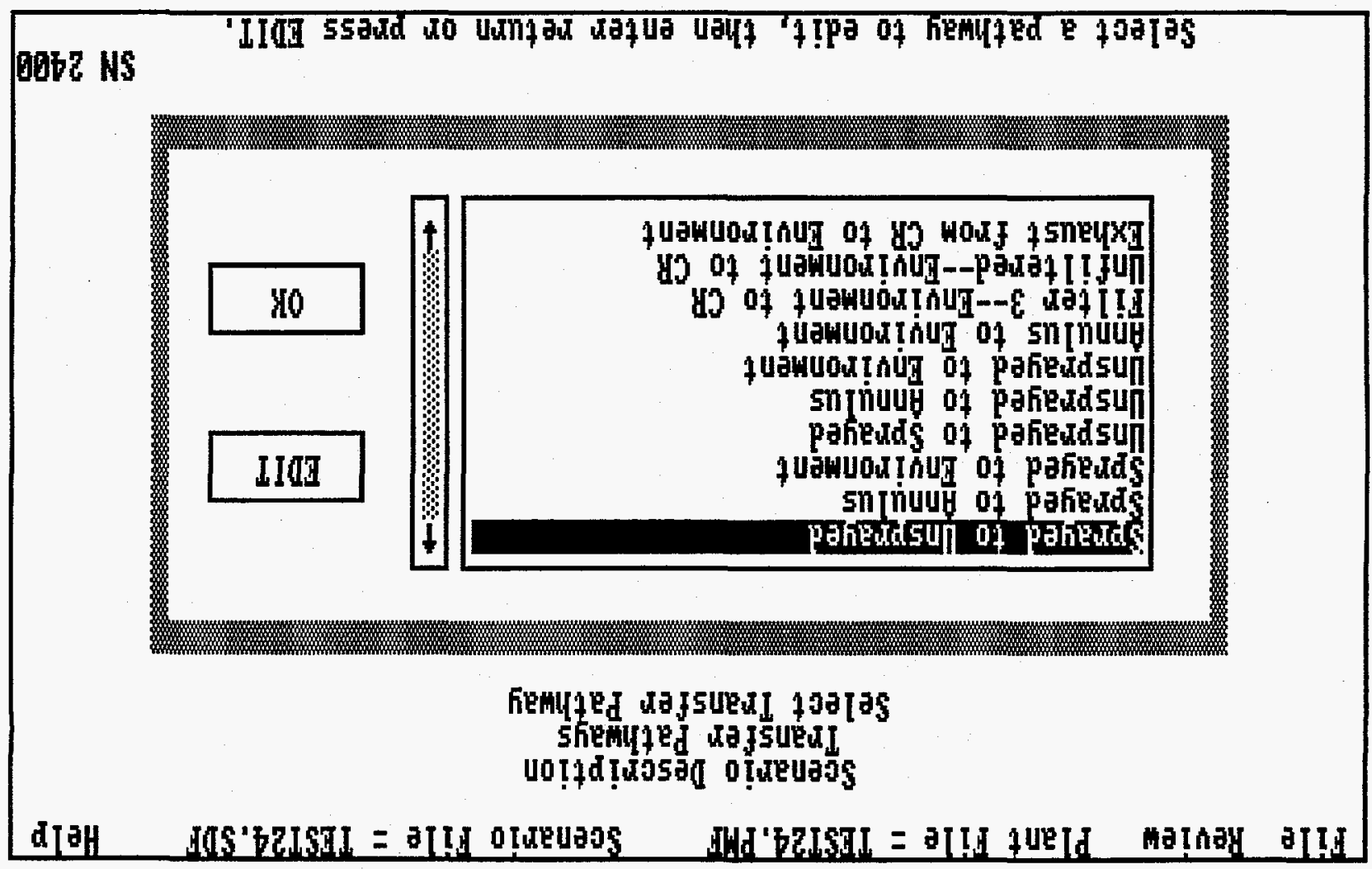




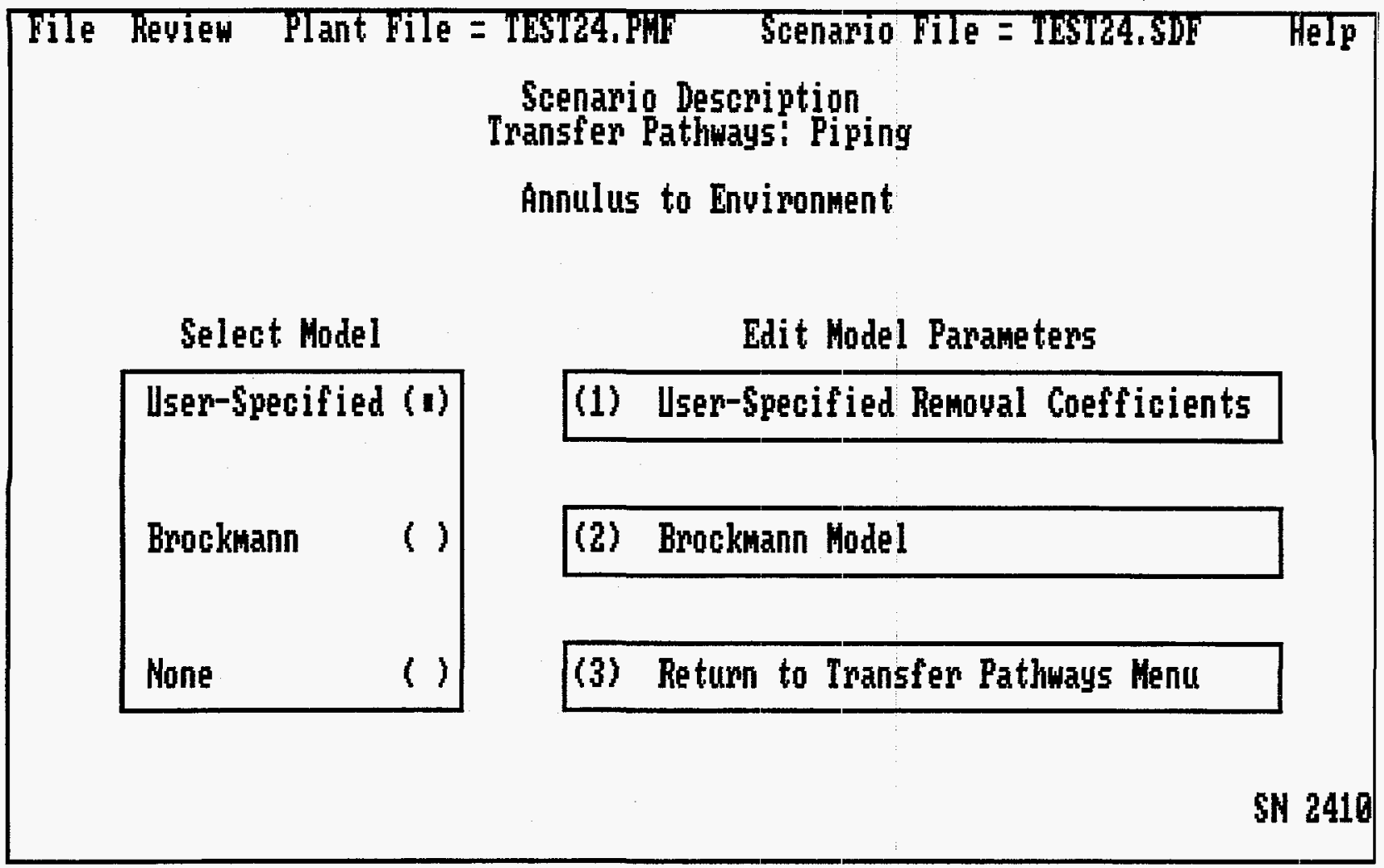

The Transfer Pathways: Piping is screen number 2410. This screen allows the user to include information on fission product removal in the piping. The selection chosen will be used to determine the deposition of aerosols, elemental iodine, and organic iodine in a pipe. The user may elect to input user-specified decontamination factors and volumetric flow rates as a function of time from accident initiation or may select a model. The user may only specify one of these methods for providing the code with decontamination factors. Selecting Return to Transfer Pathways Menu returns the user to Select Transfer Pathway (screen number 2400). 


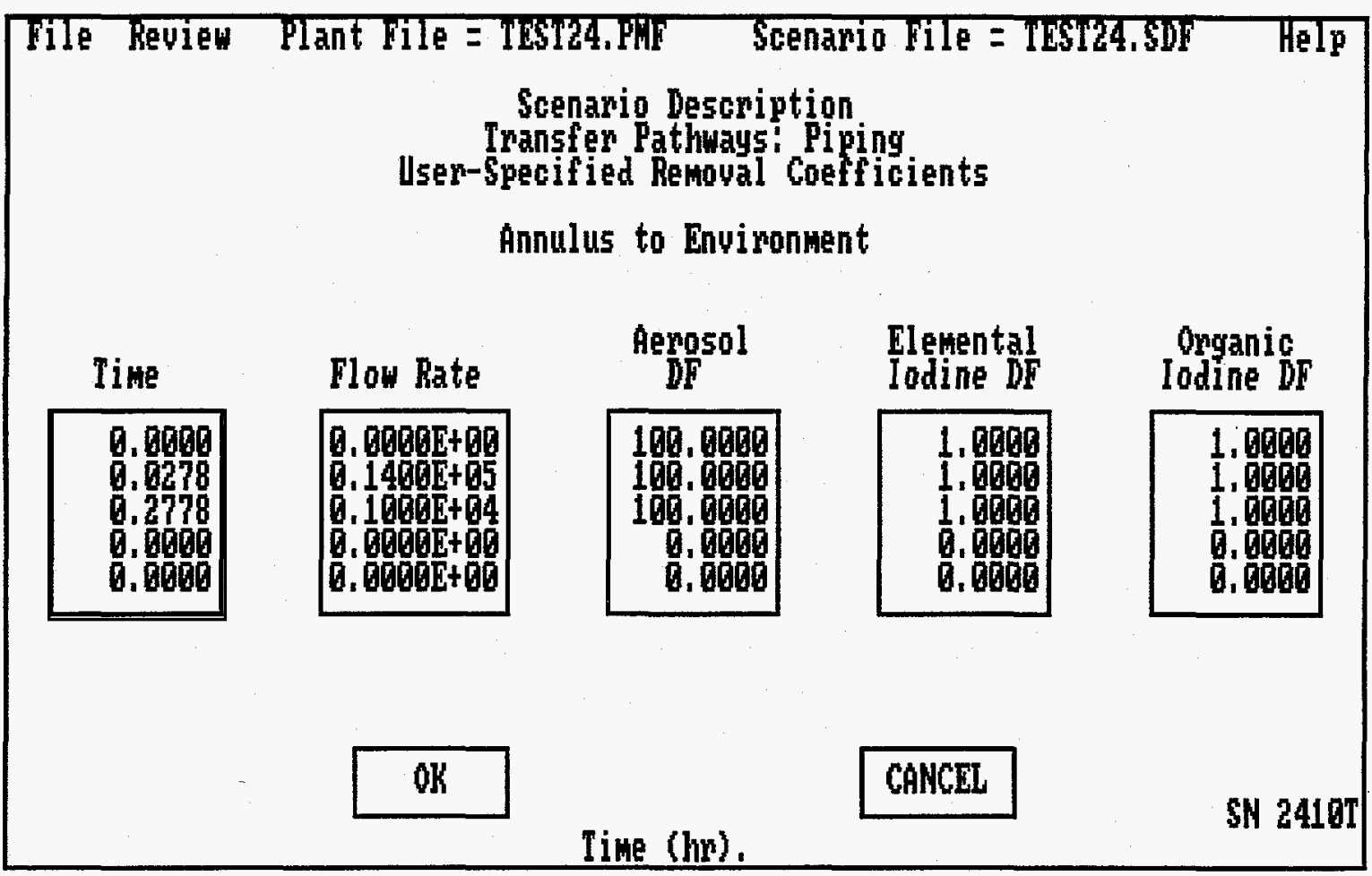

The User-Specified Removal Coefficients are screen number 2410T. This screen allows the user to specify the time dependence of the aerosol, elemental iodine, and organic iodine decontamination factors and the volumetric flow rate through the pipe. The values for Flow Rate (cfm), Aerosol, Elemental Iodine, and Organic Iodine Decontamination Factors are changed in a stepwise fashion at each Time that they are defined to change. Decontamination factors are always $\geq 1.0$. This option was exercised in acceptance test cases 13 and 13B. In these cases, a detailed analysis using the Cline model was carried out at Pacific Northwest National Laboratory and the effective efficiencies necessary to duplicate the results were input in terms of decontamination factors. In that model all the aerosols and elementals as well as a varying part of the organics were deposited in the pipe. Clicking on OK or CANCEL will return the user to the Transfer Pathways: Piping menu (screen number 2410). 


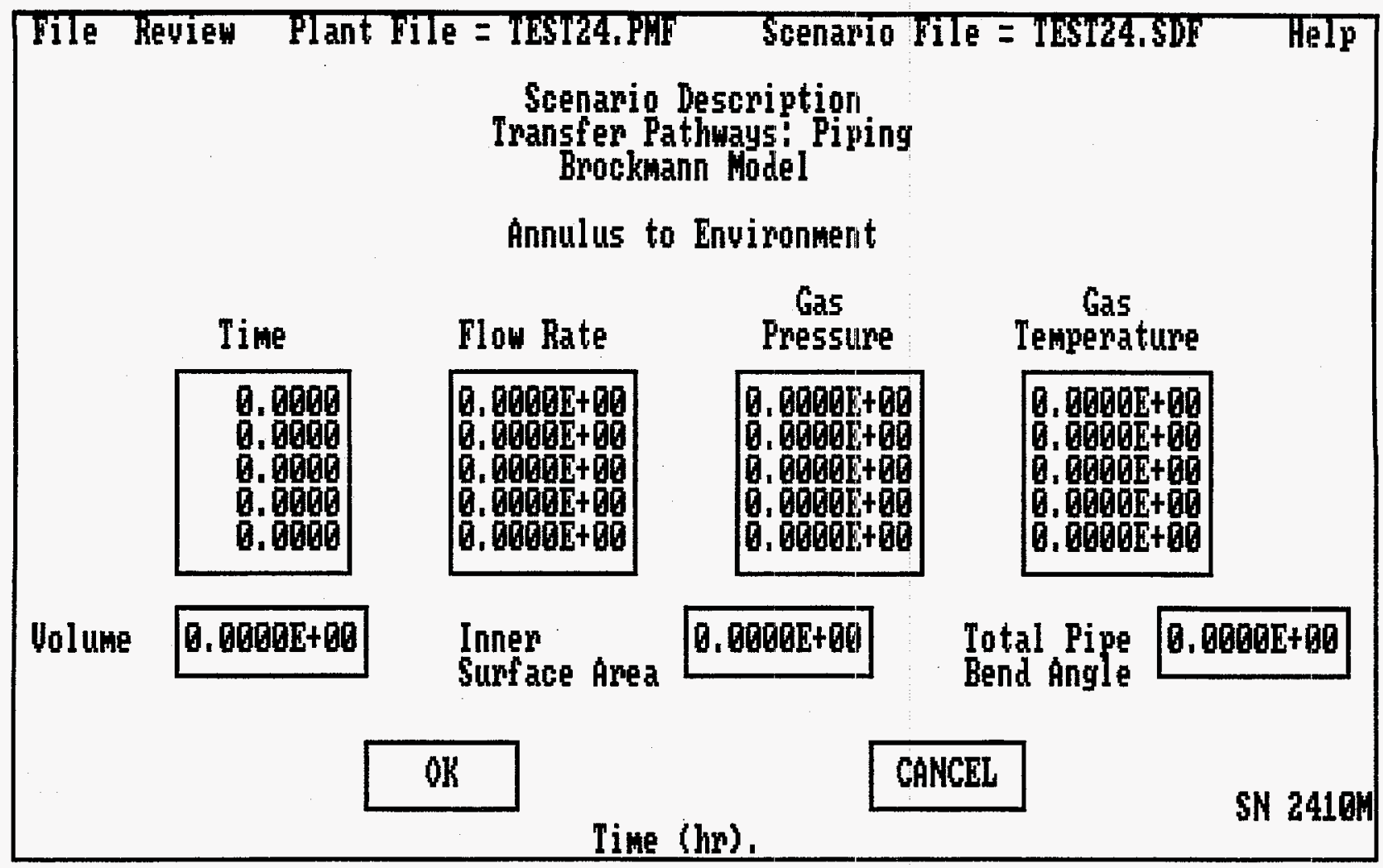

The Brockmann Model is screen number 2410M. This screen allows the user to enter data for the Brockmann-Bixler [Br91, Bi95] models described in Section 2.2.6. The model needs the total surface area $\left(\mathrm{ft}^{2}\right)$, volume $\left(\mathrm{ft}^{3}\right)$, and bend angle (degree) in the pipe to determine the geometric relationships. It then uses the Gas Pressure and Flow Rate variations to determine aerosol deposition. The tabular properties are changed in a stepwise fashion at each Time (hr) that they are defined to change. Although the models used in the Brockmann model are very general, the experimental base is not perfect. The pressure $(\mathrm{cfm})$, temperature $(\mathrm{F})$ and flow $(\mathrm{cfm})$ limits are not defined, but tens of atmospheres, thousands of degrees, or sonic flow are well beyond the model's capabilities, as are supersaturated conditions. The combination of Volume and Inner Surface Area is used to determine the effective diameter, which is then used to determine the length. The Total Pipe Bend Angle is the sum in degrees of all the angles that the flow moves through. Thus one $90^{\circ}$ and two $45^{\circ}$ are summed as one $180^{\circ}$, and the value 180 is input. Clicking OK or CANCEL will return the user to the Transfer Pathways: Piping menu (screen number 2410). 


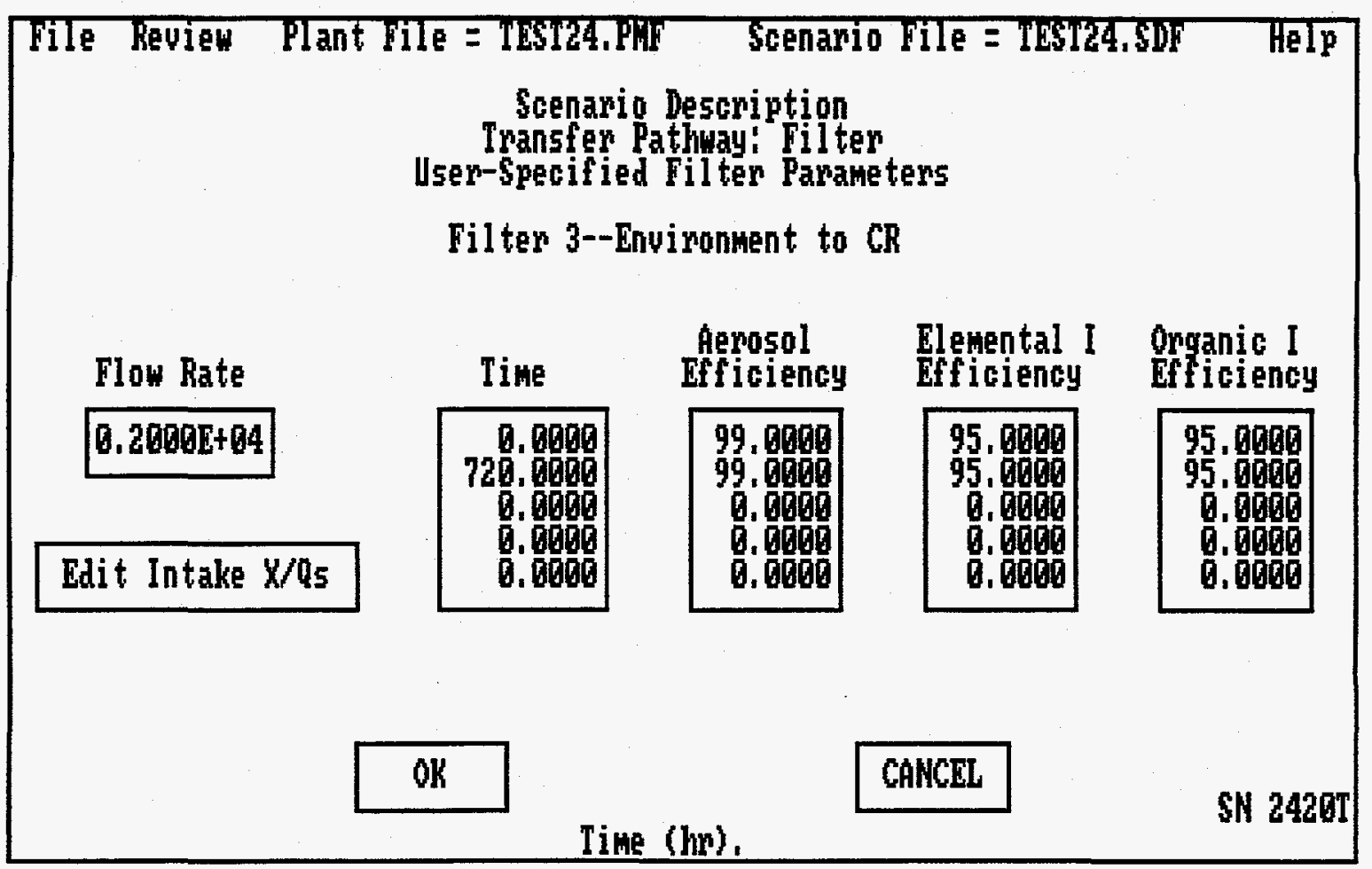

The User-Specified Filter Parameters are screen number 2420T. This screen allows the user to enter information about the removal efficiency of an air filter between compartments as a function of time ( $\mathrm{hr}$ ) and flow rate (cfm). The values for aerosol and iodine efficiencies are changed in a stepwise fashion at each Time that they are defined to change. The user must also verify the atmospheric relative concentration (dispersion), $\mathrm{X} / \mathrm{Q}$, to the intake. These values must exist at the inlet to the filter from the environment Multiple inlets to the same room will have the same X/Q. This capability is the only allowed transfer path from the environment. This option was exercised in acceptance test cases 14 and 14B. Clicking on OK or CANCEL will return the user to the Select Transfer Pathway menu (screen number 2400). 


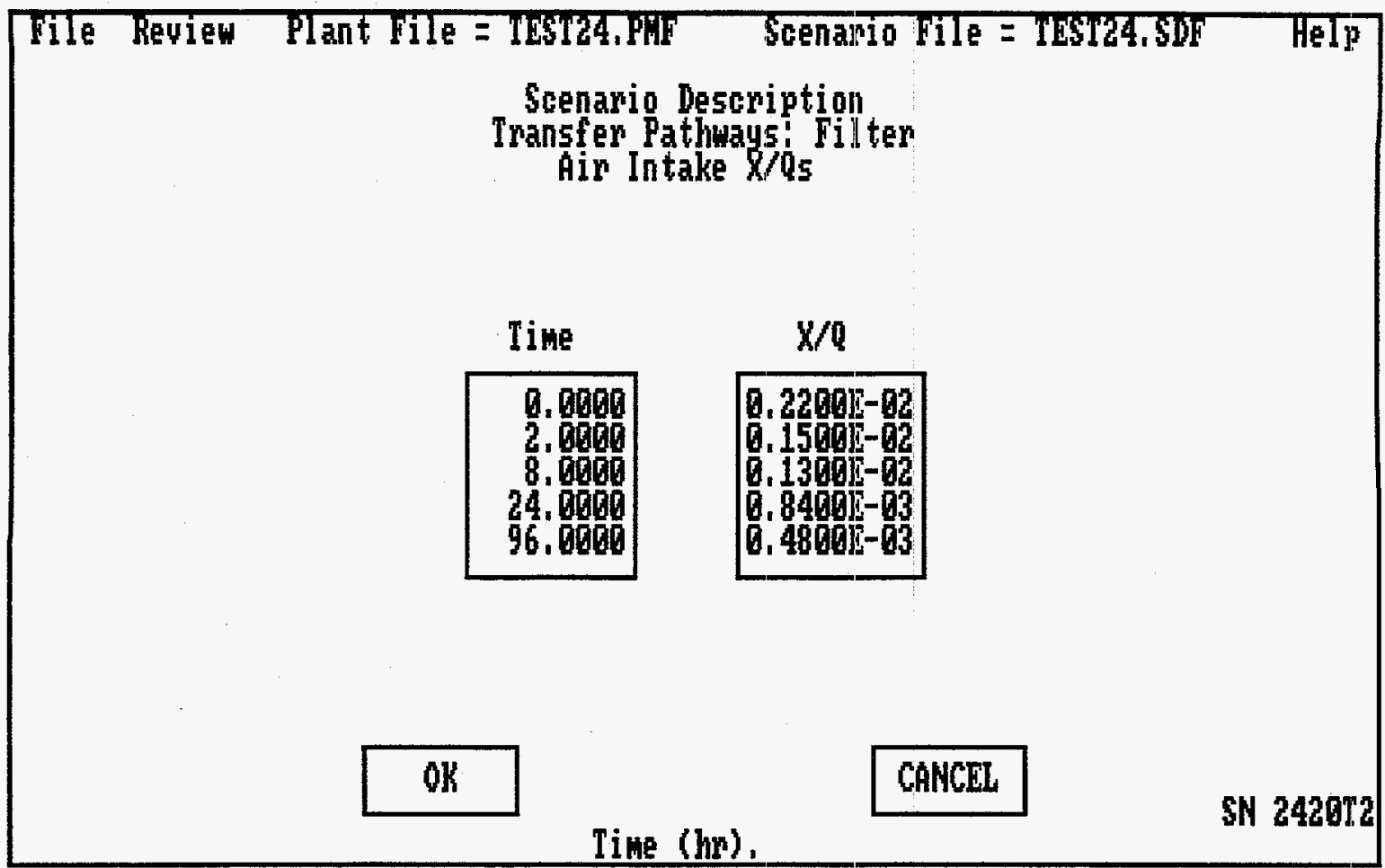

The Air Intake $X / Q$ s description is screen number 2420T2. This screen allows the user to input a set of $X / Q\left(s / \mathrm{m}^{3}\right)$ relative concentrations for the inlet. $X / Q$ has also been referred to as the atmospheric dispersion coefficient because it is a measure of the spread of the released mass from the release point to the receptor inlet. NUREG/CR-6331 [Ra95] details a method of calculation. This input allows the fission product concentration to be drawn into a compartment. Because of the code structure, all inlets from the environment to a compartment must have the same $\mathrm{X} / \mathrm{Q}$. This option was used in all acceptance tests that had control rooms. The X/Q will change in a stepwise fashion at each time that it is defined to change. Clicking on $\mathbf{O K}$ or CANCEL will return the user to the User-Specified Filter Parameters menu (screen number 2420T). 


File Review Plant File = IEST24.PhI Scenario File a IESL24.SDF Help
Scenario Description
Transfer Pathways: Suppression Pool
Suppression Pool

Select Models

Áerosol \langle\rangle
Elemental Iodine \langle\rangle
Onganic Iodine \langle\rangle

Edit Model Parameters

(1) Aerosol Renoval

(2) Elemental Iodine Removal

(3) Onganic Iodine Removal

(4) Return to Iransfer Pathways Menu

The Transfer Pathways: Suppression Pool menu is screen number 2430. This screen allows the user to include information on the removal of fission products by passing through a Suppression Pool. The user may specify information on Aerosol Removal, Elemental Iodine Removal, or Organic Iodine Removal from the pool. Any or all removal methods may be chosen. If the user does not choose a removal mechanism, the default is for no removal. Selecting Return to Transfer Pathways Menu returns the user to the Select Transfer Pathway menu (screen number 2400). 


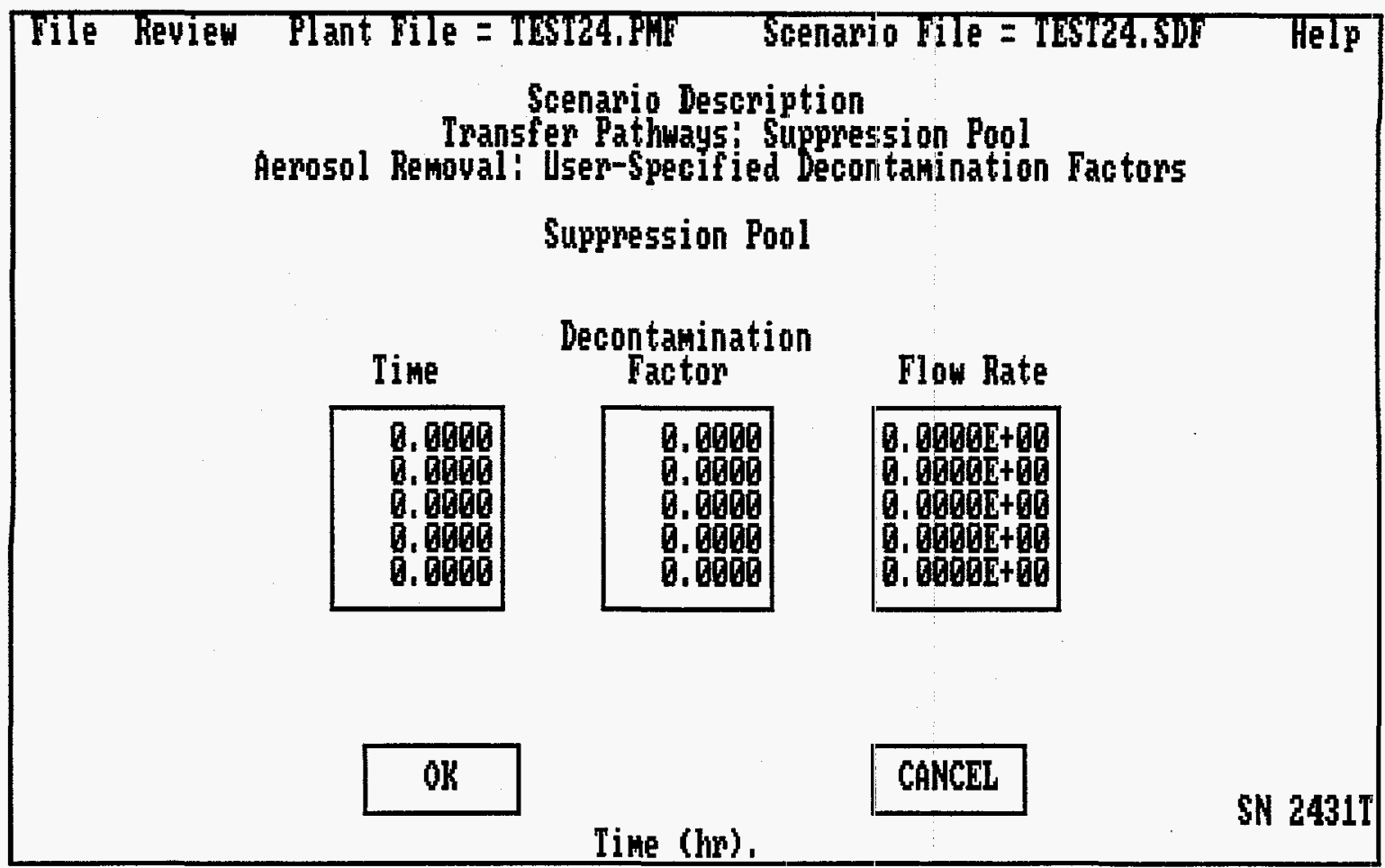

The User-Specified Decontamination Factors for aerosol removal are screen number 2431T. This allows the user to specify the time dependence of the aerosol decontamination factor for a particular aerosol flow rate in time. The Decontamination Factor and Flow Rate (cfm) will change in a stepwise fashion at each Time that they are defined to change. Decontamination factors are always $\geq 1.0$. Clicking on OK or CANCEL will return the user to the Transfer Pathways: Suppression Pool menu (screen number 2430). 


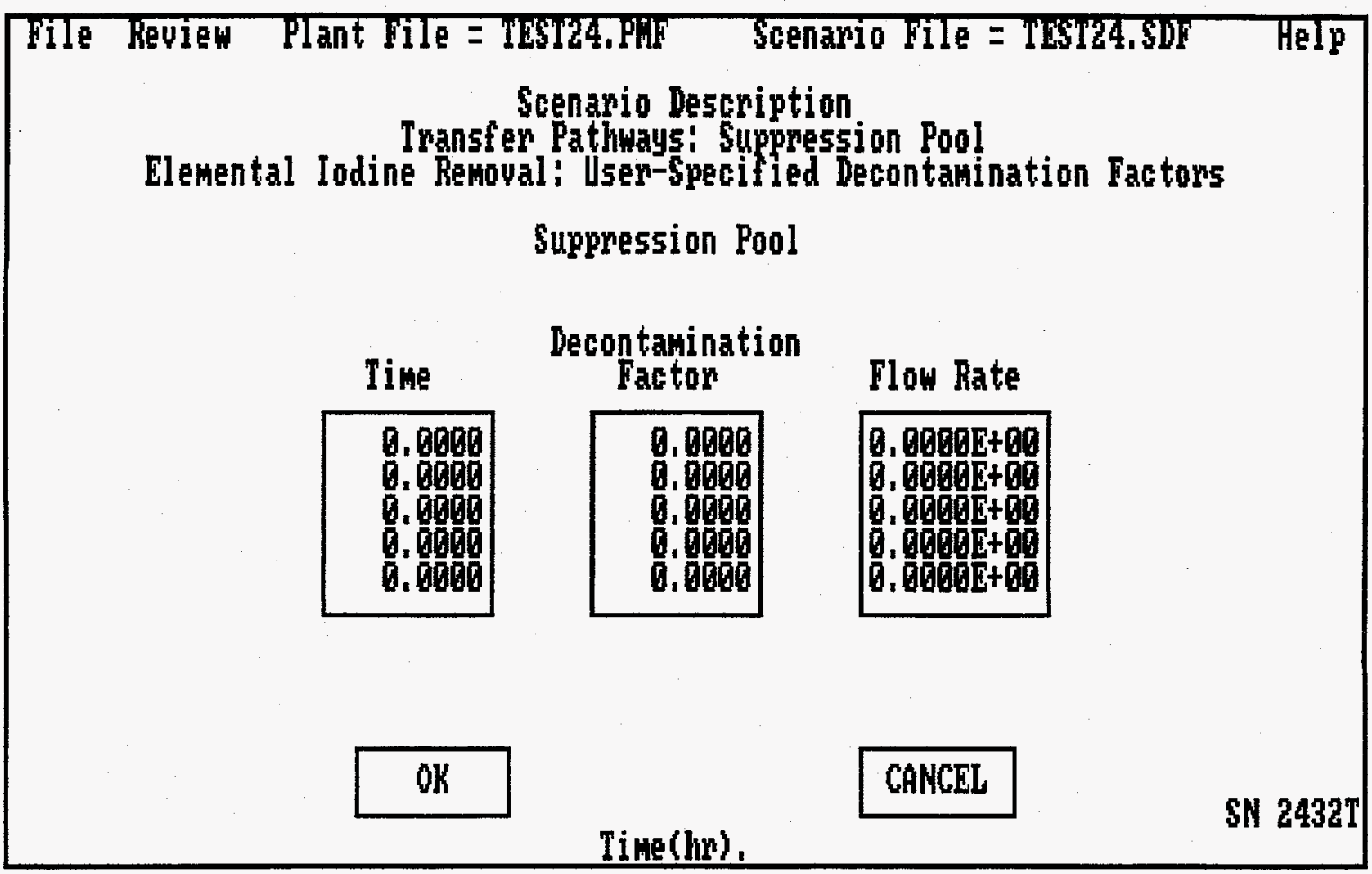

The User-Specified Decontamination Factors for elemental iodine removal are screen number 2432T. This screen allows the user to specify the time dependence of the elemental iodine decontamination factor for a particular elemental iodine flow rate $(\mathrm{cfm})$ in time. The Decontamination Factor and Flow Rate will change in a stepwise fashion at each Time they are defined to change. A minimum value of 1.0 is required for the decontamination factor. Clicking on OK or CANCEL will return the user to the Transfer Pathways: Suppression Pool menu (screen number 2430). 


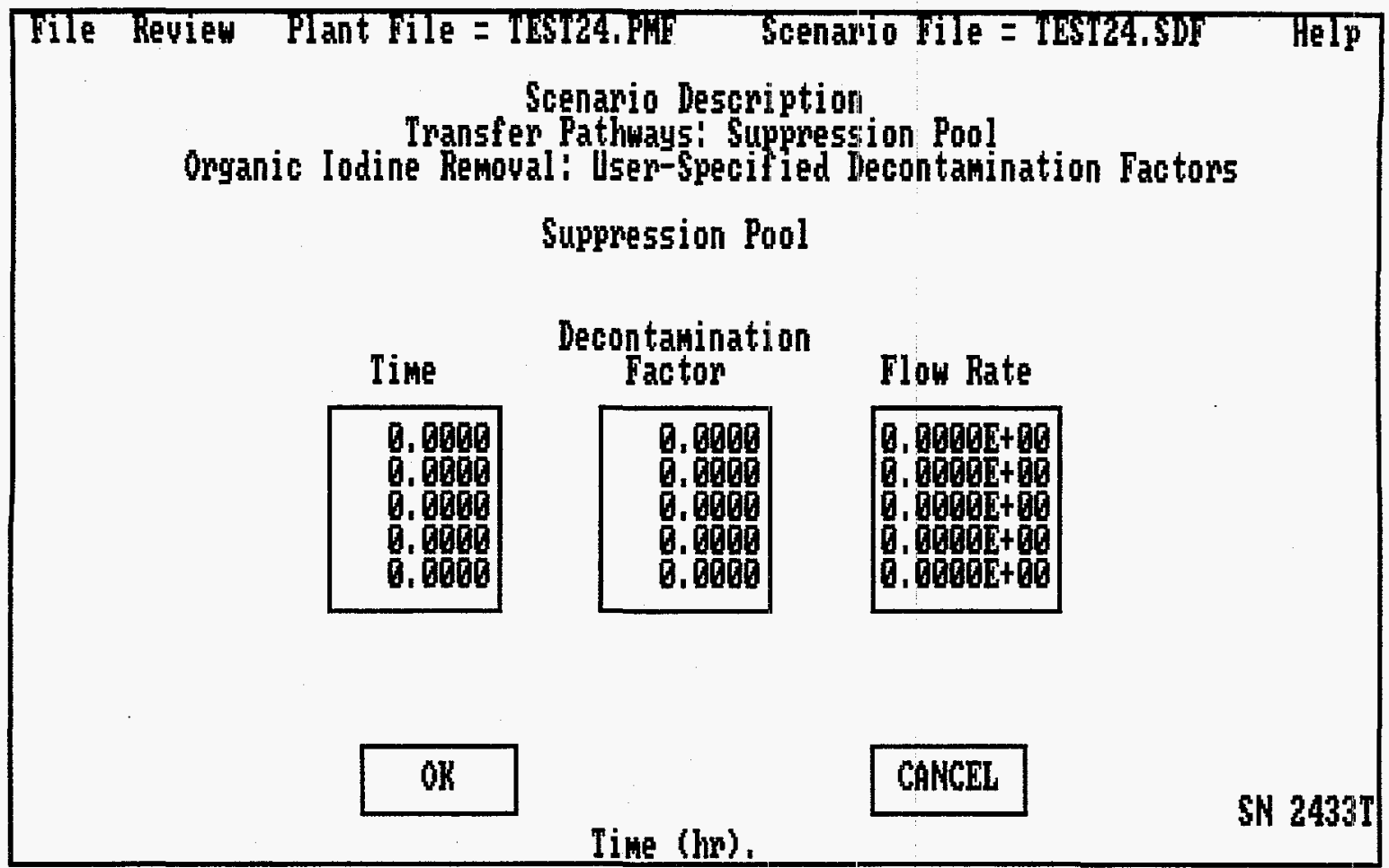

The User-Specified Decontamination Factors for organic iodine removal are screen number 2433T. This screen allows the user to specify the time dependence of the organic iodine decontamination factor for a particular organic iodine flow rate (cfm) in time. The Decontamination Factor and Flow Rate will change in a stepwise fashion at each Time they are defined to change. Clicking on OK or CANCEL will return the user to the Transfer Pathways: Suppression Pool menu (screen number 2430). 


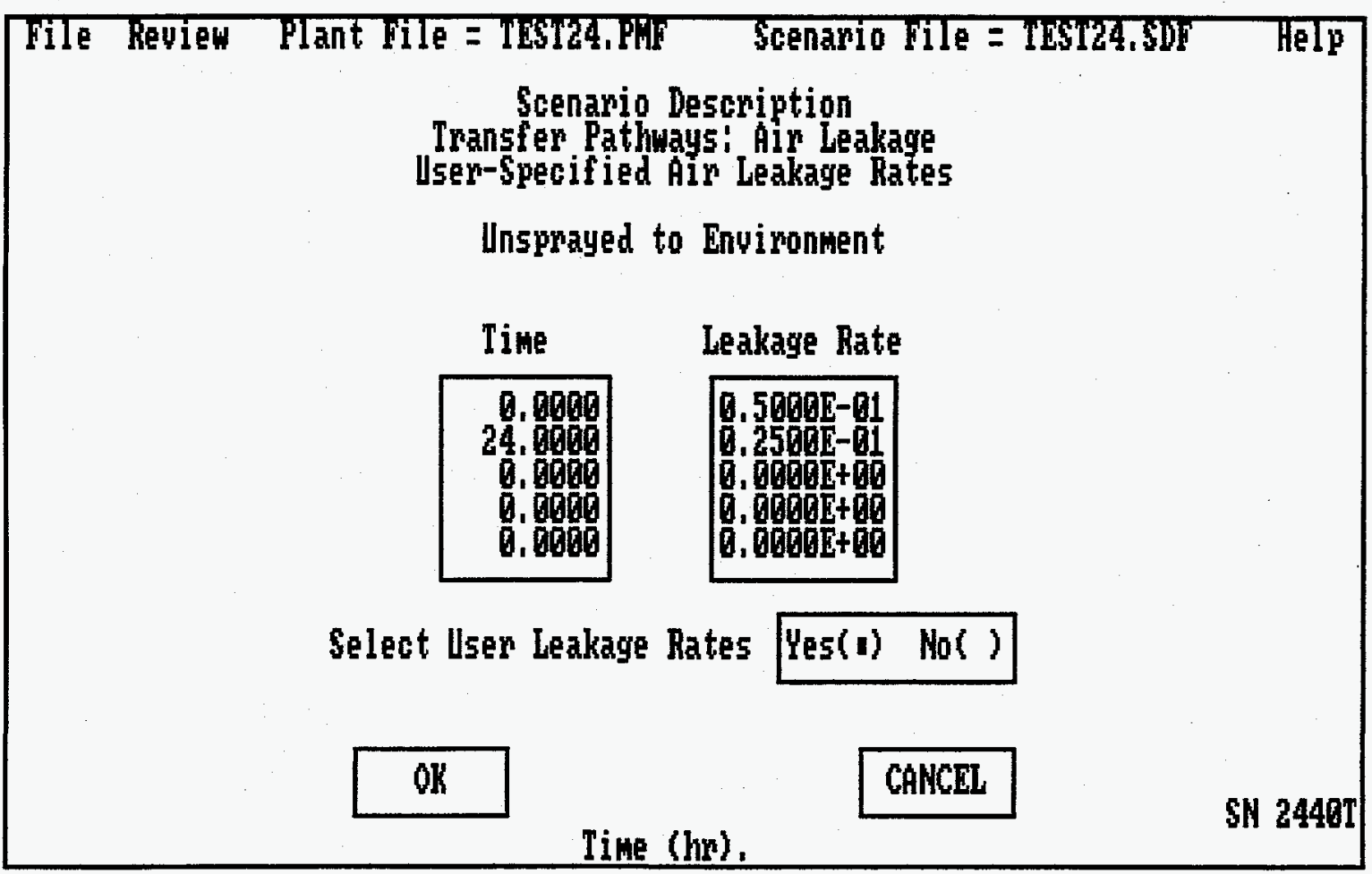

The User-Supplied Air Leakage Rates are screen number 2440T. The screen allows the user to specify the time-dependent convective flow rate from one compartment to another. This is the typical path used for containment leakage. It was used in assessment test cases 1-10 and 19-24. A typical value is $0.12 \%$ /day for a PWR and $1.8 \%$ /day for a BWR. The leakage rate (\%/day) will change in a stepwise fashion at each Time it is defined to change. The user must have the Select User Leakage Rates button on Yes for the code to use the tabular input. When it is set to No, the leak flow is always 0.0. Clicking on OK or CANCEL will return the user to the Select Transfer Pathways menu (screen number 2400). 


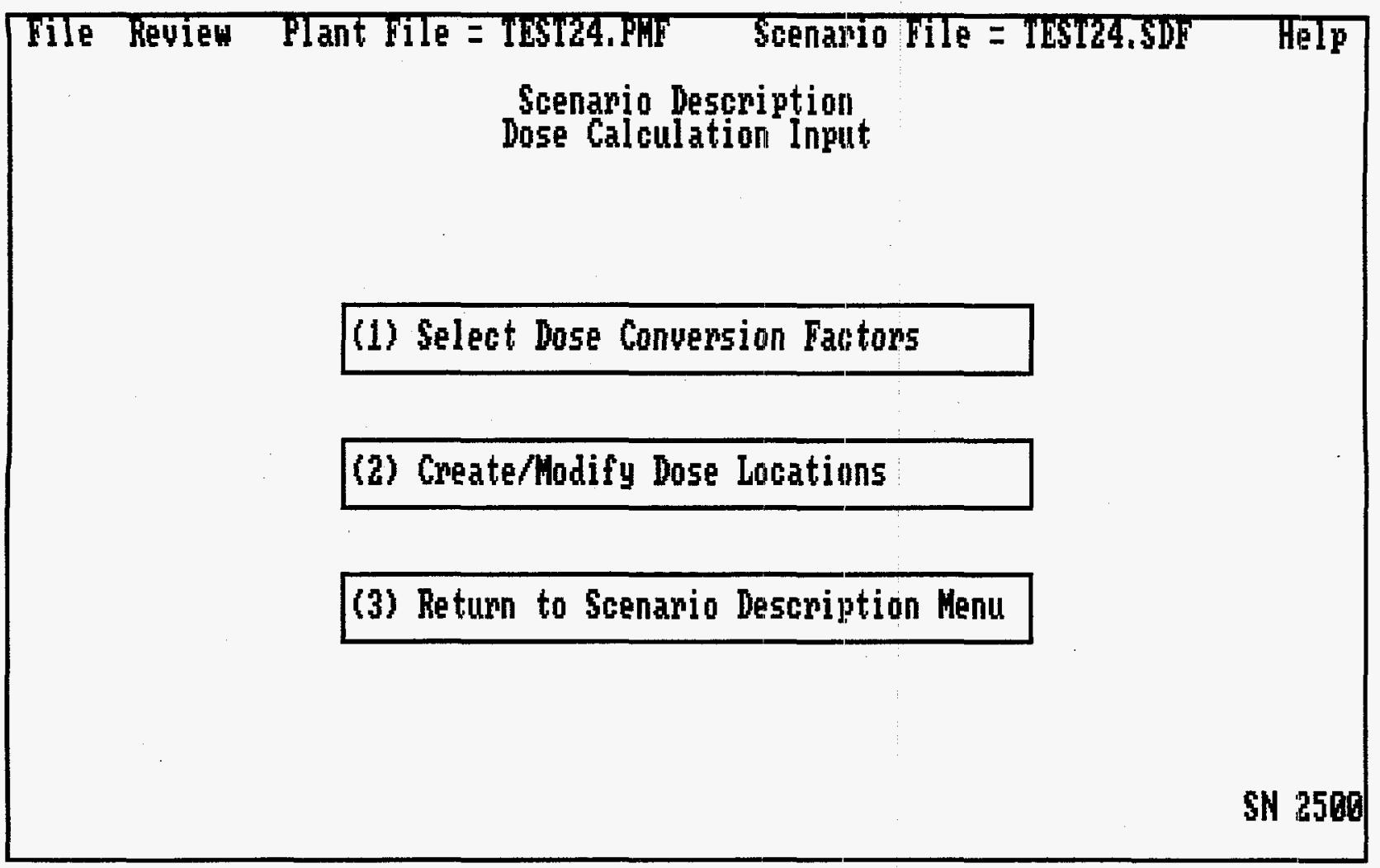

The Dose Calculation Input menu is screen 2500. This screen allows the user access to the input that can affect the dose resulting from a known concentration. The user must go through each subscreen. Selecting Return to Scenario Description Menu returns the user to screen number 2000. 


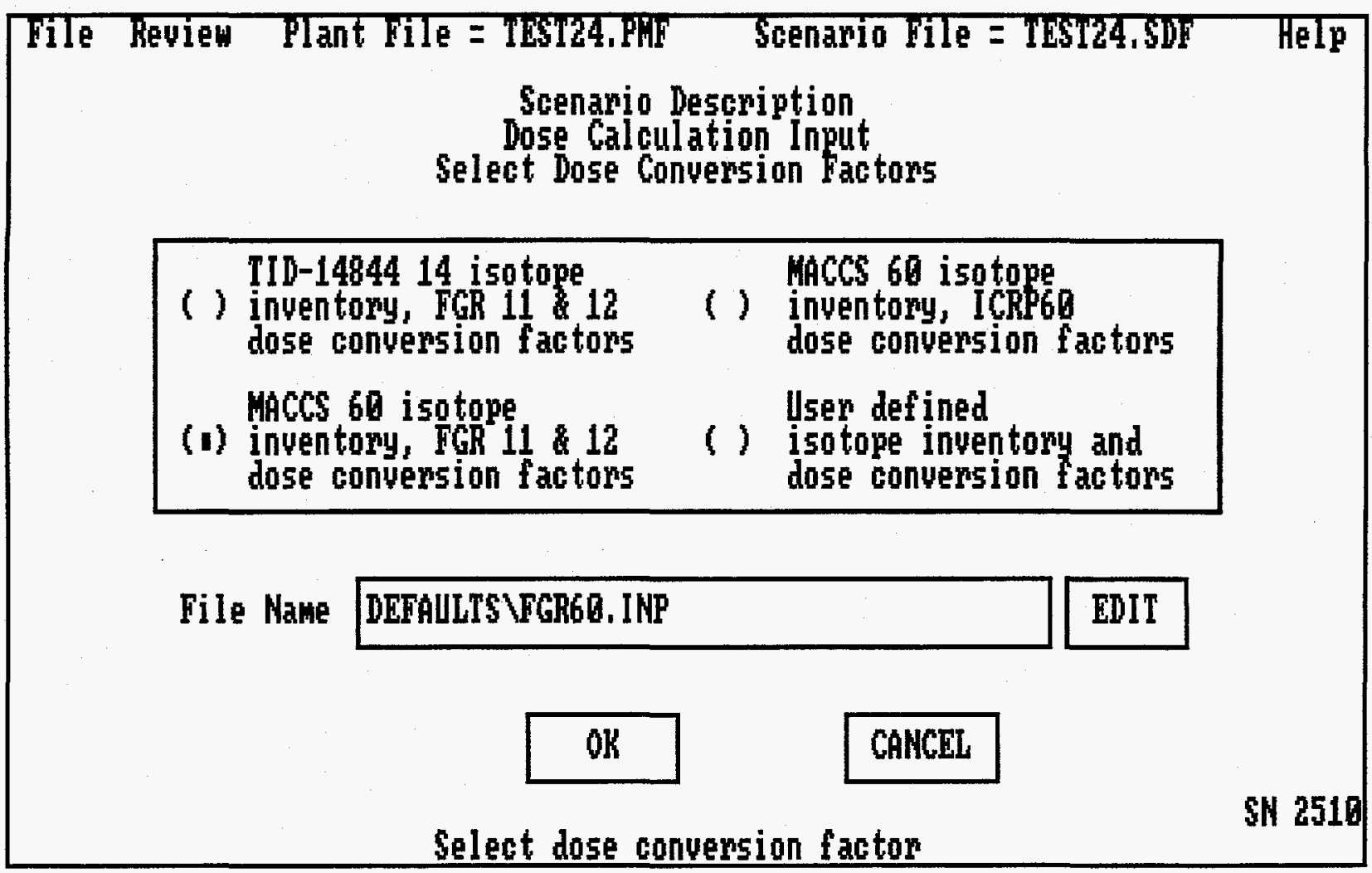

The Select Dose Conversion Factors menu is screen 2510. This screen allows the user access to the dose conversion factors used to relate concentration and dose. Typical conversion factor tables are discussed in Section 1.4.3.4. Selecting a conversion table will return the user to the Dose Calculation Input menu (screen number 2500). 


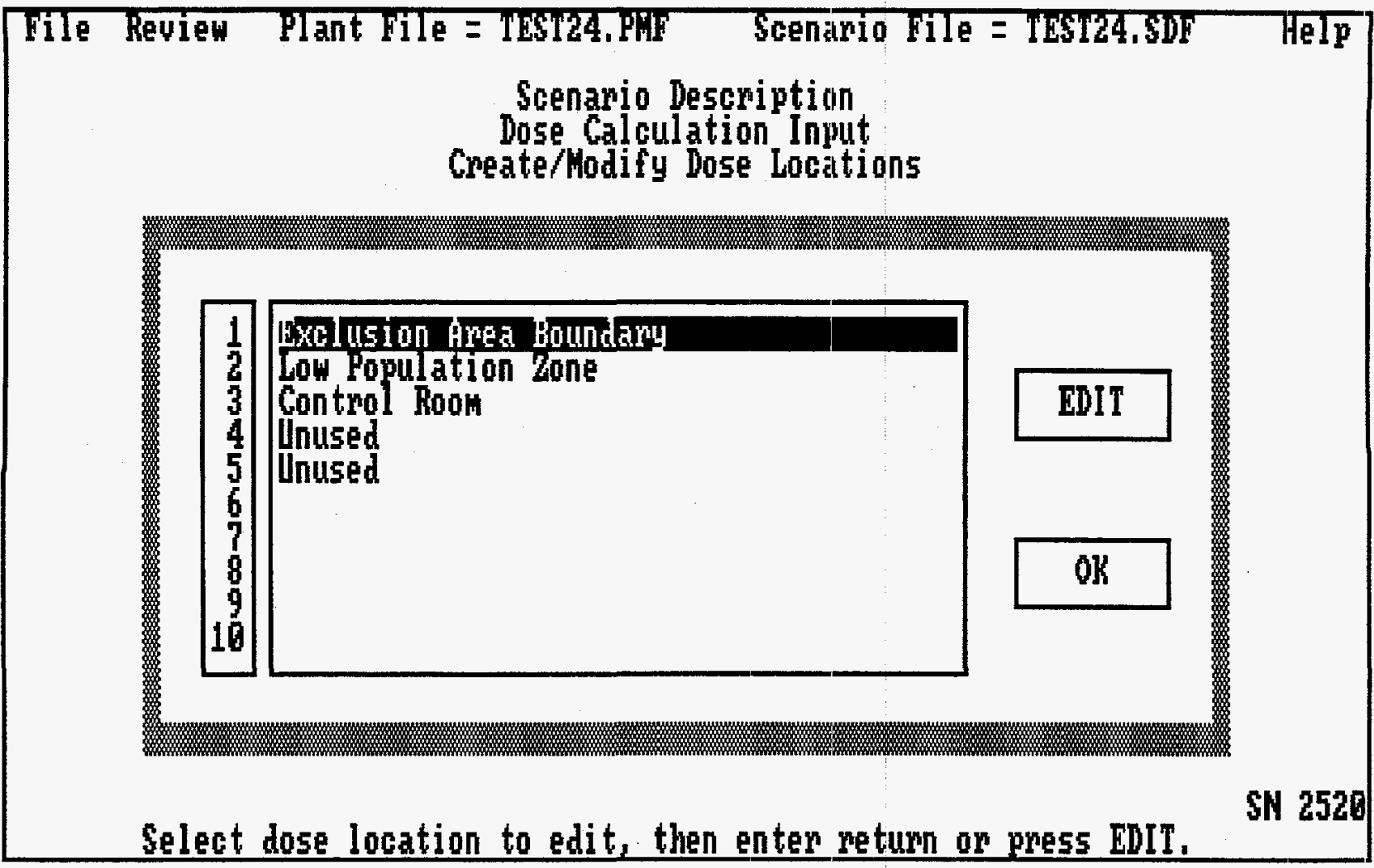

The Create/Modify Dose Locations selection menu is screen number 2520. This screen allows the user to access the locations where dose calculations are to be performed. It is invoked when the user selects (5) on the Scenario Description menu. The user will specify unique names for each of the locations. Highlighting the next "Unused" and clicking an EDIT will transfer control to a subscreen in which more detailed information is entered for that specific location. Clicking on OK will return the user to the Dose Calculation Input menu (screen number 2500). 


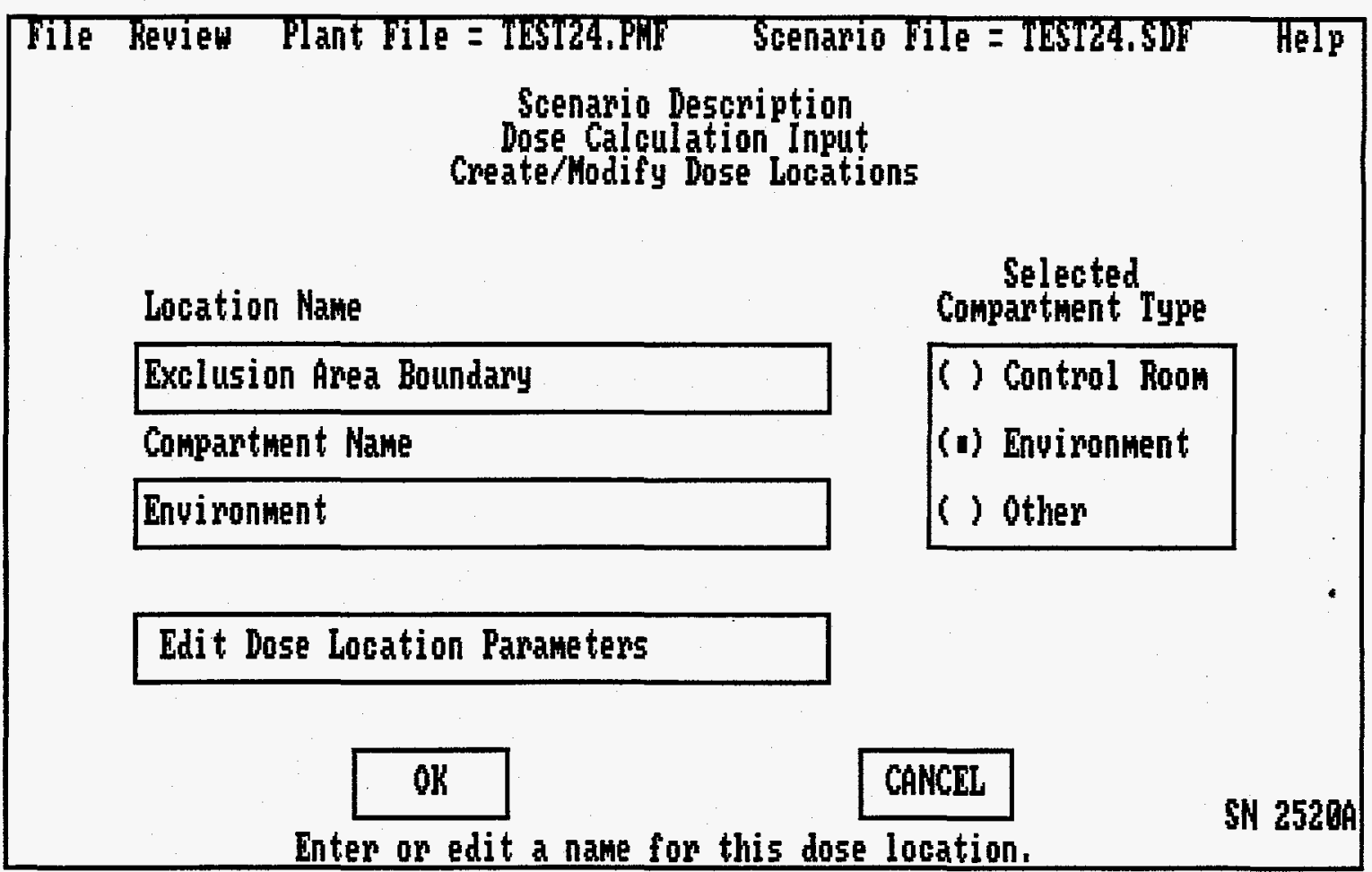

The Create/Modify Dose Locations are screen number 2520A, This screen allows the user to input dose information for a particular location, for example, the low population zone. The Location Name is chosen by the user and will be listed on the output file when detailing dose results. The compartment that contains the location is chosen from the list of compartments that is obtained by double clicking on the Compartment Name and will bring up the subscreen list. Clicking on OK or CANCEL will return the user to the Create/Modify Dose Locations selection menu (screen number 2500). 


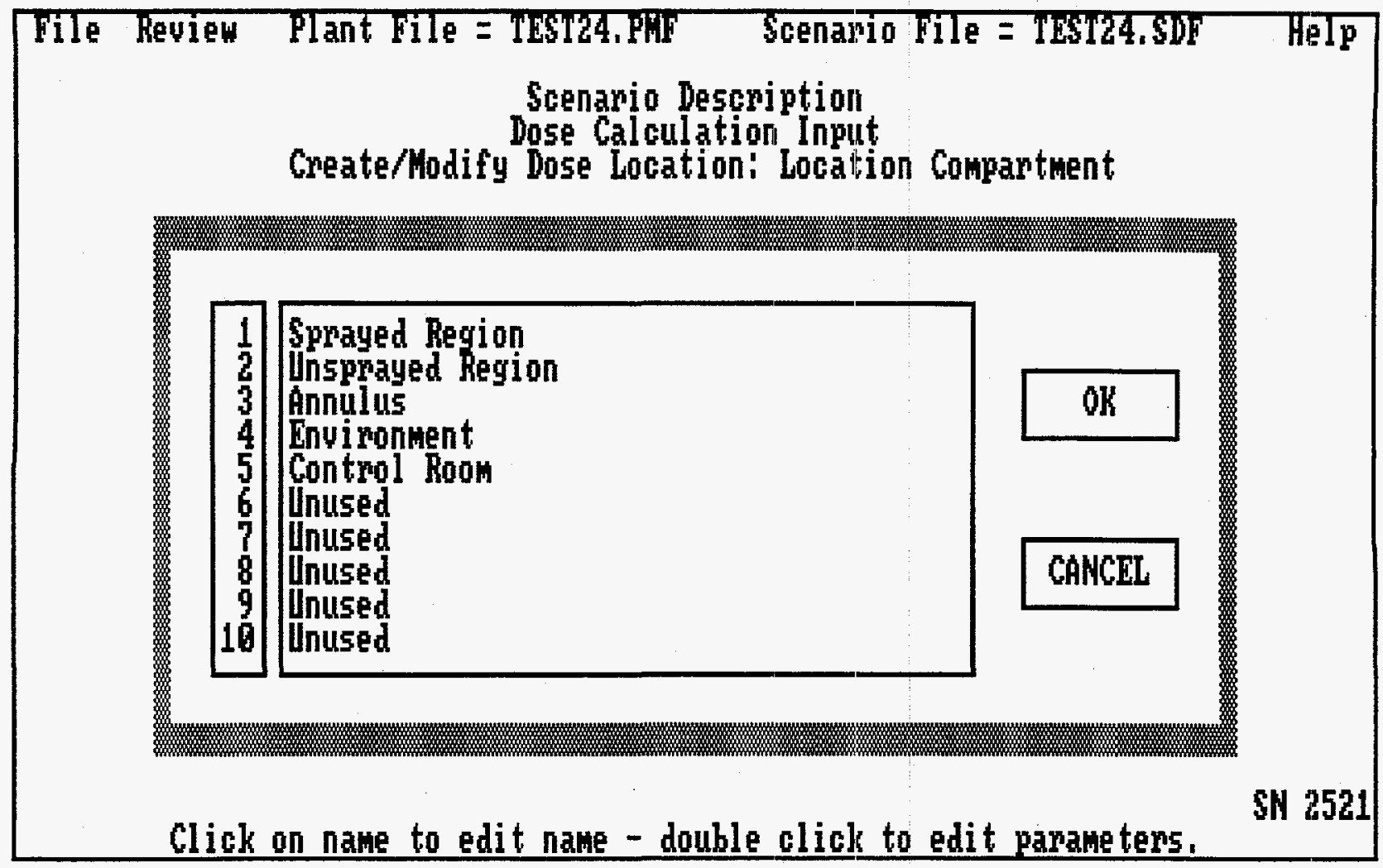

The Create/Modify Dose Location: Location Compartment menu is screen number 2521. It allows the user to select a plant model compartment within which a dose is located. Clicking on OK or CANCEL will return the user to the Create/Modify Dose Locations menu (screen number 2520A). 


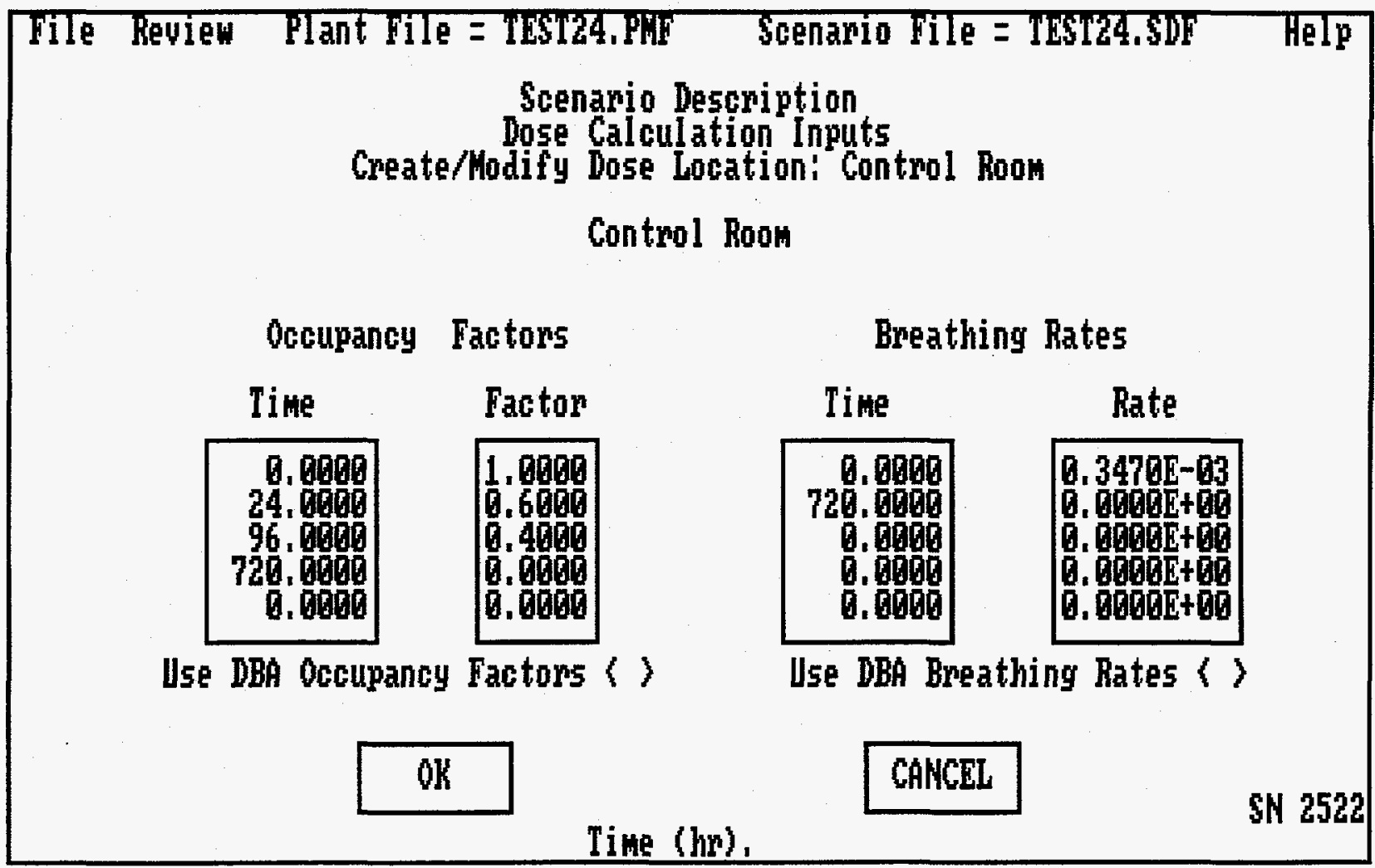

The Create/Modify Dose Location: Control Room is screen number 2522. This screen allows the user to input occupancy factors and breathing rates for a control room. The fission products typically enter through inlets. The $\mathrm{X} / \mathrm{Q}$ s to all the inlets to a control room are a constant set of time-dependent values as given on screen number 2420T2. The Breathing Rates $\left(\mathrm{m}^{3} / \mathrm{s}\right)$ and Occupancy Factors will change in a stepwise fashion each time they are defined to change. Clicking on OK or on CANCEL will return the user to the Create/Modify Dose Location screen (screen number 2520A). 


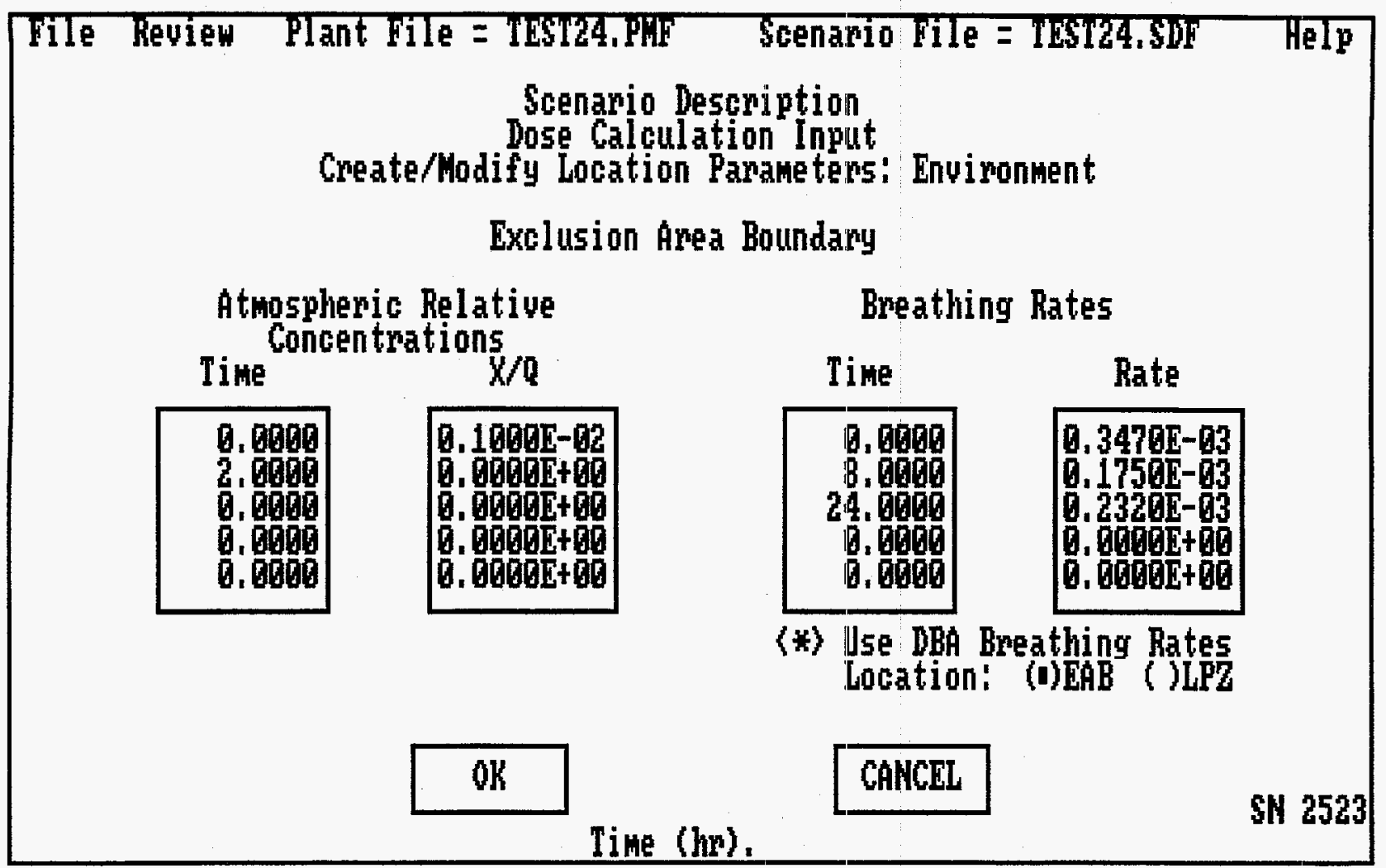

The Create/Modify Location Parameters: Environment description is screen number 2523. This screen allows the user to input the Atmospheric Relative Concentration for the environment location where a dose will be calculated. This screen is activated by selecting an environment dose and double clicking on the Edit Dose Location Parameters pad of screen 2520A. This is typically for the low population zone or exclusion area boundary. Values for X/Q vary with reactor and may be calculated using models similar to those in ARCON [Ra95]. The X/Q $\left(\mathrm{s} / \mathrm{m}^{3}\right)$ and Breathing Rate $\left(\mathrm{m}^{3} / \mathrm{s}\right)$ will change in a stepwise fashion at each time they are defined to change. Clicking on OK or CANCEL will return the user to the Create/Modify Dose Locations menu (screen number 2520A). 


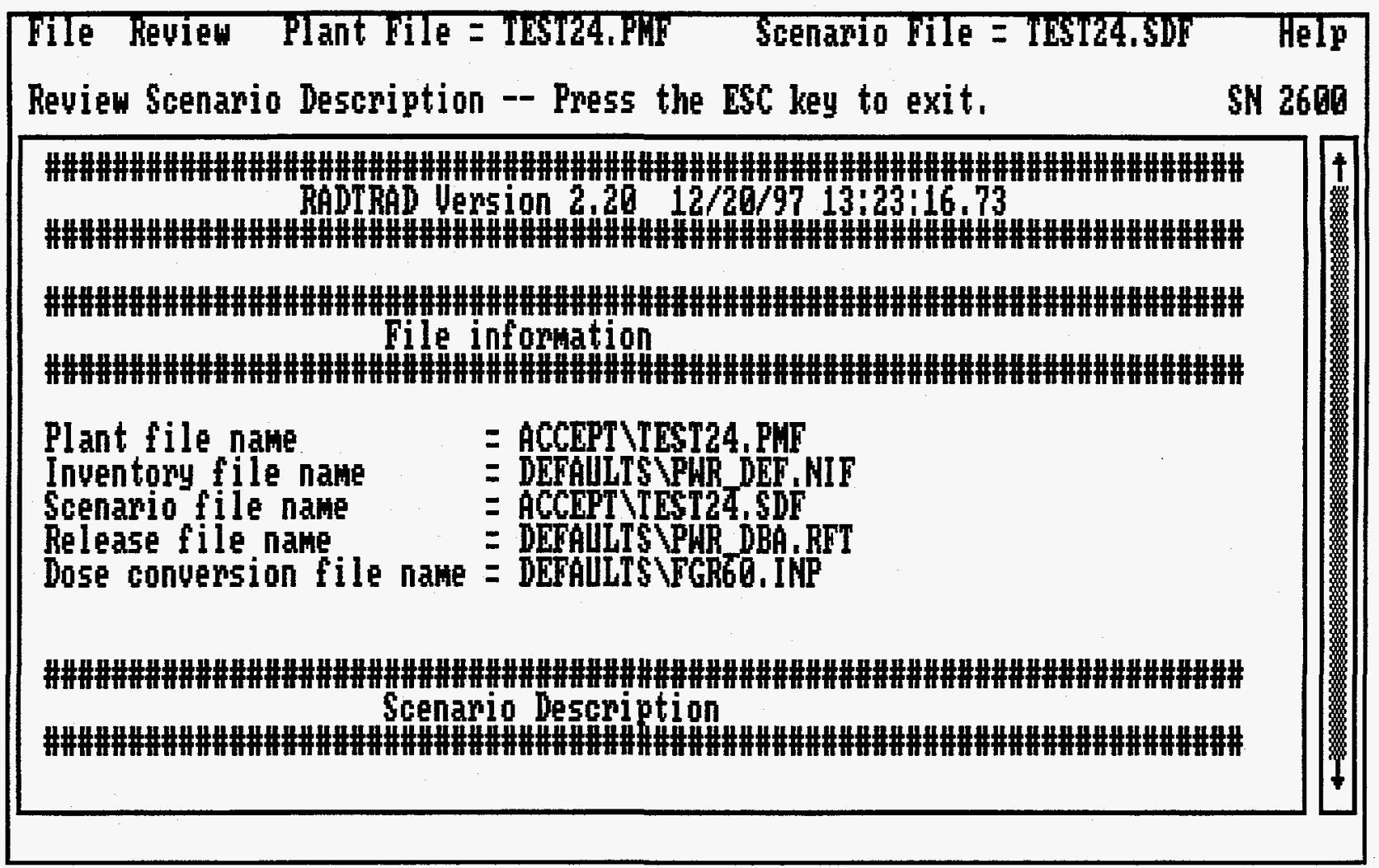

The Review Scenario Description is screen number 2600. This screen allows the user to review the current scenario. The user returns to the Scenario Description menu (screen 2000) by using the escape key (ESC) on the keyboard. 


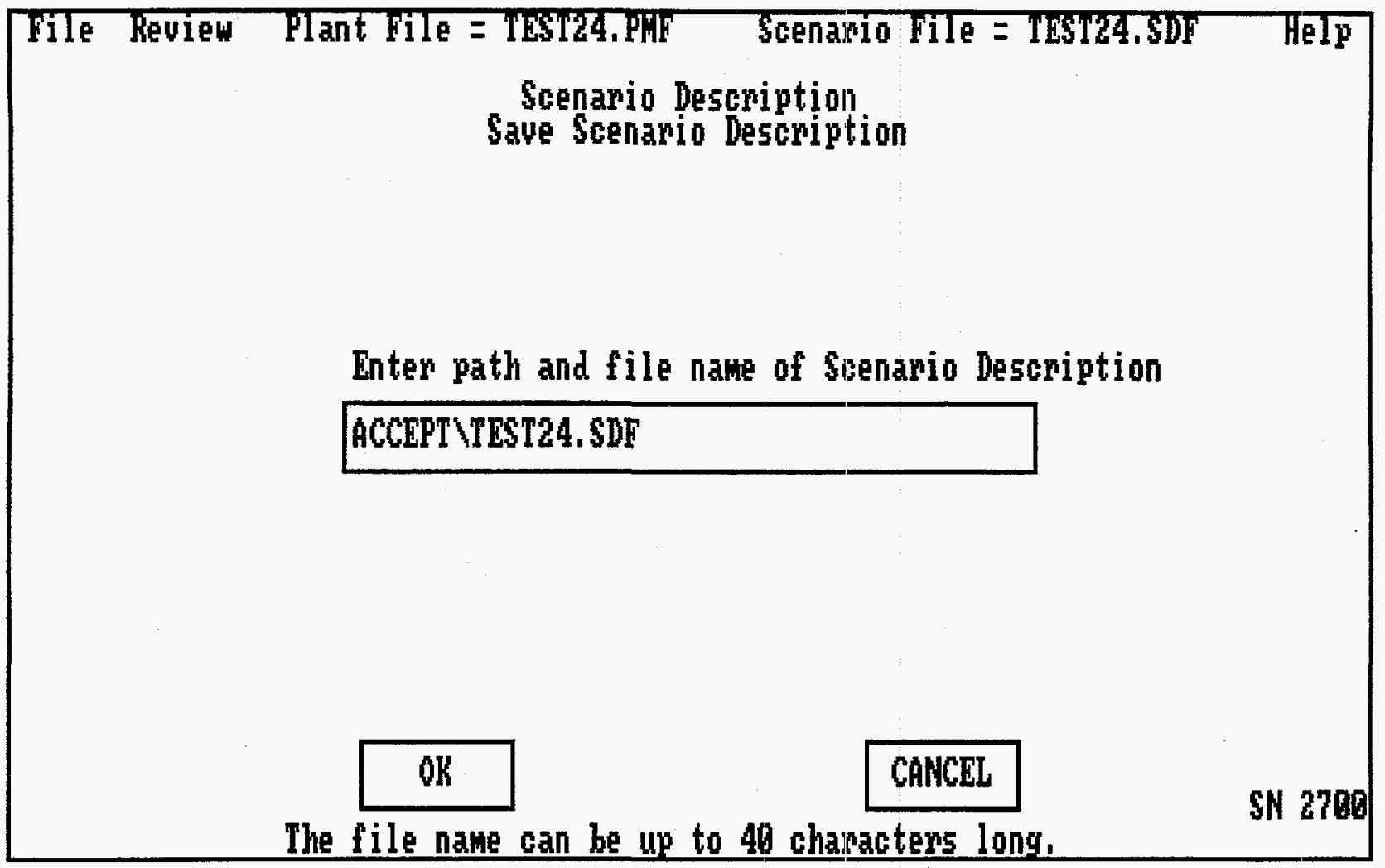

The Save Scenario Description is screen number 2700. This screen allows the user to specify a file name for the scenario description. It is invoked when the user selects Save Scenario Description on screen 2000. Note the convention of XXX.SDF where XXX is the user description and SDF stands for scenario description file. Clicking on OK or CANCEL will return the user to the Scenario Description menu (screen number 2000). 


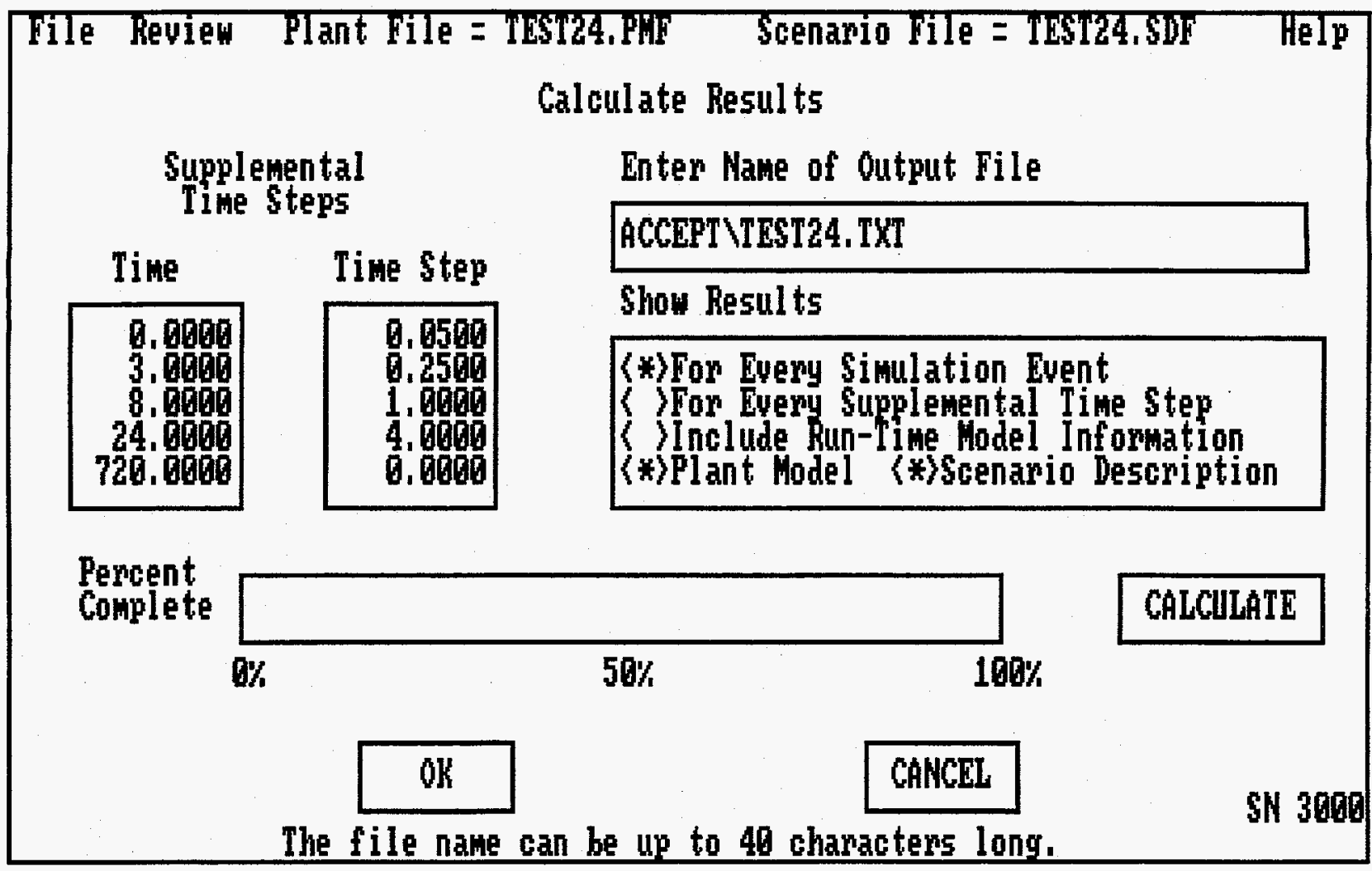

The Calculate Results menu is screen number 3000. This screen allows the user to control the execution of the code and output. The time step tables allow intermediate (supplemental) results to be calculated by making the time steps finer, for example:

$\begin{array}{rc}\text { Time } & \text { Time Step } \\ 0.0 & 0.25 \\ 4.0 & 1.0 \\ 24.0 & 4.0 \\ 720.0 & 0\end{array}$

With the table shown, the error will probably decrease by $10-15 \%$, at some increase in time. If a more accurate result is required, reduce the step accordingly, perhaps running the entire transient at $0.25 \mathrm{hr}$. However, this will significantly increase the running time.

It is recommended that the user adjust his output file name to match the PMF and SDF files used as input, perhaps XXX.TXT. The Show Results box controls the output and is explained in Section 1.5. The dose results are shown For Every Simulation Event if it is checked. Time step results can be edited by clicking the For Every Supplemental Time Step button. The Include Run Time Model Information option edits the effective removal coefficient for any models (such as the Powers spray model) that are active. The Plant Model and Scenario Description options edit the input on the output file when selected. The Percent Complete tab will go from light to dark as the calculation proceeds and allows the user to see how quickly the results are calculated. The user must click on CALCULATE to start the run. Clicking on OK or on CANCEL will return the user to the Main menu (screen number 0000). 


\section{File keview Plant file = TEST24.PHF Scenario File = IEST24.SDF Help \\ Examine Resul ts}

Enter the output file name

ACCEPT\TES24. TXT

EXAHINE

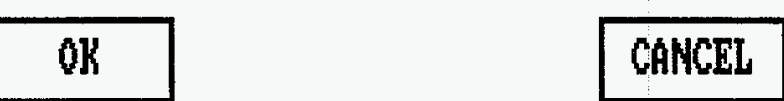

The file name can be up to 40 characters long,

The Examine Results menu is screen number 4000. This screen allows the user to edit a file, usually the output file. This screen can also be used as another way into the file directories with the ability to scan any file without leaving RADTRAD. Clicking on OK or CANCEL will return the user to the Main menu (screen number 0000). 


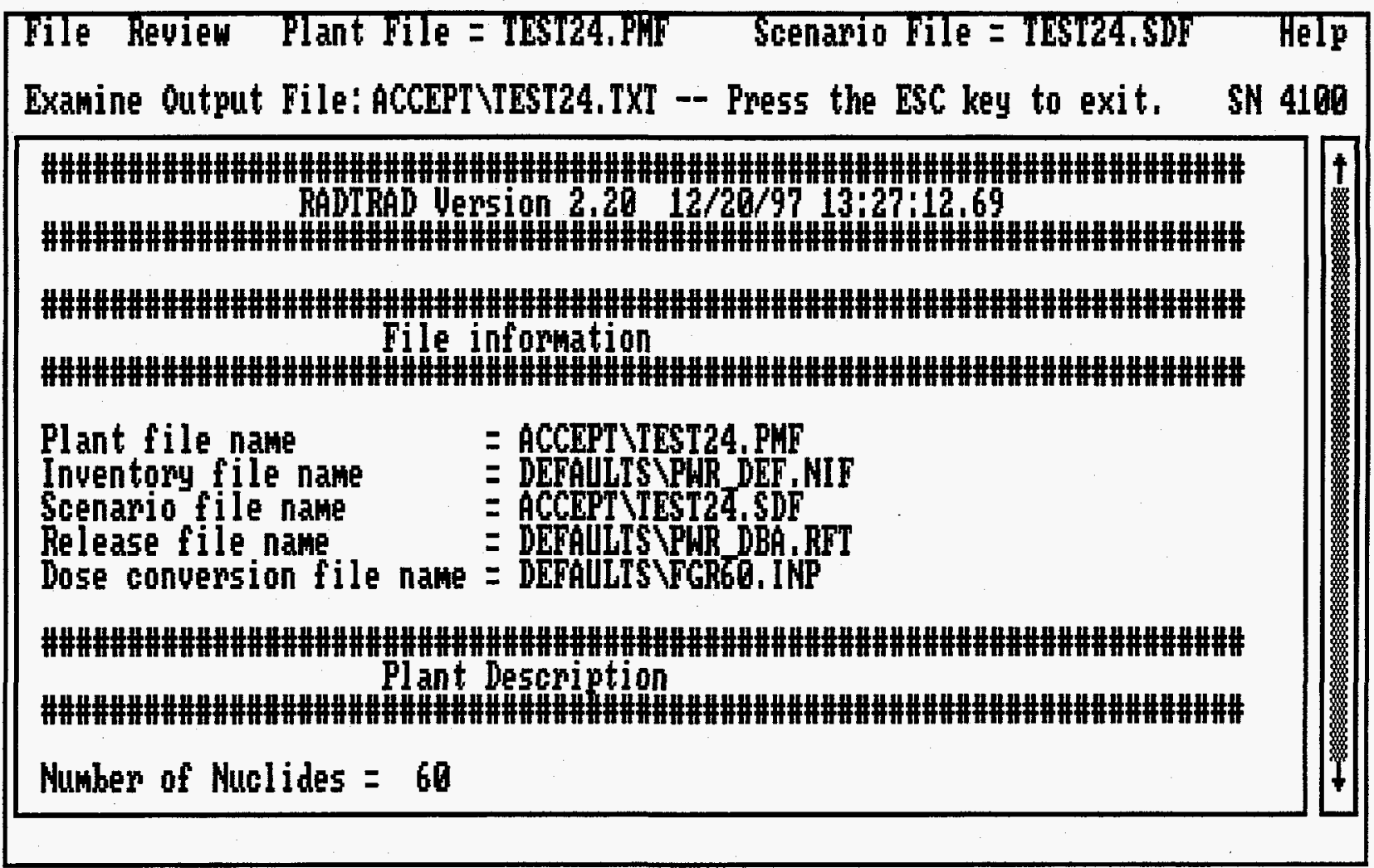

The Examine Output File is screen number 4100 . This screen allows the user to examine the output in the output file by use of the scroll bar on the right-hand side of the screen. Return to the Examine Results screen (screen number 4000) is by using the escape key (ESC) on the keyboard. 


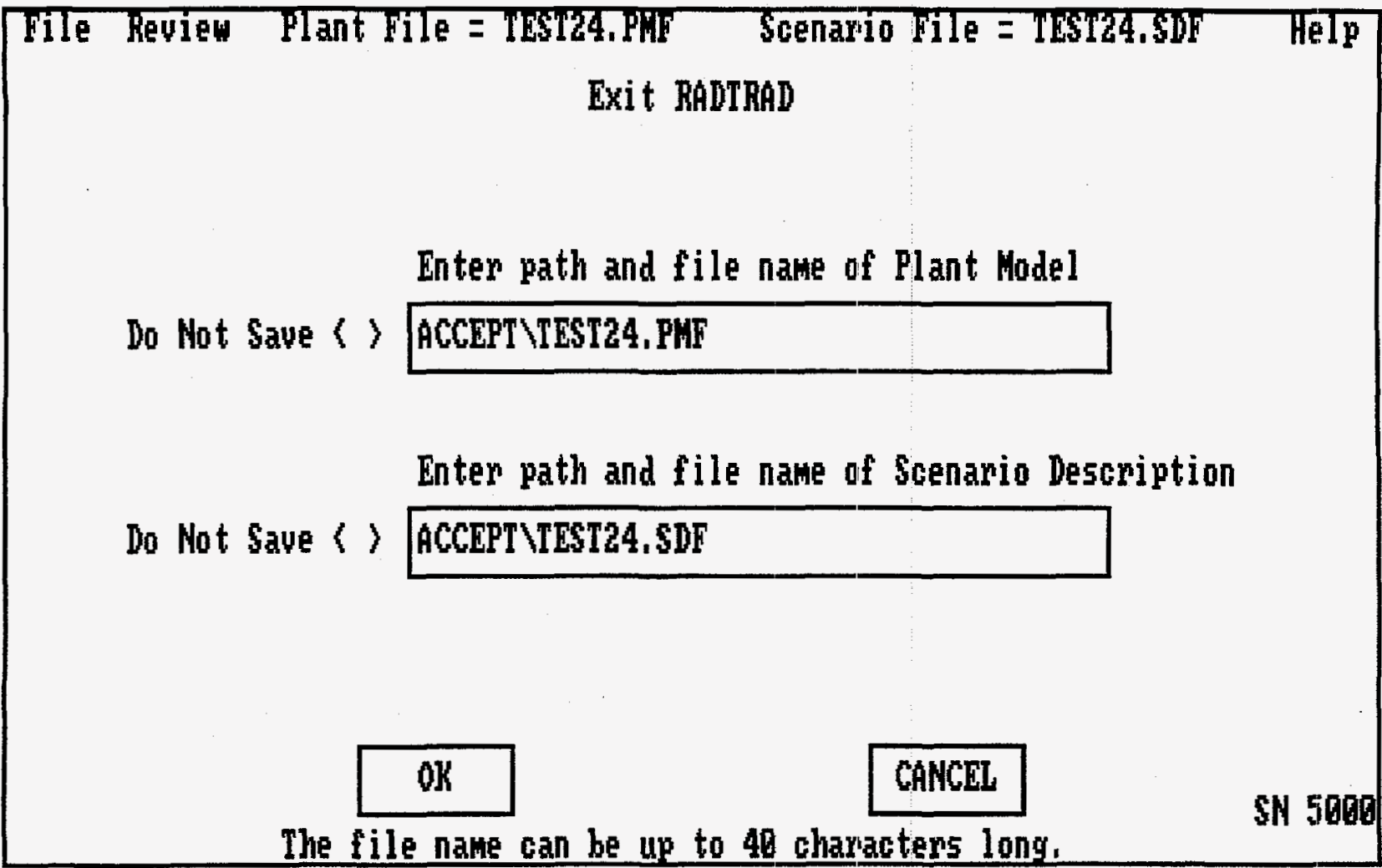

Exit RADTRAD is screen number 5000. Entering the names and clicking on OK will result in the plant model and/or scenario descriptions being saved to those files. Clicking on the Do Not Save tab and then clicking on OK will result in the information not being saved. Clicking on OK will return the user to the DOS command line, terminating RADTRAD. Clicking on CANCEL will return the user to the Main menu (screen number 0000). 


\subsubsection{Files Associated with the Source Term}

RADTRAD supports two sources for postulated releases of fission products: "Calculation of Distance Factors for Power and Test Reactor Sites," TID-14844 [Di62], and "Accident Source Terms for Light-Water Nuclear Power Plants," NUREG-1465 [So95]. These documents detail timing and release fractions of particular elemental fission products for postulated accidents. The elemental release to dose consequence calculation requires that a particular isotopic distribution be known. In the sections that follow, the release fraction data are described, then the nuclide half-life list, then sample inventories [Ca93], and then standard inventory to dose files [FGR11 and 12]. All of these files are stored as part of the defaults subdirectory when loading the code as described in Sections 1.1.1 or 1.1.2.

\subsubsection{Release Fraction and Timing Files}

Five release fraction and timing tables are normally used by RADTRAD. Chronologically, the oldest release pattern is that described in TID-14844 [Di62]. This pattern is a puff release of all the nobles and a fraction of the iodines. It is detailed in Table 1.4.3.1-1.

Tables 1.4.3.1-2 and 1.4.3.1-3 detail the release fractions and timing for a severe accident in either a PWR or a BWR as given in NUREG-1465. In these cases, a fraction of the core inventory is released during four time periods: gap, early in-vessel, ex-vessel, and late in-vessel. The last two occur simultaneously but the latter occurs for an extended period of time. Note that the difference between a design basis accident (DBA) and a severe accident is timing. In the DBA scenario, the vessel does not fail, so the last two release periods are excluded. Thus there are two PWR-1465 files: PWR_DEF, which uses four release periods, and PWR_DBA, which uses the first two. A similar pattern exists for the BWR files. 
Table 1.4.3.1-1 TID Release Fraction and Timing

Release Fraction and Timing Name:

TID, TID-14844, Table IV., w/Reg Guide $1.3 \& 1.4$ Mods

CHEMICAL GROUP GAP In-Vessel Ex-Vessel Late In-

Vessel

$0.1000 \mathrm{E}-01 \quad 0.0000 \mathrm{E}+00 \quad 0.0000 \mathrm{E}+00 \quad 0.0000 \mathrm{E}+00$

1. Noble Gases: $0.1000 \mathrm{E}+01 \quad 0.0000 \mathrm{E}+00 \quad 0.0000 \mathrm{E}+00 \quad 0.0000 \mathrm{E}+00$

2. Iodine: $\quad 0.2500 \mathrm{E}+00 \quad 0.0000 \mathrm{E}+00 \quad 0.0000 \mathrm{E}+00 \quad 0.0000 \mathrm{E}+00$

3. Cesium: $\quad 0.0000 E+00 \quad 0.0000 E+00 \quad 0.0000 E+00 \quad 0.0000 E+00$

4. Tellurium: $\quad 0.0000 \mathrm{E}+00 \quad 0.0000 \mathrm{E}+00 \quad 0.0000 \mathrm{E}+00 \quad 0.0000 \mathrm{E}+00$

5. Strontium: $\quad 0.0000 \mathrm{E}+00 \quad 0.0000 \mathrm{E}+00 \quad 0.0000 \mathrm{E}+00 \quad 0.0000 \mathrm{E}+00$

6. Barium: $\quad 0.0000 E+00 \quad 0.0000 E+00 \quad 0.0000 E+00 \quad 0.0000 E+00$

7. Ruthenium: $\quad 0.0000 \mathrm{E}+00 \quad 0.0000 \mathrm{E}+00 \quad 0.0000 \mathrm{E}+00 \quad 0.0000 \mathrm{E}+00$

8. Cerium: $\quad 0.0000 E+00 \quad 0.0000 E+00 \quad 0.0000 E+00 \quad 0.0000 E+00$

9. Lanthanum: $\quad 0.0000 \mathrm{E}+00 \quad 0.0000 \mathrm{E}+00 \quad 0.0000 \mathrm{E}+00 \quad 0.0000 \mathrm{E}+00$

Nonradioactive $\quad 0.0000 E+00 \quad 0.0000 E+00 \quad 0.0000 E+00 \quad 0.0000 E+00$ aerosols ( $\mathrm{kg})$ :

End of Release File

Note: These values are stored as file "TID_DEF.RFT" 
Table 1.4.3.1-2 PWR-NUREG-1465 Release Fraction and Timing

Release Fraction of Core Inventories and Timings:

PWR, NUREG-1465, Tables 3.13, February 1995

DURATION (hr)

CHEMICAI GROUP GAP In-Vessel Ex-Vessel Late In-

Vessel

\begin{tabular}{lllll} 
& $0.5000 \mathrm{E}+00$ & $0.1300 \mathrm{E}+01$ & $0.2000 \mathrm{E}+01$ & $0.1000 \mathrm{E}+02$ \\
\hline 1. Noble Gases: & $0.5000 \mathrm{E}-01$ & $0.9500 \mathrm{E}+00$ & $0.0000 \mathrm{E}+00$ & $0.0000 \mathrm{E}+00$ \\
2. Iodine: & $0.5000 \mathrm{E}-01$ & $0.3500 \mathrm{E}+00$ & $0.2500 \mathrm{E}+00$ & $0.1000 \mathrm{E}+00$ \\
3. Cesium: & $0.5000 \mathrm{E}-01$ & $0.2500 \mathrm{E}+00$ & $0.3500 \mathrm{E}+00$ & $0.1000 \mathrm{E}+00$ \\
4. Tellurium: & $0.0000 \mathrm{E}+00$ & $0.0500 \mathrm{E}+00$ & $0.2500 \mathrm{E}+00$ & $0.5000 \mathrm{E}-02$ \\
5. Strontium: & $0.0000 \mathrm{E}+00$ & $0.2000 \mathrm{E}-01$ & $0.1000 \mathrm{E}+00$ & $0.0000 \mathrm{E}+00$ \\
6. Barium: & $0.0000 \mathrm{E}+00$ & $0.2000 \mathrm{E}-01$ & $0.1000 \mathrm{E}+00$ & $0.0000 \mathrm{E}+00$ \\
7. Ruthenium: & $0.0000 \mathrm{E}+00$ & $0.2500 \mathrm{E}-02$ & $0.2500 \mathrm{E}-03$ & $0.0000 \mathrm{E}+00$ \\
8. Cerium: & $0.0000 \mathrm{E}+00$ & $0.5000 \mathrm{E}-03$ & $0.5000 \mathrm{E}-02$ & $0.0000 \mathrm{E}+00$ \\
9. Lanthanum: & $0.0000 \mathrm{E}+00$ & $0.2000 \mathrm{E}-03$ & $0.5000 \mathrm{E}-02$ & $0.0000 \mathrm{E}+00$ \\
$\begin{array}{l}\text { 10. } \\
\text { Nonradioactive } \\
\text { aerosols (kg): }\end{array}$ & $0.0000 \mathrm{E}+00$ & $0.0000 \mathrm{E}+00$ & $0.0000 \mathrm{E}+00$ & $0.0000 \mathrm{E}+00$
\end{tabular}

Note: These values are stored in file "PWR_DEF.RFT." A similar file with 0.0 for all values in the last 2 columns representing a design basis release is stored in file "PWR_DBA.RFT." 
Table 1.4.3.1-3 BWR NUREG-1465 Release Fraction and Timing

Release Fraction of Core Inventory and Timings:

BWR, NUREG-1465, Table 3.12

February 1995

CHEMICAL GROUP

DURATION (hr)

GAP In-Vessel Ex-Vessel Late In-

Vessel

$\begin{array}{llll}0.5000 E+00 & 0.1500 E+01 & 0.3000 E+01 & 0.1000 E+02\end{array}$

1. Noble Gases: $\quad 0.5000 \mathrm{E}-0 \mathrm{I} \quad 0.9500 \mathrm{E}+00 \quad 0.0000 \mathrm{E}+00$

2. Iodine: $\quad 0.5000 \mathrm{E}-01 \quad 0.2500 \mathrm{E}+00 \quad 0.3000 \mathrm{E}+00 \quad 0.1000 \mathrm{E}-01$

3. Cesium:

$0.5000 E-01$

$0.2000 E+00$

$0.3500 E+00$

$0.1000 E-01$

4. Tellurium:

$0.0000 E+00$

$0.5000 E-01$

$0.2500 E+00$

$0.5000 \mathrm{E}-02$

5. Strontium:

$0.0000 \mathrm{E}+00$

$0.2000 E-01$

$0.1000 \mathrm{E}+00$

$0.0000 E+00$

6. Barium:

$0.0000 \mathrm{E}+00$

$0.2000 E-01$

$0.1000 E+00$

$0.0000 \mathrm{E}+00$

7. Ruthenium:

$0.0000 E+00$

$0.2500 E-02$

$0.2500 E-02$

$0.0000 E+00$

8. Cerium:

$0.0000 E+00$

$0.5000 E-03$

$0.5000 E-02$

$0.0000 E+00$

9. Lanthanum:

$0.0000 E+00$

$0.2000 E-03$

$0.5000 \mathrm{E}-02$

$0.0000 \mathrm{E}+00$

10.

$0.0000 E+00$

$0.0000 E+00$

$0.0000 E+00$

$0.0000 E+00$

Nonradioactive aerosols (kg):

Note: These values are stored in file "BWR_DEF.RFT." A similar file with 0.0 for all values in the last 2 columns representing a design basis release is stored in file "BWR_DBA.RFT." 


\subsubsection{Nuclide Inventory}

The initial mass $\left(c_{1}\right)$ of each isotope is required to calculate a dose. Given the initial mass and a fractional release, the concentration of an isotope at a location can be determined. The masses are stored in these tables as a function of the reactor power so they can be adjusted for the power level of the reactor under consideration. The fractional releases are given in the previous section as a function of the chemical group, and within these tables, the chemical group as a function of the isotope is given. These tables were also used to relate the isotope to the element and to provide decay and daughter data. The data have been taken from the TID-14844 report and from sample calculations used by MACCS [Ch 90] that are based upon ORIGEN calculations.

There are three default files: the 14-isotope, 3-element TID-14844-related data (Table 1.4.3.2-1) and the PWR and BWR 60-isotope, 9-element NUREG-1465 data (Tables 1.4.3.2-2.3). The isotopes chosen are those selected in WASH-1400 [75] with the additional 6 isotopes added by Alpert [A186] and used in the MACCS code [Ch90]. In addition to the TID and NUREG-1465 sample inventories, a set of inventories containing only ${ }^{131} \mathrm{I}$ is also stored in the defaults subdirectory.

The structure of the files is:

Nuclide inventory name

NUREG or TID

Power level

1.0 Normalized core power

Number of nuclides

$\mathrm{XX}$

Nuclide number

Isotope

Chemical group (from Tables 1.4.3.1-1.3)

Half-life

Atomic weight

Inventory/Power (Ci/MWth)

Daughter isotope

Fraction

Daughter isotope

Fraction

Daughter isotope

Fraction

Next Nuclide Number 
Table 1.4.3.2-1

Nuclide Inventory Name:

TID-14844 Example Normalizeä Core Inventory

Power Level:

$0.1000 E+01$

Nuclides :

14

Nuclide 001 :

I-13I

2

$0.6946560000 E+06$

$0.1310 \mathrm{E}+03$

$0.2453 \mathrm{E}+05$

$\mathrm{Xe}-131 \mathrm{~m} \quad 0.1100 \mathrm{E}-01$

none

$0.0000 E+00$

none

$0.0000 E+00$

Nuclide 002 :

I-132

2

$0.8280000000 E+04$

$0.1320 E+03$

$0.9527 E+05$

none

$0.0000 \mathrm{E}+00$

none

$0.0000 E+00$

none

Nuclide 003 :

$0.0000 E+00$

I-133

2

$0.7488000000 E+05$

$0.1330 \mathrm{E}+03$

$0.5651 E+05$
$\mathrm{Xe}-133 \mathrm{~m}$
$0.2900 E-01$
$\mathrm{Xe}-133$
$0.9700 E+00$
none
$0.0000 E+00$

Nuclide 004:

$I-134$

$$
2
$$

$0.3156000000 E+04$

$0.1340 \mathrm{E}+03$

$0.6445 E+05$

none

$0.0000 E+00$

none

$0.0000 E+00$

none

$0.0000 E+00$

Nuclide 005 :

$I-135$

2

$0.2379600000 \mathrm{E}+05$

$0.1350 \mathrm{E}+03$

$0.5225 \mathrm{E}+05$

$\mathrm{Xe}-135 \mathrm{~m}$

$0.1500 \mathrm{E}+00$

$\mathrm{Xe}-135$

none

$0.8500 E+00$

Nuclide 006 :

$0.0000 E+00$

$\mathrm{Xe}-131 \mathrm{~m}$

1

$0.1028160000 E+07$

$0.1310 E+03$

$0.2487 \mathrm{E} \div 03$

none

$0.0000 E+00$ 


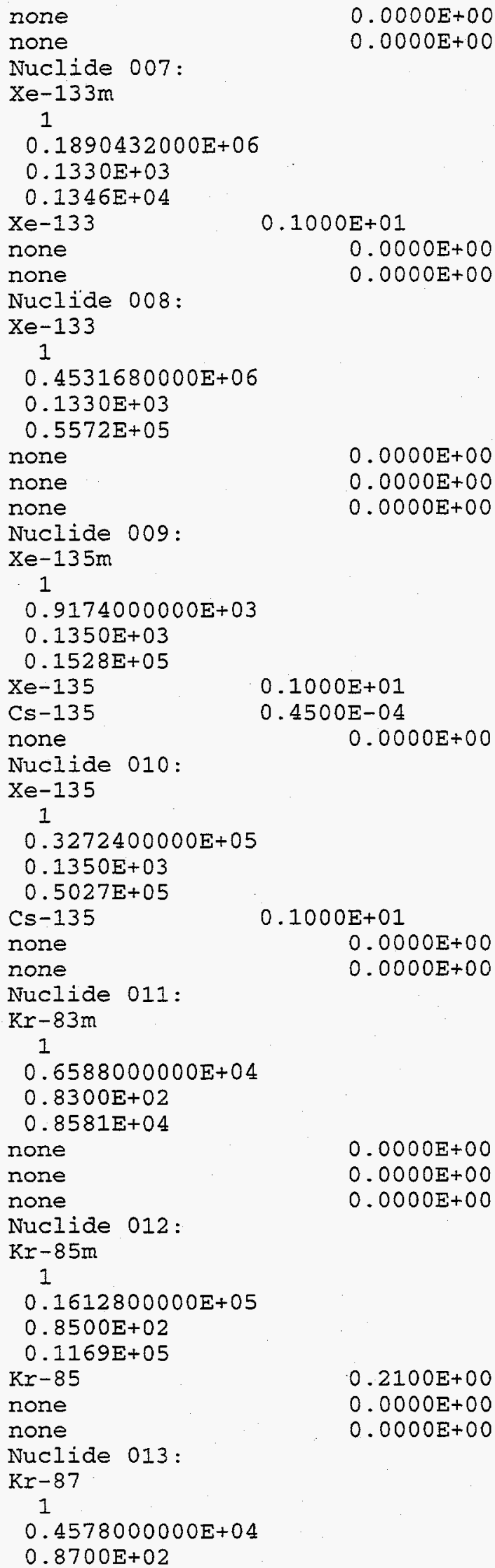




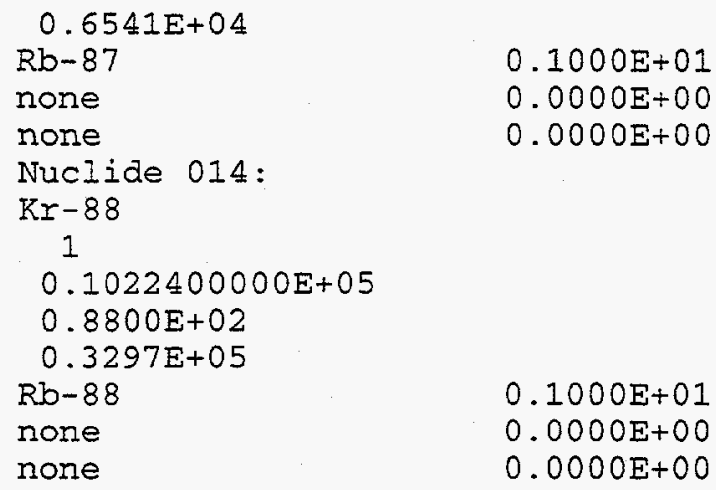


Table 1.4.3.2-2

Nuclide Inventory Name:

Normalized MACCS Sample 3412 MWth PWR Core Inventory Power Level:

$0.1000 \mathrm{E}+01$

Nuclides:

60

Nuclide 001 :

Co-58

7

$0.6117120000 E+07$

$0.5800 \mathrm{E}+02$

$0.2553 E+03$

none

$0.0000 E+00$

none

$0.0000 \mathrm{E}+00$

none

$0.0000 \mathrm{E}+00$

Nuclide 002 :

Co- 60

7

$0.1663401096 \mathrm{E}+09$

$0.6000 E+02$

$0.1953 E+03$

none

none

$0.0000 E+00$

none

$0.0000 E+00$

Nuclide 003 :

$0.0000 E+00$

$\mathrm{K} r-85$

1

$0.3382974720 E+09$

$0.8500 E+02$

$0.1960 E+03$

none

none

none

Nuclide 004:

$\mathrm{Kr}-85 \mathrm{~m}$

1

$0.1612800000 E+05$

$0.8500 E+02$

$0.9181 E+04$

$\mathrm{Kr}-85$

none

none

Nuclide 005 :

$\mathrm{Kr}-87$

1

$0.4578000000 E+04$

$0.8700 \mathrm{E}+02$

$0.1678 \mathrm{E} \div 05$

$\mathrm{Rb}-87$

$0.1000 E+01$

none

$0.0000 E+00$

none

$0.0000 E+00$

Nuclide 006 :

$\mathrm{Kr}-88$

1

$0.1022400000 \mathrm{E}+05$

$0.8800 E+02$

$0.2269 E+05$

$\mathrm{Rb}-88$

$0.0000 E+00$
$0.0000 E+00$
$0.0000 E+00$

$0.2100 E+00$

$0.0000 E+00$

$0.0000 E+00$

$0.1000 E+01$ 


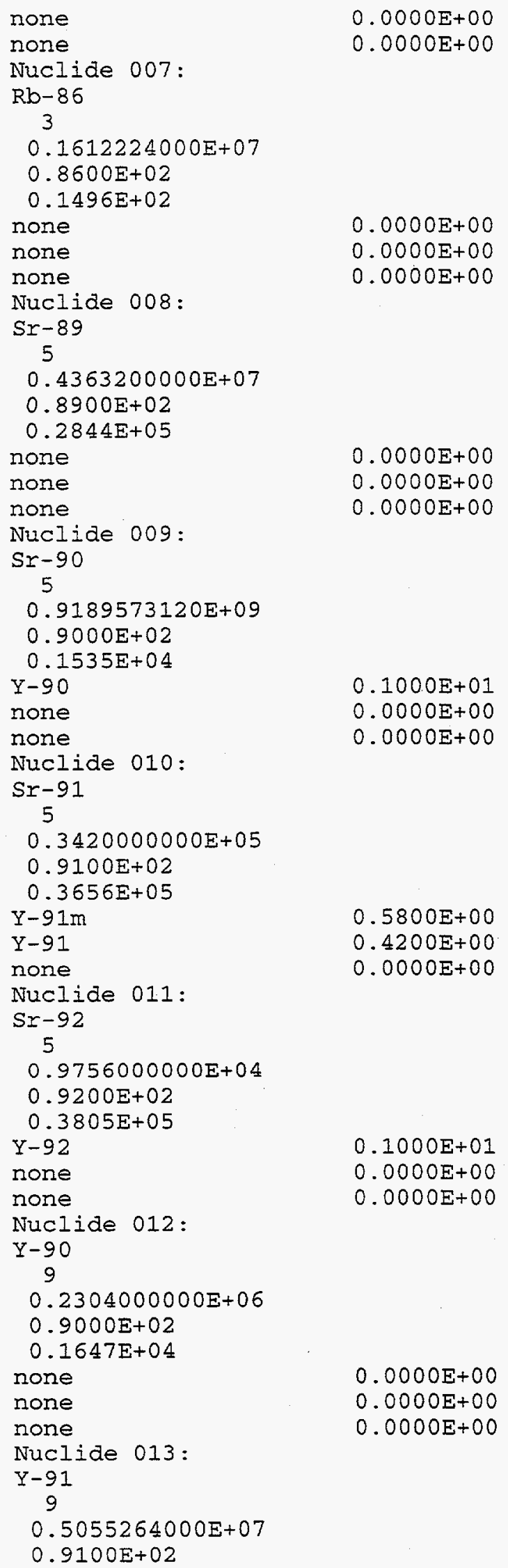

$0.0000 E+00$

$0.0000 E+00$

$0.0000 E+00$

$0.1000 E+01$

$0.0000 E+00$

$0.0000 E+00$

$0.5800 E+00$

$0.4200 \mathrm{E}+00$

$0.0000 E+00$

$0.1000 E+01$

$0.0000 E+00$

$0.0000 E+00$

$0.0000 E+00$
$0.0000 E+00$
$0.0000 E+00$ 
$0.3465 E+05$

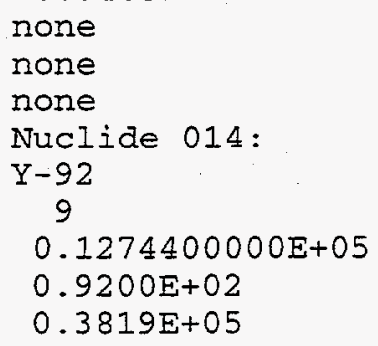

none

$0.0000 E+00$

none

$0.0000 E+00$

none

$0.0000 \mathrm{E}+00$

Nuclide 015:

$\mathrm{Y}-93$

$$
9
$$

$0.3636000000 E+05$

$0.9300 \mathrm{E}+02$

$0.4320 E+05$

Zr-93

$0.1000 E+01$

none

$0.0000 E+00$

none

Nuclide 016:

$0.0000 \mathrm{E}+00$

$\mathrm{Zr}-95$

9

$0.5527872000 \mathrm{E}+07$

$0.9500 E+02$

$0.4377 E+05$

$\mathrm{Nb}-95 \mathrm{~m}$

$0.7000 \mathrm{E}-02$

$0.9900 E+00$

$\mathrm{Nb}-95$

$0.0000 E+00$

Nuclide 017:

Zr -97

9

$0.6084000000 E+05$

$0.9700 E+02$

$0.4562 \mathrm{E}+05$

$\mathrm{Nb}-97 \mathrm{~m}$

$0.9500 E+00$

$\mathrm{Nb}-97$

$0.5300 \mathrm{E}-01$

none

Nuclide 018:

$0.0000 E+00$

$\mathrm{Nb}-95$

9

$0.3036960000 E+07$

$0.9500 E+02$

$0.4138 E+05$

none

none

none

Nuclide 019:

Mo-99

7

$0.2376000000 E+06$

$0.9900 E+02$

$0.4830 E+05$

$\mathrm{TC}-99 \mathrm{~m}$

TC-99

$0.8800 E+00$

$0.1200 E+00$

none

$0.0000 E+00$

$0.0000 E+00$

$0.0000 E+00$

Nuclide 020:

Tc-99m

$0.0000 E+00$ 


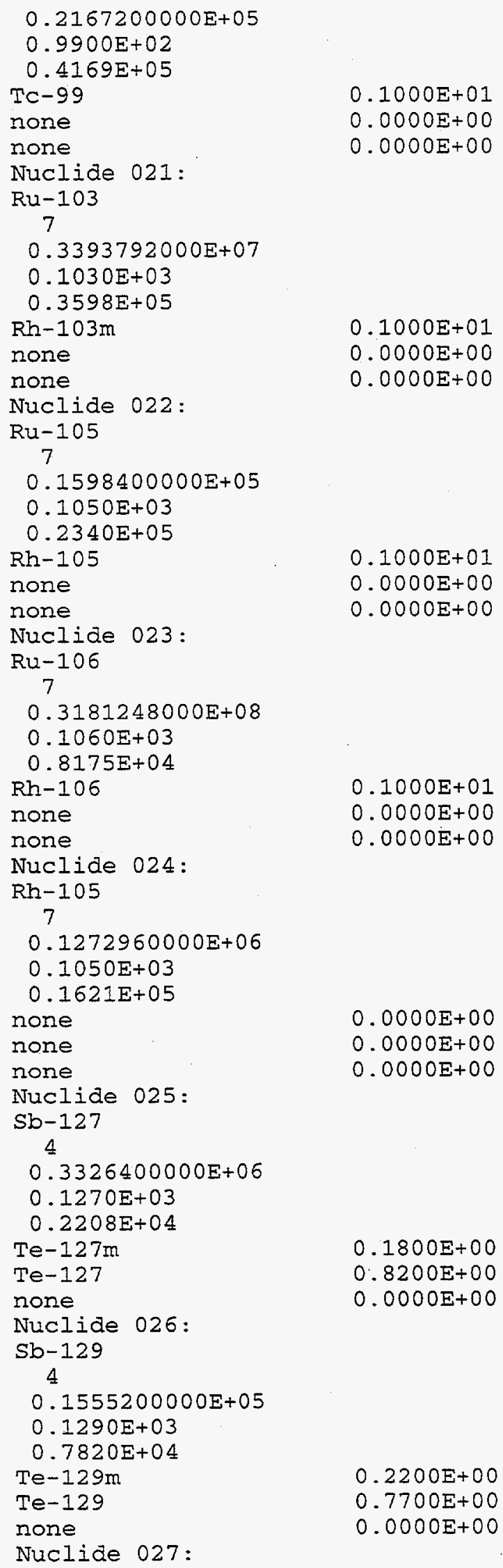

$0.1000 E+01$

$0.0000 E+00$

$0.0000 E+00$

$0.0000 E+00$

$0.0000 \mathrm{E}+00$

$0.0000 E+00$ 

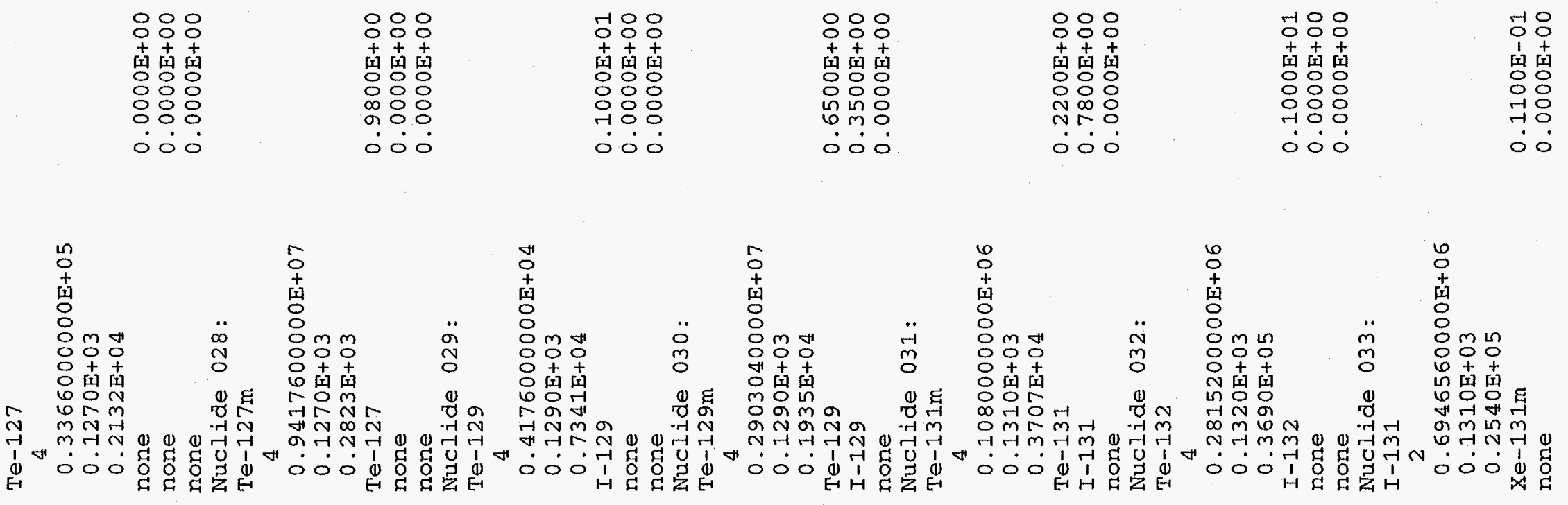


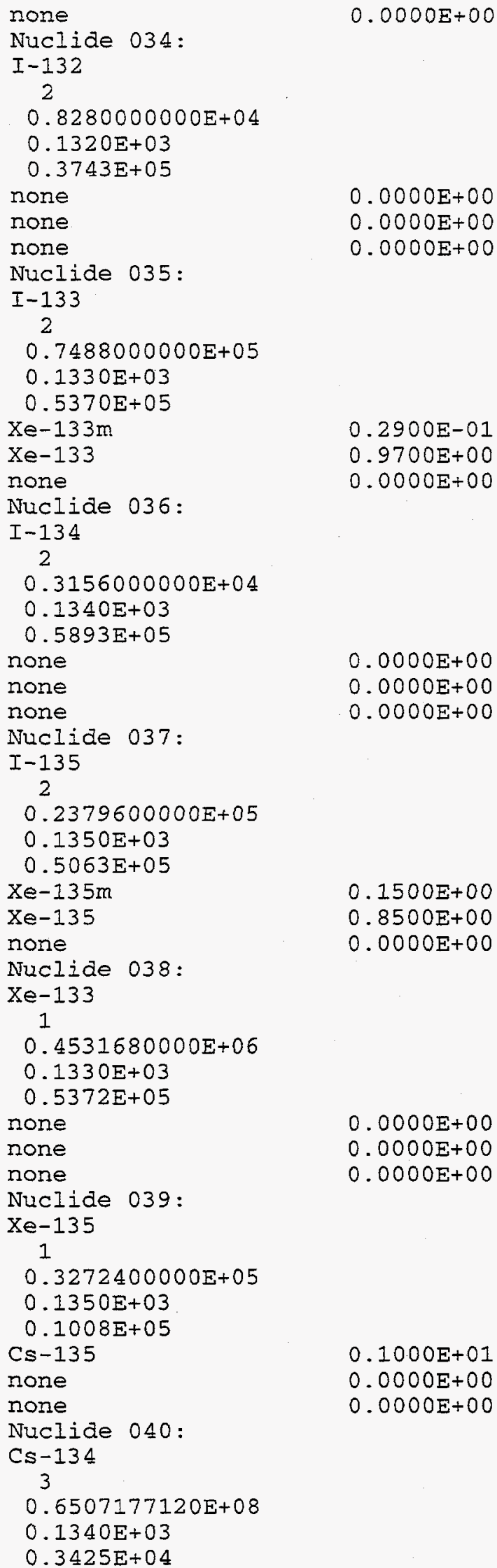

$0.0000 E+00$

$0.0000 E+00$

$0.0000 E+00$

$0.2900 E-01$

$0.9700 \mathrm{E}+00$

$0.0000 E+00$

$0.0000 E+00$

$0.0000 E+00$

$0.0000 E+00$

$0.1500 E+00$

$0.8500 E+00$

$0.0000 \mathrm{E}+00$

$0.0000 E+00$

$0.0000 E+00$

$0.0000 E+00$

$0.1000 E+01$

$0.0000 E+00$

$0.0000 E+00$ 


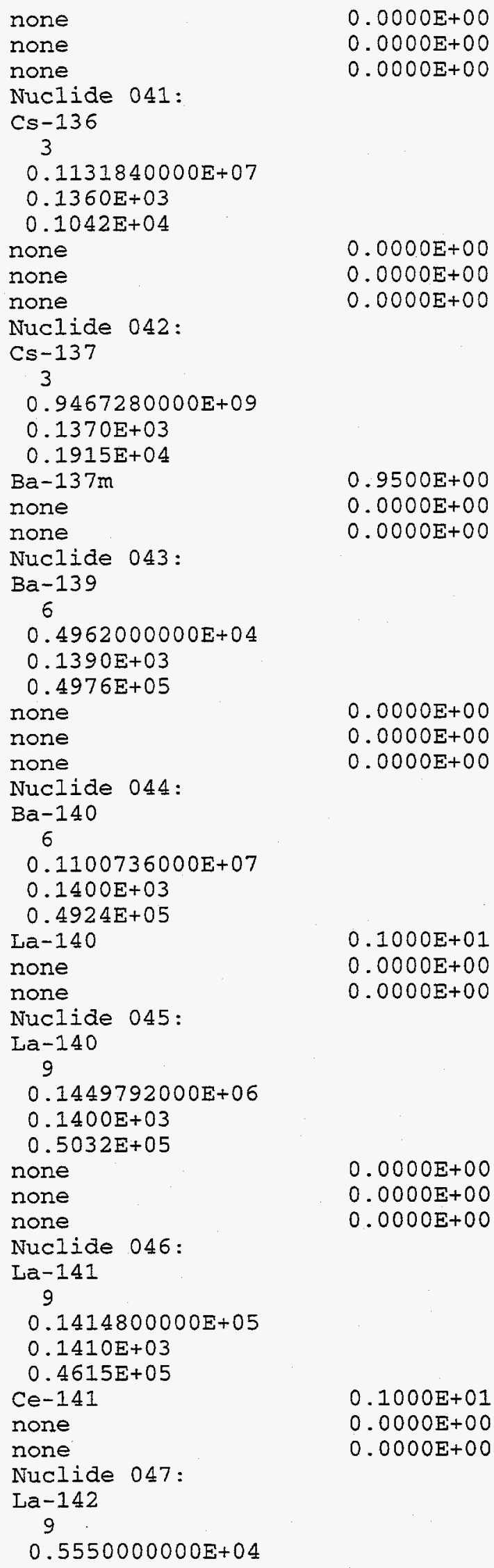


$0.1420 \mathrm{E} \div 03$

$0.4449 E+05$

$$
\text { none }
$$

none

none

Nuclide 048:

$\mathrm{Ce}-141$

$$
8
$$

$0.2808086400 \mathrm{E}+07$

$0.1410 E+03$

$0.4476 E+05$

none

none

none

Nuclide 049:

$\mathrm{Ce}-143$

8

$0.1188000000 E+06$

$0.1430 E+03$

$0.4352 E+05$

Pr-143

none

none

Nuclide 050:

$\mathrm{Ce}-144$

8

$0.2456352000 E+08$

$0.1440 \mathrm{E}+03$

$0.2697 \mathrm{E}+05$

Pr-144m

Pr-144

none

Nuclide 051 :

Pr-143

9

$0.1171584000 E+07$

$0.1430 E+03$

$0.4273 E+05$

none

none

none

Nuclide 052 :

Nd-147

$$
9
$$

$0.9486720000 E+06$

$0.1470 E+03$

$0.1911 E+05$

Pm-147

none

none

Nuclide 053 :

$\mathrm{Np}-239$

8

$0.2034720000 E+06$

$0.2390 E+03$

$0.5120 E+06$

Pu-239

none

none

Nuclide 054:

Pu-238
$0.0000 E+00$
$0.0000 E+00$
$0.0000 E+00$

$0.0000 E+00$

$0.0000 E+00$

$0.0000 E+00$

$0.1000 E+01$

$0.0000 E+00$

$0.0000 E+00$

$0.1800 E-01$

$0.9800 E+00$

$0.0000 E+00$

$0.0000 E+00$

$0.0000 \mathrm{E}+00$

$0.0000 E+00$

$0.1000 E+01$

$0.0000 E+00$

$0.0000 E+00$

$0.1000 E+01$
$0.0000 E+00$
$0.0000 E+00$ 


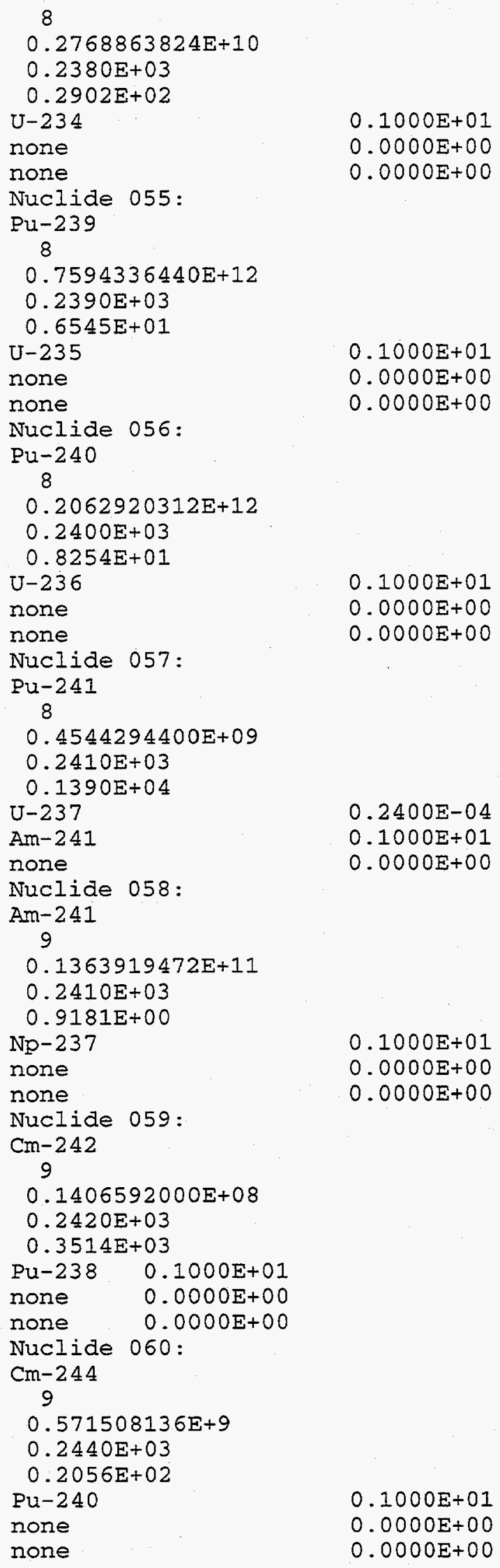

$0.1000 E+01$

$0.0000 E+00$

$0.0000 E+00$

$0.1000 E+01$

$0.0000 E+00$

$0.0000 E+00$

$0.2400 \mathrm{E}-04$

$0.1000 E+01$

$0.0000 \mathrm{E}+00$

$0.1000 E+01$

$0.0000 E+00$

$0.0000 \mathrm{E}+00$

none 
End of Nuclear Inventory File

Note: These values are stored in file "PWR_DEF.NIF." 
Table 1.4.3.2-3

Nuclide Inventory Name:

Normalized MACCS Sample 3578 MWth BWR Core Inventory Power Level:

$0.1000 \mathrm{E}+01$

Nuclides:

60

Nuclide 001 :

Co-58

7

$0.6117120000 \mathrm{E}+07$

$0.5800 \mathrm{E}+02$

$0.1529 \mathrm{E}+03$

none

none

$0.0000 E+00$

$0.0000 E+00$

none

$0.0000 \mathrm{E}+00$

Nuclide 002 :

Co-60

7

$0.1663401096 \mathrm{E}+09$

$0.6000 E+02$

$0.1830 E+03$

none

$0.0000 E+00$

none

$0.0000 E+00$

none

$0.0000 E+00$

Nuclide 003 :

$\mathrm{Kr}-85$

1

$0.3382974720 E+09$

$0.8500 \mathrm{E}+02$

$0.2506 E+03$

none

$0.0000 E+00$

none

$0.0000 \mathrm{E}+00$

none

Nuclide 004 :

$0.0000 E+00$

$\mathrm{Kr}-85 \mathrm{~m}$

1

$0.1612800000 E+05$

$0.8500 E+02$

$0.9110 E+04$

$\mathrm{Kr}-85$

$0.2100 \mathrm{E}+00$

none

$0.0000 \mathrm{E}+00$

none

Nuclide 005:

$0.0000 \mathrm{E}+00$

$\mathrm{Kr}-87$

1

$0.4578000000 E+04$

$0.8700 \mathrm{E}+02$

$0.1657 E+05$

$\mathrm{Rb}-87$

$0.1000 E+01$

none

$0.0000 \mathrm{E}+00$

none

$0.0000 E+00$

Nuclide 006:

$\mathrm{Kr}-88$

1

$0.1022400000 \mathrm{E}+05$

$0.8800 \mathrm{E}+02$

$0.2236 \mathrm{E}+05$

$\mathrm{Rb}-88$

$0.1000 E+01$ 


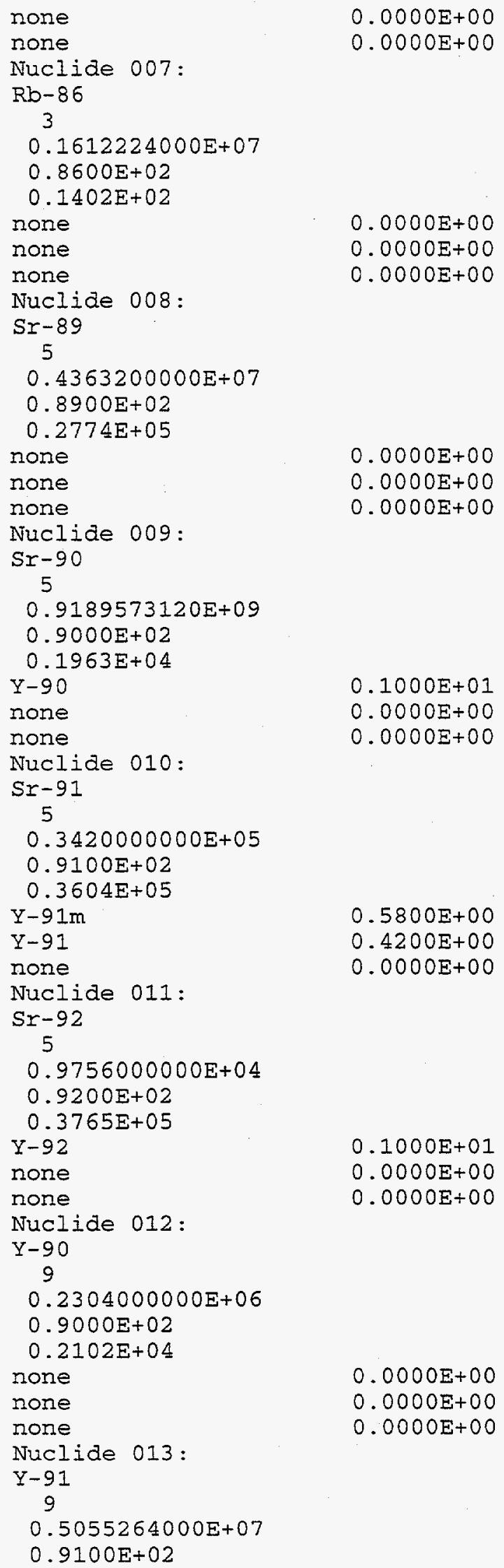

$0.1000 E+01$

$0.0000 E+00$

$0.0000 E+00$

$0.5800 \mathrm{E}+00$

$0.4200 E+00$

$0.0000 E+00$

$0.1000 E+01$

$0.0000 E+00$

$0.0000 E+00$

$0.0000 E+00$

$0.0000 \mathrm{E}+00$

$0.0000 E+00$ 


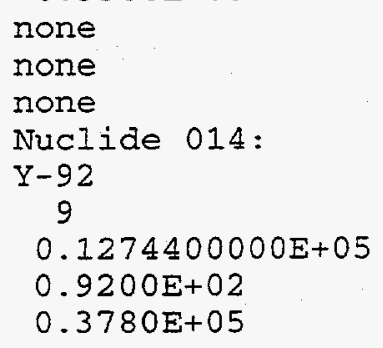

none

Nuclide 015 :

$\mathrm{Y}-93$
9
$0.3636000000 E+05$
$0.9300 \mathrm{E}+02$

$0.4298 \mathrm{E}+05$

$$
\mathrm{Zr}-93
$$

none

none

Nuclide 016:

$\mathrm{Zr}-95$

9
$0.5527872000 E+07$
$0.9500 \mathrm{E}+02$
$0.4456 \mathrm{E}+05$

$\mathrm{Nb}-95 \mathrm{~m}$

$0.0000 E+00$

$0.0000 E+00$

$0.0000 E+00$

$\mathrm{Nb}-95$

none

Nuclide 017:

$\mathrm{Zx}-97$

9

$0.6084000000 E+05$

$0.9700 \mathrm{E}+02$

$0.4587 \mathrm{E}+05$

$\mathrm{Nb}-97 \mathrm{~m}$

$\mathrm{Nb}-97$

none

Nuclide 018:

$\mathrm{Nb}-95$

9

$0.3036960000 E+07$

$0.9500 \mathrm{E}+02$

$0.4216 \mathrm{E}+05$

none

none

none

Nuclide 019:

$0.1000 E+01$

$0.0000 E+00$

$0.0000 \mathrm{E}+00$

$0.7000 E-02$

$0.9900 E+00$

$0.0000 \mathrm{E}+00$

Mo-99

7

$0.2376000000 E+06$

$0.9900 \mathrm{E}+02$

$0.4862 \mathrm{E}+05$

Tc-99m

$0.8800 E+00$

Tc-99

$0.9500 E+00$

$0.5300 E-01$

$0.0000 E+00$

none

Nuclide 020:

$0.1200 E+00$

$0.0000 E+00$

$0.0000 E+00$

$0.0000 E+00$

$0.0000 \mathrm{E}+00$

Tc-99m

7 
$0.2167200000 E+05$

$0.9900 E+02$

$0.4195 E+05$

TC-99

$0.1000 E+01$

none

$0.0000 E+00$

none

$0.0000 E+00$

Nuclide 021:

Ru-103

7

$0.3393792000 E+07$

$0.1030 E+03$

$0.3691 \mathrm{E}+05$

$\mathrm{Rh}-103 \mathrm{~m}$

$0.1000 E+01$

none

$0.0000 E \div 00$

none

Nuclide 022:

$0.0000 E+00$

Ru-105

7

$0.1598400000 \mathrm{E}+05$

$0.1050 E+03$

$0.2458 \mathrm{E}+05$

$\mathrm{Rh}-105$

none

$0.1000 E+01$

$0.0000 E+00$

none

$0.0000 E+00$

Nuclide 023:

Ru-106

7

$0.3181248000 E+08$

$0.1060 \mathrm{E}+03$

$0.1002 E+05$

Rh-106

none

none

Nuclide 024:

$\mathrm{Rh}-105$

7

$0.1272960000 E+06$

$0.1050 \mathrm{E}+03$

$0.1835 \mathrm{E}+05$

none

$0.0000 E+00$

$0.0000 E+00$

none

$0.0000 E+00$

Nuclide 025 :

$0.1000 E+01$

$0.0000 \mathrm{E}+00$

$0.0000 E+00$

$\mathrm{Sb}-127$

4
$0.3326400000 E+06$
$0.1270 \mathrm{E}+03$
$0.2324 \dot{E}+04$

Te-127m

$0.1800 E+00$

$\mathrm{Te}-127$

none

Nuclide 026:

$0.8200 E+00$

$0.0000 E+00$

Sb-129

4

$\begin{array}{ll}0.1555200000 \mathrm{E}+05 & \\ 0.1290 \mathrm{E}+03 & \\ 0.8067 \mathrm{E}+04 & \\ \text { Te-129m } & 0.2200 \mathrm{~m}+00 \\ \text { Te- } 129 & 0.7700 \mathrm{E}+00 \\ \text { none } & 0.0000 \mathrm{E}+00\end{array}$

Nuclide 027 : 


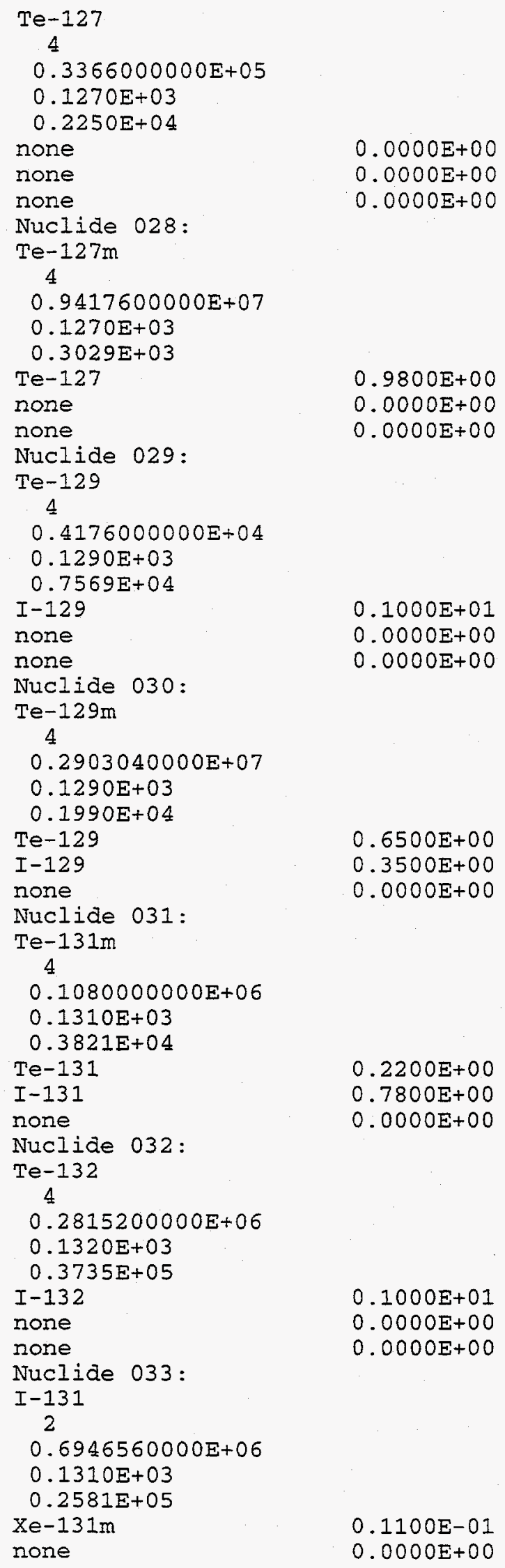




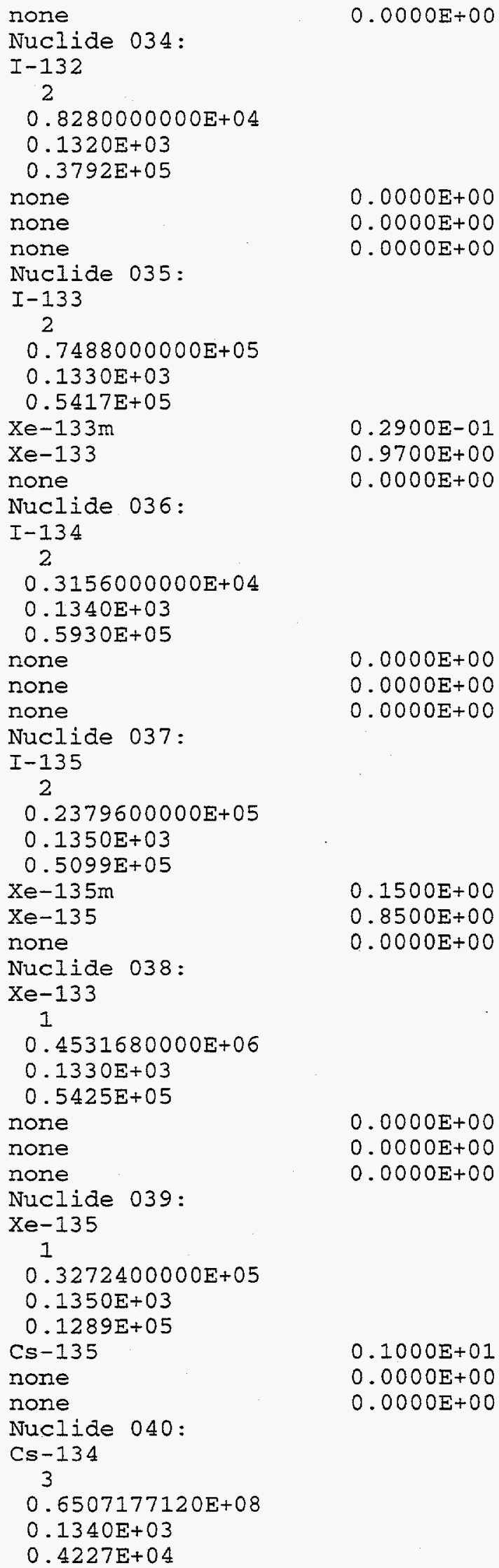




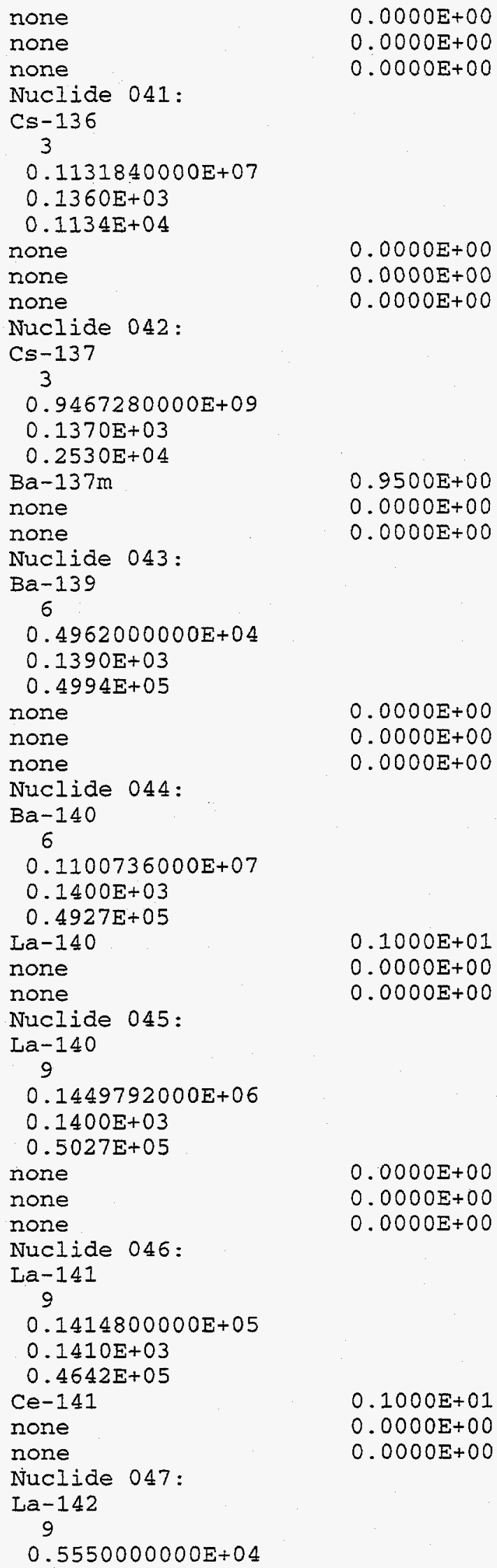




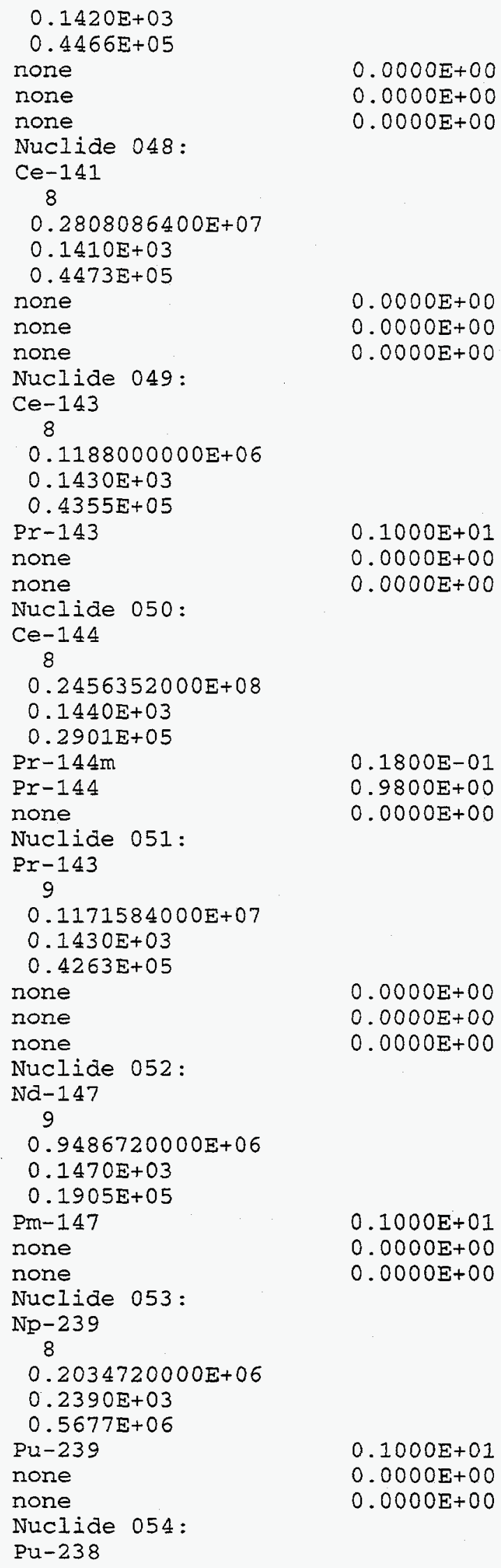




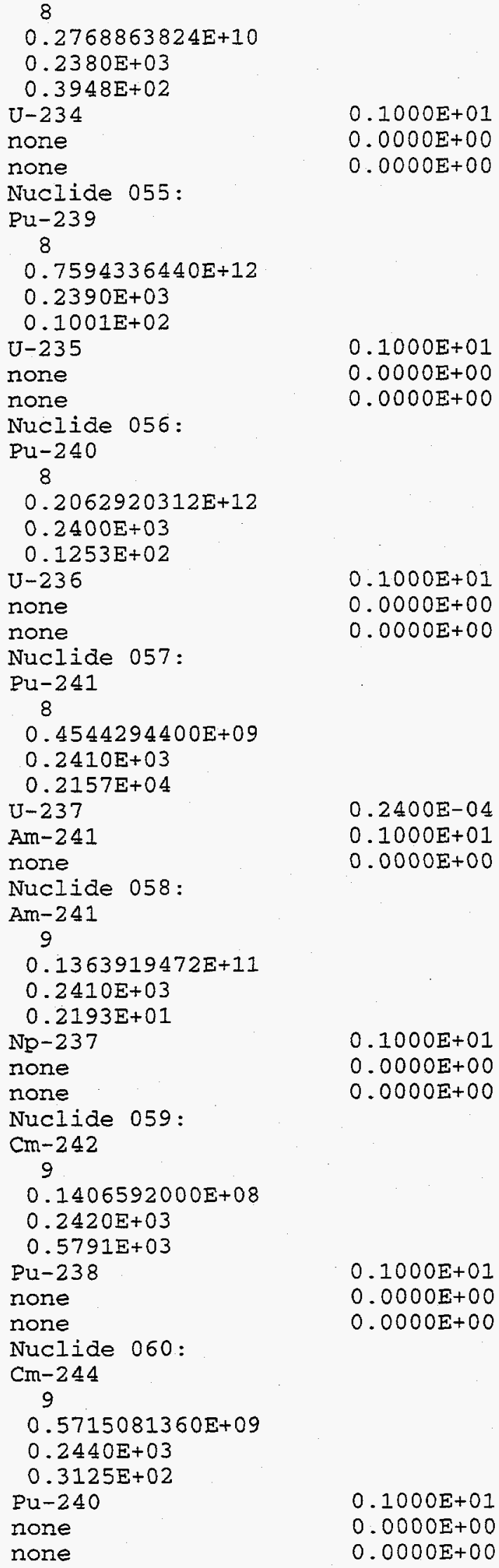


End of Nuclear Inventory File

Note: These values are stored in file "PWR_DEF.NIF."

\subsubsection{Conversion Factors File}

The relationship between isotopic concentration and dose is detailed in Section 2.4.3. Using the method detailed requires the isotope concentration to dose relationship given in these files. These files correspond to the previous inventory files. The data were derived from Federal Guidance Reports 11 and 12 [FGR11, FGR12]. Table 1.3.3.3-1 contains the data for the 14isotope, 3-element TID source term analysis. Table 1.3.3.3-2 gives similar information for the NUREG source term.

There are two files: the 14-isotope, 3-element TID-14844-related data and the 60-isotope, 9element NUREG-1465 data. The structure of the files is:

2 title cards

Number of organs

Organ list in alphanumeric form

Number of nuclides

Nuclide list in alphanumeric form (half-life units also displayed)

For each nuclide and for each organ

Dose conversion factors for 4 types of cloudshine, 2 inhalation, and 1 ingestion

(RADTRAD uses only the cloudshine, or submersion, and the inhalation effects).

Dose conversion factors $(\mathrm{Sv} / \mathrm{Bq})$ for inhalation and $\left(\mathrm{Sv}-\mathrm{s} / \mathrm{Bq} \mathrm{m}^{3}\right)$ for submersion 


\section{Table 1.4.3.3-1 Dose Conversion Factors for TID-14844 Nuclides}

FGRDCF 10/24/95 02:55:27 beta-test version 1.10, minor Fortran fixes 5/4/95 Implicit daughter half-lives $(\mathrm{m})$ less than 90 and less than 0.100 of parent 9 ORGANS DEFINED IN THIS FILE:

GONADS

BREAST

LUNGS

RED MARR

BONE SUR

THYROID

REMAINDER

EFFECTIVE

$\operatorname{SKIN~(FGR)~}$

$I-131$

14 NUCLIDES DEFINED IN THIS FILE:

$I-132$

$I-133$

$I-134$

$I-135$

$\mathrm{Xe}-13 \mathrm{Im}$

$\mathrm{xe}-133 \mathrm{~m}$

$\mathrm{xe}-133$

$\mathrm{xe}-135 \mathrm{~m}$

$\mathrm{Xe}-135$

$\mathrm{Kr}-83 \mathrm{~m}$

$\mathrm{Kr}-85 \mathrm{~m}$

$\mathrm{Kr}-87$

$\mathrm{K} x-88$

D

$\mathrm{D}$

$D$

D

D Including: $\mathrm{Xe}-135 \mathrm{~m}$

$I-131$

GONADS

BREAST

LUNGS

RED MARR

BONE SUR

THYROID

REMAINDER

EFFECTIVE

SKIN (FGR)

I-132

GONADS

BREAST

IUNGS

RED MARR

BONE SUR

THYROID

REMAINDER

EFFECTIVE

SKIN (FGR)

I-133

GONADS

BREAST

IUNGS

RED MARR

BONE SUR

THYROID

REMAINDER

EFFECTIVE

SKIN (FGR)

$I-134$

GONADS

BREAST

IUNGS

CLOUDSHINE GROUND GROUND GROUND INHALED INHALED INGESTION SHINE 8HR SHINE TDAY SHINE RATE ACUTE CHRONIC

$1.780 \mathrm{E}-14 \quad 1.119 \mathrm{E}-11 \quad 1.789 \mathrm{E}-10 \quad 3.940 \mathrm{E}-16-1.000 \mathrm{E}+00 \quad 2.530 \mathrm{E}-11 \quad 4.070 \mathrm{E}-11$ $2.040 \mathrm{E}-141.082 \mathrm{E}-11 \quad 1.730 \mathrm{E}-10 \quad 3.810 \mathrm{E}-16-1.000 \mathrm{E}+00 \quad 7.880 \mathrm{E}-11 \quad 1.210 \mathrm{E}-10$ $1.760 \mathrm{E}-141.016 \mathrm{E}-1111.626 \mathrm{E}-10 \quad 3.580 \mathrm{E}-16-1.000 \mathrm{E}+00 \quad 6.570 \mathrm{E}-10 \quad 1.020 \mathrm{E}-10$

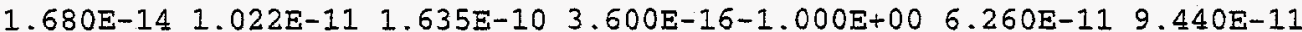
$3.450 \mathrm{E}-141.675 \mathrm{E}-112.679 \mathrm{E}-10 \quad 5.900 \mathrm{E}-16-1.000 \mathrm{E}+00 \quad 5.730 \mathrm{E}-11 \quad 8.720 \mathrm{E}-11$ $\begin{array}{lllll}1.810 E-14 & 1.053 \mathrm{E}-11 & 1.685 \mathrm{E}-10 & 3.710 \mathrm{E}-16-1.000 \mathrm{E}+00 & 2.920 \mathrm{E}-07 \quad 4.760 \mathrm{E}-07\end{array}$ $1.670 \mathrm{E}-14 \quad 9.908 \mathrm{E}-12 \quad 1.585 \mathrm{E}-10 \quad 3.490 \mathrm{E}-16-1.000 \mathrm{E}+00 \quad 8.030 \mathrm{E}-11 \quad 1.570 \mathrm{E}-10$ $1.820 \mathrm{E}-14 \quad 1.067 \mathrm{E}-11 \quad 1.707 \mathrm{E}-10 \quad 3.760 \mathrm{E}-16-1.000 \mathrm{E}+00 \quad 8.890 \mathrm{E}-09 \quad 1.440 \mathrm{E}-08$ $2.980 \mathrm{E}-14 \quad 1.825 \mathrm{E}-112.920 \mathrm{E}-10 \quad 6.430 \mathrm{E}-16-1.000 \mathrm{E}+00 \quad 0.000 \mathrm{E}+00 \quad 0.000 \mathrm{E}+00$

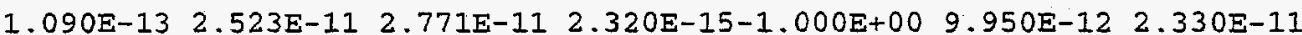
$\begin{array}{llllll}1.240 \mathrm{E}-13 & 2.414 \mathrm{E}-11 & 2.652 \mathrm{E}-11 & 2.220 \mathrm{E}-15-1.000 \mathrm{E}+00 & 1.410 \mathrm{E}-11 & 2.520 \mathrm{E}-11\end{array}$

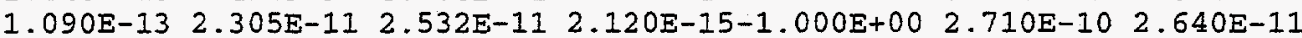

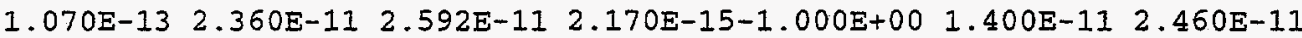

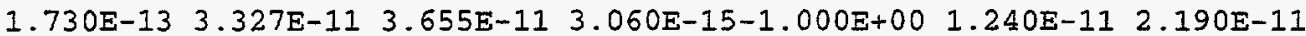

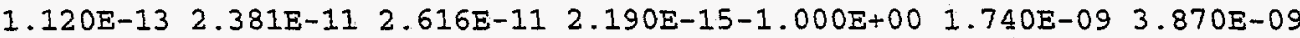

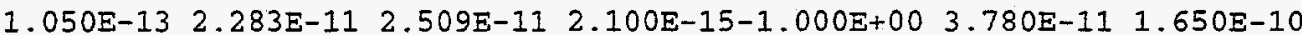
$\begin{array}{llllll}1.120 \mathrm{E}-13 & 2.403 \mathrm{E}-11 & 2.640 \mathrm{E}-11 & 2.210 \mathrm{E}-15-1.000 \mathrm{E}+00 & 1.030 \mathrm{E}-10 & 1.820 \mathrm{E}-10\end{array}$

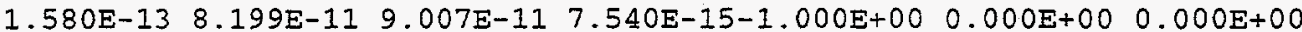

$2.870 \mathrm{E}-141.585 \mathrm{E}-11 \quad 6.748 \mathrm{E}-11 \quad 6.270 \mathrm{E}-16-1.000 \mathrm{E}+00 \quad 1.950 \mathrm{E}-11 \quad 3.630 \mathrm{E}-11$ $3.280 \mathrm{E}-141.519 \mathrm{E}-11 \quad 6.468 \mathrm{E}-11 \quad 6.010 \mathrm{E}-16-1.000 \mathrm{E}+00 \quad 2.940 \mathrm{E}-11 \quad 4.680 \mathrm{E}-11$ $2.860 \mathrm{E}-141.446 \mathrm{E}-11 \quad 6.156 \mathrm{E}-11 \quad 5.720 \mathrm{E}-16-1.000 \mathrm{E}+00 \quad 8.200 \mathrm{E}-10 \quad 4.530 \mathrm{E}-11$ $2.770 \mathrm{E}-14$ 1.466E-11 $6.242 \mathrm{E}-11 \quad 5.800 \mathrm{E}-16-1.000 \mathrm{E}+00 \quad 2.720 \mathrm{E}-11 \quad 4.300 \mathrm{E}-11$ $\begin{array}{llllll}4.870 E-14 & 2.161 E-11 & 9.202 E-11 & 8.550 E-16-1.000 E+00 ~ & 2.520 E-11 & 4.070 E-11\end{array}$ $2.930 \mathrm{E}-14$ 1.502E-11 $6.393 \mathrm{E}-11 \quad 5.940 \mathrm{E}-16-1.000 \mathrm{E}+00 \quad 4.860 \mathrm{E}-08$ 9.100E-08 $2.730 \mathrm{E}-14$ 1.418E-11 $6.038 \mathrm{E}-11 \quad 5.610 \mathrm{E}-16-1.000 \mathrm{E}+00 \quad 5.000 \mathrm{E}-11 \quad 1.550 \mathrm{E}-10$ $2.940 \mathrm{E}-14 \quad 1.509 \mathrm{E}-11 \quad 6.425 \mathrm{E}-11 \quad 5.970 \mathrm{E}-16-1.000 \mathrm{E}+00 \quad 1.580 \mathrm{E}-09 \quad 2.800 \mathrm{E}-09$ $5.830 E-14 \quad 1.150 E-10 \quad 4.897 E-10 \quad 4.550 E-15-1.000 E+00 \quad 0.000 E+00 \quad 0.000 E+00$

$1.270 \mathrm{E}-13 \quad 1.200 \mathrm{E}-11 \quad 1.202 \mathrm{E}-11 \quad 2.640 \mathrm{E}-15-1.000 \mathrm{E}+00 \quad 4.250 \mathrm{E}-121.100 \mathrm{E}-11$

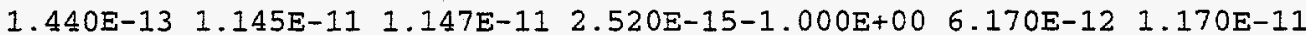
$1.270 \mathrm{E}-13$ 1.100E-11 $1.102 \mathrm{E}-11$ 2.420E-15-1.000E+00 $1.430 \mathrm{E}-10 \quad 1.260 \mathrm{E}-11$ RED MARR $1.250 \mathrm{E}-13 \quad 1.127 \mathrm{E}-11 \quad 1.129 \mathrm{E}-11 \quad 2.480 \mathrm{E}-15-1.000 \mathrm{E}+00 \quad 6.080 \mathrm{E}-12 \quad 1.090 \mathrm{E}-11$ 
BONE SUR THYROID REMAINDER EFFECTIVE SKIN (FGR) $I-135$ GONADS BREAST LUNGS RED MARR BONE SUR THYROID REMAINDER EFFECTIVE SKIN (FGR) Xe-131m GONADS BREAST LUNTGS RED MARR BONE SUR THYROID REMAINDER EFFECTIVE SKIN (FGR) $\mathrm{Xe}-133 \mathrm{~m}$ GONADS BREAST LUNGS RED MARR BONE SUR THYROID REMAINDER EFFECTIVE SKIN (FGR) $\mathrm{Xe}-133$ GONADS BREAST LUNGS RED MARR BONE SUR THYROID REMAINDER EFFECTIVE SKIN (FGR) $\mathrm{Xe}-135 \mathrm{~m}$ GONADS BREAST LUNGS

RED MARR BONE SUR THYROID REMAINDER EFFECTIVE SKIN (FGR) $\mathrm{Xe}-135$ GONADS BREAST LUNGS RED MARR BONE SUR. THYROID REMAINDER EFFECTIVE SKIN (FGR) $\mathrm{Kr}-83 \mathrm{~m}$ GONADS
$1.960 \mathrm{E}-13 \quad 1.568 \mathrm{E}-11 \quad 1.571 \mathrm{E}-11 \quad 3.450 \mathrm{E}-15-1.000 \mathrm{E}+00 \quad 5.310 \mathrm{E}-12 \quad 9.320 \mathrm{E}-12$

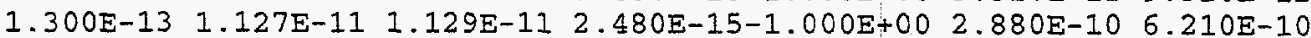
$1.220 \mathrm{E}-13$ 1.091E-11 $1.093 \mathrm{E}-112.400 \mathrm{E}-15-1.000 \mathrm{E}+002.270 \mathrm{E}-111.340 \mathrm{E}-10$

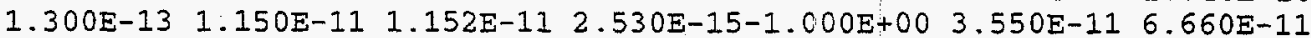

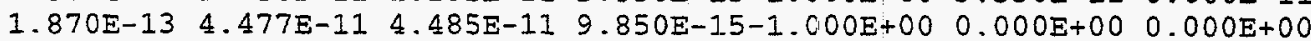

8.078E-14 3.113E-11 5.489E-11 1.599E-15-1.000E+00 1.700E-11 3.610E-11 $9.143 \mathrm{E}-14 \quad 2.971 \mathrm{E}-11 \quad 5.240 \mathrm{E}-11 \quad 1.526 \mathrm{E}-15-1.000 \mathrm{E}+00 \quad 2.340 \mathrm{E}-113.850 \mathrm{E}-11$ $8.145 \mathrm{E}-142.886 \mathrm{E}-11 \mathrm{5}$ 5.089E-11 $1.482 \mathrm{E}-15-1.000 \mathrm{E}+00 \quad 4.410 \mathrm{E}-10 \quad 3.750 \mathrm{E}-11$ 8.054E-14 2.965E-11 $5.228 E-11 \quad 1.523 E-15-1.000 E+00 \quad 2.240 E-11 \quad 3.650 E-11$ $1.184 \mathrm{E}-13 \quad 3.983 \mathrm{E}-11 \quad 7.024 \mathrm{E}-112.046 \mathrm{E}-15-1.000 \mathrm{E}+00 \quad 2.010 \mathrm{E}-113.360 \mathrm{E}-11$ $8.324 \mathrm{E}-14 \quad 2.852 \mathrm{E}-11 \quad 5.030 \mathrm{E}-11 \quad 1.465 \mathrm{E}-15-1.000 \mathrm{E}+00 \quad 8.460 \mathrm{E}-09 \quad 1.790 \mathrm{E}-08$ $7.861 \mathrm{E}-142.883 \mathrm{E}-11 \quad 5.084 \mathrm{E}-11 \quad 1.481 \mathrm{E}-15-1.000 \mathrm{E}+00 \quad 4.700 \mathrm{E}-11 \quad 1.540 \mathrm{E}-10$ 8.294E-14 2.989E-11 $5.271 \mathrm{E}-11 \quad 1.535 \mathrm{E}-15-1.000 \mathrm{E}+00 \quad 3.320 \mathrm{E}-10 \quad 6.080 \mathrm{E}-10$

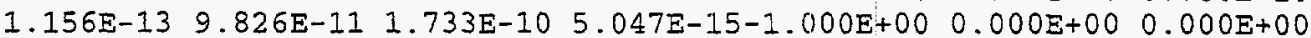

$4.570 \mathrm{E}-16 \quad 7.872 \mathrm{E}-13 \quad 1.371 \mathrm{E}-11 \quad 2.760 \mathrm{E}-17-1.000 \mathrm{E}+00 \quad 0.000 \mathrm{E}+00 \quad 0.000 \mathrm{E}+00$ $6.020 \mathrm{E}-16 \quad 8.471 \mathrm{E}-13 \quad 1.475 \mathrm{E}-11 \quad 2.970 \mathrm{E}-17-1.000 \mathrm{E}+00 \quad 0.000 \mathrm{E}+00 \quad 0.000 \mathrm{E}+00$ $2.670 \mathrm{E}-16 \quad 3.565 \mathrm{E}-13 \quad 6.209 \mathrm{E}-12 \quad 1.250 \mathrm{E}-17-1.000 \mathrm{E}+00 \quad 0.000 \mathrm{E}+00 \quad 0.000 \mathrm{E}+00$ $2.270 \mathrm{E}-16 \quad 2.792 \mathrm{E}-13 \quad 4.863 \mathrm{E}-12 \quad 9.790 \mathrm{E}-18-1.000 \mathrm{E}+00 \quad 0.000 \mathrm{E}+00 \quad 0.000 \mathrm{E}+00$ $1.060 \mathrm{E}-15 \quad 1.677 \mathrm{E}-12 \quad 2.920 \mathrm{E}-11 \quad 5.880 \mathrm{E}-17-1.000 \mathrm{E}+00 \quad 0.000 \mathrm{E}+00 \quad 0.000 \mathrm{E}+00$ $3.910 \mathrm{E}-16 \quad 5.220 \mathrm{E}-13 \quad 9.089 \mathrm{E}-12 \quad 1.830 \mathrm{E}-17-1.000 \mathrm{E}+00 \quad 0.000 \mathrm{E}+00 \quad 0.000 \mathrm{E}+00$ $2.710 \mathrm{E}-16 \quad 3.993 \mathrm{E}-13 \quad 6.954 \mathrm{E}-12 \quad 1.400 \mathrm{E}-17-1.000 \mathrm{E}+00 \quad 0.000 \mathrm{E}+00 \quad 0.000 \mathrm{E}+00$ $3.890 \mathrm{E}-16 \quad 5.876 \mathrm{E}-13 \quad 1.023 \mathrm{E}-11 \quad 2.060 \mathrm{E}-17-1.000 \mathrm{E}+00 \quad 0.000 \mathrm{E}+00 \quad 0.000 \mathrm{E}+00$

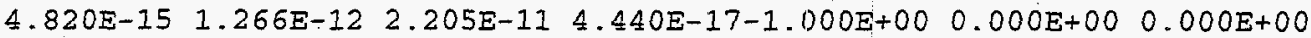

$1.420 \mathrm{E}-15 \quad 1.337 \mathrm{E}-12 \quad 1.188 \mathrm{E}-11 \quad 4.890 \mathrm{E}-17-1.000 \mathrm{E}+00 \quad 0.000 \mathrm{E}+00 \quad 0.000 \mathrm{E}+00$ $1.700 \mathrm{E}-15 \quad 1.383 \mathrm{E}-12 \quad 1.230 \mathrm{E}-11 \quad 5.060 \mathrm{E}-17-1.000 \mathrm{E}+00 \quad 0.000 \mathrm{E}+00 \quad 0.000 \mathrm{E}+00$ $1.190 \mathrm{E}-15 \quad 8.609 \mathrm{E}-13 \quad 7.656 \mathrm{E}-12 \quad 3.150 \mathrm{E}-17-1.000 \mathrm{E}+00 \quad 0.000 \mathrm{E}+00 \quad 0.000 \mathrm{E}+00$ $1.100 \mathrm{E}-15 \quad 7.844 \mathrm{E}-13 \quad 6.975 \mathrm{E}-12 \quad 2.870 \mathrm{E}-17-1.000 \mathrm{E}+00 \quad 0.000 \mathrm{E}+00 \quad 0.000 \mathrm{E}+00$ $3.230 \mathrm{E}-15 \quad 2.599 \mathrm{E}-12 \quad 2.311 \mathrm{E}-11 \quad 9.510 \mathrm{E}-17-1.000 \mathrm{E}+00 \quad 0.000 \mathrm{E}+00 \quad 0.000 \mathrm{E}+00$

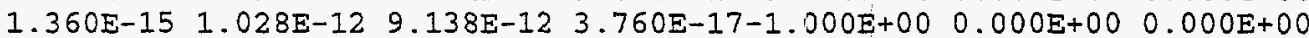
$1.150 \mathrm{E}-15 \quad 8.855 \mathrm{E}-13 \quad 7.874 \mathrm{E}-12 \quad 3.240 \mathrm{E}-17-1.000 \mathrm{E}+00 \quad 0.000 \mathrm{E}+00 \quad 0.000 \mathrm{E}+00$

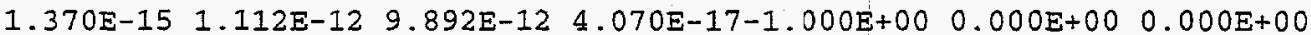
$1.040 \mathrm{E}-141.894 \mathrm{E}-12 \quad 1.684 \mathrm{E}-11 \quad 6.930 \mathrm{E}-17-1.000 \mathrm{E}+00 \quad 0.000 \mathrm{E}+00 \quad 0.000 \mathrm{E}+00$

$\begin{array}{llllll}1.610 \mathrm{E}-15 & 1.465 \mathrm{E}-12 & 2.052 \mathrm{E}-11 & 5.200 \mathrm{E}-17-1.000 \mathrm{E}+00 & 0.000 \mathrm{E}+00 \quad 0.000 \mathrm{E}+00\end{array}$ $\begin{array}{llllll}1.960 E-15 & 1.505 E-12 & 2.107 E-11 & 5.340 E-17-1.000 E+00 & 0.000 E+00 & 0.000 E+00\end{array}$ $1.320 \mathrm{E}-15$ 1.045E-12 $1.464 \mathrm{E}-11 \quad 3.710 \mathrm{E}-17-1.000 \mathrm{E}+00 \quad 0.000 \mathrm{E}+00 \quad 0.000 \mathrm{E}+00$

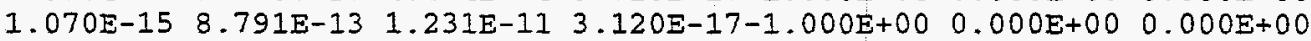
$5.130 \mathrm{E}-15 \quad 4.254 \mathrm{E}-12 \quad 5.958 \mathrm{E}-11 \quad 1.510 \mathrm{E}-16-1.000 \mathrm{E}+00 \quad 0.000 \mathrm{E}+00 \quad 0.000 \mathrm{E}+00$ $1.510 \mathrm{E}-15$ 1.181E-12 $1.653 \mathrm{E}-11 \quad 4.190 \mathrm{E}-17-1.000 \mathrm{E}+00 \quad 0.000 \mathrm{E}+00 \quad 0.000 \mathrm{E}+00$ $\begin{array}{llllll}1.240 E-15 & 1.042 E-12 & 1.460 E-11 & 3.700 E-17-1.000 E+00 & 0.000 E+00 & 0.000 E+00\end{array}$ $1.560 \mathrm{E}-15$ 1.299E-12 $1.819 \mathrm{E}-11$ 4.610E-17-1.000E+00 $0.000 \mathrm{E}+00 \quad 0.000 \mathrm{E}+00$ $\begin{array}{llllll}4.970 E-15 & 1.953 E-12 & 2.734 E-11 & 6.930 E-17-1.000 E+00 & 0.000 E+00 & 0.000 E+00\end{array}$

$2.000 E-14 \quad 5.929 E-13 \quad 5.929 E-13 \quad 4.480 E-16-I .000 E+00 \quad 0.000 E+00 \quad 0.000 E+00$ $2.290 \mathrm{E}-145.691 \mathrm{E}-13 \quad 5.691 \mathrm{E}-13 \quad 4.300 \mathrm{E}-16-1.000 \mathrm{E}+00 \quad 0.000 \mathrm{E}+00 \quad 0.000 \mathrm{E}+00$ $1.980 E-14 \quad 5.347 E-13 \quad 5.347 E-13 \quad 4.040 E-16-1.000 E+00 \quad 0.000 E+00 \quad 0.000 E+00$ $1.910 \mathrm{E}-145.400 \mathrm{E}-13 \quad 5.400 \mathrm{E}-13 \quad 4.080 \mathrm{E}-16-1.000 \mathrm{E}+00 \quad 0.000 \mathrm{E}+00 \quad 0.000 \mathrm{E}+00$ $3.500 E-14 \quad 8.246 E-13 \quad 8.246 E-13 \quad 6.230 E-16-1.000 E+00 \quad 0.000 E+00 \quad 0.000 E+00$ $2.040 \mathrm{E}-14 \quad 5.612 \mathrm{E}-13 \quad 5.612 \mathrm{E}-13 \quad 4.240 \mathrm{E}-16-1.000 \mathrm{E}+00 \quad 0.000 \mathrm{E}+00 \quad 0.000 \mathrm{E}+00$ $1.890 \mathrm{E}-14 \quad 5.241 \mathrm{E}-13 \quad 5.241 \mathrm{E}-13 \quad 3.960 \mathrm{E}-16-1.000 \mathrm{E}+00 \quad 0.000 \mathrm{E}+00 \quad 0.000 \mathrm{E}+00$ $2.040 \mathrm{E}-14 \quad 5.612 \mathrm{E}-13 \quad 5.612 \mathrm{E}-13 \quad 4.240 \mathrm{E}-16-1.000 \mathrm{E}+00 \quad 0.000 \mathrm{E}+00 \quad 0.000 \mathrm{E}+00$ $2.970 \mathrm{E}-14 \quad 1.866 \mathrm{E}-12 \quad 1.866 \mathrm{E}-12 \quad 1.410 \mathrm{E}-15-1.000 \mathrm{E}+00 \quad 0.000 \mathrm{E}+00 \quad 0.000 \mathrm{E}+00$

$\begin{array}{llllll}1.170 E-14 & 5.455 \mathrm{E}-12 & 1.194 \mathrm{E}-11 & 2.530 \mathrm{E}-16-1.000 \mathrm{E}+00 & 0.000 \mathrm{E}+00 & 0.000 \mathrm{E}+00\end{array}$ $1.330 \mathrm{E}-14 \quad 5.325 \mathrm{E}-12 \quad 1.166 \mathrm{E}-11 \quad 2.470 \mathrm{E}-16-1.000 \mathrm{E}+00 \quad 0.000 \mathrm{E}+00 \quad 0.000 \mathrm{E}+00$ $1.130 \mathrm{E}-14 \quad 4.959 \mathrm{E}-12 \quad 1.086 \mathrm{E}-11 \quad 2.300 \mathrm{E}-16-1.000 \mathrm{E}+00 \quad 0.000 \mathrm{E}+00 \quad 0.000 \mathrm{E}+00$ $\begin{array}{llllll}1.070 E-14 & 4.959 \mathrm{E}-12 & 1.086 \mathrm{E}-11 & 2.300 \mathrm{E}-16-1.000 \mathrm{E}+00 & 0.000 \mathrm{E}+00 & 0.000 \mathrm{E}+00\end{array}$ $2.570 \mathrm{E}-14 \quad 9.120 \mathrm{E}-12 \quad 1.997 \mathrm{E}-11 \quad 4.230 \mathrm{E}-16-1.000 \mathrm{E}+00 \quad 0.000 \mathrm{E}+00 \quad 0.000 \mathrm{E}+00$ $\begin{array}{llllll}1.180 E-14 & 5.023 E-12 & 1.100 E-11 & 2.330 E-16-1.000 E+00 & 0.000 E+00 & 0.000 E+00\end{array}$

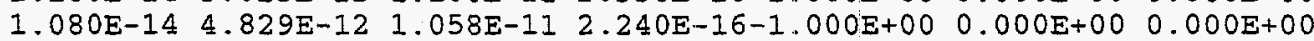

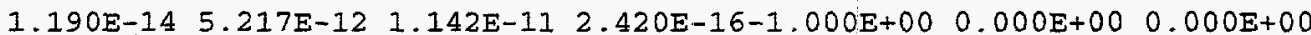

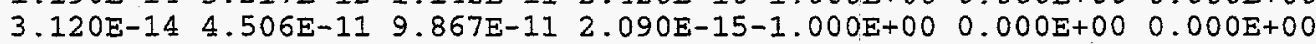
$\begin{array}{llllll}1.710 E-18 & 5.572 E-15 & 5.855 E-15 & 6.160 E-19-1.000 E+00 & 0.000 E+00 & 0.000 E+00\end{array}$ 
BREAST

LUNGS

RED MARR

BONE SUR

THYROID

REMAINDER

EFFECTIVE

SKIN (FGR)

$\mathrm{Kr}-85 \mathrm{~m}$

GONADS

BREAST

IUNGS

RED MARR

BONE SUR

THYROID

REMAINDER

EFFECTIVE

$\operatorname{SKIN~(FGR)~}$

$\mathrm{Kr}-87$

GONADS

BREAST

LUNGS

RED MARR

BONE SUR

THYROID

REMAINDER

EFFECTIVE

SKIN (FGR)

$\mathrm{K} r-88$

GONADS

BREAST

LUNGS

RED MARR

BONE SUR

THYROID

REMAINDER

EFFECTIVE

$\operatorname{SKIN~(FGR)~}$ $\begin{array}{llllll}5.050 E-18 & 9.498 E-15 & 9.980 E-15 & 1.050 E-18-1.000 E+00 & 0.000 E+00 & 0.000 E+00\end{array}$ $1.640 \mathrm{E}-19$ 1.266E-16 $1.331 \mathrm{E}-16 \quad 1.400 \mathrm{E}-20-1.000 \mathrm{E}+00 \quad 0.000 \mathrm{E}+00 \quad 0.000 \mathrm{E}+00$ $3.830 \mathrm{E}-195.617 \mathrm{E}-16 \quad 5.902 \mathrm{E}-16 \quad 6.210 \mathrm{E}-20-1.000 \mathrm{E}+00 \quad 0.000 \mathrm{E}+00 \quad 0.000 \mathrm{E}+00$ $2.250 \mathrm{E}-18 \quad 3.437 \mathrm{E}-15 \quad 3.612 \mathrm{E}-15 \quad 3.800 \mathrm{E}-19-1.000 \mathrm{E}+00 \quad 0.000 \mathrm{E}+00 \quad 0.000 \mathrm{E}+00$ $6.430 \mathrm{E}-19 \quad 7.698 \mathrm{E}-16 \quad 8.088 \mathrm{E}-16 \quad 8.510 \mathrm{E}-20-1.000 \mathrm{E}+00 \quad 0.000 \mathrm{E}+00 \quad 0.000 \mathrm{E}+00$ $5.300 E-19$ 1.393E-15 $1.464 E-15 \quad 1.540 E-19-1.000 E+00 \quad 0.000 E+00 \quad 0.000 E+00$ $\begin{array}{llllll}1.500 E-18 & 3.437 E-15 & 3.612 E-15 & 3.800 E-19-1.000 E+00 & 0.000 E+00 & 0.000 E+00\end{array}$ $3.560 \mathrm{E}-17 \quad 1.167 \mathrm{E}-13 \quad 1.226 \mathrm{E}-13 \quad 1.290 \mathrm{E}-17-1.000 \mathrm{E}+00 \quad 0.000 \mathrm{E}+00 \quad 0.000 \mathrm{E}+00$

$7.310 E-152.594 E-12 \quad 3.653 E-12 \quad 1.570 E-16-1.000 E+00 \quad 0.000 E+00 \quad 0.000 E+00$ $\begin{array}{llllll}8.410 \mathrm{E}-15 & 2.527 \mathrm{E}-12 & 3.560 \mathrm{E}-12 & 1.530 \mathrm{E}-16-1.000 \mathrm{E}+00 & 0.000 \mathrm{E}+00 & 0.000 \mathrm{E}+00\end{array}$ $\begin{array}{lllllll}7.040 E-15 & 2.379 E-12 & 3.351 E-12 & 1.440 E-16-1.000 E+00 & 0.000 E+00 & 0.000 E+00\end{array}$ $\begin{array}{llllll}6.430 \mathrm{E}-15 & 2.346 \mathrm{E}-12 & 3.304 \mathrm{E}-12 & 1.420 \mathrm{E}-16-1.000 \mathrm{E}+00 & 0.000 \mathrm{E}+00 & 0.000 \mathrm{E}+00\end{array}$ $1.880 \mathrm{E}-145.286 \mathrm{E}-12 \quad 7.446 \mathrm{E}-12 \quad 3.200 \mathrm{E}-16-1.000 \mathrm{E}+00 \quad 0.000 \mathrm{E}+00 \quad 0.000 \mathrm{E}+00$

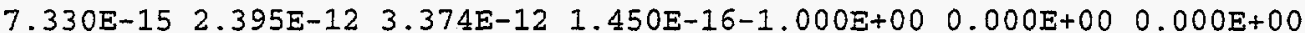
$6.640 \mathrm{E}-15 \quad 2.313 \mathrm{E}-12 \quad 3.257 \mathrm{E}-12 \quad 1.400 \mathrm{E}-16-1.000 \mathrm{E}+00 \quad 0.000 \mathrm{E}+00 \quad 0.000 \mathrm{E}+00$ $\begin{array}{lllllll}7.480 E-15 & 2.511 E-12 & 3.537 E-12 & 1.520 E-16-1.000 E+00 & 0.000 E+00 & 0.000 E+00\end{array}$ $2.240 E-142.247 E-11 \quad 3.164 E-11 \quad 1.360 E-15-1.000 E+00 \quad 0.000 E+00 \quad 0.000 E+00$

$4.000 \mathrm{E}-14 \quad 4.962 \mathrm{E}-12 \quad 5.026 \mathrm{E}-12 \quad 7.610 \mathrm{E}-16-1.000 \mathrm{E}+00 \quad 0.000 \mathrm{E}+00 \quad 0.000 \mathrm{E}+00$ $4.500 \mathrm{E}-14 \quad 4.740 \mathrm{E}-12 \quad 4.802 \mathrm{E}-12 \quad 7.270 \mathrm{E}-16-1.000 \mathrm{E}+00 \quad 0.000 \mathrm{E}+00 \quad 0.000 \mathrm{E}+00$ $4.040 \mathrm{E}-14$ 4.603E-12 $4.663 \mathrm{E}-12 \quad 7.060 \mathrm{E}-16-1.000 \mathrm{E}+00 \quad 0.000 \mathrm{E}+00 \quad 0.000 \mathrm{E}+00$ $\begin{array}{lllllll}4.000 E-14 & 4.708 E-12 & 4.769 E-12 & 7.220 E-16-1.000 E+00 & 0.000 E+00 & 0.000 E+00\end{array}$ $\begin{array}{lllllll}6.020 E-14 & 6.514 E-12 & 6.598 E-12 & 9.990 E-16-1.000 E+00 & 0.000 E+00 & 0.000 E+00\end{array}$ $4.130 \mathrm{E}-14 \quad 4.473 \mathrm{E}-12 \quad 4.531 \mathrm{E}-12 \quad 6.860 \mathrm{E}-16-1.000 \mathrm{E}+00 \quad 0.000 \mathrm{E}+00 \quad 0.000 \mathrm{E}+00$ $3.910 \mathrm{E}-14 \quad 4.590 \mathrm{E}-12 \quad 4.650 \mathrm{E}-12 \quad 7.040 \mathrm{E}-16-1.000 \mathrm{E}+00 \quad 0.000 \mathrm{E}+00 \quad 0.000 \mathrm{E}+00$ $4.120 \mathrm{E}-14 \quad 4.773 \mathrm{E}-12 \quad 4.835 \mathrm{E}-12 \quad 7.320 \mathrm{E}-16-1.000 \mathrm{E}+00 \quad 0.000 \mathrm{E}+00 \quad 0.000 \mathrm{E}+00$ $1.370 \mathrm{E}-13 \quad 8.802 \mathrm{E}-11 \quad 8.916 \mathrm{E}-11 \quad 1.350 \mathrm{E}-14-1.000 \mathrm{E}+00 \quad 0.000 \mathrm{E}+00 \quad 0.000 \mathrm{E}+00$

$9.900 \mathrm{E}-142.278 \mathrm{E}-1122.655 \mathrm{E}-11 \quad 1.800 \mathrm{E}-15-1.000 \mathrm{E}+00 \quad 0.000 \mathrm{E}+00 \quad 0.000 \mathrm{E}+00$ $1.110 \mathrm{E}-13 \quad 2.177 \mathrm{E}-11 \quad 2.537 \mathrm{E}-11 \quad 1.720 \mathrm{E}-15-1.000 \mathrm{E}+00 \quad 0.000 \mathrm{E}+00 \quad 0.000 \mathrm{E}+00$ $\begin{array}{llllll}1.010 E-13 & 2.139 \mathrm{E}-11 & 2.493 \mathrm{E}-11 & 1.690 \mathrm{E}-15-1.000 \mathrm{E}+00 & 0.000 \mathrm{E}+00 & 0.000 \mathrm{E}+00\end{array}$ $\begin{array}{llllll}1.000 E-13 & 2.190 \mathrm{E}-11 & 2.552 \mathrm{E}-11 & 1.730 \mathrm{E}-15-1.000 \mathrm{E}+00 & 0.000 \mathrm{E}+00 & 0.000 \mathrm{E}+00\end{array}$ $\begin{array}{lllllll}1.390 E-13 & 2.886 E-11 & 3.363 E-11 & 2.280 E-15-1.000 E+00 & 0.000 E+00 & 0.000 E+00\end{array}$ $\begin{array}{llllll}1.030 \mathrm{E}-13 & 2.012 \mathrm{E}-11 & 2.345 \mathrm{E}-11 & 1.590 \mathrm{E}-15-1.000 \mathrm{E}+00 & 0.000 \mathrm{E}+00 & 0.000 \mathrm{E}+00\end{array}$ $9.790 \mathrm{E}-14.2 .139 \mathrm{E}-112.493 \mathrm{E}-11 \quad 1.690 \mathrm{E}-15-1.000 \mathrm{E}+00 \quad 0.000 \mathrm{E}+00 \quad 0.000 \mathrm{E}+00$

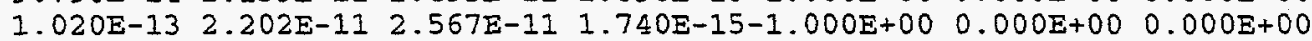
$\begin{array}{llllll}1.350 E-13 & 5.607 E-1 I & 6.534 E-11 & 4.430 E-15-1.000 E+00 & 0.000 E+00 & 0.000 E+00\end{array}$

Note: These values are stored as file "TID.INP." The corresponding file that contains the atomic weights and halflives for these isotopes is "TID.NIF." 
Table 1.4.3.3-2 Dose Conversion Factors for NUREG-1465 Nuclides

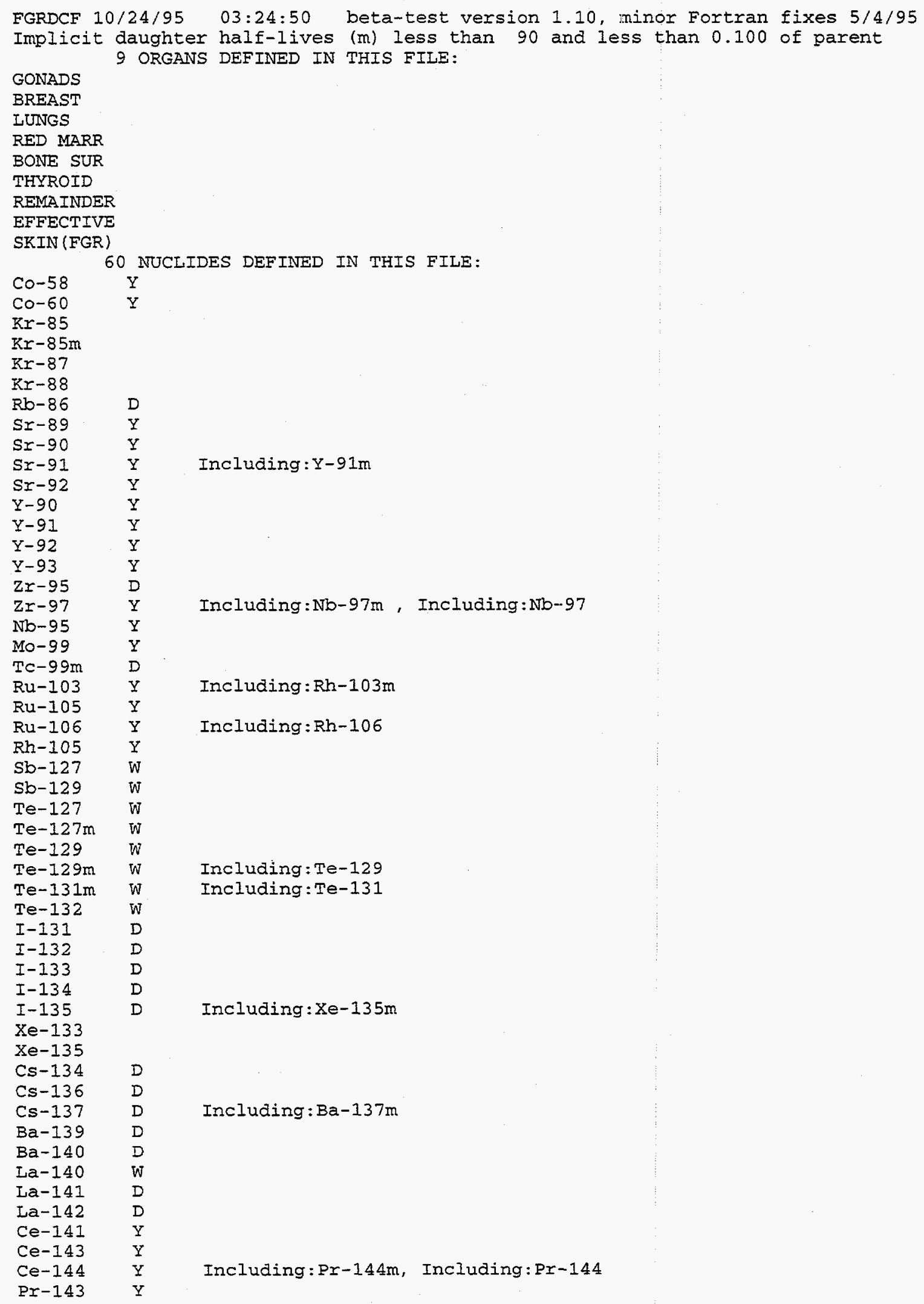


Co-58

GONADS

BREAST

LUNGS

RED MARR

BONE SUR

THYROID

REMAINDER

EFFECTIVE

SKIN (FGR)

Co- 60

GONADS

BREAST

LUNGS

RED MARR

BONE SUR

THYROID

REMAINDER

EFFECTIVE

SKIN (FGR)

$\mathrm{Kr}-85$

GONADS

BREAST

LUNGS

RED MARR

BONE SUR

THYROID

REMAINDER

EFFECTIVE

SKIN (FGR)

$\mathrm{Kr}-85 \mathrm{~m}$

GONADS

BREAST

LUNGS

RED MARR

BONE SUR

THYROID

REMAINDER

EFFECTIVE

SKIN (FGR)

$\mathrm{Kr}-87$

GONADS

BREAST

LUNGS

RED MARR

BONE SUR

THYROID

REMAINDER

EFFECTIVE

SKIN (FGR)

$\mathrm{K} r-88$

GONADS

BREAST

IUNGS

RED MARR

BONE SUR

$Y$
$W$
$Y$
$Y$
$Y$
$Y$
$W$
$W$
$W$

CLOUDSHINE GROUND GROUND GROUND INHALED
SHINE 8HR SHINE 7DAY SHINE RATE ACUTE

$4.660 \mathrm{E}-14 \quad 2.867 \mathrm{E}-11 \quad 5.828 \mathrm{E}-10 \quad 9.970 \mathrm{E}-16-1.000 \mathrm{E}+00 \quad 6.170 \mathrm{E}-10 \quad 1.040 \mathrm{E}-09$ $5.300 \mathrm{E}-14 \quad 2.737 \mathrm{E}-11 \quad 5.565 \mathrm{E}-10 \quad 9.520 \mathrm{E}-16-1.000 \mathrm{E}+00 \quad 9.370 \mathrm{E}-10 \quad 1.790 \mathrm{E}-10$ $4.640 \mathrm{E}-14 \quad 2.617 \mathrm{E}-11 \quad 5.319 \mathrm{E}-10 \quad 9.100 \mathrm{E}-16-1.000 \mathrm{E}+00 \quad 1.600 \mathrm{E}-08 \quad 8.530 \mathrm{E}-11$ $4.530 \mathrm{E}-142.671 \mathrm{E}-11 \quad 5.430 \mathrm{E}-10 \quad 9.290 \mathrm{E}-16-1.000 \mathrm{E}+00 \quad 9.230 \mathrm{E}-10 \quad 2.600 \mathrm{E}-10$ $7.410 \mathrm{E}-14 \quad 3.795 \mathrm{E}-11 \quad 7.716 \mathrm{E}-10 \quad 1.320 \mathrm{E}-15-1.000 \mathrm{E}+00 \quad 6.930 \mathrm{E}-10 \quad 1.250 \mathrm{E}-10$

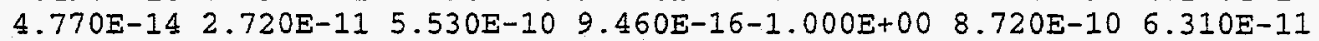
$4.440 \mathrm{E}-142.585 \mathrm{E}-11 \quad 5.255 \mathrm{E}-10 \quad 8.990 \mathrm{E}-16-1.000 \mathrm{E}+00 \quad 1.890 \mathrm{E}-09$ 1.580E-09 $4.760 E-142.732 E-11 \quad 5.553 E-10 \quad 9.500 E-16-1.000 E+00 \quad 2.940 E-09 \quad 8.090 E-10$ $5.580 \mathrm{E}-14 \quad 3.278 \mathrm{E}-11 \quad 6.664 \mathrm{E}-10 \quad 1.140 \mathrm{E}-15-1.000 \mathrm{E}+00 \quad 0.000 \mathrm{E}+00 \quad 0.000 \mathrm{E}+00$

$1.230 \mathrm{E}-13 \quad 7.056 \mathrm{E}-11 \quad 1.480 \mathrm{E}-09 \quad 2.450 \mathrm{E}-15-1.000 \mathrm{E}+00 \quad 4.760 \mathrm{E}-09 \quad 3.190 \mathrm{E}-09$ $1.390 \mathrm{E}-13 \quad 6.739 \mathrm{E}-11 \mathrm{1} 1.413 \mathrm{E}-09 \quad 2.340 \mathrm{E}-15-1.000 \mathrm{E}+00 \quad 1.840 \mathrm{E}-08 \quad 1.100 \mathrm{E}-09$ $1.240 \mathrm{E}-13 \quad 6.537 \mathrm{E}-11 \quad 1.371 \mathrm{E}-09 \quad 2.270 \mathrm{E}-15-1.000 \mathrm{E}+00 \quad 3.450 \mathrm{E}-07 \quad 8.770 \mathrm{E}-10$

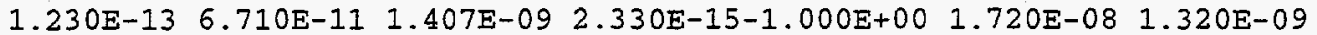
$1.780 \mathrm{E}-13 \quad 8.956 \mathrm{E}-11 \quad 1.879 \mathrm{E}-09 \quad 3.110 \mathrm{E}-15-1.000 \mathrm{E}+00$ 1.350E-08 $9.390 \mathrm{E}-10$ $1.270 \mathrm{E}-13 \quad 6.480 \mathrm{E}-111.359 \mathrm{E}-092.250 \mathrm{E}-15-1.000 \mathrm{E}+00 \quad 1.620 \mathrm{E}-08.7 .880 \mathrm{E}-10$ $1.200 \mathrm{E}-13 \quad 6.508 \mathrm{E}-11$ 1.365E-09 2.260E-15-1.000E+00 3.600E-08 $4.970 \mathrm{E}-09$ $1.260 \mathrm{E}-13 \quad 6.768 \mathrm{E}-11 \quad 1.419 \mathrm{E}-09 \quad 2.350 \mathrm{E}-15-1.000 \mathrm{E}+00 \quad 5.910 \mathrm{E}-08 \quad 2.770 \mathrm{E}-09$ $1.450 E-13 \quad 7.948 E-11 \quad 1.667 E-09 \quad 2.760 E-15-1.000 E+00 \quad 0.000 E+00 \quad 0.000 E+00$

$\begin{array}{llllll}1.170 E-16 & 8.121 E-14 & 1.704 E-12 & 2.820 E-18-1.000 E+00 & 0.000 E+00 & 0.000 E+00\end{array}$ $\begin{array}{lllllll}1.340 E-16 & 7.891 E-14 & 1.656 E-12 & 2.740 E-18-1.000 E+00 & 0.000 E+00 & 0.000 E+00\end{array}$ $1.140 \mathrm{E}-16 \quad 7.056 \mathrm{E}-14 \quad 1.481 \mathrm{E}-12 \quad 2.450 \mathrm{E}-18-1.000 \mathrm{E}+00 \quad 0.000 \mathrm{E}+00 \quad 0.000 \mathrm{E}+00$ $1.090 \mathrm{E}-16 \quad 6.998 \mathrm{E}-14 \quad 1.469 \mathrm{E}-12 \quad 2.430 \mathrm{E}-18-1.000 \mathrm{E}+00 \quad 0.000 \mathrm{E}+00 \quad 0.000 \mathrm{E}+00$ $2.200 E-16 \quad 1.287 E-13 \quad 2.702 E-12 \quad 4.470 E-18-1.000 E+00 \quad 0.000 E+00 \quad 0.000 E+00$ $1.180 \mathrm{E}-16 \quad 7.459 \mathrm{E}-14 \quad 1.565 \mathrm{E}-12 \quad 2.590 \mathrm{E}-18-1.000 \mathrm{E}+00 \quad 0.000 \mathrm{E}+00 \quad 0.000 \mathrm{E}+00$ $\begin{array}{lllllll}1.090 E-16 & 6.941 \mathrm{E}-14 & 1.457 \mathrm{E}-12 & 2.410 \mathrm{E}-18-1.000 \mathrm{E}+00 & 0.000 \mathrm{E}+00 & 0.000 \mathrm{E}+00\end{array}$ $1.190 \mathrm{E}-16 \quad 7.603 \mathrm{E}-14 \quad 1.596 \mathrm{E}-12 \quad 2.640 \mathrm{E}-18-1.000 \mathrm{E}+00 \quad 0.000 \mathrm{E}+00 \quad 0.000 \mathrm{E}+00$ $\begin{array}{llllll}1.320 \mathrm{E}-14 & 2.304 \mathrm{E}-11 & 4.835 \mathrm{E}-10 & 8.000 \mathrm{E}-16-1.000 \mathrm{E}+00 & 0.000 \mathrm{E}+00 & 0.000 \mathrm{E}+00\end{array}$

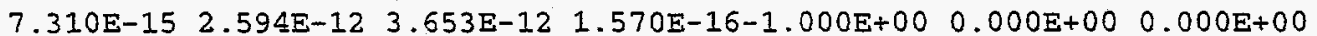
$\begin{array}{llllll}8.410 E-15 & 2.527 E-12 & 3.560 E-12 & 1.530 E-16-1.000 E+00 & 0.000 E+00 & 0.000 E+00\end{array}$ $\begin{array}{llllll}7.040 E-15 & 2.379 E-12 & 3.351 E-12 & 1.440 E-16-1.000 E+00 & 0.000 E+00 & 0.000 E+00\end{array}$

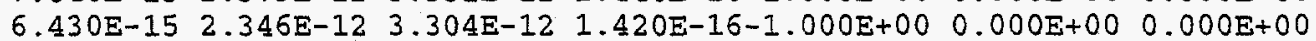
$1.880 \mathrm{E}-14 \quad 5.286 \mathrm{E}-12 \quad 7.446 \mathrm{E}-12 \cdot 3.200 \mathrm{E}-16-1.000 \mathrm{E}+00 \quad 0.000 \mathrm{E}+00 \quad 0.000 \mathrm{E}+00$ $\begin{array}{llllll}7.330 E-15 & 2.395 E-12 & 3.374 E-12 & 1.450 E-16-1.000 E+00 & 0.000 E+00 & 0.000 E+00\end{array}$

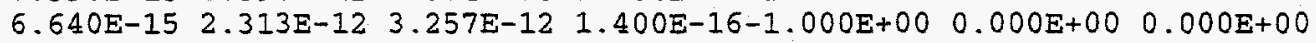

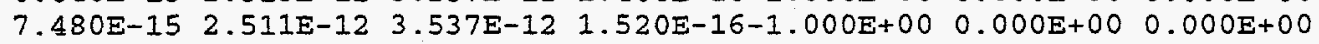
$2.240 \mathrm{E}-14 \quad 2.247 \mathrm{E}-11 \quad 3.164 \mathrm{E}-11 \quad 1.360 \mathrm{E}-15-1.000 \mathrm{E}+00 \quad 0.000 \mathrm{E}+00 \quad 0.000 \mathrm{E}+00$

$4.000 \mathrm{E}-14 \quad 4.962 \mathrm{E}-12 \quad 5.026 \mathrm{E}-12 \quad 7.610 \mathrm{E}-16-1.000 \mathrm{E}+00 \quad 0.000 \mathrm{E}+00 \quad 0.000 \mathrm{E}+00$ $4.500 \mathrm{E}-14 \quad 4.740 \mathrm{E}-12 \quad 4.802 \mathrm{E}-12 \quad 7.270 \mathrm{E}-16-1.000 \mathrm{E}+00 \quad 0.000 \mathrm{E}+00 \quad 0.000 \mathrm{E}+00$ $4.040 \mathrm{E}-14 \quad 4.603 \mathrm{E}-12 \quad 4.663 \mathrm{E}-12 \quad 7.060 \mathrm{E}-16-1.000 \mathrm{E}+00 \quad 0.000 \mathrm{E}+00 \quad 0.000 \mathrm{E}+00$ $\begin{array}{lllllll}4.000 E-14 & 4.708 E-12 & 4.769 E-12 & 7.220 E-16-1.000 E+00 & 0.000 E+00 & 0.000 E+00\end{array}$ $6.020 \mathrm{E}-14 \quad 6.514 \mathrm{E}-12 \quad 6.598 \mathrm{E}-12 \quad 9.990 \mathrm{E}-16-1.000 \mathrm{E}+00 \quad 0.000 \mathrm{E}+00 \quad 0.000 \mathrm{E}+00$ $\begin{array}{lllllll}4.130 E-14 & 4.473 E-12 & 4.531 E-12 & 6.860 E-16-1.000 E+00 & 0.000 E+00 & 0.000 E+00\end{array}$ $3.910 \mathrm{E}-14 \quad 4.590 \mathrm{E}-12 \quad 4.650 \mathrm{E}-12 \quad 7.040 \mathrm{E}-16-1.000 \mathrm{E}+00 \quad 0.000 \mathrm{E}+00 \quad 0.000 \mathrm{E}+00$ $4.120 E-14 \quad 4.773 E-12 \quad 4.835 E-12 \quad 7.320 E-16-1.000 E+00 \quad 0.000 E+00 \quad 0.000 E+00$ $\begin{array}{lllll}1.370 E-13 & 8.802 \mathrm{E}-11 & 8.916 \mathrm{E}-11 & 1.350 \mathrm{E}-14-1.000 \mathrm{E}+00 \quad 0.000 \mathrm{E}+00 & 0.000 \mathrm{E}+00\end{array}$

$9.900 E-14 \quad 2.278 E-11 \quad 2.655 E-11 \quad 1.800 E-15-1.000 E+00 \quad 0.000 E+00 \quad 0.000 E+00$ $\begin{array}{llllll}1.110 E-13 & 2.177 E-11 & 2.537 E-11 & 1.720 E-15-1.000 E+00 & 0.000 E+00 & 0.000 E+00\end{array}$ $\begin{array}{llllll}1.010 E-13 & 2.139 E-11 & 2.493 E-11 & 1.690 E-15-1.000 E+00 & 0.000 E+00 & 0.000 E+00\end{array}$ $\begin{array}{llllll}1.000 E-13 & 2.190 E-11 & 2.552 E-11 & 1.730 E-15-1.000 E+00 & 0.000 E+00 & 0.000 E+00\end{array}$

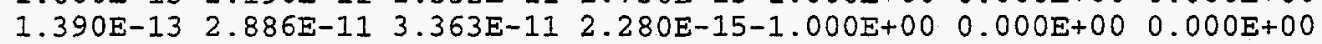


THYROID REMAINDER EFFECTIVE SKIN (FGR) $\mathrm{Rb}-86$ GONADS BREAST IUNGS RED MARR BONE SUR THYROID REMAINDER EFFECTIVE SKIN (FGR) Sx-89 GONADS BREAST IUNGS RED MARR BONE SUR THYROID REMA INDER EFFECTIVE SKIN (FGR) Sr-90 GONADS BREAST LUNGS RED MARR BONE SUR THYROID REMAINDER EFFECTIVE SKIN (FGR) Sr-91

GONADS BREAST LUNGS RED MARR BONE SUR THYROID REMAINDER EFFECTIVE SKIN (FGR) Sr-92

GONADS BREAST LUNGS RED MARR BONE SUR THYROID REMAINDER EFFECTIVE SKIN (FGR) Y-90 GONADS BREAST LUNGS RED MARR BONE SUR THYROID REMAINDER EFFECTIVE SKIN (FGR) $\mathrm{Y}-91$ GONADS BREAST
$1.030 \mathrm{E}-132.012 \mathrm{E}-112.345 \mathrm{E}-11 \quad 1.590 \mathrm{E}-15-1.000 \mathrm{E}+00 \quad 0.000 \mathrm{E}+00 \quad 0.000 \mathrm{E}+00$ $9.790 E-142.139 E-112.493 E-11 \quad 1.690 E-15-1.000 E+00 \quad 0.000 E+00 \quad 0.000 E+00$ $\begin{array}{llllll}1.020 \mathrm{E}-13 & 2.202 \mathrm{E}-11 & 2.567 \mathrm{E}-11 & 1.740 \mathrm{E}-15-1.000 \mathrm{E}+00 & 0.000 \mathrm{E}+00 & 0.000 \mathrm{E}+00\end{array}$ $1.350 \mathrm{E}-13 \quad 5.607 \mathrm{E}-11 \quad 6.534 \mathrm{E}-11 \quad 4.430 \mathrm{E}-15-1.000 \mathrm{E}+00 \quad 0.000 \mathrm{E}+00 \quad 0.000 \mathrm{E}+00$

$4.710 \mathrm{E}-15 \quad 2.788 \mathrm{E}-12 \quad 5.187 \mathrm{E}-11 \quad 9.740 \mathrm{E}-17-1.000 \mathrm{E}+00 \quad 1.340 \mathrm{E}-09 \quad 2.150 \mathrm{E}-09$ $5.340 E-15 \quad 2.662 E-12 \quad 4.953 E-11 \quad 9.300 E-17-1.000 E+00 \quad 1.330 E-092.140 E-09$ $4.710 \mathrm{E}-15 \quad 2.553 \mathrm{E}-12 \quad 4.750 \mathrm{E}-11 \quad 8.920 \mathrm{E}-17-1.000 \mathrm{E}+00 \quad 3.300 \mathrm{E}-09 \quad 2.140 \mathrm{E}-09$ $\begin{array}{llllll}4.640 \mathrm{E}-15 & 2.619 \mathrm{E}-12 & 4.873 \mathrm{E}-11 & 9.150 \mathrm{E}-17-1.000 \mathrm{E}+00 & 2.320 \mathrm{E}-09 & 3.720 \mathrm{E}-09\end{array}$ $7.050 \mathrm{E}-15 \quad 3.635 \mathrm{E}-12 \quad 6.764 \mathrm{E}-11 \quad 1.270 \mathrm{E}-16-1.000 \mathrm{E}+00 \quad 4.270 \mathrm{E}-09 \quad 6.860 \mathrm{E}-09$ $4.840 \mathrm{E}-15 \quad 2.599 \mathrm{E}-12 \quad 4.836 \mathrm{E}-11 \quad 9.080 \mathrm{E}-17-1.000 \mathrm{E}+00 \quad 1.330 \mathrm{E}-09 \quad 2.140 \mathrm{E}-09$

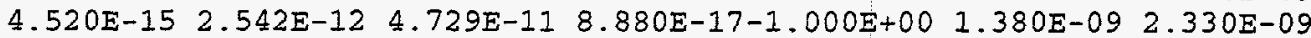
$4.810 \mathrm{E}-15 \quad 2.665 \mathrm{E}-12 \quad 4.958 \mathrm{E}-11$ 9.310E-17-1.000E+00 1.790E-09 $2.530 \mathrm{E}-09$ $4.850 \mathrm{E}-142.210 \mathrm{E}-10 \quad 4.111 \mathrm{E}-09 \quad 7.720 \mathrm{E}-15-1.000 \mathrm{E}+00 \quad 0.000 \mathrm{E}+00 \quad 0.000 \mathrm{E}+00$

$7.730 \mathrm{E}-17 \quad 7.155 \mathrm{E}-14 \quad 1.436 \mathrm{E}-12 \quad 2.490 \mathrm{E}-18-1.000 \mathrm{E}+00 \quad 7.950 \mathrm{E}-12 \quad 8.050 \mathrm{E}-12$ $9.080 \mathrm{E}-17 \quad 7.212 \mathrm{E}-14 \quad 1.447 \mathrm{E}-12 \quad 2.510 \mathrm{E}-18-1.000 \mathrm{E}+00 \quad 7.960 \mathrm{E}-12 \quad 7.980 \mathrm{E}-12$ $7.080 \mathrm{E}-17 \quad 5.689 \mathrm{E}-14 \quad 1.142 \mathrm{E}-12 \quad 1.980 \mathrm{E}-18-1.000 \mathrm{E}+00 \quad 8.350 \mathrm{E}-08 \quad 7.970 \mathrm{E}-12$ $6.390 \mathrm{E}-17 \quad 5.345 \mathrm{E}-14 \quad 1.073 \mathrm{E}-12 \quad 1.860 \mathrm{E}-18-1.000 \mathrm{E}+00 \quad 1.070 \mathrm{E}-10 \quad 1.080 \mathrm{E}-10$ $1.940 \mathrm{E}-16 \quad 1.560 \mathrm{E}-13 \quad 3.131 \mathrm{E}-12 \quad 5.430 \mathrm{E}-18-1.000 \mathrm{E}+00 \quad 1.590 \mathrm{E}-10 \quad 1.610 \mathrm{E}-10$ $7.600 \mathrm{E}-17 \quad 6.063 \mathrm{E}-14 \quad 1.217 \mathrm{E}-12 \quad 2.110 \mathrm{E}-18-1.000 \mathrm{E}+00 \quad 7.960 \mathrm{E}-12 \quad 7.970 \mathrm{E}-12$ $6.710 \mathrm{E}-17 \quad 5.603 \mathrm{E}-14 \quad 1.124 \mathrm{E}-12 \quad 1.950 \mathrm{E}-18-1.000 \mathrm{E}+00 \quad 3.970 \mathrm{E}-09 \quad 8.250 \mathrm{E}-09$

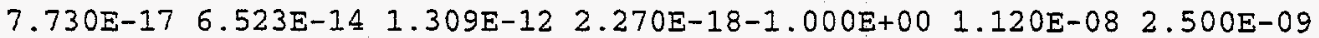

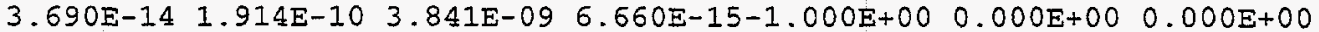

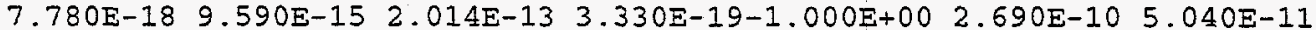
$9.490 \mathrm{E}-18 \quad 1.008 \mathrm{E}-14 \quad 2.116 \mathrm{E}-13 \quad 3.500 \mathrm{E}-19-1.000 \mathrm{E}+00 \quad 2.690 \mathrm{E}-10 \quad 5.040 \mathrm{E}-11$ $6.440 \mathrm{E}-18 \quad 6.307 \mathrm{E}-15 \quad 1.324 \mathrm{E}-13 \quad 2.190 \mathrm{E}-19-1.000 \mathrm{E}+00 \quad 2.860 \mathrm{E}-06 \quad 5.040 \mathrm{E}-11$ $5.440 \mathrm{E}-18 \quad 5.558 \mathrm{E}-15 \quad 1.167 \mathrm{E}-13 \quad 1.930 \mathrm{E}-19-1.000 \mathrm{E}+00 \quad 3.280 \mathrm{E}-08 \quad 6.450 \mathrm{E}-09$ $2.280 \mathrm{E}-17 \quad 2.393 \mathrm{E}-14 \quad 5.025 \mathrm{E}-13 \quad 8.310 \mathrm{E}-19-1.000 \mathrm{E}+00 \quad 7.090 \mathrm{E}-08 \quad 1.390 \mathrm{E}-08$ $\begin{array}{llllll}7.330 \mathrm{E}-18 & 7.171 \mathrm{E}-15 & 1.506 \mathrm{E}-13 & 2.490 \mathrm{E}-19-1.000 \mathrm{E}+00 & 2.690 \mathrm{E}-10 & 5.040 \mathrm{E}-11\end{array}$ $6.110 \mathrm{E}-18 \quad 6.422 \mathrm{E}-15 \quad 1.348 \mathrm{E}-13 \quad 2.230 \mathrm{E}-19-1.000 \mathrm{E}+00 \quad 5.730 \mathrm{E}-09 \quad 6.700 \mathrm{E}-09$

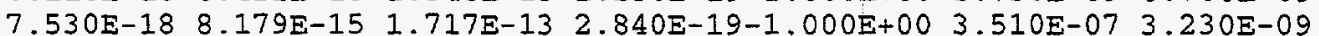
$9.200 \mathrm{E}-15 \quad 4.032 \mathrm{E}-12 \quad 8.465 \mathrm{E}-11 \quad 1.400 \mathrm{E}-16-1.000 \mathrm{E}+00 \quad 0.000 \mathrm{E}+00 \quad 0.000 \mathrm{E}+00$

$4.819 \mathrm{E}-142.155 \mathrm{E}-11 \quad 5.062 \mathrm{E}-11 \quad 1.026 \mathrm{E}-15-1.000 \mathrm{E}+00 \quad 5.669 \mathrm{E}-112.520 \mathrm{E}-10$ $5.477 \mathrm{E}-142.059 \mathrm{E}-11 \quad 4.838 \mathrm{E}-11 \quad 9.806 \mathrm{E}-16-1.000 \mathrm{E}+00 \quad 1.775 \mathrm{E}-113.676 \mathrm{E}-11$ $4.803 \mathrm{E}-14$ 1.970E-11 $4.626 \mathrm{E}-11 \quad 9.376 \mathrm{E}-16-1.000 \mathrm{E}+00 \quad 2.170 \mathrm{E}-09 \quad 1.055 \mathrm{E}-11$ $4.691 \mathrm{E}-142.011 \mathrm{E}-11 \quad 4.722 \mathrm{E}-11$ 9.570E-16-1.000E+00 2.275E-11 $5.659 \mathrm{E}-11$

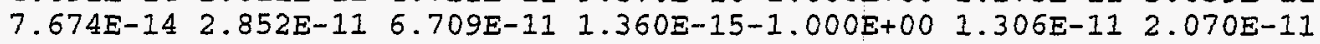
$\begin{array}{lllllll}4.938 \mathrm{E}-14 & 2.035 \mathrm{E}-11 & 4.782 \mathrm{E}-11 & 9.693 \mathrm{E}-16-1.000 \mathrm{E}+00 & 9.930 \mathrm{E}-12 & 1.968 \mathrm{E}-12\end{array}$ $4.610 \mathrm{E}-14$ 1.948E-11. 4.573E-11 9.268E-16-1.000E+00 5.802E-10 2.557E-09 $4.924 \mathrm{E}-142.057 \mathrm{E}-11 \quad 4.832 \mathrm{E}-11 \quad 9.793 \mathrm{E}-16-1.000 \mathrm{E}+00 \quad 4.547 \mathrm{E}-10 \quad 8.455 \mathrm{E}-10$ $9.938 \mathrm{E}-141.748 \mathrm{E}-10 \quad 3.987 \mathrm{E}-10 \quad 8.080 \mathrm{E}-15-1.000 \mathrm{E}+00 \quad 0.000 \mathrm{E}+00 \quad 0.000 \mathrm{E}+00$

$6.610 \mathrm{E}-141.593 \mathrm{E}-111.830 \mathrm{E}-11 \quad 1.300 \mathrm{E}-15-1.000 \mathrm{E}+00 \quad 1.020 \mathrm{E}-118.180 \mathrm{E}-11$ $7.480 \mathrm{E}-14 \quad 1.520 \mathrm{E}-11 \quad 1.745 \mathrm{E}-11 \quad 1.240 \mathrm{E}-15-1.000 \mathrm{E}+00 \quad 6.490 \mathrm{E}-12 \quad 1.700 \mathrm{E}-11$ $6.670 \mathrm{E}-141.483 \mathrm{E}-11 \quad 1.703 \mathrm{E}-11$ 1.210E-15-1.000E+00 $1.050 \mathrm{E}-09 \quad 7.220 \mathrm{E}-12$ $6.620 \mathrm{E}-14 \quad 1.520 \mathrm{E}-111.745 \mathrm{E}-11 \quad 1.240 \mathrm{E}-15-1.000 \mathrm{E}+00 \quad 6.980 \mathrm{E}-12 \quad 2.290 \mathrm{E}-11$ $9.490 \mathrm{E}-142.010 \mathrm{E}-112 \mathrm{2} 2.308 \mathrm{E}-11 \quad 1.640 \mathrm{E}-15-1.000 \mathrm{E}+00 \quad 4.360 \mathrm{E}-12 \quad 8.490 \mathrm{E}-12$ $6.820 \mathrm{E}-14 \quad 1.446 \mathrm{E}-11 \quad 1.661 \mathrm{E}-11 \quad 1.180 \mathrm{E}-15-1.000 \mathrm{E}+00 \quad 3.920 \mathrm{E}-12 \quad 1.300 \mathrm{E}-12$ $6.450 \mathrm{E}-141.471 \mathrm{E}-11 \quad 1.689 \mathrm{E}-11 \quad 1.200 \mathrm{E}-15-1.000 \mathrm{E}+00 \quad 2.900 \mathrm{E}-10 \quad 1.720 \mathrm{E}-09$ $6.790 \mathrm{E}-141.532 \mathrm{E}-111.759 \mathrm{E}-11 \quad 1.250 \mathrm{E}-15-1.000 \mathrm{E}+00 \quad 2.180 \mathrm{E}-10 \quad 5.430 \mathrm{E}-10$ $\begin{array}{llllll}8.560 \mathrm{E}-14 & 2.280 \mathrm{E}-11 & 2.618 \mathrm{E}-11 & 1.860 \mathrm{E}-15-1.000 \mathrm{E}+00 & 0.000 \mathrm{E}+00 & 0.000 \mathrm{E}+00\end{array}$

$1.890 \mathrm{E}-16 \quad 1.586 \mathrm{E}-13 \quad 1.601 \mathrm{E}-12 \quad 5.750 \mathrm{E}-18-1.000 \mathrm{E}+00 \quad 5.170 \mathrm{E}-13 \quad 1.430 \mathrm{E}-14$ $2.200 \mathrm{E}-16 \quad 1.578 \mathrm{E}-13 \quad 1.593 \mathrm{E}-12 \quad 5.720 \mathrm{E}-18-1.000 \mathrm{E}+00 \quad 5.170 \mathrm{E}-13 \quad 1.270 \mathrm{E}-14$ $1.770 \mathrm{E}-16 \quad 1.313 \mathrm{E}-13 \quad 1.326 \mathrm{E}-12 \quad 4.760 \mathrm{E}-18-1.000 \mathrm{E}+00 \quad 9.310 \mathrm{E}-09 \quad 1.260 \mathrm{E}-14$ $1.620 \mathrm{E}-16 \quad 1.261 \mathrm{E}-13 \quad 1.273 \mathrm{E}-12 \quad 4.570 \mathrm{E}-18-1.000 \mathrm{E}+00 \quad 1.520 \mathrm{E}-11 \quad 3.700 \mathrm{E}-13$ $4.440 \mathrm{E}-16 \quad 3.228 \mathrm{E}-13 \quad 3.259 \mathrm{E}-12 \quad 1.170 \mathrm{E}-17-1.000 \mathrm{E}+00 \quad 1.510 \mathrm{E}-11 \quad 3.670 \mathrm{E}-13$ $1.870 \mathrm{E}-16 \quad 1.385 \mathrm{E}-13 \quad 1.398 \mathrm{E}-12 \quad 5.020 \mathrm{E}-18-1.000 \mathrm{E}+00 \quad 5.170 \mathrm{E}-13 \quad 1.260 \mathrm{E}-14$ $1.680 \mathrm{E}-16 \quad 1.291 \mathrm{E}-13 \quad 1.303 \mathrm{E}-12 \quad 4.680 \mathrm{E}-18-1.000 \mathrm{E}+00 \quad 3.870 \mathrm{E}-09 \quad 9.680 \mathrm{E}-09$ $1.900 \mathrm{E}-16 \quad 1.468 \mathrm{E}-13 \quad 1.482 \mathrm{E}-12 \quad 5.320 \mathrm{E}-18-1.000 \mathrm{E}+00 \quad 2.280 \mathrm{E}-092.910 \mathrm{E}-09$ $6.240 \mathrm{E}-142.897 \mathrm{E}-10 \quad 2.924 \mathrm{E}-09 \quad 1.050 \mathrm{E}-14-1.000 \mathrm{E}+00 \quad 0.000 \mathrm{E}+00 \quad 0.000 \mathrm{E}+00$

$2.560 \mathrm{E}-16 \quad 1.756 \mathrm{E}-13 \quad 3.546 \mathrm{E}-12 \quad 6.110 \mathrm{E}-18-1.000 \mathrm{E}+00 \quad 8.200 \mathrm{E}-12 \quad 3.540 \mathrm{E}-12$ $2.930 \mathrm{E}-16 \quad 1.713 \mathrm{E}-13 \quad 3.459 \mathrm{E}-12 \quad 5.960 \mathrm{E}-18-1.000 \mathrm{E}+00 \quad 8.920 \mathrm{E}-12 \quad 5.540 \mathrm{E}-13$ 
IUNGS

RED MARR

BONE SUR

THYROID

REMAINDER

EFFECTIVE

SKIN (FGR)

Y-92

GONADS

BREAST

LUNGS

RED MARR

BONE SUR

THYROID

REMAINDER

EFFECTIVE

SKIN (FGR)

$Y-93$

GONADS

BREAST

IUNGS

RED MARR

BONE SUR

THYROID

REMAINDER

EFFECTIVE

SKIN (FGR)

Zr -95

GONADS

BREAST

LUNGS

RED MARR

BONE SUR

THYROID

REMAINDER

EFFECTIVE

$\operatorname{SKIN~(FGR)~}$

Zr-97

GONADS

BREAST

IUNGS

RED MARR

BONE SUR

THYROID

REMA INDER

EFFECTIVE

SKIN (FGR)

$\mathrm{Nb}-95$

GONADS

BREAST

IUNGS

RED MARR.

BONE SUR

THYROID

REMAINDER

EFFECTIVE

SKIN (FGR)

Mo-99

GONADS

BREAST

LUNGS

RED MARR

BONE SUR

THYROID

REMAINDER

EFFECTIVE

SKIN (FGR)
$2.500 \mathrm{E}-16 \quad 1.526 \mathrm{E}-13 \quad 3.082 \mathrm{E}-12 \quad 5.310 \mathrm{E}-18-1.000 \mathrm{E}+00 \quad 9.870 \mathrm{E}-08 \quad 2.020 \mathrm{E}-13$ $2.410 \mathrm{E}-16 \quad 1.521 \mathrm{E}-13 \quad 3.070 \mathrm{E}-12 \quad 5.290 \mathrm{E}-18-1.000 \mathrm{E}+00 \quad 3.190 \mathrm{E}-106.590 \mathrm{E}-12$ $4.560 \mathrm{E}-16 \quad 2.903 \mathrm{E}-13 \quad 5.862 \mathrm{E}-12 \quad 1.010 \mathrm{E}-17-1.000 \mathrm{E}+00 \quad 3.180 \mathrm{E}-10 \quad 6.130 \mathrm{E}-12$ $2.600 E-16 \quad 1.564 E-13 \quad 3.157 E-12 \quad 5.440 E-18-1.000 E+00 \quad 8.500 E-12 \quad 1.290 E-13$ $2.390 \mathrm{E}-16 \quad 1.509 \mathrm{E}-13 \quad 3.047 \mathrm{E}-12 \quad 5.250 \mathrm{E}-18-1.000 \mathrm{E}+00 \quad 4.200 \mathrm{E}-09 \quad 8.570 \mathrm{E}-09$ $2.600 \mathrm{E}-16 \quad 1.650 \mathrm{E}-13 \quad 3.332 \mathrm{E}-12 \quad 5.740 \mathrm{E}-18-1.000 \mathrm{E}+00$ 1.320E-08 $2.570 \mathrm{E}-09$ $3.850 \mathrm{E}-14 \quad 1.989 \mathrm{E}-10 \quad 4.016 \mathrm{E}-09 \quad 6.920 \mathrm{E}-15-1.000 \mathrm{E}+00 \quad 0.000 \mathrm{E}+00 \quad 0.000 \mathrm{E}+00$

$1.270 \mathrm{E}-14 \quad 3.855 \mathrm{E}-12 \quad 4.872 \mathrm{E}-12 \quad 2.650 \mathrm{E}-16-1.000 \mathrm{E}+00 \quad 2.610 \mathrm{E}-12 \quad 1.960 \mathrm{E}-11$ $1.440 \mathrm{E}-14 \quad 3.580 \mathrm{E}-12 \quad 4.652 \mathrm{E}-12 \quad 2.530 \mathrm{E}-16-1.000 \mathrm{E}+00 \quad 1.500 \mathrm{E}-12 \quad 3.550 \mathrm{E}-12$ $1.270 \mathrm{E}-14 \quad 3.535 \mathrm{E}-12 \quad 4.468 \mathrm{E}-12 \quad 2.430 \mathrm{E}-16-1.000 \mathrm{E}+00 \quad 1.240 \mathrm{E}-09 \quad 1.390 \mathrm{E}-12$ $1.250 \mathrm{E}-14 \quad 3.608 \mathrm{E}-12 \quad 4.560 \mathrm{E}-12 \quad 2.480 \mathrm{E}-16-1.000 \mathrm{E}+00 \quad 2.070 \mathrm{E}-12 \quad 4.910 \mathrm{E}-12$ $1.950 E-14 \quad 5.091 \mathrm{E}-12 \quad 6.435 \mathrm{E}-12 \quad 3.500 \mathrm{E}-16-1.000 \mathrm{E}+00 \quad 1.510 \mathrm{E}-12 \quad 1.750 \mathrm{E}-12$ $1.300 \mathrm{E}-14 \cdot 3.579 \mathrm{E}-12 \quad 4.523 \mathrm{E}-12 \quad 2.460 \mathrm{E}-16-1.000 \mathrm{E}+00 \quad 1.050 \mathrm{E}-12 \quad 1.770 \mathrm{E}-13$ $1.220 E-14 \quad 3.506 E-12 \quad 4.431 E-12 \quad 2.410 E-16-1.000 E+002.030 E-101.700 E-09$ $1.300 \mathrm{E}-14 \quad 3.680 \mathrm{E}-12 \quad 4.652 \mathrm{E}-12 \quad 2.530 \mathrm{E}-16-1.000 \mathrm{E}+00 \quad 2.110 \mathrm{E}-10 \quad 5.150 \mathrm{E}-10$

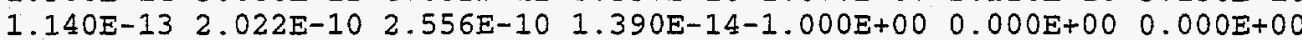

4.670E-15 2.108E-12 4.989E-12 9.510E-17-1.000E+00 5.310E-12 2.200E-11 $5.300 E-15 \quad 2.026 E-12 \quad 4.794 E-12 \quad 9.140 E-17-1.000 E+00 \quad 1.740 E-12 \quad 3.130 E-12$ $4.680 E-15 \quad 1.937 E-12 \quad 4.585 E-12 \quad 8.740 E-17-1.000 E+002.520 E-098.670 E-13$ $4.580 \mathrm{E}-15 \quad 1.972 \mathrm{E}-12 \quad 4.669 \mathrm{E}-12 \quad 8.900 \mathrm{E}-17-1.000 \mathrm{E}+00 \quad 4.040 \mathrm{E}-12 \quad 4.930 \mathrm{E}-12$ $7.580 E-15 \quad 2.948 E-12 \quad 6.977 E-12 \quad 1.330 E-16-1.000 E+00 \quad 3.140 E-12 \quad 1.730 E-12$ $4.790 \mathrm{E}-15 \quad 1.908 \mathrm{E}-12 \quad 4.516 \mathrm{E}-12 \quad 8.610 \mathrm{E}-17-1.000 \mathrm{E}+00 \quad 9.260 \mathrm{E}-13 \quad 1.260 \mathrm{E}-13$ $4.510 \mathrm{E}-15 \quad 1.919 \mathrm{E}-12 \quad 4.543 \mathrm{E}-12 \quad 8.660 \mathrm{E}-17-1.000 \mathrm{E}+00 \quad 9.250 \mathrm{E}-10 \quad 4.090 \mathrm{E}-09$ $4.800 \mathrm{E}-15 \quad 2.021 \mathrm{E}-12 \quad 4.784 \mathrm{E}-12 \quad 9.120 \mathrm{E}-17-1.000 \mathrm{E}+00 \quad 5.820 \mathrm{E}-10 \quad 1.230 \mathrm{E}-09$ $8.500 \mathrm{E}-142.726 \mathrm{E}-10 \quad 6.452 \mathrm{E}-10 \quad 1.230 \mathrm{E}-14-1.000 \mathrm{E}+00 \quad 0.000 \mathrm{E}+00 \quad 0.000 \mathrm{E}+00$

$3.530 \mathrm{E}-142.182 \mathrm{E}-11 \quad 4.421 \mathrm{E}-10 \quad 7.590 \mathrm{E}-16-1.000 \mathrm{E}+00 \quad 1.880 \mathrm{E}-098.160 \mathrm{E}-10$ $4.010 \mathrm{E}-14 \quad 2.084 \mathrm{E}-11 \quad 4.223 \mathrm{E}-10 \quad 7.250 \mathrm{E}-16-1.000 \mathrm{E}+00 \quad 1.910 \mathrm{E}-09 \quad 1.050 \mathrm{E}-10$ $3.510 E-14$ 1.989E-11 4.030E-10 6.920E-16-1.000E+00 2.170E-09 2.340E-11 $3.430 \mathrm{E}-142.030 \mathrm{E}-11 \quad 4.112 \mathrm{E}-10 \quad 7.060 \mathrm{E}-16-1.000 \mathrm{E}+00 \quad 1.300 \mathrm{E}-08 \quad 2.140 \mathrm{E}-10$ $5.620 \mathrm{E}-14 \quad 2.875 \mathrm{E}-11 \quad 5.824 \mathrm{E}-10 \quad 1.000 \mathrm{E}-15-1.000 \mathrm{E}+00 \quad 1.030 \mathrm{E}-07 \quad 4.860 \mathrm{E}-10$ $3.610 \mathrm{E}-142.076 \mathrm{E}-11$ 4.205E-10 $7.220 \mathrm{E}-16-1.000 \mathrm{E}+00$ 1.440E-09 $8.270 \mathrm{E}-12$ $3.360 E-14 \quad 1.963 E-11 \quad 3.978 E-10 \quad 6.830 E-16-1.000 E+00 \quad 2.280 E-09 \quad 2.530 E-09$ $3.600 \mathrm{E}-142.078 \mathrm{E}-11$ 4.211E-10 $7.230 \mathrm{E}-16-1.000 \mathrm{E}+00 \quad 6.390 \mathrm{E}-09$ 1.020E-09 $4.500 \mathrm{E}-142.561 \mathrm{E}-115.190 \mathrm{E}-10 \quad 8.910 \mathrm{E}-16-1.000 \mathrm{E}+00 \quad 0.000 \mathrm{E}+00 \quad 0.000 \mathrm{E}+00$

$4.331 \mathrm{E}-142.179 \mathrm{E}-11 \quad 7.799 \mathrm{E}-11 \quad 9.253 \mathrm{E}-16-1.000 \mathrm{E}+00 \quad 1.840 \mathrm{E}-10 \quad 6.228 \mathrm{E}-10$ $4.928 \mathrm{E}-142.083 \mathrm{E}-11 \quad 7.455 \mathrm{E}-11 \quad 8.846 \mathrm{E}-16-1.000 \mathrm{E}+00 \quad 4.706 \mathrm{E}-11 \quad 8.137 \mathrm{E}-11$ $4.322 \mathrm{E}-14 \quad 1.992 \mathrm{E}-11$ 7.127E-11 8.456E-16-1.000E+00 4.108E-09 $1.770 \mathrm{E}-11$ $4.224 \mathrm{E}-142.034 \mathrm{E}-11$ 7.279E-11 $8.634 \mathrm{E}-16-1.000 \mathrm{E}+00 \quad 6.376 \mathrm{E}-11 \quad 1.302 \mathrm{E}-10$ $6.897 \mathrm{E}-142.881 \mathrm{E}-11$ 1.031E-10 $1.224 \mathrm{E}-15-1.000 \mathrm{E}+00 \quad 3.504 \mathrm{E}-11 \mathrm{l}$ 4.558E-11 4.443E-14 2.061E-11 7.377E-11 8.755E-16-1.000E+00 2.315E-11 $2.671 E-12$ $4.139 \mathrm{E}-14$ 1.966E-11 $7.035 \mathrm{E}-11 \quad 8.345 \mathrm{E}-16-1.000 \mathrm{E}+00 \quad 2.041 \mathrm{E}-096.990 \mathrm{E}-09$

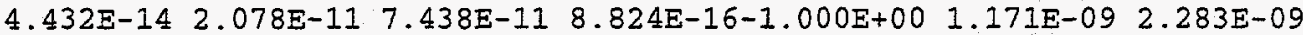
$9.835 \mathrm{E}-142.281 \mathrm{E}-10 \quad 8.148 \mathrm{E}-10 \quad 9.587 \mathrm{E}-15-1.000 \mathrm{E}+00 \quad 0.000 \mathrm{E}+00 \quad 0.000 \mathrm{E}+00$

$3.660 \mathrm{E}-1422.253 \mathrm{E}-11 \quad 4.435 \mathrm{E}-10 \quad 7.850 \mathrm{E}-16-1.000 \mathrm{E}+00 \quad 4.320 \mathrm{E}-10 \quad 8.050 \mathrm{E}-10$ $4.160 \mathrm{E}-14$ 2.150E-11 $4.231 \mathrm{E}-10 \quad 7.490 \mathrm{E}-16-1.000 \mathrm{E} \div 00 \quad 4.070 \mathrm{E}-10 \quad 1.070 \mathrm{E}-10$ $3.650 \mathrm{E}-14 \quad 2.055 \mathrm{E}-11 \mathrm{4} \quad 4.045 \mathrm{E}-10 \quad 7.160 \mathrm{E}-16-1.000 \mathrm{E}+00 \quad 8.320 \mathrm{E}-092.740 \mathrm{E}-11$ $3.560 E-142.101 E-11 \quad 4.135 E-10 \quad 7.320 E-16-1.000 E+00 \quad 4.420 E-10 \quad 1.990 E-10$ $5.790 \mathrm{E}-142.957 \mathrm{E}-115.819 \mathrm{E}-10 \quad 1.030 \mathrm{E}-15-1.000 \mathrm{E}+00 \quad 5.130 \mathrm{E}-10 \quad 2.940 \mathrm{E}-10$ $3.750 \mathrm{E}-142.144 \mathrm{E}-11 \quad 4.220 \mathrm{E}-10 \quad 7.470 \mathrm{E}-16-1.000 \mathrm{E}+00 \quad 3.580 \mathrm{E}-10 \quad 1.180 \mathrm{E}-11$ $3.490 \mathrm{E}-142.032 \mathrm{E}-11$ 4.000E-10 7.080E-16-1.000E+00 1.070E-09 1.470E-09 $3.740 \mathrm{E}-142.147 \mathrm{E}-11$ 4.226E-10 $7.480 \mathrm{E}-16-1.000 \mathrm{E}+00 \quad 1.570 \mathrm{E}-09 \quad 6.950 \mathrm{E}-10$ $4.300 \mathrm{E}-14 \quad 2.598 \mathrm{E}-11 \quad 5.112 \mathrm{E}-10 \quad 9.050 \mathrm{E}-16-1.000 \mathrm{E}+00 \quad 0.000 \mathrm{E}+00 \quad 0.000 \mathrm{E}+00$

$7.130 \mathrm{E}-15 \quad 4.282 \mathrm{E}-12 \quad 4.403 \mathrm{E}-11 \quad 1.550 \mathrm{E}-16-1.000 \mathrm{E}+00 \quad 9.510 \mathrm{E}-112.180 \mathrm{E}-10$ $8.130 \mathrm{E}-15 \quad 4.116 \mathrm{E}-12 \quad 4.233 \mathrm{E}-11$ 1.490E-16-1.000E+00 $2.750 \mathrm{E}-113.430 \mathrm{E}-11$ $7.060 \mathrm{E}-15 \quad 3.867 \mathrm{E}-12 \quad 3.977 \mathrm{E}-11 \quad 1.400 \mathrm{E}-16-1.000 \mathrm{E}+00 \quad 4.290 \mathrm{E}-09 \quad 1.510 \mathrm{E}-11$ $6.820 \mathrm{E}-15 \quad 3.923 \mathrm{E}-12 \quad 4.034 \mathrm{E}-11$ 1.420E-16-1.000E+00 5.240E-11 $8.320 \mathrm{E}-11$

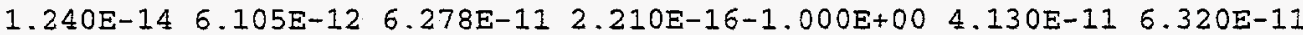
$7.270 \mathrm{E}-15 \quad 4.033 \mathrm{E}-12 \quad 4.147 \mathrm{E}-11 \quad 1.460 \mathrm{E}-16-1.000 \mathrm{E}+00$ 1.520E-11 $1.030 \mathrm{E}-11$ $6.740 \mathrm{E}-15 \quad 3.812 \mathrm{E}-12 \quad 3.920 \mathrm{E}-11 \quad 1.380 \mathrm{E}-16-1.000 \mathrm{E}+00 \quad 1.740 \mathrm{E}-09 \quad 4.280 \mathrm{E}-09$ $7.280 \mathrm{E}-15 \quad 4.061 \mathrm{E}-12 \quad 4.176 \mathrm{E}-11 \quad 1.470 \mathrm{E}-16-1.000 \mathrm{E}+00 \quad 1.070 \mathrm{E}-09 \quad 1.360 \mathrm{E}-09$ $3.140 \mathrm{E}-141.039 \mathrm{E}-10 \quad 1.068 \mathrm{E}-09 \quad 3.760 \mathrm{E}-15-1.000 \mathrm{E}+00 \quad 0.000 \mathrm{E}+00 \quad 0.000 \mathrm{E}+00$ 
Tc-99m GONADS BREAST LUNGS RED MARR BONE SUR THYROID REMAINDER EFFECTIVE SKIN (FGR) Ru-103 GONADS BREAST LUNGS RED MARR BONE SUR THYROID REMAINDER EFFECTIVE $\operatorname{SKIN~(FGR)~}$

Ru-105 GONADS BREAST IUNGS RED MARR BONE SUR THYROID REMAINDER EFFECTIVE SKIN (FGR) Ru-106 GONADS BREAST IUNGS RED MARR BONE SUR THYROID REMAINDER EFFECTIVE SKIN (FGR) Rh-105 GONADS BREAST LUNGS RED MARR BONE SUR THYROID REMAINDER EFFECTIVE SKIN (FGR) Sb- 127 GONADS BREAST LUNGS RED MARR BONE SUR THYROID REMAINDER EFFECTIVE SKIN (FGR) Sb-129 GONADS BREAST LUNGS RED MARR BONE SUR THYROID $\begin{array}{lllllll}5.750 \mathrm{E}-15 & 2.334 \mathrm{E}-12 & 3.877 \mathrm{E}-12 & 1.240 \mathrm{E}-16-1.000 \mathrm{E}+00 & 2.770 \mathrm{E}-12 & 9.750 \mathrm{E}-12\end{array}$ $6.650 \mathrm{E}-152.258 \mathrm{E}-12 \quad 3.752 \mathrm{E}-12 \quad 1.200 \mathrm{E}-16-1.000 \mathrm{E}+00 \quad 2.150 \mathrm{E}-12 \quad 3.570 \mathrm{E}-12$ $5.490 \mathrm{E}-15 \quad 2.127 \mathrm{E}-12 \cdot 3.533 \mathrm{E}-12 \quad 1.130 \mathrm{E}-16-1.000 \mathrm{E}+00 \quad 2.280 \mathrm{E}-11 \quad 3.140 \mathrm{E}-12$ $4.910 \mathrm{E}-15 \quad 2.070 \mathrm{E}-12 \quad 3.439 \mathrm{E}-12 \quad 1.100 \mathrm{E}-16-1.000 \mathrm{E}+00 \quad 3.360 \mathrm{E}-12 \quad 6.290 \mathrm{E}-12$ $1.630 E-14 \quad 5.383 E-12 \quad 8.942 E-12 \quad 2.860 E-16-1.000 E+00 \quad 2.620 E-12 \quad 4.060 E-12$ $5.750 \mathrm{E}-15 \quad 2.145 \mathrm{E}-12 \quad 3.564 \mathrm{E}-12 \quad 1.140 \mathrm{E}-16-1.000 \mathrm{E}+00 \quad 5.010 \mathrm{E}-11 \quad 8.460 \mathrm{E}-11$ $5.150 \mathrm{E}-15 \quad 2.070 \mathrm{E}-12 \quad 3.439 \mathrm{E}-12 \quad 1.100 \mathrm{E}-16-1.000 \mathrm{E}+00 \quad 1.020 \mathrm{E}-11 \quad 3.340 \mathrm{E}-11$ $5.890 \mathrm{E}-15 \quad 2.277 \mathrm{E}-12 \quad 3.783 \mathrm{E}-12 \quad 1.210 \mathrm{E}-16-1.000 \mathrm{E}+00 \quad 8.800 \mathrm{E}-12 \quad 1.680 \mathrm{E}-11$ $7.140 \mathrm{E}-152.710 \mathrm{E}-12 \quad 4.502 \mathrm{E}-12 \quad 1.440 \mathrm{E}-16-1.000 \mathrm{E}+00 \quad 0.000 \mathrm{E}+00 \quad 0.000 \mathrm{E}+00$

$2.191 \mathrm{E}-141.404 \mathrm{E}-112.783 \mathrm{E}-10 \quad 4.892 \mathrm{E}-16-1.000 \mathrm{E}+00 \quad 3.070 \mathrm{E}-10 \quad 5.720 \mathrm{E}-10$ $2.512 \mathrm{E}-14$ 1.350E-11 $2.677 \mathrm{E}-10 \quad 4.705 \mathrm{E}-16-1.000 \mathrm{E}+00$ 3.1IOE-10 $1.200 \mathrm{E}-10$ $2.180 \mathrm{E}-14 \quad 1.273 \mathrm{E}-112.522 \mathrm{E}-10 \quad 4.432 \mathrm{E}-16-1.000 \mathrm{E}+00 \quad 1.561 \mathrm{E}-08 \quad 7.310 \mathrm{E}-11$ $2.100 \mathrm{E}-14$ 1.287E-11 2.551E-10 4.483E-16-1.000E+00 3.190E-10 1.660E-10 $3.892 \mathrm{E}-14 \quad 1.958 \mathrm{E}-11 \quad 3.882 \mathrm{E}-10 \quad 6.823 \mathrm{E}-16-1.000 \mathrm{E}+00 \quad 2.370 \mathrm{E}-10 \quad 9.631 \mathrm{E}-11$ $2.241 E-14$ 1.331E-I1 $2.639 \mathrm{E}-10 \quad 4.638 \mathrm{E}-16-1.000 \mathrm{E}+00 \quad 2.570 \mathrm{E}-10 \quad 6.250 \mathrm{E}-11$ $2.080 \mathrm{E}-14$ 1.248E-11 $2.472 \mathrm{E}-10 \quad 4.346 \mathrm{E}-16-1.000 \mathrm{E}+00 \quad 1.250 \mathrm{E}-092.110 \mathrm{E}-09$ $2.251 \mathrm{E}-141.332 \mathrm{E}-112.641 \mathrm{E}-10 \quad 4.642 \mathrm{E}-16-1.000 \mathrm{E}+00 \quad 2.421 \mathrm{E}-09 \quad 8.271 \mathrm{E}-10$ $2.774 E-14 \quad 1.785 E-11 \quad 3.543 E-10 \quad 6.229 E-16-1.000 E+00 \quad 0.000 E+00 \quad 0.000 E+00$

$3.720 \mathrm{E}-141.327 \mathrm{E}-11 \quad 1.861 \mathrm{E}-11$ 8.070E-16-1.000E+00 1.590E-11 9.670E-11 $4.240 \mathrm{E}-141.271 \mathrm{E}-11 \mathrm{1} 1.783 \mathrm{E}-11 \quad 7.730 \mathrm{E}-16-1.000 \mathrm{E}+00 \quad 6.610 \mathrm{E}-121.590 \mathrm{E}-11$ $3.700 \mathrm{E}-14$ 1.210E-I1 $1.697 \mathrm{E}-11 \quad 7.360 \mathrm{E}-16-1.000 \mathrm{E}+00 \quad 5.730 \mathrm{E}-10 \quad 6.210 \mathrm{E}-12$ $3.590 \mathrm{E}-141.230 \mathrm{E}-11 \quad 1.725 \mathrm{E}-11 \quad 7.480 \mathrm{E}-16-1.000 \mathrm{E}+00 \quad 7.700 \mathrm{E}-12 \quad 2.350 \mathrm{E}-11$ $6.280 \mathrm{E}-14 \quad 1.809 \mathrm{E}-112.537 \mathrm{E}-11 \quad 1.100 \mathrm{E}-15-1.000 \mathrm{E}+00 \quad 4.620 \mathrm{E}-12 \quad 8.890 \mathrm{E}-12$ $3.800 \mathrm{E}-141.260 \mathrm{E}-11$ 1.766E-11 $7.660 \mathrm{E}-16-1.000 \mathrm{E}+00 \quad 4.150 \mathrm{E}-12 \quad 1.820 \mathrm{E}-12$ $3.540 \mathrm{E}-14$ 1.189E-11 1.667E-11 7.230E-16-1.000E+00 1.610E-10 8.540E-10 $3.810 \mathrm{E}-141.265 \mathrm{E}-111.773 \mathrm{E}-11 \quad 7.690 \mathrm{E}-16-1.000 \mathrm{E}+00 \quad 1.230 \mathrm{E}-102.870 \mathrm{E}-10$ $6.730 \mathrm{E}-14 \quad 7.368 \mathrm{E}-11 \quad 1.033 \mathrm{E}-10 \quad 4.480 \mathrm{E}-15-1.000 \mathrm{E}+00 \quad 0.000 \mathrm{E}+00 \quad 0.000 \mathrm{E}+00$

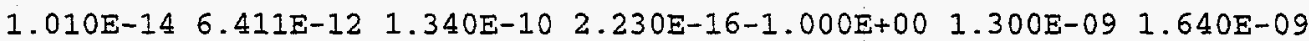
$1.160 \mathrm{E}-146.152 \mathrm{E}-12 \quad 1.286 \mathrm{E}-10 \quad 2.140 \mathrm{E}-16-1.000 \mathrm{E}+00 \quad 1.780 \mathrm{E}-09 \quad 1.440 \mathrm{E}-09$ $1.010 \mathrm{E}-14 \quad 5.836 \mathrm{E}-12 \quad 1.220 \mathrm{E}-10 \quad 2.030 \mathrm{E}-16-1.000 \mathrm{E}+00 \quad 1.040 \mathrm{E}-06 \quad 1.420 \mathrm{E}-09$ $9.750 \mathrm{E}-15 \quad 5.893 \mathrm{E}-12 \quad 1.232 \mathrm{E}-10 \quad 2.050 \mathrm{E}-16-1.000 \mathrm{E}+00 \quad 1.760 \mathrm{E}-09 \quad 1.460 \mathrm{E}-09$ $1.720 \mathrm{E}-14 \quad 8.883 \mathrm{E}-12 \quad 1.856 \mathrm{E}-10 \quad 3.090 \mathrm{E}-16-1.000 \mathrm{E}+00 \quad 1.610 \mathrm{E}-091.430 \mathrm{E}-09$ $1.030 \mathrm{E}-14 \quad 6.066 \mathrm{E}-12 \quad 1.268 \mathrm{E}-10 \quad 2.110 \mathrm{E}-16-1.000 \mathrm{E}+00 \quad 1.720 \mathrm{E}-09 \quad 1.410 \mathrm{E}-09$ $9.630 \mathrm{E}-15 \quad 5.721 \mathrm{E}-12 \quad 1.196 \mathrm{E}-10 \quad 1.990 \mathrm{E}-16-1.000 \mathrm{E}+00 \quad 1.200 \mathrm{E}-08 \quad 2.110 \mathrm{E}-08$ $1.040 E-14 \quad 6.095 E-12 \quad 1.274 E-10 \quad 2.120 E-16-1.000 E \div 00 \quad 1.290 E-07 \quad 7.400 E-09$ $1.090 \mathrm{E}-13 \quad 4.082 \mathrm{E}-10 \quad 8.531 \mathrm{E}-09 \quad 1.420 \mathrm{E}-14-1.000 \mathrm{E}+00 \quad 0.000 \mathrm{E}+00 \quad 0.000 \mathrm{E}+00$

3.640E-15 2.127E-12 1.411E-11 7.980E-17-1.000E+00 2.110E-11 5.800E-11 $4.160 \mathrm{E}-15 \quad 2.063 \mathrm{E}-12 \quad 1.369 \mathrm{E}-11 \quad 7.740 \mathrm{E}-17-1.000 \mathrm{E}+00 \quad 5.610 \mathrm{E}-12 \quad 8.970 \mathrm{E}-12$ $3.570 \mathrm{E}-15 \quad 1.935 \mathrm{E}-12 \quad 1.284 \mathrm{E}-11 \quad 7.260 \mathrm{E}-17-1.000 \mathrm{E}+00 \quad 9.580 \mathrm{E}-10 \quad 3.860 \mathrm{E}-12$ $3.380 E-15 \quad 1.946 E-12 \quad 1.291 E-11 \quad 7.300 E-17-1.000 E+00 \quad 7.770 E-12 \quad 1.47 .0 E-11$ $7.530 \mathrm{E}-15 \quad 3.332 \mathrm{E}-12 \quad 2.210 \mathrm{E}-11 \quad 1.250 \mathrm{E}-16-1.000 \mathrm{E}+00 \quad 4.460 \mathrm{E}-12 \quad 6.750 \mathrm{E}-12$ $3.680 \mathrm{E}-15 \quad 1.983 \mathrm{E}-12 \quad 1.316 \mathrm{E}-11 \quad 7.440 \mathrm{E}-17-1.000 \mathrm{E}+00 \quad 2.880 \mathrm{E}-12 \quad 2.910 \mathrm{E}-12$ $3.390 \mathrm{E}-15$ 1.885E-12 $1.250 \mathrm{E}-11 \quad 7.070 \mathrm{E}-17-1.000 \mathrm{E}+00 \quad 4.530 \mathrm{E}-10$ 1.270E-09 $3.720 \mathrm{E}-15 \quad 2.031 \mathrm{E}-12 \quad 1.347 \mathrm{E}-11 \quad 7.620 \mathrm{E}-17-1.000 \mathrm{E}+00 \quad 2.580 \mathrm{E}-10 \quad 3.990 \mathrm{E}-10$ $1.070 \mathrm{E}-14 \quad 4.691 \mathrm{E}-12 \quad 3.112 \mathrm{E}-11 \quad 1.760 \mathrm{E}-16-1.000 \mathrm{E}+00 \quad 0.000 \mathrm{E}+00 \quad 0.000 \mathrm{E}+00$

$3.260 \mathrm{E}-14141.985 \mathrm{E}-1122.441 \mathrm{E}-10 \quad 7.100 \mathrm{E}-16-1.000 \mathrm{E}+00 \quad 2.520 \mathrm{E}-10 \quad 6.140 \mathrm{E}-10$ $3.720 \mathrm{E}-14 \quad 1.904 \mathrm{E}-11 \quad 2.341 \mathrm{E}-10 \quad 6.810 \mathrm{E}-16-1.000 \mathrm{E}+00 \quad 9.120 \mathrm{E}-117.600 \mathrm{E}-11$ 3.240E-14 1.809E-11 2.224E-10 6.470E-16-1.000E+00 6.940E-09 $1.570 \mathrm{E}-11$ $3.140 \mathrm{E}-141.834 \mathrm{E}-112.255 \mathrm{E}-10 \quad 6.560 \mathrm{E}-16-1.000 \mathrm{E}+00 \quad 1.610 \mathrm{E}-10 \quad 1.330 \mathrm{E}-10$ $5.520 \mathrm{E}-142.720 \mathrm{E}-11 \quad 3.345 \mathrm{E}-10 \quad 9.730 \mathrm{E}-16-1.000 \mathrm{E}+00 \quad 1.340 \mathrm{E}-10 \quad 5.240 \mathrm{E}-11$ $3.330 \mathrm{E}-14 \quad 1.884 \mathrm{E}-11 \quad 2.317 \mathrm{E}-10 \quad 6.740 \mathrm{E}-16-1.000 \mathrm{E}+00 \quad 6.150 \mathrm{E}-11 \quad 4.640 \mathrm{E}-12$ $3.090 \mathrm{E}-14 \quad 1.775 \mathrm{E}-11 \quad 2.183 \mathrm{E}-10 \quad 6.350 \mathrm{E}-16-1.000 \mathrm{E}+00 \quad 2.330 \mathrm{E}-09 \quad 5.870 \mathrm{E}-09$ $3.330 \mathrm{E}-14 \quad 1.890 \mathrm{E}-112.324 \mathrm{E}-10 \quad 6.760 \mathrm{E}-16-1.000 \mathrm{E}+00 \quad 1.630 \mathrm{E}-09 \quad 1.950 \mathrm{E}-09$ $5.580 \mathrm{E}-14 \quad 7.967 \mathrm{E}-11 \quad 9.799 \mathrm{E}-10 \quad 2.850 \mathrm{E}-15-1.000 \mathrm{E}+00 \quad 0.000 \mathrm{E}+00 \quad 0.000 \mathrm{E}+00$

$6.970 \mathrm{E}-142.336 \mathrm{E}-11 \quad 3.231 \mathrm{E}-11 \quad 1.440 \mathrm{E}-15-1.000 \mathrm{E}+00 \quad 2.150 \mathrm{E}-11 \quad 1.510 \mathrm{E}-10$ $7.910 \mathrm{E}-142.222 \mathrm{E}-11$ 3.074E-11 $1.370 \mathrm{E}-15-1.000 \mathrm{E}+00 \quad 1.280 \mathrm{E}-112.560 \mathrm{E}-11$ $6.980 \mathrm{E}-142.141 \mathrm{E}-11 \quad 2.962 \mathrm{E}-11 \quad 1.320 \mathrm{E}-15-1.000 \mathrm{E}+00 \quad 8.980 \mathrm{E}-10 \quad 9.390 \mathrm{E}-12$ $6.860 E-142.190 E-11 \quad 3.029 E-11 \quad 1.350 E-15-1.000 E+00 \quad 1.700 E-11 \quad 3.670 E-11$ $1.070 \mathrm{E}-13$ 3.033E-11 $4.196 \mathrm{E}-11 \quad 1.870 \mathrm{E}-15-1.000 \mathrm{E}+00 \quad 1.460 \mathrm{E}-11 \quad 1.340 \mathrm{E}-11$ $7.160 \mathrm{E}-142.174 \mathrm{E}-11 \quad 3.007 \mathrm{E}-11 \quad 1.340 \mathrm{E}-15-1.000 \mathrm{E}+00 \quad 9.720 \mathrm{E}-12 \quad 1.470 \mathrm{E}-12$ 
REMAINDER EFFECTIVE SKIN (FGR) Te-127 GONADS BREAST LUNGS RED MARR BONE SUR THYROID REMAINDER EFFECTIVE SKIN (FGR) Te-127m GONADS BREAST LUNGS RED MARR BONE SUR THYROID REMAINDER EFFECTIVE SKIN (FGR) Te-129 GONADS BREAST LUNGS RED MARR BONE SUR THYROID REMAINDER EFFECTIVE SKIN (FGR) $\mathrm{Te}-129 \mathrm{~m}$ GONADS BREAST LUTNGS RED MARR BONE SUR THYROID REMAINDER EFFECTIVE SKIN (FGR) $\mathrm{Te}-131 \mathrm{~m}$ GONADS BREAST LUNGS RED MARR BONE SUR THYROID REMAINDER EFFECTIVE SKIN (FGR) Te-132 GONADS BREAST IUNGS RED MARR BONE SUR THYROID REMAINDER EFFECTIVE SKIN (FGR) I-131 GONADS BREAST LUNGS
$6.710 \mathrm{E}-142.125 \mathrm{E}-1122.939 \mathrm{E}-11 \quad 1.310 \mathrm{E}-15-1.000 \mathrm{E}+00 \quad 1.870 \mathrm{E}-10 \quad 1.450 \mathrm{E}-09$ $7.140 \mathrm{E}-142.238 \mathrm{E}-11 \quad 3.096 \mathrm{E}-11 \quad 1.380 \mathrm{E}-15-1.000 \mathrm{E}+00 \quad 1.740 \mathrm{E}-10 \quad 4.840 \mathrm{E}-10$ $1.050 \mathrm{E}-13 \quad 8.273 \mathrm{E}-11 \cdot 1.144 \mathrm{E}-10 \quad 5.100 \mathrm{E}-15-1.000 \mathrm{E}+00 \quad 0.000 \mathrm{E}+00 \quad 0.000 \mathrm{E}+00$

$2.370 \mathrm{E}-16 \quad 1.191 \mathrm{E}-13 \quad 2.661 \mathrm{E}-13 \quad 5.480 \mathrm{E}-18-1.000 \mathrm{E}+00 \quad 2.020 \mathrm{E}-12 \quad 4.020 \mathrm{E}-12$ $2.730 \mathrm{E}-16 \quad 1.158 \mathrm{E}-13 \quad 2.588 \mathrm{E}-13 \quad 5.330 \mathrm{E}-18-1.000 \mathrm{E}+00 \quad 1.880 \mathrm{E}-12 \quad 3.000 \mathrm{E}-12$ $2.320 \mathrm{E}-16 \quad 1.060 \mathrm{E}-13 \quad 2.370 \mathrm{E}-13 \quad 4.880 \mathrm{E}-18-1.000 \mathrm{E}+00 \quad 4.270 \mathrm{E}-10 \quad 2.890 \mathrm{E}-12$ $2.210 \mathrm{E}-16 \quad 1.058 \mathrm{E}-13 \quad 2.365 \mathrm{E}-13.4 .870 \mathrm{E}-18-1.000 \mathrm{E}+00 \quad 4.090 \mathrm{E}-12 \quad 6.570 \mathrm{E}-12$ $4.650 \mathrm{E}-16 \quad 1.862 \mathrm{E}-13 \quad 4.162 \mathrm{E}-13 \quad 8.570 \mathrm{E}-18-1.000 \mathrm{E}+00 \quad 4.090 \mathrm{E}-12 \quad 6.460 \mathrm{E}-12$ $2.400 \mathrm{E}-16 \quad 1.106 \mathrm{E}-13 \quad 2.472 \mathrm{E}-13 \quad 5.090 \mathrm{E}-18-1.000 \mathrm{E}+00 \quad 1.840 \mathrm{E}-12 \quad 2.860 \mathrm{E}-12$ $2.210 \mathrm{E}-16 \quad 1.036 \mathrm{E}-13 \quad 2.316 \mathrm{E}-13 \quad 4.770 \mathrm{E}-18-1.000 \mathrm{E}+00 \quad 1.110 \mathrm{E}-10 \quad 6.130 \mathrm{E}-10$ $2.420 \mathrm{E}-16 \quad 1.125 \mathrm{E}-13 \quad 2.515 \mathrm{E}-13 \quad 5.180 \mathrm{E}-18-1.000 \mathrm{E}+00 \quad 8.600 \mathrm{E}-11 \quad 1.870 \mathrm{E}-10$ $1.140 \mathrm{E}-14 \quad 1.173 \mathrm{E}-112.622 \mathrm{E}-11 \quad 5.400 \mathrm{E}-16-1.000 \mathrm{E}+00 \quad 0.000 \mathrm{E}+00 \quad 0.000 \mathrm{E}+00$

$1.900 \mathrm{E}-16 \quad 4.689 \mathrm{E}-13 \quad 9.642 \mathrm{E}-12 \quad 1.630 \mathrm{E}-17-1.000 \mathrm{E}+00 \quad 1.100 \mathrm{E}-10 \quad 1.250 \mathrm{E}-10$ $2.690 \mathrm{E}-16 \quad 5.150 \mathrm{E}-13 \quad 1.059 \mathrm{E}-11 \quad 1.790 \mathrm{E}-17-1.000 \mathrm{E}+00 \quad 1.100 \mathrm{E}-10 \quad 9.740 \mathrm{E}-11$ $7.620 \mathrm{E}-17 \quad 1.602 \mathrm{E}-13 \quad 3.295 \mathrm{E}-12 \quad 5.570 \mathrm{E}-18-1.000 \mathrm{E}+00 \quad 3.340 \mathrm{E}-08 \quad 9.620 \mathrm{E}-11$ $6.430 \mathrm{E}-17 \quad 1.249 \mathrm{E}-13 \quad 2.567 \mathrm{E}-12 \quad 4.340 \mathrm{E}-18-1.000 \mathrm{E}+00 \quad 5.360 \mathrm{E}-09 \quad 5.430 \mathrm{E}-09$ $3.940 \mathrm{E}-16 \quad 9.005 \mathrm{E}-13 \quad 1.852 \mathrm{E}-11 \quad 3.130 \mathrm{E}-17-1.000 \mathrm{E}+00 \quad 2.040 \mathrm{E}-08 \quad 2.070 \mathrm{E}-08$ $1.500 \mathrm{E}-16 \quad 2.779 \mathrm{E}-13 \quad 5.714 \mathrm{E}-12 \quad 9.660 \mathrm{E}-18-1.000 \mathrm{E}+00 \quad 9.660 \mathrm{E}-11 \quad 9.430 \mathrm{E}-11$ 8.640E-17 1.999E-13 4.111E-12 6.950E-18-1.000E+00 1.660E-09 2.980E-09

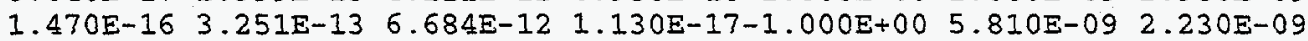
8.490E-16 1.496E-12 $3.076 \mathrm{E}-11 \quad 5.200 \mathrm{E}-17-1.000 \mathrm{E}+00 \quad 0.000 \mathrm{E}+00 \quad 0.000 \mathrm{E}+00$

$2.710 \mathrm{E}-15 \quad 3.889 \mathrm{E}-13 \quad 3.922 \mathrm{E}-13 \quad 6.510 \mathrm{E}-17-1.000 \mathrm{E}+00 \quad 5.050 \mathrm{E}-13 \quad 1.590 \mathrm{E}-12$ $3.120 \mathrm{E}-15 \quad 3.800 \mathrm{E}-13 \quad 3.832 \mathrm{E}-13 \quad 6.360 \mathrm{E}-17-1.000 \mathrm{E}+00 \quad 5.390 \mathrm{E}-13 \quad 6.050 \mathrm{E}-13$ $2.640 \mathrm{E}-15 \quad 3.298 \mathrm{E}-13 \quad 3.326 \mathrm{E}-13 \quad 5.520 \mathrm{E}-17-1.000 \mathrm{E}+00 \quad 1.530 \mathrm{E}-10 \quad 4.910 \mathrm{E}-13$ $2.540 \mathrm{E}-15 \quad 3.298 \mathrm{E}-13 \quad 3.326 \mathrm{E}-13 \quad 5.520 \mathrm{E}-17-1.000 \mathrm{E}+00 \quad 6.190 \mathrm{E}-13 \quad 7.640 \mathrm{E}-13$ $4.880 E-15 \quad 5.753 E-13 \quad 5.802 E-13 \quad 9.630 E-17-1.000 E+00 \quad 6.220 E-13 \quad 5.400 E-13$ $2.740 \mathrm{E}-15 \quad 3.525 \mathrm{E}-13 \quad 3.555 \mathrm{E}-13 \quad 5.900 \mathrm{E}-17-1.000 \mathrm{E}+00 \quad 5.090 \mathrm{E}-13 \quad 3.360 \mathrm{E}-13$ $2.520 \mathrm{E}-15 \quad 3.262 \mathrm{E}-13 \quad 3.289 \mathrm{E}-13 \quad 5.460 \mathrm{E}-17-1.000 \mathrm{E}+00 \quad 7.280 \mathrm{E}-12 \quad 1.790 \mathrm{E}-10$ $2.750 \mathrm{E}-15 \quad 3.590 \mathrm{E}-13 \quad 3.621 \mathrm{E}-13 \quad 6.010 \mathrm{E}-17-1.000 \mathrm{E}+00 \quad 2.090 \mathrm{E}-11 \quad 5.450 \mathrm{E}-11$ $3.570 E-14 \quad 3.429 E-113.458 E-11 \quad 5.740 E-15-1.000 E+00 \quad 0.000 E+00 \quad 0.000 E+00$

$3.321 E-15 \quad 2.206 E-12 \quad 4.799 E-11 \quad 8.561 E-17-1.000 E+00 \quad 1.783 E-10 \quad 2.420 E-10$ $3.838 \mathrm{E}-15 \quad 2.181 \mathrm{E}-12 \quad 4.739 \mathrm{E}-11 \quad 8.454 \mathrm{E}-17-1.000 \mathrm{E}+00 \quad 1.694 \mathrm{E}-10 \quad 1.664 \mathrm{E}-10$ $3.176 \mathrm{E}-15$ 1.741E-12 $3.815 \mathrm{E}-11 \quad 6.808 \mathrm{E}-17-1.000 \mathrm{E}+00$ 4.040E-08 $1.593 \mathrm{E}-10$ $3.071 \mathrm{E}-15 \quad 1.729 \mathrm{E}-12 \quad 3.793 \mathrm{E}-11 \quad 6.768 \mathrm{E}-17-1.000 \mathrm{E}+00 \quad 3.100 \mathrm{E}-09 \quad 3.500 \mathrm{E}-09$ 5.772E-15 $3.287 \mathrm{E}-12 \quad 7.147 \mathrm{E}-11$ 1.275E-16-1.000E+00 $7.050 \mathrm{E}-09 \quad 7.990 \mathrm{E}-09$ $3.341 \mathrm{E}-15 \quad 1.923 \mathrm{E}-12 \quad 4.201 \mathrm{E}-11 \quad 7.495 \mathrm{E}-17-1.000 \mathrm{E}+00 \quad 1.563 \mathrm{E}-10 \quad 1.572 \mathrm{E}-10$ $3.048 E-15$ 1.746E-12 $3.822 \mathrm{E}-11 \quad 6.819 \mathrm{E}-17-1.000 \mathrm{E}+00 \quad 3.275 \mathrm{E}-09 \quad 7.196 \mathrm{E}-09$ $3.337 \mathrm{E}-15 \quad 1.974 \mathrm{E}-12 \quad 4.308 \mathrm{E}-11 \quad 7.686 \mathrm{E}-17-1.000 \mathrm{E}+00 \quad 6.484 \mathrm{E}-09 \quad 2.925 \mathrm{E}-09$ $3.811 \mathrm{E}-14 \quad 1.501 \mathrm{E}-10 \quad 3.360 \mathrm{E}-09 \quad 6.001 \mathrm{E}-15-1.000 \mathrm{E}+00 \quad 0.000 \mathrm{E}+00 \quad 0.000 \mathrm{E}+00$

$7.292 \mathrm{E}-14 \quad 4.020 \mathrm{E}-112.343 \mathrm{E}-10 \quad 1.535 \mathrm{E}-15-1.000 \mathrm{E}+00 \quad 2.345 \mathrm{E}-10 \quad 7.415 \mathrm{E}-10$ $8.286 \mathrm{E}-143.853 \mathrm{E}-112.246 \mathrm{E}-10 \quad 1.472 \mathrm{E}-15-1.000 \mathrm{E}+00 \quad 9.309 \mathrm{E}-111.361 \mathrm{E}-10$ $7.265 \mathrm{E}-14 \quad 3.657 \mathrm{E}-112.131 \mathrm{E}-10 \quad 1.397 \mathrm{E}-15-1.000 \mathrm{E}+00 \quad 2.296 \mathrm{E}-09 \quad 6.335 \mathrm{E}-11$ $7.097 \mathrm{E}-14 \quad 3.736 \mathrm{E}-112.178 \mathrm{E}-10 \quad 1.427 \mathrm{E}-15-1.000 \mathrm{E}+00 \quad 1.417 \mathrm{E}-10 \quad 2.435 \mathrm{E}-10$ $1.174 E-13 \quad 5.467 E-11 \quad 3.189 E-10 \quad 2.090 E-15-1.000 E+00 \quad 2.276 E-10 \quad 3.248 E-10$ $7.471 \mathrm{E}-14 \quad 3.741 \mathrm{E}-11 \quad 2.181 \mathrm{E}-10 \quad 1.429 \mathrm{E}-15-1.000 \mathrm{E}+00 \quad 3.669 \mathrm{E}-08 \quad 4.383 \mathrm{E}-08$ $6.965 \mathrm{E}-143.626 \mathrm{E}-11 \quad 2.113 \mathrm{E}-10 \quad 1.385 \mathrm{E}-15-1.000 \mathrm{E}+00 \quad 9.509 \mathrm{E}-10 \quad 3.153 \mathrm{E}-09$

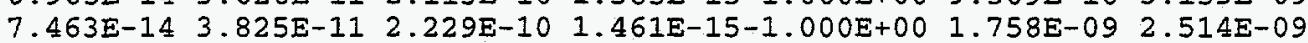
$1.038 \mathrm{E}-13 \quad 1.033 \mathrm{E}-10 \quad 6.188 \mathrm{E}-10 \quad 4.056 \mathrm{E}-15-1.000 \mathrm{E}+00 \quad 0.000 \mathrm{E}+00 \quad 0.000 \mathrm{E}+00$

$1.020 \mathrm{E}-14 \quad 6.812 \mathrm{E}-12 \quad 7.706 \mathrm{E}-11 \quad 2.450 \mathrm{E}-16-1.000 \mathrm{E}+00 \quad 4.150 \mathrm{E}-10 \quad 5.410 \mathrm{E}-10$ $1.180 \mathrm{E}-14 \quad 6.756 \mathrm{E}-12 \cdot 7.643 \mathrm{E}-11 \quad 2.430 \mathrm{E}-16-1.000 \mathrm{E}+00 \quad 3.630 \mathrm{E}-10 \quad 3.500 \mathrm{E}-10$

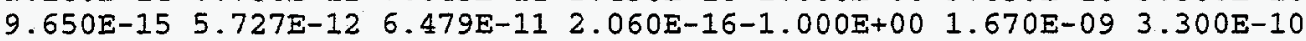
$8.950 \mathrm{E}-15 \quad 5.588 \mathrm{E}-12 \quad 6.322 \mathrm{E}-11 \quad 2.010 \mathrm{E}-16-1.000 \mathrm{E}+00 \quad 4.270 \mathrm{E}-10 \quad 4.440 \mathrm{E}-10$ $2.420 \mathrm{E}-14$ 1.273E-11 $1.441 \mathrm{E}-10 \quad 4.580 \mathrm{E}-16-1.000 \mathrm{E}+00 \quad 7.120 \mathrm{E}-10 \quad 8.300 \mathrm{E}-10$

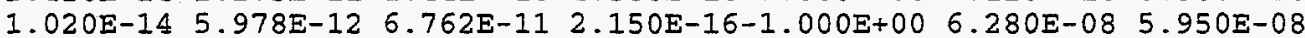
$9.160 \mathrm{E}-15 \quad 5.644 \mathrm{E}-12 \quad 6.385 \mathrm{E}-11$ 2.030E-16-1.000E+00 $7.890 \mathrm{E}-10 \quad 1.490 \mathrm{E}-09$ $1.030 \mathrm{E}-14 \quad 6.339 \mathrm{E}-12 \quad 7.171 \mathrm{E}-1122.280 \mathrm{E}-16-1.000 \mathrm{E}+00 \quad 2.550 \mathrm{E}-09 \quad 2.540 \mathrm{E}-09$

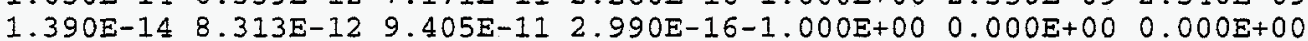

$1.780 \mathrm{E}-14 \quad 1.119 \mathrm{E}-11 \quad 1.789 \mathrm{E}-10 \quad 3.940 \mathrm{E}-16-1.000 \mathrm{E}+00 \quad 2.530 \mathrm{E}-11 \quad 4.070 \mathrm{E}-11$ $2.040 \mathrm{E}-141.082 \mathrm{E}-11 \quad 1.730 \mathrm{E}-10 \quad 3.810 \mathrm{E}-16-1.000 \mathrm{E}+00 \quad 7.880 \mathrm{E}-11 \quad 1.210 \mathrm{E}-10$ $1.760 \mathrm{E}-14 \quad 1.016 \mathrm{E}-11 \quad 1.626 \mathrm{E}-10 \quad 3.580 \mathrm{E}-16-1.000 \mathrm{E}+00 \quad 6.570 \mathrm{E}-10 \quad 1.020 \mathrm{E}-10$ 
RED MARR BONE SUR THYROID REMAINDER EFFECTIVE SKIN (FGR) I- 132 GONADS BREAST LUNGS RED MARR BONE SUR THYROID REMAINDER EFFECTIVE SKIN (FGR) I-133 GONADS BREAST IUNGS RED MARR. BONE SUR THYROID REMAINDER EFFECTIVE SKIN (FGR) I-134 GONADS BREAST IUNGS RED MARR BONE SUR THYROID REMAINDER EFFECTIVE SKIN (FGR) I- 135 GONADS BREAST IUNGS RED MARR BONE SUR THYROID REMAINDER EFFECTIVE SKIN (FGR) Xe-133 GONADS BREAST LUNGS RED MARR BONE SUR. THYROID REMAINDER EFFECTIVE SKIN (FGR) $\mathrm{Xe}-135$ GONADS BREAST LUNGS RED MARR BONE SUR THYROID REMAINDER EFFECTIVE SKIN (FGR) Cs -134
$1.680 \mathrm{E}-141.022 \mathrm{E}-11 \quad 1.635 \mathrm{E}-10 \quad 3.600 \mathrm{E}-16-1.000 \mathrm{E}+00 \quad 6.260 \mathrm{E}-11 \quad 9.440 \mathrm{E}-11$ $3.450 \mathrm{E}-14 \quad 1.675 \mathrm{E}-112.679 \mathrm{E}-10 \quad 5.900 \mathrm{E}-16-1.000 \mathrm{E}+00 \quad 5.730 \mathrm{E}-11 \quad 8.720 \mathrm{E}-11$ $1.810 \mathrm{E}-14 \quad 1.053 \mathrm{E}-11 \quad 1.685 \mathrm{E}-10 \quad 3.710 \mathrm{E}-16-1.000 \mathrm{E}+00 \quad 2.920 \mathrm{E}-07 \quad 4.760 \mathrm{E}-07$ $1.670 \mathrm{E}-14 \quad 9.908 \mathrm{E}-12 \quad 1.585 \mathrm{E}-10 \quad 3.490 \mathrm{E}-16-1.000 \mathrm{E}+00 \quad 8.030 \mathrm{E}-111.570 \mathrm{E}-10$ $1.820 \mathrm{E}-14 \quad 1.067 \mathrm{E}-11 \mathrm{I} \quad 1.707 \mathrm{E}-10 \quad 3.760 \mathrm{E}-16-1.000 \mathrm{E}+00 \quad 8.890 \mathrm{E}-09 \quad 1.440 \mathrm{E}-08$ $2.980 E-14 \quad 1.825 E-1122.920 E-10 \quad 6.430 E-16-1.000 E+00 \quad 0.000 E+00 \quad 0.000 E+00$

$\begin{array}{llllll}1.090 E-13 & 2.523 E-11 & 2.771 E-11 & 2.320 E-15-1.000 E+00 & 9.950 E-12 & 2.330 E-11\end{array}$

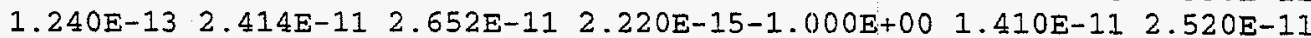

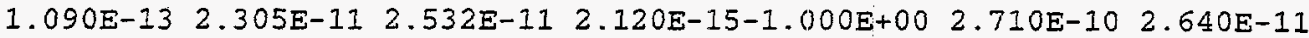

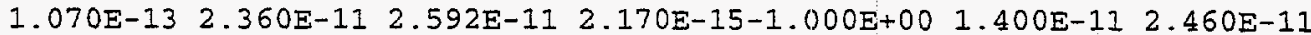
$1.730 \mathrm{E}-13 \quad 3.327 \mathrm{E}-11 \quad 3.655 \mathrm{E}-11 \quad 3.060 \mathrm{E}-15-1.000 \mathrm{E}+00 \quad 1.240 \mathrm{E}-112.190 \mathrm{E}-11$

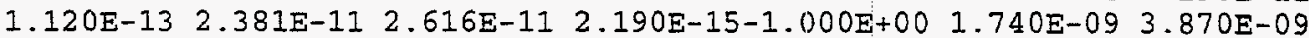

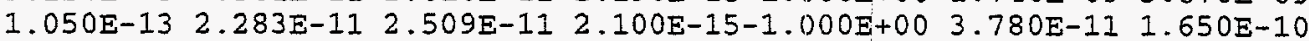

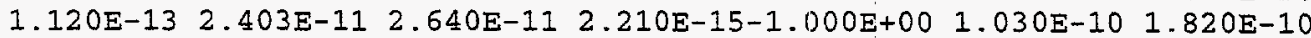
$1.580 \mathrm{E}-13 \quad 8.199 \mathrm{E}-11 \quad 9.007 \mathrm{E}-11 \quad 7.540 \mathrm{E}-15-1.000 \mathrm{E}+00 \quad 0.000 \mathrm{E}+00 \quad 0.000 \mathrm{E}+00$

$2.870 \mathrm{E}-14 \quad 1.585 \mathrm{E}-11 \quad 6.748 \mathrm{E}-11 \quad 6.270 \mathrm{E}-16-1.000 \mathrm{E}+00 \quad 1.950 \mathrm{E}-11 \quad 3.630 \mathrm{E}-11$ $3.280 \mathrm{E}-141.519 \mathrm{E}-11 \quad 6.468 \mathrm{E}-11 \quad 6.010 \mathrm{E}-16-1.000 \mathrm{E}+00 \quad 2.940 \mathrm{E}-11 \quad 4.680 \mathrm{E}-11$ $2.860 \mathrm{E}-141.446 \mathrm{E}-11 \quad 6.156 \mathrm{E}-11 \quad 5.720 \mathrm{E}-16-1.000 \mathrm{E}+00 \quad 8.200 \mathrm{E}-10 \quad 4.530 \mathrm{E}-11$ $2.770 E-14$ I.466E-11 6.242E-11 5.800E-16-1.000E+00 2.720E-11 4.300E-11 $4.870 \mathrm{E}-142.161 \mathrm{E}-11 \quad 9.202 \mathrm{E}-11 \quad 8.550 \mathrm{E}-16-1.000 \mathrm{E}+00 \quad 2.520 \mathrm{E}-11 \quad 4.070 \mathrm{E}-11$ $2.930 E-14 \quad 1.502 E-11 \quad 6.393 E-11 \quad 5.940 E-16-1.000 E+00 \quad 4.860 E-08 \quad 9.100 E-08$ $2.730 \mathrm{E}-141.418 \mathrm{E}-11$ 6.038E-11 5.610E-16-1.000E+00 5.000E-11 $1.550 \mathrm{E}-10$ $2.940 E-14 \quad 1.509 E-11 \quad 6.425 E-11 \quad 5.970 E-16-1.000 E+00 \quad 1.580 E-092.800 E-09$ $5.830 \mathrm{E}-14 \quad 1.150 \mathrm{E}-10 \quad 4.897 \mathrm{E}-10 \quad 4.550 \mathrm{E}-15-1.000 \mathrm{E}+00 \quad 0.000 \mathrm{E}+00 \quad 0.000 \mathrm{E}+00$

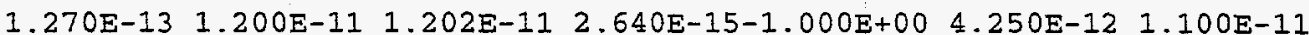
$1.440 \mathrm{E}-13 \quad 1.145 \mathrm{E}-11 \quad 1.147 \mathrm{E}-11 \quad 2.520 \mathrm{E}-15-1.000 \mathrm{E}+00 \quad 6.170 \mathrm{E}-12 \quad 1.170 \mathrm{E}-11$ $1.270 \mathrm{E}-13$ 1.100E-11 $1.102 \mathrm{E}-112.420 \mathrm{E}-15-1.000 \mathrm{E}+00$ 1.430E-10 $1.260 \mathrm{E}-11$ $1.250 \mathrm{E}-13$ 1.127E-11 $1.129 \mathrm{E}-112.480 \mathrm{E}-15-1.000 \mathrm{E}+00 \quad 6.080 \mathrm{E}-12$ 1.090E-11 $1.960 \mathrm{E}-13 \quad 1.568 \mathrm{E}-11 \quad 1.571 \mathrm{E}-11 \quad 3.450 \mathrm{E}-15-1.000 \mathrm{E}+00 \quad 5.310 \mathrm{E}-12 \quad 9.320 \mathrm{E}-12$ $1.300 \mathrm{E}-13$ 1.127E-11 $1.129 \mathrm{E}-112.480 \mathrm{E}-15-1.000 \mathrm{E}+00 \quad 2.880 \mathrm{E}-10 \quad 6.210 \mathrm{E}-10$ $1.220 \mathrm{E}-13$ 1.091E-11 $1.093 \mathrm{E}-112.400 \mathrm{E}-15-1.000 \mathrm{E}+00 \quad 2.270 \mathrm{E}-11$ 1.340E-10

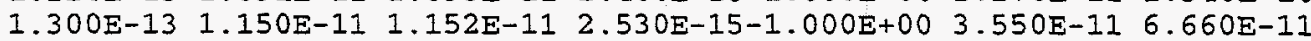
$1.870 E-13 \quad 4.477 \Xi-11 \quad 4.485 E-11 \quad 9.850 E-15-1.000 E+00 \quad 0.000 E+00 \quad 0.000 E+00$

$8.078 \mathrm{E}-143.113 \mathrm{E}-11 \quad 5.489 \mathrm{E}-11 \quad 1.599 \mathrm{E}-15-1.000 \mathrm{E}+00 \quad 1.700 \mathrm{E}-113.610 \mathrm{E}-11$ $9.143 \mathrm{E}-142.971 \mathrm{E}-11 \quad 5.240 \mathrm{E}-11 \quad 1.526 \mathrm{E}-15-1.000 \mathrm{E}+00 \quad 2.340 \mathrm{E}-113.850 \mathrm{E}-11$ $8.145 \mathrm{E}-142.886 \mathrm{E}-11 \quad 5.089 \mathrm{E}-11$ 1.482E-15-1.000E+00 $4.410 \mathrm{E}-10 \quad 3.750 \mathrm{E}-11$ $8.054 \mathrm{E}-142.965 \mathrm{E}-11 \quad 5.228 \mathrm{E}-11$ 1.523E-15-1.000E+00 $2.240 \mathrm{E}-11 \quad 3.650 \mathrm{E}-11$

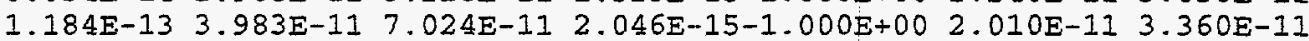
8.324E-14 2.852E-11 5.030E-11 1.465E-15-1.000E+00 8.460E-09 $1.790 \mathrm{E}-08$ $7.861 \mathrm{E}-142.883 \mathrm{E}-11 \mathrm{5}$.084E-11 $1.481 \mathrm{E}-15-1.000 \mathrm{E}+00$ 4.700E-11 $1.540 \mathrm{E}-10$ 8.294E-14 2.989E-11 5.271E-11 $1.535 \mathrm{E}-15-1.000 \mathrm{E}+00 \quad 3.320 \mathrm{E}-10 \quad 6.080 \mathrm{E}-10$

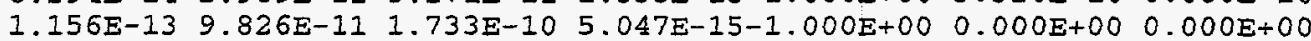

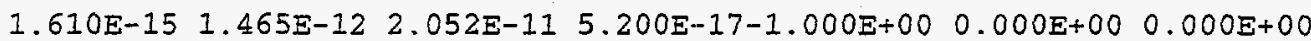
$\begin{array}{llllll}1.960 \mathrm{E}-15 & 1.505 \mathrm{E}-12 & 2.107 \mathrm{E}-11 & 5.340 \mathrm{E}-17-1.000 \mathrm{E}+00 & 0.000 \mathrm{E}+00 & 0.000 \mathrm{E}+00\end{array}$ $1.320 \mathrm{E}-15$ 1.045E-12 $1.464 \mathrm{E}-11 \quad 3.710 \mathrm{E}-17-1.000 \mathrm{E}+00 \quad 0.000 \mathrm{E}+00 \quad 0.000 \mathrm{E}+00$ $1.070 \mathrm{E}-15 \quad 8.791 \mathrm{E}-13 \quad 1.231 \mathrm{E}-11 \quad 3.120 \mathrm{E}-17-1.000 \mathrm{E}+00 \quad 0.000 \mathrm{E}+00 \quad 0.000 \mathrm{E}+00$ $5.130 \mathrm{E}-15 \quad 4.254 \mathrm{E}-12 \quad 5.958 \mathrm{E}-11 \quad 1.510 \mathrm{E}-16-1.000 \mathrm{E}+00 \quad 0.000 \mathrm{E}+00 \quad 0.000 \mathrm{E}+00$ $1.510 \mathrm{E}-15 \quad 1.181 \mathrm{E}-12 \quad 1.653 \mathrm{E}-11 \quad 4.190 \mathrm{E}-17-1.000 \mathrm{E}+00 \quad 0.000 \mathrm{E}+00 \quad 0.000 \mathrm{E}+00$

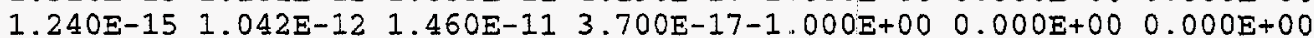
$1.560 \mathrm{E}-15 \quad 1.299 \mathrm{E}-12 \quad 1.819 \mathrm{E}-11 \quad 4.610 \mathrm{E}-17-1.000 \mathrm{E}+00 \quad 0.000 \mathrm{E}+00 \quad 0.000 \mathrm{E}+00$ $4.970 \mathrm{E}-15 \quad 1.953 \mathrm{E}-12 \quad 2.734 \mathrm{E}-11 \quad 6.930 \mathrm{E}-17-1.000 \mathrm{E}+00 \quad 0.000 \mathrm{E}+00 \quad 0.000 \mathrm{E}+00$

$1.170 \mathrm{E}-14 \quad 5.455 \mathrm{E}-12 \quad 1.194 \mathrm{E}-112.530 \mathrm{E}-16-1.000 \mathrm{E}+00 \quad 0.000 \mathrm{E}+00 \quad 0.000 \mathrm{E}+00$ $\begin{array}{llllll}1.330 \mathrm{E}-14 & 5.325 \mathrm{E}-12 & 1.166 \mathrm{E}-11 & 2.470 \mathrm{E}-16-1.000 \mathrm{E}+00 & 0.000 \mathrm{E}+00 & 0.000 \mathrm{E}+00\end{array}$ $1.130 \mathrm{E}-14 \quad 4.959 \mathrm{E}-12 \quad 1.086 \mathrm{E}-11 \quad 2.300 \mathrm{E}-16-1.000 \mathrm{E}+00 \quad 0.000 \mathrm{E}+00 \quad 0.000 \mathrm{E}+00$ $1.070 \mathrm{E}-14 \quad 4.959 \mathrm{E}-12 \quad 1.086 \mathrm{E}-112.300 \mathrm{E}-16-1.000 \mathrm{E}+00 \quad 0.000 \mathrm{E}+00 \quad 0.000 \mathrm{E}+00$ $2.570 \mathrm{E}-14 \quad 9.120 \mathrm{E}-12 \quad 1.997 \mathrm{E}-11 \quad 4.230 \mathrm{E}-16-1.000 \mathrm{E}+00 \quad 0.000 \mathrm{E}+00 \quad 0.000 \mathrm{E}+00$

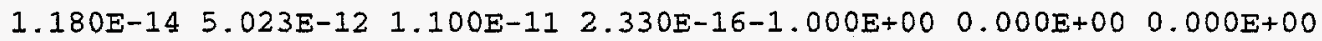
$\begin{array}{lllllll}1.080 \mathrm{E}-14 & 4.829 \mathrm{E}-12 & 1.058 \mathrm{E}-11 & 2.240 \mathrm{E}-16-1.000 \mathrm{E}+00 & 0.000 \mathrm{E}+00 & 0.000 \mathrm{E}+00\end{array}$

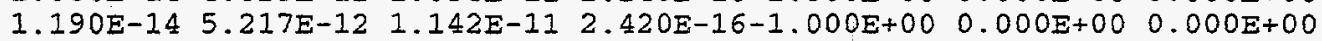
$3.120 \mathrm{E}-14 \quad 4.506 \mathrm{E}-11 \quad 9.867 \mathrm{E}-11 \quad 2.090 \mathrm{E}-15-1.000 \mathrm{E}+00 \quad 0.000 \mathrm{E}+00 \quad 0.000 \mathrm{E}+00$ 
GONADS BREAST IUNGS RED MARR BONE SUR THYROID REMAINDER EFFECTIVE SKIN (FGR) Cs-136 GONADS BREAST LUNGS RED MARR BONE SUR THYROID REMAINDER EFFECTIVE SKIN (FGR) Cs -137 GONADS BREAST LUNGS RED MARR BONE SUR THYROID REMAINDER EFFECTIVE SKIN (FGR) $\mathrm{B} a-139$ GONADS BREAST LUNGS RED MARR BONE SUR THYROID REMAINDER EFFECTIVE SKIN (FGR) $\mathrm{Ba}-140$ GONADS BREAST LUNGS RED MARR BONE SUR THYROID REMAINDER EFFECTIVE SKIN (FGR) La-140 GONADS BREAST LUNGS RED MARR BONE SUR THYROID REMAINDER EFFECTIVE SKIN (FGR) La-141 GONADS BREAST IUNGS RED MARR BONE SUR THYROID REMAINDER
$7.400 \mathrm{E}-14 \quad 4.607 \mathrm{E}-11 \quad 9.646 \mathrm{E}-10 \quad 1.600 \mathrm{E}-15-1.000 \mathrm{E}+00 \quad 1.300 \mathrm{E}-08 \quad 2.060 \mathrm{E}-08$ $8.430 \mathrm{E}-14 \quad 4.406 \mathrm{E}-11 \quad 9.224 \mathrm{E}-10 \quad 1.530 \mathrm{E}-15-1.000 \mathrm{E}+00 \quad 1.080 \mathrm{E}-08 \quad 1.720 \mathrm{E}-08$ $7.370 \mathrm{E}-14$ 4.204E-11 8.802E-10 1.460E-15-1.000E+00 1.180E-08 $1.760 \mathrm{E}-08$ $7.190 \mathrm{E}-14 \quad 4.262 \mathrm{E}-11 \quad 8.922 \mathrm{E}-10$ 1.480E-15-1.000E+00 1.180E-08 $1.870 \mathrm{E}-08$ $1.200 \mathrm{E}-13 \quad 6.105 \mathrm{E}-11 \quad 1.278 \mathrm{E}-09 \quad 2.120 \mathrm{E}-15-1.000 \mathrm{E}+00 \quad 1.100 \mathrm{E}-08 \quad 1.740 \mathrm{E}-08$ $7.570 \mathrm{E}-14 \quad 4.377 \mathrm{E}-11 \quad 9.163 \mathrm{E}-10$ 1.520E-15-1.000E+00 $1.110 \mathrm{E}-08 \quad 1.760 \mathrm{E}-08$ $7.060 \mathrm{E}-14 \quad 4.147 \mathrm{E}-11 \quad 8.681 \mathrm{E}-10$ 1.440E-15-1.000E+00 1.390E-08 2.210E-08 $7.570 \mathrm{E}-14 \quad 4.377 \mathrm{E}-11 \quad 9.163 \mathrm{E}-10 \quad 1.520 \mathrm{E}-15-1.000 \mathrm{E}+00$ 1.250E-08 $1.980 \mathrm{E}-08$ $9.450 \mathrm{E}-14 \quad 6.249 \mathrm{E}-11 \quad 1.308 \mathrm{E}-09 \quad 2.170 \mathrm{E}-15-1.000 \mathrm{E}+00 \quad 0.000 \mathrm{E}+00 \quad 0.000 \mathrm{E}+00$

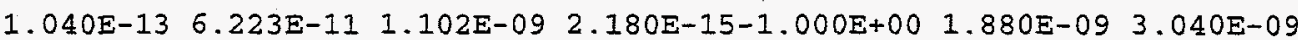
$1.180 \mathrm{E}-13 \quad 5.966 \mathrm{E}-11$ 1.056E-09 2.090E-15-1.000E+00 1.670E-09 2.650E-09 $1.040 \mathrm{E}-13$ 5.710E-11 $1.011 \mathrm{E}-09 \quad 2.000 \mathrm{E}-15-1.000 \mathrm{E}+00 \quad 2.320 \mathrm{E}-09 \quad 2.620 \mathrm{E}-09$ $1.010 \mathrm{E}-13 \quad 5.824 \mathrm{E}-111.031 \mathrm{E}-09 \quad 2.040 \mathrm{E}-15-1.000 \mathrm{E}+00 \quad 1.860 \mathrm{E}-092.950 \mathrm{E}-09$

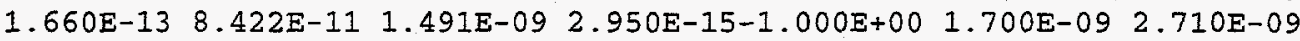
$1.070 \mathrm{E}-13 \quad 5.852 \mathrm{E}-111.036 \mathrm{E}-09 \quad 2.050 \mathrm{E}-15-1.000 \mathrm{E}+00 \quad 1.730 \mathrm{E}-09 \quad 2.740 \mathrm{E}-09$ $9.950 \mathrm{E}-14 \quad 5.652 \mathrm{E}-11$ 1.001E-09 $1.980 \mathrm{E}-15-1.000 \mathrm{E}+00 \quad 2.190 \mathrm{E}-09 \quad 3.520 \mathrm{E}-09$ 1. $060 \mathrm{E}-13 \quad 5.966 \mathrm{E}-11$ 1.056E-09 2.090E-15-1.000E+00 1.980E-09 3.040E-09

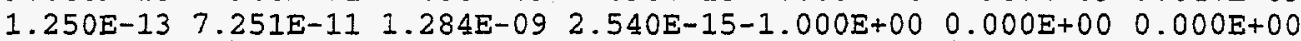

$2.669 \mathrm{E}-141.669 \mathrm{E}-11 \quad 3.530 \mathrm{E}-10 \quad 5.840 \mathrm{E}-16-1.000 \mathrm{E}+00 \quad 8.760 \mathrm{E}-09 \quad 1.390 \mathrm{E}-08$ $3.047 \mathrm{E}-14 \quad 1.596 \mathrm{E}-1113.376 \mathrm{E}-10 \quad 5.585 \mathrm{E}-16-1.000 \mathrm{E}+00 \quad 7.840 \mathrm{E}-09 \quad 1.240 \mathrm{E}-08$ $2.649 \mathrm{E}-14$ 1.517E-11 $3.209 \mathrm{E}-10 \quad 5.309 \mathrm{E}-16-1.000 \mathrm{E}+00 \quad 8.820 \mathrm{E}-09$ 1.270E-08 $2.583 E-14 \quad 1.542 E-11 \quad 3.260 E-10 \quad 5.394 E-16-1.000 E+00 \quad 8.300 E-09 \quad 1.320 E-08$ $4.382 \mathrm{E}-142.238 \mathrm{E}-11 \mathrm{4}$ 2.734E-10 $7.832 \mathrm{E}-16-1.000 \mathrm{E}+00 \quad 7.940 \mathrm{E}-09$ 1.260E-08 $2.725 \mathrm{E}-14 \quad 1.588 \mathrm{E}-11 \quad 3.358 \mathrm{E}-10 \quad 5.556 \mathrm{E}-16-1.000 \mathrm{E}+00 \quad 7.930 \mathrm{E}-09 \quad 1.260 \mathrm{E}-08$ $2.536 \mathrm{E}-141.490 \mathrm{E}-11 \quad 3.152 \mathrm{E}-10 \quad 5.215 \mathrm{E}-16-1.000 \mathrm{E}+00 \quad 9.120 \mathrm{E}-09 \quad 1.450 \mathrm{E}-08$ $2.725 \mathrm{E}-141.585 \mathrm{E}-11 \quad 3.353 \mathrm{E}-10 \quad 5.546 \mathrm{E}-16-1.000 \mathrm{E}+00 \quad 8.630 \mathrm{E}-09$ 1.350E-08 $4.392 E-14 \quad 5.253 E-11 \quad 1.110 E-09 \quad 1.836 E-15-1.000 E+00 \quad 0.000 E+00 \quad 0.000 E+00$

$2.130 \mathrm{E}-15 \quad 3.368 \mathrm{E}-13 \quad 3.429 \mathrm{E}-13 \quad 4.790 \mathrm{E}-17-1.000 \mathrm{E}+00 \quad 2.560 \mathrm{E}-12 \quad 1.560 \mathrm{E}-12$ $2.450 \mathrm{E}-15 \quad 3.297 \mathrm{E}-13 \quad 3.357 \mathrm{E}-13 \quad 4.690 \mathrm{E}-17-1.000 \mathrm{E}+00 \quad 2.460 \mathrm{E}-12 \quad 5.170 \mathrm{E}-13$ $2.030 \mathrm{E}-15 \quad 3.002 \mathrm{E}-13 \quad 3.057 \mathrm{E}-13 \quad 4.270 \mathrm{E}-17-1.000 \mathrm{E}+00 \quad 2.530 \mathrm{E}-10 \quad 3.890 \mathrm{E}-13$ $1.870 \mathrm{E}-15 \quad 2.932 \mathrm{E}-13 \quad 2.985 \mathrm{E}-13 \quad 4.170 \mathrm{E}-17-1.000 \mathrm{E}+00 \quad 3.410 \mathrm{E}-12 \quad 8.590 \mathrm{E}-13$

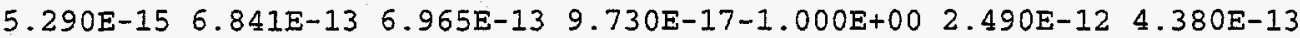
$2.130 \mathrm{E}-15 \quad 3.044 \mathrm{E}-13 \quad 3.100 \mathrm{E}-13 \quad 4.330 \mathrm{E}-17-1.000 \mathrm{E}+00 \quad 2.400 \mathrm{E}-12 \quad 2.660 \mathrm{E}-13$

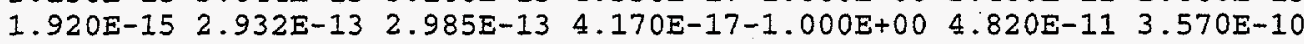
$2.170 \mathrm{E}-15 \quad 3.227 \mathrm{E}-13 \quad 3.286 \mathrm{E}-13 \quad 4.590 \mathrm{E}-17-1.000 \mathrm{E}+00 \quad 4.640 \mathrm{E}-11 \quad 1.080 \mathrm{E}-10$ $6.160 E-14 \quad 7.241 E-11 \quad 7.373 E-11 \quad 1.030 E-14-1.000 E+00 \quad 0.000 E+00 \quad 0.000 E+00$

8.410E-15 5.451E-12 9.607E-11 1.910E-16-1.000E+00 4.300E-10 9.960E-10 $9.640 \mathrm{E}-15$. $5.280 \mathrm{E}-12 \quad 9.305 \mathrm{E}-11 \quad 1.850 \mathrm{E}-16-1.000 \mathrm{E}+00 \quad 2.870 \mathrm{E}-10 \quad 1.590 \mathrm{E}-10$ $8.270 \mathrm{E}-15$ 4.852E-12 $8.550 \mathrm{E}-11$ 1.700E-16-1.000E+00 $1.660 \mathrm{E}-096.630 \mathrm{E}-11$ $7.930 E-15 \quad 4.880 E-12 \quad 8.601 E-11 \quad 1.710 E-16-1.000 E+00 \quad 1.290 E-09 \quad 4.390 E-10$ $1.550 \mathrm{E}-14$ 8.020E-12 $1.413 \mathrm{E}-10 \quad 2.810 \mathrm{E}-16-1.000 \mathrm{E}+00 \quad 2.410 \mathrm{E}-09 \quad 5.530 \mathrm{E}-10$ 8.530E-15 5.109E-12 9.003E-11 1.790E-16-1.000E+00 2.560E-10 5.250E-11 $7.890 \mathrm{E}-15 \quad 4.766 \mathrm{E}-12 \quad 8.399 \mathrm{E}-11 \quad 1.670 \mathrm{E}-16-1.000 \mathrm{E}+00 \quad 1.410 \mathrm{E}-09 \quad 7.370 \mathrm{E}-09$ 8.580E-15 5.137E-12 $9.053 E-11 \quad 1.800 E-16-1.000 E+00 \quad 1.010 E-09 \quad 2.560 E-09$ $2.520 \mathrm{E}-14 \quad 5.565 \mathrm{E}-11 \quad 9.808 \mathrm{E}-10 \quad 1.950 \mathrm{E}-15-1.000 \mathrm{E}+00 \quad 0.000 \mathrm{E}+00 \quad 0.000 \mathrm{E}+00$

$1.140 \mathrm{E}-13 \quad 6.027 \mathrm{E}-11 \quad 4.425 \mathrm{E}-10 \quad 2.240 \mathrm{E}-15-1.000 \mathrm{E}+00 \quad 4.540 \mathrm{E}-10 \quad 1.340 \mathrm{E}-09$ $1.290 \mathrm{E}-13 \quad 5.758 \mathrm{E}-11 \quad 4.228 \mathrm{E}-10 \quad 2.140 \mathrm{E}-15-1.000 \mathrm{E}+00 \quad 1.450 \mathrm{E}-10 \quad 1.800 \mathrm{E}-10$ $1.150 \mathrm{E}-13$ 5.596E-11 4.109E-10 2.080E-15-1.000E+00 4.210E-09 4.010E-11 $1.140 \mathrm{E}-13 \quad 5.731 \mathrm{E}-11 \quad 4.208 \mathrm{E}-10 \quad 2.130 \mathrm{E}-15-1.000 \mathrm{E}+00 \quad 2.140 \mathrm{E}-10.2 .810 \mathrm{E}-10$ $1.690 \mathrm{E}-13 \quad 7.776 \mathrm{E}-11 \quad 5.709 \mathrm{E}-10 \quad 2.890 \mathrm{E}-15-1.000 \mathrm{E}+00 \quad 1.410 \mathrm{E}-10 \quad 9.770 \mathrm{E}-11$ $1.180 \mathrm{E}-13$ 5.462E-11 $4.010 \mathrm{E}-10 \quad 2.030 \mathrm{E}-15-1.000 \mathrm{E}+00 \quad 6.870 \mathrm{E}-11 \quad 6.400 \mathrm{E}-12$ $\begin{array}{llllll}1.110 \mathrm{E}-13 & 5.569 \mathrm{E}-11 & 4.089 \mathrm{E}-10 & 2.070 \mathrm{E}-15-1.000 \mathrm{E}+00 & 2.120 \mathrm{E}-09 & 6.260 \mathrm{E}-09\end{array}$ $\begin{array}{llllll}1.170 E-13 & 5.812 \mathrm{E}-11 & 4.267 \mathrm{E}-10 & 2.160 \mathrm{E}-15-1.000 \mathrm{E}+00 & 1.310 \mathrm{E}-09 & 2.280 \mathrm{E}-09\end{array}$

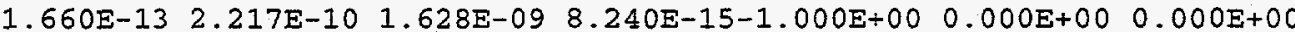

$2.330 \mathrm{E}-15 \quad 7.315 \mathrm{E}-13 \quad 9.675 \mathrm{E}-13 \quad 4.740 \mathrm{E}-17-1.000 \mathrm{E}+00 \quad 1.010 \mathrm{E}-11 \quad 3.770 \mathrm{E}-12$ 2.640E-15 7.007E-13 $9.267 \mathrm{E}-13 \quad 4.540 \mathrm{E}-17-1.000 \mathrm{E}+00 \quad 9.840 \mathrm{E}-12 \quad 7.070 \mathrm{E}-13$ $2.340 E-15 \quad 6.713 E-13 \quad 8.879 E-13 \quad 4.350 E-17-1.000 E+00 \quad 6.460 E-10 \quad 2.720 E-13$ $2.310 \mathrm{E}-15 \quad 6.852 \mathrm{E}-13 \quad 9.063 \mathrm{E}-13 \quad 4.440 \mathrm{E}-17-1.000 \mathrm{E}+00 \quad 2.930 \mathrm{E}-11 \quad 1.070 \mathrm{E}-12$ $3.490 \mathrm{E}-15 \quad 9.923 \mathrm{E}-13 \quad 1.312 \mathrm{E}-12 \quad 6.430 \mathrm{E}-17-1.000 \mathrm{E}+00 \quad 1.200 \mathrm{E}-10 \quad 6.060 \mathrm{E}-13$ $2.390 \mathrm{E}-15 \quad 6.590 \mathrm{E}-13 \quad 8.716 \mathrm{E}-13 \quad 4.270 \mathrm{E}-17-1.000 \mathrm{E}+00 \quad 9.400 \mathrm{E}-12 \quad 5.290 \mathrm{E}-14$ $2.260 \mathrm{E}-15 \quad 6.682 \mathrm{E}-13 \quad 8.838 \mathrm{E}-13 \quad 4.330 \mathrm{E}-17-1.000 \mathrm{E}+00 \quad 2.280 \mathrm{E}-10 \quad 1.240 \mathrm{E}-09$ 
EFFECTIVE SKIN (FGR)

Ia-142

GONADS

BREAST

LUNGS

RED MARR

BONE SUR

THYROID

REMAINDER

EFFECTIVE

SKIN (FGR)

Ce-141

GONADS

BREAST

IUNTGS

RED MARR

BONE SUR

THYROID

REMAINDER

EFFECTIVE

SKIN (FGR)

$\mathrm{Ce}-143$

GONADS

BREAST

LUNGS

RED MARR

BONE SUR

THYROID

REMAINDER

EFFECTIVE

SKIN (FGR)

$\mathrm{Ce}-144$

GONADS

BREAST

LUNGS

RED MARR

BONE SUR

THYROID

REMAINDER

EFFECTIVE

SKIN (FGR)

Pr-143

GONADS

BREAST

LUNGS

RED MARR

BONE SUR

THYROID

REMAINDER

EFFECTIVE

SKIN (FGR)

Nd-147

GONADS

BREAST

LUNGS

RED MARR

BONE SUR

THYROID

REMAINDER

EFFECTIVE

SKIN (FGR)

Np-239

GONADS

BREAST

LUNGS

RED MARR
$2.390 E-15 \quad 7.007 E-13 \quad 9.267 E-13 \quad 4.540 E-17-1.000 E+00 \quad 1.570 E-10 \quad 3.740 E-10$ $6.580 \mathrm{E}-141.667 \mathrm{E}-10 \quad 2.204 \mathrm{E}-10 \quad 1.080 \mathrm{E}-14-1.000 \mathrm{E}+00 \quad 0.000 \mathrm{E}+00 \quad 0.000 \mathrm{E}+00$

$1.400 \mathrm{E}-13 \quad 1.978 \mathrm{E}-112.034 \mathrm{E}-11 \quad 2.540 \mathrm{E}-15-1.000 \mathrm{E}+00 \quad 1.660 \mathrm{E}-11 \quad 6.990 \mathrm{E}-11$ $1.570 \mathrm{E}-13$ 1.885E-11 $1.938 \mathrm{E}-112.420 \mathrm{E}-15-1.000 \mathrm{E}+00$ 1.130E-11 $1.540 \mathrm{E}-11$ $1.420 \mathrm{E}-13$ 1.846E-11 $1.898 \mathrm{E}-11 \quad 2.370 \mathrm{E}-15-1.000 \mathrm{E}+00 \quad 3.010 \mathrm{E}-10 \quad 8.400 \mathrm{E}-12$ 1. $420 \mathrm{E}-13$ 1.900E-11 $1.954 \mathrm{E}-112.440 \mathrm{E}-15-1.000 \mathrm{E}+00$ 1.360E-11 $1.930 \mathrm{E}-11$ $1.950 \mathrm{E}-132.484 \mathrm{E}-112.554 \mathrm{E}-11 \quad 3.190 \mathrm{E}-15-1.000 \mathrm{E}+00$ 1.110E-11 $7.400 \mathrm{E}-12$

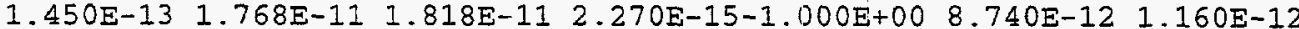
$1.380 \mathrm{E}-13$ 1.853E-11 $1.906 \mathrm{E}-112.380 \mathrm{E}-15-1.000 \mathrm{E}+00 \quad 8.070 \mathrm{E}-115.200 \mathrm{E}-10$ $1.440 \mathrm{E}-13$ 1.916E-11 $1.970 \mathrm{E}-11 \quad 2.460 \mathrm{E}-15-1.000 \mathrm{E}+00 \quad 6.840 \mathrm{E}-111.790 \mathrm{E}-10$ $2.160 E-139.111 E-11 \quad 9.368 E-11 \quad 1.170 E-14-1.000 E+00 \quad 0.000 E+00 \quad 0.000 E+00$

$3.380 E-15 \quad 2.213 E-12 \quad 4.332 E-11 \quad 7.710 E-17-1.000 E+00 \quad 5.540 E-11 \quad 1.080 E-10$ $3.930 \mathrm{E}-15 \quad 2.170 \mathrm{E}-12 \quad 4.247 \mathrm{E}-11 \quad 7.560 \mathrm{E}-17-1.000 \mathrm{E}+00 \quad 4.460 \mathrm{E}-11 \quad 1.110 \mathrm{E}-11$ 3. $170 \mathrm{E}-15 \quad 1.951 \mathrm{E}-12 \quad 3.820 \mathrm{E}-11$ 6.800E-17-1.000E+00 $1.670 \mathrm{E}-08 \quad 1.430 \mathrm{E}-12$ $2.830 \mathrm{E}-15 \quad 1.860 \mathrm{E}-12 \quad 3.641 \mathrm{E}-11 \quad 6.480 \mathrm{E}-17-1.000 \mathrm{E}+00 \quad 8.960 \mathrm{E}-11 \quad 3.390 \mathrm{E}-11$ $9.410 \mathrm{E}-15$ 5.166E-12 $1.011 \mathrm{E}-10 \quad 1.800 \mathrm{E}-16-1.000 \mathrm{E}+00 \quad 2.540 \mathrm{E}-10 \quad 2.300 \mathrm{E}-11$ $3.350 E-15 \quad 2.003 E-12 \quad 3.922 E-11 \quad 6.980 E-17-1.000 E+00 \quad 2.550 E-11 \quad 1.800 E-13$ $2.980 \mathrm{E}-15 \quad 1.894 \mathrm{E}-12 \quad 3.708 \mathrm{E}-11 \quad 6.600 \mathrm{E}-17-1.000 \mathrm{E}+00 \quad 1.260 \mathrm{E}-09 \quad 2.500 \mathrm{E}-09$ $3.430 \mathrm{E}-15 \quad 2.118 \mathrm{E}-12 \quad 4.146 \mathrm{E}-11 \quad 7.380 \mathrm{E}-17-1.000 \mathrm{E}+00 \quad 2.420 \mathrm{E}-09 \quad 7.830 \mathrm{E}-10$ $1.020 \mathrm{E}-14 \quad 3.788 \mathrm{E}-12 \quad 7.416 \mathrm{E}-11 \quad 1.320 \mathrm{E}-16-1.000 \mathrm{E}+00 \quad 0.000 \mathrm{E}+00 \quad 0.000 \mathrm{E}+00$

$1.280 \mathrm{E}-14 \quad 7.900 \mathrm{E}-12 \quad 4.958 \mathrm{E}-112.980 \mathrm{E}-16-1.000 \mathrm{E}+00 \quad 7.530 \mathrm{E}-112.120 \mathrm{E}-10$ $1.470 \mathrm{E}-14 \quad 7.688 \mathrm{E}-12 \quad 4.825 \mathrm{E}-112.900 \mathrm{E}-16-1.000 \mathrm{E}+00$ 1.660E-II $2.320 \mathrm{E}-11$ $1.230 \mathrm{E}-14 \quad 6.893 \mathrm{E}-12 \quad 4.325 \mathrm{E}-11 \quad 2.600 \mathrm{E}-16-1.000 \mathrm{E} \div 00 \quad 3.880 \mathrm{E}-09 \quad 3.820 \mathrm{E}-12$ $1.170 E-14 \quad 6.787 E-12 \quad 4.259 E-112.560 E-16-1.000 E+00 \quad 2.960 E-115.070 E-11$ $2.520 \mathrm{E}-14$ 1.323E-11 8.302E-11 4.990E-16-1.000E +00 1.640E-11 $1.610 \mathrm{E}-11$ $1.280 \mathrm{E}-14 \quad 7.211 \mathrm{E}-12 \quad 4.525 \mathrm{E}-11 \quad 2.720 \mathrm{E}-16-1.000 \mathrm{E}+00 \quad 6.230 \mathrm{E}-124.350 \mathrm{E}-13$ $1.170 \mathrm{E}-14 \quad 6.734 \mathrm{E}-12 \quad 4.226 \mathrm{E}-11 \quad 2.540 \mathrm{E}-16-1.000 \mathrm{E}+00$ 1.420E-09 $3.890 \mathrm{E}-09$ $1.290 \mathrm{E}-14 \quad 7.396 \mathrm{E}-12 \quad 4.642 \mathrm{E}-11 \quad 2.790 \mathrm{E}-16-1.000 \mathrm{E}+00 \quad 9.160 \mathrm{E}-10 \quad 1.230 \mathrm{E}-09$ $3.960 \mathrm{E}-14 \quad 1.058 \mathrm{E}-10 \quad 6.638 \mathrm{E}-10 \quad 3.990 \mathrm{E}-15-1.000 \mathrm{E}+00 \quad 0.000 \mathrm{E}+00 \quad 0.000 \mathrm{E}+00$

$2.725 \mathrm{E}-15 \quad 6.328 \mathrm{E}-13 \quad 1.319 \mathrm{E}-11 \quad 6.088 \mathrm{E}-17-1.000 \mathrm{E}+00 \quad 2.390 \mathrm{E}-10 \quad 6.987 \mathrm{E}-11$ $3.129 \mathrm{E}-15 \quad 6.274 \mathrm{E}-13 \quad 1.307 \mathrm{E}-11 \quad 5.922 \mathrm{E}-17-1.000 \mathrm{E}+00 \quad 3.480 \mathrm{E}-10 \quad 1.223 \mathrm{E}-11$ $2.639 \mathrm{E}-15 \quad 5.228 \mathrm{E}-13 \quad 1.089 \mathrm{E}-11 \quad 5.362 \mathrm{E}-17-1.000 \mathrm{E}+00 \quad 7.911 \mathrm{E}-07 \quad 6.551 \mathrm{E}-12$ $2.507 \mathrm{E}-15 \quad 4.755 \mathrm{E}-13 \quad 9.907 \mathrm{E}-12 \quad 5.247 \mathrm{E}-17-1.000 \mathrm{E}+00 \quad 2.880 \mathrm{E}-09 \quad 8.923 \mathrm{E}-11$ $5.441 E-15 \quad 1.646 E-12 \quad 3.429 E-11 \quad 1.127 E-16-1.000 E+00 \quad 4.720 E-09 \quad 1.280 E-10$ $2.753 \mathrm{E}-15 \quad 5.529 \mathrm{E}-13 \quad 1.152 \mathrm{E}-11 \quad 5.418 \mathrm{E}-17-1.000 \mathrm{E}+00 \quad 2.920 \mathrm{E}-10 \quad 5.154 \mathrm{E}-12$ $2.534 E-15 \quad 5.086 E-13 \quad 1.060 E-11 \quad 5.283 E-17-1.000 E+00 \quad 1.910 E-08 \quad 1.890 E-08$ $2.773 \mathrm{E}-15$ 5.909E-13 $1.231 \mathrm{E}-11$ 5.766E-17-1.000E+00 1.010E-07 5.711E-09 $8.574 \mathrm{E}-14 \quad 7.648 \mathrm{E}-13 \quad 1.594 \mathrm{E}-11 \quad 1.250 \mathrm{E}-14-1.000 \mathrm{E}+00 \quad 0.000 \mathrm{E}+00 \quad 0.000 \mathrm{E}+00$ $2.130 \mathrm{E}-17 \quad 2.264 \mathrm{E}-14 \quad 4.032 \mathrm{E}-13 \quad 7.930 \mathrm{E}-19-1.000 \mathrm{E}+00 \quad 4.370 \mathrm{E}-18 \quad 8.990 \mathrm{E}-18$ $2.550 \mathrm{E}-17 \quad 2.330 \mathrm{E}-14 \quad 4.149 \mathrm{E}-13 \quad 8.160 \mathrm{E}-19-1.000 \mathrm{E}+00 \quad 2.220 \mathrm{E}-18 \quad 1.090 \mathrm{E}-18$ $1.860 \mathrm{E}-17$ 1.642E-14 2.923E-13 5.750E-19-1.000E+00 1.330E-08 $1.910 \mathrm{E}-19$ $1.620 \mathrm{E}-17$ 1.493E-14 2.659E-13 5.230E-19-1.000E+00 1.480E-11 $1.030 \mathrm{E}-12$ $5.930 \mathrm{E}-17 \quad 5.454 \mathrm{E}-14 \quad 9.711 \mathrm{E}-13 \quad 1.910 \mathrm{E}-18-1.000 \mathrm{E}+00 \quad 1.490 \mathrm{E}-11 \quad 1.030 \mathrm{E}-12$ $2.050 \mathrm{E}-17 \quad 1.802 \mathrm{E}-14 \quad 3.208 \mathrm{E}-13 \quad 6.310 \mathrm{E}-19-1.000 \mathrm{E}+00 \quad 1.680 \mathrm{E}-18 \quad 2.660 \mathrm{E}-20$ $1.760 \mathrm{E}-17$ 1.642E-14 $2.923 \mathrm{E}-13 \quad 5.750 \mathrm{E}-19-1.000 \mathrm{E}+00$ 1.970E-09 $4.220 \mathrm{E}-09$ $2.100 E-17 \quad 2.002 E-14 \quad 3.564 E-13 \quad 7.010 E-19-1.000 E+00 \quad 2.190 E-09 \quad 1.270 E-09$ $1.760 \mathrm{E}-14 \quad 5.711 \mathrm{E}-11 \quad 1.017 \mathrm{E}-09 \quad 2.000 \mathrm{E}-15-1.000 \mathrm{E}+00 \quad 0.000 \mathrm{E}+00 \quad 0.000 \mathrm{E}+00$

$6.130 \mathrm{E}-15 \quad 4.218 \mathrm{E}-12 \quad 7.235 \mathrm{E}-11 \quad 1.480 \mathrm{E}-16-1.000 \mathrm{E}+00 \quad 8.410 \mathrm{E}-11 \quad 1.790 \mathrm{E}-10$ $7.120 \mathrm{E}-15 \quad 4.132 \mathrm{E}-12 \quad 7.088 \mathrm{E}-11 \quad 1.450 \mathrm{E}-16-1.000 \mathrm{E}+00 \quad 3.450 \mathrm{E}-111.870 \mathrm{E}-11$ $5.820 \mathrm{E}-15 \quad 3.648 \mathrm{E}-12 \quad 6.257 \mathrm{E}-11$ 1.280E-16-1.000E+00 $1.060 \mathrm{E}-08 \quad 2.440 \mathrm{E}-12$ $5.400 \mathrm{E}-15 \quad 3.505 \mathrm{E}-12 \quad 6.013 \mathrm{E}-11$ 1.230E-16-1.000E+00 9.190E-11 $5.050 \mathrm{E}-11$

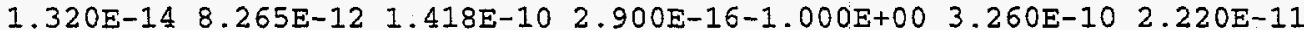
$6.120 \mathrm{E}-15 \quad 3.876 \mathrm{E}-12 \quad 6.648 \mathrm{E}-11 \quad 1.360 \mathrm{E}-16-1.000 \mathrm{E}+00 \quad 1.820 \mathrm{E}-112.640 \mathrm{E}-13$ $5.530 \mathrm{E}-15 \quad 3.562 \mathrm{E}-12 \quad 6.111 \mathrm{E}-11$ 1.250E-16-1.000E+00 $1.760 \mathrm{E}-09 \quad 3.760 \mathrm{E}-09$ $6.190 \mathrm{E}-15$ 3.961E-12 $6.795 \mathrm{E}-11$ 1.390E-16-1.000E+00 $1.850 \mathrm{E}-09 \quad 1.180 \mathrm{E}-09$ $1.950 \mathrm{E}-14 \quad 3.135 \mathrm{E}-11 \quad 5.377 \mathrm{E}-10 \quad 1.100 \mathrm{E}-15-1.000 \mathrm{E}+00 \quad 0.000 \mathrm{E}+00 \quad 0.000 \mathrm{E}+00$

$7.530 E-15 \quad 4.691 E-124.380 E-11 \quad 1.710 E-16-1.000 E+007.450 E-11 \quad 1.620 E-10$ $8.730 \mathrm{E}-15 \quad 4.636 \mathrm{E}-12 \quad 4.329 \mathrm{E}-11$ 1.690E-16-1.000E+00 $1.630 \mathrm{E}-11 \quad 1.720 \mathrm{E}-11$ $7.180 \mathrm{E}-15 \quad 4.115 \mathrm{E}-12 \quad 3.842 \mathrm{E}-11 \quad 1.500 \mathrm{E}-16-1.000 \mathrm{E}+00 \quad 2.360 \mathrm{E}-09 \quad 2.400 \mathrm{E}-12$ $6.500 \mathrm{E}-15$ 4.005E-12 3.740E-11 1.460E-16-1.000E+00 2.080E-10 4.660E-11 
BONE SUR

THYROID

REMAINDER

EFFECTIVE

SKIN (FGR)

$\mathrm{Pu}-238$

GONADS

BREAST

LUNGS

RED MARR

BONE SUR

THYROID

REMAINDER

EFFECTIVE

SKIN (FGR)

Pu-239

GONADS

BREAST

IUNGS

RED MARR

BONE SUR

THYROID

REMAINDER

EFFECTIVE

SKIN (FGR)

Pu-240

GONADS

BREAST

LUNGS

RED MARR

BONE SUR

THYROID

REMAINDER

EFFECTIVE

SKIN (FGR)

Pu-241

GONADS

BREAST

LUNGS

RED MARR

BONE SUR

THYROID

REMAINDER

EFFECTIVE

SKIN (FGR)

Am-241

GONADS

BREAST

LUNGS

RED MARR

BONE SUR

THYROID

REMAINDER

EFFECTIVE

SKIN (FGR)

$\mathrm{Cm}-242$

GONADS

BREAST

LUNGS

RED MARR

BONE SUR

THYROID

REMAINDER

EFFECTIVE

SKIN (FGR)

Cm-244

GONADS
$2.000 E-14 \quad 1.001 E-11 \quad 9.349 E-11 \quad 3.650 E-16-1.000 E+00 \quad 2.030 E-09 \quad 3.590 E-11$ $7.520 \mathrm{E}-15 \quad 4.197 \mathrm{E}-12 \quad 3.919 \mathrm{E}-11 \quad 1.530 \mathrm{E}-16-1.000 \mathrm{E}+00 \quad 7.620 \mathrm{E}-12 \quad 2.070 \mathrm{E}-13$ $6.760 \mathrm{E}-15 \quad 4.005 \mathrm{E}-12 \quad 3.740 \mathrm{E}-11 \quad 1.460 \mathrm{E}-16-1.000 \mathrm{E}+00 \quad 9.590 \mathrm{E}-10 \quad 2.770 \mathrm{E}-09$ $7.690 \mathrm{E}-15 \quad 4.471 \mathrm{E}-12 \quad 4.175 \mathrm{E}-11 \quad 1.630 \mathrm{E}-16-1.000 \mathrm{E}+00 \quad 6.780 \mathrm{E}-10 \quad 8.820 \mathrm{E}-10$

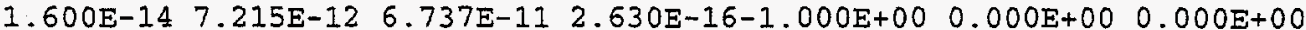

$6.560 \mathrm{E}-18 \quad 4.291 \mathrm{E}-14 \quad 9.011 \mathrm{E}-13 \quad 1.490 \mathrm{E}-18-1.000 \mathrm{E}+00 \quad 1.040 \mathrm{E}-05 \quad 2.330 \mathrm{E}-09$ $1.270 \mathrm{E}-17 \quad 5.558 \mathrm{E}-14 \quad 1.167 \mathrm{E}-12 \quad 1.930 \mathrm{E}-18-1.000 \mathrm{E}+00 \quad 4.400 \mathrm{E}-10 \quad 1.800 \mathrm{E}-13$ $1.060 \mathrm{E}-18 \quad 2.267 \mathrm{E}-15 \quad 4.759 \mathrm{E}-14 \quad 7.870 \mathrm{E}-20-1.000 \mathrm{E}+00 \quad 3.200 \mathrm{E}-04 \quad 8.640 \mathrm{E}-14$ $1.680 \mathrm{E}-18 \quad 5.587 \mathrm{E}-15 \quad 1.173 \mathrm{E}-13 \quad 1.940 \mathrm{E}-19-1.000 \mathrm{E}+00 \quad 5.800 \mathrm{E}-05 \quad 1.270 \mathrm{E}-08$ $9.300 \mathrm{E}-18 \quad 3.514 \mathrm{E}-14 \quad 7.378 \mathrm{E}-13 \quad 1.220 \mathrm{E}-18-1.000 \mathrm{E}+00 \quad 7.250 \mathrm{E}-04 \quad 1.580 \mathrm{E}-07$

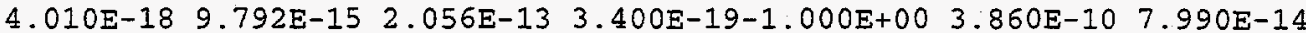

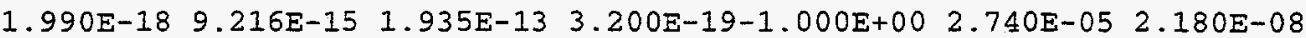
$4.880 \mathrm{E}-18 \quad 2.413 \mathrm{E}-14 \quad 5.068 \mathrm{E}-13 \quad 8.380 \mathrm{E}-19-1.000 \mathrm{E}+00 \quad 7.790 \mathrm{E}-05 \quad 1.340 \mathrm{E}-08$ $4.090 E-172.776 E-13 \quad 5.830 E-12 \quad 9.640 E-18-1.000 E+00 \quad 0.000 E+00 \quad 0.000 E+00$

$4.840 \mathrm{E}-18 \quad 1.768 \mathrm{E}-14 \quad 3.713 \mathrm{E}-13 \quad 6.140 \mathrm{E}-19-1.000 \mathrm{E}+00 \quad 1.200 \mathrm{E}-05 \quad 2.640 \mathrm{E}-09$ $7.550 \mathrm{E}-18 \quad 2.238 \mathrm{E}-14 \quad 4.699 \mathrm{E}-13 \quad 7.770 \mathrm{E}-19-1.000 \mathrm{E}+00 \quad 3.990 \mathrm{E}-10 \quad 1.210 \mathrm{E}-13$ $2.650 \mathrm{E}-18 \quad 2.267 \mathrm{E}-15 \quad 4.760 \mathrm{E}-14 \quad 7.870 \mathrm{E}-20-1.000 \mathrm{E}+00 \quad 3.230 \mathrm{E}-04 \quad 7.890 \mathrm{E}-14$ $2.670 \mathrm{E}-18 \quad 3.456 \mathrm{E}-15 \quad 7.258 \mathrm{E}-14 \quad 1.200 \mathrm{E}-19-1.000 \mathrm{E}+00 \quad 6.570 \mathrm{E}-05 \quad 1.410 \mathrm{E}-08$ $9.470 \mathrm{E}-18 \quad 1.673 \mathrm{E}-14 \quad 3.514 \mathrm{E}-13 \quad 5.810 \mathrm{E}-19-1.000 \mathrm{E}+00 \quad 8.210 \mathrm{E}-04 \quad 1.760 \mathrm{E}-07$ $3.880 \mathrm{E}-18 \quad 5.126 \mathrm{E}-15 \quad 1.077 \mathrm{E}-13 \quad 1.780 \mathrm{E}-19-1.000 \mathrm{E}+00 \quad 3.750 \mathrm{E}-10 \quad 7.500 \mathrm{E}-14$ $2.860 E-18 \quad 4.838 E-15 \quad 1.016 E-13 \quad 1.680 E-19-1.000 E+00 \quad 3.020 E-05 \quad 2.120 E-08$

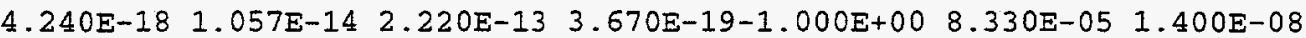
$1.860 \mathrm{E}-17 \quad 1.057 \mathrm{E}-13 \quad 2.220 \mathrm{E}-12 \quad 3.670 \mathrm{E}-18-1.000 \mathrm{E}+00 \quad 0.000 \mathrm{E}+00 \quad 0.000 \mathrm{E}+00$

$6.360 \mathrm{E}-18 \quad 4.118 \mathrm{E}-14 \quad 8.649 \mathrm{E}-13 \quad 1.430 \mathrm{E}-18-1.000 \mathrm{E}+00 \quad 1.200 \mathrm{E}-05 \quad 2.640 \mathrm{E}-09$ $1.230 \mathrm{E}-17 \quad 5.328 \mathrm{E}-14 \quad 1.119 \mathrm{E}-12 \quad 1.850 \mathrm{E}-18-1.000 \mathrm{E}+00 \quad 4.330 \mathrm{E}-10 \quad 1.730 \mathrm{E}-13$ $1.090 \mathrm{E}-18 \quad 2.249 \mathrm{E}-15 \quad 4.723 \mathrm{E}-14 \quad 7.810 \mathrm{E}-20-1.000 \mathrm{E}+00 \quad 3.230 \mathrm{E}-04 \quad 8.220 \mathrm{E}-14$ $\begin{array}{llllll}1.650 \mathrm{E}-18 & 5.386 \mathrm{E}-15 & 1.131 \mathrm{E}-13 & 1.870 \mathrm{E}-19-1.000 \mathrm{E}+00 & 6.570 \mathrm{E}-05 & 1.410 \mathrm{E}-08\end{array}$ $9.260 \mathrm{E}-18 \quad 3.398 \mathrm{E}-14 \quad 7.137 \mathrm{E}-13 \quad 1.180 \mathrm{E}-18-1.000 \mathrm{E}+00 \quad 8.210 \mathrm{E}-04 \quad 1.760 \mathrm{E}-07$ $3.920 \mathrm{E}-18 \quad 9.446 \mathrm{E}-15 \quad 1.984 \mathrm{E}-13 \quad 3.280 \mathrm{E}-19-1.000 \mathrm{E}+00 \quad 3.760 \mathrm{E}-10 \quad 7.510 \mathrm{E}-14$

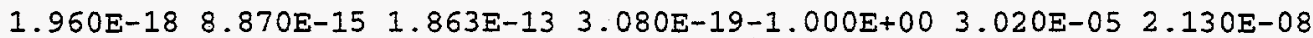
$4.750 \mathrm{E}-18 \quad 2.313 \mathrm{E}-14 \quad 4.857 \mathrm{E}-13 \quad 8.030 \mathrm{E}-19-1.000 \mathrm{E}+00 \quad 8.330 \mathrm{E}-05 \quad 1.400 \mathrm{E}-08$ $3.920 E-17 \quad 2.644 E-13 \quad 5.552 E-12 \quad 9.180 E-18-1.000 E+00 \quad 0.000 E+00 \quad 0.000 E+00$

$7.190 \mathrm{E}-20 \quad 6.653 \mathrm{E}-17 \quad 1.396 \mathrm{E}-15 \quad 2.310 \mathrm{E}-21-1.000 \mathrm{E}+00 \quad 2.760 \mathrm{E}-07 \quad 5.660 \mathrm{E}-11$ $8.670 \mathrm{E}-20 \quad 7.229 \mathrm{E}-17 \quad 1.517 \mathrm{E}-15.2 .510 \mathrm{E}-21-1.000 \mathrm{E}+00 \quad 2.140 \mathrm{E}-112.790 \mathrm{E}-15$ $6.480 E-20 \quad 4.090 E-17 \quad 8.584 E-16 \quad 1.420 E-21-1.000 E+00 \quad 3.180 E-06 \quad 4.480 E-15$ $5.630 \mathrm{E}-20 \quad 4.003 \mathrm{E}-17 \quad 8.403 \mathrm{E}-16 \quad 1.390 \mathrm{E}-21-1.000 \mathrm{E}+00 \quad 1.430 \mathrm{E}-06 \quad 2.780 \mathrm{E}-10$ $2.190 \mathrm{E}-19 \quad 1.385 \mathrm{E}-16 \quad 2.908 \mathrm{E}-15 \quad 4.810 \mathrm{E}-21-1.000 \mathrm{E}+00 \quad 1.780 \mathrm{E}-05 \quad 3.480 \mathrm{E}-09$ $6.980 \mathrm{E}-20 \quad 4.522 \mathrm{E}-17 \quad 9.491 \mathrm{E}-16 \quad 1.570 \mathrm{E}-21-1.000 \mathrm{E}+00 \quad 9.150 \mathrm{E}-12 \quad 1.010 \mathrm{E}-15$ $6.090 \mathrm{E}-20 \quad 4.291 \mathrm{E}-179.007 \mathrm{E}-16 \quad 1.490 \mathrm{E}-21-1.000 \mathrm{E}+00 \quad 6.020 \mathrm{E}-07 \quad 1.850 \mathrm{E}-10$ $7.250 \mathrm{E}-20 \quad 5.558 \mathrm{E}-17 \quad 1.167 \mathrm{E}-15 \quad 1.930 \mathrm{E}-21-1.000 \mathrm{E}+00 \quad 1.340 \mathrm{E}-06 \quad 2.070 \mathrm{E}-10$

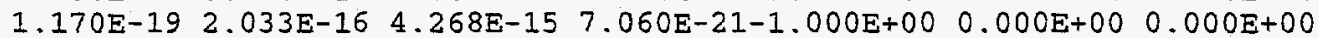

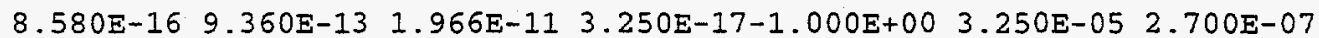

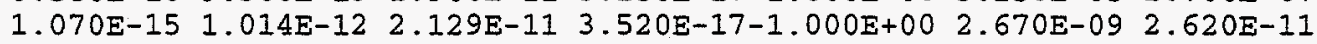
$6.740 \mathrm{E}-16 \quad 5.789 \mathrm{E}-13 \quad 1.216 \mathrm{E}-11 \quad 2.010 \mathrm{E}-17-1.000 \mathrm{E}+00 \quad 1.840 \mathrm{E}-05 \quad 3.360 \mathrm{E}-11$ $5.210 \mathrm{E}-16 \quad 4.838 \mathrm{E}-13 \cdot 1.016 \mathrm{E}-11 \quad 1.680 \mathrm{E}-17-1.000 \mathrm{E}+00$ 1.740E-04 $1.450 \mathrm{E}-06$ $2.870 \mathrm{E}-15 \quad 2.678 \mathrm{E}-12 \quad 5.625 \mathrm{E}-11 \quad 9.300 \mathrm{E}-17-1.000 \mathrm{E}+00 \quad 2.170 \mathrm{E}-03 \quad 1.810 \mathrm{E}-05$ $7.830 \mathrm{E}-16 \quad 6.365 \mathrm{E}-13 \quad 1.337 \mathrm{E}-11 \quad 2.210 \mathrm{E}-17-1.000 \mathrm{E}+00 \quad 1.600 \mathrm{E}-09 \quad 1.320 \mathrm{E}-11$ $6.340 E-16 \quad 5.933 E-13 \quad 1.246 E-11 \quad 2.060 E-17-1.000 E+00 \quad 7.820 E-05 \quad 6.660 E-07$

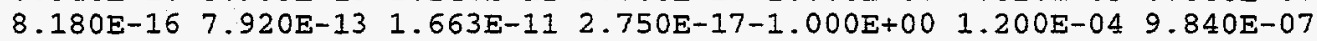
$1.280 \mathrm{E}-15 \quad 2.396 \mathrm{E}-12 \quad 5.032 \mathrm{E}-11 \quad 8.320 \mathrm{E}-17-1.000 \mathrm{E}+00 \quad 0.000 \mathrm{E}+00 \quad 0.000 \mathrm{E}+00$

$7.830 E-18 \quad 4.893 E-14 \quad 1.013 E-12 \quad 1.700 E-18-1.000 E+00 \quad 5.700 E-07 \quad 5.200 E-09$ $1.480 \mathrm{E}-17 \quad 6.159 \mathrm{E}-14 \quad 1.275 \mathrm{E}-12 \quad 2.140 \mathrm{E}-18-1.000 \mathrm{E}+00 \quad 9.440 \mathrm{E}-10 \quad 8.950 \mathrm{E}-12$ $1.130 \mathrm{E}-18 \quad 3.022 \mathrm{E}-15 \quad 6.257 \mathrm{E}-14 \quad 1.050 \mathrm{E}-19-1.000 \mathrm{E}+00 \quad 1.550 \mathrm{E}-05 \quad 8.840 \mathrm{E}-12$ $1.890 \mathrm{E}-18 \quad 6.562 \mathrm{E}-15 \quad 1.359 \mathrm{E}-13 \quad 2.280 \mathrm{E}-19-1.000 \mathrm{E}+00 \quad 3.900 \mathrm{E}-06 \quad 3.570 \mathrm{E}-08$ $1.060 \mathrm{E}-17 \quad 4.231 \mathrm{E}-14 \quad 8.759 \mathrm{E}-13 \quad 1.470 \mathrm{E}-18-1.000 \mathrm{E}+00 \quad 4.870 \mathrm{E}-05 \quad 4.460 \mathrm{E}-07$ $4.910 E-18 \quad 1.261 E-14 \quad 2.610 E-13 \quad 4.380 E-19-1.000 E+00 \quad 9.410 E-10 \quad 8.820 E-12$ $2.270 E-18 \quad 1.079 E-14 \quad 2.235 E-13 \quad 3.750 E-19-1.000 E+00 \quad 2.450 E-06 \quad 4.020 E-08$ $5.690 \mathrm{E}-18 \quad 2.751 \mathrm{E}-14 \quad 5.697 \mathrm{E}-13 \quad 9.560 \mathrm{E}-19-1.000 \mathrm{E}+00 \quad 4.670 \mathrm{E}-06 \quad 3.100 \mathrm{E}-08$ $4.290 \mathrm{E}-172.700 \mathrm{E}-13 \quad 5.589 \mathrm{E}-12 \quad 9.380 \mathrm{E}-18-1.000 \mathrm{E}+00 \quad 0.000 \mathrm{E}+00 \quad 0.000 \mathrm{E}+00$ $6.900 E-18 \quad 4.522 E-14 \quad 9.492 E-13 \quad 1.570 E-18-1.000 E+00 \quad 1.590 E-05 \quad 1.330 E-07$ 
BREAST

LUNGS

RED MARR BONE SUR

THYROID REMAINDER EFFECTIVE $\operatorname{SKIN~(FGR)~}$
$1.330 \mathrm{E}-17 \quad 5.702 \mathrm{E}-14 \quad 1.197 \mathrm{E}-12 \quad 1.980 \mathrm{E}-18-1.000 \mathrm{E}+00 \quad 1.040 \mathrm{E}-09 \quad 8.820 \mathrm{E}-12$ $7.080 \mathrm{E}-192.592 \mathrm{E}-15 \quad 5.441 \mathrm{E}-14 \quad 9.000 \mathrm{E}-20-1.000 \mathrm{E}+00 \quad 1.930 \mathrm{E}-05 \quad 8.810 \mathrm{E}-12$ $1.460 \mathrm{E}-18 \quad 5.875 \mathrm{E}-15 \quad 1.233 \mathrm{E}-13 \quad 2.040 \mathrm{E}-19-1.000 \mathrm{E}+00 \quad 9.380 \mathrm{E}-05 \quad 7.820 \mathrm{E}-07$ $8.820 \mathrm{E}-18 \quad 3.859 \mathrm{E}-14 \quad 8.101 \mathrm{E}-13 \quad 1.340 \mathrm{E}-18-1.000 \mathrm{E}+00 \quad 1.170 \mathrm{E}-03 \quad 9.770 \mathrm{E}-06$ $4.190 \mathrm{E}-18 \quad 1.146 \mathrm{E}-142.406 \mathrm{E}-13 \quad 3.980 \mathrm{E}-19-1.000 \mathrm{E}+00$ 1.010E-09 $8.440 \mathrm{E}-12$

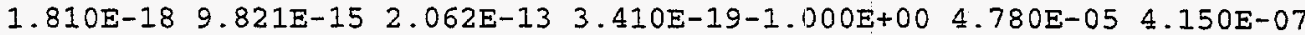
$4.910 \mathrm{E}-18 \quad 2.529 \mathrm{E}-14 \quad 5.308 \mathrm{E}-13 \quad 8.780 \mathrm{E}-19-1.000 \mathrm{E}+00 \quad 6.700 \mathrm{E}-05 \quad 5.450 \mathrm{E}-07$ $3.910 E-172.506 E-13 \quad 5.260 E-12 \quad 8.700 E-18-1.000 E+00 \quad 0.000 E+00 \quad 0.000 E+00$

These values are stored in file "FGR60. INP".

The half lives and atomic weights for these 60 isotopes are stored in file "FGR60.NIF." 


\subsubsection{Plant Model File}

After the input to screens 0000 through 1900 discussed in Section 1.4 has been entered, it is saved as a plant model file. The user may use this file as one of the two files needed to run RADTRAD in batch mode. The format of the file is detailed below and a sample file is then listed.

Plant model file name format (standard MS-DOS rules apply): filename.PMF

Plant model file format and variable names

Plain text or variable name

Format

Comments

Plant Model Name:

$1 \mathrm{x}, \mathrm{a} 19$

plant_model_name

a 40

printable ASCII characters

Nuclide Inventory File:

$1 \mathrm{x}, \mathrm{a} 21$

inventory_filename

a9

file name suffix must be NIF and standard MS-DOS device name, path, and file name rules apply

Plant Power Level:

$1 \mathrm{x}, \mathrm{a} 20$

plant_power_level

f11.4

units $=$ megawatts - thermal valid range $\geq>0.0$

Compartments:

$1 \mathrm{x}, \mathrm{a} 25$

number_of_compartments

i2

valid range $=0$ to 10 inclusive

For each compartment $\mathrm{n}=1$ to number_of_compartments

\begin{tabular}{|c|c|c|}
\hline Compartment $n:$ & $1 \mathrm{x}, \mathrm{a} 12, \mathrm{i} 1, \mathrm{a} 1$ & \\
\hline compartment_name $(\mathrm{n})$ & a40 & printable ASCII characters \\
\hline compartment_type(n) & $1 \mathrm{x}, \mathrm{i} 1$ & $\begin{array}{l}1=\text { normal compartment } \\
2=\text { control room, } \\
3=\text { environment }\end{array}$ \\
\hline compartment_volume $(\mathrm{n})$ & $1 \mathrm{x}, \mathrm{e} 11.4$ & $\begin{array}{l}\text { units }=\text { meter }^{3} \\
\text { valid range }>0.0\end{array}$ \\
\hline compartment_sprays(n) & $1 \mathrm{x}, \mathrm{i} 1$ & $\begin{array}{l}0=\text { sprays not present } \\
1=\text { sprays present }\end{array}$ \\
\hline compartment_sump $(\mathrm{n})$ & $1 x, i 1$ & $\begin{array}{l}0=\text { sump not present } \\
1=\text { sump present }\end{array}$ \\
\hline compartment_filters( $\mathrm{n})$ & $1 \mathrm{x}, \mathrm{i} 1$ & $\begin{array}{l}0=\text { recirculating filters not present } \\
1=\text { recirculating filters present }\end{array}$ \\
\hline
\end{tabular}


compartment_deposition(n)

compartment_opool( $n)$
$1 \mathrm{x}, \mathrm{i} 1$

$0=$ natural deposition does not occur

1 = natural deposition does occur

$0=$ natural deposition does not occur

1 = natural deposition does occur

Finish for all compartments used

Pathways:

number_of_pathways
$1 \mathrm{x}$, a9

i2

For each compartment-to-compartment pathway $n=1$ to number of pathways
$\operatorname{Pathway}(\mathrm{n})=$
$1 \mathrm{x}, \mathrm{a} 9, \mathrm{I} 2, \mathrm{a} 4$
pathway_name (1)
a40
printable ASCII characters
pathway from(1)
i2
valid range $=1$ to
number_of_compartments
inclusive
pathway_to(I)
i2
valid range $=1$ to
number_of_compartments
inclusive
pathway_type(1)
I1

$$
\begin{aligned}
& 1=\text { piping } \\
& 2=\text { filtered pathway } \\
& 3=\text { suppression pool } \\
& 4=\text { natural convection }
\end{aligned}
$$

Finish for all pathways used

End of plant model file 


\subsection{Acceptance Test Case \#24 PMF File}

Plant Model Name:

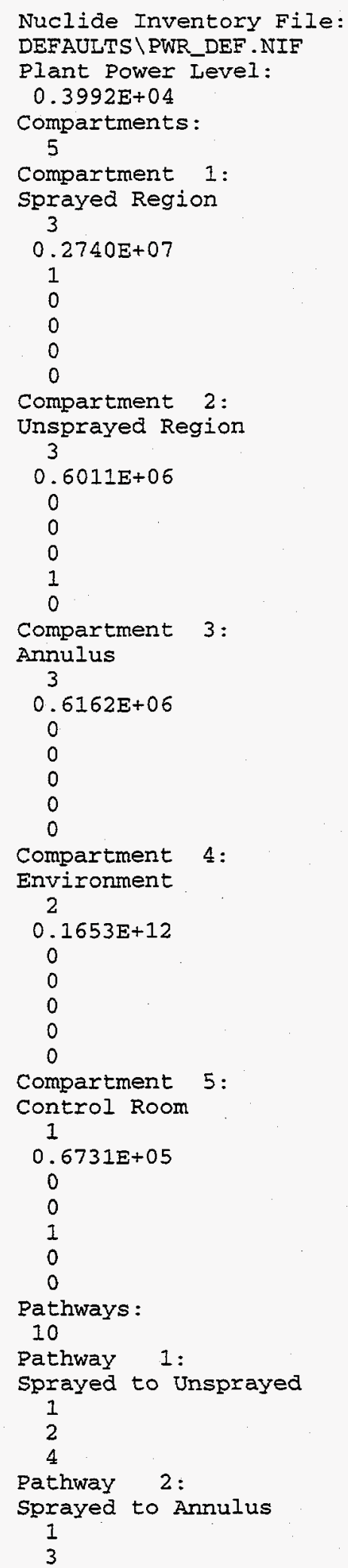


Pathway 3:

Sprayed to Environment

1

4

4

Pathway

4:

Unsprayed to Sprayed

2

1

Pathway 5:

Unsprayed to Annulus

2

3

4

Pathway 6:

Unsprayed to Environment

2

4

Pathway 7

Annulus to Environment

3

4

1

Pathway

Filter 3--Environment to CR

4

5

2

Pathway

9 :

Unfiltered--Environment to $C R$

4

5

2

Pathway 10

Exhaust from CR to Environment 5

4

End of Plant Model File 


\subsubsection{Scenario Description File}

After the input to screens 2000 through 2900 discussed in Section 1.4 has been entered, it is saved as a scenario description file. The user may use this file as one of the two files needed to run RADTRAD in batch mode. The format of the file is detailed below and a sample file is then listed.

Scenario description file name format (standard MS-DOS rules apply): filename.SDF

Scenario description file format and variable names

Plain text or variable name

Scenario Description Name:

scenario_name

Plant Model File Name:

plant_model_filename

Source Term:

source_term_compartment

dose_conversion_filename

release_filename

delay_time

calculate_daughters

i_fraction_aerosol

NUREG/CR-6604
Format

$1 \mathrm{x}, \mathrm{a} 26$

$1 \mathrm{x}, \mathrm{a} 40$

$1 \mathrm{x}, \mathrm{a} 21$

$1 \mathrm{x}, \mathrm{a} 40$

$1 \mathrm{x}, \mathrm{a} 12$

$1 \mathrm{x}, \mathrm{i} 3$

$1 \mathrm{x}, \mathrm{a} 18$

$1 \mathrm{x}, \mathrm{a} 40$

$1 \mathrm{x}, \mathrm{e} 11.4$

$1 x$, i3

$1 \mathrm{x}, \mathrm{e} 11.4$
Comments

printable ASCII characters

file name suffix must be PMF and standard MS-DOS device name, path, and file name rules apply

valid range $=1$ to

number_of_compartments file names suffix must be DAT and standard MS-DOS device name, path, and file name rules apply.

file name suffix must be RFT and standard MS-DOS device name, path, and file name rules apply

valid range $=>0.0$ hours

$0=$ no daughters are calculated

$1=$ all daughters are calculated

valid range $=0.0$ to 1.0 
i_fraction_elemental

ifraction_organic

i_fraction_radioactive

Overlying Pool:

op_decay

op_initial_volume

op_aerosol_model $\mathrm{e} 11.4$

el1.4

e11.4

$1 \mathrm{x}, \mathrm{a} 15$

$1 \mathrm{x}, \mathrm{i} 3$

$1 \mathrm{x}, \mathrm{e} 11.4$

$1 x$, i3 valid range $=0.0$ to 1.0

valid range $=0.0$ to 1.0

these three variables should total 1.0

valid range $=0.0$ to 1.0

these four variables appear on the same line in the file

$0=$ no decay chain processing

$1=$ decay chain processing

units $=$ cubic meters

valid range $>=0.0$

$0=$ no aerosol removal

1 = user-specified decontamination

factors

2 = decontamination factors

calculated using the Powers model

These lines will be included if and only if the user-specified (op_aerosol_model $=1$ ) decontamination factors are used.
op_aerosol_count
$1 \mathrm{x}, \mathrm{i} 3$
valid range $=1$ to 25 inclusive
$o p \_a e r o s o l \_t i m e(n)$
units $=$ hours
$o p \_a e r o s o l \_d f(n)$
$2(1 \mathrm{x}, \mathrm{e} 11.4)$ valid range $=0.0$ to 1.0
these two variables appear in the file on the same line. Enter for $\mathrm{n}=1, \mathrm{op}$ _aerosol_count

End of user-specified decontamination factors.

These lines will be included if the Powers model (op_aerosol_model $=2$.) for calculating the decontamination factors is used.
op_aerosol_percentile
$1 \mathrm{x}, \mathrm{e} 11.4$
$10.0=10^{\text {th }}$ percentile model
$50.0=50^{\text {th }}$ percentile model
$90.0=90^{\text {th }}$ percentile model
op_aerosol_count
$1 x$, i3
valid range $=1$ to 25 inclusive
op_aerosol_time $(n)$
units $=$ hours
op_aerosol_height $(n)$
units $=$ meters 


$$
\begin{array}{ll}
\text { op_aerosol_cooling }(n) \quad 3(1 \mathrm{x}, \mathrm{e} 11.4) & \begin{array}{l}
\text { units }=\mathrm{K} \\
\text { these three variables appear on the } \\
\text { same line in the file }
\end{array}
\end{array}
$$

End of Powers model-calculated decontamination factors.

op_elemental_model

$1 x, \mathrm{i} 3$
$0=$ no elemental iodine removal
1 = user-specified decontamination factors
$2=$ decontamination factors calculated using a model (no model is available at this time)

These lines will be included if and only if user-specified (op_elemental_model $=1$ ) decontamination factors are used.

$$
\begin{aligned}
& \text { op_elemental_count } \\
& \text { op_elemental_time }(1) \\
& o p \_e l e m e n t a l \_d f(1) \\
& \text { op_elemental_time( }(n) \\
& o p \_e l e m e n t a l \_d f(n)
\end{aligned}
$$$$
1 \mathrm{x}, \mathrm{i} 3
$$$$
\text { valid range }=1 \text { to } 25 \text { inclusive }
$$$$
2(1 \mathrm{x}, \mathrm{e} 11.4)
$$$$
\text { units }=\text { hours }
$$$$
\text { valid range }=0.0 \text { to } 1.0
$$
these two variables appear on the same line in the file

$$
\mathrm{n}=o p \_e l e m e n t a l \_d f \_c o u n t
$$

End of user-specified decontamination factors.

op_organic_model

$$
1 x, i 3
$$

$$
\begin{aligned}
& 0=\text { no organic iodine removal } \\
& 1=\text { user-specified decontamination } \\
& \text { factors } \\
& 2=\text { decontamination factors } \\
& \text { calculated using a model (no model } \\
& \text { is available at this time) }
\end{aligned}
$$

These lines will be included if and only if user-specified (op_organic_model $=1$ ) decontamination factors are used.
op_organic_count
$1 \mathrm{x}, \mathrm{i} 3$
valid range $=0$ to 25 inclusive
$0=$ no organic iodine decontamination 1-25 = number of decontamination factors 
op_organic_time $(n)$

$o p \_o r g a n i c \_d f n 1$ ) units $=$ hours

$2(1 \mathrm{x}$, e11.4) valid range $=0.0$ to 1.0

These two variables appear on the same line in the file; input for $\mathrm{n}=1$,op_organic_count

End of user-specified decontamination factors.

op_volatilization_model

Compartments:

number_of_compartments

Compartment n:

compartment_detail( $n)$

compartment_decay $(n)$

sprays_aerosol_count(n)
$1 \mathrm{x}, \mathrm{i3}$

$1 \mathrm{x}, \mathrm{a} 13$

$1 x$, i3 valid range $=$ the number of compartments specified in the plant model file

$1 \mathrm{x}, \mathrm{a} 12, \mathrm{i2}, \mathrm{al}$ valid range $=1$ to number_of_compartments

$1 x$, i3

$1 x$, i3

$0=$ no detail output printed

1 = detail output printed

$0=$ no decay chain processing

$1=$ decay chain processing

$1 \mathrm{x}, \mathrm{i3}$

removal

1-25 = number of aerosol removal coefficients supplied or calculated

These lines will be included if the user-specified (sprays_aerosol_count $(n)>0$ )
sprays_aerosol_model(n)
$1 \mathrm{x}, \mathrm{i3}$
$1=$ user-specified aerosol removal coefficients
2 = aerosol removal coefficients calculated using the Powers model

These lines will be included if the user-specified (sprays_aerosol_model $(n)=1)$ removal coefficients are used and sprays_aerosol_count( $n)$ is nonzero.

sprays_aerosol_time $(n)(m)$

$\operatorname{sprays\_ aerosol}(n)(m)$

$$
\text { units }=\text { hours }
$$

$2(1 \mathrm{x}, \mathrm{e} 11.4) \quad$ units $=$ per second

These two variables appear on the 
same line in the file; input for up to 5 sets of data.

End of user-specified decontamination factors.

These lines will be included if the Powers model (sprays_aerosol_model $(1)=2$.) for calculating the decontamination factors is used and sprays_aerosol_count(1) is nonzero.

\begin{tabular}{|c|c|c|}
\hline sprays_aerosol_percentile(1) & $1 \mathrm{x}, \mathrm{e} 11.4$ & $10.0=10^{\text {th }}$ percentile model \\
\hline & & $50.0=50^{\text {th }}$ percentile model \\
\hline & & $90.0=90^{\text {th }}$ percentile model \\
\hline sprays_aerosol_time $(n)(m)$ & & units $=$ hours \\
\hline sprays_aerosol_flux $(n)(m)$ & & units $=$ cubic meters of water per \\
\hline sprays_aerosol_height $(n)(m)$ & $3(1 x, e 11.4)$ & $\begin{array}{l}\text { square meter per second } \\
\text { units = meters } \\
\text { these three variables appear on the } \\
\text { same line in the file }\end{array}$ \\
\hline
\end{tabular}

End of Powers model-calculated removal coefficients.

sprays_elemental_count( $n$ )

sprays_elemental_time $(1)(1)$

sprays_elemental $(1)(1)$

sprays_elemental_time $(I)(n)$

sprays_elemental $(1)(n)$

sprays_organic_count(1)

sprays_organic_time $(1)(1)$
$1 \mathrm{x}, \mathrm{i} 3$

$2(1 \mathrm{x}, \mathrm{e} 11.4)$ valid range $=0$ to 25 inclusive $0=$ no elemental iodine removal $1-25=$ number of removal coefficients

units $=$ hours

valid range $=0.0$ to 1.0

these two variables appear on the same line in the file

$\mathrm{n}=$ sprays_elemental_count $(1)$

$1 \mathrm{x}$, i3 valid range $=0$ to 25 inclusive

$0=$ no organic iodine

decontamination

$1-25=$ number of decontamination factors supplied

units $=$ hours 
sprays_organic(l)(1)

sprays_organic_time $(1)(n)$

sprays_organic(l)(n)

sump_volatilization_count(1)

c_filter_norm_flow_rate( 1 )

c_filter_eff_count( 1 )

c_filter_aerosol_eff(1)(1)

c_filter_elemental_eff $(I)(1)$

c_filter_organic_eff(1)(1)

c_filter_aerosol_eff $(1)(n)$

c_filter_elemental_eff $(I)(n)$

$c$ filter_organic_eff $(1)(n)$

deposit_aerosol_count(1)

deposit_aerosol_model(1)
$2(1 \mathrm{x}$, e11.4) valid range $=0.0$ to 1.0 these two variables appear on the same line in the file

$\mathrm{n}=$ sprays_organic_count $(I)$

$1 x$, i3 $\quad 0=$ no iodine volatilization

$1 \mathrm{x}, \mathrm{e} 11.4 \quad$ units $=$ per second

$1 x$, i3 valid range $=0$ to 25 inclusive

$0=$ no filter action

$1-25=$ number of filter efficiencies

units $=$ percent

valid range $=0.0$ to 100.0 inclusive

units $=$ percent

valid range $=0.0$ to 100.0 inclusive

$3(1 \mathrm{x}, \mathrm{e} 11.4) \quad$ units $=$ percent

valid range $=0.0$ to 100.0 inclusive these three variables appear on the same line in the file

$\mathrm{n}=c$ filter_eff_count $(1)$

$1 \mathrm{x}$, i3 valid range $=0$ to 25 inclusive

$0=$ no aerosol removal

$1-25=$ number of aerosol removal coefficients supplied or calculated

$1 \mathrm{x}$, i3 valid range $=1$ to 2 inclusive 1 = user-specified aerosol removal coefficients

$2=$ aerosol removal coefficients calculated using the Henry model

These lines will be included if the user-specified (deposit_aerosol_model $(1)=1$ ) removal coefficients are used and deposit_aerosol_count( $(I)$ is nonzero.

units $=$ hours 
deposit_aerosol(1)(1)

$2(1 \mathrm{x}, \mathrm{e} 11.4) \quad$ units $=$ per second

these two variables appear on the same line in the file

deposit_aerosol_time $(1)(n)$

deposit_aerosol( $I)(n)$ $\mathrm{n}=$ deposit_aerosol_count $(1)$

End of user-specified decontamination factors.

No lines will be included if the Henry model (deposit_aerosol_model $(1)=2$.) for calculating the decontamination factors is used or sprays_aerosol_count(1) is zero.

deposit_elemental_count(1)

deposit_elemental_time $(1)(1)$

deposit_elemental( $(1)(1)$

deposit_elemental_time $(1)(n)$

deposit_elemental(1)(n)

\section{Compartment m:}

compartment_detail(m)

compartment_decay $(m)$

deposit_elemental_time $(m)(n)$

deposit_elemental $(m)(n)$

Pathways:

number_of_pathways

Pathway 1:

pathway_detail( $(1)$
$1 \mathrm{x}, \mathrm{i} 3$

2(1x, e11.4) valid range $=0$ to 25 inclusive $0=$ no elemental iodine removal $1-25=$ number of aerosol removal coefficients supplied or calculated

units $=$ hours

units $=$ per second

these two variables appear on the same line in the file

$\mathrm{n}=$ deposit_elemental_count $(1)$

$$
\begin{aligned}
& \mathrm{m}=\text { number_of_compartments } \\
& \mathrm{n}=\text { deposit_elemental_count }(m)
\end{aligned}
$$

$1 \mathrm{x}, \mathrm{a} 9$

$1 x$, i3 valid range $=$ the number of pathways specified in the plant model file

$1 \mathrm{x}, \mathrm{a} 10$

$1 x$, i3 valid range $=0$ to 1 inclusive

$0=$ no detailed output printed

$1=$ detailed output printed 
pathway_decay $(1)$

piping_aerosol_model(1)
$1 \mathrm{x}, \mathrm{i3}$

valid range $=0$ to 1 inclusive $0=$ no decay chain processing

$1=$ decay chain processing

$1 \mathrm{x}, \mathrm{i3} \quad$ valid range $=0$ to 2

inclusive

$0=$ no aerosol removal

1 = user-specified decontamination factors

2 = decontamination factors calculated using a model (no model is available at this time)

These lines will be included if and only if the user-specified (piping_aerosol_model =1) decontamination factors are used.

piping_aerosol_count $(1)$
piping_aerosol_time $(1)(1)$
piping_aerosol_df $(1)(1)$
piping_aerosol_flow $(1)(1)$

$1 \mathrm{x}, \mathrm{i} 3$

$3(1 \mathrm{x}, \mathrm{e} 11.4)$ valid range $=1$ to 25 inclusive

$$
\text { units }=\text { hours }
$$

units $=$ per second

units = cubic meters per second these three variables appear on the same line in the file

$$
\mathrm{n}=\text { piping_aerosol_count }(1)
$$

piping_aerosol_time $(1)(n)$

piping_aerosol_df $(1)(n)$

piping_aerosol_flow $(1)(n)$

End of user-specified decontamination factors.

piping_elemental_model(1)

$1 x$, i3

valid range $=0$ to 2 inclusive

$0=$ no elemental iodine removal

1 = user-specified elemental iodine decontamination factors

2 = elemental iodine decontamination factors calculated using a model (no model is available at this time)

These lines will be included if and only if the user-specified (piping_elemental_model =1) decontamination factors are used.

$\begin{array}{lll}\text { piping_elemental_count }(1) & 1 \mathrm{x}, \mathrm{i} 3 & \begin{array}{l}\text { valid range }=1 \text { to } 25 \text { inclusive } \\ \text { units }=\text { hours }\end{array} \\ \begin{array}{l}\text { piping_elemental_time }(1)(1) \\ \text { piping_elemental_df }(1)(1)\end{array} & \begin{array}{l}\text { units = per second } \\ \text { piping_elemental_flow }(1)(1)\end{array} & \begin{array}{l}\text { units = cubic meters per second } \\ \text { these three variables appear on the } \\ \text { same line in the file }\end{array}\end{array}$


piping_elemental_time $(1)(n)$

piping_elemental_df $(1)(n)$

piping_elemental_flow $(1)(n)$

$\mathrm{n}=$ piping_elemental_count $(1)$

End of user-specified decontamination factors.

piping_organic_model( 1 1) $1 \mathrm{x}, \mathrm{i} 3$

valid range $=0$ to 2 inclusive

$0=$ no aerosol removal

1 = user-specified aerosol

decontamination factors

2 = aerosol decontamination

factors calculated using a model

(no model is available at this time)

These lines will be included if and only if the user-specified (piping_elemental_model =1) decontamination factors are used.

piping_organic_count(1)

piping_organic_time $(1)(1)$

piping_organic_df $(1)(1)$

piping_organic_flow $(1)(1)$
$1 x$, i3

$3(1 \mathrm{x}, \mathrm{e} 11.4)$ valid range $=1$ to 25 inclusive

units $=$ hours

units $=$ per second

units = cubic meters per second

these three variables appear on the same line in the file

$\mathrm{n}=$ piping_organic_count $(1)$

piping_organic_time $(1)(n)$

piping_organic_df $(1)(n)$

piping_organic_flow $(1)(n)$

End of user-specified decontamination factors.

p_filter_norm_flow_rate(1)

$1 \mathrm{x}, \mathrm{e} 11.4$

$1 \mathrm{x}, \mathrm{i} 3$

$p \_$filter_eff_count( $(1)$

p_filter_aerosol_eff(1)(1)

p_filter_elemental_eff $(1)(1)$

p_filter_organic_eff(1)(1)

$1 x, 13$

$3(1 \mathrm{x}, \mathrm{e} 11.4)$ units $=$ per second

valid range $=0$ to 25 inclusive

$0=$ no filter action

$1-25=$ number of filter efficiencies

units = percent

valid range $=0.0$ to 100.0 inclusive units $=$ percent

valid range $=0.0$ to 100.0 inclusive

units $=$ percent

valid range $=0.0$ to 100.0 inclusive these three variables appear on the same line in the file 
$p$ filter_aerosol_eff $(1)(n)$

$p$ filter_elemental_eff(1)(n)

$p$ filter_organic_eff $(1)(n)$

$\mathrm{n}=$ filter_eff_count $(1)$

$s p \_a e r o s o l \_m o d e l(1)$

$1 x, \mathrm{i} 3$

valid range $=0$ to 2 inclusive

$0=$ no aerosol removal

$1=$ user-specified decontamination

factors

$2=$ decontamination factors

calculated using a model (no model is available at this time)

These lines will be included if and only if the user-specified $\left(s p \_a e r o s o l \_m o d e l(1)=1\right)$ decontamination factors are used.

$s p \_i n i t i a l \_v o l u m e(1)$

sp_aerosol_count(1)

$s p \_a e r o s o l \_t i m e(1)(1)$

$s p \_a e r o s o l \_d f(1)(1)$

$s p \_a e r o s o l \_t i m e(l)(n)$

$s p \_a e r o s o l \_d f(1)(n)$

End of user-specified decontamination factors.

sp_elemental_model(1)

$1 x, \mathrm{i} 3$

$$
1 \mathrm{x}, \mathrm{e} 11.4
$$

$1 x$, i3

$2(1 \mathrm{x}, \mathrm{e} 11.4)$

units $=$ cubic meters

valid range $\geq 0.0$

$0.0=$ no suppression pool

valid range $=1$ to 25 inclusive

units $=$ hours

valid range $=0.0$ to 1.0

these two variables appear on the

same line in the file

$\mathrm{n}=s p \_a e r o s o l \_c o u n t(1)$

valid range $=0$ to 2 inclusive

$0=$ no elemental iodine removal

1 = user-specified decontamination

factors

2 = decontamination factors

calculated using a model (no model is available at this time)

These lines will be included if and only if user-specified (sp_elemental_model $(1)=1$ ) decontamination factors are used.
$s p \_e l e m e n t a l \_c o u n t(I)$
$1 x$, i3
valid range $=1$ to 25 inclusive
sp_elemental_time $(1)(1)$
units $=$ hours
$s p \_e l e m e n t a l \_d f(1)(1)$
$2(1 \mathrm{x}, \mathrm{e} 11.4)$
valid range $=0.0$ to 1.0
these two variables appear on the same line in the file 
sp_elemental_time $(1)(n)$

$s p \_e l e m e n t a l \_d f(1)(n)$

$\mathrm{n}=s p \_$_elemental_df_count

End of user-specified decontamination factors.

$s p \_$organic_model $(1) \quad 1 \mathrm{x}, \mathrm{i} 3 \quad$ valid range $=0$ to 2 inclusive

$0=$ no organic iodine removal

1 = user-specified decontamination

factors

2 = decontamination factors

calculated using a model (no model is available at this time)

These lines will be included if and only if user-specified (sp_organic_model $(1)=1$ ) decontamination factors are used.

sp_organic_count (1)

$1 \mathrm{x}, \mathrm{i} 3$

valid range $=0$ to 25 inclusive

$0=$ no organic iodine

decontamination

$1-25=$ number of decontamination

factors

sp_organic_time $(1)(1)$

units $=$ hours

$s p \_o r g a n i c \_d f(1)(1)$

$2(1 \mathrm{x}$, e11.4) valid range $=0.0$ to 1.0

these two variables appear on the same line in the file

$\mathrm{n}=s p \_$organic_count $(1)$

$s p \_o r g a n i c \_d f(1)(n)$

End of user-specified decontamination factors.

sp_volatilization_model( 1$)$

convection_model( $(1)$
$1 \mathrm{x}, \mathrm{i3}$

$1 \mathrm{x}, \mathrm{i} 3$ valid range $=0$ to 2 inclusive

$0=$ no natural convection

$1=$ user-specified normalized flow rates

$2=$ normalized flow rates

calculated using a model (no model is available at this time) 
These lines will be included if and only if the user-specified (convection_model $(I)=1$ ) decontamination factors are used.

$\begin{array}{lll}\begin{array}{l}\text { convection_count }(1) \\ \text { convection_norm_flow_rate }(I)(1)\end{array} & \begin{array}{l}1 \mathrm{x}, \mathrm{i} 3 \\ 1 \mathrm{x}, \mathrm{e} 11.4\end{array} & \begin{array}{l}\text { valid range }=1 \text { to } 25 \text { inclusive } \\ \text { units }=\text { per second }\end{array} \\ \begin{array}{ll}\text { convection_norm_flow_rate }(1)(n) & \mathrm{n}=\text { convection_count }(1)\end{array}\end{array}$

End of user-specified normalized flow rates.

water_leakage_model $(1)$

$1 x, \mathrm{i} 3$

$0=$ no water leakage

End of user-specified normalized flow rates.

\section{Pathway m:}

pathway_detail( $(m)$

pathway_decay $(m)$

water_leakage_rate $(m)(n)$

$\mathrm{m}=$ number_of_pathways

$\mathrm{n}=$ water_leakage_count $(\mathrm{m})$

Dose Locations:

number_of_locations

Location 1:

location_name(1)

compartment_number(1)

pathway_count(1)

pathway_number(1)(1)

pathway_exp_time $(1)(1)$

pathway_xq_model $(1)(1)$
$1 \mathrm{x}, \mathrm{a} 15$

$1 \mathrm{x}, \mathrm{i} 3$

$1 \mathrm{x}, \mathrm{a} 11$

$1 \mathrm{x}, \mathrm{a} 40$

$1 x, \mathrm{i} 3$

$1 \mathrm{x}, \mathrm{i} 3$

$1 \mathrm{x}, \mathrm{i} 3$

$1 \mathrm{x}, \mathrm{e} 11.4$

$1 \mathrm{x}, \mathrm{i} 3$ valid range $=0$ to 10

valid range $=$ printable $\mathrm{ASCU}$ characters from 32 to 126 inclusive valid range $=1$ to $\mathrm{n}$ inclusive the number of the compartment that the location is in.

valid range $=0$ to 10

valid range $=1$ to 100

units $=$ hours

valid range $=0$ to 2 inclusive $0=\mathrm{X} / \mathrm{Q}$ is a constant 1.0

$1=$ user-specified $\mathrm{X} / \mathrm{Q}$

$2=\mathrm{X} / \mathrm{Q}$ calculated using a model

(no model is available at this time) 
These lines will be included if and only if the user-specified (pathway_xq_model $(1)(1)=1) \mathrm{X} / \mathrm{Q}$ factors are used.

pathway_xq_count(1)(1)

pathway_xq_time $(1)(1)(1)$

pathway_xq(l)(1)(1)
$1 x, \mathrm{i} 3$

$2(1 \mathrm{x}, \mathrm{e} 11.4)$ valid range $=1$ to 25

units $=$ hours

units $=$ seconds per cubic meters these two variables appear on the same line in the file

$\mathrm{n}=$ pathway_xq_count $(1)(1)$

pathway_xq_time $(1)(1)(n)$

pathway_xq(1)(1)(n)

End of user-specified X/Q factors.

pathway_number $(1)(m)$

pathway_exp_time $(1)(m)$

pathway_xq_model $(1)(m)$

pathway_xq(1)(m)(1)

pathway_xq(1)(m)(n)

$\mathrm{m}=$ pathway_count $(1)$

$\mathrm{n}=$ pathway_x $q_{-} \operatorname{count}(1)(\mathrm{m})$

\section{Location $\ell$ :}

location_name $(t)$

compartment_number( $\ell$ )

pathway_xq_model $(\ell)(m)$

pathway_xq( $\operatorname{lo}(m)(1)$

pathway_xq( $\ell)(m)(n)$

$$
\begin{aligned}
& \mathrm{l}=\text { number_of_locations } \\
& \mathrm{m}=\text { pathway_count }(\ell) \\
& \mathrm{n}=\text { pathway_xq_count }(\ell)(\mathrm{m})
\end{aligned}
$$


Scenario Description Name:

Plant Model Filename:

ACCEPT \TEST24.SDF

Source Term:

1

DEFAULTS $\backslash F G R 60 . N I S \quad$ DEFAULTS $\backslash F G R 60$. INP DEFAULTS $\backslash$ PWR_DBA.RFT

$0.0000 \mathrm{E}+00$

$0.9500 \mathrm{E}+00$

\section{$0.4850 \mathrm{E}-01 \quad 0.1500 \mathrm{E}-02 \quad 0.1000 \mathrm{E}+01$} overlying Pool

0

$0.0000 \mathrm{E}+00$

0

0

Compartments :

$\vec{w}$ compa

0

2

$0.0000 \mathrm{E}+00$

$0.1000 \mathrm{E}+01$

$0.5000 \mathrm{E}+02$

2

$0.0000 \mathrm{E}+00$

$0.3000 \mathrm{E}+01$

1

$0.0000 \mathrm{E}+00$

$0.0000 \mathrm{E}+00$

$0.5000 \mathrm{E}+00$

$0.1800 \mathrm{E}+01$

$0.2000 \mathrm{E}+01$

$0.8000 \mathrm{E}+01$

$0.3940 E-01$

$0.0000 \mathrm{E}+00$

$0.8500 \mathrm{E}+02$

$0.8500 \mathrm{E}+02$

$0.1500 \mathrm{E}+01$

$0.1200 E+02$

$0.5000 \mathrm{E}+01$

$0.2000 \mathrm{E}+01$

$0.4000 \mathrm{E}+00$
Compartment 2

0

0

0

0
1

2

$0.0000 \mathrm{E}+00 \quad 0.1500 \mathrm{E}+00$

$0.7200 \mathrm{E}+03 \quad 0.1500 \mathrm{E}+00$

Compartment 3 :

0

0

0

0

0

0

0

Compartment 4 :

0

0

0

0

0

0

Compartment 5 :

1

$0.4000 \mathrm{E}+04$

$0.0000 \mathrm{E}+00$

0.7200

$0.9900 \mathrm{E}+02$

$0.9900 \mathrm{E}+02$

$0.9500 \mathrm{E}+02$

$0.9500 \mathrm{E}+02$

$0.9500 \mathrm{E}+02$

$0.9500 \mathrm{E}+02$ 


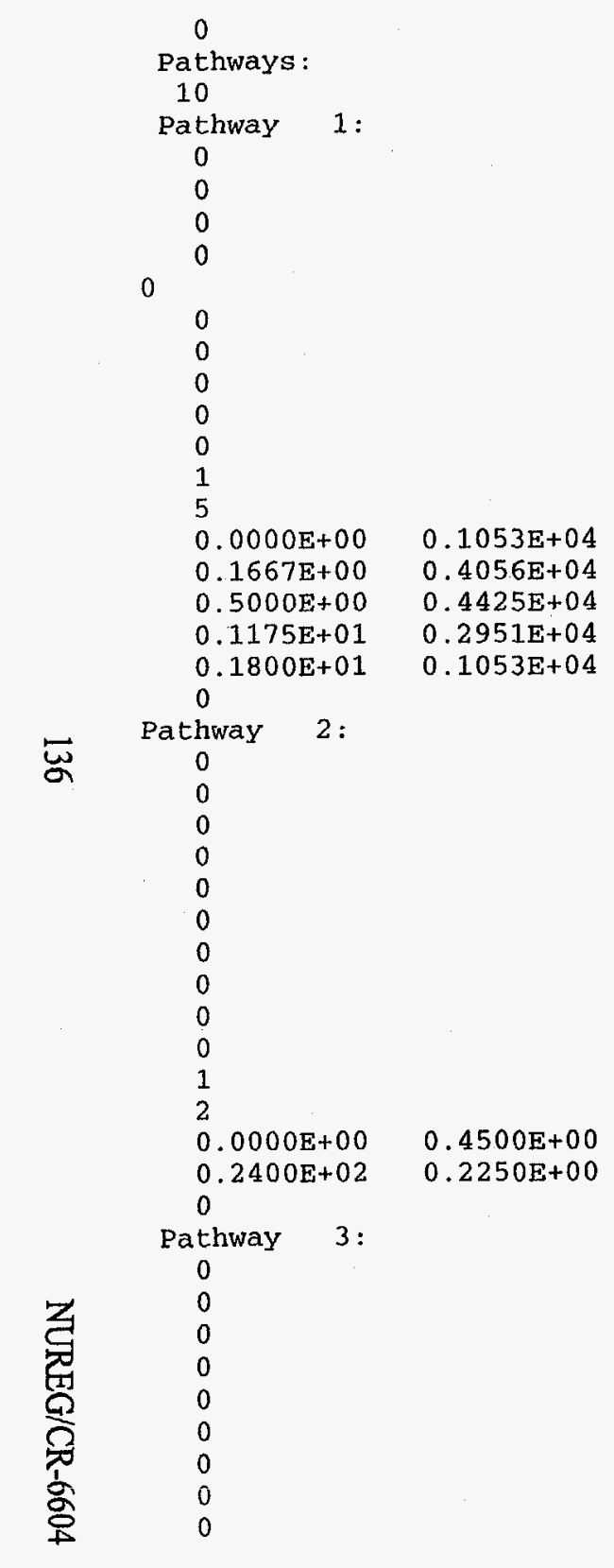

$0.0000 \mathrm{E}+00 \quad 0.5000 \mathrm{E}-01$ $0.2400 \mathrm{E}+02 \quad 0.2500 \mathrm{E}-01$ 0

Pathway 4

0

0

0

0

0

0

0

0

$0.0000 \mathrm{E}+00 \quad 0.4800 \mathrm{E}+04$

$0.1667 \mathrm{E}+00 \quad 0.1845 \mathrm{E}+05$ 

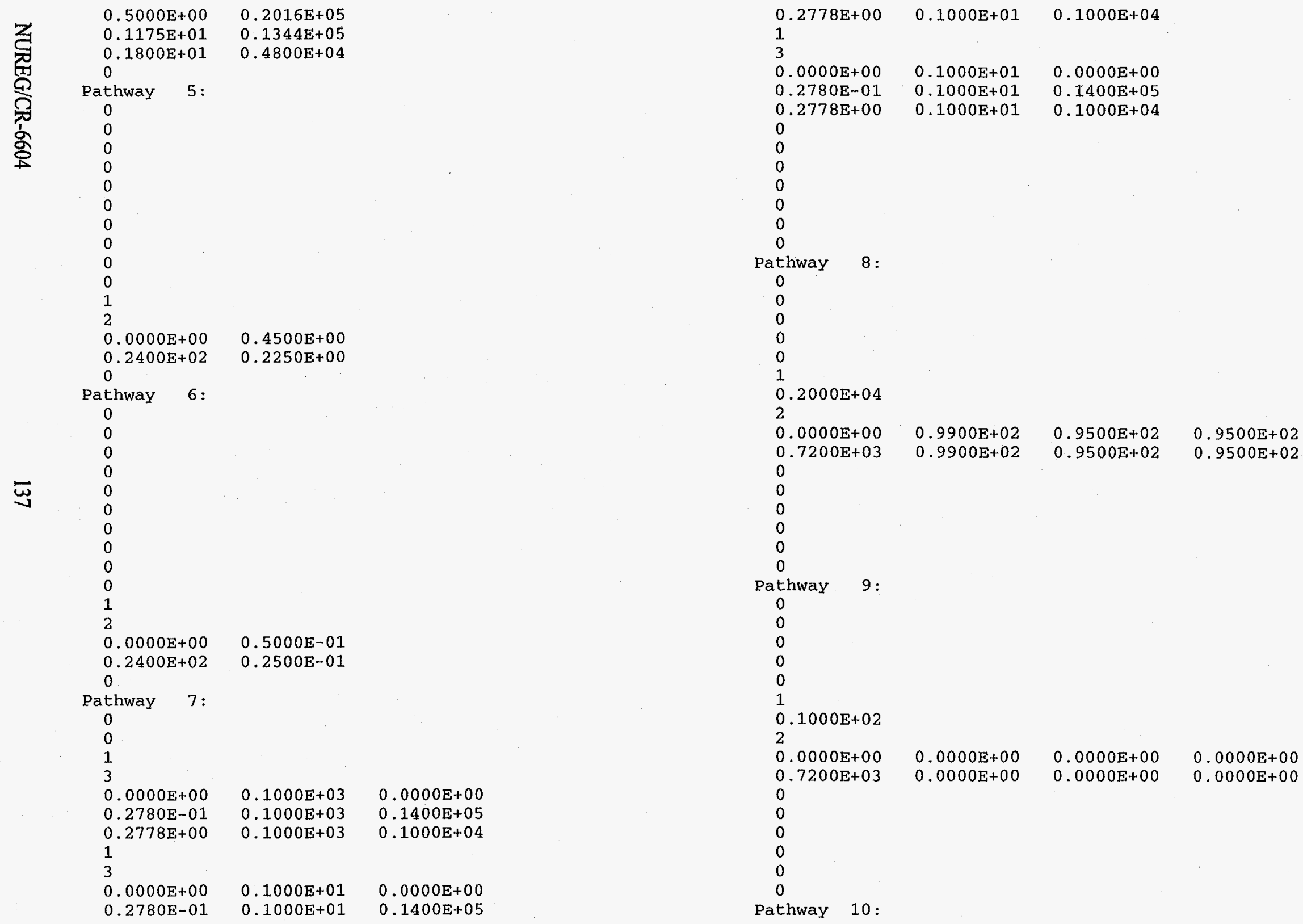


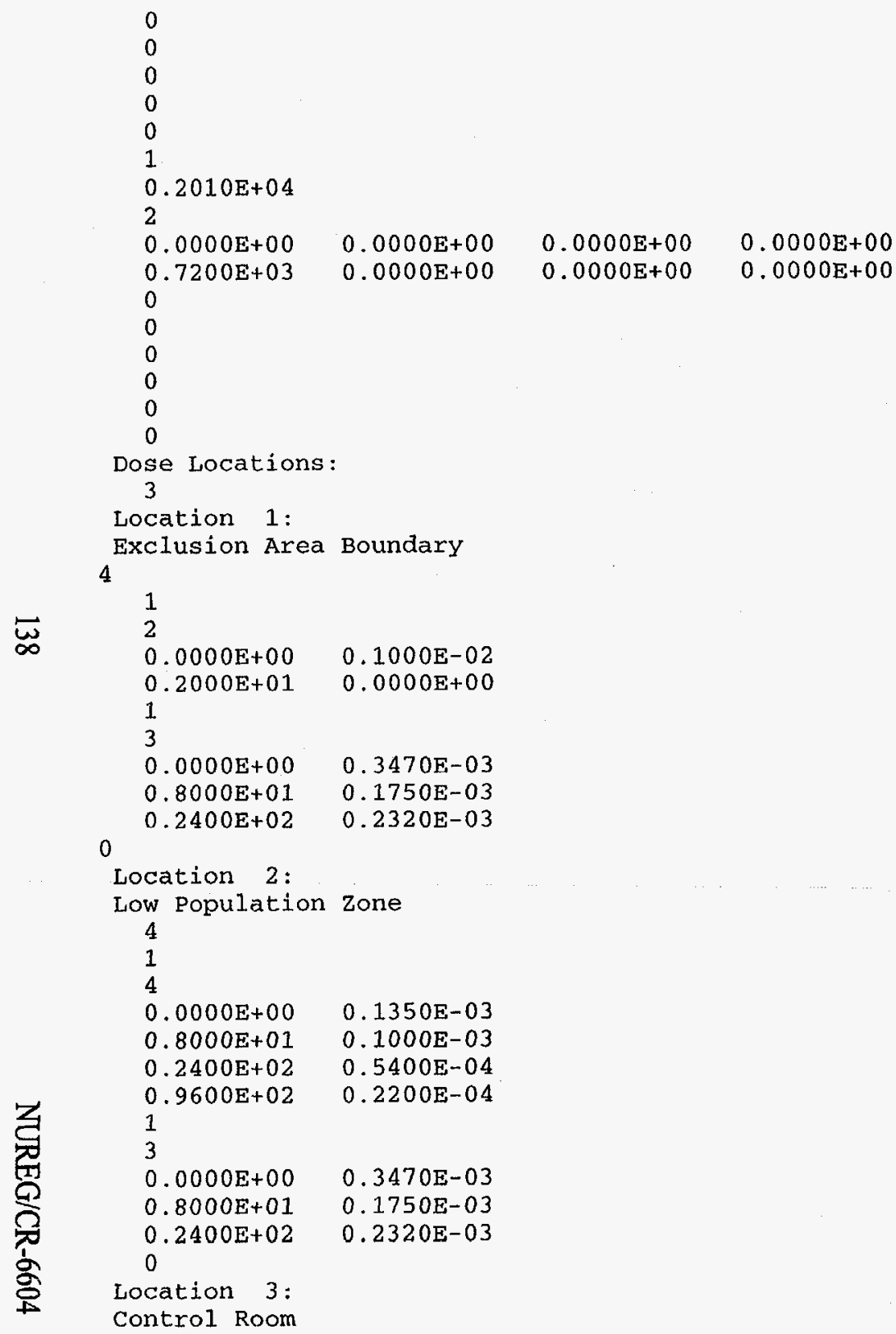

0
0

0

0
0

1.

$0.2010 E+04$

2

$0.0000 \mathrm{E}+00$

$0.7200 \mathrm{E}+03$

0

0

0
0

0

Dose Locations:

3

Location 1:

Exclusion Area Boundary

$\omega_{\infty}$

1

$0.0000 \mathrm{E}+00 \quad 0.1000 \mathrm{E}-02$

$0.2000 \mathrm{E}+01 \quad 0.0000 \mathrm{E}+00$

1

$0.0000 \mathrm{E}+00 \quad 0.3470 \mathrm{E}-03$

$0.8000 \mathrm{E}+01 \quad 0.1750 \mathrm{E}-03$

$0.2400 \mathrm{E}+02 \quad 0.2320 \mathrm{E}-03$

Location 2 :

Low Population zone 4

1

$0.0000 \mathrm{E}+00$

$0.1350 \mathrm{E}-03$

$0.8000 \mathrm{E}+01 \quad 0.1000 \mathrm{E}-03$

$0.2400 \mathrm{E}+02 \quad 0.5400 \mathrm{E}-04$

录

$0.0000 \mathrm{E}+00 \quad 0.0000 \mathrm{E}+00$

$0.0000 \mathrm{E}+00 \quad 0.0000 \mathrm{E}+00$

1

$0.0000 \mathrm{E}+00 \quad 0.3470 \mathrm{E}-03$

$0.8000 E+01 \quad 0.1750 E-03$

$0.2400 \mathrm{E}+02 \quad 0.2320 \mathrm{E}-03$

0

Location 3 :

Control Room

5

$0.0000 \mathrm{E}+00 \quad 0.3470 \mathrm{E}-03$

$0.7200 \mathrm{E}+03 \quad 0.0000 \mathrm{E}+00$

$0.0000 \mathrm{E}+00 \quad 0.1000 \mathrm{E}+01$

$0.2400 \mathrm{E}+02 \quad 0.6000 \mathrm{E}+00$

$0.9600 \mathrm{E}+02 \quad 0.4000 \mathrm{E}+00$

$0.0000 \mathrm{E}+00$

Effective Volume Location: 1

$0.0000 \mathrm{E}+00 \quad 0.2200 \mathrm{E}-02$

$0.2000 \mathrm{E}+01 \quad 0.1500 \mathrm{E}-02$

$0.8000 \mathrm{E}+01 \quad 0.1300 \mathrm{E}-02$

$0.2400 \mathrm{E}+02 \quad 0.8400 \mathrm{E}-03$

$0.9600 \mathrm{E}+02 \quad 0.4800 \mathrm{E}-03$

Simulation Parameters:

5

$0.0000 \mathrm{E}+00 \quad 0.5000 \mathrm{E}-01$

$0.3000 \mathrm{E}+01 \quad 0.2500 \mathrm{E}+00$

$0.8000 \mathrm{E}+01 \quad 0.1000 \mathrm{E}+01$

$0.2400 \mathrm{E}+02 \quad 0.4000 \mathrm{E}+01$

$0.7200 \mathrm{E}+03 \quad 0.0000 \mathrm{E}+00$

output Filename:

ACCEPT \TEST24.TXT

1
1

0

0

End of Scenario File 


\subsection{Output Description}

The software output is of three types. The first type is summary output and is always generated by the software. It consists of a brief description of the scenario that was being analyzed, as well as the time-dependent, whole-body, thyroid, and total effective dose equivalent (TEDE) doses resulting from releases during user-specified time periods. This is followed by summary tables for the whole-body TEDE and thyroid dose, as well as the ${ }^{131}$ I concentrations at all user-selected locations. This information may be browsed at any time via the user interface. A hard copy may be generated at any time during such an examination. Alternatively, the summary output file can be written to an ASCII text file which can be imported into a word processor or spreadsheet.

The second type of output is detailed output that is intended to be used to record, as functions of time, the intermediate results used in the calculations. The start and end times to be used for generating these results are under the control of the user and typically include the entire accident progression. The specification of which results to store is under the control of the user but all of these selections must be made prior to execution of the scenario. The detailed output is generated in ASCII text format and written to the output file. A list of the intermediate results that can be generated is:

1. the total radioactivity, as well as the number of radioactive and stable atoms in a compartment (a "compartment" in this section refers not just to the enclosed atmosphere, but to all locations in the volume and its surfaces that can hold radioactive material, for example, the surfaces, filters, and pools associated with each compartment defined in the plant model);

2. the number of atoms and radioactivity of each fission product in a compartment; and

3. the transport rate of total radioactivity, as well as the number of radioactive and stable atoms passed to the environment.

The third type of output is primarily used by the developers to debug a subroutine or model. Within the logic for each subroutine are embedded print statements that are controlled by a variable named Level XXX where XXX is the subroutine name. The value of this variable indicates the quantity of additional information required; a $0=$ no additional and a $9=$ all input and calculated values. Intermediate values will get intermediate prints. These variables can be initialized by the user in data set FLAG.DAT, as discussed in Section 4.2.3.

\subsubsection{Sample Output}

Typical output is shown on the following pages and is described here.

In the beginning of the output is an annotated list of the input. In the example provided, the full listing of the input takes several pages because a detailed problem was chosen. In most cases, the user will specify fewer volumes with less flow paths, resulting in a shorter listing.

After the header File Information, the output lists the external files the case will use for background information such as dose conversion factors and to store input and output results. 
After the header Plant Description, the output details the plant as described by the user. This section is also displayed in screen 1800 if chosen by the user during the input mode. In this example the user has described 5 volumes; the first one contains the source from the reactor coolant system. Each path to other compartments is listed, as well as the flow direction. There is flow in both directions between volumes 1 and 2 as indicated by the pathway to and pathway from lines. For each volume, its size and any active removal devices, for example, sprays or recirculating filters, are also noted. With this information a plant is described that can be used for a variety of accident scenarios.

After the header Scenario Description, the output details the scenario chosen for this example. The first part gives the information for generating the source term, such as plant power, the iodine chemical form, and the decay and daughtering selections. The next part details information about each compartment. For example, in compartment 1 the user selected the Powers spray model; hence the input from screen 2311B is edited. In compartment 5 , the control room, the capabilities of the recirculating filter are edited. The third part details the pathway data. In this part the input for each pathway is detailed. For example, the flow between compartments 1 and 2 is given under pathway 1 for flow from 1 to 2 and under pathway 4 for flow from 2 to 1 . In this example, volume 1 is the sprayed region of the containment and volume 2 is the unsprayed region, hence the mixing flows. Pathway number 8 is between the environment and the control room. Note that a filter exists on this path with the capability of removing most aerosols, elemental iodines, and organic iodines. The fourth part of this section details the dose location information as controlled from screens associated with screen 2500 . In this part, the $\mathrm{X} / \mathrm{Q}$, breathing rate, and occupancy information are edited. The fifth section details the time-step limitation information the user has selected; in this case a $900-\mathrm{s}(0.25-\mathrm{hr})$ limit is imposed between the start of the accident and $8 \mathrm{hr}$.

After the header Dose, Detailed Model and Detailed Inventory Output, the full output is presented. In this case only the first step dose results and the last step detailed dose and mass distribution results are shown. The user has control over the quantity of output, most noticeably by requesting detailed output on screen 2300 . Without this selection, only the dose results would be edited. In this example, the user has selected detailed mass distributions for several volumes, the environment and the control room. The nuclide distributions are given for the volume in a variety of units. These are also summed by chemical group following the nuclide detailed output. Note that for the environment compartment, the nuclide release rates are also given. After the volume or compartment information is detailed, all pathways linked to that compartment are also detailed for each chemical group mass. Thus one can see the mass retained by the filter between the environment and the control room and between the annulus and the environment. Following the nuclide inventory in the controll room are the chemical group masses in the control room and in the recirculating filter, as well as the masses in the pathways connected to the control room. This is followed by the dose and ${ }^{131} \mathrm{I}$ concentration summary tables. 
Plant filename

$=$ accept $\backslash$ test $24 a \cdot \mathrm{pmf}$

Inventory filename

$=$ DEFAULTS $\backslash$ PWR_DEF.NIF

Scenario filename $\quad=$ accept 1 test 24 a.sdf

Release filename = DEFAULTS $\backslash$ PWR_DBAA.RF'

Dose conversion filename = DEFAULTS $\backslash F G R 60$. INP

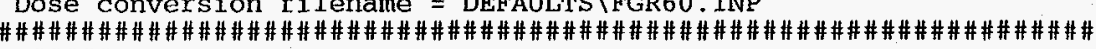
\#\#\#\#\#\#\#

plant Description

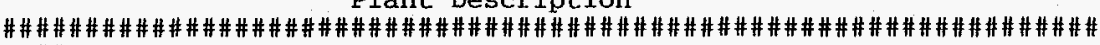
\#\#\#\#\#\#\#

Number of Nuclides $=60$

$\mp \quad$ Inventory Power $=0.1000 \mathrm{E}+01$ MWth

I P1ant Power Level $=0.3992 \mathrm{E}+04$ MWth

Number of compartments $=5$

Compartment information

Compartment number 1 (Source term compartment)

Name: Sprayed Region

Compartment volume $=0.2740 \mathrm{E}+07$ (Cubic feet)

Removal devices within compartment:

$$
\text { Spray (s) }
$$

Pathways into and out of compartment 1

Pathway to compartment number 2: Sprayed to Unsprayed Pathway to compartment number 3: Sprayed to Annulus pathway to compartment number 4 : Sprayed to Environment Pathway from compartment number 2: Unsprayed to Sprayed

Compartment number 2

Name: Unsprayed Region

Compartment volume $=0.6011 \mathrm{E}+06$ (Cubic feet)

Removal devices within compartment: Deposition

Pathways into and out of compartment 2

Pathway to compartment number 1: Unsprayed to Sprayed

Pathway to compartment number 3: Unsprayed to Annulus

Pathway to compartment number 4: Unsprayed to Environment

Pathway from compartment number 1: Sprayed to Unsprayed

Compartment number 3
Cathways into and out of compartment

Pathway to compartment number 4 : Annulus to Environment Pathway from compartment number 1: Sprayed to Annulus

Pathway from compartment number 2 : Unsprayed to Annulus

Compartment number

Name: Environment

Pathways into and out of compartment

$\mathrm{CR}$

CR

5: Filter 3---Environment to

Pathway from compartment number 1: Sprayed to Environment

Pathway from compartment number 2: Unsprayed to Environment

Pathway from compartment number 3: Annulus to Environment Environment

Compartment number 5

Name: Control Room

Compartment volume $=0.6731 \mathrm{E}+05$ (Cubic feet)

Removal devices within compartment:

$$
\text { Filter (s) }
$$

Pathways into and out of compartment 5

Pathway to compartment number 4 : Exhaust from $\mathrm{CR}$ to

Environment

Pathway from compartment number 4: Filter 3--Environment to

$\mathrm{CR}$

CR

Pathway from compartment number 4: Unfiltered--Environment to

Total number of pathways $=10$

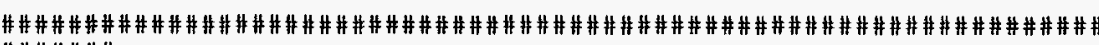
\#\#\#\#\#\#

Scenario Description

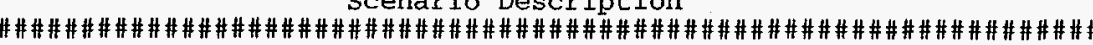

\#\#\#\#\#\#

Iodine fractions

$$
\begin{array}{ll}
\text { Aerosol } & =0.9500 \mathrm{E}+00 \\
\text { Elemental } & =0.4850 \mathrm{E}-01 \\
\text { Organic } & =0.1500 \mathrm{E}-02
\end{array}
$$

COMPARTMENT DATA

Compartment number 1: Sprayed Region

Sprays: Aerosol Removal Data

alpha $=0.1000 \mathrm{E}+01$

percentile $=50$ 


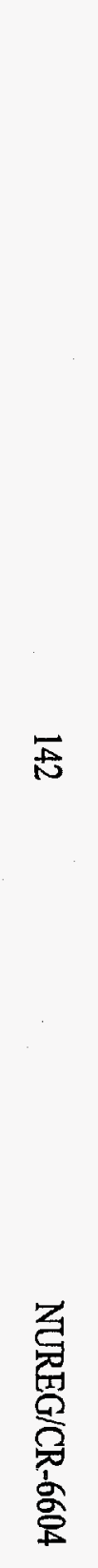

$\begin{array}{lcl}\text { Time (hr) } & \text { Flux }\left(\mathrm{cf}^{*} \mathrm{ft}^{\wedge}-2^{*} \mathrm{~min}^{\wedge}-1\right) & \text { Height (ft) } \\ 0.0000 \mathrm{E}+00 & 0.3940 \mathrm{E}-01 & 0.8500 \mathrm{E}+02 \\ 0.3000 \mathrm{E}+01 & 0.0000 \mathrm{E}+00 & 0.8500 \mathrm{E}+02\end{array}$

Sprays: Elemental Removal Data

Time (hr)

$0.0000 \mathrm{E}+00$

$0.5000 \mathrm{E}+00$

$0.1800 \mathrm{E}+01$

$0.8000 \mathrm{E}+01$

DF $\left(\mathrm{hr}^{\wedge}-1\right)$

$0.1500 \mathrm{E}+01$

$0.1200 \mathrm{E}+02$

$0.5000 \mathrm{E}+01$

$0.4000 \mathrm{E}+00$

Compartment number 2: Unsprayed Region

$$
\begin{array}{ll}
\text { Natural Deposition: Aerosol data } \\
\text { Time (hr) } & \text { DF (hr } \left.\mathrm{hr}^{\wedge}-1\right) \\
0.0000 \mathrm{E}+00 & 0.1500 \mathrm{E}+00 \\
0.7200 \mathrm{E}+03 & 0.1500 \mathrm{E}+00
\end{array}
$$

Compartment number 3: Annulus

Compartment number 4: Environment

Compartment number 5: Control Room

Compartment Filter Data

Flow rate $=0.4000 \mathrm{E}+04(\mathrm{cfm})$

\begin{tabular}{llll} 
Time $(\mathrm{hr})$ & \multicolumn{3}{c}{ Filter } \\
& Aerosiciencies & $(\mathrm{g})$ \\
$0.0000 \mathrm{E}+00$ & $0.9900 \mathrm{E}+02$ & $0.9500 \mathrm{E}+02$ & $0.9500 \mathrm{E}+02$ \\
$0.7200 \mathrm{E}+03$ & $0.9900 \mathrm{E}+02$ & $0.9500 \mathrm{E}+02$ & $0.9500 \mathrm{E}+02$
\end{tabular}

PATHWAY DATA

Pathway number 1: Sprayed to Unsprayed

$\begin{array}{cr}\begin{array}{c}\text { Convection Data } \\ \text { Time (hr) }\end{array} & \text { Flow Rate (\% / day) } \\ 0.0000 \mathrm{E}+00 & 0.1053 \mathrm{E}+04 \\ 0.1667 \mathrm{E}+00 & 0.4056 \mathrm{E}+04 \\ 0.5000 \mathrm{E}+00 & 0.4425 \mathrm{E}+04 \\ 0.1175 \mathrm{E}+01 & 0.2951 \mathrm{E}+04 \\ 0.1800 \mathrm{E}+01 & 0.1053 \mathrm{E}+04\end{array}$

Pathway number 2: Sprayed to Annulus

$$
\begin{array}{cc}
\text { Time (hr) } & \text { Flow Rate (q / day) } \\
0.0000 \mathrm{E}+00 & 0.4500 \mathrm{E}+00 \\
0.2400 \mathrm{E}+02 & 0.2250 \mathrm{E}+00
\end{array}
$$

Pathway number 3: Sprayed to Environment

Convection Data Time (hr)

Flow Rate ( $\%$ / day)
$0.0000 \mathrm{E}+00$
$0.5000 \mathrm{E}-01$
$0.2400 \mathrm{E}+02$

Pathway number 4: Unsprayed to Sprayed

$\begin{array}{lr}\text { Convection Data } & \\ \text { Time (hr) } & \text { Flow Rate (\%) d day) } \\ 0.0000 \mathrm{E}+00 & 0.4800 \mathrm{E}+04 \\ 0.1667 \mathrm{E}+00 & 0.1845 \mathrm{E}+05 \\ 0.5000 \mathrm{E}+00 & 0.2016 \mathrm{E}+05 \\ 0.1175 \mathrm{E}+01 & 0.1344 \mathrm{E}+05 \\ 0.1800 \mathrm{E}+01 & 0.4800 \mathrm{E}+04\end{array}$

Pathway number 5: Unsprayed to Annulus

$\begin{array}{lc}\text { Convection Data } & \\ \text { Time (hr) } & \text { Flow Rate }(8 / \text { day }) \\ 0.0000 \mathrm{E}+00 & 0.4500 \mathrm{E}+00 \\ 0.2400 \mathrm{E}+02 & 0.2250 \mathrm{E}+00\end{array}$

Pathway number 6: Unsprayed to Environment

$\begin{array}{cc}\text { Convection Data } & \text { Flow Rate (\% / day) } \\ \text { Time (hr) } & 0.5000 \mathrm{E}-01 \\ 0.0000 \mathrm{E}+00 & 0.2500 \mathrm{E}-01 \\ 0.2400 \mathrm{E}+02 & \end{array}$

Pathway number 7: Annulus to Environment

Piping: Removal Data

$\begin{array}{ccll}\text { Time }(\mathrm{hr}) & \text { Flow Rate }(\mathrm{cfm}) & & \begin{array}{l}\text { DF }\left(\mathrm{s}^{\wedge}-1\right) \\ \text { Elemental }\end{array} \\ \begin{array}{c}\text { Organic } \\ 0.0000 \mathrm{E}+00\end{array} & 0.2118 \mathrm{E}+00 & 0.3600 \mathrm{E}+06 & 0.2778 \mathrm{E}-03 \\ \begin{array}{c}0.2778 \mathrm{E}-03 \\ 0.2780 \mathrm{E}-01\end{array} & 0.1400 \mathrm{E}+05 & 0.3600 \mathrm{E}+06 & 0.2778 \mathrm{E}-03 \\ \begin{array}{c}0.2778 \mathrm{E}-03 \\ 0.2778 \mathrm{E}+00\end{array} & 0.1000 \mathrm{E}+04 & 0.3600 \mathrm{E}+06 & 0.2778 \mathrm{E}-03 \\ 0.2778 \mathrm{E}-03 & & & \end{array}$

Pathway number 8: Filter 3--Environment to $\mathrm{CR}$

Pathway Filter: Removal Data

Filter flow rate $=0.2000 \mathrm{E}+04(\mathrm{cfm})$
Time (hr)
Filter efficiency $(8)$
$0.0000 E+00$
Aerosol
Organic
$0.7200 \mathrm{E}+03$
$0.9900 \mathrm{E}+02$
$0.9500 \mathrm{E}+02$
$0.9500 \mathrm{E}+02$
$0.9500 \mathrm{E}+02$

Pathway number 9: Unfiltered--Environment to $\mathrm{CR}$

Pathway Filter: Removal Data

Filter flow rate $=0.1000 \mathrm{E}+02(\mathrm{cfm})$ 


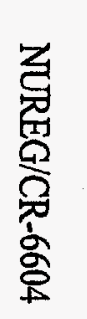

Time (hr)

$0.0000 \mathrm{E}+00$

$0.7200 \mathrm{E}+03$

Filter
$0.0000 \mathrm{E}+00$
$0.0000 \mathrm{E}+00$

(

Pathway number 10: Exhaust from $C R$ to Environment

Pathway Filter: Removal Data

Filter flow rate $=0.2010 \mathrm{E}+04(\mathrm{cfm})$

Time (hr)

$0.0000 \mathrm{E}+00$

Fil
$0.0000 \mathrm{E}+00$
$0.0000 \mathrm{E}+00$

Filter efficiency $(\%)$

$0.7200 \mathrm{E}+03$

$0.0000 \mathrm{E}+00 \quad 0.0000 \mathrm{E}+00$

Organic

$0.0000 \mathrm{E}+00$

LOCATION DATA

Location Exclusion Area Boundary

is in compartment

Location X/Q Data

$$
\begin{array}{cc}
\text { Location } & \mathrm{h} \text { ) } \\
\text { Time (hr) } & \mathrm{x} / \mathrm{Q}\left(\mathrm{s} * \mathrm{~m}^{\wedge}-3\right) \\
0.0000 \mathrm{E}+00 & 0.1000 \mathrm{E}-02 \\
0.2000 \mathrm{E}+01 & 0.0000 \mathrm{E}+00
\end{array}
$$

Location Breathing Rate Data

Time (hr)
$0.0000 \mathrm{E}+00$

$\begin{array}{ll}0.0000 \mathrm{E}+00 & 0.3470 \mathrm{E}-03 \\ 0.8000 \mathrm{E}+01 & 0.1750 \mathrm{E}-03\end{array}$

$0.8000 \mathrm{E}+01 \quad 0.1750 \mathrm{E}-03$

Location $\mathrm{X} / \mathrm{Q}$ Data

$\begin{array}{lc}\text { Location } X / Q \text { Data } & \\ \text { Time (hr) } & \mathrm{x} / \mathrm{Q}\left(\mathrm{s} * \mathrm{~m}^{\wedge}-3\right) \\ 0.0000 \mathrm{E}+00 & 0.1350 \mathrm{E}-03 \\ 0.8000 \mathrm{E}+01 & 0.1000 \mathrm{E}-03 \\ 0.2400 \mathrm{E}+02 & 0.5400 \mathrm{E}-04 \\ 0.9600 \mathrm{E}+02 & 0.2200 \mathrm{E}-04\end{array}$

$0.9600 \mathrm{E}+02 \quad 0.2200 \mathrm{E}-04$

Location Breathing Rate Data

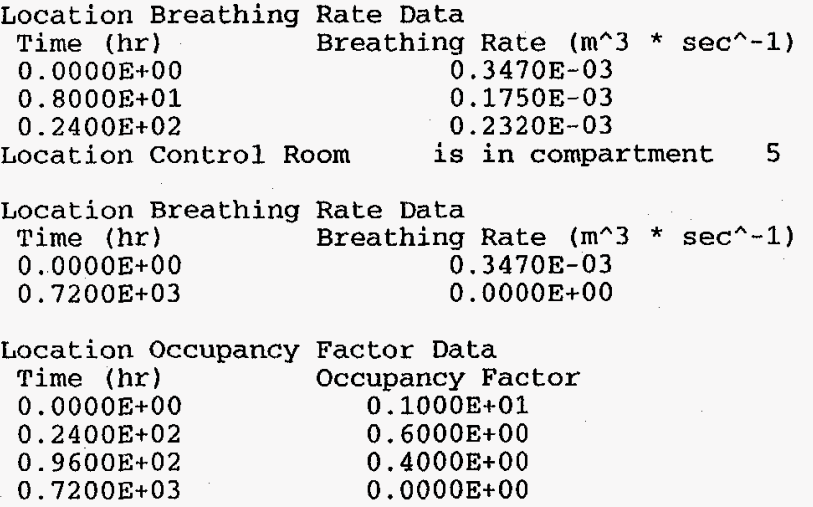

USER SPECIFIED TIME STEP DATA - SUPPLEMENTAL TIME STEPS

$\begin{array}{ll}\text { Time } & \text { Time step } \\ 0.0000 \mathrm{E}+00 & 0.5000 \mathrm{E}-01 \\ 0.3000 \mathrm{E}+01 & 0.2500 \mathrm{E}+00 \\ 0.8000 \mathrm{E}+01 & 0.1000 \mathrm{E}+01 \\ 0.2400 \mathrm{E}+02 & 0.4000 \mathrm{E}+01 \\ 0.7200 \mathrm{E}+03 & 0.0000 \mathrm{E}+00\end{array}$

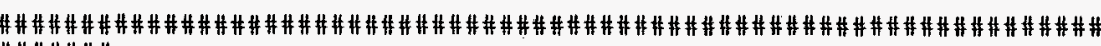
\#\#\#\#\#\#

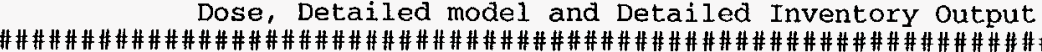
$\# \# \# \# \# \#$

Exclusion Area Boundary Doses:

Time $(\mathrm{h})=0.0278$ Whole Body Thyroid TEDE $\begin{array}{llll}\text { Delta dose (rem) } & 0.2437 \mathrm{E}-03 & 0.4064 \mathrm{E}-01 & 0.1758 \mathrm{E}-02 \\ \text { Accumulated dose (rem) } & 0.2437 \mathrm{E}-03 & 0.4064 \mathrm{E}-01 & 0.1758 \mathrm{E}-02\end{array}$

Low Population Zone Doses:

Time $(\mathrm{h})=0.0278$ Whole Body Thyroid TEDE $\begin{array}{llll}\text { Delta dose (rem) } & 0.3290 \mathrm{E}-04 & 0.5487 \mathrm{E}-02 & 0.2373 \mathrm{E}-03\end{array}$

Control Room Doses:

\begin{tabular}{|c|c|c|c|}
\hline $\operatorname{Time}_{\mathrm{Kr}-85}(\mathrm{~h})=0.0278$ & $\begin{array}{c}\text { Atoms } \\
0.1138 \mathrm{E}+17\end{array}$ & $\frac{\mathrm{kg}}{0.1606 \mathrm{E}-08}$ & $\begin{array}{c}\mathrm{Ci} \\
0.6299 \mathrm{E}-03\end{array}$ \\
\hline $0.2331 \mathrm{E}+08$ & & & \\
\hline $\begin{array}{l}\mathrm{Kr}-85 \mathrm{~m} \\
0.1092 \mathrm{E}+10\end{array}$ & $0.2540 \mathrm{E}+14$ & $0.3585 \mathrm{E}-11$ & $0.2951 \mathrm{E}-01$ \\
\hline $\begin{array}{l}\mathrm{Kr}-87 \\
0.1995 \mathrm{E}+10\end{array}$ & $0.1318 E+14$ & $0.1904 \mathrm{E}-11$ & $0.5393 \mathrm{E}-01$ \\
\hline $\begin{array}{l}\mathrm{Kr}-88 \\
0.2698 \mathrm{E}+10\end{array}$ & $0.3980 \mathrm{E}+14$ & $0.5815 E-11$ & $0.7292 \mathrm{E}-01$ \\
\hline $\begin{array}{l}\mathrm{Rb}-86 \\
0.1649 \mathrm{E}+07\end{array}$ & $0.3835 \mathrm{E}+13$ & $0.5477 \mathrm{E}-12$ & $0.4457 \mathrm{E}-04$ \\
\hline $\begin{array}{l}\mathrm{I}-131 \\
0.2809 \mathrm{E}+10\end{array}$ & $0.2815 \mathrm{E}+16$ & $0.6123 E-09$ & $0.7591 \mathrm{E}-01$ \\
\hline $\begin{array}{l}I-132 \\
0.4139 \mathrm{E}+10\end{array}$ & $0.4944 \mathrm{E}+14$ & $0.1084 \mathrm{E}-10$ & $0.1119 \mathrm{E}+00$ \\
\hline $\begin{array}{l}I-133 \\
0.5938 E+10\end{array}$ & $0.6415 \mathrm{E}+15$ & $0.1417 \mathrm{E}-09$ & $0.1605 \mathrm{E}+00$ \\
\hline $\begin{array}{l}I-134 \\
0.6517 \mathrm{E}+10\end{array}$ & $0.2967 \mathrm{E}+14$ & $0.6602 \mathrm{E}-11$ & $0.1761 \mathrm{E}+00$ \\
\hline $\begin{array}{l}I-135 \\
0.5599 \mathrm{E}+10\end{array}$ & $0.1922 \mathrm{E}+15$ & $0.4309 \mathrm{E}-10$ & $0.1513 E+00$ \\
\hline $\begin{array}{c}\mathrm{Xe}-133 \\
0.6388 \mathrm{E}+10\end{array}$ & $0.4176 \mathrm{E}+16$ & $0.9223 \mathrm{E}-09$ & $0.1726 \mathrm{E}+00$ \\
\hline $\begin{array}{c}\mathrm{Xe}-135 \\
0.1199 \mathrm{E}+10\end{array}$ & $0.5659 \mathrm{E}+14$ & $0.1269 \mathrm{E}-10$ & $0.3240 \mathrm{E}-01$ \\
\hline
\end{tabular}

Time $(h)=0.0278$ whole Body Thyroid TEDE $\begin{array}{llll}\text { Delta dose (rem) (rem) } 0.8335 \mathrm{E}-07 & 0.3609 \mathrm{E}-04 & 0.1403 \mathrm{E}-05\end{array}$

Environment Atmosphere Nuclide Inventory: 


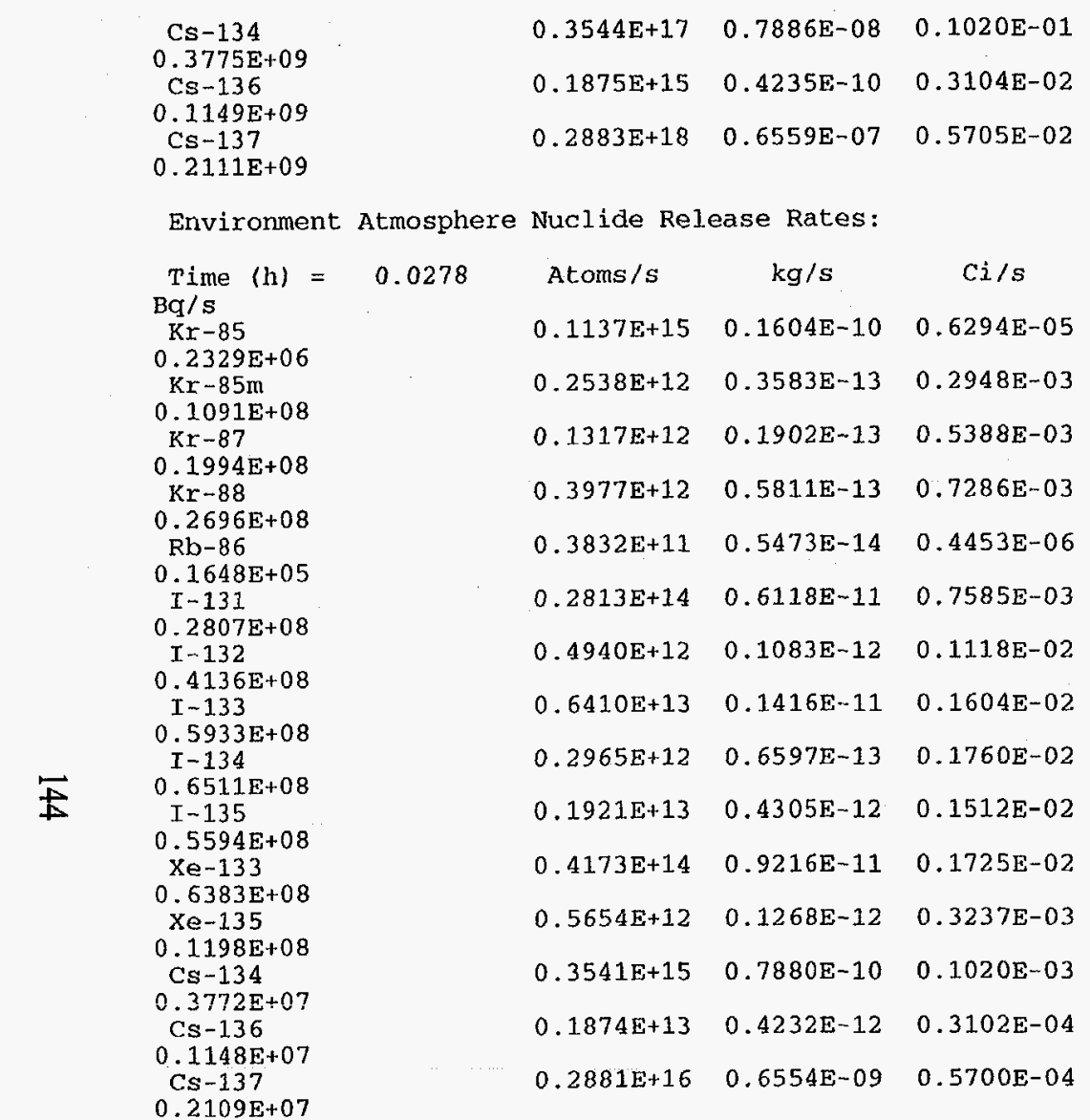

Environment Transport Group Inventory:

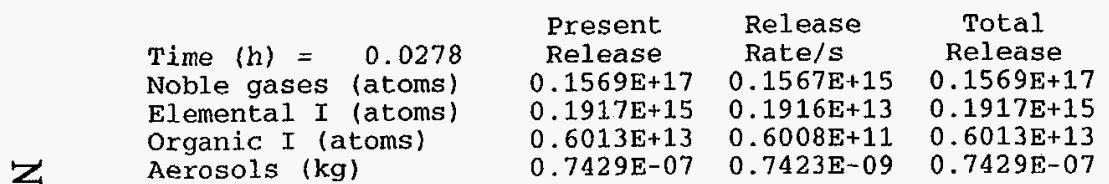

anic I (atoms)

$\begin{array}{lll}0.7429 \mathrm{E}-07 & 0.7423 \mathrm{E}-09 & 0.7429 \mathrm{E}-07\end{array}$

Annulus to Environment Transport: Group Inventory:

Time $(\mathrm{h})=0.0278$

Noble gases (atoms)

Elemental I (atoms)

Organic I (atoms)

Pipe Walls

$0.0000 \mathrm{E}+00$

$0.2638 \mathrm{E}+02$

$0.1265 \mathrm{E}-12$
Filter 3--Environment to CR Transport Group Inventory:

$\begin{array}{lc}\text { Time (h) }=\quad 0.0278 & \text { Pathway } \\ \text { Filter } \\ \text { Noble gases (atoms) } & 0.0000 \mathrm{E}+00 \\ \text { Elemental I (atoms) } & 0.5736 \mathrm{E}+16 \\ \text { Organic I (atoms) } & 0.1799 \mathrm{E}+15 \\ \text { Aerosols (kg) } & 0.2316 \mathrm{E}-05\end{array}$

Unfiltered--Envixonment to CR Txansport Group Inventory:

$\begin{array}{lc}\text { Time }(\mathrm{h})=\quad 0.0278 & \text { Pathway } \\ \text { Noble gases (atoms) } & 0.0000 \mathrm{E}+00 \\ \text { Elemental I (atoms) } & 0.0000 \mathrm{E}+00 \\ \text { Organic I (atoms) } & 0.0000 \mathrm{E}+00 \\ \text { Aerosols (kg) } & 0.0000 \mathrm{E}+00\end{array}$

Aerosols (kg) $\quad 0.0000 \mathrm{E}+00$

Exhaust from $\mathrm{CR}$ to Environment Transport Group Inventory:

$\begin{array}{ll}\text { Time }(\mathrm{h})=\text { Pathway } \\ \text { Noble gases (atoms) } & 0.0278 \\ \text { Elemental I (atoms) } & 0.0000 \mathrm{E}+00 \\ \text { Organic I (atoms) } & 0.0000 \mathrm{E}+00 \\ \text { Aerosols (kg) } & 0.0000 \mathrm{E}+00\end{array}$

Aerosols (kg) $\quad 0.0000 \mathrm{E}+00$

Control Room Atmosphere Nuclide Inventory:

\begin{tabular}{|c|c|c|c|}
\hline$\underset{K r-85}{\operatorname{Time}}(\mathrm{h})=0.0278$ & $\begin{array}{c}\text { Atoms } \\
0.2335 \mathrm{E}+14\end{array}$ & $\begin{array}{c}\mathrm{kg} \\
0.3296 \mathrm{E}-11\end{array}$ & $\begin{array}{c}\mathrm{Ci} \\
0.1293 \mathrm{E}-05\end{array}$ \\
\hline $0.4784 \mathrm{E}+05$ & & & \\
\hline $\mathrm{Kr}-85 \mathrm{~m}$ & $0.5214 \mathrm{E}+11$ & $0.7360 \mathrm{E}-14$ & $0.6057 \mathrm{E}-04$ \\
\hline $\begin{array}{l}0.2241 \mathrm{E}+07 \\
\mathrm{Kr}-87\end{array}$ & $0.2705 E+11$ & $0.3908 \mathrm{E}-14$ & $0.1107 \mathrm{E}-03$ \\
\hline $0.4096 \mathrm{E}+07$ & & & \\
\hline $\begin{array}{c}\mathrm{Kr}-88 \\
0.5538 \mathrm{E}+07\end{array}$ & $0.8169 \mathrm{E}+11$ & $0.1194 \mathrm{E}-13$ & $0.1497 \mathrm{E}-03$ \\
\hline $\begin{array}{l}\mathrm{Rb}-86 \\
0.4888 \mathrm{E}+02\end{array}$ & $0.1137 \mathrm{E}+09$ & $0.1623 \mathrm{E}-16$ & $0.1321 \mathrm{E}-08$ \\
\hline $\begin{array}{l}I-131 \\
0.9506 \mathrm{E}+05\end{array}$ & $0.9527 \mathrm{E}+11$ & $0.2072 \mathrm{E}-13$ & $0.2569 \mathrm{E}-05$ \\
\hline $\begin{array}{l}I-132 \\
0.1401 \mathrm{E}+06\end{array}$ & $0.1673 \mathrm{E}+10$ & $0.3668 \mathrm{E}-15$ & $0.3786 \mathrm{E}-05$ \\
\hline $\begin{array}{l}I-133 \\
0.2010 \mathrm{E}+06\end{array}$ & $0.2171 \mathrm{E}+11$ & $0.4795 \mathrm{E}-14$ & $0.5432 \mathrm{E}-05$ \\
\hline $\begin{array}{l}I-134 \\
0.2206 E+06\end{array}$ & $0.1004 \mathrm{E}+10$ & $0.2234 \mathrm{E}-15$ & $0.5961 E-05$ \\
\hline $\begin{array}{l}I-135 \\
0.1895 \mathrm{E}+06\end{array}$ & $0.6505 \mathrm{E}+10$ & $0.1458 \mathrm{E}-14$ & $0.5121 \mathrm{E}-05$ \\
\hline $\begin{array}{c}\mathrm{Xe}-133 \\
0.1311 \mathrm{E}+08\end{array}$ & $0.8573 \mathrm{E}+13$ & $0.1893 \mathrm{E}-11$ & $0.3544 \mathrm{E}-03$ \\
\hline $\begin{array}{c}\mathrm{Xe}-135 \\
0.2460 \mathrm{E}+07\end{array}$ & $0.1162 E+12$ & $0.2604 \mathrm{E}-13$ & $0.6650 \mathrm{E}-04$ \\
\hline $\mathrm{Cs}-134$ & $0.1050 \mathrm{E}+13$ & $0.2337 \mathrm{E}-12$ & $0.3024 \mathrm{E}-06$ \\
\hline
\end{tabular}




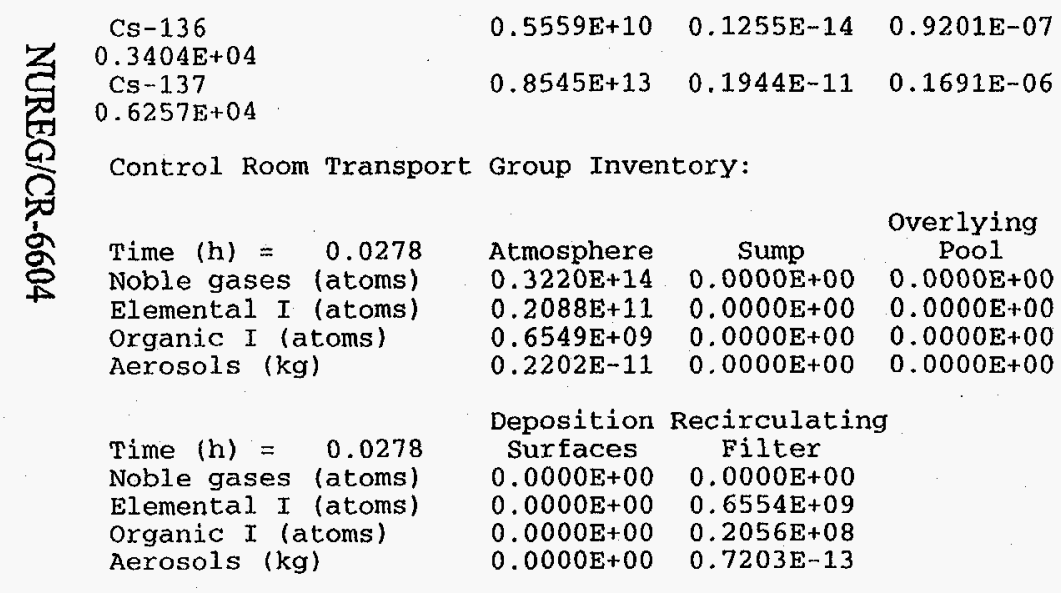

Filter 3--Environment to CR Transport Group Inventory:

$\begin{array}{lc}\text { Time }(\mathrm{h})=\quad 0.0278 & \begin{array}{c}\text { Pathway } \\ \text { Filter }\end{array} \\ \text { Noble gases (atoms) } & 0.0000 \mathrm{E}+00 \\ \text { Elemental I (atoms) } & 0.5736 \mathrm{E}+16 \\ \text { Organic I (atoms) } & 0.1799 \mathrm{E}+15 \\ \text { Aerosols }(\mathrm{kg}) & 0.2316 \mathrm{E}-05\end{array}$

\& Unfiltered--Environment to CR Transport Group Inventory:

$\begin{array}{lc}\text { Time }(\mathrm{h})=\quad 0.0278 & \text { Pathway } \\ \text { Noble gases (atoms) } & 0.0000 \mathrm{E}+00 \\ \text { Elemental I (atoms) } & 0.0000 \mathrm{E}+00 \\ \text { Organic I (atoms) } & 0.0000 \mathrm{E}+00 \\ \text { Aerosols }(\mathrm{kg}) & 0.0000 \mathrm{E}+00\end{array}$

Exhaust from CR to Environment Transport Group Inventory:

$\begin{array}{lc}\text { Time }(\mathrm{h})=\quad 0.0278 & \text { Pathway } \\ \text { Noble gases (atoms) } & 0.0000 \mathrm{E}+00 \\ \text { Elemental I (atoms) } & 0.0000 \mathrm{E}+00 \\ \text { Organic I (atoms) } & 0.0000 \mathrm{E}+00 \\ \text { Aerosols (kg) } & 0.0000 \mathrm{E}+00\end{array}$

\section{*** Intermediate time steps not edited $* * *$}

Exclusion Area Boundary Doses:

Time $(h)=720.0000$ Whole Body Thyroid TEDE $\begin{array}{llll}\text { Delta dose (rem) } & 0.0000 \mathrm{E}+00 & 0.0000 \mathrm{E}+00 & 0.0000 \mathrm{E}+00 \\ \text { Accumulated dose (rem) } & 0.1663 \mathrm{E}+01 & 0.6858 \mathrm{E}+02 & 0.4539 \mathrm{E}+01\end{array}$ Low Population zone Doses:

Time $(\mathrm{h})=720.0000$ Whole Body Thyroid TEDE $\begin{array}{llll}\text { Delta dose (rem) } & 0.6654 \mathrm{E}+02 & 0.2970 \mathrm{E}+02 & 0.6748 \mathrm{E}+02\end{array}$ $\begin{array}{lllll}\text { Accumulated dose } & (\mathrm{rem}) \quad 0.1038 \mathrm{E}+03 & 0.6043 \mathrm{E}+02 & 0.1058 \mathrm{E}+03\end{array}$

Control Room Doses:

Time $(\mathbf{h})=720.0000 \quad$ Whole Body Thyroid TEDE Delta dose $(\mathrm{rem}) \quad 0.2119 \mathrm{E}+02 \quad 0.7350 \mathrm{E}+01 \quad 0.2143 \mathrm{E}+02$

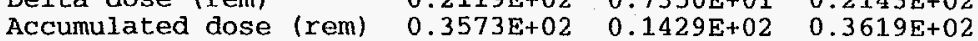

Environment Atmosphere Nuclide Inventory:

\begin{tabular}{|c|c|c|c|}
\hline $\begin{array}{l}\text { Time } \\
\text { Co-58 }\end{array}$ & $\begin{array}{c}\text { Atoms } \\
0.1104 \mathrm{E}+15\end{array}$ & $\frac{\mathrm{kg}}{0.1063 \mathrm{E}-10}$ & $\begin{array}{c}\mathrm{Ci} \\
0.3380 \mathrm{E}-03\end{array}$ \\
\hline $0.1251 \mathrm{E}+08$ & & & \\
\hline $\begin{array}{c}C 0-60 \\
0.9568 E+07\end{array}$ & $0.2296 E+16$ & $0.2288 \mathrm{E}-09$ & $0.2586 \mathrm{E}-03$ \\
\hline $\mathrm{Kr}-85$ & $0.8796 \mathrm{E}+24$ & $0.1242 \mathrm{E}+00$ & $0.4871 \mathrm{E}+05$ \\
\hline $0.1802 E+16$ & & & \\
\hline $\begin{array}{c}\mathrm{Kr}-85 \mathrm{~m} \\
0.8442 \mathrm{E}+17\end{array}$ & $0.1964 \mathrm{E}+22$ & $0.2773 \mathrm{E}-03$ & $0.2282 \mathrm{E}+07$ \\
\hline $\begin{array}{l}\mathrm{Kr}-87 \\
0.1543 \mathrm{E}+18\end{array}$ & $0.1019 \mathrm{E}+22$ & $0.1472 \mathrm{E}-03$ & $0.4170 \mathrm{E}+07$ \\
\hline $\begin{array}{l}\mathrm{Kr}-88 \\
0.2086 \mathrm{E}+18\end{array}$ & $0.3078 \mathrm{E}+22$ & $0.4497 \mathrm{E}-03$ & $0.5639 \mathrm{E}+07$ \\
\hline $\begin{array}{l}\mathrm{Rb}-86 \\
0.7338 \mathrm{E}+08\end{array}$ & $0.1707 \mathrm{E}+15$ & $0.2437 \mathrm{E}-10$ & $0.1983 \mathrm{E}-02$ \\
\hline $\begin{array}{l}S r-89 \\
0.1115 \mathrm{E}+11\end{array}$ & $0.7016 \mathrm{E}+17$ & $0.1037 E-07$ & $0.3013 \mathrm{E}+00$ \\
\hline $\begin{array}{l}\mathrm{Sr}-90 \\
0.6016 \mathrm{E}+09\end{array}$ & $0.7976 \mathrm{E}+18$ & $0.1192 \mathrm{E}-06$ & $0.1626 \mathrm{E}-01$ \\
\hline $\begin{array}{l}\text { Sr-91 } \\
0.1433 E+11\end{array}$ & $0.7070 \mathrm{E}+15$ & $0.1068 \mathrm{E}-09$ & $0.3873 \mathrm{E}+00$ \\
\hline $\begin{array}{l}S r-92 \\
0.1491 \mathrm{E}+11\end{array}$ & $0.2099 \mathrm{E}+15$ & $0.3207 \mathrm{E}-10$ & $0.4030 \mathrm{E}+00$ \\
\hline $\begin{array}{l}Y-90 \\
0.6455 \mathrm{E}+07\end{array}$ & $0.2146 \mathrm{E}+13$ & $0.3207 \mathrm{E}-12$ & $0.1745 \mathrm{E}-03$ \\
\hline $\begin{array}{l}Y-91 \\
0.1358 \mathrm{E}+09\end{array}$ & $0.9904 \mathrm{E}+15$ & $0.1497 \mathrm{E}-09$ & $0.3670 \mathrm{E}-02$ \\
\hline $\begin{array}{l}Y-92 \\
0.1497 \mathrm{E}+09\end{array}$ & $0.2752 \mathrm{E}+13$ & $0.4204 \mathrm{E}-12$ & $0.4045 \mathrm{E}-02$ \\
\hline $\begin{array}{l}Y-93 \\
0.1693 E+09\end{array}$ & $0.8882 \mathrm{E}+13$ & $0.1372 \mathrm{E}-11$ & $0.4576 \mathrm{E}-02$ \\
\hline $\begin{array}{l}\mathrm{Zr-95} \\
0.1715 \mathrm{E}+09\end{array}$ & $0.1368 \mathrm{E}+16$ & $0.2158 \mathrm{E}-09$ & $0.4636 \mathrm{E}-02$ \\
\hline $\begin{array}{c}2 x-97 \\
0.1788 \mathrm{E}+09\end{array}$ & $0.1569 \mathrm{E}+14$ & $0.2528 \mathrm{E}-11$ & $0.4832 \mathrm{E}-02$ \\
\hline $\begin{array}{l}\mathrm{Nb}-95 \\
0.1622 \mathrm{E}+09\end{array}$ & $0.7106 \mathrm{E}+15$ & $0.1121 \mathrm{E}-09$ & $0.4383 \mathrm{E}-02$ \\
\hline $\begin{array}{l}\text { Mo-99 } \\
0.2366 \mathrm{E}+10\end{array}$ & $0.8111 \mathrm{E}+15$ & $0.1333 \mathrm{E}-09$ & $0.6395 \mathrm{E}-01$ \\
\hline $\begin{array}{l}\mathrm{TC}-99 \mathrm{~m} \\
0.2042 \mathrm{E}+10\end{array}$ & $0.6386 \mathrm{E}+14$ & $0.1050 \mathrm{E}-10$ & $0.5520 \mathrm{E}-01$ \\
\hline
\end{tabular}




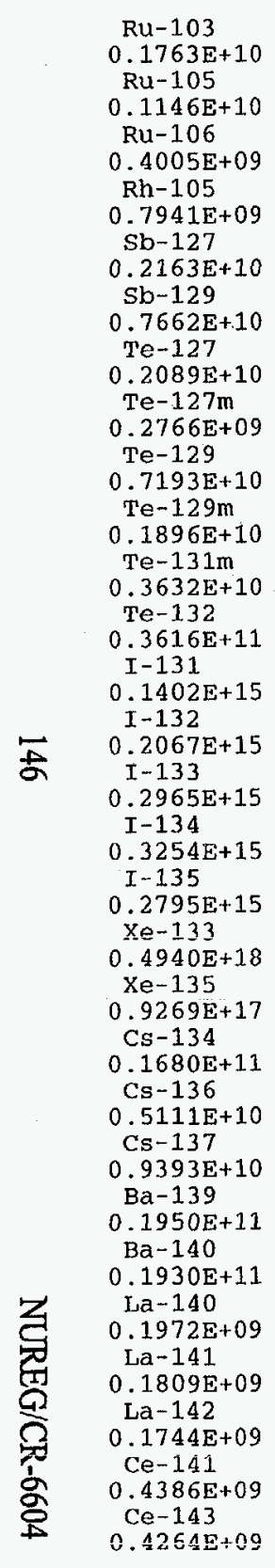

$\begin{array}{lll}0.8630 \mathrm{E}+16 & 0.1476 \mathrm{E}-08 & 0.4764 \mathrm{E}-01 \\ 0.2644 \mathrm{E}+14 & 0.4609 \mathrm{E}-11 & 0.3098 \mathrm{E}-01 \\ 0.1838 \mathrm{E}+17 & 0.3235 \mathrm{E}-08 & 0.1082 \mathrm{E}-01 \\ 0.1458 \mathrm{E}+15 & 0.2543 \mathrm{E}-10 & 0.2146 \mathrm{E}-01 \\ 0.1038 \mathrm{E}+16 & 0.2190 \mathrm{E}-09 & 0.5847 \mathrm{E}-01 \\ 0.1719 \mathrm{E}+15 & 0.3683 \mathrm{E}-10 & 0.2071 \mathrm{E}+00 \\ 0.1014 \mathrm{E}+15 & 0.2139 \mathrm{E}-10 & 0.5646 \mathrm{E}-01 \\ 0.3758 \mathrm{E}+16 & 0.7925 \mathrm{E}-09 & 0.7476 \mathrm{E}-02 \\ 0.4333 \mathrm{E}+14 & 0.9283 \mathrm{E}-11 & 0.1944 \mathrm{E}+00 \\ 0.7941 \mathrm{E}+16 & 0.1701 \mathrm{E}-08 & 0.5124 \mathrm{E}-01 \\ 0.5659 \mathrm{E}+15 & 0.1231 \mathrm{E}-09 & 0.9817 \mathrm{E}-01 \\ 0.1468 \mathrm{E}+17 & 0.3219 \mathrm{E}-08 & 0.9772 \mathrm{E}+00 \\ 0.1405 \mathrm{E}+21 & 0.3057 \mathrm{E}-04 & 0.3790 \mathrm{E}+04 \\ 0.2469 \mathrm{E}+19 & 0.5411 \mathrm{E}-06 & 0.5585 \mathrm{E}+04 \\ 0.3203 \mathrm{E}+20 & 0.7074 \mathrm{E}-05 & 0.8013 \mathrm{E}+04 \\ 0.1481 \mathrm{E}+19 & 0.3296 \mathrm{E}-06 & 0.8794 \mathrm{E}+04 \\ 0.9597 \mathrm{E}+19 & 0.2151 \mathrm{E}-05 & 0.7555 \mathrm{E}+04 \\ 0.3230 \mathrm{E}+24 & 0.7132 \mathrm{E}-01 & 0.1335 \mathrm{E}+08 \\ 0.4376 \mathrm{E}+22 & 0.9810 \mathrm{E}-03 & 0.2505 \mathrm{E}+07 \\ 0.1577 \mathrm{E}+19 & 0.3509 \mathrm{E}-06 & 0.4541 \mathrm{E}+00 \\ 0.8346 \mathrm{E}+16 & 0.1885 \mathrm{E}-08 & 0.1381 \mathrm{E}+00 \\ 0.1283 \mathrm{E}+20 & 0.2919 \mathrm{E}-05 & 0.2539 \mathrm{E}+00 \\ 0.1396 \mathrm{E}+15 & 0.3222 \mathrm{E}-10 & 0.5271 \mathrm{E}+00 \\ 0.3065 \mathrm{E}+17 & 0.7125 \mathrm{E}-08 & 0.5216 \mathrm{E}+00 \\ 0.4125 \mathrm{E}+14 & 0.9590 \mathrm{E}-11 & 0.5330 \mathrm{E}-02 \\ 0.3692 \mathrm{E}+13 & 0.8644 \mathrm{E}-12 & 0.4888 \mathrm{E}-02 \\ 0.1396 \mathrm{E}+13 & 0.3292 \mathrm{E}-12 & 0.4713 \mathrm{E}-02 \\ 0.177 \mathrm{E}+16 & 0.4160 \mathrm{E}-09 & 0.1185 \mathrm{E}-01 \\ 0.14 & 0.1735 \mathrm{E}-10 & 0.1152 \mathrm{E}-01\end{array}$

\begin{tabular}{|c|c|c|c|}
\hline $\begin{array}{c}\mathrm{Ce}-144 \\
0.2643 \mathrm{E}+09\end{array}$ & $0.9365 \mathrm{E}+16$ & $0.2239 \mathrm{E}-08$ & $0.7142 \mathrm{E}-02$ \\
\hline $\begin{array}{c}0.2643 E+09 \\
\operatorname{Pr}-143\end{array}$ & $0.2831 \mathrm{E}+15$ & $0.6722 \mathrm{E}-10$ & $0.4526 \mathrm{E}-02$ \\
\hline $0.1675 \mathrm{E}+09$ & & & \\
\hline $\begin{array}{l}\mathrm{Nd}-147 \\
0.7490 \mathrm{E}+08\end{array}$ & $0.1025 E+15$ & $0.2502 \mathrm{E}-10$ & $0.2024 \mathrm{E}-02$ \\
\hline $\begin{array}{c}N p-239 \\
0.5017 \mathrm{E}+10\end{array}$ & $0.1473 E+16$ & $0.5844 \mathrm{E}-09$ & $0.1356 \mathrm{E}+00$ \\
\hline $\begin{array}{c}0.5017 \mathrm{E}+10 \\
\mathrm{Pu}-238 \\
0.2843 \mathrm{E}+06\end{array}$ & $0.1136 \mathrm{E}+16$ & $0.4489 \mathrm{E}-09$ & $0.7685 \mathrm{E}-05$ \\
\hline $\begin{array}{c}\mathrm{Pu}-239 \\
0.6413 \mathrm{E}+05\end{array}$ & $0.7026 \mathrm{E}+17$ & $0.2788 \mathrm{E}-07$ & $0.1733 \mathrm{E}-05$ \\
\hline $\begin{array}{c}\mathrm{Pu}-240 \\
0.8087 \mathrm{E}+05\end{array}$ & $0.2407 E+17$ & $0.9592 \mathrm{E}-08$ & $0.2186 \mathrm{E}-05$ \\
\hline $\begin{array}{c}\mathrm{Pu}-241 \\
0.1362 \mathrm{E}+08\end{array}$ & $0.8929 E+16$ & $0.3573 \mathrm{E}-08$ & $0.3681 \mathrm{E}-03$ \\
\hline $\begin{array}{c}\mathrm{Am}-241 \\
0.3598 \mathrm{E}+04\end{array}$ & $0.7080 \mathrm{E}+14$ & $0.2834 \mathrm{E}-10$ & $0.9725 \mathrm{E}-07$ \\
\hline $\begin{array}{c}\mathrm{Cm}-242 \\
0.1377 \mathrm{E}+07\end{array}$ & $0.27 .95 \mathrm{E}+14$ & $0.1123 \mathrm{E}-10$ & $0.3722 \mathrm{E}-04$ \\
\hline $\begin{array}{c}\mathrm{Cm}-244 \\
0.8058 \mathrm{E}+05\end{array}$ & $0.6644 \mathrm{E}+14$ & $0.2692 \mathrm{E}-10$ & $0.2178 \mathrm{E}-05$ \\
\hline
\end{tabular}

Environment Atmosphere Nuclide Release Rates:

\begin{tabular}{|c|c|c|c|}
\hline$\underset{\substack{\text { Time } \\
\mathrm{g} / \mathrm{s}}}{ }(\mathrm{h})=720.0000$ & Atoms/s & $\mathrm{kg} / \mathrm{s}$ & $\mathrm{Ci} / \mathrm{s}$ \\
\hline $\mathrm{Co}-58$ & $0.4914 \mathrm{E}+08$ & $0.4732 \mathrm{E}-1.7$ & $0.1505 \mathrm{E}-09$ \\
\hline $\begin{array}{l}0.5568 \mathrm{E}+01 \\
\mathrm{CO}-60\end{array}$ & & & \\
\hline $\begin{array}{c}\mathrm{CO}-60 \\
0.4259 \mathrm{E}+01\end{array}$ & $0.1022 \mathrm{E}+10$ & $0.1018 \mathrm{E}-15$ & $0.1151 \mathrm{E}-09$ \\
\hline $\begin{array}{l}\mathrm{Kr}-85 \\
0.8023 \mathrm{E}+09\end{array}$ & $0.3916 \mathrm{E}+18$ & $0.5527 \mathrm{E}-07$ & $0.2168 \mathrm{E}-01$ \\
\hline $0.8023 E+09$ & $0.8744 \mathrm{E}+15$ & $0.1234 \mathrm{E}-09$ & $0.1016 \mathrm{E}+01$ \\
\hline $\begin{array}{l}0.3758 \mathrm{E}+11 \\
\mathrm{Kr}-87\end{array}$ & $0.4537 E+15$ & $0.6554 \mathrm{E}-10$ & $0.1856 \mathrm{E}+01$ \\
\hline $\begin{array}{l}0.6869 \mathrm{E}+11 \\
\mathrm{Kr}-88\end{array}$ & $0.1370 \mathrm{E}+16$ & $0.2002 E-09$ & $0.2510 \mathrm{E}+01$ \\
\hline $\begin{array}{c}0.9288 \mathrm{E}+11 \\
\mathrm{Rb}-86\end{array}$ & $0.7598 \mathrm{E}+08$ & $0.1085 \mathrm{E}-16$ & $0.8829 \mathrm{E}-09$ \\
\hline $\begin{array}{l}0.3267 \mathrm{E}+02 \\
5 x-89\end{array}$ & $0.3123 \mathrm{E}+11$ & $0.4616 \mathrm{E}-14$ & $0.1341 \mathrm{E}-06$ \\
\hline $\begin{array}{l}0.4962 \mathrm{E}+04 \\
\mathrm{Sr}-90 \\
0.2678 \mathrm{E}+03\end{array}$ & $0.3551 \mathrm{E}+12$ & $0.5306 \mathrm{E}-13$ & $0.7238 \mathrm{E}-08$ \\
\hline $\begin{array}{c}S r-91 \\
0.6379 E+04\end{array}$ & $0.3147 E+09$ & $0.4756 E-16$ & $0.1724 \mathrm{E}-06$ \\
\hline $\begin{array}{l}S r-92 \\
0.6639 \mathrm{E}+04\end{array}$ & $0.9344 \mathrm{E}+08$ & $0.1427 \mathrm{E}-16$ & $0.1794 \mathrm{E}-06$ \\
\hline $\begin{array}{l}Y-90 \\
0.2873 \mathrm{E}+01\end{array}$ & $0.9551 \mathrm{E}+06$ & $0.1427 E-1.8$ & $0.7766 \mathrm{E}-10$ \\
\hline $\begin{array}{l}Y-91 \\
0.6045 \mathrm{E}+02\end{array}$ & $0.4409 \mathrm{E}+09$ & $0.6662 \mathrm{E}-16$ & $0.1634 \mathrm{E}-08$ \\
\hline $\begin{array}{l}Y-92 \\
0.6663 \mathrm{E}+02\end{array}$ & $0.1225 E+07$ & $0.1871 \mathrm{E}-18$ & $0.1801 E-08$ \\
\hline$Y-93$ & $0.3954 E+07$ & $0.6106 E-18$ & $0.2037 E-08$ \\
\hline
\end{tabular}




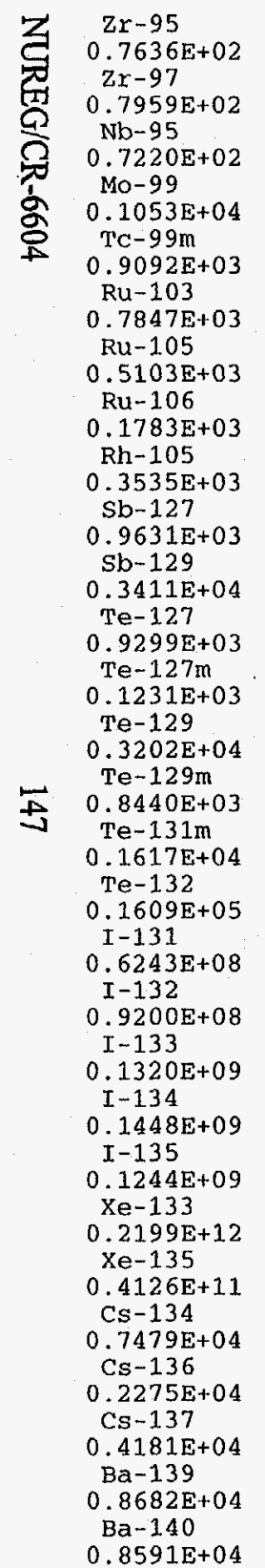

\begin{abstract}
$0.6090 \mathrm{E}+09 \quad 0.9607 \mathrm{E}-16 \quad 0.2064 \mathrm{E}-08$
$0.6986 \mathrm{E}+07 \quad 0.1125 \mathrm{E}-17 \quad 0.2151 \mathrm{E}-08$

$0.3163 \mathrm{E}+09 \quad 0.4990 \mathrm{E}-16 \quad 0.1951 \mathrm{E}-08$

$0.3611 \mathrm{E}+09 \quad 0.5936 \mathrm{E}-16 \quad 0.2847 \mathrm{E}-07$

$0.2843 \mathrm{E}+08 \quad 0.4673 \mathrm{E}-17 \quad 0.2457 \mathrm{E}-07$

$0.3842 \mathrm{E}+10 \quad 0.6571 \mathrm{E}-15 \quad 0.2121 \mathrm{E}-07$

$0.1177 \mathrm{E}+08 \quad 0.2052 \mathrm{E}-17 \quad 0.1379 \mathrm{E}-07$

$0.8183 \mathrm{E}+10 \quad 0.1440 \mathrm{E}-14 \quad 0.4819 \mathrm{E}-08$

$0.6492 \mathrm{E}+08 \quad 0.1132 \mathrm{E}-16 \quad 0.9555 \mathrm{E}-08$

$0.4622 \mathrm{E}+09 \quad 0.9747 \mathrm{E}-16 \quad 0.2603 \mathrm{E}-07$

$0.7653 \mathrm{E}+08 \quad 0.1639 \mathrm{E}-16 \quad 0.9219 \mathrm{E}-07$

$0.4516 \mathrm{E}+08 \quad 0.9523 \mathrm{E}-17 \quad 0.2513 \mathrm{E}-07$

$0.1673 \mathrm{E}+10 \quad 0.3528 \mathrm{E}-15 \quad 0.3328 \mathrm{E}-08$

$0.1929 \mathrm{E}+08 \quad 0.4132 \mathrm{E}-17 \quad 0.8654 \mathrm{E}-07$

$0.3535 \mathrm{E}+10 \quad 0.7572 \mathrm{E}-15 \quad 0.2281 \mathrm{E}-07$

$\begin{array}{llll}0.2519 \mathrm{E}+09 & 0.5480 \mathrm{E}-16 & 0.4370 \mathrm{E}-07\end{array}$

$0.6537 \mathrm{E}+10 \quad 0.1433 \mathrm{E}-14 \quad 0.4350 \mathrm{E}-06$

$0.6256 \mathrm{E}+14 \quad 0.1361 \mathrm{E}-10 \quad 0.1687 \mathrm{E}-02$

$0.1099 \mathrm{E}+13 \quad 0.2409 \mathrm{E}-12 \quad 0.2486 \mathrm{E}-02$

$0.1426 \mathrm{E}+14 \quad 0.3149 \mathrm{E}-11 \quad 0.3567 \mathrm{E}-02$

$0.6595 \mathrm{E}+12 \quad 0.1467 \mathrm{E}-12 \quad 0.3915 \mathrm{E}-02$

$0.4272 \mathrm{E}+13 \quad 0.9577 \mathrm{E}-12 \quad 0.3363 \mathrm{E}-02$

$0.1438 \mathrm{E}+18 \quad 0.3175 \mathrm{E}-07 \quad 0.5943 \mathrm{E}+01$

$0.1948 \mathrm{E}+16 \quad 0.4367 \mathrm{E}-09 \quad 0.1115 \mathrm{E}+01$

$0.7021 \mathrm{E}+12 \quad 0.1562 \mathrm{E}-12 \quad 0.2021 \mathrm{E}-06$

$0.3715 \mathrm{E}+10 \quad 0.8390 \mathrm{E}-15 \quad 0.6149 \mathrm{E}-07$

$0.5711 \mathrm{E}+13 \quad 0.1299 \mathrm{E}-11 \quad 0.1130 \mathrm{E}-06$

$0.6215 \mathrm{E}+08 \quad 0.1434 \mathrm{E}-16 \quad 0.2346 \mathrm{E}-06$

$0.1364 \mathrm{E}+11 \quad 0.3172 \mathrm{E}-14 \quad 0.2322 \mathrm{E}-06$
\end{abstract}

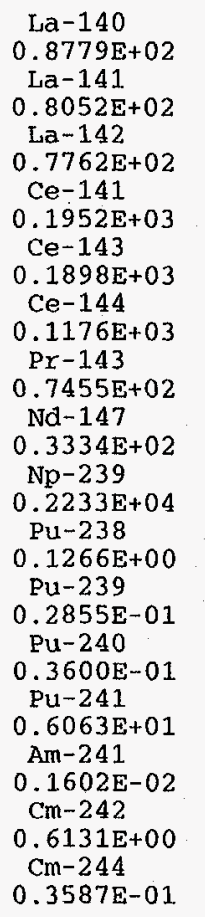

$\begin{array}{lll}0.1836 \mathrm{E}+08 & 0.4269 \mathrm{E}-17 & 0.2373 \mathrm{E}-08 \\ 0.1643 \mathrm{E}+07 & 0.3848 \mathrm{E}-18 & 0.2176 \mathrm{E}-08 \\ 0.6215 \mathrm{E}+06 & 0.1465 \mathrm{E}-18 & 0.2098 \mathrm{E}-08 \\ 0.7909 \mathrm{E}+09 & 0.1852 \mathrm{E}-15 & 0.5276 \mathrm{E}-08 \\ 0.3253 \mathrm{E}+08 & 0.7725 \mathrm{E}-17 & 0.5130 \mathrm{E}-08 \\ 0.4169 \mathrm{E}+10 & 0.9968 \mathrm{E}-15 & 0.3179 \mathrm{E}-08 \\ 0.1260 \mathrm{E}+09 & 0.2992 \mathrm{E}-16 & 0.2015 \mathrm{E}-08 \\ 0.4563 \mathrm{E}+08 & 0.1114 \mathrm{E}-16 & 0.9011 \mathrm{E}-09 \\ 0.6556 \mathrm{E}+09 & 0.2602 \mathrm{E}-15 & 0.6036 \mathrm{E}-07 \\ 0.5056 \mathrm{E}+09 & 0.1998 \mathrm{E}-15 & 0.3421 \mathrm{E}-11 \\ 0.3128 \mathrm{E}+11 & 0.1241 \mathrm{E}-13 & 0.7716 \mathrm{E}-12 \\ 0.1071 \mathrm{E}+11 & 0.4270 \mathrm{E}-14 & 0.9730 \mathrm{E}-12 \\ 0.3975 \mathrm{E}+10 & 0.1591 \mathrm{E}-14 & 0.1639 \mathrm{E}-09 \\ 0.3152 \mathrm{E}+08 & 0.1261 \mathrm{E}-16 & 0.4329 \mathrm{E}-13 \\ 0.1244 \mathrm{E}+08 & 0.5000 \mathrm{E}-17 & 0.1657 \mathrm{E}-10 \\ 0.2958 \mathrm{E}+08 & 0.1198 \mathrm{E}-16 & 0.9695 \mathrm{E}-12\end{array}$

Environment Transport Group Inventory:

$\begin{array}{lccc}\text { Time }(\mathrm{h})=720.0000 & \begin{array}{c}\text { Present } \\ \text { Release }\end{array} & \begin{array}{l}\text { Release } \\ \text { Rate/s }\end{array} & \begin{array}{c}\text { Total } \\ \text { Release }\end{array} \\ \text { Noble gases (atoms) } & 0.1213 \mathrm{E}+25 & 0.5400 \mathrm{E}+18 & 0.1432 \mathrm{E}+25 \\ \text { Elemental I (atoms) } & 0.2714 \mathrm{E}+15 & 0.1208 \mathrm{E}+09 & 0.3045 \mathrm{E}+19 \\ \text { Organic I (atoms) } & 0.1859 \mathrm{E}+21 & 0.8275 \mathrm{E}+14 & 0.2195 \mathrm{E}+21 \\ \text { Aerosols }(\mathrm{kg}) & 0.1296 \mathrm{E}-03 & 0.5767 \mathrm{E}-10 & 0.5367 \mathrm{E}-02\end{array}$

Annulus to Environment Transport Group Inventory:

$\begin{array}{ll}\text { Time }(\mathrm{h})=720.0000 & \text { Pipe Walls } \\ \text { Noble gases (atoms) } & 0.0000 \mathrm{E}+00 \\ \text { Elemental I (atoms) } & 0.2192 \mathrm{E}+12 \\ \text { Organic I (atoms) } & 0.1578 \mathrm{E}+14 \\ \text { Aerosols (kg) } & 0.4387 \mathrm{E}-01\end{array}$

Filter 3--Environment to CR Transport Group Inventory:

$\begin{array}{lc}\text { Time }(\mathrm{h})=720.0000 & \text { Pathway } \\ \text { Noble gases (atoms) } & 0.0000 \mathrm{E}+00 \\ \text { Elemental I (atoms) } & 0.4348 \mathrm{E}+22 \\ \text { Organic I (atoms) } & 0.1372 \mathrm{E}+25 \\ \text { Aerosols (kg) } & 0.7988 \mathrm{E}+01\end{array}$


Unfiltered--Environment to CR Transport Group Inventory:

$\begin{array}{lc}\text { Pime }(\mathrm{h})=720.0000 & \begin{array}{c}\text { Pathway } \\ \text { Filter }\end{array} \\ \text { Noble gases (atoms) } & 0.0000 \mathrm{E}+00 \\ \text { Elemental I (atoms) } & 0.0000 \mathrm{E}+00 \\ \text { Organic I (atoms) } & 0.0000 \mathrm{E}+00 \\ \text { Aerosols }(\mathrm{kg}) & 0.0000 \mathrm{E}+00\end{array}$

Exhaust from CR to Environment Transport Group Inventory:

$\begin{array}{lc}\text { Pime (h) }=720.0000 & \begin{array}{c}\text { Pathway } \\ \text { Filter }\end{array} \\ \text { Noble gases (atoms) } & 0.0000 \mathrm{E}+00 \\ \text { Elemental I (atoms) } & 0.0000 \mathrm{E}+00 \\ \text { Organic I (atoms) } & 0.0000 \mathrm{E}+00 \\ \text { Aerosols (kg) } & 0.0000 \mathrm{E}+00\end{array}$

Control Room Atmosphere Nuclide Inventory:

Time $(h)=720.0000$

Co-58

$0.3032 \mathrm{E}-07$

Co- 60

$0.2320 \mathrm{E}-07$

$\mathrm{Kr}-85$

$0.7105 \mathrm{E}+09$

$\mathrm{Kr}-85 \mathrm{~m}$
$0.3328 \mathrm{E}+11$

$\mathrm{Kr}-87$

$0.6082 \mathrm{E}+11$

$\mathrm{Kr} \sim 88$

$0.8225 \mathrm{E}+11$

$\mathrm{Rb}-86$

$0.1779 \mathrm{E}-06$

Sr-89

$0.2702 E-04$
Sr -90

$0.1459 \mathrm{E}-05$

sr-91

$0.3474 \mathrm{E}-04$

$0.3474 \mathrm{E}-04$
$\mathrm{Sr}-92$

$0.3616 \mathrm{E}-04$

Y-90

$0.1565 \mathrm{E}-07$

$\mathrm{Y}-91$

$0.3293 E-06$

$\mathrm{Y}-92$

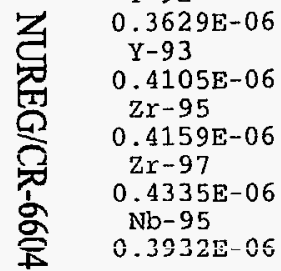

$\begin{array}{ccc}\text { Atoms } & \mathrm{kg} & \mathrm{Ci} \\ 0.2676 \mathrm{E}+00 & 0.2577 \mathrm{E}-25 & 0.8196 \mathrm{E}-18 \\ 0.5567 \mathrm{E}+01 & 0.5546 \mathrm{E}-24 & 0.6270 \mathrm{E}-18 \\ 0.3467 \mathrm{E}+18 & 0.4894 \mathrm{E}-07 & 0.1920 \mathrm{E}-01 \\ 0.7743 \mathrm{E}+15 & 0.1093 \mathrm{E}-09 & 0.8994 \mathrm{E}+00 \\ 0.4017 \mathrm{E}+15 & 0.5804 \mathrm{E}-10 & 0.1644 \mathrm{E}+01 \\ 0.1213 \mathrm{E}+16 & 0.1773 \mathrm{E}-09 & 0.2223 \mathrm{E}+01 \\ 0.4138 \mathrm{E}+00 & 0.5909 \mathrm{E}-25 & 0.4808 \mathrm{E}-17 \\ 0.1701 \mathrm{E}+03 & 0.2514 \mathrm{E}-22 & 0.7304 \mathrm{E}-15 \\ 0.1934 \mathrm{E}+04 & 0.2890 \mathrm{E}-21 & 0.3942 \mathrm{E}-16 \\ 0.1714 \mathrm{E}+01 & 0.2590 \mathrm{E}-24 & 0.9389 \mathrm{E}-15 \\ 0.5089 \mathrm{E}+00 & 0.7774 \mathrm{E}-25 & 0.9772 \mathrm{E}-15 \\ 0.5202 \mathrm{E}-02 & 0.7774 \mathrm{E}-27 & 0.4230 \mathrm{E}-18 \\ 0.2401 \mathrm{E}+01 & 0.3629 \mathrm{E}-24 & 0.8899 \mathrm{E}-17 \\ 0.6672 \mathrm{E}-02 & 0.1019 \mathrm{E}-26 & 0.9808 \mathrm{E}-17 \\ 0.2153 \mathrm{E}-01 & 0.3325 \mathrm{E}-26 & 0.1109 \mathrm{E}-16 \\ 0.3317 \mathrm{E}+01 & 0.5232 \mathrm{E}-24 & 0.1124 \mathrm{E}-16 \\ 0.3805 \mathrm{E}-01 & 0.6129 \mathrm{E}-26 & 0.1172 \mathrm{E}-16 \\ 0.1723 \mathrm{E}+01 & 0.2718 \mathrm{E}-24 & 0.1063 \mathrm{E}-16\end{array}$

Mo-99

$0.5737 \mathrm{E}-05$

TC-99m

$0.4952 \mathrm{E}-05$

$\mathrm{Ru}-103$

$0.4274 \mathrm{E}-05$

$\mathrm{Ru}-105$

$0.2779 \mathrm{E}-05$

$\mathrm{Ru}-106$

$0.9710 \mathrm{E}-06$

Rh-105

$0.1925 \mathrm{E}-05$

Sb-127

$0.5245 \mathrm{E}-05$

$\mathrm{Sb}-129$

$0.1858 \mathrm{E}-04$

$\mathrm{Te}-127$

$0.5065 \mathrm{E}-05$

$\mathrm{Te}-127 \mathrm{~m}$

$0.6706 \mathrm{E}-06$

Te-129

$\mathrm{Bq}$

$0.1744 E-04$

$\mathrm{Te}-129 \mathrm{~m}$

$0.4597 \mathrm{E}-05$

Te-131m

$0.8806 \mathrm{E}-05$

Te-132

$0.8766 \mathrm{E}-04$

I-131

$0.1045 \mathrm{E}+07$

I-132

$0.1540 \mathrm{E}+07$

I -133

$0.2210 \mathrm{E}+07$

I-134

$0.2425 \mathrm{E}+07$

I-135

$0.2084 \mathrm{E}+07$

Xe-133

$0.1947 E+12$

$\mathrm{Xe}-135$

$0.3654 \mathrm{E}+11$

Cs-134

$0.4073 \mathrm{E}-04$

Cs-136

$0.1239 \mathrm{E}-04$

Cs-137

0.2277 E-04

$\mathrm{Ba}-139$

$0.4728 \mathrm{E}-04$

$\mathrm{Ba}-140$

$0.4679 \mathrm{E}-04$

La-140

$0.4781 \mathrm{E}-06$

Ia-141

$0.4385 \mathrm{E}-06$

La -142

$0.4228 \mathrm{E}-06$
$0.1967 \mathrm{E}+01 \quad 0.3233 \mathrm{E}-24 \quad 0.1551 \mathrm{E}-15$

$0.1548 \mathrm{E}+00 \quad 0.2545 \mathrm{E}-25 \quad 0.1338 \mathrm{E}-15$

$0.2092 \mathrm{E}+02 \quad 0.3579 \mathrm{E}-23 \quad 0.1155 \mathrm{E}-15$

0.6409E-01 0.1117E-25 0.7512E-16

$0.4456 \mathrm{E}+02 \quad 0.7844 \mathrm{E}-23 \quad 0.2624 \mathrm{E}-16$

$0.3536 \mathrm{E}+00 \quad 0.6165 \mathrm{E}-25 \quad 0.5204 \mathrm{E}-16$

$0.2517 \mathrm{E}+01 \quad 0.5308 \mathrm{E}-24 \quad 0.1418 \mathrm{E}-15$

$0.4168 \mathrm{E}+00 \quad 0.8928 \mathrm{E}-25 \quad 0.5021 \mathrm{E}-15$

$0.2459 \mathrm{E}+00 \quad 0.5187 \mathrm{E}-25 \quad 0.1369 \mathrm{E}-15$

$0.9111 \mathrm{E}+01 \quad 0.1922 \mathrm{E}-23 \quad 0.1812 \mathrm{E}-16$

$0.1051 \mathrm{E}+00 \quad 0.2251 \mathrm{E}-25 \quad 0.4713 \mathrm{E}-15$

$0.1925 \mathrm{E}+02 \quad 0.4124 \mathrm{E}-23 \quad 0.1242 \mathrm{E}-15$

$0.1372 \mathrm{E}+01 \quad 0.2985 \mathrm{E}-24 \quad 0.2380 \mathrm{E}-15$

$0.3560 \mathrm{E}+02 \quad 0.7804 \mathrm{E}-23 \quad 0.2369 \mathrm{E}-14$

$0.1048 \mathrm{E}+13 \quad 0.2279 \mathrm{E}-12 \quad 0.2825 \mathrm{E}-04$

$0.1840 \mathrm{E}+11 \quad 0.4034 \mathrm{E}-14 \quad 0.4163 \mathrm{E}-04$

$0.2388 \mathrm{E}+12 \quad 0.5273 \mathrm{E}-13 \quad 0.5973 \mathrm{E}-04$

$0.1104 \mathrm{E}+11 \quad 0.2457 \mathrm{E}-14 \quad 0.6555 \mathrm{E}-04$

$0.7154 \mathrm{E}+11 \quad 0.1604 \mathrm{E}-13 \quad 0.5632 \mathrm{E}-04$

$0.1273 \mathrm{E}+18 \quad 0.2812 \mathrm{E}-07 \quad 0.5263 \mathrm{E}+01$

$0.1725 \mathrm{E}+16 \quad 0.3867 \mathrm{E}-09 \quad 0.9875 \mathrm{E}+00$

$0.3824 \mathrm{E}+04 \quad 0.8508 \mathrm{E}-21 \quad 0.1101 \mathrm{E}-14$

$0.2023 \mathrm{E}+02 \quad 0.4569 \mathrm{E}-23 \quad 0.3349 \mathrm{E}-15$

$0.3110 \mathrm{E}+05 \quad 0.7076 \mathrm{E}-20 \quad 0.6155 \mathrm{E}-15$

$0.3385 \mathrm{E}+00 \quad 0.7813 \mathrm{E}-25 \quad 0.1278 \mathrm{E}-14$

$0.7430 \mathrm{E}+02 \quad 0.1727 \mathrm{E}-22 \quad 0.1265 \mathrm{E}-14$

$0.1000 \mathrm{E}+00 \quad 0.2325 \mathrm{E}-25 \quad 0.1292 \mathrm{E}-16$

$0.8951 \mathrm{E}-02 \quad 0.2096 \mathrm{E}-26 \quad 0.1185 \mathrm{E}-16$

$0.3385 \mathrm{E}-02 \quad 0.7982 \mathrm{E}-27 \quad 0.1143 \mathrm{E}-16$ 


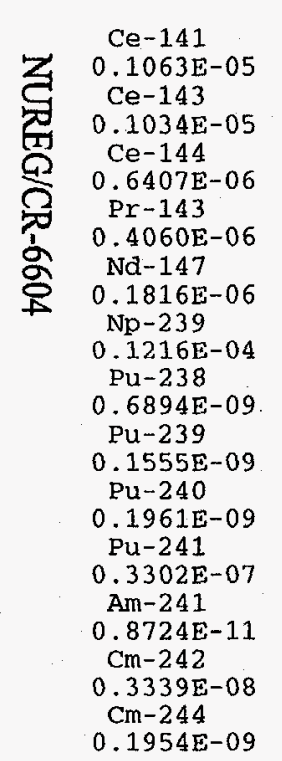

$\begin{array}{lll}0.4308 \mathrm{E}+01 & 0.1009 \mathrm{E}-23 & 0.2874 \mathrm{E}-16 \\ 0.1772 \mathrm{E}+00 & 0.4208 \mathrm{E}-25 & 0.2794 \mathrm{E}-16 \\ 0.2270 \mathrm{E}+02 & 0.5429 \mathrm{E}-23 & 0.1732 \mathrm{E}-16 \\ 0.6863 \mathrm{E}+00 & 0.1630 \mathrm{E}-24 & 0.1097 \mathrm{E}-16 \\ 0.2485 \mathrm{E}+00 & 0.6067 \mathrm{E}-25 & 0.4908 \mathrm{E}-17 \\ 0.3570 \mathrm{E}+01 & 0.1417 \mathrm{E}-23 & 0.3287 \mathrm{E}-15 \\ 0.2754 \mathrm{E}+01 & 0.1088 \mathrm{E}-23 & 0.1863 \mathrm{E}-19 \\ 0.1703 \mathrm{E}+03 & 0.6761 \mathrm{E}-22 & 0.4202 \mathrm{E}-20 \\ 0.5836 \mathrm{E}+02 & 0.2326 \mathrm{E}-22 & 0.5299 \mathrm{E}-20 \\ 0.2165 \mathrm{E}+02 & 0.8663 \mathrm{E}-23 & 0.8924 \mathrm{E}-18 \\ 0.1717 \mathrm{E}+00 & 0.6870 \mathrm{E}-25 & 0.2358 \mathrm{E}-21 \\ 0.6776 \mathrm{E}-01 & 0.2723 \mathrm{E}-25 & 0.9024 \mathrm{E}-19 \\ 0.1611 \mathrm{E}+00 & 0.6527 \mathrm{E}-25 & 0.5280 \mathrm{E}-20\end{array}$

Control Room Transport Group Inventory:

$\begin{array}{llcc}\text { Time }(\mathrm{h})=720.0000 & \text { Atmosphere } & \text { Sump } & \begin{array}{c}\text { Overlying } \\ \text { Pool }\end{array} \\ \text { Noble gases (atoms) } & 0.4782 \mathrm{E}+18 & 0.0000 \mathrm{E}+00 & 0.0000 \mathrm{E}+00 \\ \text { Elemental I (atoms) } & 0.5310 \mathrm{E}-18 & 0.0000 \mathrm{E}+00 & 0.0000 \mathrm{E}+00 \\ \text { Organic I (atoms) } & 0.1387 \mathrm{E}+13 & 0.0000 \mathrm{E}+00 & 0.0000 \mathrm{E}+00 \\ \text { Aerosols (kg) } & 0.3141 \mathrm{E}-18 & 0.0000 \mathrm{E}+00 & 0.0000 \mathrm{E}+00 \\ & \text { Deposition } & \text { Recirculating } \\ & \text { Surfaces } & \text { Filter } \\ \text { Time (h) }=720.0000 & \text { Sulting } & \\ \text { Noble gases (atoms) } & 0.0000 \mathrm{E}+00 & 0.0000 \mathrm{E}+00 & \\ \text { Elemental I (atoms) } & 0.0000 \mathrm{E}+00 & 0.1462 \mathrm{E}+15 & \\ \text { Organic I (atoms) } & 0.0000 \mathrm{E}+00 & 0.3992 \mathrm{E}+16 & \\ \text { Aerosols (kg) } & 0.0000 \mathrm{E}+00 & 0.8697 \mathrm{E}-07\end{array}$

Filter 3--Environment to CR Transport Group Inventory:

$\begin{array}{lc}\text { Time }(\mathrm{h})=720.0000 & \text { Pathway } \\ \text { Filter } \\ \text { Noble gases (atoms) } & 0.0000 \mathrm{E}+00 \\ \text { Elemental I (atoms) } & 0.4348 \mathrm{E}+22 \\ \text { Organic I (atoms) } & 0.1372 \mathrm{E}+25 \\ \text { Aerosols (kg) } & 0.7988 \mathrm{E}+01\end{array}$

Unfiltered--Environment to CR Transport Group Inventory:

$\begin{array}{lc}\text { Time }(h)=720.0000 & \text { Pathway } \\ \text { Noble gases (atoms) } & 0.0000 \mathrm{E}+00 \\ \text { Elemental I (atoms) } & 0.0000 \mathrm{E}+00\end{array}$
Organic I (atoms)
$0.0000 \mathrm{E}+00$

Exhaust from CR to Environment Transport Group Inventory:

$\begin{array}{lc}\text { Time }(\mathrm{h})=720.0000 & \text { Pathway } \\ \text { Filter } \\ \text { Noble gases (atoms) } & 0.0000 \mathrm{E}+00 \\ \text { Elemental I (atoms) } & 0.0000 \mathrm{E}+00 \\ \text { Organic I (atoms) } & 0.0000 \mathrm{E}+00 \\ \text { Aerosols (kg) } & 0.0000 \mathrm{E}+00\end{array}$

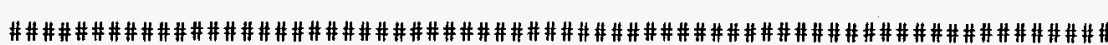
\#\#\#\#\#\#\#

Cumulative Dose Summary

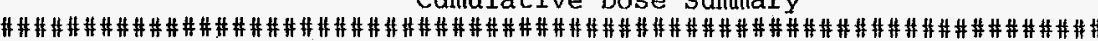
\#\#\#\#\#\#\#

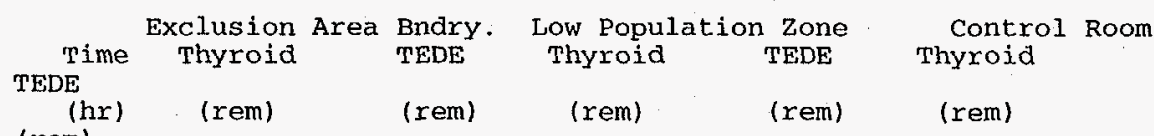

$\begin{array}{ccccc}\text { (hr) } & \text { (rem) (rem) (rem) } & \text { (rem) } & \text { (rem) }\end{array}$

$\begin{array}{llllll}0.03 & 0.4064 \mathrm{E}-01 & 0.1758 \mathrm{E}-02 & 0.5487 \mathrm{E}-02 & 0.2373 \mathrm{E}-03 & 0.3609 \mathrm{E}-04\end{array}$ $0.1403 \mathrm{E}-05$

$\begin{array}{llllll}0.17 & 0.1120 \mathrm{E}+01 & 0.4931 \mathrm{E}-01 & 0.1512 \mathrm{E}+00 & 0.6657 \mathrm{E}-02 & 0.4047 \mathrm{E}-02\end{array}$ $0.1623 \mathrm{E}-03$

$\begin{array}{llllll}0.28 & 0.2653 \mathrm{E}+01 & 0.1186 \mathrm{E}+00 & 0.3582 \mathrm{E}+00 & 0.1601 \mathrm{E}-01 & 0.1517 \mathrm{E}-01\end{array}$ $0.6257 \mathrm{E}-03$

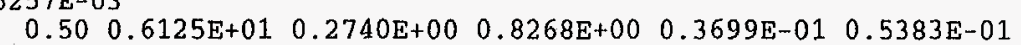
$0.2309 \mathrm{E}-02$

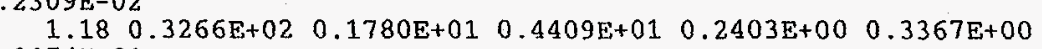
$0.2074 \mathrm{E}-01$

$\begin{array}{cccccc}1.80 & 0.6228 \mathrm{E}+02 & 0.3907 \mathrm{E}+01 & 0.8408 \mathrm{E}+01 & 0.5275 \mathrm{E}+00 & 0.7241 \mathrm{E}+00\end{array}$ $0.6661 \mathrm{E}-01$

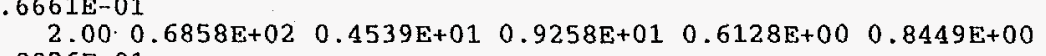
$0.8826 \mathrm{E}-01$

$\begin{array}{llllll}3.00 & 0.6858 \mathrm{E}+02 & 0.4539 \mathrm{E}+01 & 0.1056 \mathrm{E}+02 & 0.9600 \mathrm{E}+00 & 0.1077 \mathrm{E}+01\end{array}$ $.2004 \mathrm{E}+00$

$\begin{array}{llllll}8.00 & 0.6858 \mathrm{E}+02 & 0.4539 \mathrm{E}+01 & 0.1482 \mathrm{E}+02 & 0.3902 \mathrm{E}+01 & 0.1804 \mathrm{E}+01\end{array}$ $0.1258 \mathrm{E}+01$

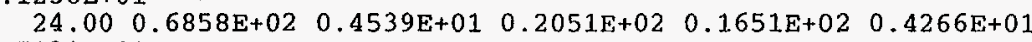

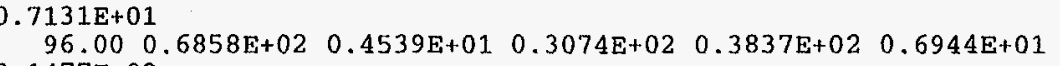
$0.1477 \mathrm{E}+02$

$\begin{array}{llllll}720.00 & 0.6858 \mathrm{E}+02 & 0.4539 \mathrm{E}+01 & 0.6043 \mathrm{E}+02 & 0.1058 \mathrm{E}+03 & 0.1429 \mathrm{E}+02\end{array}$ $0.3619 \mathrm{E}+02$

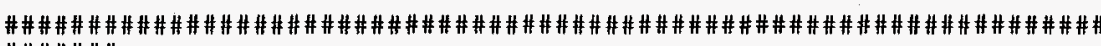

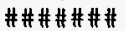

I-131 Summary

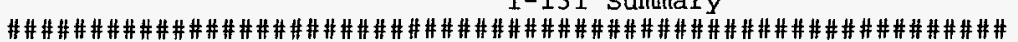

Annulus Sprayed Region Unsprayed Region 


\begin{tabular}{|c|c|c|}
\hline Time (hr) & I-131 (Curies) & I-131 (Curies) \\
\hline $\begin{array}{r}\text { (Curies) } \\
0.03\end{array}$ & $0.2513 \mathrm{E}+06$ & $0.1560 \mathrm{E}+04$ \\
\hline $0.6832 \mathrm{E}+00$ & & \\
\hline 0.17 & $0.9177 \mathrm{E}+06$ & $0.3603 E+05$ \\
\hline $\begin{array}{c}0.1652 \mathrm{E}+02 \\
0.28\end{array}$ & $0.1074 \mathrm{E}+07$ & $0.1421 \mathrm{E}+06$ \\
\hline $0.3544 \mathrm{E}+02$ & & \\
\hline $\begin{array}{c}0.50 \\
0.9123 E+02\end{array}$ & $0.1249 \mathrm{E}+07$ & $0.2381 \mathrm{E}+06$ \\
\hline 1.18 & $0.3194 \mathrm{E}+07$ & $0.6762 \mathrm{E}+06$ \\
\hline $0.4944 \mathrm{E}+03$ & & \\
\hline $\begin{array}{c}1.80 \\
0.9105 \mathrm{E}+03\end{array}$ & $0.3248 \mathrm{E}+07$ & $0.6933 E+06$ \\
\hline $\begin{array}{c}2.00 \\
0.9845 \mathrm{E}+03\end{array}$ & $0.9303 \mathrm{E}+06$ & $0.5760 \mathrm{E}+06$ \\
\hline $\begin{array}{c}3.00 \\
0.9857 \mathrm{E}+03\end{array}$ & $0.9191 \mathrm{E}+05$ & $0.9890 \mathrm{E}+05$ \\
\hline $\begin{array}{c}.903 .00 \\
8.00\end{array}$ & $0.1290 \mathrm{E}+06$ & $0.2730 \mathrm{E}+05$ \\
\hline $\begin{array}{c}0.7281 \mathrm{E}+03 \\
24.00\end{array}$ & $0.1020 \mathrm{E}+06$ & $0.2170 \mathrm{E}+05$ \\
\hline $\begin{array}{c}0.3584 \mathrm{E}+03 \\
96.00\end{array}$ & $0.5746 \mathrm{~F}+05$ & $0.1250 E+05$ \\
\hline $0.7104 \mathrm{E}+02$ & & \\
\hline $\begin{array}{c}720.00 \\
0.5428 \mathrm{E}+02\end{array}$ & $0.4618 \mathrm{E}+05$ & $0.1013 \mathrm{E}+05$ \\
\hline & Environment & Control Room \\
\hline $\begin{array}{c}\text { Time }(\mathrm{hr}) \\
0.03\end{array}$ & I-131 (Curies) & I-131 (Curies) \\
\hline 0.03 & $0.7591 \mathrm{E}-01$ & $0.2569 E-05$ \\
\hline 0.17 & $0.3496 \mathrm{E}+00$ & $0.6307 \mathrm{E}-04$ \\
\hline 0.28 & $0.7994 \mathrm{E}+00$ & $0.1374 \mathrm{E}-03$ \\
\hline 0.50 & $0.1572 \mathrm{E}+01$ & $0.2072 \mathrm{E}-03$ \\
\hline 1.18 & $0.2093 \mathrm{E}+01$ & $0.5508 \mathrm{E}-03$ \\
\hline 1.80 & $0.4373 \mathrm{E}+01$ & $0.634 \hat{9 E}-03$ \\
\hline 2.00 & $0.2042 \mathrm{E}+01$ & $0.5077 \mathrm{E}-03$ \\
\hline 3.00 & $0.5646 \mathrm{E}+00$ & $0.1388 \mathrm{E}-03$ \\
\hline 8.00 & $0.3073 \mathrm{E}+01$ & $0.1555 \mathrm{E}-03$ \\
\hline 24.00 & $0.1356 \mathrm{E}+02$ & $0.1618 \mathrm{E}-03$ \\
\hline 96.00 & $0.2558 \mathrm{E}+02$ & $0.5299 \mathrm{E}-04$ \\
\hline 720.00 & $0.2349 \mathrm{E}+02$ & $0.2825 \mathrm{E}-04$ \\
\hline
\end{tabular}




\subsection{Example Cases}

\subsubsection{Example Problem 1 Description}

Example problem 1 is a containment leak from a pressurized water reactor (see Figure 1.6.1-1). It uses the TID-14844 release for the source term. As the iodine is released from the containment, the code calculates the dose at the low population zone. The sample is similar to acceptance test case 2, which has an analytic solution. The data used will vary according to plant and will be known by the user. In this sample problem, the user will be guided through each screen to familiarize him or her with the general process.

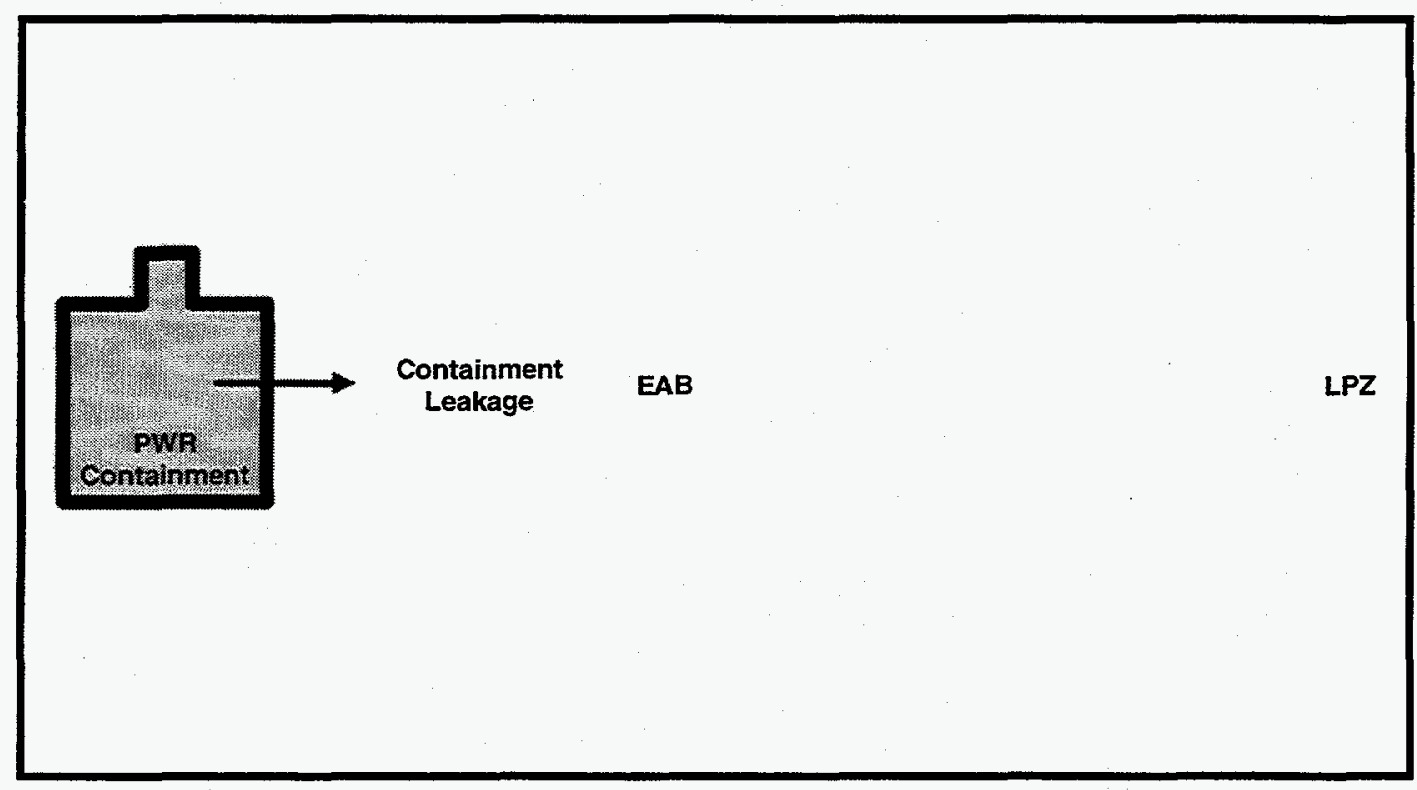

Figure 1.6.1-1. PWR Containment Leakage

\section{Source Term}

Release fractions and timing: TID-14844, Table IV with modifications per NRC Regulatory Guides 1.3 and 1.4 (removes mixed fission products and details iodine fractions) and further modified as described below.

Start of release:

$0.0000 \mathrm{hr}$

Iodine fractions:

Fraction of released iodine chemical form:

Elemental: $\quad 0.9100$

Organic: $\quad 0.0400$

Aerosol: $\quad 0.0500$

Inventory:

TID-14844 example normalized core inventory.

Reactor power:

$1932 \mathrm{MWt}$ 
Plant Model

Containment volume: $\quad 0.1730 \mathrm{E}+07 \mathrm{ft}^{3}$

Environment

Containment leakage: Air leakage from: Containment to: Environment

Scenario Description

Containment leak rate: $\quad 0.1200 \% /$ day

Dose Parameters

$\begin{array}{lll}\text { LPZ X/Q values: } & \text { Time }(\mathrm{hr}) & \mathrm{X} / \mathrm{Q}\left(\mathrm{s} / \mathrm{m}^{3}\right) \\ & 0.0000 & 0.1350 \mathrm{E}-03 \\ 8.0000 & 0.1000 \mathrm{E}-03 \\ 24.0000 & 0.5400 \mathrm{E}-04 \\ 96.0000 & 0.2200 \mathrm{E}-04\end{array}$

Environmental

Breathing Rates: Time (hr) Breathing Rate $\left(\mathrm{m}^{3} / \mathrm{s}\right)$

$0.0000 \quad 0.3470 \mathrm{E}-03$

$8.0000 \quad 0.1750 \mathrm{E}-03$

$24.0000 \quad 0.2320 \mathrm{E}-03$

\subsubsection{RADTRAD Screen Input}

As noted earlier, the input should be entered in a sequential order, as opposed to partially entering the data on a screen, moving on to another screen, and then returning. While the code has been heavily tested, all possible patterns of inputting data have not.

To run this example problem, the following steps should be followed:

Go to the RADTRAD directory and type RADTRAD; this will bring up a welcome screen.

Touch any alphanumeric key; this will bring up screen number 0000 , which allows the user to input, execute, or examine a case.

Enter the number 1, or click on the pad labeled Plant Model. This will bring up screen number 1000 , the Plant Model menu.

Since this is a new case, there is no reason to touch the first pad and retrieve an old plant model. Enter the number 2, or click on the pad labeled Nuclide Inventory. This will bring up screen number 1200.

The nuclide inventory menu allows the user to initialize the core radionuclide inventory. There are several defaults shown that are detailed in Section 1.4.3.2. The user should select the button under Default TID Inventory. The plant power is used as a multiplier on the inventory; hence a 
3000-MWt plant will have three times the inventory of radionuclides as a $1000 \mathrm{MWt}$. For this case, the user should input $1.932 E+03$ on the pad next to the label Plant Power. When the user selected the TID button for the default inventory the location of the file came up on the File Name pad. If the user wants to follow only the ${ }^{131}$ I nuclide, there is a TID inventory file available called "DEFAULTSITID_I131.NIF". The user should leave the file name alone for this example and click on OK. This will return the user to screen 1000.

The user should continue developing the plant model by entering the number 3 , or clicking on the pad labeled Compartment Description. This will bring up screen 1300.

Screen 1300 is the Select Compartment screen and at this point should show Unused for all 10 compartments. The first unused is highlighted; the user should click on EDIT or hit RETURN. This will bring up screen 1310.

Screen 1310 allows the user to identify the compartments. The user should enter Containment on the pad following the label Compartment Name. A containment is neither a control room nor an environment, so the user should select the button next to Other on the pad next to the label Compartment Type. The user should input $0.1730 E+07$ on the pad next to the label Compartment Volume. In this example problem, no engineered or natural safety features will be activated, hence the user should click on $\mathbf{O K}$. This will return the user to screen number 1300.

Screen number 1300 now has the label Containment in the first row and the second row should be highlighted. The user should again click on EDIT to add the next compartment for this problem. This will return the user to screen 1310.

On screen 1310, the user should enter Environment on the pad following the label Compartment Name. The user should select the button next to Environment on the pad next to Compartment Type. Note that both the volume and features options have disappeared. This is because the environment does not have either a set volume or engineered safety features. The user should click on OK. This will return the user to screen number 1300 .

Screen 1300 now has the two labeled volumes, Containment and Environment, that this example requires. The user should click on $\mathbf{O K}$. This will return the user to screen number 1000 .

The user should continue developing the plant model by entering the number 4 , or clicking on the pad labeled Transfer Pathways. This will bring up screen 1400.

Screen 1400 is the Select Pathway screen, which allows the user to define how radionuclides will move between the compartments just defined. The user should highlight the first "Unused" pathway and click on EDIT. This will bring up screen 1410.

Screen 1410 is the Transfer Pathway Description screen. The user should enter Air Leakage on the pad beneath the label Pathway Name. In this example both air and radionuclides will leak from the containment to the environment. The user should now double click on the pad beneath the label Pathway from Compartment. This will bring up screen number 1411. 
Screen 1411 has the list of the compartments the user defined previously. The user should select the first compartment, Containment, and then click an $\mathbf{O K}$ to return to screen 1410. The input system now knows where the flow is coming from.

Screen 1410 now has the name Containment on the pad below the Pathway from Compartment. The user should double click on the pad beneath the label F'athway to Compartment to identify the environment as the receiving volume of the flow. This will bring up screen 1411 , where the user should highlight the environment compartment and click $\mathrm{OK}$ to return to screen 1410 . The user should select the button next to the Air Leakage label on the list of Transfer Mechanisms. This will activate the correct linkages for later input. The user should click on $\mathbf{O K}$. This will return the user to screen number 1400 .

Screen 1400 now shows the only transport path, Air Leakage. The user should click on OK. This will return the user to screen number 1000.

The user may enter the number 5, or click on the pad labeled Review Plant Model, to look at the input selected for the plant model. Screen number 1500 cannot be edited; therefore any changes to the input can only be made by returning to screen 1000 and selecting the appropriate subscreen to change the data. The return to screen 1000 from this screen is accomplished by hitting the ESC key on the keyboard.

The user should enter the number 6, or click on the pad labeled Save Plant Model, to save input data. This will activate screen number 1600 . It is recommended that the user select an appropriate name with the convention of XXX.PMF where the PMF stands for plant model file. For this example use SAMPLE1.PMF. Clicking on OK will bring up screen number 1000.

The plant model is now complete. Enter the number 7 , or click on the pad labeled Return to Main Menu to return to screen number 0000 and continue the input process.

The user should enter the number 2, or click on the pad labeled Scenario Description to continue the development of the input example. This will bring up screen number 2000 .

Screen 2000 is the controlling screen for describing the scenario. As with the plant model description, there is no model to retrieve, so the user should enter the number 2 , or click on the pad labeled Source Term Parameters. This will bring up screen 2200.

Screen 2200 lets the user describe the radionuclide release. The user should enter the number 1 , or click on the pad labeled Source Term Compartment; this will bring up screen 2210 . Screen 2210 allows the user to choose where the source is located. In this case, the user should highlight the Containment compartment and click on $\mathrm{OK}$ to return to screen 2200. The user should enter the number 2, or click on the pad labeled Release Fractions and Timing. This will bring up screen 2220.

Screen 2220 allows the user to choose from several default: release systems, or to design his or her own. The TID Release Type should be selected. Note that it has been preselected because this example chose the TID inventory for the plant model. There is no delay time between reactor shutdown and radionuclide release in this example, so the default value of $0.0 \mathrm{hr}$ is 
correct. Note that the TID release fraction file table has been listed on the file name pad. The user may change it by clicking on EDIT to the right. For this example, the user should click on OK to return to screen 2200 .

This case will not activate the decay chain processing, nor the daughtering system, so the user will skip pad 3 and enter the number 4, or click on the pad labeled lodine Chemical Form. This will activate screen number 2240. The user should enter $0.91,0.04$, and 0.05 for elemental, organic, and aerosol iodine chemical fractions for this example. These values are typical of the TID source term assumptions. The user should click on OK to return to screen 2200 . The source term form has now been described. The user should enter the number 5 , or click on the pad labeled Return to Scenario Description to return to screen number 2000.

The user should continue developing the scenario model by entering the number 3 , or clicking on the pad labeled Compartment Features. This will bring up screen 2300 . Screen 2300 lists the two compartments described in the plant model. The user should highlight the Containment compartment and click on EDIT. This will bring up screen number $2300 \mathrm{~A}$.

Screen 2300A allows the user access to the removal features selected in the plant model. If a feature was selected, an $\mathrm{X}$ will be in the box to the left of the appropriate pad, indicating that it had been selected and that the pad could be activated. Detailed output is not needed for this case so the default is correct. The user should select $\mathbf{O K}$, which will return to screen number 2300 . There is also nothing to add to the environment volume, so the user should select $\mathbf{O K}$ again and return to screen number 2000 .

The user should continue developing the scenario model by entering the number 4, or clicking on the pad labeled Transfer Pathways. This will bring up screen 2400 . Screen 2400 lists the air leakage pathway described by the plant model. Highlighting this pathway and clicking on EDIT will bring up the screen associated with this transfer pathway, in this case screen number $2440 \mathrm{~T}$.

Screen $2440 \mathrm{~T}$ allows the user to enter the leakage flow versus time. In this example the user should select the top row of Time and then enter 0.0,0.12,720.0, and 0.12, with a return after each number. This should fill the table with two time entries $(0.0$ and $720 \mathrm{hr})$ and two leakage entries (both 0.12\%/day). The user should select the Yes button on the pad next to the label Select User Leakage Rates. Yes allows the table to be saved and be used by the code, a No only allows it to be saved. The user should click on OK to return to screen 2400. Because this example uses only one transport path, the user should click on $\mathbf{O K}$ again to return to screen number 2000.

The user should continue developing the scenario model by entering the number 5 , or clicking on the pad labeled Dose Parameters. This will bring up screen 2500. Screen 2500 controls the dose calculation. The user should enter the number 1, or click on the pad labeled Select Dose Conversion Factors. This will bring up screen 2510.

Screen 2510 allows the user to select the dose conversion factors to be used in the calculation. Several default tables are given and the user should verify that the button associated with the 
FGR 11 and 12 label has been selected. The default tables are listed in Section 1.4.3.3. The user should click on the pad labeled TID-14844 and the OK button to return to screen 2500 .

The user should enter the number 2, or click on the pad labeled Create/Modify Dose Locations to continue the input of dose information. This action will bring up screen 2520 , which will eventually list all the locations where the user will want a dose to be calculated. Typically, the user seeks to calculate doses in the control room, the exclusion area boundary, and at the low population zone. In this example calculation, only the low population zone information will be input. Clicking on EDIT will bring up screen 2520A and allow the user to input this data.

On screen 2520A the user should enter Low Population Zone on the pad beneath the label Location Name. The user should then double click on the pad beneath the label Compartment Name. This action will bring up screen 2521 and a list of all compartments. The user should highlight the Environment compartment and click on OK. This will return the user to screen 2520A. The user should verify that the button labeled Environment under Selected Type has been selected. The user should double click on Edit Dose Location Parameters to finish the dose input. This action will bring up screen 2523.

Screen 2523 allows the user to input dilution and breathing rates. If the user selects the top row of the left Time table and inputs, $0.0 \quad 0.1350 E-03 \quad 0.0 \quad 0.3470 e-03 \quad 8.0 \quad 0.1000 e-03 \quad 8.0$

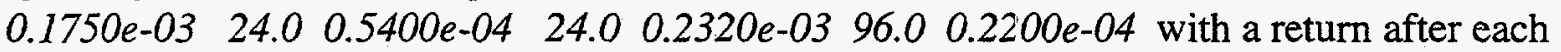
value, the result should be two tables. The concentration table should have four time and value entries and the breathing rate table should have three entries. The user selects $\mathbf{O K}$ to return to screen 2520A. The user selects OK again to return to screen 2500.

The user enters 3, or clicks on the pad labeled Return to Scenario Description Menu to move from screen 2500 to screen 2000 . The user may enter the number 6 , or click on the pad labeled Review Scenario Description to look at the input selected for the scenario description. Screen number 2600 cannot be edited; therefore any changes to the input can only be made by returning to screen 2000 and selecting the appropriate subscreen to change the data. The return to screen 2000 from this screen is obtained by using the ESC key on the keyboard.

The user should enter the number 7, or click on the pad labeled Save Scenario Description to save input data. This will activate screen number 2700 . It is recommended that the user select an appropriate name with the convention of XXX.SDF where the SDF stands for scenario description file. It is recommended that the XXX used for the scenario description file be the same as the XXX for the plant model file. For this example use SAMPLE1.SDF. Clicking on OK will bring up screen number 2000.

The user enters the number 8, or clicks on the pad labeled Return to Main Menu to return to screen number 0000 . The input has now been entered and the user should enter the number 3, or click on the pad labeled Calculate Results to move to screen 3000 and start execution.

Screen 3000 allows the user to control several items including:

1. The name of the output file. The default is OUTPUT.TXT 
2. The supplemental time steps. The user adds additional time steps to the calculation using the maximum time step allowed table. For most cases, a time step of $0.25 \mathrm{hr}(15 \mathrm{~min})$ for the first $4 \mathrm{hr}$, a 1-hr time step between 4 and $24 \mathrm{hr}$, and a 4-hr time step between 1 day and 30 days are reasonable.

3. Amount of output printed. This depends on whether the user wants information only when a phenomenon changes (for example, the breathing rate changes at 8 and $24 \mathrm{hr}$ ) or wants the output for all time steps (this includes those from the supplemental time steps).

4. Type of output printed. The run-time removal coefficients are included when the Include Model Run-Time Information button is selected. In addition, the output of the information input is controlled from the plant model and the scenario description.

5. Execution initialization. The user should click on the CALCULATE button to begin execution. The pad to the left of the button should change from empty to full at a speed related to the execution speed (in this case very quickly; a few seconds). The output file will be displayed on screen when the calculation terminates.

The general output is described in Section 1.6.2. In this case, the user should compare the resulting output with the output in the following section. The user may exit RADTRAD by pulling down the file menu and clicking on EXIT. This action will bring up screen number 5000 , which can be used to save the input files. When the user clicks on OK, RADTRAD will return to the DOS command line. 


\subsubsection{Example Problem 1 Output}

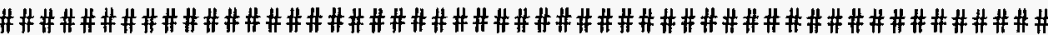 \#\#\#\#\#\#\#\# \\ RADTRAD Version $2.12 \quad 06 / 19 / 97$}

$12: 00: 52.94$

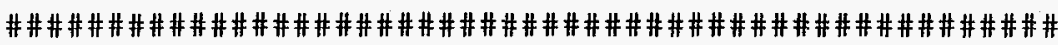
\#\#\#\#\#\#\#\#\#

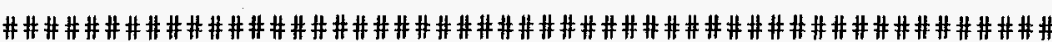
\#\#\#\#\#\#\#\#

File information

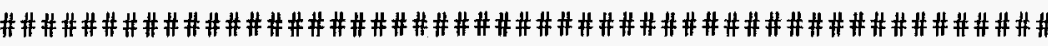
\#\#\#\#\#\#\#\#

\section{Plant filename}

Inventory filename

Scenario filename

Release filename

$=\mathrm{D}: \backslash$ RADMANUA $\backslash$ SAMPLE1. PMF

$=$ DEFAULTS $\backslash T I D \_D E F . N I F$

= D: \RADMANUA \SAMPLE1.SDF

$=$ DEFAUITS $\backslash T I D$ DEF .RFT

Dose conversion filename $=$ DEFAULTS $\backslash T I D 14$. INP

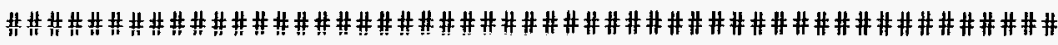
\#\#\#\#\#\#\#\#\#

$$
\text { Plant Description }
$$

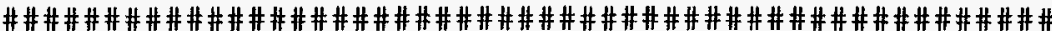
\#\#\#\#\#\#\#\#

Number of Nuclides $=14$

Inventory Power $=0.1000 \mathrm{E}+01 \mathrm{MWth}$

Plant Power Level $=0.1932 \mathrm{E}+04 \mathrm{MWth}$

\section{Compartment information}

Compartment number 1 (Source term compartment)

Name: Containment

Compartment volume $=0.1730 \mathrm{E}+07$ (Cubic feet)
Pathways into and out of compartment 1

Pathway to compartment number 2: Air Leakage

Compartment number 2

Name: Environment

Pathways into and out of compartment 2

Pathway from compartment number 1: Air Leakage

Total number of pathways $=1$

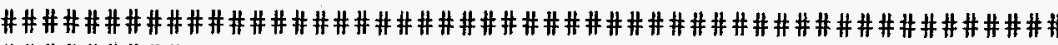
\#\#\#\#\#\#\#\#

Scenario Description

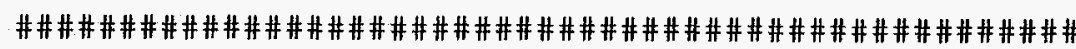
\#\#\#\#\#\#\#\#

Iodine fractions

$$
\begin{array}{ll}
\text { Aerosol } & =0.5000 \mathrm{E}-01 \\
\text { Elemental } & =0.9100 \mathrm{E}+00 \\
\text { Organic } & =0.4000 \mathrm{E}-01
\end{array}
$$

COMPARTMENT DATA

Compartment number 1: Containment

Compartment number 2: Environment

PATHWAY DATA

Pathway number 1: Air Leakage

$$
\begin{array}{lr}
\text { Convection Data } \\
\text { Time (hr) } & \text { Flow Rate (\% / day) } \\
0.0000 \mathrm{E}+00 & 0.1200 \mathrm{E}+00 \\
0.7200 \mathrm{E}+03 & 0.1200 \mathrm{E}+00
\end{array}
$$

LOCATION DATA

Location Low Population Zone is in compartment 2

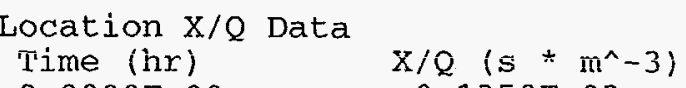




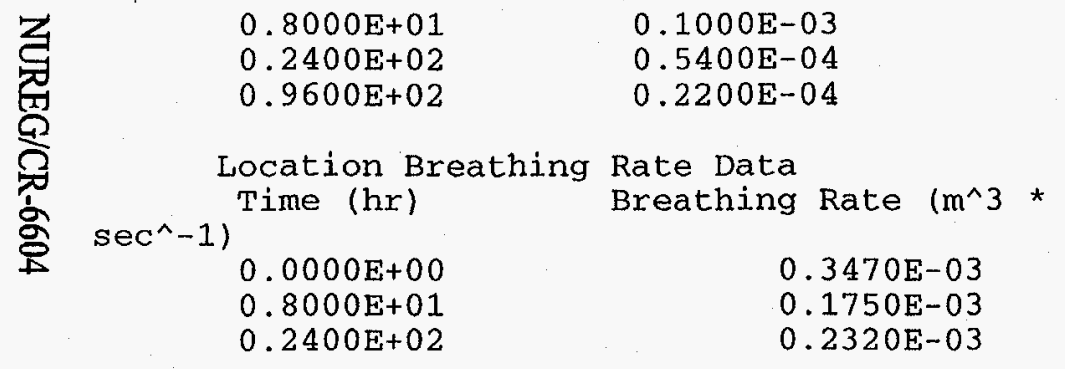

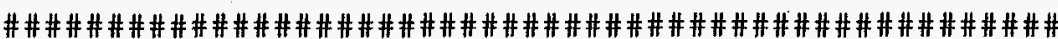
\#\#\#\#\#\#\#\#

Dose Output

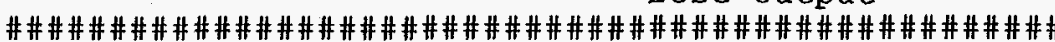
\#\#\#\#\#\#\#\#\#

Low Population Zone Doses:

$\begin{array}{lll}\text { Time }(\mathrm{h})=0.0100 & \text { Whole Body } & \text { Thyroid } \\ \text { TEDE } & 0.2676 \mathrm{E}-02 & 0.2205 \mathrm{E}+00 \\ \text { Delta dose (rem) } & & \\ 0.9725 \mathrm{E}-02 & & \\ \text { Accumulated dose (rem) } & 0.2676 \mathrm{E}-02 & 0.2205 \mathrm{E}+00 \\ 0.9725 \mathrm{E}-02 & & \end{array}$

Low Population Zone Doses:

$\begin{array}{lll}\text { Time }(\mathrm{h})=8.0000 & \text { Whole Body } & \text { Thyroid } \\ \text { TEDE } & 0.4275 \mathrm{E}+01 & 0.3523 \mathrm{E}+03 \\ \text { Delta dose (rem) } & & \\ 0.1554 \mathrm{E}+02 & & \\ \text { Accumulated dose (rem) } & 0.4278 \mathrm{E}+01 & 0.3525 \mathrm{E}+03 \\ 0.1555 \mathrm{E}+02 & & \end{array}$

Low Population Zone Doses:

$\begin{array}{lcl}\text { Time }(\mathrm{h})=24.0000 & \text { Whole Body } & \text { Thyroid } \\ \text { TEDE } & 0.6338 \mathrm{E}+01 & 0.2634 \mathrm{E}+03 \\ \text { Delta dose (rem) } & 0.1062 \mathrm{E}+02 & 0.6159 \mathrm{E}+03 \\ 0.1476 \mathrm{E}+02 & & \\ \text { Accumulated dose (rem) } & 0.030 \mathrm{E}+02\end{array}$

Low Population Zone Doses:

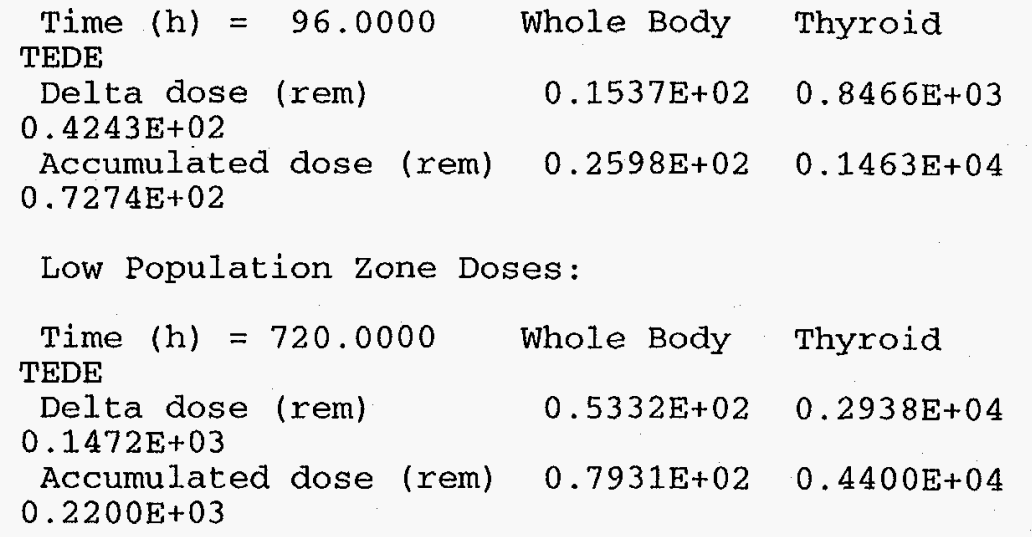

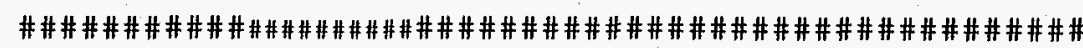
\#\#\#\#\#\#\#\#\#\#\#\#\#\#\#

Cumulative Dose Summary

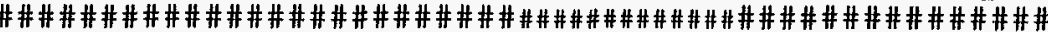

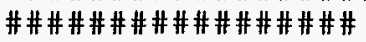

\begin{tabular}{|c|c|c|}
\hline Time & $\begin{array}{l}\text { Low Population } \\
\text { Thyroid }\end{array}$ & TEDE \\
\hline$(h r)$ & (rem) & (rem) \\
\hline 0.01 & $0.2205 E+00$ & $0.9725 E-02$ \\
\hline 8.00 & $0.3525 E+03$ & $0.1555 E+02$ \\
\hline 24.00 & $0.6159 \mathrm{E}+03$ & $0.3030 \mathrm{E}+02$ \\
\hline 96.00 & $0.1463 \mathrm{E}+04$ & $0.7274 \mathrm{E}+02$ \\
\hline 720.00 & $0.4400 \mathrm{E}+04$ & $0.2200 \mathrm{E}+03$ \\
\hline
\end{tabular}

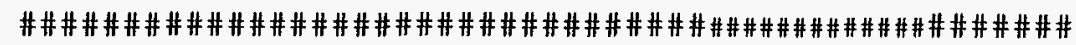

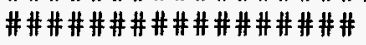

I-131 Summary

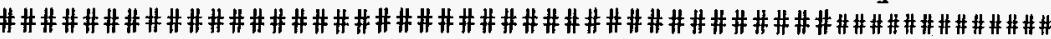

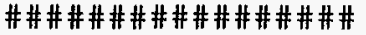

$\begin{array}{rrr} & \text { Containment } \\ \text { Environment } & \text { I-131 (Curies) } & \text { I-131 (Curies) } \\ \text { Time (hr) } & 0.1185 \mathrm{E}+08 & 0.2962 \mathrm{E}+01 \\ 0.01 & 0.1184 \mathrm{E}+08 & 0.4732 \mathrm{E}+04 \\ 8.00 & 0.1183 \mathrm{E}+08 & 0.9471 \mathrm{E}+04 \\ 24.00 & 0.1179 \mathrm{E}+08 & 0.4253 \mathrm{E}+05 \\ 96.00 & 0.1143 \mathrm{E}+08 & 0.3622 \mathrm{E}+06\end{array}$




\subsubsection{Example Problem 2 Description}

The second example problem is a boiling water reactor with a specified $\operatorname{TID}^{131}$ I leak through the main steam line to the condenser and then to the environment (see Figure 1.6.2-1). In this example there will be a specified deposition and reevolution of the iodine on the pipe walls. After the release from the turbine building, the code will calculate the doses at the exclusion area boundary, the low population zone, and in the control room. This case was also used for acceptance test case 13 .

The data below should be known by the user. In general the user should define the plant model first, that is, those values going into screen numbers between 1000 and 1600 , and then define the scenario. After developing the model, the user can compare the input with that provided in Section 1.6.2.1.

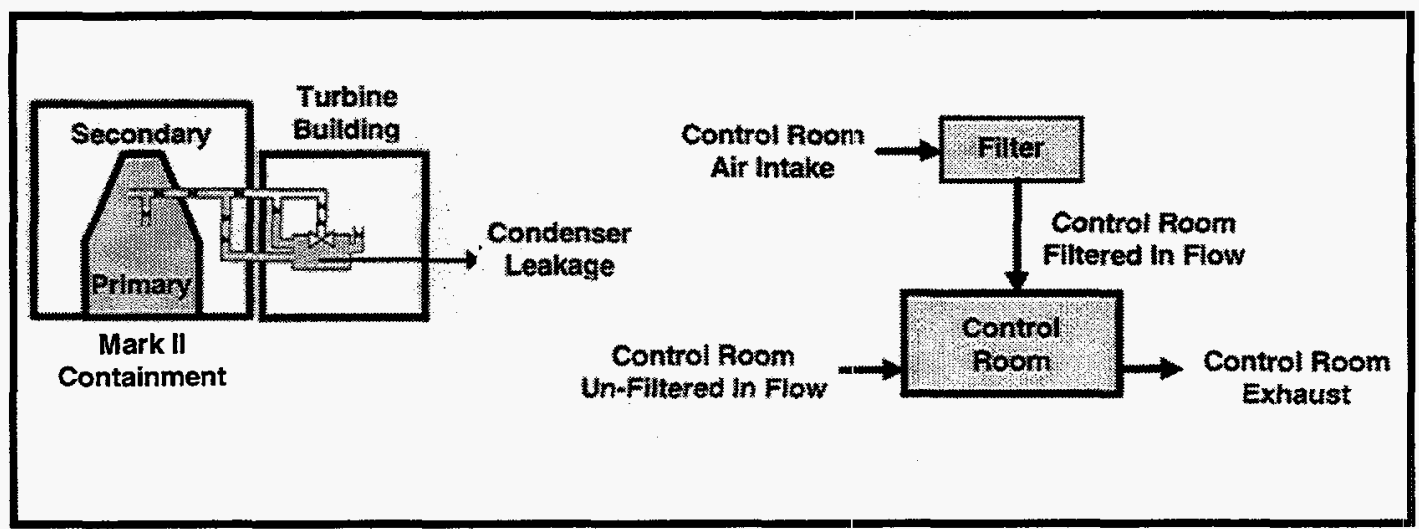

Figure 1.6.2-1. BWR-Mark II Containment Control Room Detail

\section{Source Term}

Release fractions and timing:

TID-14844, Table IV with modifications per NRC Regulatory Guide 1.3 and further modified as described below.

[screen number 2220]

Start of release: $\quad 0.0000 \mathrm{hr}$

[screen number 2220]

Iodine fractions: 
Fraction of released iodine chemical form:

$\begin{array}{ll}\text { Elemental: } & 0.8760 \\ \text { Organic: } & 0.0740 \\ \text { Aerosol: } & 0.0500\end{array}$

[Note that these values are not the standard $0.91,0.04$ and 0.05 . In this case there is a specified iodine deposition. The iodine deposition was obtained from the Cline model, which accounts for deposition and reevolution due to chemisorption. This results in the growth of organic iodines over time at the expense of elemental iodines. To implement these effects in RADTRAD, it was necessary to generate the maximum organic iodine inventory from the fraction released plus the fraction produced and then to deposit decreasing amounts of the organics over time. Therefore the environmental release is initially a small amount of organic iodines followed by a larger amount of organics, thus duplicating the Cline results.]

[screen number 2240]

Inventory: $\quad$ TID-14844 example normalized core inventory modified as follows:

${ }^{131}$ I only [Note that this is not one of the default options. The group of inventory files supplied with RADTRAD is available in the Defaults subdirectory.] The user should use file TID_I131.NIF for this case

[screen number 1200]

Reactor power: $3441 \mathrm{MWt}$

[screen number 1200]

\section{Plant Model}

Containment volume:

$0.3892 \mathrm{E}+06 \mathrm{ft}^{3}$

Effective condenser volume:

$0.9860 \mathrm{E}+05 \mathrm{ft}^{3}$

Control room volume:

$0.1100 \mathrm{E}+06 \mathrm{ft}^{3}$

Environment

[all four values for name and the three values for volume are on screen number 1310]

$\begin{array}{llll}\text { Containment leakage: } & \text { Air leakage } & \text { from: Containment } & \text { to: Condenser } \\ \text { Condenser leakage: } & \text { Piping } & \text { from: Condenser } & \text { to: Environment } \\ \text { Filter: } & \text { Filtered pathway } & \text { from: Environment } & \text { to: Control room } \\ \text { CR unfiltered inflow: } & \text { Filtered Pathway } & \text { from: Environment } & \text { to: Control room }\end{array}$


CR exhaust:

Filtered Pathway from: Control room to: Environment

[All five transfer pathways are defined (name, from and to compartments, and transfer mechanism) on screen number 1410.]

\section{Scenario Description}

Containment leak rate: $1.851 \% /$ day

[screen number 2440T]

Condenser leak rate: $\quad 0.7666 \mathrm{cfm}$

[screen number 2410T]

Condenser

Pipe deposition: $\quad$ Time $(\mathrm{hr})$

Decontamination Factors

Efficiency (\%) Aerosol Elemental Organic

$0-48$

900.

900.

5.555

48-96

900.

900.

2.707

96-200

900.

900.

1.639

200-300

900.

900.

1.219

$300-$

900.

900.

1.0417

4

[A decontamination factor of 900 is the equivalent of a $100 \%$ efficient deposition, as assumed in the Cline model. The effect of reducing deposition efficiency for the organics reflects reevolution due to chemisorption effects, which releases organic iodine from the interaction of elemental iodine and the material on the wall surface. These values, when used with larger organic release fractions, replicate the results of the Cline model for release to the environment through a pipe and condenser. Screen number 2410T]

CR filter flow rate: $\quad 5800 \mathrm{cfm}$

[screen number 2420T]

CR filter efficiencies:

$\begin{array}{ll}\text { Elemental iodine } & 99.0000 \% \\ \text { Organic iodine } & 0.0000 \% \\ \text { Aerosols } & 99.0000 \%\end{array}$

[screen number 2420T] 
CR unfiltered inflow:

$10 \mathrm{cfm}$

[This accounts for leakage into the room through doorways and other unfiltered leaks. All the efficiencies will be $0.0 \%$ for this example. Screen number 2420T]

CR exhaust:

$5810 \mathrm{cfm}$

[This exhaust is through filters and will not affect the environmental dose. All the efficiencies are set at $100 \%$ for this example. Screen number 2420T]

\section{Dose Parameters}

$\begin{array}{lll}\text { EAB X/Q values: } & \text { Time (hr) } & \text { X/Q }\left(\mathrm{s} / \mathrm{m}^{3}\right) \\ & 0.0000 & 0.1100 \mathrm{E}-02 \\ 2.0000 & 0.0000 \mathrm{E}+00 \\ & & \\ & & \\ \text { LPZ X/Q values: } & \text { Time (hr) } & \text { X/Q }\left(\mathrm{s} / \mathrm{m}^{3}\right) \\ & 0.0000 & 0.5200 \mathrm{E}-04 \\ & 8.0000 & 0.3600 \mathrm{E}-04 \\ & 24.0000 & 0.1600 \mathrm{E}-04 \\ & 96.0000 & 0.5300 \mathrm{E}-05\end{array}$

Environmental

breathing rates:

$\begin{array}{ll}\text { Time }(\mathrm{hr}) & \text { Breathing Rate }\left(\mathrm{m}^{3} / \mathrm{s}\right) \\ 0.0000 & 0.3470 \mathrm{E}-03 \\ 8.0000 & 0.1750 \mathrm{E}-03 \\ 24.0000 & 0.2320 \mathrm{E}-03\end{array}$

[The locations are on screen number 2520 and the values are on screen number 2523.]

$\begin{array}{lll}\text { CR filter X/Q values: } & \text { Time }(\mathrm{hr}) & \mathrm{X} / \mathrm{Q}\left(\mathrm{s} / \mathrm{m}^{3}\right) \\ & 0.0000 & 0.3320 \mathrm{E}-03 \\ 8.0000 & 0.1960 \mathrm{E}-03 \\ & 24.0000 & 0.7640 \mathrm{E}-04 \\ & 96.0000 & 0.2190 \mathrm{E}-04\end{array}$

[These values are also used for the unfiltered inlets and are input on screen number $2420 \mathrm{~T} 2$ with the efficiencies and flows.] 
$\begin{array}{lll}\mathrm{CR} \text { breathing rates: } & \text { Time }(\mathrm{hr}) & \text { Breathing Rate }\left(\mathrm{m}^{3} / \mathrm{s}\right) \\ & 0.0000 & 0.3470 \mathrm{E}-03\end{array}$

CR occupancy factor: Time (hr) Occupancy Fiactor

$\begin{array}{ll}0.0000 & 1.000 \\ 24.000 & 0.600 \\ 96.0000 & 0.400\end{array}$

[The locations are on screen number 2520 and the values are on screen number 2522.] 
$\mathrm{T} 0+\mathrm{H} 058 \mathrm{~T} \cdot 0$

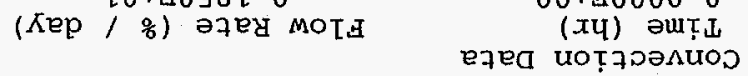

$00+30000^{\circ} 0$

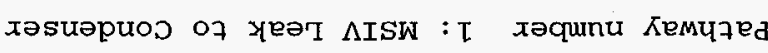

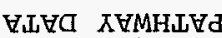

$$
\begin{aligned}
& \text { mooy roxzuos : }
\end{aligned}
$$

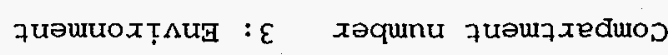

$$
\begin{aligned}
& \text { xәsuәрuор : } z \text { xәqumu quawzxeđuros }
\end{aligned}
$$

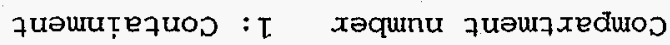

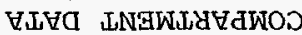

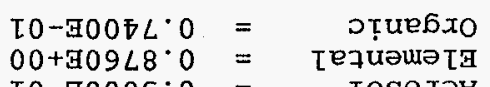

$$
\begin{aligned}
& \text { T. } 0-30005 \cdot 0=\text { tosoxay } \\
& \text { suoț7oexf ouṭpoI }
\end{aligned}
$$

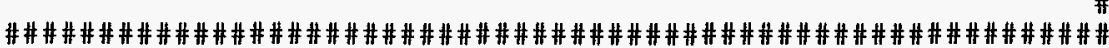

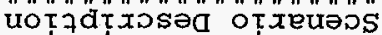

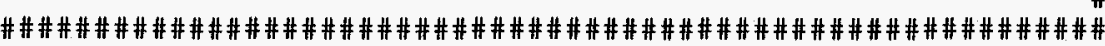

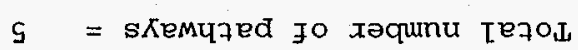

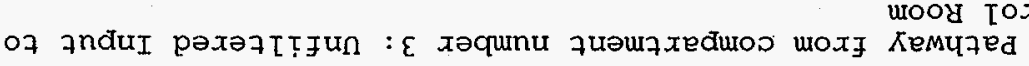

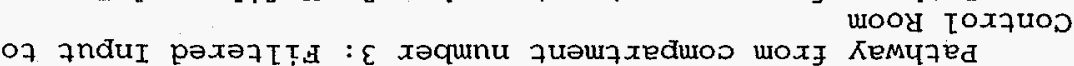

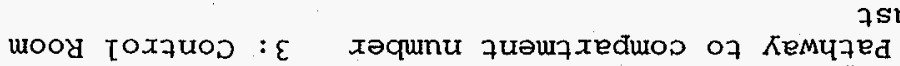
7 sneyxg

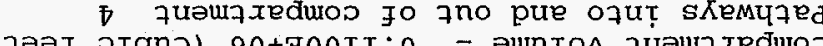

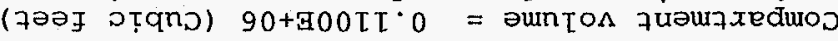
mooy toxzuos : כuren

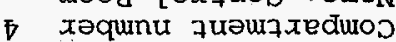

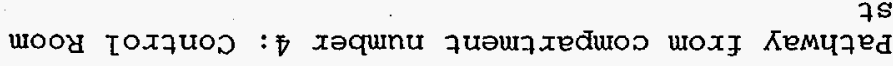

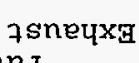

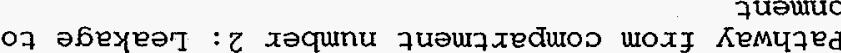
uroy roxpuos

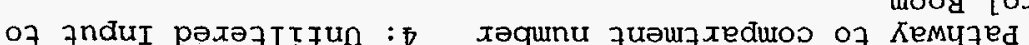
wooy roxzuop of znduI pәxә7ттн : $\varepsilon$ zuәuдxedusos jo zno pue ozut sरемyzed zuวurtox $x \wedge$ ư : :ureN

$\varepsilon$ xәqunu zuawajeduro?

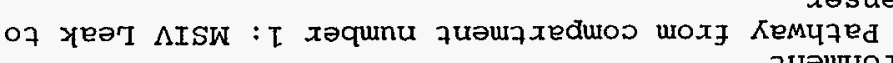

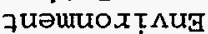

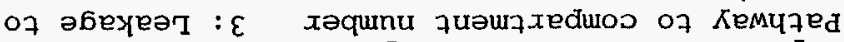

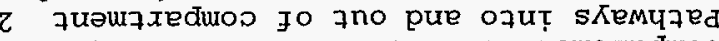

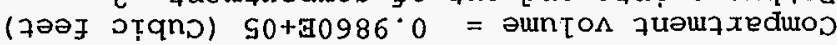
jasuopuoj : auren
2 xəquinu zuəuraxeduoD

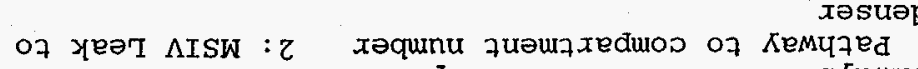
I queuraxeduros fo 7 no pue ofut sКemuzed

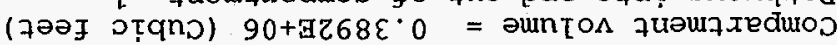
zuourtequos : aureN

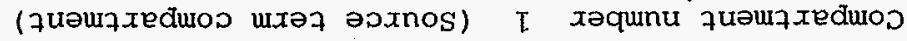

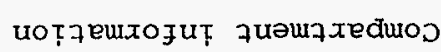

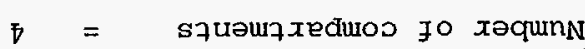

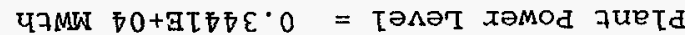

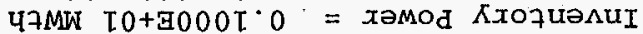

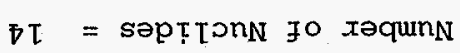

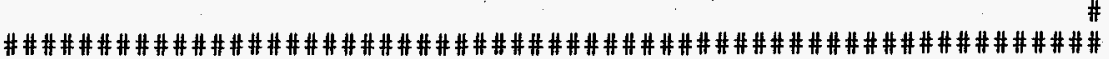

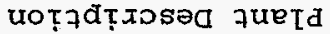

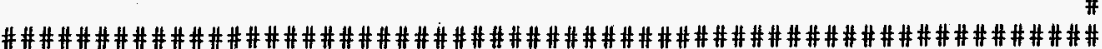

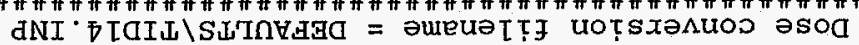

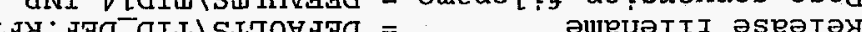

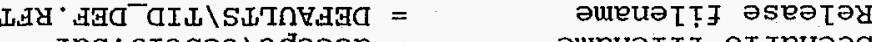

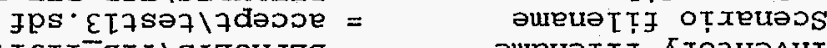

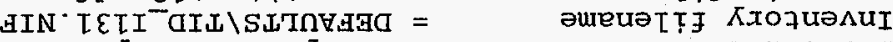

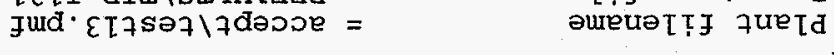

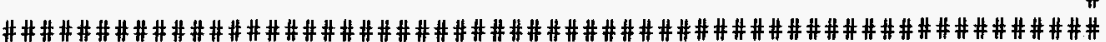

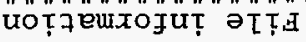

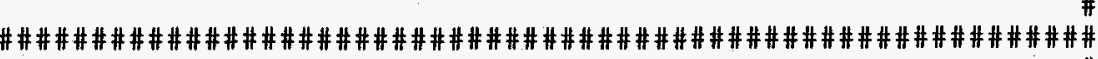

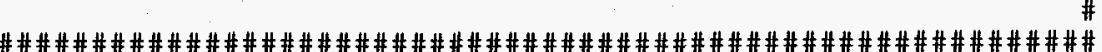

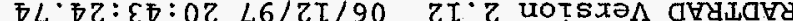


$0.7200 \mathrm{E}+03$

$0.1850 \mathrm{E}+01$

Pathway number 2: Leakage to Environment

Piping: Removal Data

Time (hr) Flow Rate (cfm) Organic

$4628 \mathrm{E}-03$

DF $\left(s^{\wedge}-1\right)$

Elemental

$0.4628 \mathrm{E}-0$

$0.4000 \mathrm{E}+02$

$0.7666 \mathrm{E}+00$

$0.3240 \mathrm{E}+07$

$0.2500 \mathrm{E}+00$

$\mathrm{E}-03$

$0.7666 \mathrm{E}+00$

$0.3240 \mathrm{E}+07$

$0.2500 \mathrm{E}+00$

$0.3267 \mathrm{E}-03$

$0.2000 \mathrm{E}+03$

$0.2925 \mathrm{E}-03$

$0.4000 \mathrm{E}+03$

$0.2778 \mathrm{E}-03$

$0.7666 \mathrm{E}+00 \quad 0.3240 \mathrm{E}+07 \quad 0.2500 \mathrm{E}+00$

$0.7666 \mathrm{E}+00 \quad 0.3240 \mathrm{E}+07 \quad 0.2500 \mathrm{E}+00$

$0.7666 \mathrm{E}+00$

$0.3240 E+07$

$0.2500 \mathrm{E}+00$

Pathway number 3: Filtered Input to Control Room

$\overline{2}$

Pathway Filter: Removal Data

Filter flow rate $=0.5800 \mathrm{E}+04(\mathrm{cfm})$

Time $(\mathrm{hr})$

Filter efficiency

(8)

Organic

$0.0000 \mathrm{E}+0000 \mathrm{E}+00$

$0.9900 \mathrm{E}+02 \quad 0.9900 \mathrm{E}+02$

$0.7200 \mathrm{E}+03$
$0.0000 \mathrm{E}+00$

$0.9900 \mathrm{E}+02 \quad 0.9900 \mathrm{E}+02$

Pathway number 4: Unfiltered Input to Control Room

Pathway Filter: Removal Data

Filter flow rate $=0.1000 \mathrm{E}+02(\mathrm{cfm})$

Time (hr)

$$
\text { Aerosol } \begin{gathered}
\text { Filter efficiency } \\
\text { Elemental }
\end{gathered}
$$

Organic

$0.0000 \mathrm{E}+00$

$0.0000 \mathrm{E}+00 \quad 0.0000 \mathrm{E}+00$

$0.0000 \mathrm{E}+00$

$0.0000 \mathrm{E}+00 \quad 0.0000 \mathrm{E}+00$

Pathway number 5: Control Room Exhaust

Pathway Filter: Removal Data

Filter flow rate $=0.5810 \mathrm{E}+04(\mathrm{cfm})$

Time (hr)

Organic

$$
\begin{gathered}
0.0000 \mathrm{E}+00 \\
0.0000 \mathrm{E}+00
\end{gathered}
$$

$0.0000 \mathrm{E}+00$
Aerosol Filter efficienc

$0.0000 \mathrm{E}+00$

$0.0000 \mathrm{E}+00$

$0.0000 \mathrm{E}+00$

$0.0000 \mathrm{E}+00$

\section{LOCATION DATA}

Location Exclusion Area Bndry is in compartment

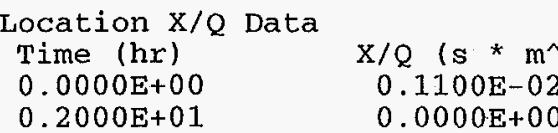

Location Breathing Rate Data

Time (hr) Breathing Rate $\left(m^{\wedge} 3 * \sec ^{\wedge}-1\right)$

$0.0000 \mathrm{E}+00$

$0.8000 \mathrm{E}+01$

$0.2400 \mathrm{E}+02$

$0.3470 \mathrm{E}-03$

$0.1750 \mathrm{E}-03$

$0.2320 \mathrm{E}-03$

3.

$\begin{array}{lc}\begin{array}{c}\text { Location } X / Q \text { Data } \\ \text { Time (hx) }\end{array} & \left.\text { X/Q (s * } \mathrm{m}^{\wedge}-3\right) \\ 0.0000 \mathrm{E}+00 & 0.5200 \mathrm{E}-04 \\ 0.8000 \mathrm{E}+01 & 0.3600 \mathrm{E}-04 \\ 0.2400 \mathrm{E}+02 & 0.1600 \mathrm{E}-04 \\ 0.9600 \mathrm{E}+02 & 0.5300 \mathrm{E}-05\end{array}$

Location Breathing Rate Data

Time (hr)

$0.0000 \mathrm{E}+00$

$0.2400 \mathrm{E}+02$

Location Control Room

Breathing Rate $\left(\mathrm{m}^{\wedge} 3 * \sec ^{\wedge}-1\right)$

$0.3470 \mathrm{E}-03$

$0.1750 \mathrm{E}-03$

$0.2320 \mathrm{E}-03$

is in compartment 4

Location Breathing Rate Data

Time (hr)

$0.0000 \mathrm{E}+00$

Breathing Rate $\left(\mathrm{m}^{\wedge} 3 * \sec ^{\wedge}-1\right)$

$0.7200 \mathrm{E}+03$

$0.3470 \mathrm{E}-03$

$0.0000 \mathrm{E}+00$

Location Occupancy Factor Data

Time (hr)

$0.2400 \mathrm{E}+02$

$0.9600 \mathrm{E}+02$

$0.7200 \mathrm{E}+03$

Occupancy Factor

$0.1000 \mathrm{E}+01$

$0.6000 \mathrm{E}+00$

$0.4000 \mathrm{E}+00$

$0.0000 \mathrm{E}+00$ 
USER SPECIFIED TIME STEP DATA - SUPPLEMENTAL TIME STEPS

Time
$0.0000 \mathrm{E}+00$
$0.8000 \mathrm{E}+01$
$0.2400 \mathrm{E}+02$

$0.7200 \mathrm{E}+03$

Time step

$0.2500 \mathrm{E}+00$

$0.1000 \mathrm{E}+01$

$0.4000 \mathrm{E}+01$

$0.0000 \mathrm{E}+00$

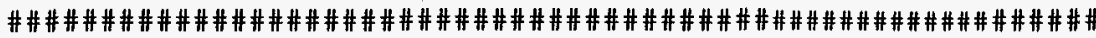

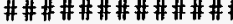

\section{Output}

Dose, Detailed model and Detailed Inventory

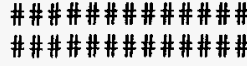

Exclusion Area Bndry Doses:

$\begin{array}{lcclc}\text { Time }(\mathrm{h})=0.0100 & \text { Whole Body } & \text { Thyroid } & \text { TEDE } \\ \text { Delta dose (rem) } & 0.9846 \mathrm{E}-08 & 0.5481 \mathrm{E}-04 & 0.1679 \mathrm{E}-05 \\ \text { Accumulated dose (rem) } & 0.9846 \mathrm{E}-08 & 0.5481 \mathrm{E}-04 & 0.1679 \mathrm{E}-05\end{array}$

Low Population Zone Doses:

$\begin{array}{lclll}\text { Time }(h)=\quad 0.0100 & \text { Whole Body } & \text { Thyroid } & \text { TEDE } \\ \text { Delta dose (rem) } & 0.4654 \mathrm{E}-09 & 0.2591 \mathrm{E}-05 & 0.7935 \mathrm{E}-07 \\ \text { Accumulated dose (rem) } & 0.4654 \mathrm{E}-09 & 0.2591 \mathrm{E}-05 & 0.7935 \mathrm{E}-07\end{array}$
Elemental I (atoms) Organic I (atoms)

Aerosols $(\mathrm{kg})$

$0.0000 \mathrm{E}+00 \quad 0.0000 \mathrm{E}+00$

$0.0000 \mathrm{E}+00 \quad 0.0000 \mathrm{E}+00$

Filtered Input to Control Room Transport Group Inventory:

$\begin{array}{lc}\text { Time }(\mathrm{h})=\quad 0.0100 & \begin{array}{c}\text { Pathway } \\ \text { Filter }\end{array} \\ \text { Noble gases (atoms) } & 0.0000 \mathrm{E}+00 \\ \text { Elemental I (atoms) } & 0.3133 \mathrm{E}+13 \\ \text { Organic I (atoms) } & 0.0000 \mathrm{E}+00 \\ \text { Aerosols (kg) } & 0.6506 \mathrm{E}-16\end{array}$

Aerosols (kg)

Unfiltered Input to Control Room Transport Group Inventory:

$\begin{array}{ll}\text { Time }(\mathrm{h})=\quad 0.0100 & \begin{array}{c}\text { Pathway } \\ \text { Filter }\end{array} \\ \text { Noble gases (atoms) } & 0.0000 \mathrm{E}+00 \\ \text { Elemental I (atoms) } & 0.0000 \mathrm{E}+00 \\ \text { Organic I (atoms) } & 0.0000 \mathrm{E}+00 \\ \text { Aerosols (kg) } & 0.0000 \mathrm{E}+00\end{array}$

Aerosols (kg) $0.0000 \mathrm{E}+00$

Control Room Exhaust Transport Group Inventory:

$\begin{array}{ll}\text { Time }(\mathrm{h})=\text {. } 0.0100 & \begin{array}{c}\text { Pathway } \\ \text { Noble gases (atoms) }\end{array} \\ \text { Elemental I (atoms) } & 0.0000 \mathrm{E}+00 \\ \text { Organic I (atoms) } & 0.0000 \mathrm{E}+00 \\ \text { Aerosols (kg) } & 0.000 \mathrm{E}+00 \\ \end{array}$

*** Intermediate time steps not edited $* * *$

Exclusion Area Bndry Doses:

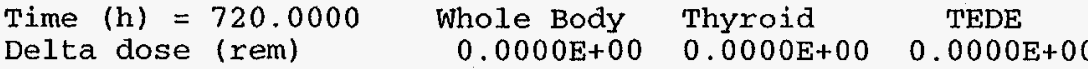
$\begin{array}{llll}\text { Delta dose (rem) } & 0.0000 \mathrm{E}+00 & 0.0000 \mathrm{E}+00 & 0.0000 \mathrm{E}+00 \\ \text { Accumulated dose (rem) } & 0.5083 \mathrm{E}-04 & 0.2830 \mathrm{E}+00 & 0.8666 \mathrm{E}-02\end{array}$ Low Population Zone Doses:

Time $(h)=720.0000$ Whole Body Thyroid TEDE Delta dose (rem) $0.2558 \mathrm{E}-01 \quad 0.9523 \mathrm{E}+02 \quad 0.2925 \mathrm{E}+01$ Accumulated dose (rem) $0.3957 \mathrm{E}-01 \quad 0.1472 \mathrm{E}+03 \quad 0.4521 \mathrm{E}+01$

Control Room Doses: 
Time $(\mathrm{h})=720.0000$

Delta dose (rem)

Accumulated dose (rem)

Whole Body

$0.1797 \mathrm{E}-0$

Thyroid

$0.2321 \mathrm{E}+03$

$0.3762 \mathrm{E}+03$

TEDE

$0.7067 \mathrm{E}+01$

$0.1146 \mathrm{E}+02$

Control Room Atmosphere Nuclide Inventory:

Time $(\mathrm{h})=720.0000$

Atoms

$\mathrm{kg}$

$\mathrm{Ci}$

$\mathrm{Bq}$

$I-131$
$0.1819 \mathrm{E}+09$

$0.1823 \mathrm{E}+15 \quad 0.3966 \mathrm{E}-10$

$0.4917 \mathrm{E}-02$

Control Room Transport Group Inventory:

Time $(\mathrm{h})=720.0000$

Noble gases (atoms)

Elemental I (atoms)

Organic I (atoms)

Aerosols $(\mathrm{kg})$

Time $(\mathrm{h})=720.0000$

Noble gases (atoms)

Elemental I (atoms)

Organic I (atoms)

Aerosols $(\mathrm{kg})$

$\begin{array}{cc}\text { Atmosphere } & \text { Sump } \\ 0.0000 \mathrm{E}+00 & 0.0000 \mathrm{E}+00 \\ 0.2785 \mathrm{E}+11 & 0.0000 \mathrm{E}+00 \\ 0.1823 \mathrm{E}+15 & 0.0000 \mathrm{E}+00 \\ 0.3458 \mathrm{E}-15 & 0.0000 \mathrm{E}+00 \\ & \\ \text { Deposition } & \text { Recirculating } \\ \text { Surfaces } & \text { Filter } \\ 0.0000 \mathrm{E}+00 & 0.0000 \mathrm{E}+00 \\ 0.0000 \mathrm{E}+00 & 0.0000 \mathrm{E}+00 \\ 0.0000 \mathrm{E}+00 & 0.0000 \mathrm{E}+00 \\ 0.0000 \mathrm{E}+00 & 0.0000 \mathrm{E}+00\end{array}$

overlying Pool $0.0000 \mathrm{E}+00$ $0.0000 \mathrm{E}+00$ $0.0000 \mathrm{E}+00$ $0.0000 \mathrm{E}+00$

Filtered Input to Contro1 Room Transport Group Inventory:

$\begin{array}{lc}\text { Pathway } \\ \text { Time (h) }=720.0000 & \text { Filter } \\ \text { Noble gases (atoms) } & 0.0000 \mathrm{E}+00 \\ \text { Elemental I (atoms) } & 0.1033 \mathrm{E}+25 \\ \text { Organic I (atoms) } & 0.0000 \mathrm{E}+00 \\ \text { Aerosols (kg) } & 0.1283 \mathrm{E}-01\end{array}$

Unfiltered Input to Control Room Transport Group Inventory:

Time $(\mathrm{h})=720.0000$ Noble gases (atoms)

Elemental I (atoms)

Organic I (atoms)

Aerosols ( $\mathrm{kg}$ )

Pathway

Filter

$0.0000 \mathrm{E}+00$

$0.0000 E+00$

$0.0000 \mathrm{E}+00$

$0.0000 \mathrm{E}+00$

Control Room Exhaust Transport Group Inventory:

Time $(\mathrm{h})=720.0000$

Noble gases (atoms)

Elemental I (atoms)
$0.0000 \mathrm{E}+00$

$0.0000 \mathrm{E}+00$
Organic I (atoms) $0.0000 \mathrm{E}+00$

Aerosols (kg) $0.0000 \mathrm{E}+00$

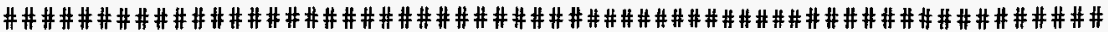

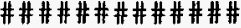

Cumulative Dose Summary

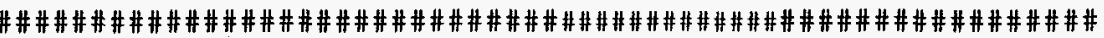

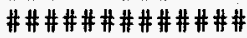

Control Room

Exclusion Area Bndry Low Population Zone

Time Thyroid TEDE Thyroid TEDE

Thyroid

TEDE

(rem)

(rem)

(rem)

(rem)

(rem)

(rem)

$07 \quad 0.3337 \mathrm{E}-09$

$010.1871 \mathrm{E}-02$

$8.00 \quad 0.2830 \mathrm{E}+00 \quad 0.8666 \mathrm{E}-02 \quad 0.2143 \mathrm{E}+00 \quad 0.6563 \mathrm{E}-02$

$0.1237 \mathrm{E}+01 \quad 0.3766 \mathrm{E}-01$

$24.00 \quad 0.2830 \mathrm{E}+00 \quad 0.8666 \mathrm{E}-02 \quad 0.8089 \mathrm{E}+00 \quad 0.2488 \mathrm{E}-01$

$0.7460 \mathrm{E}+01 \quad 0.2272 \mathrm{E}+00$

$\begin{array}{lllll}40.00 & 0.2830 \mathrm{E}+00 & 0.8666 \mathrm{E}-02 & 0.1503 \mathrm{E}+01 & 0.4619 \mathrm{E}-01\end{array}$

$0.1076 \mathrm{E}+02 \quad 0.3278 \mathrm{E}+00$

$\begin{array}{lllll}96.00 & 0.2830 \mathrm{E}+00 & 0.8666 \mathrm{E}-02 & 0.7362 \mathrm{E}+01 & 0.2261 \mathrm{E}+00\end{array}$

$0.3520 \mathrm{E}+02 \quad 0.1072 \mathrm{E}+01$

$\begin{array}{lllll}100.00 & 0.2830 \mathrm{E}+00 & 0.8666 \mathrm{E}-02 & 0.7558 \mathrm{E}+01 & 0.2322 \mathrm{E}+00\end{array}$

$0.3626 \mathrm{E}+02 \quad 0.1104 \mathrm{E}+01$

$200.00 \quad 0.2830 E \div 00 \quad 0.8656 \mathrm{E}-02 \quad 0.1634 \mathrm{E} \div 02 \quad 0.5020 \mathrm{E}+00$

$0.5753 \mathrm{E}+02 \quad 0.1752 \mathrm{E}+01$

$\begin{array}{lllll}400.00 & 0.2830 \mathrm{E}+00 & 0.8666 \mathrm{E}-02 & 0.5195 \mathrm{E}+02 & 0.1596 \mathrm{E}+01\end{array}$

$0.1442 \mathrm{E}+03 \quad 0.4390 \mathrm{E}+01$

$\begin{array}{lllll}720.00 & 0.2830 \mathrm{E}+00 & 0.8666 \mathrm{E}-02 & 0.1472 \mathrm{E}+03 & 0.4521 \mathrm{E}+01\end{array}$

$0.3762 \mathrm{E}+03 \quad 0.1146 \mathrm{E}+02$

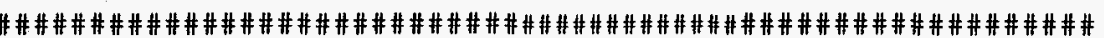

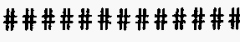

I-131 Summary

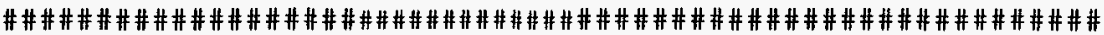

\#\#\#\#\#\#\#\#\#\#\#\#\#

Environment

Containment

Condenser

$\begin{aligned} & \text { Time } \text { (hr) } \\ & \text { I-131 (Curies) }\end{aligned}$

$$
0.01
$$

I-131 (Curies)

$0.2110 \mathrm{E}+08$

I-131 (Curies)

$0.1329 \mathrm{E}-03$ 


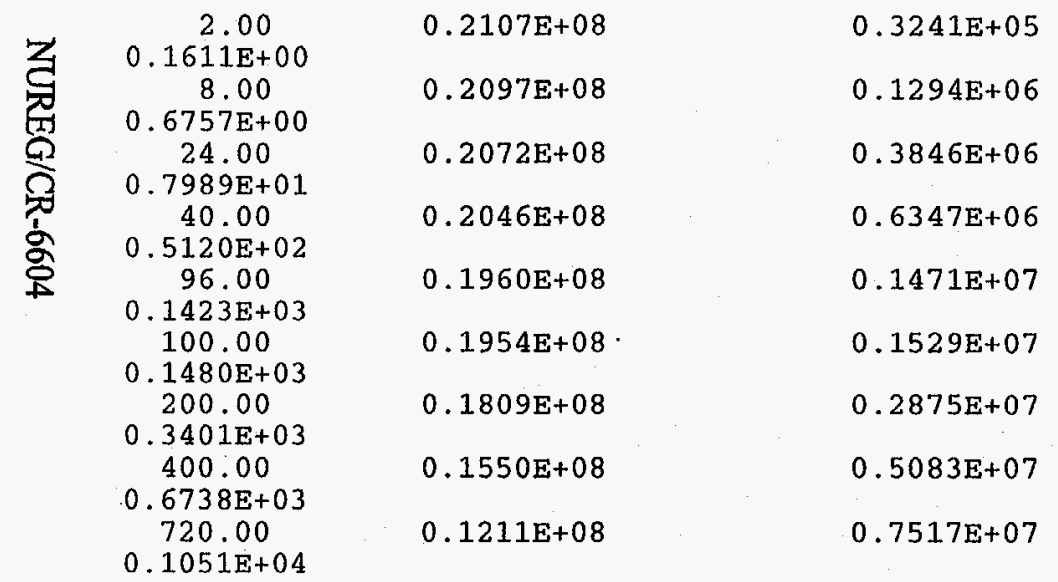




\subsubsection{Example Problem 3 Description}

The third example problem is an advanced PWR design with a specified NUREG-1465 source term released into a sprayed zone. Only part of the containrnent is sprayed and there is mixing between the two parts and natural deposition in the unsprayed part. There is an annulus surrounding the containment that has an exhaust filter (see Figure 1.6.3-1). There is a release to the environment from the annulus and both the sprayed and unsprayed portions of the containment. The code will calculate the doses at the exclusion area boundary, the low population zone, and in the control room. This case was also used for acceptance test case 23.

The data below should be known by the user. In general the user should define the plant model first, that is, those values going into screen numbers starting with 1 , and then define the scenario. After developing the model, the user can compare the input with that provided in Section 1.6.3.1.

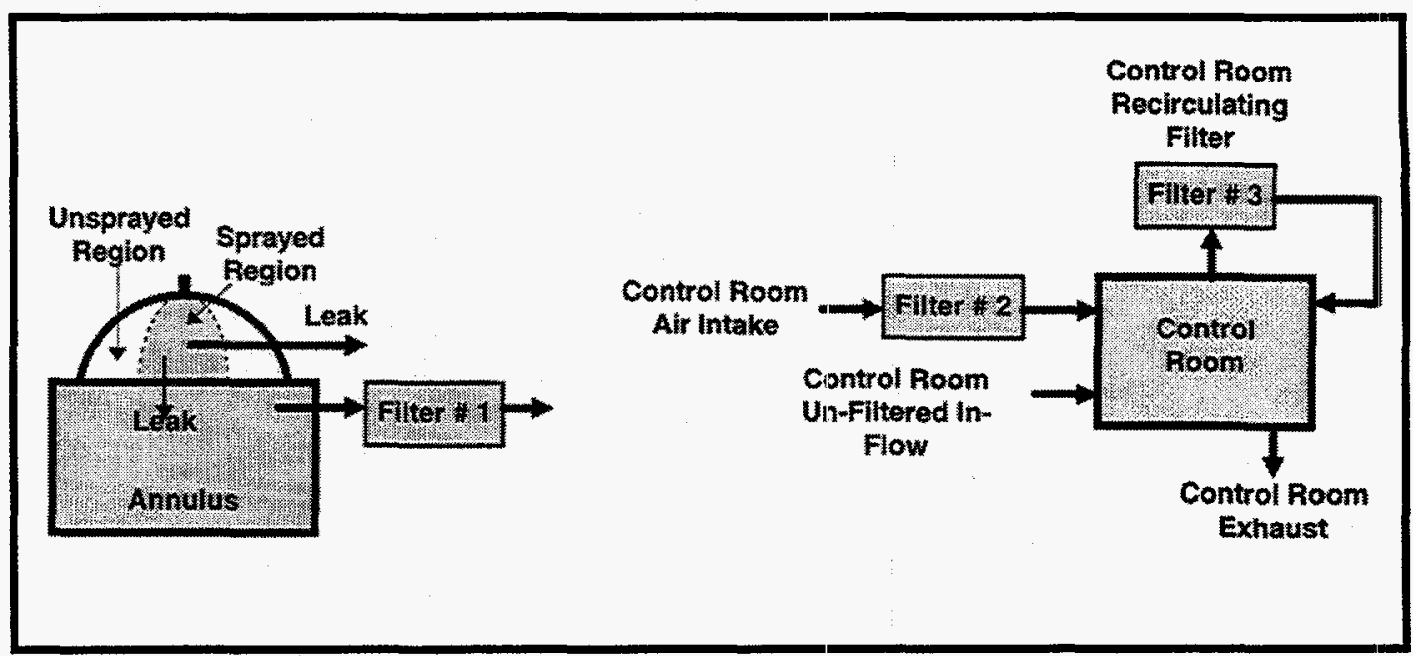

Figure 1.6.3-1. Advanced PWR Control Room Detail

\section{Source Term}

Release fractions and timing:

NUREG-1465, Tables 3.12 and 3.13 .

[screen number 2220]

Start of release: $\quad 0.0000 \mathrm{hr}$

[screen number 2220]

Iodine fractions: Fraction of released iodine chemical form:
Aerosol:
0.9500
Elemental: $\quad 0.0485$
Organic: $\quad 0.0015$ 
[screen number 2240]

Inventory: $\quad$ Normalized MACCS Sample 3412 MWt PWR core inventory.

Reactor power: $3992 \mathrm{MWt}$

[screen number 1200]

\section{Plant Model}

Sprayed containment volume:

$0.2740 \mathrm{E}+07 \mathrm{ft}^{3}$

Unsprayed containment volume:

$0.6011 \mathrm{E}+06 \mathrm{ft}^{3}$

Annulus volume:

$0.6162 \mathrm{E}+06 \mathrm{ft}^{3}$

Control room volume:

$0.6731 \mathrm{E}+05 \mathrm{ft}^{3}$

Filter \#3:

Recirculation filter from: Control Room to: Control Room

Environment

[All five values for name, four values of the volume, the presence of sprays and natural deposition in the containment, and the recirculation filter feature for the control room use screen number 1310.]

Sprayed containment leakage:

Bypass:

Annulus:

Unsprayed containment leakage:

Bypass:

Annulus:

Air leakage

Air leakage

Containment mixing:

Sprayed:

Unsprayed:

Filter \#1:

Filter \#2:

CR exhaust:

$\mathrm{CR}$ unfiltered inflow:

Air Leakage

Air Leakage
Air leakage

Air leakage from: Sprayed

from: Sprayed

from: Unsprayed

from: Unsprayed

Filtered Pathway from: Annulus

from: Sprayed

from: Unsprayed

Filtered Pathway from: Environment

Filtered Pathway from: Control room

Filtered Pathway from: Environment to: Environment

to: Annulus

to: Environment

to: Annulus

to: Unsprayed

to: Sprayed

to: Environment

to: Control Room

to: Environment

to: Control Room

[All 10 transfer pathways are defined (name, from and to compartment, and transfer mechanism) on screen number 1410.] 


\section{Scenario Description}

Containment mixing

$\begin{array}{ll}\begin{array}{l}\text { Time } \\ \text { (hr) }\end{array} & \begin{array}{l}\text { Sprayed to } \\ \text { unsprayed (\%/day) }\end{array} \\ 0.000 & 0.1053 E+04 \\ 0.1667 & 0.4056 \mathrm{E}+04 \\ 0.500 & 0.4915 E+04 \\ 1.175 & 0.2951 E+04 \\ 1.800 & 0.1053 E+04\end{array}$

Unsprayed

to sprayed (\%/day)

$0.5555 \mathrm{E}+04$

$0.2139 \mathrm{E}+05$

$2333 \mathrm{E}+05$

$0.1555 \mathrm{E}+05$

$0.5555 \mathrm{E}+04$

Sprayed zone leak rate: Time (hr) Bypass rate (\%/day)

Annulus rate (\%/day)

$\begin{array}{ll}0.0000 & 0.5000 \mathrm{E}-01 \\ 24.0000 & 0.2500 \mathrm{E}-01\end{array}$

$0.4500 \mathrm{E}+00$

$0.2250 \mathrm{E}+00$

Unsprayed zone leak rate: Time (hr) Bypass rate (\%/day)

Annulus rate (\%/day)
0.0000
$0.5000 \mathrm{E}-01$
$0.4500 \mathrm{E}+00$
24.0000
$0.2500 \mathrm{E}-01$
$0.2250 \mathrm{E}+00$

[All three sets of mixing flows use screen number 2440A.]

Spray removal coefficients: Time

(hr)

0.00

0.50

1.80

2.00

8.00

Time

(hr)

0.0
Aerosol and

Elemental Removal Coefficients $\left(\mathrm{hr}^{-1}\right)$

1.50

12.0

5.00

2.00

0.40

Organic Removal

Coefficients

0.0

[Aerosols are on screen number 2311A, elementals on screen number 2312A, and organics are on screen number 2314A. Remember to set the Select User Parameters to Yes.]

Unsprayed zone aerosol natural deposition rate:

$$
0.4167 \mathrm{E}-04 \mathrm{~s}^{-1}
$$

[Screen number 2341A; the values for elementals and organics will default to 0.0 .]

Annulus exhaust:

Time (hr)

0.0000

0.0278

0.2778
Filter \#1 Flow rate (cfm)

$0.0000 \mathrm{E}+00$

$0.1400 \mathrm{E}+05$

$1.0000 \mathrm{E}+03$ 
Filter \#1 efficiencies:

$\begin{array}{ll}\text { Elemental iodine } & 0.0000 \% \\ \text { Organic iodine } & 0.0000 \% \\ \text { Aerosols } & 99.0000 \%\end{array}$

[screen number $2420 \mathrm{~A}$ ]

$\mathrm{CR}$ filter \#2 flow rate: $\quad 0.2000 \mathrm{E}+04 \mathrm{cfm}$

CR filter \#2 efficiencies:

$\begin{array}{ll}\text { Elemental iodine } & 95.0000 \% \\ \text { Organic iodine } & 95.0000 \% \\ \text { Aerosols } & 99.0000 \%\end{array}$

[screen number $2420 \mathrm{~A}$ ]

$\mathrm{CR}$ filter \#3 flow rate:

$0.4000 \mathrm{E}+04 \mathrm{cfm}$

CR filter \#3 efficiencies:

$\begin{array}{lr}\text { Elemental iodine } & 95.0000 \% \\ \text { Organic iodine } & 95.0000 \% \\ \text { Aerosols } & 99.0000 \%\end{array}$

[screen number $2330 \mathrm{~A}$ ]

CR unfiltered inflow:

$10 \mathrm{cfm}$

[This accounts for leakage into the room through doorways and other unfiltered leaks. All the efficiencies will be $0.0 \%$ for this example. Screen number 2420A.]

CR exhaust:

$0.2010 \mathrm{E}+04 \mathrm{cfm}$

[This exhaust is through filters and will not affect the environmental dose. All the efficiencies are set at $100 \%$ for this example. Screen number 2420T.] 


\section{Dose Parameters}

EAB X/Q values: $\quad$ Time (hr) X/Q (s/m $\left.\mathrm{m}^{3}\right)$
0.0000
$0.1100 \mathrm{E}-02$
2.0000
$0.0000 \mathrm{E}+00$

LPZ X/Q values:

$\begin{array}{ll}\text { Time }(\mathrm{hr}) & \mathrm{X} / \mathrm{Q}\left(\mathrm{s} / \mathrm{m}^{3}\right) \\ 0.0000 & 0.5200 \mathrm{E}-04 \\ 8.0000 & 0.3600 \mathrm{E}-04 \\ 24.0000 & 0.1600 \mathrm{E}-04 \\ 96.0000 & 0.5300 \mathrm{E}-05\end{array}$

Environmental

breathing rates:

Time (hr) Breathing Rate $\left(\mathrm{m}^{3} / \mathrm{s}\right)$

$\begin{array}{ll}0.0000 & 0.3470 \mathrm{E}-03 \\ 8.0000 & 0.1750 \mathrm{E}-03 \\ 24.0000 & 0.2320 \mathrm{E}-03\end{array}$

[The locations are on screen number $2520 \&$ the values are on screen number 2523.]

CR filter X/Q values: $\quad$ Time (hr) $\quad X / Q\left(\mathrm{~s} / \mathrm{m}^{3}\right)$

$\begin{array}{ll}0.0000 & 0.3320 \mathrm{E}-03 \\ 8.0000 & 0.1960 \mathrm{E}-03 \\ 24.0000 & 0.7640 \mathrm{E}-04 \\ 96.0000 & 0.2190 \mathrm{E}-04\end{array}$

[These values are also used for the unfiltered inlets and are input on screen number $24.20 \mathrm{~T} 2$ with the efficiencies and flows.]

CR breathing rates: Time (hr) Breathing Rate $\left(\mathrm{m}^{3} / \mathrm{s}\right)$

$0.0000 \quad 0.3470 \mathrm{E}-03$

CR occupancy factor: Time (hr) Occupancy Factor

$\begin{array}{ll}0.0000 & 1.000 \\ 24.000 & 0.600 \\ 96.0000 & 0.400\end{array}$

[The locations are on screen number 2520 and the values are on screen number 2522.] 


\subsubsection{Example Problem 3 Output}

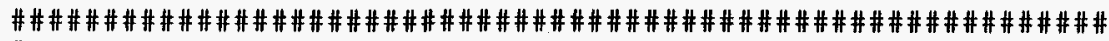

RADTRAD Version 2.12 06/12/97 21:29:27.44

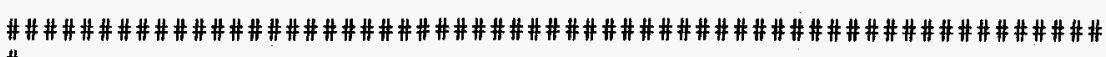

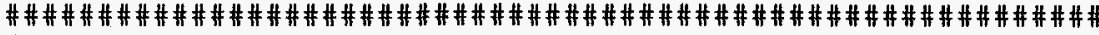

File information

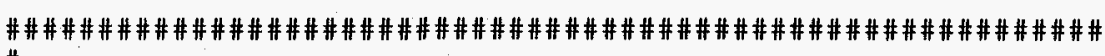

Plant filename

Inventory filename

Scenario filename

Release filename
$=$ accept $\backslash$ test $23 \cdot \mathrm{pmf}$
$=$ DEFAULTS $\backslash$ PWR_DEF.NIF
$=$ accept $\backslash$ test $2 \overline{3} \cdot$.sdf
$=$ DEFAULTS $\backslash$ PWR DBA.RFT

Dose conversion filename $=$ DEFAULTS $\backslash F G R \overline{6} 0$. INP

Plant Description

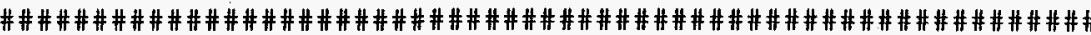

Number of Nuclides $=60$

Inventory Power $=0.1000 \mathrm{E}+01$ MWth

Plant Power Level $=0.3992 \mathrm{E}+04 \mathrm{MWth}$

Number of compartments $=5$

Compartment information

Compartment number 1 (Source term compartment)

Name: Sprayed Region

Compartment volume $=0.2740 \mathrm{E}+07$ (Cubic feet)

Removal devices within compartment Spray (s)

Pathways into and out of compartment 1 Unsprayed
Pathway to compartment number 3: Sprayed to Annulus Pathway to compartment number 4: Sprayed to Environment

Pathway from compartment number 2: Unsprayed to sprayed

Compartment number 2

Name: Unsprayed Region

Compartment volume $=0.6011 \mathrm{E}+06$ (Cubic feet)

Removal devices within compartment: Deposition

Pathways into and out of compartment 2

Pathway to compartment number 1: Unsprayed to Sprayed

Pathway to compartment number 3: Unsprayed to Annulus

Pathway to compartment number 4: Unsprayed to Environment

Pathway from compartment number 1: Sprayed to Unsprayed

Compartment number 3

Name: Annulus

Compartment volume $=0.6162 \mathrm{E}+06$ (Cubic feet)

Pathways into and out of compartment

Pathway to compartment number 4: Annulus to Environment

Pathway from compartment number 1: Sprayed to Annulus Annulus

Pathway from compartment number 2: Unsprayed to

Compartment number 4

Name: Environment

Pathways into and out of compartment Pathway to compartment number 5: Filter 2-Environment to $\mathrm{CR}$

Pathway to compartment number 5: Unfiltered--

Environment to $\mathrm{CR}$

Pathway from compartment number 1: Sprayed to Environment

Pathway from compartment number 2: Unsprayed to Environment

Pathway from compartment number 3: Annulus to Environment

Pathway from compartment number 5: Exhaust from $\mathrm{CR}$ to Environment

Compartment number 5

Name: Control Room

Compartment volume $=0.6731 \mathrm{E}+05$ (Cubic feet)

Removal devices within compartment:

$$
\text { Filter(s) }
$$


Pathways into and out of compartment 5

Pathway to compartment number 4 : Exhaust from $\mathrm{CR}$ to Environment

Pathway from compartment number 4: Filter 2-Environment to $\mathrm{CR}$

Pathway from compartment number 4: Unfiltered-Environment to $\mathrm{CR}$

Total number of pathways $=10$

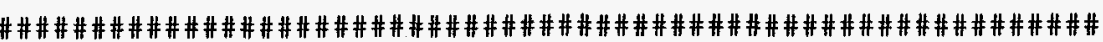

Scenario Description

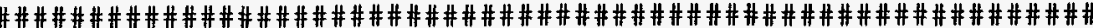

Iodine fractions

$$
\begin{array}{ll}
\text { Aerosol } & =0.9500 \mathrm{E}+00 \\
\text { Elemental } & =0.4850 \mathrm{E}-01 \\
\text { Organic } & =0.1500 \mathrm{E}-02
\end{array}
$$

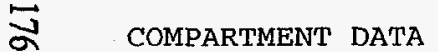

Compartment number 1: Sprayed Region
Compartment number 4: Environment

Compartment number 5: Control Room

Compartment Filter Data

Flow rate $=0.4000 \mathrm{E}+04(\mathrm{cfm})$

Time $(\mathrm{hr})$

$0.0000 \mathrm{E}+00$

$0.9500 \mathrm{E}+02$

$0.7200 \mathrm{E}+03$

$0.9500 \mathrm{E}+02$

\begin{tabular}{lll}
\multicolumn{1}{c}{ Filter } & $\begin{array}{l}\text { Efficiencies } \\
\text { Elemental } \\
\text { Aeroso1 }\end{array}$ & Organic \\
$0.9900 \mathrm{E}+02$ & $0.9500 \mathrm{E}+02$ & \\
$0.9900 \mathrm{E}+02$ & $0.9500 \mathrm{E}+02$ &
\end{tabular}

PATHWAY DATA

Pathway number 1: Sprayed to Unsprayed

$\begin{array}{cr}\text { Convection Data } & \\ \text { Time (hr) } & \text { Flow Rate (\% / day) } \\ 0.0000 \mathrm{E}+00 & 0.1053 \mathrm{E}+04 \\ 0.1667 \mathrm{E}+00 & 0.4056 \mathrm{E}+04 \\ 0.5000 \mathrm{E}+00 & 0.4425 \mathrm{E}+04 \\ 0.1175 \mathrm{E}+01 & 0.2951 \mathrm{E}+04 \\ 0.1800 \mathrm{E}+01 & 0.1053 \mathrm{E}+04\end{array}$

pathway number 2: Sprayed to Annulus

$\begin{array}{cc}\text { Convection Data } & \\ \text { Time (hr) } & \text { Flow Rate (\% / day) } \\ 0.0000 \mathrm{E}+00 & 0.4500 \mathrm{E}+00 \\ 0.2400 \mathrm{E}+02 & 0.2250 \mathrm{E}+00\end{array}$

Pathway number 3: Sprayed to Environment

$\begin{array}{cc}\text { Convection Data } & \\ \text { Time (hr) } & \text { Flow Rate (\% / day) } \\ 0.0000 \mathrm{E}+00 & 0.5000 \mathrm{E}-01 \\ 0.2400 \mathrm{E}+02 & 0.2500 \mathrm{E}-01\end{array}$

Pathway number 4: Unsprayed to Sprayed

$\begin{array}{cr}\begin{array}{c}\text { Convection Data } \\ \text { Time (hr) }\end{array} & \text { Flow Rate (\% / day) } \\ 0.0000 \mathrm{E}+00 & 0.4800 \mathrm{E}+04 \\ 0.1667 \mathrm{E}+00 & 0.1845 \mathrm{E}+05 \\ 0.5000 \mathrm{E}+00 & 0.2015 \mathrm{E}+05 \\ 0.1175 \mathrm{E}+01 & 0.1344 \mathrm{E}+05 \\ 0.1800 \mathrm{E}+01 & 0.4800 \mathrm{E}+04\end{array}$
DF $\left(h r^{\wedge}-1\right)$ $0.1500 \mathrm{E}+01$

$0.1200 \mathrm{E}+02$

$\begin{array}{ll}0.1800 \mathrm{E}+01 & 0.5000 \mathrm{E}+01 \\ 0.2000 \mathrm{E}+01 & 0.2000 \mathrm{E}+01\end{array}$

$0.8000 \mathrm{E}+01 \quad 0.4000 \mathrm{E}+00$

Compartment number 2: Unsprayed Region

$\begin{array}{cl}\text { Natural Deposition: } & \text { Aerosol data } \\ \text { Time (hr) } & \text { DF (hr^-1) } \\ 0.0000 \mathrm{E}+00 & 0.1500 \mathrm{E}+00 \\ 0.7200 \mathrm{E}+03 & 0.1500 \mathrm{E}+00\end{array}$

$0.1500 \mathrm{E}+00$

Compartment number 3: Annulus 
Pathway number 5: Unsprayed to Annulus

$\begin{array}{lc}\text { Convection Data } & \text { Flow Rate (\% / day) } \\ \text { Time (hr) } & 0.4500 \mathrm{E}+00 \\ 0.0000 \mathrm{E}+00 & 0.2250 \mathrm{E}+00\end{array}$

Pathway number 6: Unsprayed to Environment

$\begin{array}{lr}\text { Convection Data } & \\ \text { Time (hr) } & \text { Flow Rate ( } \% / \text { day }) \\ 0.0000 \mathrm{E}+00 & 0.5000 \mathrm{E}-01 \\ 0.2400 \mathrm{E}+02 & 0.2500 \mathrm{E}-01\end{array}$

Pathway number 7: Annulus to Environment

Piping: Removal Data

Time (hr) Flow Rate (cfm) organic

$0.2778 \mathrm{E}-03$

$$
0.2780 \mathrm{E}-01
$$

$0.0000 \mathrm{E}+00$

Aerosol

$0.2778 \mathrm{E}-03$

\section{$0.2778 \mathrm{E}+00$}

$0.1400 \mathrm{E}+05$

$0.2778 \mathrm{E}-03$

$0.2778 \mathrm{E}-03$

$0.1000 \mathrm{E}+04$

$0.3600 \mathrm{E}+06$

$0.3600 \mathrm{E}+06$

$0.2778 \mathrm{E}-03$

$0.2778 \mathrm{E}-03$

$0.2778 \mathrm{E}-03$

$0.1000 \mathrm{E}+04$

$0.3600 \mathrm{E}+06$

$0.2778 \mathrm{E}-03$

Pathway Filter: Removal Data

\section{Filter flow rate $=0.1000 \mathrm{E}+02(\mathrm{cfm})$}

$$
\text { Time (hr) }
$$

Organic

$0.0000 \mathrm{E}+00$

$0.0000 \mathrm{E}+00$

$0.0000 \mathrm{E}+00$

$$
0.7200 \mathrm{E}+03
$$$$
\text { Aerosol }
$$$$
\text { Filter efficiency }(\%)
$$$$
\text { Elemental }
$$
$0.0000 \mathrm{E}+00$
$0.0000 \mathrm{E}+00$
$0.0000 \mathrm{E}+00$
$0.0000 \mathrm{E}+00$

Pathway Filter: Removal Data

\section{Filter flow rate $=0.2010 \mathrm{E}+04(\mathrm{cfm})$}

$$
\text { Time (hr) }
$$

Organic

$0.0000 E+00$

$0.0000 \mathrm{E}+00$

$0.7200 \mathrm{E}+03$

$$
\text { Aerosol Filter efficiency ( } 8 \text { ) }
$$

$0.0000 \mathrm{E}+00 \quad 0.0000 \mathrm{E}+00$

$0.0000 \mathrm{E}+00 \quad 0.0000 \mathrm{E}+00$

\section{LOCATION DATA}

Location Exclusion Area Boundary is in compartment 4

Pathway Filter: Removal Data

Filter flow rate $=0.2000 \mathrm{E}+04(\mathrm{cfm})$

Time (hr)

Organic

$0.0000 \mathrm{E}+00$

0.000
$\mathbf{E}+02$

$0.9500 \mathrm{E}+0.4800 \mathrm{E}+02$

$0.7200 \mathrm{E}+02$
$0.9500 \mathrm{E}+02$

$0.1200 \mathrm{E}+03$

$0.9500 \mathrm{E}+02$

$0.2400 \mathrm{E}+03$

$0.9500 \mathrm{E}+02$

Pathway number

\begin{tabular}{ll}
\multicolumn{2}{c}{ Filter efficiency } \\
Elemental \\
$0.9900 \mathrm{E}+02$ & $0.9500 \mathrm{E}+02$ \\
$0.9900 \mathrm{E}+02$ & $0.9500 \mathrm{E}+02$ \\
$0.9900 \mathrm{E}+02$ & $0.9500 \mathrm{E}+02$ \\
$0.9900 \mathrm{E}+02$ & $0.9500 \mathrm{E}+02$ \\
$0.9900 \mathrm{E}+02$ & $0.9500 \mathrm{E}+02$
\end{tabular}

$(8)$

: Unfiltered--Environment to $C R$

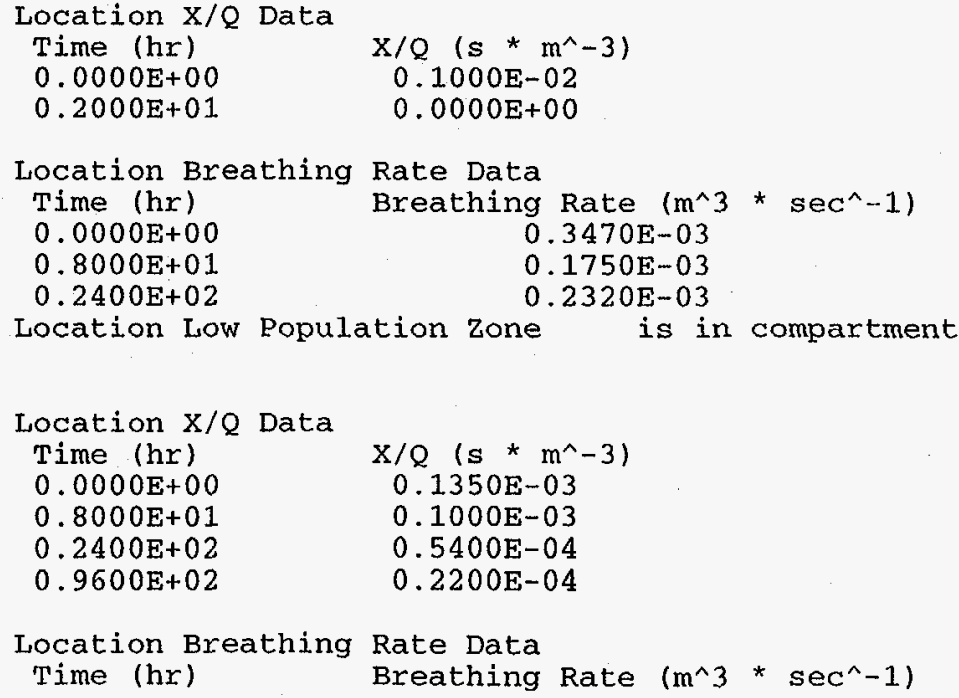




$\begin{array}{lc}0.0000 \mathrm{E}+00 & 0.3470 \mathrm{E}-03 \\ 0.8000 \mathrm{E}+01 & 0.1750 \mathrm{E}-03 \\ 0.2400 \mathrm{E}+02 & 0.2320 \mathrm{E}-03 \\ \text { Location Contxo1 Room } & \text { is in compartment } 5 \\ & \\ \text { Location Breathing Rate Data } & \\ \text { Time (hr) } & \text { Breathing Rate }\left(\mathrm{m}^{\wedge} 3 * \mathrm{sec}^{\wedge}-1\right) \\ 0.0000 \mathrm{E}+00 & 0.3470 \mathrm{E}-03 \\ 0.7200 \mathrm{E}+03 & 0.0000 \mathrm{E}+00 \\ & \\ \text { Location Occupancy } & \text { Factor Data } \\ \text { Time (hr) } & \text { Occupancy Factor } \\ 0.0000 \mathrm{E}+00 & 0.1000 \mathrm{E}+01 \\ 0.2400 \mathrm{E}+02 & 0.6000 \mathrm{E}+00 \\ 0.9600 \mathrm{E}+02 & 0.4000 \mathrm{E}+00 \\ 0.7200 \mathrm{E}+03 & 0.0000 \mathrm{E}+00\end{array}$

USER SPECIFIED TIME STEP DATA - SUPPLEMENTAL TIME STEPS

$\begin{array}{ll}\text { Time } & \text { Time step } \\ 0.0000 \mathrm{E}+00 & 0.2500 \mathrm{E}+00 \\ 0.4000 \mathrm{E}+01 & 0.1000 \mathrm{E}+01 \\ 0.2400 \mathrm{E}+02 & 0.4000 \mathrm{E}+01 \\ 0.7200 \mathrm{E}+03 & 0.0000 \mathrm{E}+00\end{array}$

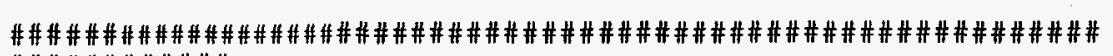
\#\#\#\#\#\#\#\#\#\#\#

Output

\author{
Dose, Detailed model and Detailed Inventory
}

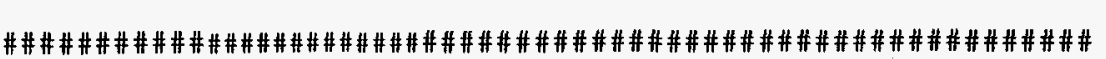
\#\#\#\#\#\#\#\#\#\#\#\#

Exclusion Area Boundary Doses:

$\begin{array}{lccll}\text { Time }(\mathrm{h})= & 0.0278 & \text { Whole Body } & \text { Thyroid } & \text { TEDE } \\ \text { Delta dose (rem) } & 0.2561 \mathrm{E}-03 & 0.4311 \mathrm{E}-01 & 0.1863 \mathrm{E}-02 \\ \text { Accumulated dose (rem) } & 0.2561 \mathrm{E}-03 & 0.4311 \mathrm{E}-01 & 0.1863 \mathrm{E}-02\end{array}$
Low Population Zone Doses:

$$
\begin{array}{lcccc}
\text { Time }(\mathrm{h})=0.0278 & \text { Whole Body } & \text { Thyroid } & \text { TEDE } \\
\text { Delta dose (rem) } & 0.3458 \mathrm{E}-04 & 0.5819 \mathrm{E}-02 & 0.2515 \mathrm{E}-03 \\
\text { Accumulated dose (rem) } & 0.3458 \mathrm{E}-04 & 0.5819 \mathrm{E}-02 & 0.2515 \mathrm{E}-03
\end{array}
$$

Control Room Doses:

Time $(\mathrm{h})=0.0278$

Delta dose (rem)

Whole Body Thyroid

$0.8371 \mathrm{E}-07 \quad 0.3803 \mathrm{E}-04$

\begin{tabular}{|c|c|c|c|c|}
\hline Time $(h)=$ & 0.0278 & Atoms & $\mathrm{kg}$ & $\mathrm{Ci}$ \\
\hline $\mathrm{Bq}$ & & $0.1024 \mathrm{E}+18$ & $0.1445 \mathrm{E}-07$ & $0.5669 \mathrm{E}-02$ \\
\hline $\begin{array}{l}0.2098 E+09 \\
K r-85 m\end{array}$ & & $0.2286 \mathrm{E}+15$ & $0.3227 \mathrm{E}-10$ & $0.2655 \mathrm{E}+00$ \\
\hline $\begin{array}{l}\mathrm{Kr}-87 \\
0.1796 \mathrm{E}+11\end{array}$ & & $0.1186 \mathrm{E}+15$ & $0.1713 \mathrm{E}-10$ & $0.4853 E+00$ \\
\hline $\begin{array}{c}\mathrm{Kr}-88 \\
0.2428 \mathrm{E}+11\end{array}$ & & $0.3582 E+15$ & $0.5234 \mathrm{E}-10$ & $0.6563 \mathrm{E}+00$ \\
\hline $\begin{array}{l}\mathrm{Rb}-86 \\
0.1579 \mathrm{E}+08\end{array}$ & & $0.3673 E+14$ & $0.5245 \mathrm{E}-11$ & $0.4268 \mathrm{E}-03$ \\
\hline $\begin{array}{c}I-131 \\
0.2681 \mathrm{E}+11\end{array}$ & & $0.2687 \mathrm{E}+17$ & $0.5845 E-08$ & $0.7246 \mathrm{E}+00$ \\
\hline $\begin{array}{c}I-132 \\
0.3951 \mathrm{E}+11\end{array}$ & & $0.4719 \mathrm{E}+15$ & $0.1034 E-09$ & $0.1068 \mathrm{E}+01$ \\
\hline $\begin{array}{c}I-133 \\
0.5668 E+11\end{array}$ & & $0.6123 E+16$ & $0.1352 \mathrm{E}-08$ & $0.1532 \mathrm{E}+01$ \\
\hline $\begin{array}{c}I-134 \\
0.6220 E+11\end{array}$ & & $0.2832 \mathrm{E}+15$ & $0.6302 \mathrm{E}-10$ & $0.1681 \mathrm{E}+01$ \\
\hline $\begin{array}{c}I-135 \\
0.5344 \mathrm{E}+11\end{array}$ & & $0.1835 \mathrm{E}+16$ & $0.4113 \mathrm{E}-09$ & $0.1444 \mathrm{E}+01$ \\
\hline $\begin{array}{c}\mathrm{Xe}-133 \\
0.5749 \mathrm{E}+11\end{array}$ & & $0.3759 \mathrm{E}+17$ & $0.8301 \mathrm{E}-08$ & $0.1554 \mathrm{E}+01$ \\
\hline $\begin{array}{c}\mathrm{Xe}-135 \\
0.1079 \mathrm{E}+11\end{array}$ & & $0.5093 E+15$ & $0.1142 E-09$ & $0.2915 E+00$ \\
\hline $\begin{array}{c}\mathrm{Cs}-134 \\
0.3615 \mathrm{E}+10\end{array}$ & & $0.3394 \mathrm{E}+18$ & $0.7552 \mathrm{E}-07$ & $0.9770 \mathrm{E}-01$ \\
\hline $\begin{array}{c}C s-136 \\
0.1100 \mathrm{E}+10\end{array}$ & & $0.1796 \mathrm{E}+16$ & $0.4056 \mathrm{E}-09$ & $0.2972 \mathrm{E}-01$ \\
\hline Cs- & & $0.2761 \mathrm{E}+19$ & $0.6280 \mathrm{E}-06$ & $0.5463 E-01$ \\
\hline
\end{tabular}

TEDE

Accumulated dose (rem)

$0.8371 E-07 \quad 0.3803 E-04$

$.1476 \mathrm{E}-05$

Annulus Atmosphere Nuclide Inventory:
0. $20 \hat{2} 1 \mathrm{E}+10$

Annulus Transport Group Inventory:

Time $(\mathrm{h})=0.0278$ Noble gases (atoms) Elemental I (atoms) Organic I (atoms) Aerosols (kg)

Time $(h)=0.0278$ Noble gases (atoms) Elemental I (atoms) Organic I (atoms) Aerosols ( $\mathrm{kg})$

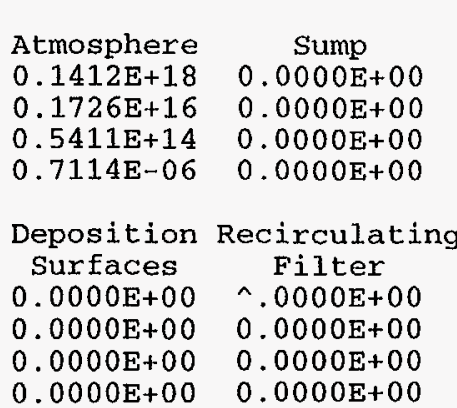

Overlying Pool

$0.0000 \mathrm{E}+00$ $0.0000 \mathrm{E}+00$ $0.0000 \mathrm{E}+00$

Annulus to Environment Transport Group Inventory: 


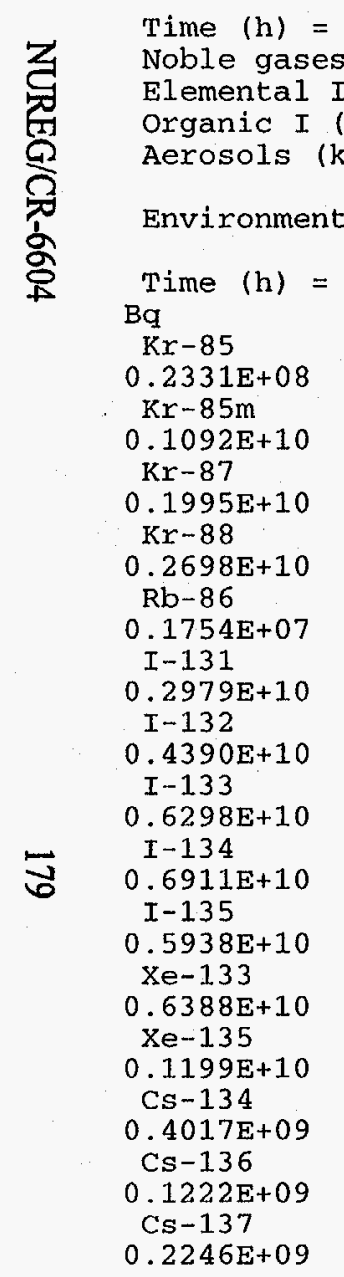

Pipe Walls

$0.0000 \mathrm{E}+00$

$0.0000 \mathrm{E}+00$

$0.0000 \mathrm{E}+00$

$0.0000 \mathrm{E}+00$

Environment Atmosphere Nuclide Inventory:

Time $(\mathrm{h})=0.0278 \quad$ Atoms $\quad \mathrm{kg}$

$\mathrm{K} r-85$

$0.2331 \mathrm{E}+08$

$0.1092 \mathrm{E}+10$

$\mathrm{Kr}-87$

$0.1137 \mathrm{E}+17 \quad 0.1606 \mathrm{E}-08 \quad 0.6299 \mathrm{E}-03$

Ci.

$0.2540 \mathrm{E}+14 \quad 0.3585 \mathrm{E}-11 \quad 0.2951 \mathrm{E}-01$

$0.1318 \mathrm{E}+14 \quad 0.1904 \mathrm{E}-11 \quad 0.5393 \mathrm{E}-01$

$0.3980 E+14 \quad 0.5815 E-11 \quad 0.7292 E-01$

$0.4081 \mathrm{E}+13 \quad 0.5828 \mathrm{E}-12 \quad 0.4742 \mathrm{E}-04$

$0.2985 \mathrm{E}+16 \quad 0.6494 \mathrm{E}-09 \quad 0.8051 \mathrm{E}-01$

$0.5244 \mathrm{E}+14 \quad 0.1149 \mathrm{E}-10 \quad 0.1186 \mathrm{E}+00$

$0.6804 E+15 \quad 0.1503 E-09 \quad 0.1702 E+00$

$0.3147 \mathrm{E}+14 \quad 0.7002 \mathrm{E}-11 \quad 0.1868 \mathrm{E}+00$

$0.2038 \mathrm{E}+15 \quad 0.4570 \mathrm{E}-10 \quad 0.1605 \mathrm{E}+00$

$0.4176 \mathrm{E}+16 \quad 0.9223 \mathrm{E}-09 \quad 0.1726 \mathrm{E}+00$

$0.5659 E+14 \quad 0.1269 E-10 \quad 0.3239 E-01$

$0.3771 \mathrm{E}+17 \quad 0.8391 \mathrm{E}-08 \quad 0.1086 \mathrm{E}-01$

$0.1995 \mathrm{E}+15 \quad 0.4506 \mathrm{E}-10 \quad 0.3303 \mathrm{E}-02$

$0.3067 \mathrm{E}+18 \quad 0.6978 \mathrm{E}-07 \quad 0.6070 \mathrm{E}-02$

Environment Atmosphere Nuclide Release Rates:

$\begin{array}{llccc}\text { Time }(\mathrm{h})= & 0.0278 & \text { Atoms } / \mathrm{s} & \mathrm{kg} / \mathrm{s} & \mathrm{Ci} / \mathrm{s} \\ \mathrm{Bq} / \mathrm{s} & & 0.1137 \mathrm{E}+15 & 0.1604 \mathrm{E}-10 & 0.6294 \mathrm{E}-05 \\ \mathrm{Kr}-85 & & 0.2538 \mathrm{E}+12 & 0.3582 \mathrm{E}-13 & 0.2948 \mathrm{E}-03 \\ 0.2329 \mathrm{E}+06 & & 0.1317 \mathrm{E}+12 & 0.1902 \mathrm{E}-13 & 0.5388 \mathrm{E}-03 \\ \mathrm{Kr}-85 \mathrm{~m} & & & \end{array}$

$\mathrm{Kr}-88$

$0.2696 \mathrm{E}+08$

$\mathrm{Rb}-86$

I-131

$0.2977 \mathrm{E}+08$

I- 132

$0.4386 \mathrm{E}+08$

$\mathrm{I}-133$

$0.6293 \mathrm{E}+08$

$\mathrm{I}-134$

$0.6906 \mathrm{E}+08$

I-135

$0.5933 \mathrm{E}+08$

$\mathrm{Xe}-133$

$0.6383 \mathrm{E}+08$

\begin{tabular}{l}
$\mathrm{Xe}-135$ \\
\hline
\end{tabular}

$\mathrm{Cs}-134$

Cs -136

$0.1221 \mathrm{E}+07$

Cs -137

$0.2244 \mathrm{E}+07$
$0.1753 \mathrm{E}+05$

$0.1198 \mathrm{E}+08$

$0.4014 \mathrm{E}+07$

\begin{abstract}
$0.3976 \mathrm{E}+12 \quad 0.5811 \mathrm{E}-13 \quad 0.7286 \mathrm{E}-03$
$0.4078 \mathrm{E}+11 \quad 0.5823 \mathrm{E}-14 \quad 0.4738 \mathrm{E}-06$

$0.2983 E+14 \quad 0.6489 E-11 \quad 0.8045 E-03$

$0.5240 \mathrm{E}+12 \quad 0.1148 \mathrm{E}-12 \quad 0.1185 \mathrm{E}-02$

$0.6798 \mathrm{E}+13 \quad 0.1501 \mathrm{E}-11 \quad 0.1701 \mathrm{E}-02$

$0.3144 \mathrm{E}+12 \quad 0.6996 \mathrm{E}-13 \quad 0.1866 \mathrm{E}-02$

$0.2037 \mathrm{E}+13 \quad 0.4566 \mathrm{E}-12 \quad 0.1604 \mathrm{E}-02$

$0.4173 \mathrm{E}+14 \quad 0.9216 \mathrm{E}-11 \quad 0.1725 \mathrm{E}-02$

$0.5654 \mathrm{E}+12 \quad 0.1268 \mathrm{E}-12 \quad 0.3237 \mathrm{E}-03$

$0.3768 \mathrm{E}+15 \quad 0.8384 \mathrm{E}-10 \quad 0.1085 \mathrm{E}-03$

$0.1994 \mathrm{E}+13 \quad 0.4503 \mathrm{E}-12 \quad 0.3300 \mathrm{E}-04$

$0.3065 \mathrm{E}+16 \quad 0.6973 \mathrm{E}-09 \quad 0.6065 \mathrm{E}-04$
\end{abstract}

Environment Transport Group Inventory:

$\begin{array}{llllc}\text { Time }(\mathrm{h})= & \begin{array}{l}\text { Present } \\ \text { Release }\end{array} & \begin{array}{c}\text { Release } \\ \text { Rate/s }\end{array} & \begin{array}{c}\text { Total } \\ \text { Release }\end{array} \\ \text { Noble gases (atoms) } & 0.1569 \mathrm{E}+17 & 0.1567 \mathrm{E}+15 & 0.1569 \mathrm{E}+17 \\ \text { Elemental I (atoms) } & 0.1917 \mathrm{E}+15 & 0.1916 \mathrm{E}+13 & 0.1917 \mathrm{E}+15 \\ \text { Organic I (atoms) } & 0.6013 \mathrm{E}+13 & 0.6008 \mathrm{E}+11 & 0.6013 \mathrm{E}+13 \\ \text { Aerosols (kg) } & 0.7904 \mathrm{E}-07 & 0.7898 \mathrm{E}-09 & 0.7904 \mathrm{E}-07\end{array}$

Annulus to Environment Transport Group Inventory:

Time $(\mathrm{h})=0.0278 \quad$ Pipe walls

Noble gases (atoms) $0.0000 \mathrm{E}+00$

Elemental I (atoms) $0.0000 E+00$

Organic I (atoms) $0.0000 \mathrm{E}+00$

$0.0000 \mathrm{E}+00$

Filter 2--Environment to CR Transport Group Inventory:

$\begin{array}{lc}\text { Time }(\mathrm{h})=\quad 0.0278 & \begin{array}{c}\text { Pathway } \\ \text { Filter }\end{array} \\ \text { Noble gases (atoms) } & 0.0000 \mathrm{E}+00 \\ \text { Elemental I (atoms) } & 0.5736 \mathrm{E}+16 \\ \text { Organic I (atoms) } & 0.1799 \mathrm{E}+15 \\ \text { Aerosols (kg) } & 0.2464 \mathrm{E}-05\end{array}$

Unfiltered--Environment to CR Transport Group Inventory: 
Time $(\mathrm{h})=0.0278$

Noble gases (atoms) Elemental I (atoms) organic I (atoms)

Aerosols ( $\mathrm{kg})$

Exhaust from CR to Environment Transport Group Inventory:

$\begin{array}{ll}\text { Time }(\mathrm{h})=\quad 0.0278 & \begin{array}{c}\text { Pathway } \\ \text { Filter }\end{array} \\ \text { Noble gases (atoms) } & 0.0000 \mathrm{E}+00 \\ \text { Elemental I (atoms) } & 0.0000 \mathrm{E}+00 \\ \text { Organic I (atoms) } & 0.0000 \mathrm{E}+00 \\ \text { Aerosols (kg) } & 0.0000 \mathrm{E}+00\end{array}$

Control Room Atmosphere Nuclide Inventory:

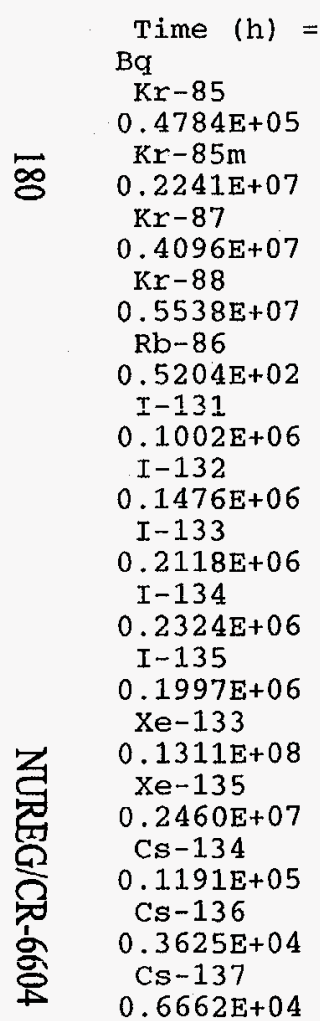

Control Room Transport Group Inventory:

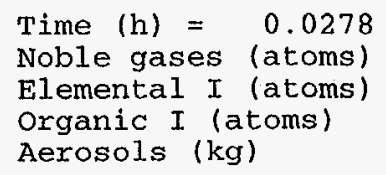

Time $(\mathrm{h})=0.0278$

Noble gases (atoms) Elemental I (atoms) Organic I. (atoms) Aerosols $(\mathrm{kg})$

Time $(\mathrm{h})=0.0278$ Noble gases (atoms) Elemental I (atoms) Organic I (atoms) Aerosols ( $\mathrm{kg})$

Filter 2--Environment to CR Transport Group Inventory:

$\begin{array}{lc}\text { Time }(\mathbf{h})=\quad 0.0278 & \begin{array}{c}\text { Pathway } \\ \text { Filter }\end{array} \\ \text { Noble gases (atoms) } & 0.0000 \mathrm{E}+00 \\ \text { Elemental I (atoms) } & 0.5736 \mathrm{E}+16 \\ \text { Organic I (atoms) } & 0.1799 \mathrm{E}+15 \\ \text { Aerosols }(\mathrm{kg}) & 0.2464 \mathrm{E}-05\end{array}$
$0.2335 \mathrm{E}+14 \quad 0.3296 \mathrm{E}-11 \quad 0.1293 \mathrm{E}-05$
$0.5214 E+11 \quad 0.7360 E-14 \quad 0.6057 E-04$

$0.2705 \mathrm{E}+11 \quad 0.3908 \mathrm{E}-14 \quad 0.1107 \mathrm{E}-03$

$0.8169 \mathrm{E}+11 \quad 0.1194 \mathrm{E}-13 \quad 0.1497 \mathrm{E}-03$

$0.1210 \mathrm{E}+09 \quad 0.1729 \mathrm{E}-16 \quad 0.1407 \mathrm{E}-08$

$0.1004 \mathrm{E}+12 \quad 0.2184 \mathrm{E}-13 \quad 0.2707 \mathrm{E}-05$

$0.1763 \mathrm{E}+10 \quad 0.3865 \mathrm{E}-15 \quad 0.3989 \mathrm{E}-05$

$0.2288 E+11 \quad 0.5053 E-14 \quad 0.5724 E-05$

$0.1058 \mathrm{E}+10 \quad 0.2355 \mathrm{E}-15 \quad 0.6281 \mathrm{E}-05$

$0.6855 \mathrm{E}+10 \quad 0.1537 \mathrm{E}-14 \quad 0.5396 \mathrm{E}-05$

$0.8573 \mathrm{E}+13 \quad 0.1893 \mathrm{E}-11 \quad 0.3544 \mathrm{E}-03$

$0.1162 \mathrm{E}+12 \quad 0.2604 \mathrm{E}-13 \quad 0.6650 \mathrm{E}-04$

$0.1119 \mathrm{E}+13 \quad 0.2489 \mathrm{E}-12 \quad 0.3220 \mathrm{E}-06$

$0.5919 \mathrm{E}+10 \quad 0.1337 \mathrm{E}-14 \quad 0.9797 \mathrm{E}-07$

$0.9099 \mathrm{E}+13 \quad 0.2070 \mathrm{E}-11 \quad 0.1800 \mathrm{E}-06$

$\begin{array}{lc}\text { Pime }(\mathrm{h})=\quad 0.0278 & \begin{array}{c}\text { Pathway } \\ \text { Filter }\end{array} \\ \text { Noble gases (atoms) } & 0.0000 \mathrm{E}+00 \\ \text { Elemental I (atoms) } & 0.0000 \mathrm{E}+00 \\ \text { Organic I (atoms) } & 0.0000 \mathrm{E}+00 \\ \text { Aerosols }(\mathrm{kg}) & 0.0000 \mathrm{E}+00\end{array}$

$\begin{array}{ccc}\text { Atmosphexe } & \text { Sump } & \begin{array}{c}\text { Overlying } \\ \text { POOI }\end{array} \\ 0.3220 \mathrm{E}+14 & 0.0000 \mathrm{E}+00 & 0.0000 \mathrm{E}+00 \\ 0.2088 \mathrm{E}+11 & 0.0000 \mathrm{E}+00 & 0.0000 \mathrm{E}+00 \\ 0.6549 \mathrm{E}+09 & 0.0000 \mathrm{E}+00 & 0.0000 \mathrm{E}+00 \\ 0.2345 \mathrm{E}-11 & 0.0000 \mathrm{E}+00 & 0.0000 \mathrm{E}+00\end{array}$

Deposition Recirculating

surfaces Filter

$0.0000 \mathrm{E}+00 \quad 0.0000 \mathrm{E}+00$

$0.0000 \mathrm{E}+00 \quad 0.6554 \mathrm{E}+09$

$0.0000 \mathrm{E}+00 \quad 0.2056 \mathrm{E}+08$

$0.0000 \mathrm{E}+00 \quad 0.7669 \mathrm{E}-13$
Unfiltered--Environment to CR Transport Group Inventory:

Exhaust from $C R$ to Environment Transport Group Inventory:

$\begin{array}{lll} & \text { Pathway } \\ \text { Time }(\mathrm{h})=\quad 0.0278 & \mathrm{Filter} & \\ \text { Noble gases (atoms) } & 0.0000 \mathrm{E}+00 & \\ \text { Elemental I (atoms) } & 0.0000 \mathrm{E}+00 & 0.0000 \mathrm{E}+00 \\ \text { Organic I (atoms) } & 0.0000 \mathrm{E}+00 & \\ \text { Aerosols (kg) } & & \\ \text { Organic I (atoms) } & 0.0000 \mathrm{E}+00 & 0.0000 \mathrm{E}+00 \\ \text { Aerosols (kg) } & 0.0000 \mathrm{E}+00 & 0.0000 \mathrm{E}+00\end{array}$

*** Intermediate time steps not edited $\star * *$ 


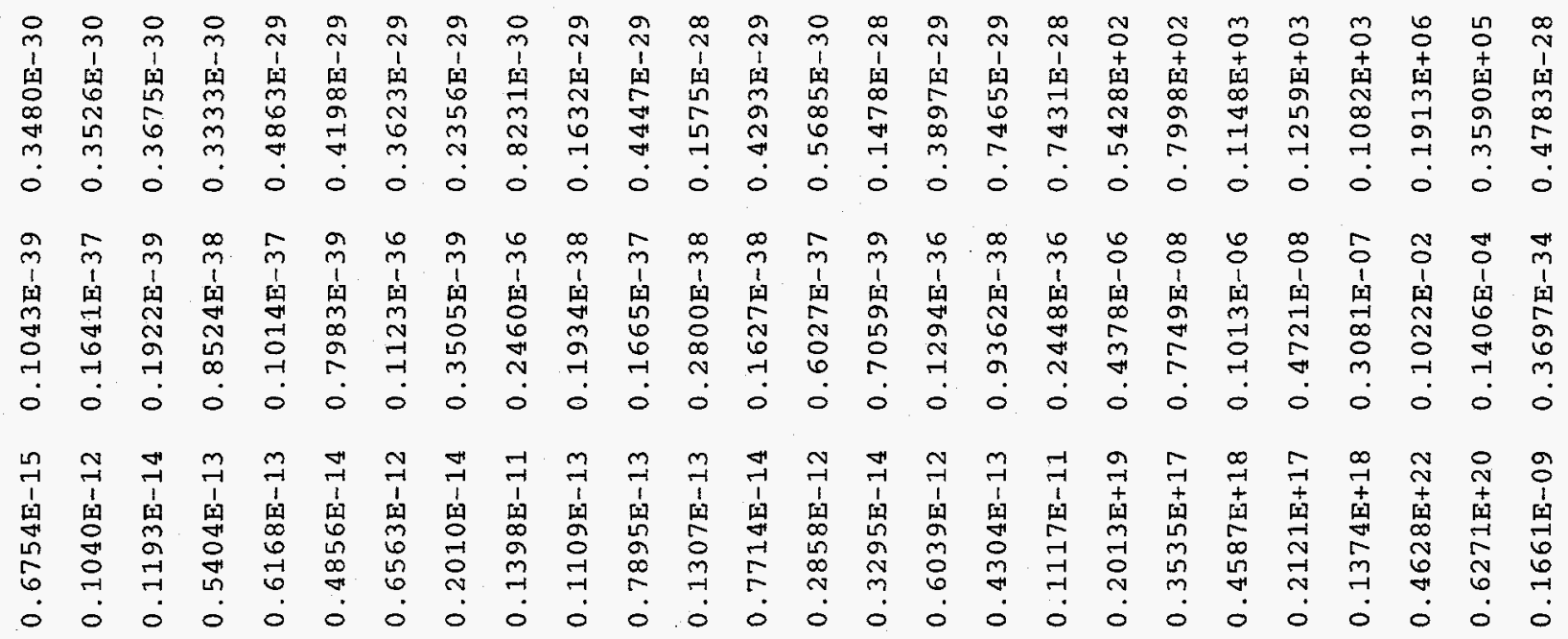

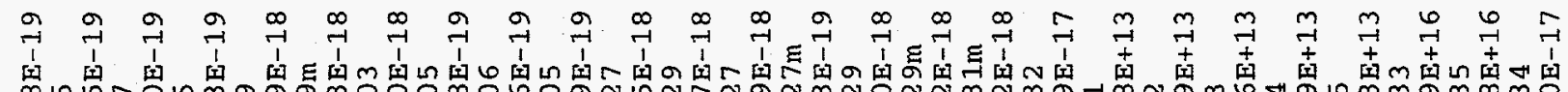

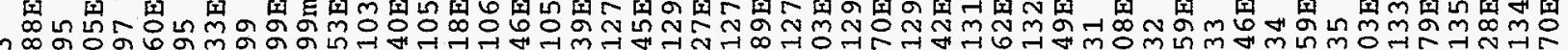
mon

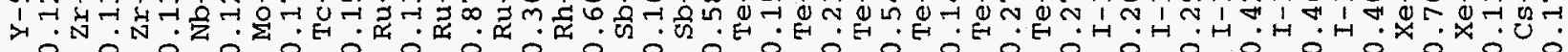

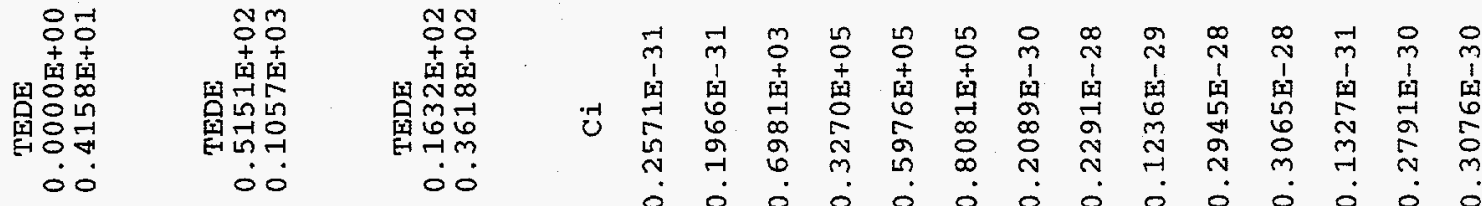

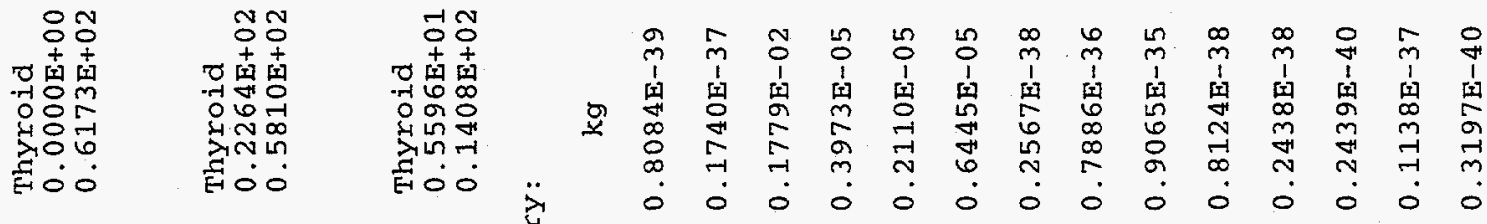

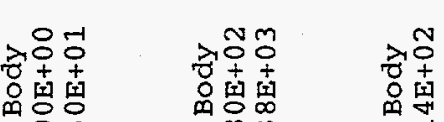

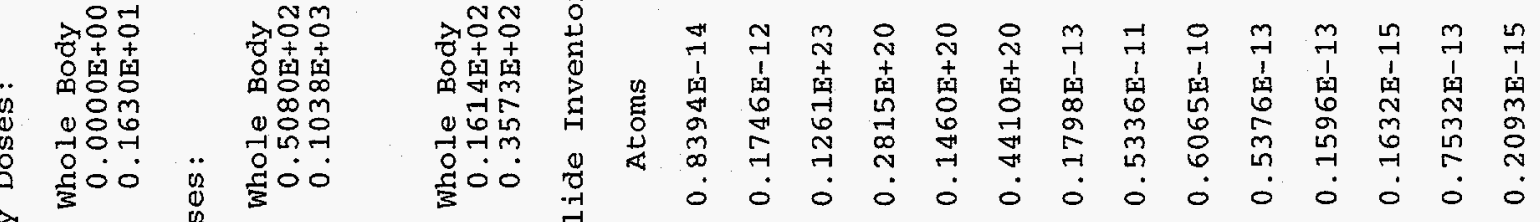

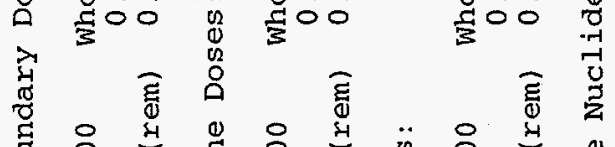

ఏ్t

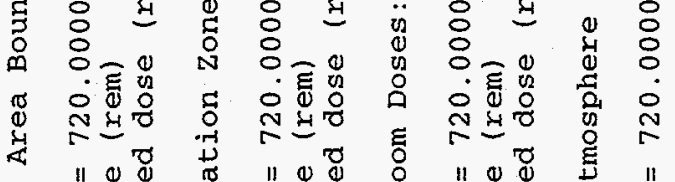

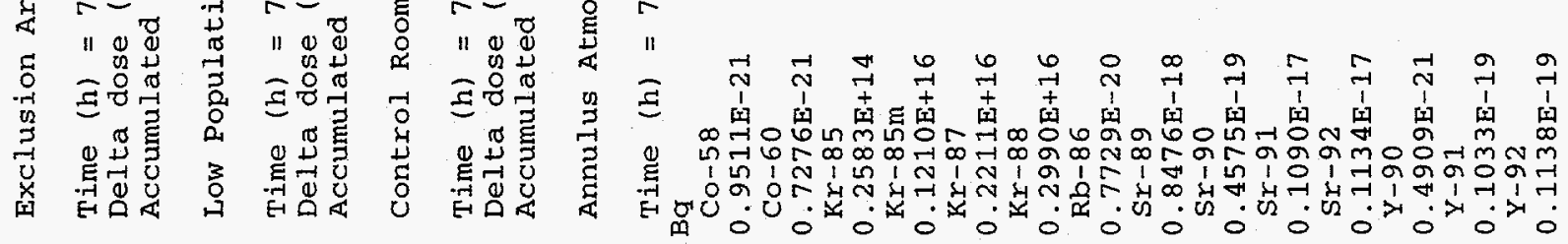




$$
\begin{gathered}
\text { Cs-136 } \\
0.5384 \mathrm{E}-18 \\
\text { Cs-137 } \\
0.9894 \mathrm{E}-18 \\
\mathrm{Ba}-139 \\
0.1483 \mathrm{E}-17 \\
\mathrm{Ba}-140 \\
0.1468 \mathrm{E}-17 \\
\mathrm{La}-140 \\
0.1500 \mathrm{E}-19 \\
\mathrm{La}-141 \\
0.1375 \mathrm{E}-19 \\
\mathrm{La}-142 \\
0.1326 \mathrm{E}-19 \\
\mathrm{Ce}-141 \\
0.3335 \mathrm{E}-19 \\
\mathrm{Ce}-143 \\
0.3243 \mathrm{E}-19 \\
\mathrm{Ce}-144 \\
0.2010 \mathrm{E}-19 \\
\mathrm{Pr}-143 \\
0.1274 \mathrm{E}-19 \\
\mathrm{Nd}-147 \\
0.5696 \mathrm{E}-20 \\
\mathrm{~Np}-239 \\
0.3815 \mathrm{E}-18 \\
\mathrm{Pu}-238 \\
0.2162 \mathrm{E}-22 \\
\mathrm{Pu}-239 \\
0.4877 \mathrm{E}-23 \\
\mathrm{Pu}-240 \\
0.6150 \mathrm{E}-23 \\
\mathrm{Pu}-241 \\
0.1036 \mathrm{E}-20 \\
\mathrm{Am}-241 \\
0.2736 \mathrm{E}-24 \\
\mathrm{Cm}-242 \\
0.1047 \mathrm{E}-21 \\
\mathrm{Cm}-244 \\
0.6128 \mathrm{E}-23 \\
\\
\hline 0
\end{gathered}
$$

$0.8791 \mathrm{E}-12 \quad 0.1985 \mathrm{E}-36 \quad 0.1455 \mathrm{E}-28$

$0.1351 \mathrm{E}-08 \quad 0.3074 \mathrm{E}-33 \quad 0.2674 \mathrm{E}-28$

$0.1062 \mathrm{E}-13 \quad 0.2451 \mathrm{E}-38 \quad 0.4008 \mathrm{E}-28$

$0.2331 \mathrm{E}-11 \quad 0.5418 \mathrm{E}-36 \quad 0.3966 \mathrm{E}-28$

$0.3137 \mathrm{E}-14 \quad 0.7293 \mathrm{E}-39 \quad 0.4053 \mathrm{E}-30$

$0.2808 \mathrm{E}-15 \quad 0.6573 \mathrm{E}-40 \quad 0.3718 \mathrm{E}-30$

$0.1062 \mathrm{E}-15 \quad 0.2504 \mathrm{E}-40 \quad 0.3584 \mathrm{E}-30$

$0.1351 \mathrm{E}-12 \quad 0.3163 \mathrm{E}-37 \quad 0.9014 \mathrm{E}-30$

$0.5558 E-14 \quad 0.1320 E-38 \quad 0.8764 E-30$

$0.7121 \mathrm{E}-12 \quad 0.1703 \mathrm{E}-36 \quad 0.5431 \mathrm{E}-30$

$0.2153 \mathrm{E}-13 \quad 0.5111 \mathrm{E}-38 \quad 0.3442 \mathrm{E}-30$

$0.7795 \mathrm{E}-14 \quad 0.1903 \mathrm{E}-38 \quad 0.1539 \mathrm{E}-30$

$0.1120 \mathrm{E}-12 \quad 0.4444 \mathrm{E}-37 \quad 0.1031 \mathrm{E}-28$

$0.8638 \mathrm{E}-13 \quad 0.3414 \mathrm{E}-37 \quad 0.5844 \mathrm{E}-33$

$0.5343 \mathrm{E}-11 \quad 0.2121 \mathrm{E}-35 \quad 0.1318 \mathrm{E}-33$

$0.1830 \mathrm{E}-11 \quad 0.7295 \mathrm{E}-36 \quad 0.1662 \mathrm{E}-33$

$0.6790 \mathrm{E}-12 \quad 0.2717 \mathrm{E}-36 \quad 0.2799 \mathrm{E}-31$

$0.5384 \mathrm{E}-14 \quad 0.2155 \mathrm{E}-38 \quad 0.7396 \mathrm{E}-35$

$0.2125 \mathrm{E}-14 \quad 0.8541 \mathrm{E}-39 \quad 0.2831 \mathrm{E}-32$

$0.5052 \mathrm{E}-14 \quad 0.2047 \mathrm{E}-38 \quad 0.1656 \mathrm{E}-33$

Annulus Transport Group Inventory:
Time $(h)=720.0000$ Noble gases (atoms) Elementa1 I (atoms) Organic I (atoms) Aerosols $(\mathrm{kg})$

Time $(h)=720.0000$

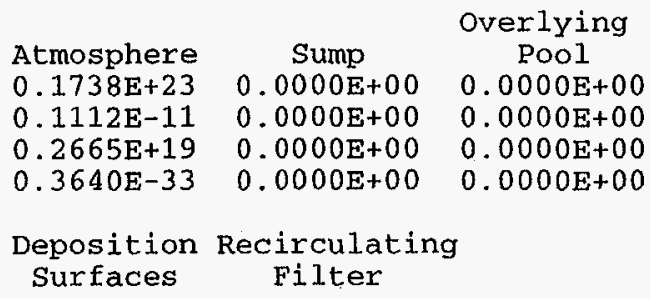

Noble gases (atoms) Elemental I (atoms) Organic I (atoms) Aerosols (kg)

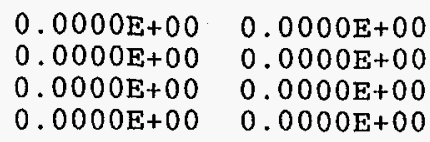

Annulus to Environment Transport Group Inventory:

Time $(h)=720.0000$ Noble gases (atoms) Elemental I (atoms) Organic I (atoms) Aerosols ( $\mathrm{kg})$

Pipe Walls $0.0000 \mathrm{E}+00$ $0.2193 \mathrm{E}+12$ $0.1578 \mathrm{E}+14$ $0.8976 \mathrm{E}-03$

Environment Atmosphere Nuclide Inventory:

Time $(h)=720.0000$ Atoms

$\mathrm{kg}$

$\mathrm{Ci}$

Bq $\mathrm{Co}-58$

$0.1889 \mathrm{E}-02$ Co-60

$0.1445 \mathrm{E}-02$ $\mathrm{Kr}-85$

$0.1376 \mathrm{E}+16$

$\mathrm{Kr}-85 \mathrm{~m}$

$0.6445 \mathrm{E}+17$

$\mathrm{Kr}-87$

$0.1178 \mathrm{E}+18$

$\mathrm{Kr}-88$

$0.1593 \mathrm{E}+18$

$\mathrm{Rb}-86$

$0.1535 \mathrm{E}-01$

Sr-89

$0.1684 \mathrm{E}+01$

$\mathrm{Sr}-90$

$0.9088 \mathrm{E}-01$

$\mathrm{Sr}-91$

$0.2165 \mathrm{E}+01$

$\mathrm{Sr}-92$

$0.2253 \mathrm{E}+01$

$\mathrm{X}-90$

$0.9751 \mathrm{E}-03$

$\mathrm{Y}-91$

$0.2052 \mathrm{E}-01$

$\mathrm{Y}-92$

$0.2261 \mathrm{E}-01$

$\mathrm{Y}-93$

$0.2558 \mathrm{E}-01$

$\mathrm{Zr}-95$

$0.2591 \mathrm{E}-01$

$\mathrm{Zr}-97$

$0.2701 \mathrm{E}-01$
$0.1667 \mathrm{E}+05 \quad 0.1606 \mathrm{E}-20 \quad 0.5107 \mathrm{E}-13$

$0.3469 \mathrm{E}+06 \quad 0.3456 \mathrm{E}-19 \quad 0.3906 \mathrm{E}-13$

$0.6715 \mathrm{E}+24 \quad 0.9478 \mathrm{E}-01 \quad 0.3719 \mathrm{E}+05$

$0.1500 E+22 \quad 0.2117 E-03 \quad 0.1742 E+07$

$0.7780 \mathrm{E}+21 \quad 0.1124 \mathrm{E}-03 \quad 0.3184 \mathrm{E}+07$

$0.2349 \mathrm{E}+22 \quad 0.3433 \mathrm{E}-03 \quad 0.4305 \mathrm{E}+07$

$0.3571 \mathrm{E}+05 \quad 0.5100 \mathrm{E}-20 \quad 0.4150 \mathrm{E}-12$

$0.1060 \mathrm{E}+08 \quad 0.1566 \mathrm{E}-17 \quad 0.4551 \mathrm{E}-10$

$0.1205 \mathrm{E}+09 \quad 0.1801 \mathrm{E}-16 \quad 0.2456 \mathrm{E}-11$

$0.1068 \mathrm{E}+06 \quad 0.1614 \mathrm{E}-19 \quad 0.5850 \mathrm{E}-10$

$0.3171 \mathrm{E}+05 \quad 0.4844 \mathrm{E}-20 \quad 0.6089 \mathrm{E}-10$

$0.3241 \mathrm{E}+03 \quad 0.4844 \mathrm{E}-22 \quad 0.2635 \mathrm{E}-13$

$0.1496 \mathrm{E}+06 \quad 0.2261 \mathrm{E}-19 \quad 0.5545 \mathrm{E}-12$

$0.4157 \mathrm{E}+03 \quad 0.6351 \mathrm{E}-22 \quad 0.6111 \mathrm{E}-12$

$0.1342 \mathrm{E}+04 \quad 0.2072 \mathrm{E}-21 \quad 0.6913 \mathrm{E}-12$

$0.2067 \mathrm{E}+06 \quad 0.3260 \mathrm{E}-19 \quad 0.7004 \mathrm{E}-12$

$0.2371 \mathrm{E}+04 \quad 0.3819 \mathrm{E}-21 \quad 0.7300 \mathrm{E}-12$ 


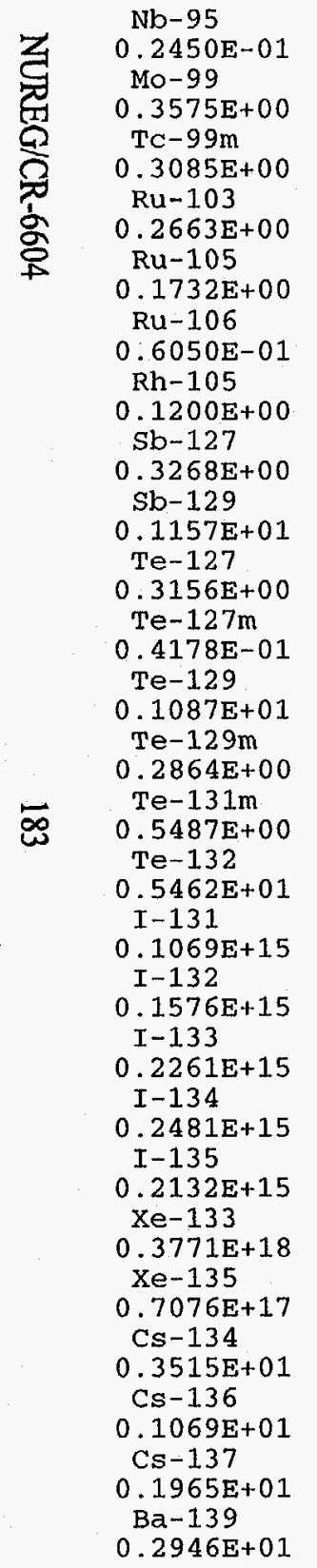
$\begin{array}{llll}0.1073 \mathrm{E}+06 & 0.1693 \mathrm{E}-19 & 0.6622 \mathrm{E}-12 & \mathrm{Ba}-140 \\ 0.2915 \mathrm{E}+01\end{array}$
$0.1225 \mathrm{E}+06 \quad 0.2014 \mathrm{E}-19 \quad 0.9661 \mathrm{E}-11 \quad$ La-140
$0.9647 \mathrm{E}+04 \quad 0.1586 \mathrm{E}-20 \quad 0.8339 \mathrm{E}-11 \quad \begin{gathered}0.2979 \mathrm{E}-01 \\ \mathrm{La}-141\end{gathered}$
$0.1304 \mathrm{E}+07 \quad 0.2230 \mathrm{E}-18 \quad 0.7197 \mathrm{E}-11 \quad 0.2732 \mathrm{E}-01$

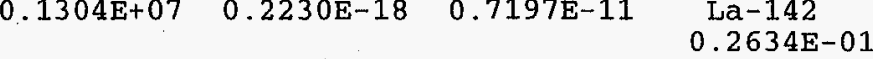
$0.3994 \mathrm{E}+04 \quad 0.6963 \mathrm{E}-21 \quad 0.4681 \mathrm{E}-11 \quad \mathrm{Ce}-141$
$\begin{array}{llll}0.2777 \mathrm{E}+07 & 0.4888 \mathrm{E}-18 & 0.1635 \mathrm{E}-11 & 0.6625 \mathrm{E}-01 \\ \mathrm{Ce}-143\end{array}$
$0.6442 \mathrm{E}-01$
$0.6442 \mathrm{E}-01$
0.3992E-01
Pr-143
$0.2530 \mathrm{E}-01$
Nd-147
$0.1131 \mathrm{E}-01$
$\mathrm{Np}-239$
$0.7578 \mathrm{E}+00$
$0.7578 E+00$
Pu-238
$0.4295 \mathrm{E}-04$
Pu-239
$0.9688 \mathrm{E}-05$
Pu-240
$0.1222 \mathrm{E}-04$
$\mathrm{Pu}-241$
0.2057 E-02
Am-241
$0.5436 \mathrm{E}-06$
Cm-242
$0.2081 \mathrm{E}-03$
Cm-244
$0.1217 \mathrm{E}-04$

$0.2442 \mathrm{E}+20 \quad 0.5394 \mathrm{E}-05 \quad 0.6110 \mathrm{E}+04$

Environment Atmosphere Nuclide Release Rates:

$0.1130 \mathrm{E}+19 \quad 0.2514 \mathrm{E}-06 \quad 0.6705 \mathrm{E}+04$

$0.7318 \mathrm{E}+19 \quad 0.1640 \mathrm{E}-05 \quad 0.5761 \mathrm{E}+04$

$0.2465 E+24 \quad 0.5445 E-01 \quad 0.1019 E+08$

$0.3341 E+22 \quad 0.7489 E-03 \quad 0.1912 E+07$

Time $(h)=720.0000$

$\mathrm{Bq} / \mathrm{s}$

$\mathrm{CO}-58$
$0.1093 \mathrm{E}-08$

$\mathrm{C} 0-60$
$0.1093 \mathrm{E}-0$

$0.8364 \mathrm{E}-09$

Kr -85

$\begin{array}{cccc}0.3300 \mathrm{E}+09 & 0.7343 \mathrm{E}-16 & 0.9501 \mathrm{E}-10 & 0.7962 \mathrm{E}+09 \\ & & & \mathrm{Kr}-85 \mathrm{~m} \\ 0.1746 \mathrm{E}+07 & 0.3944 \mathrm{E}-18 & 0.2890 \mathrm{E}-10 & 0.3730 \mathrm{E}+11\end{array}$

$\begin{array}{cccc}0.3300 \mathrm{E}+09 & 0.7343 \mathrm{E}-16 & 0.9501 \mathrm{E}-10 & 0.7962 \mathrm{E}+09 \\ & & & \mathrm{Kr}-85 \mathrm{~m} \\ 0.1746 \mathrm{E}+07 & 0.3944 \mathrm{E}-18 & 0.2890 \mathrm{E}-10 & 0.3730 \mathrm{E}+11\end{array}$

$\mathrm{Kr}-87$

$0.2685 \mathrm{E}+10 \quad 0.6107 \mathrm{E}-15 \quad 0.5312 \mathrm{E}-10$

$0.6817 \mathrm{E}+11$

$\mathrm{Kr}-88$

$0.2109 \mathrm{E}+05 \quad 0.4868 \mathrm{E}-20 \quad 0.7962 \mathrm{E}-10 \quad 0.9218 \mathrm{E}+11$

\begin{abstract}
$0.4630 \mathrm{E}+07 \quad 0.1076 \mathrm{E}-17 \quad 0.7879 \mathrm{E}-10$
$0.6231 \mathrm{E}+04 \quad 0.1449 \mathrm{E}-20 \quad 0.8052 \mathrm{E}-12$

$0.5577 \mathrm{E}+03 \quad 0.1306 \mathrm{E}-21 \quad 0.7385 \mathrm{E}-12$

$0.2109 \mathrm{E}+03 \quad 0.4973 \mathrm{E}-22 \quad 0.7119 \mathrm{E}-12$

$0.2684 \mathrm{E}+06 \quad 0.6284 \mathrm{E}-19 \quad 0.1791 \mathrm{E}-11$

$0.1104 \mathrm{E}+05 \quad 0.2622 \mathrm{E}-20 \quad 0.1741 \mathrm{E}-11$

$0.1415 \mathrm{E}+07 \quad 0.3383 \mathrm{E}-18 \quad 0.1079 \mathrm{E}-11$

$0.4276 \mathrm{E}+05 \quad 0.1015 \mathrm{E}-19 \quad 0.6838 \mathrm{E}-12$

$0.1549 \mathrm{E}+05 \quad 0.3780 \mathrm{E}-20 \quad 0.3058 \mathrm{E}-12$

$0.2225 \mathrm{E}+06 \quad 0.8829 \mathrm{E}-19 \quad 0.2048 \mathrm{E}-10$

$0.1716 \mathrm{E}+06 \quad 0.6781 \mathrm{E}-19 \quad 0.1161 \mathrm{E}-14$

$0.1061 \mathrm{E}+08 \quad 0.4212 \mathrm{E}-17 \quad 0.2618 \mathrm{E}-15$

$0.3636 E+07 \quad 0.1449 E-17 \quad 0.3302 E-15$

$0.1349 \mathrm{E}+07 \quad 0.5398 \mathrm{E}-18 \quad 0.5561 \mathrm{E}-13$

$0.1070 \mathrm{E}+05 \quad 0.4280 \mathrm{E}-20 \quad 0.1469 \mathrm{E}-16$

$0.4222 \mathrm{E}+04 \quad 0.1697 \mathrm{E}-20 \quad 0.5623 \mathrm{E}-14$

$0.1004 \mathrm{E}+05 \quad 0.4067 \mathrm{E}-20 \quad 0.3290 \mathrm{E}-15$
\end{abstract}

$\begin{array}{ccc}\text { Atoms } / \mathrm{s} & \mathrm{kg} / \mathrm{s} & \mathrm{Ci} / \mathrm{s} \\ 0.9650 \mathrm{E}-02 & 0.9294 \mathrm{E}-27 & 0.2955 \mathrm{E}-19 \\ 0.2007 \mathrm{E}+00 & 0.2000 \mathrm{E}-25 & 0.2261 \mathrm{E}-19 \\ 0.3886 \mathrm{E}+18 & 0.5485 \mathrm{E}-07 & 0.2152 \mathrm{E}-01 \\ 0.8678 \mathrm{E}+15 & 0.1225 \mathrm{E}-09 & 0.1008 \mathrm{E}+01 \\ 0.4502 \mathrm{E}+15 & 0.6504 \mathrm{E}-10 & 0.1842 \mathrm{E}+01 \\ 0.1360 \mathrm{E}+16 & 0.1987 \mathrm{E}-09 & 0.2491 \mathrm{E}+01\end{array}$




\begin{tabular}{|c|c|c|c|}
\hline $2067 \mathrm{E}-01$ & $2951 \mathrm{E}-26$ & $0.2402 E-18$ & \\
\hline $6134 \mathrm{E}+01$ & $9065 \mathrm{E}-24$ & $0.2634 \mathrm{E}-16$ & \\
\hline $9973 E+02$ & $1042 \mathrm{E}-22$ & $0.1421 \mathrm{E}-17$ & \\
\hline & & & \\
\hline & & & \\
\hline $1835 E-01$ & $2803 E-26$ & $.3524 \mathrm{E}-16$ & \\
\hline $1876 E-03$ & $.2803 \mathrm{E}-28$ & $0.1525 \mathrm{E}-19$ & \\
\hline $8659 \mathrm{E}-01$ & $3 \mathbf{E}$ & 18 & \\
\hline $6 \mathrm{E}-0$ & $3675 E-28$ & $0.3536 \mathrm{E}-18$ & \\
\hline $7764 \mathrm{E}-03$ & $0.1199 \mathrm{E}-27$ & $0.4000 E-18$ & \\
\hline $1196 F+00$ & $0.1887 F-25$ & 3 & \\
\hline $1372 \mathrm{E}-02$ & $.2210 \mathrm{E}-27$ & $0.4225 E-18$ & \\
\hline $12 \mathrm{E}-0$ & $9 E-26$ & $.3832 \mathrm{E}-18$ & \\
\hline $.7091 \mathrm{E}-01$ & $0.1166 \mathrm{E}-25$ & $8-17$ & \\
\hline $.5583 \mathrm{E}-02$ & $.9178 E-27$ & $0.4826 \mathrm{E}-17$ & \\
\hline $.7545 E+00$ & $0.1290 \mathrm{E}-24$ & $0.4165 \mathrm{E}-17$ & \\
\hline 231 & 27 & $0.2709 E-17$ & \\
\hline 政 & $0.2828 \bar{E}-24$ & $0.9463 \bar{E}-18$ & \\
\hline 1 & & 17 & \\
\hline $0.9076 \mathrm{E}-01$ & 2 & $0.5112 \mathrm{E}-17$ & \\
\hline $.1503 \mathrm{E}-0$ & $0.3219 \mathrm{E}-26$ & $0.1810 \mathrm{E}-16$ & \\
\hline 8 & & & $c$ \\
\hline 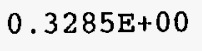 & $0.6929 \mathrm{E}-25$ & $0.6535 E-18$ & \\
\hline & & & \\
\hline $.6942 \mathrm{E}+$ & $1487 \mathrm{E}-2$ & $0.4480 \mathrm{E}-$ & \\
\hline $0.4948 \mathrm{E}-01$ & $0.1076 \mathrm{E}-25$ & $0.8582 \mathrm{E}-17$ & \\
\hline .128 & $E-1$ & -1 & $E-1$ \\
\hline
\end{tabular}

$\begin{array}{lll}0.6202 \mathrm{E}+14 & 0.1349 \mathrm{E}-10 & 0.1673 \mathrm{E}-02 \\ 0.1089 \mathrm{E}+13 & 0.2388 \mathrm{E}-12 & 0.2465 \mathrm{E}-02 \\ 0.1413 \mathrm{E}+14 & 0.3121 \mathrm{E}-11 & 0.3536 \mathrm{E}-02 \\ 0.6537 \mathrm{E}+12 & 0.1455 \mathrm{E}-12 & 0.3880 \mathrm{E}-02 \\ 0.4235 \mathrm{E}+13 & 0.9493 \mathrm{E}-12 & 0.3334 \mathrm{E}-02 \\ 0.1427 \mathrm{E}+18 & 0.3151 \mathrm{E}-07 & 0.5898 \mathrm{E}+01 \\ 0.1933 \mathrm{E}+16 & 0.4334 \mathrm{E}-09 & 0.1107 \mathrm{E}+01 \\ 0.1910 \mathrm{E}+03 & 0.4250 \mathrm{E}-22 & 0.5498 \mathrm{E}-16 \\ 0.1011 \mathrm{E}+01 & 0.2282 \mathrm{E}-24 & 0.1673 \mathrm{E}-16 \\ 0.1554 \mathrm{E}+04 & 0.3534 \mathrm{E}-21 & 0.3074 \mathrm{E}-16 \\ 0.1220 \mathrm{E}-01 & 0.2817 \mathrm{E}-26 & 0.4608 \mathrm{E}-16 \\ 0.2679 \mathrm{E}+01 & 0.6228 \mathrm{E}-24 & 0.4560 \mathrm{E}-16 \\ 0.3606 \mathrm{E}-02 & 0.8383 \mathrm{E}-27 & 0.4660 \mathrm{E}-18 \\ 0.3228 \mathrm{E}-03 & 0.7557 \mathrm{E}-28 & 0.4274 \mathrm{E}-18 \\ 0.1221 \mathrm{E}-03 & 0.2878 \mathrm{E}-28 & 0.4120 \mathrm{E}-18 \\ 0.1553 \mathrm{E}+00 & 0.3637 \mathrm{E}-25 & 0.1036 \mathrm{E}-17 \\ 0.6383 \mathrm{E}-02 & 0.1517 \mathrm{E}-26 & 0.1008 \mathrm{E}-17 \\ 0.8187 \mathrm{E}+00 & 0.1958 \mathrm{E}-24 & 0.6244 \mathrm{E}-18 \\ 0.2475 \mathrm{E}-01 & 0.5876 \mathrm{E}-26 & 0.3957 \mathrm{E}-18 \\ 0.8961 \mathrm{E}-02 & 0.2187 \mathrm{E}-26 & 0.1770 \mathrm{E}-18 \\ 0.1287 \mathrm{E}+00 & 0.5109 \mathrm{E}-25 & 0.1185 \mathrm{E}-16 \\ 0.9930 \mathrm{E}-01 & 0.3924 \mathrm{E}-25 & 0.6718 \mathrm{E}-21 \\ 0.6142 \mathrm{E}+01 & 0.2438 \mathrm{E}-23 & 0.1515 \mathrm{E}-21 \\ 0.2104 \mathrm{E}+01 & 0.8386 \mathrm{E}-24 & 0.1911 \mathrm{E}-21 \\ 0.7806 \mathrm{E}+00 & 0.3124 \mathrm{E}-24 & 0.3218 \mathrm{E}-19 \\ 0.6190 \mathrm{E}-02 & 0.2477 \mathrm{E}-26 & 0.8502 \mathrm{E}-23\end{array}$




$\mathrm{Cm}-242$
$0.1204 \mathrm{E}-09$
$\mathrm{Cm}-244$
$0.7044 \mathrm{E}-11$
Environment Transport
gime (h) = 720.0000
Noble gases (atoms)
Elemental I (atoms)
Organic I (atoms)
Aerosols (kg)

\begin{abstract}
$0.2443 E-02 \quad 0.9818 E-27 \quad 0.3254 E-20$
$0.5808 \mathrm{E}-02 \quad 0.2353 \mathrm{E}-26 \quad 0.1904 \mathrm{E}-21$
\end{abstract}

Group Inventory:

$\begin{array}{ccc}\begin{array}{c}\text { Present } \\ \text { Release }\end{array} & \begin{array}{l}\text { Release } \\ \text { Rate/s }\end{array} & \begin{array}{c}\text { Total } \\ \text { Release }\end{array} \\ 0.9260 \mathrm{E}+24 & 0.5359 \mathrm{E}+18 & 0.1432 \mathrm{E}+25 \\ 0.2209 \mathrm{E}+09 & 0.1279 \mathrm{E}+03 & 0.3045 \mathrm{E}+19 \\ 0.1419 \mathrm{E}+21 & 0.8213 \mathrm{E}+14 & 0.2195 \mathrm{E}+21 \\ 0.7230 \mathrm{E}-15 & 0.4184 \mathrm{E}-21 & 0.1097 \mathrm{E}-03\end{array}$

Annulus to Environment Transport Group Inventory:

$\begin{array}{ll}\text { Time (h) }=720.0000 & \text { Pipe Walls } \\ \text { Noble gases (atoms) } & 0.0000 \mathrm{E}+00 \\ \text { Elemental I (atoms) } & 0.2193 \mathrm{E}+12 \\ \text { Organic I (atoms) } & 0.1578 \mathrm{E}+14 \\ \text { Aerosols (kg) } & 0.8976 \mathrm{E}-03\end{array}$

Filter 2--Environment to CR Transport Group Inventory:

Time $(\mathrm{h})=720.0000$

\section{Pathway}

Filter

Noble gases (atoms) $0.0000 \mathrm{E}+00$

Elemental I (atoms) $0.5389 \mathrm{E}+22$

Organic I (atoms) $0.1374 \mathrm{E}+25$

Aerosols ( $\mathrm{kg}) \quad 0.5732 \mathrm{E}-01$

Unfiltered--Environment to CR Transport Group Inventory:

$\begin{array}{lc}\text { Time }(\mathrm{h})=720.0000 & \begin{array}{c}\text { Pathway } \\ \text { Filter }\end{array} \\ \text { Noble gases (atoms) } & 0.0000 \mathrm{E}+00 \\ \text { Elemental I (atoms) } & 0.0000 \mathrm{E}+00 \\ \text { Organic I (atoms) } & 0.0000 \mathrm{E}+00 \\ \text { Aerosols (kg) } & 0.0000 \mathrm{E}+00\end{array}$

Exhaust from CR to Environment Transport Group Inventory:

$\begin{array}{lc}\text { Time (h) }=720.0000 & \text { Pathway } \\ \text { Filter } \\ \text { Elemental I (atoms) } & 0.0000 \mathrm{E}+00 \\ \text { Organic I (atoms) } & 0.0000 \mathrm{E}+00 \\ \text { Aerosols (kg) } & 0.0000 \mathrm{E}+00 \\ & 0.0000 \mathrm{E}+00\end{array}$

Control Room Atmosphere Nuclide Inventory:
Time $(h)=720.0000$ $\mathrm{Bq}$

Co-58

$0.1205 \mathrm{E}-29$

Co-60

$0.9216 \mathrm{E}-30$
$\mathrm{~K} x-85$

$0.7105 \mathrm{E}+09$

$\mathrm{Kr}-85 \mathrm{~m}$

$0.3328 \mathrm{E}+11$

0.33287
$\mathrm{Kr}-87$

$0.6082 \mathrm{E}+11$

$\mathrm{Kr}-88$

$0.8225 \mathrm{E}+11$

0.9791E-29

Sr-89

$0.1074 \mathrm{E}-26$

$0.1074 \mathrm{E}$
$\mathrm{S} r-90$

Sr-90
$0.5795 \mathrm{E}-28$

Sr-91

$0.1380 \mathrm{E}-26$

Sr-92

$0.1436 \mathrm{E}-26$

Y-90

$0.6218 \mathrm{E}-30$

$\mathrm{Y}-91$

$0.1308 E-28$

$\mathrm{Y}-92$

$0.1442 \mathrm{E}-28$

Y-93

$0.1631 E-28$

Zx-95

$0.1652 \mathrm{E}-28$

Zr-97

0.1722 E-28

$\mathrm{Nb}-95$

$0.1562 \mathrm{E}-28$

Mo-99

$0.2279 \mathrm{E}-27$

TC $-99 \mathrm{~m}$

$0.1967 \mathrm{E}-27$

$\mathrm{Ru}-103$

$0.1698 \mathrm{E}-27$

Ru-1.05

$0.1104 \mathrm{E}-27$

Ru-106

$0.3858 \mathrm{E}-28$

Rh-105

$0.7650 \mathrm{E}-28$

Sb-127

$0.2084 \mathrm{E}-27$

\begin{tabular}{|c|c|c|}
\hline Atoms & $\mathrm{kg}$ & $\mathrm{Ci}$ \\
\hline $0.1063 \mathrm{E}-22$ & $0.1024 \mathrm{E}-47$ & $0.3256 \mathrm{E}-40$ \\
\hline $0.2212 E-21$ & $0.2204 \mathrm{E}-46$ & $0.2491 \mathrm{E}-40$ \\
\hline $0.3467 E+18$ & $0.4894 \mathrm{E}-07$ & $0.1920 \mathrm{E}-01$ \\
\hline $0.7743 \mathrm{E}+15$ & $0.1093 \mathrm{E}-09$ & $0.8994 \mathrm{E}+00$ \\
\hline $0.4017 \mathrm{E}+15$ & $0.5804 \mathrm{E}-10$ & $0.1644 \mathrm{E}+01$ \\
\hline $0.1213 \mathrm{E}+16$ & $0.1773 \mathrm{E}-09$ & $0.2223 \mathrm{E}+01$ \\
\hline $2277 \mathrm{E}-22$ & $0.3252 \mathrm{E}-47$ & $0.2646 \mathrm{E}-39$ \\
\hline $0.6759 \mathrm{E}-20$ & $0.9988 \mathrm{E}-45$ & $0.2902 E-37$ \\
\hline $0.7683 \mathrm{E}-19$ & $0.1148 \mathrm{E}-43$ & $0.1566 \mathrm{E}-38$ \\
\hline $0.6810 \mathrm{E}-22$ & $0.1029 \mathrm{E}-46$ & $0.3730 \mathrm{E}-37$ \\
\hline $0.2022 \mathrm{E}-22$ & $0.3089 E-47$ & $0.3882 E-37$ \\
\hline $0.2067 \mathrm{E}-24$ & $0.3089 E-49$ & $0.1680 \mathrm{E}-40$ \\
\hline $0.9540 \mathrm{E}-22$ & $0.1442 \mathrm{E}-46$ & $0.3535 E-39$ \\
\hline $0.2651 \mathrm{E}-24$ & $0.4050 \mathrm{E}-49$ & $0.3897 \mathrm{E}-39$ \\
\hline $0.8555 \mathrm{E}-24$ & $0.1321 \mathrm{E}-48$ & $0.4408 \mathrm{E}-39$ \\
\hline $0.1318 \mathrm{E}-21$ & $0.2079 \mathrm{E}-46$ & $0.4466 \mathrm{E}-39$ \\
\hline $0.1512 \mathrm{E}-23$ & $0.2435 E-48$ & $0.4655 \mathrm{E}-39$ \\
\hline $0.6845 \mathrm{E}-22$ & $0.1080 \mathrm{E}-46$ & $0.4222 \mathrm{E}-39$ \\
\hline $0.7813 \mathrm{E}-22$ & $0.1284 \mathrm{E}-46$ & $0.6160 \mathrm{E}-38$ \\
\hline $0.6151 \mathrm{E}-23$ & $0.1011 E-47$ & $0.5317 \mathrm{E}-38$ \\
\hline $0.8313 E-21$ & $0.1422 \mathrm{E}-45$ & $0.4589 \mathrm{E}-38$ \\
\hline $0.2546 \mathrm{E}-23$ & $0.4440 \mathrm{E}-48$ & $0.2984 E-38$ \\
\hline $0.1771 \mathrm{E}-20$ & $0.3116 \mathrm{E}-45$ & $0.1043 \mathrm{E}-38$ \\
\hline $0.1405 \mathrm{E}-22$ & $0.2449 \mathrm{E}-47$ & $0.2067 \mathrm{E}-38$ \\
\hline $1000 \mathrm{E}-21$ & $0.2109 E-46$ & $0.5632 \mathrm{E}-38$ \\
\hline
\end{tabular}

0

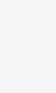




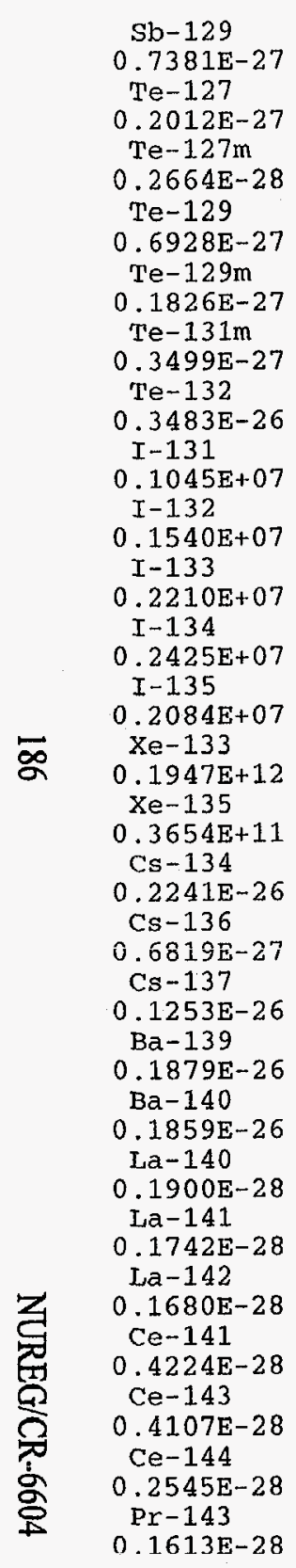

\begin{abstract}
$0.1656 \mathrm{E}-22 \quad 0.3547 \mathrm{E}-47 \quad 0.1995 \mathrm{E}-37 \quad \mathrm{Nd}-147$
$0.7214 \mathrm{E}-29$

$\begin{array}{llll}0.9771 \mathrm{E}-23 & 0.2061 \mathrm{E}-47 & 0.5438 \mathrm{E}-38 & \mathrm{~Np}-239 \\ & & & 0.4832 \mathrm{E}-27\end{array}$

$0.4832 \mathrm{E}-27$
$\mathrm{Pu}-238$

$0.3620 \mathrm{E}-21 \quad 0.7634 \mathrm{E}-46 \quad 0.7201 \mathrm{E}-39$

$0.2739 \mathrm{E}-31$

$\begin{array}{llll}0.4174 \mathrm{E}-23 & 0.8942 \mathrm{E}-48 & 0.1873 \mathrm{E}-37 & \mathrm{Pu}-239\end{array}$

$0.6177 \mathrm{E}-32$

$0.7649 \mathrm{E}-21 \quad 0.1638 \mathrm{E}-45 \quad 0.4936 \mathrm{E}-38$

$0.6177 \mathrm{E}-32$
$\mathrm{Pu}-240$

$0.7790 \mathrm{E}-32$

$0.5451 E-22 \quad 0.1186 E-46 \quad 0.9456 E-38$

$\mathrm{Pu}-241$

$0.1312 \mathrm{E}-29$

$0.1414 \mathrm{E}-20 \quad 0.3100 \mathrm{E}-45 \quad 0.9413 \mathrm{E}-37$

Am-241

$0.1048 \mathrm{E}+13 \quad 0.2279 \mathrm{E}-12 \quad 0.2825 \mathrm{E}-04$

$0.3466 \mathrm{E}-33$

$\mathrm{Cm}-242$

$0.1327 \mathrm{E}-30$

$0.1840 \mathrm{E}+110.4034 \mathrm{E}-14 \cdot 0.4163 \mathrm{E}-04$

$\mathrm{Cm}-244$

$0.7762 \mathrm{E}-32$
\end{abstract}
$0.9874 \mathrm{E}-23 \quad 0.2410 \mathrm{E}-47 \quad 0.1950 \mathrm{E}-39$
$0.1419 \mathrm{E}-21 \quad 0.5630 \mathrm{E}-46 \quad 0.1306 \mathrm{E}-37$
$0.1094 \mathrm{E}-21 \quad 0.4324 \mathrm{E}-46 \quad 0.7402 \mathrm{E}-42$
$0.6768 \mathrm{E}-20 \quad 0.2686 \mathrm{E}-44 \quad 0.1670 \mathrm{E}-42$
$0.2318 \mathrm{E}-20 \quad 0.9240 \mathrm{E}-45 \quad 0.2105 \mathrm{E}-42$
$0.8601 E-21 \quad 0.3442 E-45 \quad 0.3546 E-40$
$0.6820 \mathrm{E}-23 \quad 0.2729 \mathrm{E}-47 \quad 0.9368 \mathrm{E}-44$
$0.2692 \mathrm{E}-23 \quad 0.1082 \mathrm{E}-47 \quad 0.3585 \mathrm{E}-41$
$0.6400 E-23 \quad 0.2593 E-47 \quad 0.2098 E-42$

$0.2388 \mathrm{E}+12 \quad 0.5273 \mathrm{E}-13 \quad 0.5973 \mathrm{E}-04$

$0.1104 \mathrm{E}+11 \quad 0.2457 \mathrm{E}-14 \quad 0.6555 \mathrm{E}-04$

$0.7154 \mathrm{E}+11 \quad 0.1604 \mathrm{E}-13 \quad 0.5632 \mathrm{E}-04$

$0.1273 \mathrm{E}+18 \quad 0.2812 \mathrm{E}-07 \quad 0.5263 \mathrm{E}+01$

$0.1725 \mathrm{E}+16 \quad 0.3867 \mathrm{E}-09 \quad 0.9875 \mathrm{E}+00$

$0.2104 \mathrm{E}-18 \quad 0.4682 \mathrm{E}-43 \quad 0.6058 \mathrm{E}-37$

$0.1114 \mathrm{E}-20 \quad 0.2515 \mathrm{E}-45 \quad 0.1843 \mathrm{E}-37$

$0.1712 \mathrm{E}-17 \quad 0.3894 \mathrm{E}-42 \quad 0.3387 \mathrm{E}-37$

$0.1345 \mathrm{E}-22 \quad 0.3104 \mathrm{E}-47 \quad 0.5077 \mathrm{E}-37$

$0.2952 \mathrm{E}-20 \quad 0.6863 \mathrm{E}-45 \quad 0.5024 \mathrm{E}-37$

$0.3973 E-23 \quad 0.9237 E-48 \quad 0.5134 E-39$

$0.3556 \mathrm{E}-24 \quad 0.8326 \mathrm{E}-49 \quad 0.4709 \mathrm{E}-39$

$0.1345 \mathrm{E}-24 \quad 0.3171 \mathrm{E}-49 \quad 0.4539 \mathrm{E}-39$

$0.1711 \mathrm{E}-21 \quad 0.4007 \mathrm{E}-46 \quad 0.1142 \mathrm{E}-38$

$0.7040 \mathrm{E}-23 \quad 0.1672 \mathrm{E}-47 \quad 0.1110 \mathrm{E}-38$

$0.9020 \mathrm{E}-21 \quad 0.2157 \mathrm{E}-45 \quad 0.6880 \mathrm{E}-39$

$0.2727 \mathrm{E}-22 \quad 0.6475 \mathrm{E}-47 \quad 0.4360 \mathrm{E}-39$

Control Room Transport Group Inventory:

$\begin{array}{lccc}\text { Time }(\mathrm{h})=720.0000 & \text { Atmosphere } & \text { Sump } & \begin{array}{c}\text { Overlying } \\ \text { Pool }\end{array} \\ \text { Noble gases (atoms) } & 0.4782 \mathrm{E}+18 & 0.0000 \mathrm{E}+00 & 0.0000 \mathrm{E}+00 \\ \text { Elemental I (atoms) } & 0.5311 \mathrm{E}-18 & 0.0000 \mathrm{E}+00 & 0.0000 \mathrm{E}+00 \\ \text { Organic I (atoms) } & 0.1387 \mathrm{E}+13 & 0.0000 \mathrm{E}+00 & 0.0000 \mathrm{E}+00 \\ \text { Aerosols (kg) } & 0.4610 \mathrm{E}-42 & 0.0000 \mathrm{E}+00 & 0.0000 \mathrm{E}+00\end{array}$

(atoms)

Deposition Recirculating

Time $(h)=720.0000$

Noble gases (atoms)

Elemental I (atoms)

Organic I (atoms)

Surfaces

$0.0000 \mathrm{E}+00$ Filter

$0.0000 \mathrm{E}+00 \quad 0.0000 \mathrm{E}+00$

$0.0000 \mathrm{E}+00 \quad 0.1462 \mathrm{E}+15$

$0.0000 \mathrm{E}+00 \quad 0.3992 \mathrm{E}+16$

Aerosols ( $\mathrm{kg}$ )

$0.0000 \mathrm{E}+00 \quad 0.2111 \mathrm{E}-08$

Filter 2--Environment to $C R$ Transport Group Inventory:

Time $(h)=720.0000$

Noble gases (atoms)

Elementa1 I (atoms)

organic I (atoms)

Aerosols (kg)

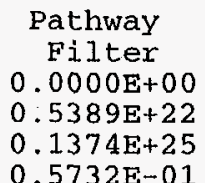

Unfiltered--Environment to CR Transport Group Inventory:

Time $(\mathrm{h})=720.0000$

Noble gases (atoms)

Elemental I (atoms)

Organic I (atoms)

Aerosols ( $\mathrm{kg})$

Pathway

Filter

$0.0000 \mathrm{E}+00$

$0.0000 \mathrm{E}+00$

$0.0000 \mathrm{E}+00$

$0.0000 \mathrm{E}+00$ 


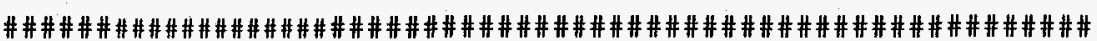
$\# \# \# \# \# \# \# \# \# \# \# \# \#$

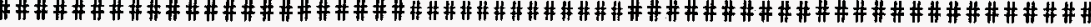 \#\#\#\#\#\#\#\#\#\#\#\#\#}

\author{
I-131 Summary
}

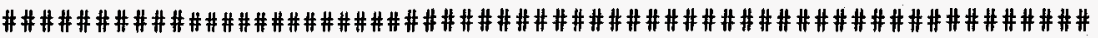

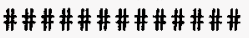

Exclusion Area Bndry Low Population zone

Control Room TEDE

TEDE

(rem)

Thyroid

TEDE

\begin{abstract}
Thyroid
\end{abstract}
TEDE

(rem)

(rem)

(rem)

$0.03 \quad 0.4311$

$040.1476 \mathrm{E}-05$

0.17
$0.2363 \mathrm{E}-03$

$$
11 \mathrm{E}-0
$$

$0.1863 E-02 \quad 0.5819 E-02$

$0.2515 E-03$ $0.2363 \mathrm{E}-03$
$0.28 \quad 0.4076$

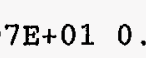

$1 \quad 0.6578 \mathrm{E}-01 \quad 0.2034 \mathrm{E}+00 \quad 0.8881 \mathrm{E}-02$

$\begin{array}{cccc}0.50 & 0.503 \mathrm{E}+00 & 0.2420 \mathrm{E}-01 & 0.2120 \mathrm{E}-0.1810 \mathrm{E}+03\end{array}$ $010.8578 \mathrm{E}-03$ $\begin{array}{rrrrr}0.8578 \mathrm{E}-03 & & 1.18 \\ 0.500 .1169 \mathrm{E}+02 & 0.5112 \mathrm{E}+00 & 0.1578 \mathrm{E}+01 & 0.6902 \mathrm{E}-01 & 0.8510 \mathrm{E}-0.5271 \mathrm{E}+03\end{array}$ $010.3501 \mathrm{E}-02$

$\begin{array}{lllll}1.18 & 0.3502 \mathrm{E}+02 & 0.1847 \mathrm{E}+01 & 0.4728 \mathrm{E}+01 & 0.2494 \mathrm{E}+00\end{array}$ $0.3807 \mathrm{E}+00 \quad 0.2242 \mathrm{E}-01$

$\begin{array}{llll}1.80 & 0.5660 \mathrm{E}+02 & 0.3583 \mathrm{E}+01 & 0.7641 \mathrm{E}+01 \quad 0.4837 \mathrm{E}+00\end{array}$ $0.6862 \mathrm{E}+00 \quad 0.6483 \mathrm{E}-01$

$2.00 \quad 0.6173 \mathrm{E}+02 \quad 0.4158 \mathrm{E}+01 \quad 0.8334 \mathrm{E}+01 \quad 0.5613 \mathrm{E}+00$ $0.7816 \mathrm{E}+00 \quad 0.8533 \mathrm{E}-01$.

$\begin{array}{lllll}8.00 & 0.6173 \mathrm{E}+02 & 0.4158 \mathrm{E}+01 & 0.1379 \mathrm{E}+02 & 0.3845 \mathrm{E}+01\end{array}$ $0.1727 \mathrm{E}+01 \quad 0.1253 \mathrm{E}+01$

$\begin{array}{lllll}24.00 & 0.6173 \mathrm{E}+02 & 0.4158 \mathrm{E}+01 & 0.1871 \mathrm{E}+02 & 0.1641 \mathrm{E}+02\end{array}$ $0.4086 \mathrm{E}+010.7122 \mathrm{E}+01$

$\begin{array}{lllll}48.00 & 0.6173 \mathrm{E}+02 & 0.4158 \mathrm{E}+01 & 0.2254 \mathrm{E}+02 & 0.2485 \mathrm{E}+02\end{array}$ $0.5175 \mathrm{E}+01 \quad 0.1022 \mathrm{E}+02$

$\begin{array}{lllll}72.00 & 0.6173 \mathrm{E}+02 & 0.4158 \mathrm{E}+01 & 0.2553 \mathrm{E}+02 & 0.3164 \mathrm{E}+02\end{array}$ $0.5966 \mathrm{E}+01 \quad 0.1252 \mathrm{E}+02$

$\begin{array}{lllll}96.00 & 0.6173 \mathrm{E}+02 & 0.4158 \mathrm{E}+01 & 0.2844 \mathrm{E}+02 & 0.3824 \mathrm{E}+02\end{array}$ $0.6734 \mathrm{E}+01 \quad 0.1476 \mathrm{E}+02$

$\begin{array}{lllll}120.00 & 0.6173 E+02 & 0.4158 E+01 & 0.2962 E+02 & 0.4092 E+02\end{array}$ $0.7043 \mathrm{E}+01 \quad 0.1566 \mathrm{E}+02$

$240.00 \quad 0.6173 \mathrm{E}+02 \quad 0.4158 \mathrm{E}+01 \quad 0.3545 \mathrm{E}+02 \quad 0.5421 \mathrm{E}+02$ $0.8486 \mathrm{E}+01 \quad 0.1987 \mathrm{E}+02$

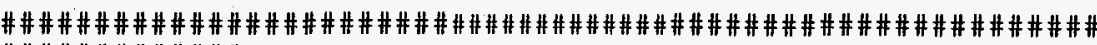

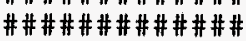

Annulus Thyroidr-131 (Curies)

$$
\begin{gathered}
0.03 \\
0.7246 \mathrm{E}+00 \\
0.17
\end{gathered}
$$
0.17 $0.3803 E-0.2261 E+02$ $681 \mathrm{E}+02$ 1.80

$$
\begin{gathered}
0.8146 \mathrm{E}+03 \\
2.00
\end{gathered}
$$

$0.8719 \mathrm{E}+03$ 8.00

$0.6441 \mathrm{E}+03$ 24.00

$0.2279 \mathrm{E}+03$ 48.00

$0.7459 \mathrm{E}+02$ 72.00

$0.5965 \mathrm{E}+02$ 96.00

$0.5808 \mathrm{E}+02$ 120.00

$0.5779 \mathrm{E}+02$ 240.00

$0.5706 \mathrm{E}+02$ 720.00 $0.5428 \mathrm{E}+02$
Sprayed Region

I-131 (Curies)

$0.2745 E+06$

$0.1450 \mathrm{E}+07$

$0.2081 \mathrm{E}+07$

$0.3152 \mathrm{E}+07$

$0.2298 \mathrm{E}+07$

$0.2317 \mathrm{E}+07$

$0.9242 E+06$

$0.5008 \mathrm{E}+05$

$0.4966 \mathrm{E}+05$

$0.4953 \mathrm{E}+05$

$0.4941 \mathrm{E}+05$

$0.4928 \mathrm{E}+05$

$0.4916 \mathrm{E}+05$

$0.4855 \mathrm{E}+05$

$0.4618 \mathrm{E}+05$
Unsprayed Region

I-131 (Curies)

$0.1656 \mathrm{E}+04$

$0.4953 E+05$

$0.2484 \mathrm{E}+06$

$0.5393 E+06$

$0.4975 \mathrm{E}+06$

$0.4953 \mathrm{E}+06$

$0.4265 E+06$

$0.1107 \mathrm{E}+05$

$0.1089 E+05$

$0.1087 \mathrm{E}+05$

$0.1084 \mathrm{E}+05$

$0.1081 E+05$

$0.1078 \mathrm{E}+05$

$0.1065 \mathrm{E}+05$

$0.1013 E+05$ 


$\begin{array}{ccc}\text { Time (hr) } & \begin{array}{c}\text { Environment } \\ \text { I-131 (Curies) }\end{array} & \begin{array}{c}\text { Contro1 Room } \\ \text { I-131 (Curies) }\end{array} \\ 0.03 & 0.8051 \mathrm{E}-01 & 0.2707 \mathrm{E}-05 \\ 0.17 & 0.2734 \mathrm{E}+01 & 0.8104 \mathrm{E}-04 \\ 0.28 & 0.1410 \mathrm{E}+01 & 0.1944 \mathrm{E}-03 \\ 0.50 & 0.1422 \mathrm{E}+02 & 0.3749 \mathrm{E}-03 \\ 1.18 & 0.1072 \mathrm{E}+02 & 0.4505 \mathrm{E}-03 \\ 1.80 & 0.3193 \mathrm{E}+01 & 0.4989 \mathrm{E}-03 \\ 2.00 & 0.9332 \mathrm{E}+01 & 0.4265 \mathrm{E}-03 \\ 8.00 & 0.1014 \mathrm{E}+02 & 0.1473 \mathrm{E}-03 \\ 24.00 & 0.1210 \mathrm{E}+02 & 0.1568 \mathrm{E}-03 \\ 48.00 & 0.2764 \mathrm{E}+02 & 0.5726 \mathrm{E}-04 \\ 72.00 & 0.2537 \mathrm{E}+02 & 0.5330 \mathrm{E}-04 \\ 96.00 & 0.2509 \mathrm{E}+02 & 0.5280 \mathrm{E}-04 \\ 120.00 & 0.2501 \mathrm{E}+02 & 0.3008 \mathrm{E}-04 \\ 240.00 & 0.2470 \mathrm{E}+02 & 0.2970 \mathrm{E}-04 \\ 720.00 & 0.2349 \mathrm{E}+02 & 0.2825 \mathrm{E}-04\end{array}$




\subsection{Problem Report Sheet}

\section{RADTRAD PROBLEM REPORT}

Return to: Sandia National Laboratories

PO Box 5800

Accident Analysis and Consequence Assessment Department Albuquerque, NM 87185-0748

fax (505) 844-3321

Name

Date

Computer

Location

Problem Summary

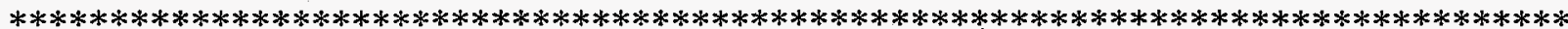

Problem Classification:

Manual Error

Desirable New Feature

Calculational Error

Small Quantitative Error
Manual Unclear

Input Error

Unexplained Termination

Large Quantitative Error

$* * * * * * * * * * * * * * * * * * * * * * * * * * * * * * * * * * * * * * * * * * * * * * * * * * * * * * * * * * * * * * * * * * * * * * * * * * * * * * * *$

Problem Description: 
[A186] D.J. Alpert, D.I. Chanin, and L.T. Ritchie, "Relative Importance of Individual Elements to Reactor Accident Consequences Assuming Equal Release Fractions," NUREG/CR-4467, SAND-85-2575, Sandia National Laboratories, Albuquerque, NM, 1986.

[Bi95] N.E. Bixler, "Simple Model of Pipe Decontamination for RADTRAD," Internal Sandia National Laboratories memorandum to D. Monroe, August 29, 1995.

[Br91] J.E. Brockmann, "Sampling and Transport of Aerosols" in Aerosol Measurement: Principles, Techniques, and Applications, K.Wilke and P.A. Baron, eds., Van Nostrand Reinhold, New York, NY, 1991.

[Ch90] D.I. Chanin, J.L. Sprung, L.T. Ritchie, and H-N Jow, "Melcor Accident Consequence Code System (MACCS) User's Guide," NUREG/CR-4691, Vol. 1, Sandia National Laboratories, Albuquerque, NM, 1990.

[C191] J.E. Cline, "MSIV Leakage Iodine Transport Analysis," NRC-03-87-029, Nuclear Regulatory Commission, Washington, DC, 1991.

[Di62] J.J. DiNunno, R.E. Baker, F.D. Anderson, and R.L. Waterfield, "Calculation of Distance Factors for Power and Test Reactor Sites," TID-14844, U.S. Atomic Energy Commission, Washington, DC, 1962.

[Fa84] Fauske \& Associates, Inc., "FAI Aerosol Correlation, An Industry Degraded Core Rulemaking (IDCOR) Program Report," Bethesda, MD, 1984.

Federal Guidance Report No. 11, "Limiting Values of Radionuclide Intake and Air Concentration and Dose Conversion Factors for Inhalation, Submersion, and Ingestion," EPA 520/1-88-020, U.S. Environmental Protection Agency, Washington, DC, 1988.

Federal Guidance Report No. 12, "External Exposure to Radionuclides in Air, Water and Soil," EPA 420-r-93-081 U.S. Environmental Protection Agency, Washington, DC, 1993.

[P092] D.A. Powers and J.L. Sprung, "A Simplified Model of Aerosol Scrubbing by a Water Pool Overlying Core Debris Interacting with Concrete," NUREG/CR-5901, SAND92-1422, Sandia National Laboratories, Albuquerque, NM, 1992.

[P093] D.A. Powers, and S.B. Burson, "A Simplified Model of Aerosol Removal by Containment Sprays," NUREG/CR-5966, SAND92-2689, Sandia National Laboratories, Albuquerque, NM, 1993. 
[Po95] D. A. Powers, K. E. Washington, S. B. Burson, and J. L. Sprung, "A Simplified Model of Aerosol Removal by Natural Process in Reactor Containments," NUREG/CR-6189, SAND940407, Sandia National Laboratories, Albuquerque, NM, 1995.

[Ra95] J. V. Ramsdell, C.A. Simonen, and S.B. Smyth, "Atmospheric Relative Concentrations in Building Wakes," NUREG/CR-6331, PNL-10521, Pacific Northwest National Laboratory, Richland, WA, 1995.

[So95] L.Soffer, S.B.Burson, C.M.Ferrell, R.Y.Lee, and J.N.Ridgely, "Accident Source Terms for Light-Water Nuclear Power Plants," NUREG-1465, Nuclear Regulatory Commission, Washington; DC, 1995.

USNRC [74] "Assumptions Used for Evaluating the Potential Radiological Consequences of a Loss of Coolant Accident for Boiling Water Reactors," Regulatory Guide 1.3, Revision 2, Nuclear Regulatory Commission, Washington, DC, 1974.

USNRC [74] "Assumptions Used for Evaluating the Potential Radiological Consequences of a Loss of Coolant Accident for Pressurized Water Reactors," Regulatory Guide 1.4, Revision 2, Nuclear Regulatory Commission, Washington, DC, 1974.

WASH-1400 [75] "Reactor Safety Study: An Assessment of Accident Risks in U.S. Commercial Nuclear Power Plants," WASH-1400, NUREG 75/014, Nuclear Regulatory Commission, Washington, DC, 1975. 


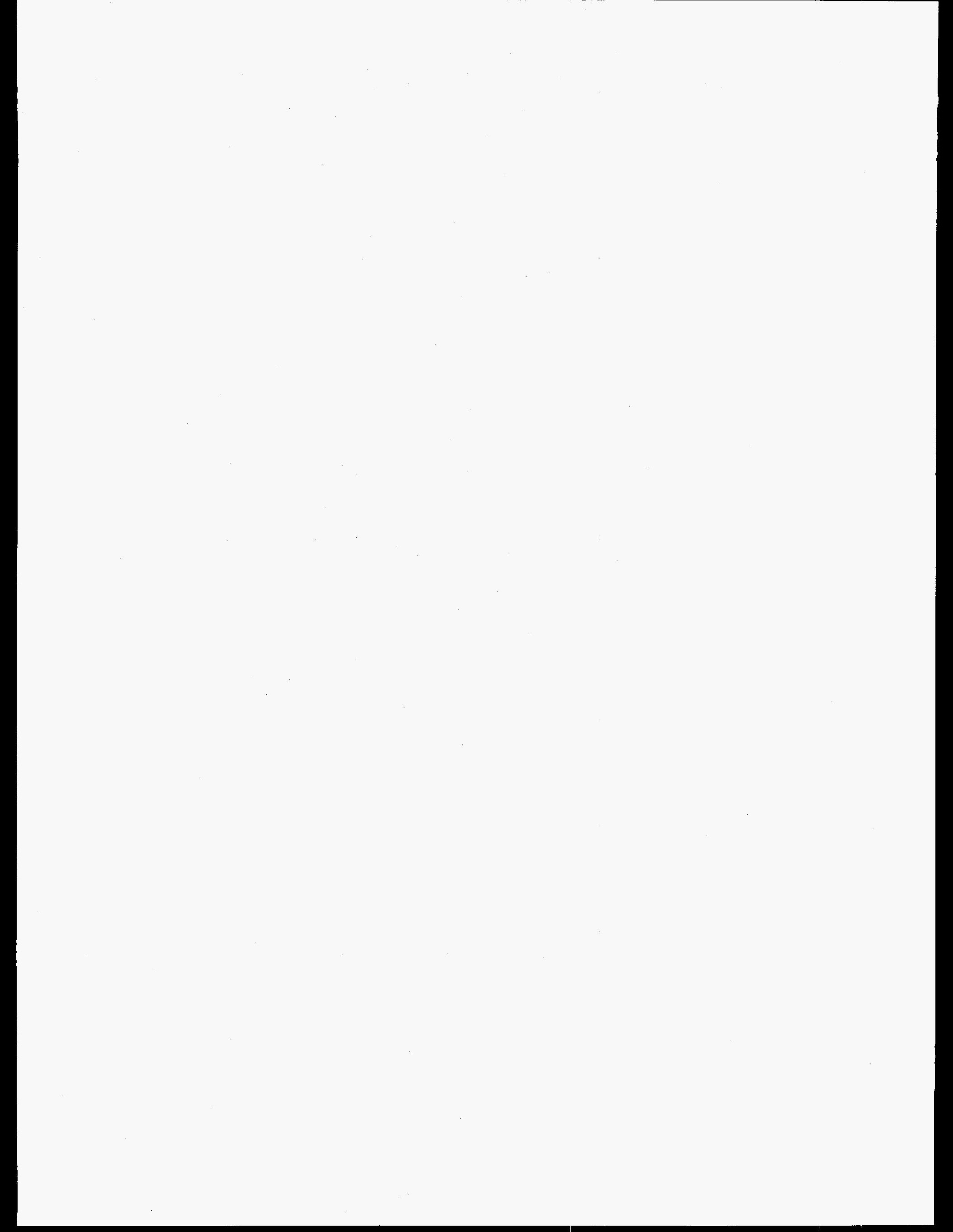




\section{Technical Bases for RADTRAD}

The radiological consequences of an accident in a nuclear reactor depend upon the quantity of the radioactive material that escapes to the environment or enters the control room. RADTRAD is designed to calculate doses at offsite locations, such as the low population zone, and in the control room. The code has two default source terms to describe fission product release to the containment: those from TID-14844 [Di62] and those from NUREG-1465 [So1995] are discussed in Section 1.4.3. As the material is transported through the containment and other buildings, credit is given for several natural and engineered removal mechanisms. Containment sprays remove aerosols, elemental iodine, and organic iodine. The flow between buildings or rooms may be through HEPA filters or a suppression pool. Leakage to the environment may occur. Aerosols can deposit on surfaces within rooms and also in connecting paths. Models are included for these different removal mechanisms. Alternatively, the user may elect to input timedependent values for the removal coefficient. In Sections 2.1 and 2.2 the technical bases for the RADTRAD model of these phenomena are discussed. After transporting the nuclides to different locations, RADTRAD calculates the dose at user-specified locations. Section 2.3 discusses the technical basis for this model.

The numerical solutions used by the code to solve these equations are discussed in Section 2.4. The code uses a Laplace transform method to solve the transport problem as discussed in Section 2.4.2. The technical basis for the radioactive decay and daughter in-growth model in the RADTRAD calculation is discussed in Section 2.4.3.

\subsection{Transport and Removal}

The basic equation for radionuclide transport and removal is the same for all compartments. However, depending on the removal mechanisms available within a compartment or between two compartments, some of the coefficients in the equation may be zero. This section describes the general equation used for the in-plant transport and removal of fission products.

\subsubsection{Governing Equations}

The governing equation for the number of atoms of nuclide $n$, in compartment $i$, during time step $m$ is provided with all source and sink terms explicitly shown for clarity. The time step $m$ refers to the scenario-dependent and supplemental time steps. Supplemental time steps are defined by the user in the Calculate Results screen (screen number 3000). Scenario-dependent time steps end when an input changes state (e.g., when sprays are turned on). 


$$
\begin{gathered}
\frac{d}{d t} N_{n, i}^{m}=\sum_{v=1}^{n-l} \beta_{n, v} N_{v, i}^{m} \lambda_{v}+S_{n, i}^{m} \\
-\left[\sum_{\substack{j=1 \\
j \neq i}}^{L}\left[F_{i, j(c o n v)}^{m}+\frac{Q_{i, j(s)}^{m}}{V o l_{i}}+\frac{Q_{i, j(p)}^{m}}{V o l_{i}}\right]+\lambda_{n}+\lambda_{s p r, n}^{m}(t)+\lambda_{d e p, n}^{m}(t)+\frac{\eta_{n: i, j}^{m}}{100} F_{i, j(f o r c e d)}^{m}\right] N_{n, i}^{m} \\
+\sum_{\substack{j=1 \\
j \neq i}}^{L}\left[\left(1-\frac{\eta_{n: i, j}^{m}}{100}\right) F_{i, j(f o r c e d)}^{m}+F_{i, j(c o n v)}^{m}+\frac{Q_{i, j(s)}^{m}}{V o l_{j} D F_{n(s)}^{m}}+\frac{Q_{i, j(p)}^{m}}{V o l_{j} D F_{n(p)}^{m}}\right] N_{n, j}^{m} \\
\lambda_{n}=1 n(2) / T_{n}^{1 / 2}
\end{gathered}
$$

where:

$N_{n, i}^{m}=\quad$ number of atoms of nuclide $\mathrm{n}$ in compartment $i$ during time step $\mathrm{m}$

$\beta_{n, v}=\quad$ fraction of nuclide $v$ that decays to nuclide $n$ (dimensionless)

$\lambda_{n}=\quad$ radiological decay constant for nuclide $n\left(\mathrm{~s}^{-1}\right)$

$T_{n}^{1 / 2}=$ half-life of nuclide $n(\mathrm{~s})$

$F_{i, j(c o n v)}^{m}=$ volume-normalized convective (leakage) air flow rate from compartment $j$ to $i\left(\mathrm{~s}^{-1}\right)$

$F_{i, j(f o r c e d)}^{m}=$ volume-normalized forced air flow rate from compartment $j$ to $i\left(\mathrm{~s}^{-1}\right)$

$L=$ number of compartments defined in the plant model

$Q_{i, j(s)}^{m}=$ volumetric flow rate from compartment $j$ to $i$ through a suppression pool $\left(\mathrm{m}^{3} / \mathrm{s}\right)$

$Q_{i, j(p)}^{m}=$ volumetric flow rate from compartment $j$ to $i$ through a pipe $\left(\mathrm{m}^{3} / \mathrm{s}\right)$

Volk $=\quad$ volume of compartment $k\left(\mathrm{~m}^{3}\right)$

$D F_{n(s)}^{m}=$ suppression pool decontamination factor for nuclide $n$ during time step $m$ (dimensionless)

$D F_{n(p)}^{m}=$ piping decontamination factor for nuclide $n$ during time step $m$ (unitless)

$\lambda_{s p r, n}^{m}(t)=$ time-dependent spray removal coefficient for nuclide $n\left(\mathrm{~s}^{-1}\right)$

$\lambda_{d e p, n}^{m}(t)=$ time-dependent natural deposition removal rate coefficient for nuclide $n\left(\mathrm{~s}^{-1}\right)$ 
$S_{n, i}^{m}=\quad$ source injection rate of nuclide $n$ to compartment $i$ during time step $m$ (atoms/s)

$\eta_{n: i, j}^{m}=\quad$ filter efficiency associated with nuclide $n$ and the pathway from $j$ to $i(\%)$

The first line of the equation relates the change in nuclide inventory within volume $i$ due to the increase from nuclide decay $\lambda_{n}$ plus the increase due to the source injection, typically from the nuclides being released during the accident from the reactor coolant system to the containment. The second line details the various removal mechanisms that can be active in volume $i$ plus the removal due to decay of nuclide $n$. These range from atoms exiting the volume due to leakage and flow through pathways, to atoms being deposited on the walls or floor of the volume. The third line details the pathways that can be used to add nuclide $n$ to the volume, typically from transport from other volumes.

Equation 1 is solved by RADTRAD using the assumption that all nuclides can be grouped according to their chemical and transport similarities. Thus four transport groups were used: noble gases, elemental iodine, organic iodine, and aerosols. These four groups transport and react with their surroundings differently, but all components of any one group act the same. This implies that all xenon and krypton isotopes will transport identically and that cesium, barium, and all other aerosol isotopes will transport identically. The user specifies the fractions for the released iodine and thus partitions the iodine within the latter three groups. The removal mechanisms are discussed in Section 2.2, the mathematical solution to the transport problems in Section 2.4, and the logic flow and program details in Section 4.

\subsection{Removal Phenomena}

Sprays, natural deposition, leakage, natural and forced convection, filters, and suppression pools are treated as mechanisms for removing radioactive material from the compartment atmospheres. In addition, during a severe accident, overlying pools can reduce the ex-vessel release into the containment atmosphere. The material that is removed from the compartment atmosphere by these mechanisms will not reappear in that compartment with the few exceptions that will be noted in the following subsections. The driving routine to determine the individual removal phenomena was implemented in COEFFS.

It is common practice to relate some removal mechanisms to particular loss terms. For example, filters are discussed in terms of efficiencies, and natural deposition in terms of removal coefficients. These are related to each other as well as to decontamination factor (DF). The decontamination factor is defined as the ratio of the total aerosol material subjected to a removal process to the total aerosol material that remains airborne. The relationship between decontamination factor and removal coefficient, $\lambda$, is:

$$
\frac{d D F}{d t}=\lambda D F
$$


The average decontamination factor is related to the mass fraction, $m_{f}$, remaining in the volume as:

$$
m_{f}=\frac{M(t)}{M(0)}=\frac{1}{D F}=\exp (-\lambda t)
$$

The decontamination factor is also related to the removal efficiency, $\eta$, as:

$$
D F=\frac{1}{1-\eta}
$$

In the first three subsections, sprays, natural deposition, and overlying pools will be discussed. These are all phenomena that occur within a volume. The following three subsections then discuss the mass removal phenomena that occur between volumes, i.e., leakage, suppression pools and deposition in piping. The last subsection discusses filters as they occur both in pathways between volumes, for example a control room intake filter, and within a volume, such as a control room recirculating filter.

\subsubsection{Sprays}

Sprays may remove aerosols and iodine. It is assumed that the noble gases are not affected by sprays. The code currently provides the user with a choice of an aerosol removal model or a userspecified aerosol removal coefficient table. Only the user-provided removal coefficient table option is available for elemental and organic iodine removal by sprays. The use of sprays will result in a user-provided sump filling with the spray water. The primary routine for spray phenomena is SPRY.

\subsubsection{Powers Model for Aerosol Removal}

The user may specify the Powers model [P093] or a table for the aerosol removal coefficient as a function of time. The extent to which sprays will decontaminate an aerosol-laden atmosphere depends on the number of spray droplets falling through the atmosphere and the distance they fall. The water flux into the containment atmosphere is time dependent and they fall distance is dependent upon the containment design. In the Powers document, a mechanistic model of aerosol removal by sprays is derived based upon how a single falling droplet would scavenge particles. Powers suggested that many of the properties and phenomena affecting the process are not accurately predictable. To account for these uncertainties, a large number of calculations were performed with the mechanistic model (with specific values for the height and water flux) and varying the uncertain parameters. Typical uncertain parameters were the containment pressure, the aerosol particle size, the water droplet distribution, the dynamic shape factors, and the properties of the water. In all, 20 parameters that were related to the phenomena were varied. An uncertainty analysis was performed using a Monte Carlo method to sample all the calculations. The results were used to construct simplified expressions for spray removal coefficients. The model input parameters are the spray water flux, $\mathrm{W}\left(\mathrm{m}^{3} \mathrm{H}_{2} \mathrm{O} / \mathrm{m}^{2} \mathrm{~s}\right)$ and the fall height of the spray droplets $H(\mathrm{~m})$. Since the model was developed from an uncertainty study, the 
user specifies a percentile. The "best estimate" value is associated with the $50^{\text {th }}$ percentile, or median values; the lower bound is associated with the $10^{\text {th }}$ percentile; and the reasonable upper bound, or largest decontamination factor, with the $90^{\text {th }}$ percentile. The model was developed using values of 10,100 , and $2500 \mathrm{~cm}^{3} \mathrm{H}_{2} \mathrm{O} / \mathrm{cm}^{2} \mathrm{~s}$ for the spray water flux, $\mathrm{W}$, and fall heights of $500,853,1000,1584,2000,3000,4000$, and $5000 \mathrm{~cm}$. The model should not be used for spray water fluxes and fall heights outside of these ranges. The aerosol removal coefficient is dependent on the fraction of the aerosol suspended in the atmosphere, $m_{f}$, which is defined as the aerosol mass in the atmosphere of a compartment at a given time, $t$, divided by the total aerosol mass released into the compartment atmosphere until this time. The model includes the effect of the unsprayed portions of the containment by incorporating the assumption that the circulation in the compartment is rapid compared with the removal rate. The equation that specifies the aerosol removal coefficient, $\lambda$, at any time is:

$$
\begin{gathered}
\lambda\left(s^{-1}\right)=\frac{1}{3600(1+\alpha)}\left(\left(a+b \log ^{10} W\right)\left\{1-\left[\frac{m_{f}(t)}{0.9}\right]^{c}\right\}+\left(\frac{m_{f}(t)}{0.9}\right)^{c}\right) \\
\exp \left(A+B \ln W+C H+D W^{2} H\right. \\
\left.+E W H^{2}+F W+G W^{2} H^{2}\right)
\end{gathered}
$$

The constants $\mathbf{A}, \mathbf{B}, \mathbf{C}, \mathbf{D}, \mathbf{E}, \mathbf{F}, \mathbf{G}, \mathbf{a}, \mathbf{b}$, and $\mathbf{c}$ are defined in Table 2.2.1.1-1. The parameter $\alpha$ is the ratio of the compartment volume not exposed to sprays to the compartment volume exposed to sprays (unsprayed volume/sprayed volume). If the assumption that the circulation is rapid compared with the removal rate is not valid, then the user can split the compartment into two separate compartments and model the flow from the unsprayed portions to the sprayed portions as a convective flow. In this case $\alpha$ would be zero for the compartment containing the sprays.

This expression was originally developed for a puff release of aerosols into a system. In those cases where there is a continuing release, the size distribution will continually be renewed by the injected particulate. The model has been extended for this case by setting coefficients $\mathbf{A}$ through $\mathbf{G}$ to zero. This makes the model assume that the size distribution has not changed in time and that most of the material suspended is fresh material provided by the source. The aerosols removed by the sprays are placed in the sump. 
Table 2.2.1.1-1. Values for constants used in equation 2 [Chapter 7, Po93]

\begin{tabular}{|c|c|c|c||}
\hline & \multicolumn{3}{|c|}{ Value } \\
\hline \hline Constant & $\mathbf{1 0}^{\text {th }}$ Percentile & $\mathbf{5 0}^{\text {th }}$ Percentile & $\mathbf{9 0}^{\text {th }}$ Percentile \\
\hline $\mathbf{A}$ & 5.5750 & 6.83707 & 7.10927 \\
\hline $\mathbf{B}$ & 0.94362 & 1.0074 & 0.92549 \\
\hline $\mathbf{C}$ & 0.0 & $-2.4045 \times 10^{-5}$ & 0.0 \\
\hline $\mathbf{D}$ & $-6.9821 \times 10^{-3}$ & $-4.1731 \times 10^{-3}$ & $-8.0868 \times 10^{-4}$ \\
\hline $\mathbf{E}$ & $-7.327 \times 10^{-7}$ & $9.006 \times 10^{-8}$ & 0.0 \\
\hline $\mathbf{F}$ & 0.0 & -1.2478 & 0.0 \\
\hline $\mathbf{G}$ & $3.555 \times 10^{-6}$ & 0.0 & 0.0 \\
\hline $\mathbf{a}$ & 0.1108 & 0.1815 & 0.3751 \\
\hline $\mathbf{b}$ & -0.00201 & -0.01153 & 0.00648 \\
\hline $\mathbf{C}$ & 0.8945 & 0.5843 & 0.2786 \\
\hline
\end{tabular}

\subsubsection{Elemental Iodine Removal}

The user may specify a table with the spray removal coefficient for elemental iodine as a function of time. Along with the aerosols removed by the sprays, the removed iodine is located in the sump.

\subsubsection{Organic Iodine Removal}

The user may specify a table with the spray removal coefficient for organic iodine removal as a function of time. Organic iodine is not usually removed by sprays, but the option is available.

\subsubsection{Natural Deposition}

Natural deposition includes many phenomena (e.g., gravitational settling, thermophoresis, diffusiophoresis). This removal mechanism is considered valid only for aerosols and perhaps elemental iodine; organic iodine and noble gases are nonreactive and therefore are not likely to be affected by these mechanisms. The user may choose a model for aerosol natural deposition or may specify time-dependent removal coefficients. A removal coefficient must be specified for the elemental iodine. The primary routine for natural deposition phenomena is XNTDEP. The removed iodine adheres to the surfaces of the volume.

\subsubsection{Aerosols}

The user may specify a model or provide a table for the natural deposition removal coefficient as a function of time. There are two choices for models of natural deposition, one based upon Henry's analysis [Fa84] and one based upon Powers' analysis [Po95]. 
2.2.2.1.1 Henry's Correlation. The correlation developed by Henry [Fa84] is based upon observation of experiments concerned with aerosol decontamination. The form of the equation is based upon the consideration that sedimentation was the dominant phenomenon observed, and the coefficients were determined from experimental data from liquid metal reactor tests. The coefficients that best follow the data are

$$
\lambda=C_{1}\left(\frac{h_{r e f}}{h}\right)\left(\frac{\rho_{P}}{\rho_{P_{r e f}}}\right)\left(\rho_{A}\right)^{K}
$$

where:

$$
C_{1}=0.0220 ; k=0.60 \text { for } \rho_{A} \geq 6.0 \times 10^{-5} \mathrm{~kg} / \mathrm{m}^{3}
$$

and:

$$
C_{1}=0.0016 ; k=0.33 \text { for } \rho_{A}<6.0 \times 10^{-5} \mathrm{~kg} / \mathrm{m}^{3}
$$

where:

$\rho_{\mathrm{A}}=$ the aerosol mass in the volume

$h_{\text {ref }}=5.0 \mathrm{~m}$, as determined from the ABCOVE test $\mathrm{AB} 5$

$\rho_{\text {Pref }}=$ theoretical sodium oxide particle density of $2270 \mathrm{~kg} / \mathrm{m}^{3}$, as determined from the ABCOVE test AB5

$\rho_{p}=$ user-provided particle density $\left(\mathrm{kg} / \mathrm{m}^{3}\right)$

$h=$ user-provided fall height (m)

Henry's correlation input can be modified to account for the influence of aerosol shape and moisture by:

$$
\rho_{P}=\alpha \rho_{T}+(1-\alpha) \rho_{w}
$$

where:

$\alpha=\quad$ ratio of the effective aerosol particle density to theoretical density

$\rho_{T}=$ theoretical density $\left(\mathrm{kg} / \mathrm{m}^{3}\right)$

$\rho_{w}=$ density of water $\left(\mathrm{kg} / \mathrm{m}^{3}\right)$

In many cases the aerosol has only $20 \%$ of its theoretical density, so that in a high steam environment its effective density would be closer to that of water. The correlation was implemented in the routine XNTDEP. 
2.2.2.1.2 Powers Model. The model developed by Powers et al.

is a continuation of the work of Otter and Vaughn [Ot 86], which followed the work of Epstein and Ellison [Ep 87]. In these models the observation of a self-preserving aerosol size distribution is a key component in predicting aerosol behavior. Powers added the effects due to turbulence and to the multiple aerosol releases specified in NUREG-1465. The modified model was then compared with a series of CONTAIN [WA 91] cases to verify the aging and aerosol size distributions due to the multiple sources. To account for the uncertainties, a large number of calculations were performed with the modified mechanistic model, with specific values for the type of reactor containment, reactor power, and the source term release phase, while varying the values of the uncertain parameters. Typical uncertain parameters were the containment pressure during the various release phases, the floor and wall surface areas, the zirconium inventory, the ratio of containment volume to thermal power, and the properties of the concrete. In all, over 20 parameters that were related to the phenomena were varied. The final, highly simplified model for aerosol decontamination is described by:

$$
\frac{d D F_{\mathrm{i}}(t)}{d t}=\lambda_{d e p, I}\left(P_{t h}, t\right) D F_{i}(t)
$$

where:

$D F_{i}(t)=\quad$ decontamination factor for the $i^{\text {th }}$ class of radioactive aerosol at time $t$ where $I$ indicates when the material was released into the containment: during the gap, invessel, ex-vessel, or late in-vessel phases of a severe reactor accident

$\lambda_{\text {dep,I }}\left(P_{t h}, t\right)=$ decontamination coefficient at time $t$ and power $P_{t h}$ for phase $\mathrm{I}$, and

$P_{t h}=\quad$ thermal power of the reactor in question.

The decontamination coefficient, $\lambda$, was solved over the various release time intervals and for the different types of reactors and various operating power levels using a Monte Carlo uncertainty analysis. The results of these analyses are detailed in Tables 2.2.2.1-1 through 5 and are given in terms of percentile decontamination factors, $\lambda_{\text {dep }}\left(N \%, P_{t h}\right)$. Since the model was developed from an uncertainty study, the user specifies a percentile, $N \%$. The "best estimate" value is associated with the $50^{\text {th }}$ percentile, or median values; the lower bound is associated with the $10^{\text {th }}$ percentile; and the reasonable upper bound, or largest decontamination factor, with the $90^{\text {th }}$ percentile. Note that the tables were developed for both design basis and severe accident scenarios for generic PWRs and BWRs. The controlling logic was implemented in routine AEROSOL. This routine determines the current decontamination coefficient, $\lambda_{\text {net }}$, from the sum of the coefficients for each phase weighted by the iodine release rate, $S_{12, I}$, for phase I. Thus,

$$
\lambda_{\text {net }}=\frac{\ln \{[D F(t+\Delta t) / D F(t)]\}}{\Delta t}
$$


where

$$
\begin{gathered}
D F(t)=\frac{\sum_{I=1}^{4} S_{I 2, I}}{\sum_{I=1}^{4} S_{I 2, I} / D F_{I}} \\
D F_{I}=\prod_{I=I}^{4} \exp \left[\lambda_{\text {dep,I }}\left(N \%, P_{t h}\right) t\right]
\end{gathered}
$$

To compare with the original Powers document, the decontamination coefficient should be weighted by each chemical group release rate. RADTRAD does not maintain the necessary weighting factors, hence a single chemical release rate was used for the weighting coefficients. For both the severe accident and the design basis accident calculations, iodine was chosen because it releases in all four phases and is the most radiologically important. If a case is developed where the most important aerosol has a release rate significantly different than the iodine release rate, then some modification to the logic would be necessary.

Table 2.2.2.1-5 provides the decontamination coefficients for a design basis accident in an advanced PWR design. These values are based upon a Powers document [Po 97b] in which it was found that the CONTAIN-related calculation results were correlated with values in the table. These correlations do not vary with reactor thermal power; hence an approximation is used when the user selects a PWR design with a different power level. The approximation used assumes that the significant difference between the PWR and the advanced pressurized water reactor (APWR) design, in terms of natural deposition, is that the wall cooling enhances the phoretic deposition. Therefore the difference in decontamination coefficient at $1800 \mathrm{MWt}$ represents this phoretic deposition and is then scaled by the power ratio. The final approximation used is:

$$
\lambda_{\text {net }}\left(A P_{-} P \text { Power }\right)=\lambda_{\text {net }}\left(P W R_{-} P_{\text {th }}\right)+\left[\lambda_{\text {net }}\left(P W R_{-} 1800\right)-\lambda_{\text {net }}\left(P W R_{-} 1800\right)\right] \frac{P_{t h}}{1800}
$$

where

$\lambda_{d e p}\left(P W R \_1800\right)=$ decontamination coefficient from the PWR-1800 calculation

$\lambda_{\text {dep }}\left(P W R \_P_{t h}\right)=$ decontamination coefficient from the PWR design basis accident calculation for the power level indicated.

\subsubsection{Elemental Iodine Removal}

The user may specify a table for the natural deposition removal coefficient for elemental iodine as a function of time. 
Table 2.2.2.1-1 Correlation of PWR effective natural deposition decontamination coefficients with reactor thermal power P(MWt) for design basis accidents [Table 36, Po95]

Release Phase Time Interval (s) Correlation ${ }^{1}\left(\mathrm{hr}^{-1}\right)$

\begin{tabular}{|c|c|c|}
\hline gap & $\begin{array}{c}0-1800 \\
(0-0.5 \mathrm{hr})\end{array}$ & $\begin{array}{l}\lambda_{\text {dcp }}(90)=0.0365+3.580 \times 10^{-6} \mathrm{P}(\mathrm{MWt}) \\
\lambda_{\text {dep }}(50)=0.0268+3.475 \times 10^{-6} \mathrm{P}(\mathrm{MWt}) \\
\lambda_{\text {dep }}(10)=0.0182+3.260 \times 10^{-6} \mathrm{P}(\mathrm{MWt})\end{array}$ \\
\hline gap & $\begin{array}{c}1800-6480 \\
(0.5-1.8 \mathrm{hr})\end{array}$ & $\begin{array}{l}\lambda_{\text {dep }}(90)=0.1036[1-\exp (-2.239 \mathrm{P}(\mathrm{MWt}) / 1000)] \\
\lambda_{\text {dep }}(50)=0.0820[1-\exp (-1.159 \mathrm{P}(\mathrm{MWt}) / 1000)] \\
\lambda_{\text {dep }}(10)=0.0645[1-\exp (-0.938 \mathrm{P}(\mathrm{MWt}) / 1000)]\end{array}$ \\
\hline early in-vessel & $\begin{array}{l}1800-6480 \\
(0.5-1.8 \mathrm{hr})\end{array}$ & $\begin{array}{l}\lambda_{\text {dep }}(90)=0.0522[1-\exp (-2.458 \mathrm{P}(\mathrm{MWt}) / 1000)] \\
\lambda_{\text {def }}(50)=0.0417[1-\exp (-1.258 \mathrm{P}(\mathrm{MWt}) / 1000)] \\
\lambda_{\text {def }}(10)=0.0326[1-\exp (-0.910 \mathrm{P}(\mathrm{MWt}) / 1000)]\end{array}$ \\
\hline $\begin{array}{c}\text { gap + } \\
\text { early in-vessel }\end{array}$ & $\begin{array}{c}6480-13,680 \\
(1.8-3.8 \mathrm{hr})\end{array}$ & $\begin{array}{l}\lambda_{\text {der }}(90)=0.421[1-\exp (-2.530 \mathrm{P}(\mathrm{MWt}) / 1000)] \\
\lambda_{\text {def }}(50)=0.196[1-\exp (-1.040 \mathrm{P}(\mathrm{MWt}) / 1000)] \\
\lambda_{\text {def }}(10)=0.094[\mathrm{I}-\exp (-0.869 \mathrm{P}(\mathrm{MWt}) / 1000)]\end{array}$ \\
\hline $\begin{array}{l}\text { gap }+ \\
\text { early in-vessel }\end{array}$ & $\begin{array}{c}13,680-49,680 \\
(3.8-13.8 \mathrm{hr})\end{array}$ & $\begin{array}{l}\lambda_{\text {dep }}(90)=0.1920-1.35 \times 10^{-6} \mathrm{P}(\mathrm{MWt}) \\
\lambda_{\text {dep }}(50)=0.1382+6.85 \times 10^{-6} \mathrm{P}(\mathrm{MWt}) \\
\lambda_{\text {dep }}(10)=0.0811+10.15 \times 10^{-6} \mathrm{P}(\mathrm{MWt})\end{array}$ \\
\hline $\begin{array}{l}\text { gap }+ \\
\text { early in-vessel }\end{array}$ & $\begin{array}{l}49,680-80,000 \\
(13.8-22.22 \mathrm{hr})\end{array}$ & $\begin{array}{l}\lambda_{\text {deps }}(90)=0.1010 \\
\lambda_{\text {dep }}(50)=0.0912 \\
\lambda_{\text {dep }}(10)=0.0860[1-\exp (-2.384 \mathrm{P}(\mathrm{MWt}) / 1000)]\end{array}$ \\
\hline
\end{tabular}

$1 \quad \lambda_{\text {dep }}(50)$ is the 50 percentile or median effective decontamination coefficient. $\lambda_{\text {dep }}(90)$ and $\lambda_{\text {dep }}(10)$ are the $90^{\text {th }}$ and $10^{\text {th }}$ percentile values, respectively. 
Table 2.2.2.1-2 Correlation of PWR effective natural deposition decontamination coefficients with reactor thermal power for severe accidents [Table 26, Po95]

\begin{tabular}{|c|c|c|}
\hline gap & $\begin{array}{c}0-1800 \\
(0-0.5 \mathrm{hr})\end{array}$ & $\begin{array}{l}\lambda_{\text {dep }}(90)=0.0349+3.755 \times 10^{-6} \mathrm{P}(\mathrm{MWt}) \\
\lambda_{\text {dep }}(50)=0.0256+3.90 \times 10^{-6} \mathrm{P}(\mathrm{MWt}) \\
\lambda_{\text {dep }}(10)=0.0167+3.25 \times 10^{-6} \mathrm{P}(\mathrm{MWt})\end{array}$ \\
\hline gap & $\begin{array}{c}1800-6-4.80 \\
(0.51 .8 \mathrm{hr})\end{array}$ & $\begin{array}{l}\lambda_{\text {dep }}(90)=0.0808+5.955 \times 10^{-6} \mathrm{P}(\mathrm{MWt}) \\
\lambda_{\text {dep }}(50)=0.0174+8.39 \times 10^{-6} \mathrm{P}(\mathrm{MWt}) \\
\lambda_{\text {dep }}(10)=0.0322+7.16 \times 10^{-6} \mathrm{P}(\mathrm{MWt})\end{array}$ \\
\hline early in-vessel & $\begin{array}{r}1800-6480 \\
(0.5-1.8 \mathrm{hr})\end{array}$ & $\begin{array}{l}\lambda_{\text {dep }}(90)=0.0505+0.94 \times 10^{-6} \mathrm{P}(\mathrm{MWt}) \\
\lambda_{\text {dep }}(50)=0.0257+3.87 \times 10^{-6} \mathrm{P}(\mathrm{MWt}) \\
\lambda_{\text {dep }}(10)=0.0166+3.49 \times 10^{-6} \mathrm{P}(\mathrm{MWt})\end{array}$ \\
\hline $\begin{array}{c}\text { gap }+ \\
\text { early in-vessel }\end{array}$ & $\begin{array}{c}6480-13,680 \\
(1.8-3.8 \mathrm{hr})\end{array}$ & $\begin{array}{l}\lambda_{\text {dep }}(90)=0.1146+371.9 / \mathrm{P}(\mathrm{MWt}) \\
\lambda_{\text {dep }}(50)=0.0948+141.2 / \mathrm{P}(\mathrm{MWt}) \\
\lambda_{\text {dep }}(10)=0.0472+62.0 / \mathrm{P}(\mathrm{MWt})\end{array}$ \\
\hline $\begin{array}{l}\text { ex-vessel + } \\
\text { late in-vessel }\end{array}$ & $\begin{array}{r}6480-13,680 \\
(1.8-3.8 \mathrm{hr})\end{array}$ & $\begin{array}{l}\lambda_{\text {dep }}(90)=0.0754+184.9 / \mathrm{P}(\mathrm{MWt}) \\
\lambda_{\mathrm{dep}}(50)=0.0551+84.65 / \mathrm{P}(\mathrm{MWt}) \\
\lambda_{\mathrm{dcp}}(10)=0.0272+42.0 / \mathrm{P}(\mathrm{MWt})\end{array}$ \\
\hline $\begin{array}{l}\text { gap + } \\
\text { early in-vessel + } \\
\text { ex-vessel }\end{array}$ & $\begin{array}{c}13,680-42,480 \\
(3.8-11.8 \mathrm{hr})\end{array}$ & $\begin{array}{l}\lambda_{\mathrm{dep}}(90)=0.378+161.6 / \mathrm{P}(\mathrm{MWt}) \\
\lambda_{\text {dep }}(50)=0.269+141.2 / \mathrm{P}(\mathrm{MWt}) \\
\lambda_{\mathrm{dep}}(10)=0.068+81.8 / \mathrm{P}(\mathrm{MWt})\end{array}$ \\
\hline late in-vessel & $\begin{array}{c}13,680-42,480 \\
(3.8-11.8 \mathrm{hr})\end{array}$ & $\begin{array}{l}\lambda_{\text {dep }}(90)=0.0829-3.40 \times 10^{-6} \mathrm{P}(\mathrm{MWt}) \\
\lambda_{\text {dep }}(50)=0.0547-0.62 \times 10^{-6} \mathrm{P}(\mathrm{MWt}) \\
\lambda_{\mathrm{dcp}}(10)=0.0222+6.44 \times 10^{-6} \mathrm{P}(\mathrm{MWt})\end{array}$ \\
\hline $\begin{array}{c}\text { gap + } \\
\text { early in-vessel + } \\
\text { ex-vessel + } \\
\text { late in-vessel }\end{array}$ & $\begin{array}{l}42,480-80,000 \\
(11.8-22.22 \mathrm{hr})\end{array}$ & $\begin{array}{l}\lambda_{\text {dep }}(90)=0.210+50.6 / \mathrm{P}(\mathrm{MWt}) \\
\lambda_{\text {dcp }}(50)=0.144 \\
\lambda_{\text {dep }}(10)=0.0915[1-\exp (-2.216 \mathrm{P}(\mathrm{MWt}) / 1000)]\end{array}$ \\
\hline $\begin{array}{c}\text { gap } \\
\text { early in-vessel + } \\
\text { ex-vessel }+ \\
\text { late in-vessel }\end{array}$ & $\begin{array}{r}80,000-100,000 \\
(22.22-27.78 \mathrm{hr})\end{array}$ & $\begin{array}{l}\lambda_{\text {dep }}(90)=0.0933+12.0 / \mathrm{P}(\mathrm{MWt}) \\
\lambda_{\text {dep }}(50)=0.0838 \\
\lambda_{\text {dep }}(10)=0.0377\end{array}$ \\
\hline $\begin{array}{l}\text { gap } \\
\text { early in-vessel + } \\
\text { ex-vessel + } \\
\text { late in-vessel }\end{array}$ & $\begin{array}{l}100,000-120,000 \\
(22.78-33.33 \mathrm{hr})\end{array}$ & $\begin{array}{l}\lambda_{\text {dep }}(90)=0.0717+10.8 / \mathrm{P}(\mathrm{MWt}) \\
\lambda_{\mathrm{dep}}(50)=0.0669 \\
\lambda_{\mathrm{dep}}(10)=0.0277\end{array}$ \\
\hline
\end{tabular}

Release Phase

$\lambda_{\mathrm{dep}}(50)$ is the median of the uncertainty distribution. The $90^{\text {th }}$ and $10^{\text {th }}$ percentile values are $\lambda_{\text {dep }}(90)$ and $\lambda_{\text {dep }}(10)$, respectively. 
Table 2.2.2.1-3 Correlations of BWR effective natural deposition decontamination coefficients with reactor thermal power for design basis accidents [Table A-19, Po97a]

\begin{tabular}{|c|c|c|}
\hline Release Phase & Time Interval (s) & Correlations $^{1}\left(h^{-1}\right)$ \\
\hline gap & $\begin{array}{c}0-1800 \\
(0-0.5 \mathrm{hr})\end{array}$ & $\begin{array}{l}\lambda_{\text {dep }}(90)=2.903[\exp (-1060 / \mathrm{P}(\mathrm{MWt})] \\
\lambda_{\text {dep }}(50)=2.252[\exp (-1977 / \mathrm{P}(\mathrm{MWt})] \\
\lambda_{\text {dep }}(10)=1.285[\exp (-2119 / \mathrm{P}(\mathrm{MWt})]\end{array}$ \\
\hline gap & $\begin{array}{l}1800-7200 \\
(0.5-2.0 \mathrm{hr})\end{array}$ & $\begin{array}{l}\lambda_{\text {dep }}(90)=1.809[\exp (-777 / \mathrm{P}(\mathrm{MWt})] \\
\lambda_{\text {dep }}(50)=1.722[\exp (-1777 / \mathrm{P}(\mathrm{MWt})] \\
\lambda_{\text {dep }}(10)=1.161[\exp (-2274 / \mathrm{P}(\mathrm{MWt})]\end{array}$ \\
\hline early in-vessel & $\begin{array}{l}1800-7200 \\
(0.5-2.0 \mathrm{hr})\end{array}$ & $\begin{array}{l}\lambda_{\text {dep }}(90)=0.746[\exp (-626 / \mathrm{P}(\mathrm{MWt})] \\
\lambda_{\text {dep }}(50)=0.752[\exp (-1565 / \mathrm{P}(\mathrm{MWt})] \\
\lambda_{\text {dep }}(10)=0.520[\exp (-2173 / \mathrm{P}(\mathrm{MWt})]\end{array}$ \\
\hline $\begin{array}{l}\text { gap + } \\
\text { early in-vessel }\end{array}$ & $\begin{array}{c}7200-18,000 \\
(2.0-5.0 \mathrm{hr})\end{array}$ & $\begin{array}{l}\lambda_{\text {dep }}(90)=1.959[\exp (-548 / \mathrm{P}(\mathrm{MWt})] \\
\lambda_{\text {dep }}(50)=1.811[\exp (-1107 / \mathrm{P}(\mathrm{MWt})] \\
\lambda_{\text {dep }}(10)=1.551[\exp (-1507 / \mathrm{P}(\mathrm{MWt})]\end{array}$ \\
\hline $\begin{array}{c}\text { gap + } \\
\text { early in-vessel }\end{array}$ & $\begin{array}{c}18,000-30,000 \\
(5.0-8.33 \mathrm{hr})\end{array}$ & $\begin{array}{l}\lambda_{\text {dep }}(90)=1.533[\exp (-573 / \mathrm{P}(\mathrm{MWt})] \\
\lambda_{\text {dep }}(50)=1.317[\exp (-1225 / \mathrm{P}(\mathrm{MWt})] \\
\lambda_{\text {dep }}(10)=0.836[\exp (-1051 / \mathrm{P}(\mathrm{MWt})]\end{array}$ \\
\hline $\begin{array}{c}\text { gap + } \\
\text { early in-vessel }\end{array}$ & $\begin{array}{l}30,000-43,200 \\
(8.33-12.0 \mathrm{hr})\end{array}$ & $\begin{array}{l}\lambda_{\text {dep }}(90)=1.524[\exp (-647 / \mathrm{P}(\mathrm{MWt})] \\
\lambda_{\text {dep }}(50)=1.339[\exp (-1474 / \mathrm{P}(\mathrm{MWt})] \\
\lambda_{\text {dep }}(10)=0.780[\exp (-1316 / \mathrm{P}(\mathrm{MWt})]\end{array}$ \\
\hline $\begin{array}{l}\text { gap + } \\
\text { early in-vessel }\end{array}$ & $\begin{array}{l}43,200-70,000 \\
(12.0-19.4 \mathrm{hr})\end{array}$ & $\begin{array}{l}\lambda_{\text {dcp }}(90)=1.564[\exp (-717 / \mathrm{P}(\mathrm{MWt})] \\
\lambda_{\text {dep }}(50)=1.373[\exp (-1634 / \mathrm{P}(\mathrm{MWt})] \\
\lambda_{\text {dep }}(10)=0.778[\exp (-1548 / \mathrm{P}(\mathrm{MWt})]\end{array}$ \\
\hline $\begin{array}{l}\text { gap }+ \\
\text { early in-vessel }\end{array}$ & $\begin{array}{l}70,000-86,400 \\
(19.4-24.0 \mathrm{hr})\end{array}$ & $\begin{array}{l}\lambda_{\text {dcp }}(90)=1.562[\exp (-719 / \mathrm{P}(\mathrm{MWt})] \\
\lambda_{\text {dep }}(50)=1.441[\exp (-1785 / \mathrm{P}(\mathrm{MWt})] \\
\lambda_{\text {dep }}(10)=0.780[\exp (-1686 / \mathrm{P}(\mathrm{MWt})]\end{array}$ \\
\hline
\end{tabular}

$\lambda_{\text {dep }}(50)$ is the 50 percentile or median effective decontamination coefficient. $\lambda_{\text {dep }}(90)$ and $\lambda_{\text {dep }}(10)$ are the $90^{\text {th }}$ and $10^{\text {th }}$ percentile values, respectively. 
Table 2.2.2.1-4 Correlations of BWR effective natural deposition decontamination coefficients with reactor thermal power, (MWt) for severe accidents [Table A-10, Po97a]

Release Phase

Time Interval (s)

Correlations $^{1}\left(h \mathbf{r}^{-1}\right)$

\begin{tabular}{|c|c|c|}
\hline gap & $\begin{array}{c}0-1800 \\
(0 .-0.5 \mathrm{hr})\end{array}$ & $\begin{array}{l}\lambda_{\text {dep }}(90)=4.074[\exp (-1165 / \mathrm{P}(\mathrm{MWt})] \\
\lambda_{\text {dep }}(50)=3.517[\exp (-2534 / \mathrm{P}(\mathrm{MWt})] \\
\lambda_{\text {dep }}(10)=1.919[\exp (-2767 / \mathrm{P}(\mathrm{MWt})]\end{array}$ \\
\hline gap & $\begin{array}{l}1800-7200 \\
(0.5-2.0 \mathrm{hr})\end{array}$ & $\begin{array}{l}\lambda_{\text {dep }}(90)=5.132[\exp (-1557 / \mathrm{P}(\mathrm{MWt})] \\
\lambda_{\text {dep }}(50)=3.155[\exp (-2407 / \mathrm{P}(\mathrm{MWt})] \\
\lambda_{\text {dep }}(10)=1.558[\exp (-2646 / \mathrm{P}(\mathrm{MWt})]\end{array}$ \\
\hline early in-vessel & $\begin{array}{c}1800-7200 \\
(0.5-2.0 \mathrm{hr})\end{array}$ & $\begin{array}{l}\lambda_{\text {dep }}(90)=1.446[\exp (-1012 / \mathrm{P}(\mathrm{MWt})] \\
\lambda_{\text {dep }}(50)=1.202[\exp (-2045 / \mathrm{P}(\mathrm{MWt})] \\
\lambda_{\text {dep }}(10)=0.724[\exp (-2498 / \mathrm{P}(\mathrm{MWt})]\end{array}$ \\
\hline $\begin{array}{c}\text { gap + } \\
\text { early in-vessel }\end{array}$ & $\begin{array}{c}7200-18,000 \\
(2.0-5.0 \mathrm{hr})\end{array}$ & $\begin{array}{l}\lambda_{\text {dep }}(90)=2.224[\exp (-116 / \mathrm{P}(\mathrm{MWt})] \\
\lambda_{\text {dep }}(50)=1.479[\exp (-500 / \mathrm{P}(\mathrm{MWt})] \\
\lambda_{\text {dep }}(10)=0.939[\exp (-616 / \mathrm{P}(\mathrm{MWt})]\end{array}$ \\
\hline $\begin{array}{l}\text { ex-vessel + } \\
\text { late in-vessel }\end{array}$ & $\begin{array}{c}7200-18,000 \\
(2.0-5.0 \mathrm{hr})\end{array}$ & $\begin{array}{l}\lambda_{\text {dep }}(90)=0.639[\exp (-62 / \mathrm{P}(\mathrm{MWt})] \\
\lambda_{\text {dep }}(50)=0.525[\exp (-302 / \mathrm{P}(\mathrm{MWt})] \\
\lambda_{\text {dep }}(10)=0.394[\exp (-429 / \mathrm{P}(\mathrm{MWt})]\end{array}$ \\
\hline $\begin{array}{l}\text { gap + } \\
\text { early in-vessel + } \\
\text { ex-vessel }\end{array}$ & $\begin{array}{c}18,000-43,200 \\
(5.0-12.0 \mathrm{hr})\end{array}$ & $\begin{array}{l}\lambda_{\text {dep }}(90)=1.037[\exp (-240 / \mathrm{P}(\mathrm{MWt})] \\
\lambda_{\text {dcp }}(50)=0.779[\exp (-252 / \mathrm{P}(\mathrm{MWt})] \\
\lambda_{\text {dep }}(10)=0.576[\exp (-172 / \mathrm{P}(\mathrm{MWt})]\end{array}$ \\
\hline late in-vessel & $\begin{array}{c}18,000-43,200 \\
(5.0-12.0 \mathrm{hr})\end{array}$ & $\begin{array}{l}\lambda_{\text {dep }}(90)=0.052 \\
\lambda_{\text {dep }}(50)=0.00075 \\
\lambda_{\text {dep }}(10)=0.000005\end{array}$ \\
\hline $\begin{array}{l}\text { gap + } \\
\text { early in-vessel + } \\
\text { ex-vessel + } \\
\text { late in-vessel }\end{array}$ & $\begin{array}{c}43,200-80,000 \\
(12.0-22.2 \mathrm{hr})\end{array}$ & $\begin{array}{l}\lambda_{\text {dep }}(90)=0.704[\exp (-468 / \mathrm{P}(\mathrm{MWt})] \\
\lambda_{\text {dep }}(50)=0.445[\exp (-630 / \mathrm{P}(\mathrm{MWt})] \\
\lambda_{\text {dep }}(10)=0.295[\exp (-849 / \mathrm{P}(\mathrm{MWt})]\end{array}$ \\
\hline $\begin{array}{l}\text { gap + } \\
\text { early in-vessel + } \\
\text { ex-vessel + } \\
\text { late in-vessel }\end{array}$ & $\begin{array}{c}80,000-100,000 \\
(22.2-27.8 \mathrm{hr})\end{array}$ & $\begin{array}{l}\lambda_{\text {dep }}(90)=0.700[\exp (-717 / \mathrm{P}(\mathrm{MWt})] \\
\lambda_{\text {dcp }}(50)=0.463[\exp (-1242 / \mathrm{P}(\mathrm{MWt})] \\
\lambda_{\text {dep }}(10)=0.217[\exp (-894 / \mathrm{P}(\mathrm{MWt})]\end{array}$ \\
\hline $\begin{array}{l}\text { gap + } \\
\text { early in-vessel + } \\
\text { ex-vessel + } \\
\text { late in-vessel }\end{array}$ & $\begin{array}{c}100,000-120,000 \\
(27.8-33.3 \mathrm{hr})\end{array}$ & $\begin{array}{l}\lambda_{\text {dep }}(90)=0.665[\exp (-643 / \mathrm{P}(\mathrm{MWt})] \\
\lambda_{\text {dep }}(50)=0.473[\exp (-1384 / \mathrm{P}(\mathrm{MWt})] \\
\lambda_{\text {dep }}(10)=0.196[\exp (-945 / \mathrm{P}(\mathrm{MWt})]\end{array}$ \\
\hline
\end{tabular}

$\lambda_{\text {dicp }}(50)$ is the 50 percentile or median effective decontamination coefficient. 
Table 2.2.2.1-5 Correlations of APWR effective natural deposition decontamination coefficients for design basis accidents [Table 15, Po97b]

Release Phase

gap

gap

early in-vessel

gap +

early in-vessel

gap +

early in-vessel

gap +

early in-vessel
Time Interval (s)

Correlations ${ }^{1}\left(h^{-1}\right)$

\author{
0.1800 \\ (0-0.5 hr)
}

1800-6480

(0.5-1.8 hr)

1800-6480

(0.5-1.8 hr)

$6480-13,680$

(1.8-3.8 hr)

$13,680-49,680$

$(3.8-13.8 \mathrm{hr})$

$49,680-86,400$

$(13.8-24.0 \mathrm{hr})$

$$
\begin{aligned}
& \lambda_{\text {dep }}(90)=0.466 \\
& \lambda_{\text {dep }}(50)=0.233 \\
& \lambda_{\text {dep }}(10)=0.029 \\
& \lambda_{\text {dep }}(90)=0.872 \\
& \lambda_{\text {dep }}(50)=0.487 \\
& \lambda_{\text {dep }}(10)=0.160
\end{aligned}
$$

$\lambda_{\mathrm{dep}}(90)=0.393$

$\lambda_{\text {dep }}(50)=0.240$

$\lambda_{\mathrm{dep}}(10)=0.100$

$\lambda_{\mathrm{dcp}}(90)=1.210$

$\lambda_{\mathrm{dep}}(50)=0.980$

$\lambda_{\text {dep }}(10)=0.731$

$\lambda_{\text {dipp }}(90)=0.594$

$\lambda_{\text {dep }}(50)=0.397$

$\lambda_{\mathrm{dep}}(10)=0.245$

$\lambda_{\text {dep }}(90)=0.598$

$\lambda_{\text {dep }}(50)=0.327$

$\lambda_{\text {dep }}(10)=0.107$

$\lambda_{\mathrm{dep}}(50)$ is the 50 percentile or median effective decontamination coefficient. $\lambda_{\text {dep }}(90)$ and $\lambda_{\text {dep }}(10)$ are the $90^{\text {th }}$ and $10^{\text {th }}$ percentile values, respectively. 


\subsubsection{Overlying Pools}

Overlying pools are modeled as an in-compartment decontamination process similar to that for sprays and natural deposition. Overlying pools only affect the ex-vessel phase of fission product release from a severe accident. A model is available for the removal of fission products during transport through the pool. Alternatively, the user may specify decontamination factors as function of time. Noble gases are assumed not to interact with the pool and are transmitted without any depletion.

\subsubsection{Powers Model for Aerosol Removal}

The Powers model [P092] was developed by performing an uncertainty study using a model for aerosol scrubbing from bubbles rising through water (the POOL model from the VANESA code, Chapter 7 [Po86]). The uncertainty analysis was performed using the Monte Carlo method to sample 15 parameters related to phenomena connected with concrete erosion, the aerosols in the pool, and pool-bubble dynamics. The results of the uncertainty study were used to construct simple correlations relating the pool depth, subcooling, and uncertainty to the decontamination factor. Since the model was developed from an uncertainty study, the user specifies a percentile. The "best estimate" value is associated with the $50^{\text {th }}$ percentile, or median values; the lower bound is associated with the $10^{\text {th }}$ percentile; and the reasonable upper bound, or largest decontamination factor, with the $90^{\text {th }}$ percentile. The equations were developed using pool depths of $30,50,100,200,300$, and $500 \mathrm{~cm}$ and subcooling levels of $0,2,5,10,20,30,50$, and $70 \mathrm{~K}$. For pools with a depth of less than $30 \mathrm{~cm}$, the decontamination factor is taken as that for a pool of $30-\mathrm{cm}$ depth. For pools with a depth greater than $500 \mathrm{~cm}$, the decontamination factor is taken as that for a pool of 500-cm depth.

For a saturated pool, the general form of the decontamination factor equation is

$$
\ln (D F)=a+b H^{1 / 2}+c H^{3 / 2}+d H^{3}
$$

where $H$ is the pool depth $(\mathrm{cm})$.

The constants $\mathbf{a}, \mathbf{b}, \mathbf{c}$, and $\mathbf{d}$ are defined in Table 2.2.3.1-1 for the three different percentiles.

Table 2.2.3.1-1. Values for the constant used in equation 16 [Chapter 5, Po92]

\begin{tabular}{|c|c|c|c|}
\hline & \multicolumn{3}{|c|}{ Values } \\
\hline \hline Constant & $\mathbf{1 0}^{\text {th }}$ Percentile & $\mathbf{5 0}^{\text {th }}$ Percentile & $\mathbf{9 0}^{\text {th }}$ Percentile \\
\hline $\mathbf{a}$ & -0.1832417 & -0.195036 & 0.114994 \\
\hline $\mathbf{b}$ & 0.0879653 & 0.17976 & 0.29587 \\
\hline $\mathbf{c}$ & $8.192503 \times 10^{-5}$ & 0.0 & 0.0 \\
\hline $\mathbf{d}$ & $-1.2281546 \times 10^{-9}$ & $4.68319 \times 10^{-9}$ & $1.087539 \times 10^{-8}$ \\
\hline
\end{tabular}


For a subcooled pool, the general equation for the decontamination factor is:

$$
\begin{gathered}
\ln (D F)=\ln [D F(H, 0)]+a+b \Delta T+c \sqrt{\Delta T}+d \Delta T^{3 / 2} \\
+e H \Delta T+f(H \Delta T)^{1 / 2}+g H^{3} \sqrt{\Delta T}+h H^{3 / 2}
\end{gathered}
$$

where $\Delta T$ is the number of degrees of subcooling $(\mathrm{K})$. Note that the first term in equation 17 is the result of equation 16 .

The constants $\mathbf{a}, \mathbf{b}, \mathbf{c}, \mathbf{d}, \mathbf{e}, \mathbf{f}, \mathbf{g}$, and $\mathbf{h}$ are shown in Table 2.2.3.1-2 for the different percentiles.

Table 2.2.3.1-2. Values for the constant used in equation 17 [Chapter 5, Po92]

\begin{tabular}{|c|c|c|c|}
\hline & \multicolumn{3}{|c|}{ Values } \\
\hline Constant & $\mathbf{1 0}^{\text {th }}$ Percentile & $\mathbf{5 0}^{\text {th }}$ Percentile & $\mathbf{9 0}^{\text {th }}$ Percentile \\
\hline $\mathbf{a}$ & 0.00993606 & -0.084 .381550 & 0.03437166 \\
\hline $\mathbf{b}$ & -0.0474108 & -0.07040774 & -0.2333505 \\
\hline $\mathbf{c}$ & 0.5696997 & 0.8238286 & 1.4415216 \\
\hline $\mathbf{d}$ & 0.0 & 0.0 & 0.01234607 \\
\hline $\mathbf{e}$ & 0.0 & 0.0 & $3.92396212 \times 10^{-4}$ \\
\hline $\mathbf{f}$ & 0.0433372 & 0.0668004 & 0.075810892 \\
\hline $\mathbf{g}$ & 0.0 & 0.0 & $1.3850581 \times 10^{-8}$ \\
\hline $\mathbf{h}$ & 0.0 & $8.2346311 \times 10^{-5}$ & 0.0 \\
\hline
\end{tabular}

\subsubsection{Elemental Iodine Removal}

The user may specify a table for the elemental iodine removal coefficient as a function of time. This removal coefficient should include the effects of reevolution of iodine once it is deposited because iodine reevolution is not explicitly modeled.

\subsubsection{Organic Iodine Removal}

The user may specify a table for the organic iodine decontamination factor as a function of time in an overlying pool.

\subsubsection{Leakage}

The user may specify the leakage between compartments or from a compartment to the environment compartment. No differentiation is made for different chemical groups or forms, thus all fission products leak at the same rate, with a removal efficiency of zero for all transport 
groups. This is the commonly used method of releasing radionuclides to the environment. The primary routine for leakage phenomena is CIN.

\subsubsection{Suppression Pools}

Suppression pools are modeled as a pathway between two compartments. As material passes through the suppression pool, some portion of that material will be retained. The retention by the pool of aerosols and elemental and organic iodine is discussed in this section. Noble gases are assumed to not be affected by the presence of a suppression pool. The primary routine for suppression pool phenomena is SUPIN, which is called for the path into the pool; SUPOUT is called for the path out of the suppression pool and determines flow rates only.

\subsubsection{Aerosol Removal}

The user may specify a table for the aerosol decontamination factor and the volumetric flow rate into the suppression pool as a function of time. As those fission products that are in aerosol form move from one compartment to another through a suppression pool, the amount retained by the pool is related to the decontamination factor as given by equation 3 . The aerosol decontamination factor is applied to iodine aerosols as well as aerosols from all other chemical groups.

\subsubsection{Elemental Iodine Removal}

The user may specify a table for the elemental iodine decontamination factor and the volumetric flow rate into in the suppression pool as a function of time. The elemental iodine retained is placed in the suppression pool.

\subsubsection{Organic Iodine Removal}

The user may specify a table for the organic iodine decontamination factor and the volumetric flow rate into the suppression pool as a function of time. The organic iodine retained is placed in the suppression pool.

\subsubsection{Deposition in Piping}

Piping is modeled as a transfer pathway where deposition can occur. A model is available for the removal of fission products during transport through pipes. Alternatively, the user may specify decontamination factors and volumetric flow rates as a function of time. Noble gases are assumed not to interact with the piping and are transmitted without any depletion. The primary routine for pipe deposition phenomena is PIPIN. The models chosen do not have documented limitations; however, the assumption is made that only primary deposition phenomena are being modeled. Scenarios in which supersaturated conditions occur, or in which very high or very low velocities are found, may give unrealistic results. 


\subsubsection{Brockmann Model for Aerosol Removal}

The user may specify a table for the aerosol decontamination factor and a volumetric flow rate as a function of time for aerosols moving through the piping. Alternatively, the user may select the Brockmann model for deposition in pipes. The variation of the Brockmann model [Br91] used in RADTRAD only examines deposition in pipe bends and that due to settling, turbulent inertia, and diffusion. The primary routine for the Brockmann model of aerosol deposition in pipes is implemented in the routine BROCK.

The mean particle diameter, $d_{p}$, is assumed to be $1 \mu$ with an average particle density, $\rho_{p}$, of $1000 \mathrm{~kg} / \mathrm{m}^{3}$. The temperature, pressure, open volume, and other parameters as noted below are provided by the user.

Particles will settle due to gravitational force and deposit on the horizontally projecting lower surface of the flow path during transport. This deposition efficiency, $\eta_{g}$, is modeled as:

$$
\begin{aligned}
& \eta_{g}=1-\exp \left(-U_{g} A_{s} / \pi Q\right) \\
& U_{g}=\tau g \\
& \tau=d_{p}^{2} \rho_{p} / 18 \mu
\end{aligned}
$$

where:

$$
\begin{array}{lll}
U_{g} & = & \text { gravitational deposition velocity }(\mathrm{m} / \mathrm{s}) \\
\tau & = & \text { particle relaxation time }(\mathrm{s}) \\
Q & = & \text { user-provided pipe gas flow }\left(\mathrm{m}^{3} / \mathrm{s}\right) \\
A_{s} & = & \text { user-provided total pipe surface area }\left(\mathrm{m}^{2}\right) \\
\mu & = & \text { viscosity of air, } 1.93 \times 10^{-5} \mathrm{~Pa}-\mathrm{s}
\end{array}
$$

If the flow is turbulent, then turbulence in the central core can propel a particle into the laminar sublayer. If the particle inertia is high, then it will penetrate the sublayer and be collected on the wall. There have been experiments on inertial deposition velocity [Li.74] and Brockmann has modeled the turbulent deposition efficiency, $\eta_{\text {turb }}$, as:

$$
\begin{aligned}
& \eta_{\text {turb }}=1-\exp \left(-U_{t} A_{s} / Q\right) \\
& U_{t}=0.01988 U_{a i r} / R e^{1 / 8} \\
& R e=\rho_{\text {air }} U_{\text {air }} d_{H} / \mu
\end{aligned}
$$


where:

$U_{t} \quad=\quad$ turbulent inertial deposition velocity $(\mathrm{m} / \mathrm{s})$

$U_{\text {air }}=$ air velocity $(\mathrm{m} / \mathrm{s})$

$d_{H} \quad=\quad$ hydraulic diameter, $4 \mathrm{vol} / A_{S}(\mathrm{~m})$

$\rho_{\text {air }}=$ air density $\left(\mathrm{kg} / \mathrm{m}^{3}\right)$

When the flow is slower such that

$$
\frac{\tau U_{a i r}}{d_{H}} \operatorname{Re}^{3 / 4}<326.6
$$

then the turbulent deposition velocity is

$$
U_{t}=1.861 \times 10^{-7}\left[\frac{\tau U_{a i r}}{d_{H}}\right]^{2} U_{a i r} \operatorname{Re}^{11 / 8}
$$

Small particles undergoing Brownian diffusion will diffuse from areas of high concentration to those of low concentration. Since the wall acts like a sink for these particles, there is net diffusion from the bulk to the wall. This phenomenon has been analyzed [Go 49] and the diffusional deposition efficiency is modeled as:

$$
\eta_{\text {diff }}=1-\exp \left(-U_{d i f f} A_{s} / Q\right)
$$

In laminar flow, this efficiency is modeled as:

$$
\begin{gathered}
\eta_{\text {diff }}=1.0-2.56 \zeta^{2 / 3}+1.2 \zeta+0.77 \zeta^{4 / 3} \\
\zeta=A_{S} D_{i f f} / Q d_{H}
\end{gathered}
$$

where

$\zeta=$ dimensionless diffusion parameter

$D_{i f f}=$ particle diffusion coefficient $\left(\mathrm{m}^{2} / \mathrm{s}\right)$

When the flow is slow or the pipe length is large such that $\zeta>0.02$, then

$$
\eta_{\text {diff }}=0.819 \exp (-3.657 \zeta)+0.097 \exp (-22.3 \zeta)+0.032 \exp (-57 \zeta)
$$


In turbulent flow, the diffusional efficiency [Fr 77] is modeled as:

$$
\eta_{\text {diff }}=1-\exp \left[-\zeta^{2 / 3} 0.0187 \operatorname{Re}^{13 / 24}\left(\frac{L}{d_{H}}\right)^{1 / 3}\right]
$$

where:

$L \quad=\quad$ path length $=A_{s} / \pi d_{H}(\mathrm{~m})$

When the direction of gas flow is diverted in a bend, an aerosol particle may deviate from the gas flow due to its inertia and deposit on the wall. The efficiency of this process is modeled as:

$$
\eta_{\text {bend }}=\frac{\tau U_{a i r} \varphi}{d_{H}}
$$

where:

$\tau=$ particle relaxation time, equation 18

$\varphi=$ the user-provided sum of the angles the flow has been diverted (radians)

When the flow is turbulent, this efficiency [Pu 87] is modeled as

$$
\eta_{\text {bend }}=1.0-\exp \frac{\left(-2.823 \tau U_{a i r} \phi\right)}{d_{H}}
$$

Thus the total aerosol deposition efficiency is modeled as:

$$
\eta_{T}=1.0-\left(1.0-\eta_{g}\right)\left(1.0-\eta_{\text {turb }}\right)\left(1.0-\eta_{\text {diff }}\right)\left(1.0-\eta_{\text {bend }}\right)
$$

\subsubsection{Bixler Model for Elemental Iodine Removal}

The user may specify an elemental iodine decontamination factor and a volumetric flow rate as a function of time. Alternatively, the user may also select the Bixler model for deposition in pipes. With this model, the elemental iodine vapor is deposited with an efficiency, $\eta_{e i}$, as:

$$
\begin{gathered}
\eta_{e i}=1-\exp \left(-A_{S} U_{e i} / 100 Q\right) \\
U_{e i}=2809 / T-12.5
\end{gathered}
$$


where:

$T=$ gas temperature $(\mathrm{K})$

The Bixler model for elemental iodine vapor deposition is based upon the Cline [C191] correlation and is implemented in the routine BIXLER1.

\subsubsection{Bixler Model for Organic Iodine Removal}

The user may specify an organic iodine decontamination factor and a volumetric flow rate as a function of time. Alternatively, the user may also select the Bixler model for deposition in pipes. With this model the organic iodine vapor is deposited with an efficiency, $\eta_{o i}$, as:

$$
\begin{gathered}
\eta_{o i}=1-\exp \left(-A_{S} U_{o i} / 100 Q\right) \\
U_{o i}=2809 / T-19.30
\end{gathered}
$$

The Bixler model for organic iodine vapor deposition is based upon the Cline [Cl 91] results and is implemented in the routine BIXLER3.

\subsubsection{Filters}

The user may specify a flow and a table of the filter efficiencies (\%) for the different transport groups and flow as a function of time. The model used for filtration assumes that the usersupplied fraction of material passing through the filter is retained. Thus, the decontamination coefficient is:

$$
\lambda_{\text {filter }}=\left(\eta_{\text {filter }} / 100\right) F
$$

where $F$ is the volume-normalized flow rate $\left(\mathrm{s}^{-1}\right)$.

The material removed is retained by the filters. Noble gases are not filtered. This type of transport path is typically used for both filtered and unfiltered inflow into a compartment. For example, both the control room leakage inflow and filtered inflow use this model. The primary routines for filter phenomena are FILTIN for inlet filters and RECIRC for recirculating filters.

\subsection{Consequence Analysis}

The user provides the atmospheric relative concentrations (X/Q) for offsite locations, for example, the exclusion area boundary or the outer perimeter of the low population zone, and the control room. In addition, the breathing rates (BR) and the control room occupancy factors are provided by the user. No atmospheric transport modeling is performed by the code at this time. Typical values for atmospheric dilution can be calculated using the PAVAN [Ba 82] or ARCON model [Ra95]. The consequence models were implemented in routine DOSES. 


\subsubsection{Offsite Consequences Analysis}

The dose to the hypothetical individual is calculated using the specified X/Qs and the amount of each nuclide released during the exposure period. The air immersion dose from each nuclide, $n$, in an environmental compartment is calculated as:

$$
D_{c, n}^{e n v}=A_{n}\left(X / Q^{)} D C F_{c, n}\right.
$$

where:

$D_{c, n}^{e n v}=$ air immersion (cloudshine) dose due to nuclide $n$ in the environment compartment (Sv)

$D C F_{c, n}=$ FGR 11 and 12 air immersion (cloudshine) dose conversion factor for nuclide $n$ as discussed in Section 1.4.3.3 ( $\left.\mathrm{Sv} \mathrm{m}^{3} / \mathrm{Bq} \mathrm{s}\right)$

$X / Q=\quad$ user-provided atmospheric relative concentration $\left(\mathrm{s} / \mathrm{m}^{3}\right)$

$A_{n}=\quad$ released activity of nuclide $n(\mathrm{~Bq})$

The activity is related to the number of atoms of nuclide $n$ as:

$$
A_{n}=N_{n} \lambda_{n}
$$

where $\lambda_{n}$ is the radiological decay constant for the nuclide as defined in equation 1.

The inhalation dose from each nuclide, $n$, is calculated as:

$$
D_{i, n}^{e n v}=A_{n}\left(X / Q^{B R L}\right) C F
$$

where:

$D_{i, n}^{e n v}=$ inhalation dose commitment due to nuclide $n$ in the environment compartment (Sv)

$B R=\quad$ user-provided breathing rate $\left(\mathrm{m}^{3} / \mathrm{s}\right)$

$D C F_{i, n}=$ user-provided inhalation dose conversion factor for nuclide $n$ as discussed in Section 1.4.3.3 (Sv/Bq)

\subsubsection{Control Room Consequence Analysis}

The dose to a hypothetical individual in the control room is calculated based on the timeintegrated concentration in the control room compartment. The air immersion dose in the control room is:

$$
D_{c, n}^{C R}=\int C_{n}(t) d t\left(D C F_{c, n} / G_{F}\right)
$$


where $C_{n}(t)$ is the instantaneous concentration of radionuclide $n$ in the compartment. The Murphy-Campe [Mu74] geometric factor, $G_{f}$, relates the dose from an infinite cloud to the dose from a cloud of volume $V$ as:

$$
G_{F}=\frac{1173}{V^{0.338}}
$$

The inhalation dose in the control room is:

$$
D_{i, n}^{C R}=\int C(t) d t\left(\frac{B R \cdot O F \cdot D C F_{i, n}}{G_{F}}\right)
$$

where $O F=$ user-supplied occupancy factor

The time-dependent atmospheric relative concentration $(\mathrm{X} / \mathrm{Q})$ provided by the user for the control room intake locations. The inhalation dose conversion factors have been taken from the output file produced by the FGRDCF code that is based on FGR 11 and 12 and are detailed in Section 1.4.3.3.

\subsection{Mathematical Solution Method}

This section addresses the mathematical solution method used to ultimately calculate the doses at various locations. The numerical solution technique is discussed in Section 2.4.1. The transport and radioactive decay calculations are discussed in Sections 2.4 .2 and 2.4.3, respectively.

\subsubsection{Numerical Solution Technique}

It is possible to define a single system of coupled ordinary differential equations (ODEs) that simultaneously represents all of the phenomena considered by the code. However, the size of this equation set, and the computational cost of its numerical integration, would make its implementation impractical on a PC. Significant economies of calculation time and computer memory size in RADTRAD are achieved by dividing the calculations into two parts: (1) transport and (2) radioactive decay.

The fundamental premise behind this approach ensures that during a time step of small enough duration, the interdependence of the two components of the calculations can be neglected. That is, the transport equations defining transfer of material between compartments during a calculational time step can be solved numerically without taking account of the radioactive decay and in-growth occurring over that period. Likewise, the radioactive decay and in-growth equations can be solved during a time step without considering the simultaneous phenomenon of transport between compartments. If the user is concerned with the assumption, the scenario can be tested by executing the code with $0.25,1.0,4.0$, and normal time steps to observe the differences; Section 2.4 .3 also discusses this effect. 
The two types of calculations are performed alternately. That is, the code begins its integration over a time step by considering the effect of radioactive decay on the inventory of all compartments. After the consideration of radioactive decay for the time step, the code considers the effect of transport between compartments during that time step. This process of alternating between decay and transport is repeated until the specified end time is reached.

During the course of its numerical integration, the code keeps track of the number of atoms of each fission product nuclide in each compartment. Compartrnents in this sense include not just atmospheric volumes within the plant, but surfaces, pools, and filters as well. By tracking all atoms in this manner, conservation of mass can be verified.

\subsubsection{Transport Calculations}

The present application is known as an "initial value problem" for the solution of ODEs. Numerical solution of the transport equations is accomplished with a Laplace transform method. The advantage of this approach is its capability to use large time intervals. The key assumption is that there is no change in any phenomenon during a time step. Thus, the code synchronizes the time step with the user-supplied phenomena, resulting in very large time steps that are limited only by the maximum transport or decay coefficient. The primary disadvantage is that this numerical technique is not as well developed and therefore is not as available in software libraries as other solutions to "initial value problems" like the Runge-Kutta. It was determined that the use of a few intermediate time steps, to account for the effect of deposition and removal, yielded a numerically stable solution. Thus RADTRAD uses on the order of 200 time intervals to cross a 720-hr calculation. If the user chooses to use fewer time steps, then the estimation error will increase by about $10 \%$ for 10 time steps. If the user chooses to use more time steps, then the estimation error will decrease by a few percent for 2,000 time steps. The time step selection was implemented in routines NECSTEP and AUTODT, and the user controls the input on screen 3000 .

Equation 1 can be rewritten in terms of the constant coefficients over the time intervals as:

$$
\frac{d}{d t} N_{\text {group }, i}^{m}=-C_{\text {group }, i} N_{\text {group }, i}^{m}+\sum_{\substack{j=1 \\ j \neq i}}^{L} C_{\text {group }, j} N_{\text {group }, j}^{-m}+R_{\text {group }, i}^{m}
$$

where

$$
\begin{array}{ll}
C_{\text {group }, i} & =\begin{array}{l}
\text { coefficients corresponding to the bracketed terms on line } 2 \text { of equation } 1 \\
\text { for each transport group in volume } i
\end{array} \\
C_{\text {group }, j} & =\quad \begin{array}{l}
\text { coefficients corresponding to the bracketed terms on line } 3 \text { of equation } 1 \\
\text { for each transport group, and volume } j
\end{array} \\
R_{\text {group }, i} & =\quad \text { source terms from line } 1 \text { for transport group in volume } i
\end{array}
$$


Note that the term "transport groups" has been substituted for nuclides. To further reduce computation time, the equations were resolved in terms of four transport groups: noble gases, elemental iodine, organic iodine, and aerosols. These four groups transport differently, but all components of any one group act the same. This implies that all xenon and krypton isotopes will transport identically and that cesium, barium, and all other aerosol isotopes will transport identically. The user specifies the release fractions for the various types of iodine and therefore determines the mass within each transport group.

Equation (34) can be transformed into Laplace space as

$s \bar{N}_{\text {group }, i}^{m}-N_{\text {group }, i}^{m}(t=0)=-C_{\text {group }, i} \bar{N}_{\text {group }, i}^{m}+\sum_{\substack{j=1 \\ j \neq i}}^{L} C_{\text {group }, j} \bar{N}_{\text {group }, j}^{m}+\frac{R_{\text {group }, i}^{m}}{s}$

where $s$ is the Laplace operator. This equation can be solved by inverting the coefficient matrix and then multiplying the constant terms. For the case of two components or volumes, the result is:

$$
\left[\begin{array}{l}
\bar{N}_{\text {group }, 1}^{m} \\
\bar{N}_{\text {group }, 2}^{m}
\end{array}\right]=\left[\begin{array}{lr}
\left(s+C_{\text {group }, 1}\right) & -C_{\text {group }, 1,2} \\
-C_{\text {group }, 2,1} & \left(s+C_{\text {group }, 2}\right)
\end{array}\right]^{-1}\left[\begin{array}{l}
N_{\text {group }, 1}^{m}(t=0)+\frac{R_{\text {group }, 1}^{m}}{s} \\
N_{\text {group }, 2}^{m}(t=0)+\frac{R_{\text {group }, 2}^{m}}{s}
\end{array}\right]
$$

As the number of compartments increases, the number of elements in the matrix increases by a factor of 4, to account for each transport group. If nuclides were used instead of transport groups, then this increase would be significantly larger for a comprehensive nuclide set. An alternative to the numerically costly matrix inversion is to solve the problem analytically. This implies a determination of the zeros in the transformed system of equations. Then, given the zeros, the solution in time will be determined by the residues at each pole. The residue is determined from the derivative in Laplace space as:

$$
a_{k}=\frac{1}{(m-1) !} \frac{d^{m-1}}{d s^{m-1}}\left[\left(s-a_{k}\right)^{m} F(s) e^{s t}\right] s_{s=a_{k}}
$$

The evaluation of the derivative can be performed numerically, that is, over many small time intervals, or it can be determined analytically. The analytic solutions, discussed in the following sections, have been derived and implemented for cases with up to 10 equivalent volumes in the routine RESADER. This limitation on equivalent volumes can be extended as the limitation on the number of compartments is extended. However, even for a model using all ten compartments, it is not expected that the equivalency limit (also called multiplicity) will be near 10. This is because the variations in the transport and removal coefficients from compartment to compartment that occur in real problems will ensure distinct roots. Only in very simple models, where there may be equivalent volumes with equivalent transport and removal coefficients, can most of the roots be equal. 
The solution uses COENUM and ZEROD to determine the poles and XNUPDT to update the nuclide inventories. Within the code this combination is solved three times, the first with the old inventories in each volume, then with the added sources, and finally as a complete inventory plus source solution. Thus the code can relate the new nuclide distribution within each transport group to the old distribution plus any additional radionuclide releases. This is implemented in RADCALC.

\subsubsection{Calculating the Transport and Removal Coefficients}

This section describes the methodology for calculating the transport and removal coefficients, $C_{\text {group }, n}$ and $C_{\text {group,n,m}}$, discussed in the previous section. Separate sets of transport coefficients are calculated for each transport group (group). Most of the discussion below applies equally to all transport groups. For clarity, only when transport groups are treated differently will the separate transport groups be discussed. The remainder of this section uses the term "nuclide" to indicate all of the atoms, molecules, or particles of a transport group within a compartment or transport path.

\subsection{Compartment Losses. Coefficients of the first form, $C_{\text {group }, n}$, represent the sum of} losses for a group from the compartment $n$. Losses arise from compartment removal mechanisms: sprays, natural deposition, and recirculating filters; and from pathway transport mechanisms: piping, filtered pathways, suppression pools, and air leaks. (Losses are the sum of flows out of compartment $n$.) All loss coefficients are in units of $\mathrm{s}^{-1}$ and represent the instantaneous fraction of nuclides in compartment $n$ lost per second, as shown in equation 40 . These coefficients appear as positive coefficients in row $n$, column $n$ (the diagonal) of the Laplacian coefficient matrix (see equation 42).

The removal coefficients for sprays and natural deposition are equal to the coefficients ( $\lambda s$ ) calculated by the models described in Section 2.2 for the user-selected the models, or to the userdefined removal coefficients if the user chose to define coefficients. For recirculating filters, the user specifies efficiencies for the recirculating filter and a flow rate. The coefficients for the recirculating filter are then calculated using equation 44 . Note: there are no removal mechanisms for the noble gas transport group.

$$
C_{\text {group }, n}^{\text {rfilter }}=\frac{r_{\text {rfilter, flow }}}{V_{n}} \times \frac{\eta}{100}
$$

where:

$$
\begin{array}{ll}
C_{\text {group, } n}^{\text {rfilter }} & =\text { recirculating filter removal coefficient } \\
r_{\text {rfilterflow }} & =\text { recirculating filter volumetric flow rate } \\
V_{n} & =\text { volume of compartment } n \\
\eta & =\text { percent filter efficiency }
\end{array}
$$


The transport coefficients representing the flow out of compartment $n$ are not transport group dependent. Some pathways remove nuclides from the flow (group dependent), but this is modeled by reducing the flow into the destination compartment (see compartment $m$ below). It does not affect the flow out of compartment $n$.

The transport coefficient for air leakage is equal to the user-defined removal coefficients. The coefficients for piping, filtered pathways, and suppression pools are calculated using equation 45 .

$$
C_{\text {group }, n}^{\text {pipe }}=\frac{r_{\text {pipe, flow }}}{V_{n}}, \quad C_{\text {group }, n}^{\text {pfiler }}=\frac{r_{\text {filter }, \text { flow }}}{V_{n}}, \quad C_{\text {group }, n}^{\text {pool }}=\frac{r_{\text {pool, flow }}}{V_{n}}
$$

where:

$C_{\text {group,n }}^{\text {pipe }}=$ piping transport coefficient

$C_{\text {group,n }}^{\text {pfilter }}=$ filtered pathway transport coefficient

$C_{\text {group, } n}^{\text {pool }}=$ suppression pool transport coefficient

$r_{\text {pipe,flow }}=$ piping pathway volumetric flow rate

$r_{\text {filterflow }}=$ filtered pathway volumetric flow rate

$r_{\text {poolffow }}=$ suppression pool volumetric flow rate

$V_{n} \quad=$ volume of compartment $n$

The sum of all the losses from compartment $n, C_{\text {group }, n}$, is equal to the sum of all of the compartment removal and pathway transport coefficients as shown by equation 46 .

$$
C_{\text {group }, n}=C_{\text {group }, n}^{\text {spray }}+C_{\text {group }, n}^{\text {dep }}+C_{\text {group }, n}^{\text {rilter }}+\sum_{i=1}^{n^{\prime}} C_{\text {group }, n}^{\text {path },}
$$

where:

$C_{\text {group, } n}=$ pathway transport coefficient for total flow from compartment $n$

$C_{\text {group }, n}^{\text {path } i}=i^{\text {th }}$ pathway transport coefficient from compartment $n$

$n^{\prime} \quad=$ number of paths from compartment $n$

The loss coefficients are calculated by the COEFFS subroutine. When calculating compartment removal mechanisms, COEFFS calls RECIRC, XNTDEP, and SPRY to calculate removal coefficients for recirculating filters, natural deposition, and sprays, respectively. For calculating 
pathway transport losses, COEFFS calls PIPOUT, FOUT, SUPOUT, and COUT for piping, filtered, suppression pool, and leakage pathways.

2.4.2.1.2 Compartment Gains. Coefficients of the second form, $C_{\text {group, }, m, m}$, represent the sum of gains to compartment $n$ from compartment $m$ due to pathway transport. All gain coefficients are in units of $\mathrm{s}^{-1}$ and represent the instantaneous fraction of nuclides in compartment $m$ transported to compartment $n$ per second, as shown in equation 40 . These coefficients appear as negative coefficients in row $n$, column $m$, of the Laplacian coefficient matrix (see equation 42 ).

The coefficients for pathway transport into compartment $n$ are equal to the negative of compartment $m$ 's matching pathway transport loss coefficient, if there was no loss in the pathway itself. This is always true for the air leakage pathway as well as the noble gas transport group. It will also be true if the user specifies no removal for the other pathways.

If the user has specified that losses are to occur in the pathway or has selected a model that calculates the losses that occur, then the pathway coefficient from compartment $m$ (the source compartment) must be multiplied by a transport efficiency factor that describes the fraction of material that makes it to compartment $n$ (the destination compartment). For filters, the transport efficiency factor is equal to one minus the filter efficiency. For piping and suppression pool pathways, the transport efficiency is equal to one divided by the decontamination factor. These relationships are shown in equation 47 . Note: these equations are valid in all cases except when compartment $n$ is connected to the environment compartment via intake filters. This case is discussed below.

$$
\begin{aligned}
& C_{\text {group, }, m}^{\text {pipe }}=\frac{C_{\text {group, } m}^{\text {pipe }}}{D F_{\text {group,pipe }}}, C_{\text {group, }, m}^{\text {pfilter }}=C_{\text {group }, m}^{\text {pfilter }}\left(1-\frac{\eta_{\text {group }, p f i l t e r}}{100}\right) \\
& C_{\text {group, }, m}^{\text {pool }}=\frac{C_{\text {group }, m}^{\text {pool }}}{D F_{\text {group, pool }}}
\end{aligned}
$$

where:

$$
\begin{array}{ll}
C_{\text {group,n,m }}^{\text {pipe }} & =\text { piping transport coefficient for flow to compartment } n \text { from compartment } m \\
C_{\text {group, } n, m}^{\text {pfilter }} & =\text { filtered pathway transport coefficient for flow to compartment } n \text { from } \\
& \text { compartment } m \\
C_{\text {group,n,m }}^{\text {pool }} & =\text { suppression pool transport coefficient for flow to compartment } n \text { from } \\
& \text { compartment } m \\
D F_{\text {group,pipe }} & =\text { piping pathway decontamination factor } \\
\eta_{\text {group,pfilter }} & =\text { filtered pathway filter efficiency (\%) }
\end{array}
$$


$D F_{\text {group,pool }}=$ suppression pool volumetric decontamination factor

If compartment $n$ is connected to the environment compartment via intake filters (at present this is the only method for transport out of the environment into another compartment), then a different algorithm must be used so that the relative atmospheric concentration $(\mathrm{X} / Q)$ at the intake of the air filter(s) can be taken into account. If the environment is compartment $l$, and a pathway exists from another compartment $m$ into the environment, then the pathway transport from compartment $m$ to compartment $n$ is given by equation 48 .

$$
C_{\text {group }, n, m}^{\text {path }}=C_{\text {group,l, } m}^{\text {path }} \frac{X}{Q} \sum_{i=1}^{n^{\prime}}\left[r_{\text {flow }, i}\left(1-\frac{\eta_{l}}{100}\right)\right]
$$

where:

$C_{\text {group }, n, m}^{\text {path }}=$ pathway transport coefficient for flow to compartment $n$ from compartment $m$

$C_{\text {group }, l m}^{\text {path }}=$ pathway transport coefficient for flow to compartment $l$ (the environment) from compartment $m$

$r_{\text {flow }, i}=i^{\text {th }}$ intake filtered pathway volumetric flow rate

$\eta_{i}=i^{\text {th }}$ intake filtered pathway percent filter efficiency

$n^{\prime} \quad=$ number of intake filters from the environment to compartment $n$

This is repeated for all paths that flow into the environment over the sum of all of the intake filters. Because all direct pathways (pathways that do not exit out of the environment) must be known before the above equation can be calculated, RADTRAD calculates the coefficients for the indirect environment pathways last.

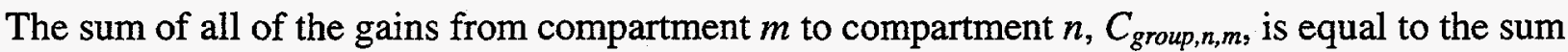
of all of the direct pathways from compartment $m$ to compartment $n$, and the sum of all of the indirect pathways through the environment as shown in equation 49 .

$$
C_{\text {group }, n, m}=\sum_{i=1}^{n^{\prime}} C_{\text {group }, n, m}^{\text {path },}+\sum_{i=1}^{n^{\prime \prime}}\left\{C_{\text {group }, h, m}^{\text {path },} \frac{X}{Q} \sum_{j=1}^{n^{\prime \prime \prime}}\left[r_{\text {flow }, j}\left(1-\frac{\eta_{j}}{100}\right)\right]\right\}
$$

where:

$C_{\text {group,n,m }}=$ pathway transport coefficient for total flow to compartment $n$ from compartment $m$

$C_{\text {group }, n, m}^{\text {path },}=i^{\text {th }}$ pathway transport coefficient for flow to compartment $n$ from compartment $m$ 


$$
\begin{aligned}
& \begin{array}{ll}
C_{g r o u p, l m}^{\text {pathi }} \quad & =i^{\text {th }} \text { pathway transport coefficient for flow to compartment } l \text { (the environment) from } \\
& \text { compartment } m .
\end{array} \\
& r_{f l o w, j}=j^{\text {th }} \text { intake filtered pathway volumetric flow rate } \\
& \eta_{j}=j^{\text {th }} \text { intake filtered pathway filter efficiency (\%) } \\
& n^{\prime} \quad=\text { number of direct paths from compartment } m \text { to compartment } n \\
& n^{\prime \prime} \quad=\quad \text { number of indirect paths through the environment, from compartment } m \text { to } \\
& \quad \text { compartment } n \\
& n^{\prime \prime \prime} \quad=\text { number of intake filters from the environment to compartment } n
\end{aligned}
$$

The gain coefficients are calculated by the COEFFS subroutine. For calculating pathway transport gains, COEFFS calls PIPIN, FILTIN, SUPIN, and CIN for piping, filtered, suppression pool, and leakage pathways.

\subsubsection{Calculating the Derivatives of the Reduced Transform}

This section describes the methodology for calculating the derivatives of the reduced transform, $F(s)$. Recall that $F(s)$ is obtained by forming the product:

$$
F(s)=\left(s-z_{\text {den }}\right)^{\mu} X(s)
$$

where $X(s)$ is the Laplace transform to be inverted, $s$ is the Laplace transform variable, $z_{d e n}$ is the denominator root in question, and $\mu$ is the multiplicity, the numbers of repetitions of the root.

Let $D(K)$ denote the value of the $K^{\text {th }}$ derivative of $F(s)$ evaluated at $s=z_{\text {den }}$. The index $K$ is assumed to range from a minimum value of zero, and the "zeroth" derivative $D(0)$ is assumed to be the value of $F\left(z_{d e n}\right)$ itself. Thus

$$
\begin{gathered}
D(0)=F^{(0)}\left(z_{\text {den }}\right), D(1)=F^{(1)}\left(z_{\text {den }}\right), D(2)=F^{(2)}\left(z_{\text {den }}\right), \ldots, \\
D(K)=F^{(K)}\left(z_{\text {den }}\right)
\end{gathered}
$$

The superscript on $F$ indicates the order of the derivative of $F(s)$ with respect to $s$.

The corresponding inverse transform, $x(t)$, is the sum of the residues for the denominator multiplicity $\mu$ under consideration. It can be expressed as follows:

$$
x(t)=\sum_{K=0}^{\mu-1}\left[\frac{D(K)}{K !}\right] \exp \left(z_{d e n} t\right)\left[\frac{t^{\mu-K-1}}{(\mu-K-1) !}\right]
$$




\subsubsection{Numerator Multiplicity of Zero}

When the numerator multiplicity is zero, the sequence of derivatives $D(K)$ is calculated using an iterative method. First, the zeroth derivative $D(0)$ is evaluated. This is the same as the value of the reduced transform $F(s)$ evaluated at the denominator root $z_{\text {den }}$ under consideration. It initializes the iteration. The expression is:

$$
D(0)=\frac{\prod_{K}\left[Z_{d e n}(J)-Z_{n u m}(K)\right]}{\prod_{L}^{\prime}\left[Z_{d e n}(J)-Z_{d e n}(L)\right]^{\mu L}}
$$

The prime on the second product symbol ( $\Pi$ ) indicates that the case $L=J$ has been excluded. The first product is represented by the complex variable TOPVAL in the code, while the second one is represented by the complex variable BOTVAL.

Given the value of $D(0)$, the higher derivatives can be obtained by using an iterative method in which each higher derivative is a linear combination of all lower-order derivatives.

$$
D(K)=\sum_{L=0}^{K-1} \frac{(K-1) !}{[(K-1-L) ! L !]} G(K-L) D(L)
$$

The sequence of quantities $G(K)$ is used to link any given derivative to derivatives of all lower orders. It is obtained by calling the complex function SUMPWR and multiplying:

$$
G(K)=(-1)^{K-1}(K-1) ! \text { SUMPWR }
$$

Like the derivatives $D(K), G(K)$ is a function of the Laplace transform variable $s$, which has been evaluated at $s=z_{d e n}$. The complex function SMPWR returns a value equal to the difference of two sums. The first sum is that of the inverse powers of $\left(s-z_{\text {num }}\right)$ with $s$ set equal to $z_{d e n}$. The power in question is $n$, the order of the derivative required. The second sum is that of the inverse powers of $\left(s-z_{d e n}\right)$ with $s$ set equal to $z_{d e n}$. Note that the powers in the second sum are weighted according to the multiplicities of the denominator roots.

$$
\text { SUMPWR }=\sum_{K=1}^{N_{\text {num }}}\left[Z_{\text {den }}(J)-Z_{\text {num }}(K)\right]^{n}-\sum_{K=1}^{N_{\text {denn }}} \mu(J)\left[Z_{\text {den }}(J)-Z_{\text {num }}(K)\right]^{-n}
$$

The only terms to be included in the summations are those for which the root differences in the square brackets are not equal to zero. Thus, if $z_{\text {den }}(J)-z_{\text {num }}(K)$ is zero for some $K$, then that value of $K$ should be excluded from the first summation. Likewise, if $z_{d e n}(J)-z_{d e n}(K)$ is zero for some $K$, then that $K$ should be excluded from the second summation.

The integer $N_{\text {num }}$, appearing in the first summation, is the number of numerators, which is the same as the number of compartments. The integer $N_{\text {den, }}$ appearing in the second summation, is 
the number of distinct denominator roots. As noted earlier, the integer $n$ appearing as an exponent in both summations is the order of the derivative desired.

\subsubsection{Numerator Multiplicity Greater than Zero}

When the numerator multiplicity (designated by $v$ ) is greater than zero, the sequence of derivatives $D(k)$ is calculated much the same way as before, but with an additional step. Initially, the reduced transform $F(s)$ is further reduced by factoring out the polynomial $\left(s-z_{\text {num }}\right)^{v}$, where $z_{\text {num }}$ is the numerator root that is equal to the denominator root $z_{\text {den }}$ under consideration.

Otherwise, the value of $F(s)$ at $s=z_{\text {den }}$ would vanish. The twice-reduced transform is designated as $F B A R(s)$.

The value of $F B A R(s)$ at $s=z_{d e n}$ is designated as the zeroth derivative $D B A R(0)$, which corresponds to $D(0)$ when the numerator multiplicity is zero $(v=0)$. In all cases, the value of $D B A R(0)$ can be expressed as follows:

$$
\operatorname{DBAR}(0)=\frac{\prod_{K}^{\prime}\left[Z_{d e n}(J)-Z_{n u m}(K)\right]}{\prod_{L}^{\prime}\left[Z_{d e n}(J)-Z_{d e n}(L)\right]^{\mu \mathrm{L}}}
$$

The prime on the first product symbol indicates that the factor involving the numerator root $z_{\text {num }}$ has been excluded from the product. The prime on the second product symbol indicates that the case $L=J$ has been excluded. As before, the first product is represented by the complex variable TOPVAL in the code, while the second one is represented by the complex variable BOTVAL.

The calculations continue, as in the case when $v=0$, for the higher derivatives $D B A R(K)$ and the derivative linking factors $G B A R(K)$. Thus for $K>0$ :

$$
D B A R(K)=\sum_{L=0}^{K-1} \frac{(K-1) !}{[(K-1-L) ! L !]} G B A R(K-L) D B A R(L)
$$

and also:

$$
\operatorname{GBAR}(K)=(-1)^{K-1}(K-1) ! S C M P W R
$$

Finally, the derivatives $D(K)$ are obtained from their counterparts $D B A R(K)$ for $\mathrm{v} \leq K$ :

$$
D(K)=\frac{K !}{[(K-v) ! v !]}
$$




\subsubsection{Radioactive Decay Calculations}

Simulation of nuclide decay in a computer model like RADTRAD required the use of the exponential decay law. Simulation of daughter in-growth is more involved and it is generally necessary to invoke one or more simplifying assumptions to keep the computational burden within reasonable bounds.

This section outlines an algorithm for implementing daughter in-growth along with parent decay within the dose-and-consequence model. It begins with a description of the simulation of decay without in-growth, followed by a description of the inclusion of in-growth.

The algorithm for daughter in-growth assumes that only one generation of daughters needs to be calculated per time step in order to provide a level of nuclide inventory accuracy commensurate with the other algorithms used in the code. This implies that the user must specify a time step commensurate with the accuracy desired when choosing daughter in-growth. The algorithm consists of two steps. In the first step, all parents undergo simple exponential decay. In the second, the decay products are apportioned to one generation of daughters. Thus ${ }^{135} \mathrm{I}$ with a halflife of $6.6 \mathrm{hr}$ will decay and form ${ }^{135} \mathrm{Xe}$ and ${ }^{135 \mathrm{~m}} \mathrm{Xe}$. The decay of the ${ }^{135 \mathrm{~m}} \mathrm{Xe}$ in $15 \mathrm{~min}$ to ${ }^{135} \mathrm{Xe}$ increases the cloudshine dose. Therefore, a time step of less than $6 \mathrm{hr}$ is needed to estimate this dose.

\subsubsection{Simulation of Decay without Daughter In-growth}

The decay of nuclide parents is simulated by applying the expression for exponential decay. If the initial amount of the $i^{\text {th }}$ nuclide is $N_{i}^{\circ}$, then the amount at time $t$ is given by the following expression:

$$
N_{i}^{t}=N_{i}^{o} \exp \left(-\lambda_{i} t\right)
$$

where $\lambda_{i}$ is the radiological decay constant for the $i^{\text {th }}$ nuclide.

The decrease in the amount of the $i^{\text {th }}$ nuclide resulting from this decay process over the time step $\Lambda t$ is given by the expression:

$$
\Delta N_{i}^{t}=N_{i}^{t-o t}-N_{i}^{t}
$$

and is always non-negative.

\subsubsection{Simulation of Daughter In-growth}

The quantity $\Delta N_{i}^{t}$ represents the amount of the $i^{\text {th }}$ nuclide that was lost during the decay time $t$. It is also the amount of the $i^{\text {th }}$ nuclide that has become available for in-growth into the corresponding daughter nuclides. This quantity is to be apportioned according to the values of the branching fractions of the daughters. The following algorithm has been adopted for simulating this in-growth process. It is simple and intuitive and does not require an extraordinary amount of computation. 
Let the $i^{\text {th }}$ nuclide be the daughter that is being "fed" by ingrowth as a result of the decay of parent nuclides. Let the $j^{\text {th }}$ nuclide be one of the parents whose decay "feeds" the daughter. Finally, let the index $k$, which ranges from 1 to 3 , denote the particular daughter associated with the $j^{\text {th }}$ nuclide (parent).

If the $k^{\text {th }}$ daughter of the $j^{\text {th }}$ nuclide is identical to the $i^{\text {th }}$ nuclide, then the amount of the $i^{\text {th }}$ nuclide is adjusted to the value $\mathrm{N}_{i}^{l+}$ according the following equation:

$$
N_{i}^{t+}=N_{i}^{t}+B F_{j, k} \Delta N_{j}^{t}
$$

Here $\mathrm{N}_{i}{ }^{t}$ is the amount of the $i^{\text {th }}$ nuclide remaining after decay and before in-growth, $\mathrm{BF}_{j, k}$ is the branching fraction daughter isotope fraction of the $k^{\text {th }}$ daughter of the $j^{\text {th }}$ nuclide detailed in Section 1.4.3.2, and $\Delta N_{i}^{t}$ is the decrease in the amount of the $j^{\text {th }}$ nuclide as a result of decay.

\subsection{References}

[Br91] J.E. Brockmann, "Sampling and Transport of Aerosols," in Aerosol Measurement: Principles, Techniques, and Applications, K. Wilke and P. A. Baron, Eds., Van Nostrand Reinhold, New York, 1991.

[Ba82] T.J. Bander, "PAVAN: An Atmospheric Dispersion Program for Evaluating Design Basis Accidental Releases of Radioactive Materials from Nuclear Power Stations," NUREG/CR-2858, PNL-4413, Pacific Northwest National Laboratory, Richland, WA, 1982.

[C191] J.E. Cline, "MSIV Leakage Iodine Transport Analysis", NRC-03-87-029, Nuclear Regulatory Commission, Washington, DC, 1991.

[Cr77] R. I. Crane and R. L. Evans, "Inertial Deposition of Farticles in a Bent Pipe," J. Aerosol Science, 8 (1977): 161-170.

[Di62] J.J. DiNunno, R.E. Baker, F.D. Anderson, and R.L. Waterfield, "Calculation of Distance Factors for Power and Test Reactor Sites," TID-14844, U.S. Atomic Energy Commission, Washington, DC, 1962.

[Ep 87] M. Epstein and P. G. Ellison, "A Principle of Similarity for Describing Aerosol Particle Size Distributions," J. Colloid and Interface Science, 119 (1987).

[Fa84] Fauske \& Associates, Inc., FAI Aerosol Correlation, An Industry Degraded Core Rulemaking (IDCOR) Program Report, Bethesda, MD, 1984.

Federal Guidance Report No. 11, "Limiting Values of Radionuclide Intake and Air Concentration and Dose Conversion Factors for Inhalation, Submersion, and Ingestion," EPA 520/1-88-020, Environmental Protection Agency, Washington, DC (1988).

Federal Guidance Report No. 12, "External Exposure to Radionuclides in Air, Water and Soil," EPA 420-r-93-081, Environmental Protection Agency, Washington, DC, 1993. 
[Fr77] S.K. Friendlander, Smoke, Dust, and Haze, Wiley, New York (1977)

[Go49] P. G. Gormley and M. Kennedy, "Diffusion from a Stream Flowing Through a Cylindrical Tube," Proc. Royal Irish Academy, 52-A (1949): 163-169.

[Li74] B.Y.H. Liu and J. K. Agarwal, "Experimental Observation of Aerosol Deposition in Turbulent Flow," J. Aerosol Science, 5(1974): 145-55.

[Mu74] K. G. Murphy and K. M. Campe, "Nuclear Power Plant Control Room Ventilation System Design for Meeting General Design Criterion 19," Proc. the $13^{\text {th }}$ AEC Air Cleaning Conference, CONF-740807, U.S. Atomic Energy Commission, Washington, DC, 1974.

[Ot86] J. M. Otter and E. U. Vaughn, "Evaluation of Aerosol Correlations," EPRI NP-4974, Rockwell International Corp., Canoga Park, CA, 1986.

[Po86] D. A. Powers, J. E. Brockmann, and A. W. Shiver, "VANESA: A Mechanistic Model of Radionuclide Release and Aerosol Generation During Core Debris Interactions with Concrete," NUREG/CR-4308, SAND85-1730, Sandia National Laboratories, Albuquerque, NM, 1986.

[P092] D. A. Powers, and J. L. Sprung, "A Simplified Model of Aerosol Scrubbing by a Water Pool Overlying Core Debris Interacting with Concrete," NUREG/CR-5901, SAND92-1422, Sandia National Laboratories, Albuquerque, NM, 1992.

[Po93] D. A. Powers and S. B. Burson, "A Simplified Model of Aerosol Removal by Containment Sprays," NUREG/CR-5966, SAND92-2689, Sandia National Laboratories, Albuquerque, NM, 1993.

[Po95] D. A. Powers, K. E. Washington, S. B. Burson, and J. L. Sprung, "A Simplified Model of Aerosol Removal by Natural Process in Reactor Containments," NUREG/CR-6189, SAND940407, Sandia National Laboratories, Albuquerque, NM, 1995

This line, left blank intentionally.

[Po97b] D. A. Powers, "Monte Carlo Uncertainty Analysis of Aerosol Behavior in the AP600 Reactor Containment Under Conditions of a Specific Design-Basis Accident," NUREG/CR6522, Sandia National Laboratories, Albuquerque, NM, 1997.

[Pu87] D. Y. H. Pui, F. Romay-Novas, and B. Y. H. Liu, "Experimental Study of Particle Deposition in Bends of Circular Cross Section," Aerosol Science Technology 7 (1987): 301-315.

[So95] L. Soffer, S.B. Burson, C.M. Ferrell, R.Y. Lee, and J.N. Ridgely, "Accident Source Terms for Light-Water Nuclear Power Plants," NUREG-1465, Nuclear Regulatory Commission, Washington, DC, 1995. 
[Ra95] J.V. Ramsdell, C.D. Simone, and S.B. Smyth, "Atmospheric Relative Concentrations in Building Wakes," NUREG/CR-6331, PNL-10521, Pacific Northwest National Laboratory, Richland, WA, 1995.

[St 95] S.A. Stage, "Computer Codes for Evaluation of Control Room Habitability (HABIT): Draft, NUREG/CR-6210, PNL-10496, Pacific Northwest National Laboratory, Richland, WA, 1995.

USNRC [74] "Assumptions Used for Evaluating the Potential Radiological Consequences of a Loss of Coolant Accident for Boiling Water Reactors," Regulatory Guide 1.3, Revision 2, June 1974.

USNRC [74] "Assumptions Used for Evaluating the Potential Radiological Consequences of a Loss of Coolant Accident for Pressurized Water Reactors," Regulatory Guide 1.4, Revision 2, June 1974.

[WA91] K.E. Washington, K.K. Murata, R.G. Gido, F. Gelbard, N.A. Russell, S.C. Billups, D.E. Carroll, R.O. Griffith, and D.L.Y. Louie, "Reference Manual for the CONTAIN 1.1 Code for Containment Severe Accident Analysis," NUREG/CR-5715, SAND 91-0835, Sandia National Laboratories, Albuquerque, NM, 1991. 


\section{Quality Assurance}

RADTRAD has been developed and tested in accordance with the requirements of ANSVANS10.4-1987, "American National Standard Guidelines for the Verification and Validation of Scientific and Engineering Computer Program for the Nuclear Industry." In addition to the use of these programming standards, the following measures were undertaken to ensure program quality:

- Individual program elements that perform calculations have been tested independently. The tests include manual checking of representative calculations. The functioning of these elements was then rechecked in conjunction with tests of other program elements when RADTRAD was run.

- Program elements involved in data input were checked by writing input data to output files and comparing the data in the input and output files. This process also checked the storage and transfer of input data within RADTRAD.

- A series of test cases were run using the full RADTRAD code. These test cases exercised the range of program options. Input and output files for each of the test cases were examined for accuracy and consistency.

- The code was placed under configuration control at the beginning of the developer's code tests. Changes in the code during the tests were logged in the code of the program element that was changed.

- The code underwent 4 months of beta testing by potential users.

- A rigorous set of acceptance tests jointly developed by SNL, NRC, PNNL, and Innovative Technology Solutions Corp. (ITS) were applied to the initial release and to subsequent releases of RADTRAD.

Section 3.1 provides a description of the individual subroutine testing and integration into the RADTRAD code. Section 3.2 gives the results of the beta testing program. In that section, 30 comments/discrepancies were reported by the PNNL team and 20 were resolved. The resulting version of RADTRAD was run through a series of about 30 acceptance tests. The tests and the comparison with other calculations are presented in Section 3.3. Section 3.4 details the last phase of quality assurance, RADTRAD configuration control. Chapter 4 discusses the coding standards used.

\subsection{Module and Integration Tests}

Module and integration tests were performed on each module as it became available and each test is detailed in the following subsections. Input screen tests are not included in this list because they are not part of the technical solution. 


\subsubsection{AEROSOL}

This subroutine determines the removal coefficient for aerosol in the containment based upon the Powers model discussed in Section 2.2.2.1.2. This routine performs two functions: selection of an appropriate subprogram for the reactor and accident sequence selected, and determination of the removal coefficient for an advanced PWR design that has a power rating other than 1800 MWt. A testing routine was developed that was called routine AEROSOL for two power ratings, three reactor designs, three bounding percentiles (lower, 10\%; median, 50\%; or high, $90 \%$ ), and the possible accident sequences [design basis accident or severe accident (SA)]. The 270 timedependent choices were:

$\begin{array}{cclll}\begin{array}{c}\text { Reactor } \\ \text { Type }\end{array} & \begin{array}{c}\text { Bounding } \\ \text { Percentile }\end{array} & \begin{array}{l}\text { Power } \\ \text { Rating } \\ \text { (MWt) }\end{array} & \begin{array}{l}\text { Accident } \\ \text { Sequence }\end{array} & \text { Time (hr } \\ \text { PWR } & 10,50,90 & 1000 & \text { DBA and SA } & 0-720 \\ & & 3000 & & \\ \text { BWR } & 10,50,90 & 1500 & \text { DBA and SA } & 0-720 \\ & & 3500 & & \\ \text { APWR } & 10,50,90 & 1800 & \text { DBA } & 0-720 \\ & & 3800 & & \end{array}$

The tests were successful from the point of view that the appropriate routines were called and the values returned gave reasonably smooth results when plotted. In addition, the APWR power increase cases caused the removal coefficients to increase in accordance with the theory given in Section 2.2.2.1.2. The routine AEROSOL calls PWRSA, PINRDBA, APWRDBA, BWRSA, or BWRDBA to generate the removal coefficients. All of these routines are programmed similarly, with the differences due only to the times and removal coefficient formulation detailed in the theory section.

\subsubsection{APWRDBA}

This subroutine determines the aerosol removal coefficient in the containment for an advanced pressurized water reactor during a design basis accident. The routine determines the average removal coefficient over the current time step. Table 2.2.2.1-5 details the variation of removal coefficient with time and the bounding percentile (lower, $10 \%$; median, $50 \%$; or high, $90 \%$ ). The routine first determines the decontamination factor for the two phases of the accident release (gap and in-vessel) at the given time by following the equation set described in Section 2.2.2.1.2. It then determines the net decontamination factor by dividing the sum of the current releases by the sum of decontamination of each of those releases. With the current decontamination factor and 
the value at the beginning of the step, the average removal coefficient is found and returned to routine AEROSOL.

There were no sample problems in the Powers documentation associated with advanced pressurized water reactors. The actual coding follows the coding for the PWRDBA routine that was compared with a sample problem.

The test cases were examined for both smoothness of the final answer (typically the residual fraction of the iodine in the containment was used as a measure) and for the absolute value of the phase-dependent decontamination coefficient as detailed in the Powers document. Within the limits of the tests, $100.0<$ Power $<4000 \mathrm{MWt}$, the answers were reasonable. As for the decontamination of all chemical species based upon the iodine decontamination, because iodine releases a larger fraction during the first two periods than the other species, the results of using the iodine removal coefficient are slightly nonconservative due to the slightly higher decontamination factor. However, the iodine is the most important radiologically and will therefore be more important to the final dose calculation.

\subsubsection{AUTODT}

This subroutine determines the next time step based on user-specified events and a manual time step control provided by the computer screen. The AUTODT subroutine was tested by being used during the acceptance test phase of the program.

\subsubsection{BIXLER1}

This subroutine calculates the decontamination coefficient for elemental iodine in a pipe as discussed in Section 2.2.6.2. It assumes that revaporization is negligible. It is called by the pipe deposition controlling the routine PIPINB.

Subroutine BIXLER1 is one of a package of subroutines that were developed as an alternative to the MSIV code for steam pipes. The other components of the package are BROCK and BIXLER3.

The purpose of BIXLER1 is to determine the transport efficiency, and thus the decontamination factor, for elemental iodine in the steam pipes. All the relevant information was detailed in Section 2.2.6.2.

The only reference is an internal memo, dated August 29, 1995, from N. E. Bixler [Bi95], which references the Cline report [ $\mathrm{Cl} 91]$.

Testing:

Subroutine BIXLER1 was first tested off-line. User-specified values were provided for the flow rate, temperature, and pressure of the vapor. The transport efficiency was obtained from the Cline correlation, and the decontamination factor was obtained as its reciprocal. The resulting values 
were compared with the curves in the Cline report. The subroutine was then integrated into RADTRAD and the results compared with HABIT calculations in acceptance tests 13 and 14 .

Note on revaporization:

Revaporization is neglected in the Bixler model for the following reason. It is assumed that at the beginning of the break, the pressure and temperature are high. Under these circumstances, revaporization is not an issue. As the accident progresses, the pressure and temperature will decrease monotonically. Under these circumstances, revaporization will occur only when both pressure and temperature are low, that is, near standard conditions. By this time, the rate of flow will also be very low, so the effects of revaporization will not have a significant impact.

\subsubsection{BIXLER3}

This subroutine calculates the decontamination coefficient for organic iodine in a pipe as discussed in Section 2.2.6.3. It assumes that revaporization is negligible. It is called by the routine controlling pipe deposition PIPINB.

Subroutine BIXLER3 is one of a package of subroutines that were developed as an alternative to the MSIV code for steam pipes. The other components of the package are BROCK and BIXLER1.

The purpose of BIXLER3 is to determine the transport efficiency and thus the decontamination factor for organic iodine in the steam pipes. All the relevant information was detailed in Section 2.2.6.3.

The only reference is an internal memo, dated August 29, 1995, from N. E. Bixler [Bi95], which references the Cline report [C191].

Testing:

Subroutine BIXLER3 was first tested off-line. User-specified values were provided for the flow rate, temperature, and pressure of the vapor. The transport efficiency was obtained from the Cline correlation, and the decontamination factor was obtained as its reciprocal. The resulting values were compared with the curves in the report. The subroutine was then integrated into RADTRAD and the results compared with HABIT calculations in acceptance tests 13 and 14 .

\subsubsection{BROCK}

This subroutine calculates the transport efficiency of aerosols for piping pathways as discussed in Section 2.2.6.1. The subroutine assumes continuous flow from the pipe inlet. It is based upon a lumped-node model description [Br91]. Subroutine BROCK includes the following effects:

1. gravitational settling, for low-flow-rate conditions;

2. turbulent inertial deposition, along straight pipe sections;

3. Brownian diffusion, along straight sections of pipe; and 
4. inertial deposition in bends.

It is called by the routine controlling pipe deposition, PIPINB. Subroutine BROCK is one of a package of subroutines that were developed as an alternative to the MSIV code for steam pipes. The other components of the package are BIXLER1 and BLXLER3.

The purpose of BROCK is to determine the transport efficiency and thus the decontamination factor for aerosols in the pipes. This subroutine considers gravitational settling, Brownian diffusion, turbulent inertial deposition, and inertial deposition at bends.

Program DRIVER was created to test subroutine BROCK. It passed the following user inputs to BROCK:

$\begin{array}{ll}\text { tube inner surface area } & 6.8101 \mathrm{E}+05 \mathrm{~cm}^{2} \\ \text { tube volume } & 1.2108 \mathrm{E}+07 \mathrm{~cm}^{3} \\ \text { flow rate } & 1.416 \mathrm{E}+06 \mathrm{~cm}^{3} / \mathrm{s} \\ \text { gas temperature } & 356.0 \mathrm{~K} \\ \text { gas pressure } & 40.0 \mathrm{~atm} \\ \text { bend angle } & 90 \text { degrees } \\ \text { length/diameter } & 1,10,100 \\ \text { tube/diameter } & 0.01,0.1,1.0 \mathrm{~m} \\ \text { gas velocity } & 0.01,0.1,1 / 0 \mathrm{~m} / \mathrm{s} \\ \text { gas temperature } & 300,500,700,900 \mathrm{~K} \\ \text { gas pressure } & 1,2 \mathrm{~atm} . \\ \text { particle size } & 0.1,0.2,0.4,0.8,1.6,3.2,6.4,13.0,26.0, \text { and } 52 \text { microns }\end{array}$

Additional parameters were derived from these. The total transport efficiency ranged from 0.0 (all aerosol would be deposited) to 1.0 (no aerosol would be deposited). The most significant deposition phenomenon was due to gravitational settling. At the higher flows and shorter lengths, the deposition due to bend was important. The diffusiophoretic had some effect for the smaller particles. The logic can be easily modified to allow the user to determine mean aerosol diameter. The current version assumes a 1-micron particle size. In addition to these results, the subroutine also provided the deposition velocity.

\subsubsection{BWRDBA}

This subroutine determines the aerosol removal coefficient in the containment for a boiling water reactor during a design basis accident. This subroutine determines the average removal coefficient over the current time step. Table 2.2.2.1-4 details the variation of removal coefficient with time, reactor thermal power, and bounding percentile (lower, $10 \%$; median, $50 \%$; or high, $90 \%$ ). This subroutine first determines the decontamination factor for the two phases of the accident release (gap and in-vessel) at a given time by following the equation set described in Section 2.2.2.1.2. It then determines the net decontamination factor by dividing the sum of the current releases by the sum of decontamination of each of those releases. With the current decontamination factor and the value at the beginning of the step, the average removal coefficient is found and returned to routine AEROSOL. 
There were no sample problems in the Powers documentation associated with boiling water reactors. The actual coding follows the coding for the PWRDBA routine, which was compared with a sample problem.

The test cases were examined for both smoothness of the final answer (typically the residual fraction of the iodine in the containment was used as a measure) and for the absolute value of the phase-dependent decontamination coefficient as detailed in the Powers document. Within the limits of the tests, $100.0<$ Power $<4000 \mathrm{MWt}$, the answers were reasonable. For the decontamination of all chemical species based upon the iodine decontamination, because iodine releases a larger fraction during the first two periods than the other species, the results of using the iodine removal coefficient are slightly nonconservative due to the slightly higher decontamination factor. However, the iodine is the most radiologically important and will therefore be more important to the final dose calculation.

\subsubsection{BWRSA}

This subroutine determines the aerosol removal coefficient in the containment for a boiling water reactor during a severe accident. This subroutine determines the average removal coefficient over the current time step. Table 2.2.2.1-3 details the variation of removal coefficient with time, reactor thermal power, and bounding percentile (lower, 10\%; median, 50\%; or high, 90\%). This subroutine first determines the decontamination factor for each phase of the accident release (gap, in-vessel, ex-vessel, and late in-vessel) at the given tine by following the equation set described in Section 2.2.2.1.2. It then determines the net decontamination factor by dividing the sum of the current releases by the sum of decontamination of each of those releases. With the current decontamination factor and the value at the beginning of the step, the average removal coefficient is found and returned to the routine AEROSOL.

There were no sample problems in the Powers documentation associated with boiling water reactors. The actual coding follows the coding for the PWRSA routine, which was compared with a sample problem.

The test cases were examined for both smoothness of the final answer (typically the residual fraction of the iodine in the containment was used as a measure) and for the absolute value of the phase-dependent decontamination coefficient as detailed in the Powers document. Within the limits of the tests, $100.0<$ Power $<4000 \mathrm{MWt}$, the answers were reasonable. For the decontamination of all chemical species based upon the iodine decontamination, because iodine releases a larger fraction during the first two periods than the other species, the results of using the iodine removal coefficient are slightly nonconservative due to the slightly higher decontamination factor. However, iodine is the most radiologically important and will therefore be more important to the final dose calculation. 


\subsubsection{CIN}

Subroutine $\mathrm{CIN}$ calculates the normalized flow into the compartment due to natural convection (air leakage). The user specifies the compartment volume and the volumetric flow rate between the compartments. These values are returned in CLIN(4).

Four values were provided for the convection flow rate. These were specified at time markers $0.0,1.0,2.0$, and $3.0 \mathrm{hr}$. The simulation time was taken to be $1.5 \mathrm{hr}$. The subroutine returned the correct values during this test.

\subsubsection{COEFFS}

Subroutine COEFFS combines the coefficients for the activated removal and transport mechanisms to obtain the system matrix for the matrix differential equation governing nuclide removal and transport. Subroutine COEFFS calls as many as eleven subroutines to obtain the removal/transport contributions due to the various removal and transport mechanisms simulated by RADTRAD. These are as follows:

for removal within a compartment:

RECIRC - recirculation filters

XNTDEP - natural deposition onto surfaces

SPRY - compartment sprays

for removal by way of a pathway:

PIPOUT - flow out by way of a piping pathway

FOUT - flow out by way of a filtered pathway

SUPOUT - flow out by way of a suppression pool

COUT - flow out by way of natural convection

for gain by way of a pathway:

PIPIN - flow in by way of a piping pathway

FILTIN - flow in by way of a filtered pathway

SUPIN - flow in by way of a suppression pool

$\mathrm{CIN}$ - flow in by way of natural convection

Dummy versions of these subroutines were created for testing COEFFS. Each one returned preset values for removal or transport coefficients, without performing any calculations.

Testing:

The following test problems used a facility model consisting of two compartments with one pathway leading from compartment 1 to compartment 2 :

00 no removal, no transport

01 recirculation filter in compartment 1

02 natural deposition in compartment 1

03 sprays in compartment 1 
04 recirculation filter in compartment 2

05 natural deposition in compartment 2

06 sprays in compartment 2

07 piping flow, pathway 1

08 filtered flow, pathway 1

09 suppression pool, pathway 1

10 natural convection, pathway 1

11 this test number was not used.

12 all removal mechanisms in both compartments

The following test problems involved a facility consisting of two compartments with four pathways:

13 all four transport methods from compartment 1 to 2

14 all four transport methods from compartment 2 to 1

The following test problem involved a facility consisting of two compartments with eight pathways:

15 all removal mechanisms in both compartments, all four transport methods from compartment 1 to 2 all four transport methods from compartment 2 to 1

The following test problem involved a still more complex facility.

16 three compartments with four pathways, one (distinct) removal mechanism for each compartment, pathway 1 from compartment 1 to compartment 2, piping pathway 2 from compartment 2 to compartment 1 , filter pathway 3 from compartment 1 to compartment 3, s-pool pathway 4 from compartment 3 to compartment 1 , natural convection

In every case, subroutine COEFFS retrieved the correct removal or transport coefficient and inserted it into the correct row and column of the system matrix.

\subsubsection{COENUM}

Subroutine COENUM computes the coefficients of the numerator polynomial in the Laplace transform of the transport group count. One such numerator exists for each compartment location.

This subroutine requires the Q-matrices obtained from subroutine INVMAT, as well as the initial quantities and release rates for each of the four transport groups in that compartment location.

The numerator polynomial $N(s)$ is a vector polynomial obtained by multiplying the matrix polynomial $Q(s)$ by the vector polynomial $\left(\mathbf{s}^{*} \mathbf{x} 0+\mathbf{v} 0\right)$. Here $s$ is the Laplace transform variable, $\mathbf{x} 0$ is the initial quantity of the transport group at each compartment location, and $\mathbf{v} 0$ is the 
release rate for that transport group at each compartment location. Note that $\mathbf{x} 0$ and $\mathbf{v} 0$ are vectors.

The coefficients of the polynomial $N(s)$ are calculated in a sequential method:

$N(k)=Q(k-1) * \mathbf{x} 0+Q(k) * b$, for $k=0$ to $k=n$

with $Q(-1)=0$ and $Q(n)=0$.

Note that $N(k)$ is the coefficient of the term in $N(s)$ with factor $s^{* *} k$.

Testing:

Four tests were performed. In each one a system matrix was given and the output of the subroutine was compared with the results available as example problems. All tests were successful.

Test Case \#1. Two compartments. Null system matrix.
$0.0000 \mathrm{E}+00 \quad 0.0000 \mathrm{E}+00$
$0.0000 \mathrm{E}+00 \quad 0.0000 \mathrm{E}+00$

Test Case \#2. Two compartments. Robust system matrix.
$0.1000 \mathrm{E}+01 \quad 0.2000 \mathrm{E}+01$
$0.3000 \mathrm{E}+01 \quad 0.4000 \mathrm{E}+01$

Test Case \#3. Three compartments. Robust system matrix.
$0.0000 \mathrm{E}+00$
$-0.1000 \mathrm{E}+01$
$-0.1000 \mathrm{E}+01$
$0.1000 \mathrm{E}+01$
$-0.2000 \mathrm{E}+01$
$-0.1000 \mathrm{E}+01$
$0.0000 \mathrm{E}+00$
$0.0000 \mathrm{E}+00$
$-0.2000 \mathrm{E}+01$

Test Case \#4. Five compartments. Robust system matrix.
$0.1000 \mathrm{E}+01$
$0.0000 \mathrm{E}+00$
$0.2000 \mathrm{E}+01$
$0.4000 \mathrm{E}+01$
$0.0000 \mathrm{E}+00$
$0.4000 \mathrm{E}+01$
$0.0000 \mathrm{E}+00$
$0.1000 \mathrm{E}+01$
$-0.3000 E+01$
$0.1000 \mathrm{E}+01$
$0.0000 \mathrm{E}+00$
$0.5000 \mathrm{E}+01$
$0.2000 \mathrm{E}+01$
$0.4000 \mathrm{E}+01$
$-0.2000 \mathrm{E}+01$
$-0.1000 E+01$
$0.0000 \mathrm{E}+00$
$0.1000 \mathrm{E}+01$
$0.3000 \mathrm{E}+01$
$0.7000 \mathrm{E}+01$
$0.1000 \mathrm{E}+01$
$0.2000 \mathrm{E}+01$
$0.3000 \mathrm{E}+01$
$0.4000 \mathrm{E}+01$
$0.1000 \mathrm{E}+01$

\subsubsection{COUT}

This subroutine calculates the removal coefficient for flow out of a compartment by way of a pathway as a result of natural convection (air leakage). The coefficients are returned in units of $1 / \mathrm{s}$. The user must provide the convection flow rate as well as corresponding time markers.

Testing:

Four values of the convection flow rate were provided in units of $1 / \mathrm{s}$. The subroutine returned the correct value of the removal coefficient. 


\subsubsection{DKNGRO}

This subroutine calculates the radioactive decay and daughter in-growth for all nuclides. It is called from the main driver routine RADCALC.

Two types of tests were performed. In the first series, only the effect of radionuclide decay was verified. This was done by first comparing the no decay case with a decay fraction of zero. In this latter case, the radiological decay constant, as discussed in Section 2.4.3.1, was set to 0.0. The decay constant was then modified to examine radionuclide disappearance. In the second series of tests, the effect of daughtering was accounted for. All tests were successful.

In addition, acceptance cases 2 and 7 were successfully comprared with HABIT runs. These cases also test the radioactive decay and daughter in-growth for all nuclides.

\subsubsection{DOSES}

The subroutine DOSES calculates the environment and control room doses for receptors located at user-defined locations (e.g., exclusion area boundary, low population zone, control room operator). The user supplies the $\mathrm{X} / Q$ s and breathing rates as a function of time for each location. The user also supplies the occupancy factors as a function of time for the control room. The subroutine is called from the main driver routine RADCALC.

Two types of tests were performed. In the first series, the compartment was varied, that is, either the control room, the environment, or a normal room was chosen using only the ${ }^{131} \mathrm{I}$ isotope. This verifies that the internal logic for location is valid. The second series varied the concentration of the nuclide and the number of nuclides in the problem to verify the dose calculation. In addition, all the acceptance test cases were successfully compared with HABIT runs on the basis of dose by location.

\subsubsection{FILTIN}

Subroutine FILTIN calculates the removal coefficient for filtering of incoming air. It provides a coefficient, measured in $1 / \mathrm{s}$, for all four transport groups. It is assumed that this coefficient is zero for noble gases. For the other three transport groups, the user must specify the volume of the "from compartment," the flow through the filter, and the efficiency of the filter for each of these transport groups.

Testing:

For purposes of testing, the filtered pathway was taken to pass from compartment 2 to compartment 1 . The volume of compartment 2 was taken to be $1.0 \mathrm{E}+05 \mathrm{~m}^{3}$. The simulation time was taken to be $1.0 \mathrm{hr}$.

Five values were provided for the filter efficiencies. The time markers were taken to be $0.0,1.0$, $2.0,3.0$, and $4.0 \mathrm{hr}$. The filter flow rates were 100.0,200.0,300.0,400.0 and 500.0 in appropriate units. The filter efficiencies for elemental iodine, organic iodine, and aerosols were taken to be $10.0,20.0,30.0,40.0$, and 50.0 percent. The subroutine returned the correct values in this test. 


\subsubsection{FOUT}

Subroutine FOUT calculates the transport rate resulting from flow out of a compartment by way of a filter. The user must provide values of the normalized flow rate.

Testing:

Four values of the filter flow rate were specified along with corresponding time markers. The subroutine returned the correct value in this test.

\subsubsection{INITALL}

Subroutine INITALL makes a sequence of calls to other subroutines that perform input and output functions. These include:

PMFOUT - output plant model file data

RDNUCD - read nuclide data

RD_NIF - read nuclide inventory file

NIFOUT - output nuclide inventory file

RDINPD - read dose conversion factor file

Testing consisted of checking to see that all referenced subroutines were properly called.

\subsubsection{INVMAT}

This subroutine uses Leverrier's algorithm, also called Fadeeva's method, for evaluating the resolvent matrix for a given system matrix. If $A$ is the system matrix, $I$ is the identity matrix, and $s$ is the Laplace transform variable, then the resolvent matrix is defined as the matrix inverse of $(s I-A)$.

The resolvent matrix is a strictly proper rational function of $s$. If the system matrix, $A$, is $n$-by- $n$, then the resolvent matrix can be expressed as:

$$
I /(s I-A)=Q(s) / p(s)
$$

where $Q(s)$ is the matrix polynomial:

$$
Q(s)=Q[n-1]^{*} s^{* *}(n-1)+Q[n-2] * s^{* *}(n-2)+\ldots+Q[1]^{*} s+Q[0]
$$

and where $p(s)$ is the scalar polynomial:

$$
p(s)=s^{* *} \mathrm{n}+p[n-1]^{*} s^{* *}(n-1)+\ldots+p[1]^{*} s+p[0] \text {. }
$$

Each $Q[i]$, with $i=0$ to $n-1$, is a constant $n$-by- $n$ matrix. 
The subroutine determines the matrices $Q[i]$ and the scalars $p[i]$ using an iterative method:

$$
\begin{aligned}
& Q[i-1]=Q[i] * \mathrm{~A}+\mathrm{p}[i] * \mathrm{I}, \text { for } i=1 \text { to } i=n-1, \text { where } Q[n-1]=I, \\
& p[i]=-1 /(n-i) * \operatorname{tr}(Q[i] * A), \text { for } i=0 \text { to } i=\mathrm{n}-1, \text { where } p[n]=1
\end{aligned}
$$

Testing:

Seven tests were performed. The system matrices used are listed below. In each case the subroutine correctly obtained the matrices $Q[i]$ and the scalars $p[i]$, as determined by available examples.

Test Case \#1. Two compartments. Null system matrix.

$$
\begin{array}{ll}
0.0000 \mathrm{E}+00 & 0.0000 \mathrm{E}+00 \\
0.0000 \mathrm{E}+00 & 0.0000 \mathrm{E}+00
\end{array}
$$

Test Case \#2. Two compartments. Diagonal system matrix.

$$
\begin{array}{ll}
0.4107 \mathrm{E}+00 & 0.0000 \mathrm{E}+00 \\
0.0000 \mathrm{E}+00 & 0.4080 \mathrm{E}+00
\end{array}
$$

Test Case \#3. Two compartments. Diagonal system matrix.

$$
\begin{array}{ll}
0.3362 \mathrm{E}+00 & 0.0000 \mathrm{E}+00 \\
0.0000 \mathrm{E}+00 & 0.2446 \mathrm{E}+00
\end{array}
$$

Test Case \#4. Two compartments. Diagonal system matrix.

$$
\begin{array}{ll}
0.6670 \mathrm{E}+00 & 0.0000 \mathrm{E}+00 \\
0.0000 \mathrm{E}+00 & 0.6652 \mathrm{E}+00
\end{array}
$$

Test Case \#5. Two compartments. Robust system matrix.

$$
\begin{array}{ll}
0.1000 \mathrm{E}+01 & 0.2000 \mathrm{E}+01 \\
0.3000 \mathrm{E}+01 & 0.4000 \mathrm{E}+01
\end{array}
$$

Test Case \#6. Three compartments. Robust system matrix.
$0.0000 \mathrm{E}+00-0.1000 \mathrm{E}+01-0.1000 \mathrm{E}+01$
$0.1000 \mathrm{E}+01-0.2000 \mathrm{E}+01-0.1000 \mathrm{E}+01$
$0.0000 \mathrm{E}+00 \quad 0.0000 \mathrm{E}+00 \quad-0.2000 \mathrm{E}+01$

Test Case \#7. Five compartments. Robust system matrix.
$0.1000 \mathrm{E}+01$
$0.0000 \mathrm{E}+00$
$0.2000 \mathrm{E}+01$
$0.4000 \mathrm{E}+01$
$-0.3000 \mathrm{E}+01$
$0.0000 \mathrm{E}+00$
$0.4000 \mathrm{E}+01$
$0.0000 \mathrm{E}+00$
$0.4000 \mathrm{E}+01$
$0.1000 \mathrm{E}+01$
$0.0000 \mathrm{E}+00 \quad 0.5000 \mathrm{E}+01$
$0.2000 \mathrm{E}+01$
$0.3000 \mathrm{E}+01$
$-0.2000 \mathrm{E}+01$
$-0.1000 \mathrm{E}+01$
$0.0000 \mathrm{E}+00$
$0.1000 \mathrm{E}+01$
$0.4000 \mathrm{E}+01$
$0.7000 \mathrm{E}+01$
$0.1000 \mathrm{E}+01$
$0.2000 \mathrm{E}+01$
$0.3000 \mathrm{E}+01$
$0.1000 \mathrm{E}+01$ 


\subsubsection{LUPC1}

The function LUPC1 performs a table lookup, based on user-specified data for the independent and dependent variables. The data are stored in one-dimensional arrays.

Testing:

Five values were specified for the independent variable $(X)$ and five for the dependent variable $(Y)$ :

$X=(1.0,2.0,3.0,4.0,5.0)$

$Y=(100.0,200.0,300.0,400.0,0.0)$

The first test consisted of allowing the value of the independent variable (X) to ramp up from a point below the specified range to a point above that range. The second test consisted of allowing the independent variable to ramp down, passing through all values in the first test, but in reverse. These two tests showed that the correct values of the dependent variable $(Y)$ were obtained in every case.

\begin{tabular}{cccc}
\multicolumn{2}{c}{ Rampup test } & \multicolumn{2}{c}{ Rampdown test } \\
x-value & $\mathrm{y}$-value & $\mathrm{x}$-value & $\mathrm{y}$-value \\
0.0 & 0.0 & 6.0 & 0.0 \\
0.5 & 0.0 & 5.5 & 0.0 \\
1.0 & 100.0 & 5.0 & 0.0 \\
1.5 & 100.0 & 4.5 & 400.0 \\
2.0 & 200.0 & 4.0 & 400.0 \\
2.5 & 200.0 & 3.5 & 300.0 \\
3.0 & 300.0 & 3.0 & 300.0 \\
3.5 & 300.0 & 2.5 & 200.0 \\
4.0 & 400.0 & 2.0 & 200.0 \\
4.5 & 400.0 & 1.5 & 100.0 \\
5.0 & 0.0 & 1.0 & 100.0 \\
5.5 & 0.0 & 0.5 & 0.0 \\
6.0 & 0.0 & 0.0 & 0.0
\end{tabular}

\subsubsection{LUPC2}

The function LUPC2 performs a table lookup based upon the compartment number, as well as user-specified data for the independent and dependent variables. The data are stored in twodimensional arrays.

Testing:

User-specified data were provided for compartment one. Five values were given for the independent variable $(\mathrm{X})$ and five for the dependent variable $(\mathrm{Y})$ :

$X=(1.0,2.0,3.0,4.0,5.0)$ 
$\mathrm{Y}=(100.0,200.0,300.0,400.0,0.0)$

The first test consisted of allowing the value of the independent variable $(\mathrm{X})$ to ramp up from a point below the specified range to a point above that range. The second test consisted of allowing the independent variable to ramp down, passing through all values in the first test, but in reverse. These two tests showed that the correct value of the dependent variable was obtained:

\begin{tabular}{cccc}
\multicolumn{2}{c}{ Rampup test } & \multicolumn{2}{c}{ Rampdown test } \\
x-value & y-value & x-value & y-value \\
0.0 & 0.0 & 6.0 & 0.0 \\
0.5 & 0.0 & 5.5 & 0.0 \\
1.0 & 100.0 & 5.0 & 0.0 \\
1.5 & 100.0 & 4.5 & 400.0 \\
2.0 & 200.0 & 4.0 & 400.0 \\
2.5 & 200.0 & 3.5 & 300.0 \\
3.0 & 300.0 & 3.0 & 300.0 \\
3.5 & 300.0 & 2.5 & 200.0 \\
4.0 & 400.0 & 2.0 & 200.0 \\
4.5 & 400.0 & 1.5 & 100.0 \\
5.0 & 0.0 & 1.0 & 100.0 \\
5.5 & 0.0 & 0.5 & 0.0 \\
6.0 & 0.0 & 0.0 & 0.0
\end{tabular}

\subsubsection{LUPL2}

This is a lookup table function based upon the location number version of a lookup for twodimensional arrays, $x p$ and yp, for example, which are the control room occupancy factor and breathing rates.

Testing:

Five values were specified for the independent variable $(X)$ and five for the dependent variable (Y):

$$
\begin{aligned}
& X=(1.0,2.0,3.0,4.0,5.0) \\
& Y=(100.0,200.0,300.0,400.0,0.0)
\end{aligned}
$$

The first test consisted of allowing the value of the independent variable $(X)$ to ramp up from a point below the specified range to a point above that range. The second test consisted of allowing the independent variable to ramp down, passing through all values in the first test, but in reverse. The correct values of the dependent variable (Y) were obtained in every case. 


\begin{tabular}{cccc}
\multicolumn{2}{c}{ Rampup test } & \multicolumn{2}{c}{ Rampdown test } \\
x-value & y-value & x-value & y-value \\
0.0 & 0.0 & 6.0 & 0.0 \\
0.5 & 0.0 & 5.5 & 0.0 \\
1.0 & 100.0 & 5.0 & 0.0 \\
1.5 & 100.0 & 4.5 & 400.0 \\
2.0 & 200.0 & 4.0 & 400.0 \\
2.5 & 200.0 & 3.5 & 300.0 \\
3.0 & 300.0 & 3.0 & 300.0 \\
3.5 & 300.0 & 2.5 & 200.0 \\
4.0 & 400.0 & 2.0 & 200.0 \\
4.5 & 400.0 & 1.5 & 100.0 \\
5.0 & 0.0 & 1.0 & 100.0 \\
5.5 & 0.0 & 0.5 & 0.0 \\
6.0 & 0.0 & 0.0 & 0.0
\end{tabular}

\subsubsection{LUPP1}

The function LUPP1 performs a table lookup based on user-specified data for the independent and dependent variables. The data are stored in one-dimensional arrays.

Testing:

Five values were specified for the independent variable $(X)$ and five for the dependent variable $(\mathrm{Y})$ :

$X=(1.0,2.0,3.0,4.0,5.0)$

$\mathrm{Y}=(100.0,200.0,300.0,400.0,0.0)$

The first test consisted of allowing the value of the independent variable $(\mathrm{X})$ to ramp up from a point below the specified range to a point above that range. The second test consisted of allowing the independent variable to ramp down, passing through all values in the first test, but in reverse. These two tests showed that the correct values of the dependent variable $(\mathrm{Y})$ were obtained in every case.

\begin{tabular}{cccc}
\multicolumn{2}{c}{ Rampup test } & \multicolumn{2}{c}{ Rampdown test } \\
x-value & y-value & x-value & y-value \\
0.0 & 0.0 & 6.0 & 0.0 \\
0.5 & 0.0 & 5.5 & 0.0 \\
1.0 & 100.0 & 5.0 & 0.0 \\
1.5 & 100.0 & 4.5 & 400.0 \\
2.0 & 200.0 & 4.0 & 400.0 \\
2.5 & 200.0 & 3.5 & 300.0 \\
3.0 & 300.0 & 3.0 & 300.0 \\
3.5 & 300.0 & 2.5 & 200.0 \\
4.0 & 400.0 & 2.0 & 200.0
\end{tabular}




$\begin{array}{rcrr}4.5 & 400.0 & 1.5 & 100.0 \\ 5.0 & 0.0 & 1.0 & 100.0 \\ 5.5 & 0.0 & 0.5 & 0.0 \\ 6.0 & 0.0 & 0.0 & 0.0\end{array}$

\subsubsection{LUPP2}

The function LUPP2 performs a table lookup based upon the pathway number, as well as userspecified data for the independent and dependent variables. The data are stored in twodimensional arrays.

Testing:

User-specified data were provided for pathway one. Five values were given for the independent variable $(\mathrm{X})$ and five for the dependent variable $(\mathrm{Y})$ :

$X=(1.0,2.0,3.0,4.0,5.0)$

$\mathrm{Y}=(100.0,200.0,300.0,400.0,0.0)$

The first test consisted of allowing the value of the independent variable (X) to ramp up from a point below the specified range to a point above that range. The second test consisted of allowing the independent variable to ramp down, passing through all values in the first test, but in reverse. The results of these two tests showed that the correct value of the dependent variable was obtained:

\begin{tabular}{cccc}
\multicolumn{2}{c}{ Rampup test } & \multicolumn{2}{c}{ Rampdown test } \\
x-value & y-value & x-value & y-value \\
0.0 & 0.0 & 6.0 & 0.0 \\
0.5 & 0.0 & 5.5 & 0.0 \\
1.0 & 100.0 & 5.0 & 0.0 \\
1.5 & 100.0 & 4.5 & 400.0 \\
2.0 & 200.0 & 4.0 & 400.0 \\
2.5 & 200.0 & 3.5 & 300.0 \\
3.0 & 300.0 & 3.0 & 300.0 \\
3.5 & 300.0 & 2.5 & 200.0 \\
4.0 & 400.0 & 2.0 & 200.0 \\
4.5 & 400.0 & 1.5 & 100.0 \\
5.0 & 0.0 & 1.0 & 100.0 \\
5.5 & 0.0 & 0.5 & 0.0 \\
6.0 & 0.0 & 0.0 & 0.0
\end{tabular}

\subsubsection{NECSTEPS}

This subroutine determines the minimum set of time steps that are necessary to capture all of the events and other system changes that are included in the user input. It is called from the main driver routine RADCALC. 


\subsubsection{OPOOL}

This subroutine calculates decontamination factors for each of the four transport groups, resulting from an overlying pool. Both user-provided and model-developed decontamination factors are implemented in this routine. The model is discussed in Section 2.2.3.

Subroutine OPOOL calculates the decontamination factors for each of the four transport groups that result from the effects of an overlying pool. It is assumed that the DF for noble gases is always unity, i.e., there is no decontamination.

The effects considered by this subroutine only apply during the ex-vessel portion of the release. Accordingly subroutine STERM should call OPOOL only during this phase of the release. The logic was corrected to ensure that this was the case. The Powers/Sprung model [Po92] is an option for the case of the aerosol DF.

\section{Testing:}

Subroutine OPOOL was originally tested in June 1995 and then exhaustively tested in September 1995. The cases of no DFs and user-specified DFs were tested for the elemental iodine, organic iodine, and aerosol transport groups. The case of the Powers/Sprung DFs was tested for aerosols. For the latter, the output from subroutine OPOOL was checked against sample problem calculations provided in tabular and graphical formats in the reference document. The DFs obtained from subroutine OPOOL were in excellent agreement with those obtained by direct calculations using the formulas provided in the reference cited.

The following caveat should be noted with regard to very large values of the DFs. Even though the model may yield DFs that are on the order of 1000 and even considerably larger, such high values may not be realizable in actual practice. This is because other phenomena, which are not modeled, come into play and result in reverse decontamination effects. See the caption to Figure 19 on page 66 of the Power reference.

\section{Verification:}

The model was independently verified as being consistently coded.

\subsubsection{PIPIN}

This subroutine calculates the removal rate for material flowing into a compartment by way of a pipeway as discussed in Section 2.2.6. The user may specify the flow rate for iodine and aerosol transport groups. In addition, the user may specify the DFs for these same transport groups or request that they be obtained using the Brockmann/Bixler algorithms. They are called from the coefficient evaluation routine, COEFFS.

\subsubsection{PIPOUT}

This subroutine calculates the normalized flow out of the given compartment by way of piping for all four transport groups. It is called from the coefficient evaluation routine, COEFF. 


\subsubsection{PWRDBA}

This subroutine determines the aerosol removal coefficient in the containment for a pressurized water reactor during a design basis accident. The routine determines the average removal coefficient over the current time step. Table 2.2.2.1-1 details the variation of the removal coefficient with time, reactor thermal power, and bounding percentile (lower, $10 \%$; median, $50 \%$; or high, $90 \%$ ). The routine first determines the decontamination factor for the two phases of the accident release (gap and in-vessel) at the given time by following the equation set described in Section 2.2.2.1.2. It then determines the net decontamination factor by dividing the sum of the current releases by the sum of decontamination of each of those releases. With the current decontamination factor and the value at the beginning of the step, the average removal coefficient is found and returned to routine AEROSOL.

The test case of $3000 \mathrm{MWt}$ and median probability at $10 \mathrm{hr}$ was used as a verification point because this case was used as example 3 in the Powers document. The code gave an average decontamination coefficient of 0.09666 whereas the document shows the value to be 0.0967 . This case should be more accurate than the preceding one because there were only two release periods and both had the release fractions that were used in the code.

The other test cases were examined for both smoothness of the final answer (typically the residual fraction of the iodine in the containment was used as a measure) and for the absolute value of the phase dependent decontamination coefficient as detailed in the Powers document. Within the limits of the tests, $100.0<$ Power $<4000 \mathrm{MWt}$, the answers were reasonable for the decontamination of all chemical species based upon the iodine decontamination. Because the iodine releases a larger fraction during the first two periods than the other species, the results of using the iodine removal coefficient are slightly non-conservative due to the slightly higher decontamination factor. However, the iodine is the most radiologically important and will therefore be more important to the final dose calculation.

\subsubsection{PWRSA}

This subroutine determines the aerosol removal coefficient in the containment for a pressurized water reactor (PWR) during a severe accident. This subroutine determines the average removal coefficient over the current time step. Table 2.2.2.1-2 details the variation of the removal coefficient with time, reactor thermal power, and bounding percentile (lower, 10\%; median, $50 \%$; or high, 90\%). This subroutine first determines the decontamination factor for each phase of the accident release (gap, in-vessel, ex-vessel, and late in-vessel) at the given time by following the equation set described in Section 2.2.2.1.2. It then determines the net decontamination factor by dividing the sum of the current releases by the sum of decontamination of each of those releases. With the current decontamination factor and the value at the beginning of the step, the average removal coefficient is found and returned to routine AEROSOL.

The test case of $3000 \mathrm{MWt}$ and median probability at $10 \mathrm{hr}$ was used as a verification point because this case was used as example 2 in the Powers document. The code gave an average decontamination coefficient of 0.1097 whereas the document shows the value to be 0.1005 . 
Note, however, that the author assumes that at $10 \mathrm{hr}$ after the start of the accident only $6.2 \mathrm{hr}$ of the late in-vessel release have passed. In the final NUREG-1465 document, $8.2 \mathrm{hr}$ would have passed and in addition the release fractions are slightly different. When these changes are made, the code and the document give almost identical results. The results were also compared with Figure 57 of the Powers document and gave the same results for all three bounding percentiles.

The other test cases were examined for both smoothness of the final answer (typically the residual fraction of the iodine in the containment was used as a measure) and for the absolute value of the phase-dependent decontamination coefficient as detailed in the Powers document. Within the limits of the tests, $100.0<$ Power $<4000 \mathrm{MWt}$, the answers were reasonable for the decontamination of all chemical species, based upon the iodine decontamination. Because the iodine releases a larger fraction during the first two periods than the other species the results of using the iodine removal coefficient are slightly nonconservative due to the slightly higher decontamination factor. However, the iodine is the most radiologically important and will therefore be more important to the final dose calculation.

\subsubsection{RADCALC}

This is the main driver routine for the calculational aspects of RADTRAD. It calculates doses to receptors having locations defined by the user. Locations may be at the site boundary, in the low population zone, and in the control room.

\subsubsection{RECIRC}

This subroutine calculates the removal coefficient for each transport group due to the recirculating filter activated in a compartment. The user must specify the filter flow rate as well as the filter efficiencies for elemental iodine, organic iodine, and aerosols. It is assumed that no removal occurs for noble gases.

Testing:

The simulation time was taken to be $1 \mathrm{hr}$. Sets of four values for each filter parameter were defined. The time markers were taken to be $0.0,1.0,2.0$, and $3.0 \mathrm{hr}$. The filter flow rates were set at $0.04,0.03,0.03$, and $0.01 \mathrm{1} / \mathrm{s}$. The efficiencies for elemental-iodine removal were 50.0, 45.0, 40.0 and $35.0 \%$. Those for organic iodine and aerosols were the same: The subroutine provided the correct value for the removal coefficient for each of the four transport groups.

\subsubsection{RESADER}

Subroutine RESADER updates each transport-group quantity (XNGRP) at each compartment location for the current time step. The first three transport groups are returned in units of atoms (molecules), while the fourth is returned in kilograms.

The subroutine begins with the Laplace transform for the transport group quantity and uses the method of residues to invert the transform. 
The most difficult aspect of the algorithm occurs when one or more zeros of the denominator occur repeatedly, that is, when their multiplicities are greater than unity. This necessitates the calculation of the derivatives of the transform.

Subroutine RESADER is an expanded version of RESWUE that uses analytical expressions for the derivatives of the Laplace transform to determine the residues. It has a number of segments appended to it by way of include statements. These perform special functions as indicated below:

RESADER0.FOR reduces the set of denominator zeros to a list of distinct zeros and their corresponding multiplicities

RESADER 1.FOR determines the residue for a denominator zero having multiplicity 1 RESADER2.FOR determines the residue for a denominator zero having multiplicity 2 RESADER3.FOR determines the residue for a denominator zero having multiplicity 3 RESADER4.FOR determines the residue for a denominator zero having multiplicity 4 RESADER5.FOR determines the residue for a denominator zero having multiplicity 5 RESADER6.FOR determines the residue for a denominator zero having multiplicity 6 RESADER7.FOR determines the residue for a denominator zero having multiplicity 7 RESADER8.FOR determines the residue for a denominator zero having multiplicity 8 RESADER9.FOR determines the residue for a denominator zero having multiplicity 9 RESADERX.FOR determines the residue for a denominator zero having multiplicity 10

Note that there are no segments to determine the residue for a denominator of zero having a multiplicity greater than 10 . This should not be a problem as long as the number of compartments does not exceed 10 , the current upper limit for RADTRAD.

The test problem sets were designed to test the ability of the subroutine to determine residues for denominator multiplicities up to and including 10, with numerator multiplicities up to one less than that of the denominator. For the sake of simplicity, the root in question was taken to be zero.

The following is a list of the actual test problem sets that were used. The Laplace transform X(s) is the ratio of two polynomials, $N(s)$ and $\mathrm{D}(\mathrm{s})$, each of which is a power of the variable $s$. The power of $s$ in $\mathrm{D}(\mathrm{s})$ is the same in the number of the test problem set and ranges from 1 to 10 . The power of $s$ in $N(s)$ ranges from 0 up to one less than the power of $s$ in $D(s)$.

Test Problem Set 1:

$\mathrm{X}(\mathrm{s})=\mathrm{N}(\mathrm{s}) / \mathrm{D}(\mathrm{s})$,

$\mathrm{D}(\mathrm{s})=\mathrm{s}$,

$N(s)=1$

Test Problem Set 2:

$$
\begin{aligned}
& X(s)=N(s) / D(s), \\
& D(s)=s^{* * 2}, \\
& N(s)=1, s
\end{aligned}
$$

Test Problem Set 3:

$$
X(s)=N(s) / D(s) \text {, }
$$




$$
\begin{aligned}
& D(s)=s * * 3 \\
& N(s)=1, s, s * * 2
\end{aligned}
$$

Test Problem Set 4:

$$
\begin{aligned}
& X(s)=N(s) / D(s), \\
& D(s)=s * * 4 \\
& N(s)=1, s, s^{* * 2}, s^{* * 3}
\end{aligned}
$$

Test Problem Set 5:

$$
\begin{aligned}
& X(s)=N(s) / D(s) \\
& D(s)=s * * 5 \\
& N(s)=1, s, s * * 2, s * 3, s * * 4
\end{aligned}
$$

Test Problem Set 6:

$$
\begin{aligned}
& X(s)=N(s) / D(s) \\
& D(s)=s * * 6 \\
& N(s)=1, s, s * * 2, s * * 3, s * * 4, s * * 5
\end{aligned}
$$

Test Problem Set 7:

$$
\begin{aligned}
& X(s)=N(s) / D(s) \\
& D(s)=s * 7 \\
& N(s)=1, s, s^{* * 2}, s^{* * 3}, s^{* * 4}, s^{* * 5}, s^{* * 6}
\end{aligned}
$$

Test Problem Set 8:

$$
\begin{aligned}
& X(s)=N(s) / D(s) \\
& D(s)=s^{* * 8} \\
& N(s)=1, s, s^{* * 2}, s^{* * 3}, s^{* * 4}, s^{* * 5}, s^{* * 6}, s^{* * 7}
\end{aligned}
$$

Test Problem Set 9:

$$
\begin{aligned}
& X(s)=N(s) / D(s) \\
& D(s)=s^{* * 9} \\
& N(s)=1, s, s^{* * 2}, s^{* * 3}, s^{* * 4}, s^{* * 5}, s^{* * 6}, s^{* * 7}, s^{* * 8}
\end{aligned}
$$

Test Problem Set 10:

$$
\begin{aligned}
& X(s)=N(s) / D(s) \\
& D(s)=s^{* * 10} \\
& N(s)=1, s, s^{* * 2}, s^{* * 3}, s^{* * 4}, s^{* * 5}, s^{* * 6}, s^{* * 7}, s^{* * 8}, s^{* * 9}
\end{aligned}
$$

\subsubsection{SORTER}

This subroutine does a sort on the elements of the real array passed to it and returns the sorted elements in the same array. Sorting means putting the elements in sequence, without eliminating duplications. The latter step is performed by subroutine NECSTEPS.

The elements in question are time markers that are defined by the scenario description. For example, the release timing file and user-input files both involve time markers. 
For the purposes of testing, a set of 116 values was generated. First, the sequence $0.0,1.0,2.0$, 3.0 was repeated 28 times, for a total of 112 values. Then the sequence $1.0,2.5,5.5,15.5$ was appended to it to yield a total of 116 elements. The subroutine successfully sorted these values.

\subsubsection{SOURCE}

This subroutine controls calculation of source material into each compartment. Source material arises from core heatup and degradation by the user's choice of inventory and release fractions. It is called from the main driver RADCALC and is discussed in Section 1.4.3. The subroutine provides RADTRAD with the total release rate for all transport groups into the compartment in question. It makes calls to STERM (source term).

Testing:

Four driver programs were developed to perform off-line testing of subroutine SOURCE. In all cases the function of subroutine STERM was simulated with the use of "dummy" subroutines. The first driver was designed to test the call to STERM, for the source term. A two-compartment model was used, with the source in the first compartment.

Off-line testing was followed by on-line testing in which SOURCE was integrated into RADTRAD and tested with PMF and SDF files.

\subsubsection{SPRY}

Subroutine SPRY determines the removal coefficients for all four transport groups due to the actions of compartment sprays. It is assumed that no removal effect occurs for noble gases. For the other three transport groups, the removal coefficient can be specified by the user.

For the aerosol transport group, the removal coefficient can also be calculated using the Powers mechanistic model [PO93]. This model requires that the user specify the following:

1. $Q$, the spray water flux, in $\mathrm{m}^{3} \mathrm{H}_{2} \mathrm{O} / \mathrm{m}^{2} / \mathrm{s}$

2. $H$, the fall height, in meters;

3. ALPHA, the ratio of unsprayed volume to sprayed volume,

4. $P C T$, the uncertainty percentile selected for the model (10th, 50 th, 90 th percentiles).

The model can handle both instantaneous "puff" releases and continuous releases. It should not be used for spray water fluxes and fall heights outside of specified ranges. For the spray water flux, this range is $1.0 \mathrm{E}-05$ to $2.5 \mathrm{E}-03 \mathrm{~m}^{3} \mathrm{H}_{2} \mathrm{O} / \mathrm{m}^{2} / \mathrm{s}$. For the fall height, it is 5.0 to $50.0 \mathrm{~m}$. In either case, if the specified value is out of range, then it will be reset to within range and a warning message will be written to the output file.

Testing:

Initial testing consisted of range checking on the spray water flux and fall height, as well as the uncertainty percentile and the fraction of aerosol suspended in the compartment atmosphere. 
Additional testing was conducted in order to duplicate results provided in the report on the Powers model [P093]. It was necessary to introduce a new subroutine SPINIT, which is called by NECSTEPS and which generates additional time markers when compartment sprays are active. It was also necessary to introduce an internal time-integration loop into the Powers model to provide acceptable accuracy in the removal coefficients for aerosols. With the addition of user time step control, this routine was removed from the code. The user should use values less than $0.1 \mathrm{hr}$ while the spray is operational.

The test-data set that was used is shown below. All tests were successful.

$\begin{array}{ccccl}\begin{array}{c}\text { Water } \\ \text { (flux) }\end{array} & \begin{array}{c}\text { Fall } \\ \text { (height) }\end{array} & \begin{array}{c}\text { Spray } \\ \text { (alpha) }\end{array} & \begin{array}{c}\text { Uncert } \\ \text { (percent) }\end{array} & \begin{array}{c}\text { Release } \\ \text { (type) }\end{array} \\ 0.0025 & 50 & 1.0 & 10 \% & \text { instant } \\ 0.0025 & 50 & 1.0 & 50 \% & \text { instant } \\ 0.0025 & 50 & 1.0 & 90 \% & \text { instant } \\ 0.0025 & 50 & 1.0 & 10 \% & \text { continuous } \\ 0.0025 & 50 & 1.0 & 50 \% & \text { continuous } \\ 0.0025 & 50 & 1.0 & 90 \% & \text { continuous }\end{array}$

\subsubsection{STERM}

This subroutine calculates the nuclide release rates and the transport-group release rates from the core inventory to the source-term compartment. Release rates for transport groups (1) noble gases, (2) elemental iodine, and (3) organic iodine are given in units of atom/s. The release rate for transport group (4), aerosols, is in units of $\mathrm{kg} / \mathrm{s}$. The reference is NUREG-1465.

Testing:

For the purposes of testing, the PWR default values for release durations and fractions were used. Nine nuclides were included in the inventory, one from each nuclide family.

User-specified data were provided for overlying pool effects. It is assumed that there is no effect on noble gases. Tables of decontamination factors were provided for elemental iodine, organic iodine, and aerosols. In addition, fall heights (from 0.3 to $5.0 \mathrm{~m}$ ), cooling temperatures (from 0 to $70 \mathrm{~K})$, and an uncertainty percentile $(10,50$, or $90 \%)$ were provided for the Powers model of aerosol removal.

Test runs were made for selected simulation times. The routine provided the correct results for each case.

$\begin{array}{ll}0.25 \mathrm{hr} & \text { release phase \#1 } \\ 1.0 & \text { release phase \#2 } \\ 2.0 & \text { release phase \#3 } \\ 8.0 & \text { release phase \#4 } \\ 14.0 & \text { after release over }\end{array}$




\subsubsection{SUMPWR}

This subroutine calculates the sum of the inverse numerator powers, minus the sum of the inverse denominator powers.

\subsubsection{SUPIN}

This subroutine calculates the removal coefficient for material flowing into a compartment through a suppression pool. The user must specify DFs for elemental iodine, organic iodine, and aerosols. It is assumed that there is no effect upon noble gases.

Testing:

Sets of four values of the DF and the flow rate were provided for elemental iodine, organic iodine, and aerosols. These were accompanied by corresponding time markers. The subroutine provides the correct values during this test.

\subsubsection{SUPOUT}

This subroutine calculates the normalized flow out of a given compartment by way of a suppression pool. The values obtained are returned in units of $1 / \mathrm{s}$. It is assumed that there is no effect upon the noble gases. For the other three transport groups, the user must provide the flow rate versus time.

Testing:

A set of four values of the flow rate was provided for elemental iodine, organic iodine, and aerosols. Corresponding time markers were also provided. The subroutine returned the correct values during this test.

\subsubsection{XNTDEP}

This subroutine calculates the removal coefficient for natural deposition. The process of natural deposition includes many phenomena, such as gravitational settling, impaction, and diffusion. The removal coefficients are returned in units of $1 / \mathrm{s}$.

The subroutine considers all four transport groups. However, natural deposition is considered. applicable only to elemental iodine and to aerosols. Noble gases and organic iodine are nonreactive and so are not affected.

The removal coefficients for elemental iodine and aerosols can be specified by the user. In the case of elemental iodine, the user should include the effects of reemission of iodine, because this effect is not modeled explicitly.

The removal coefficients for aerosols can be calculated using a correlation by Henry [Fa84]. Henry indicates that the model can be used for any compartment, rather than just the source-term 
compartment. To use the Henry correlation, the user must specify (1) the fall height in meters and (2) the particle density in $\mathrm{kg} / \mathrm{m}^{3}$. In both cases reference values are provided in data statements. For the fall height, this value is $5.0 \mathrm{~m}$ and for the sodium oxide $\left(\mathrm{Na}_{2} \mathrm{O}\right)$ particle density, it is $2270.0 \mathrm{~kg} / \mathrm{m}^{3}$. In addition, there is a reference value for the total mass of aerosols in the volume, set at $6.0 \mathrm{E}-05 \mathrm{~kg} / \mathrm{m}^{3}$.

The removal coefficients for aerosols can also be calculated using a model by Powers [Po95]. This model applies only to the source-term compartment. To use it, the user must specify (1) the reactor type (PWR, AWR, BWR), (2) the accident type (design basis accident or severe accident), and (3) the uncertainty percentile $(10 \%, 50 \%, 90 \%)$.

Testing:

Natural deposition of elemental iodine and aerosols was tested with user-specified coefficients. These tests were successful. Natural deposition of aerosols using the Henry correlation was tested by making use of tabulated data (Figure 1 on page 3) of reference Po95. Natural deposition of aerosols using the Powers model was tested off-line. See the testing report on AEROSOL.

\subsubsection{XNUPDT}

The function of subroutine XNUPDT is to convert transport-group quantities (XNRGP) into nuclide counts $(\mathrm{XN})$. This is necessary because RADTRAD simulates removal and transport of transport groups, while doses are determined from nuclide counts.

Four transport-group quantities are provided:

XNGRP $(1)=$ noble gases, atoms

$\mathrm{XNGRP}(2)=$ elemental iodines, atoms

$\mathrm{XNGRP}(3)=$ organic iodines, atoms (molecules)

$\mathrm{XNGRP}(4)=$ aerosols, kilograms

These are broken out into the nine nuclide families:

(1) noble gases, atoms

(2) iodines (elemental), atoms iodines (organic), atoms (molecules)

iodines (aerosol), kilograms

(3) cesiums (aerosol), kilograms

(4) telluriums (aerosol), kilograms

(5) strontiums (aerosol), kilograms

(6) rutheniums (aerosol), kilograms

(7) lanthanums (aerosol), kilograms

(8) ceriums (aerosol), kilograms

(9) bariums (aerosol), kilograms

In turn, these are broken out into specific nuclide counts, all measured in atoms. 

(1) $\mathrm{Xe}, \mathrm{Kr}$
(2) $\mathrm{I}, \mathrm{Br}$
(3) $\mathrm{Cs}, \mathrm{Rb}$
(4) $\mathrm{Te}, \mathrm{Sb}, \mathrm{Se}$
(5) $\mathrm{Sr}$
(6) Ru, Rh, Pd, Mo, Tc, Co
(7) $\mathrm{La}, \mathrm{Zr}, \mathrm{Nd}, \mathrm{Eu}, \mathrm{Nb}, \mathrm{Pm}, \mathrm{Pr}, \mathrm{Sm}, \mathrm{Y}, \mathrm{Am}, \mathrm{Cm}$
(8) $\mathrm{Ce}, \mathrm{Pu}, \mathrm{Np}$
(9) $\mathrm{Ba}$

\section{Testing:}

For the purposes of testing, it was assumed that there were only nine nuclides, rather than the usual sixty provided for RADTRAD. One nuclide was selected from each nuclide family and the count for that nuclide was specified.

The following list indicates those nuclides, along with the transport groups to which they belong. Note that iodine is partitioned among three transport groups.
(1) ${ }^{133} \mathrm{Xe} \quad$ noble gas
(2) ${ }^{131}$ I $1 / 3$ elemental, $1 / 3$ organic, $1 / 3$ aerosol
(3) ${ }^{134} \mathrm{Cs}$ aerosol
(4) ${ }^{127} \mathrm{Te}$ aerosol
(5) ${ }^{89} \mathrm{Sr} \quad$ aerosol
(6) ${ }^{58} \mathrm{Co} \quad$ aerosol
(7) ${ }^{90} \mathrm{Y} \quad$ aerosol
(8) ${ }^{141} \mathrm{Ce}$ aerosol
(9) ${ }^{139} \mathrm{Ba}$ aerosol

The quantities for each of the four transport groups were then calculated and passed to the subroutine XNUPDT. There the above process was inverted, that is, the nuclide counts were recovered, based only upon the quantities of each transport group. This was done successfully for every nuclide count.

\subsubsection{ZERODN}

For each transport group, subroutine ZERODN determines:

(1) the zeros of the scalar denominator,

(2) the zeros of the vector numerator.

Subroutine ZERODN accepts the coefficients of the single denominator polynomial and the NC numerator polynomials of the Laplace transform of each of the four transport groups. The value of NC is the same as the number of compartments in the facility model. 
The subroutine returns the $\mathrm{NC}+1$ roots of the denominator polynomial and the variable number of roots for each of the NC numerator polynomials. It also returns the numbers of these roots and the leading coefficients of the numerator polynomials.

Subroutine ZERODN is called by RADCALC as part of a sequence of calls comprising the mathematics package of the program. Other subroutines to which it is related are INVMAT, COENUM, and RESADER. The extraction of the roots is performed with the IMSL routine DZPORC, which works with double-precision complex arrays.

\section{Testing:}

The arrays ZERODEN and ZERONUM contain the real or complex roots of the denominator and numerators, respectively. Tests were done with these arrays declared to be single-precision complex and double-precision complex. It was found that single precision was sufficient to provide accurate roots to RADCALC.

tightly clustered zeros

zeros are

$$
\begin{aligned}
& (0.0,0.0) \\
& (1.0 \mathrm{E}-6,0.0) \\
& (1.0 \mathrm{E}-6,0.0) \\
& (0.0,1.0 \mathrm{E}-6) \\
& (0.0,1.0 \mathrm{E}-6)
\end{aligned}
$$

coefficients are

$$
\begin{aligned}
& p(0)=0.0 E+00 \\
& p(1)=-1.0 E-24 \\
& p(2)=0.0 E+00 \\
& p(3)=0.0 E+00 \\
& p(4)=0.0 E+00 \\
& p(5)=+1.0 E+00
\end{aligned}
$$

Tests of the code for the extraction of denominator roots and numerator roots were performed with the following examples:

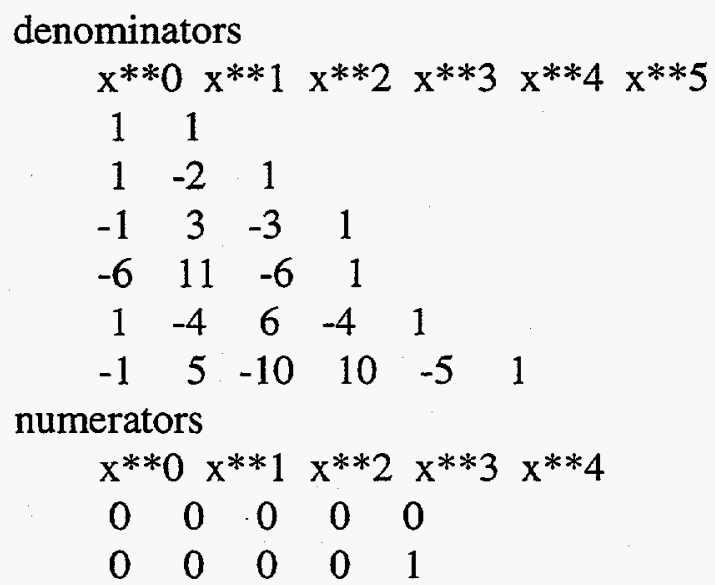




$\begin{array}{lllll}0 & 0 & 0 & 1 & 0 \\ 0 & 0 & 0 & 1 & 1 \\ 0 & 0 & 1 & 0 & 0 \\ 0 & 0 & 1 & 0 & 1 \\ 0 & 0 & 1 & 1 & 0 \\ 0 & 0 & 1 & 1 & 1 \\ 0 & 1 & 0 & 0 & 0 \\ 0 & 1 & 0 & 0 & 1 \\ 0 & 1 & 0 & 1 & 0 \\ 0 & 1 & 0 & 1 & 1 \\ 0 & 1 & 1 & 0 & 0 \\ 0 & 1 & 1 & 0 & 1 \\ 0 & 1 & 1 & 1 & 0 \\ 0 & 1 & 1 & 1 & 1 \\ 1 & 0 & 0 & 0 & 0 \\ 1 & 0 & 0 & 0 & 1 \\ 1 & 0 & 0 & 1 & 0 \\ 1 & 0 & 0 & 1 & 1 \\ 1 & 0 & 1 & 0 & 0 \\ 1 & 0 & 1 & 0 & 1 \\ 1 & 0 & 1 & 1 & 0 \\ 1 & 0 & 1 & 1 & 1 \\ 1 & 1 & 0 & 0 & 0 \\ 1 & 1 & 0 & 0 & 1 \\ 1 & 1 & 0 & 1 & 0 \\ 1 & 1 & 0 & 1 & 1 \\ 1 & 1 & 1 & 0 & 0 \\ 1 & 1 & 1 & 0 & 1 \\ 1 & 1 & 1 & 1 & 0 \\ 1 & 1 & 1 & 1 & 1\end{array}$

\subsubsection{ZPORC}

This subroutine returns the double-precision or complex zeros of a given polynomial with double-precision coefficients. The required inputs are NDEG, the degree of the polynomial, and COEFF, the vector of the double-precision coefficients (with the zeroth power occurring first). The output is ZERO, the vector of double-precision or complex roots (whose number is NDEG).

\subsubsection{ZRINV}

This routine will zero the inventories in a particular type of compartment. This routine was written for general use, but currently is used only for environment compartments (compartment type $=3$ ). The current use is to zero environment inventories for plume simulations. 


\subsection{Beta Testing Results}

Beta testing of the RADTRAD code occurred from December 1995 through March 1996.

During this time, 30 discrepancy reports were filed. Of the 30,5 were in the critical category and were all corrected. An additional 10 discrepancy reports were in the important category; 9 have been corrected and 1 has not been corrected. The uncorrected discrepancy relates to a problem that can occur when shelling out to DOS from RADTRAD. Five discrepancy reports required no changes. The remaining 10 discrepancy reports were considered cosmetic changes; of these 10,1 was corrected and the rest deferred. The following section gives the actual discrepancy reports.

\subsubsection{Discrepancy Reports}

See following pages. 


\section{RADTRAD $\beta$-Test Discrepancy Report}

RADTRAD Version: 1.03

Computer Type and Operating System: Gateway 2000 486DX2/50 MSDOS

Reported By: J.V. Ramsdell

Date: $\quad 12 / 29 / 95$

Organization: PNNL

Telephone: (509) 3726316

Problem or Requested Change: If a software problem is being reported, include a description of the input data that can be used to duplicate the problem. Attach .pmf and .sdf files if possible.

When starting data entry, there should be some indication that it is not necessary to enter names for the .PMF and .SDF files unless an existing plant/scenario design is to be modified.

Evaluation:

Summary: 3 (1 = Critical, $2=$ Important, $3=$ Cosmetic, $4=$ Documentation, $5=$ None $)$

Evaluator: Steven Humphreys Date: 3/19/96

\section{Disposition:}

Scope: _Current _ New

Code: $\quad$ Revise $\quad \checkmark$ Defer No Change Required

Documentation: _ User's Guide - Tech. Desc. No Documentation

Comments:

Minimal effort required to change $<1 \mathrm{~h}$

Date: $3 / 19 / 96$ 


\section{RADTRAD $\beta$-Test Discrepancy Report}

RADTRAD Version: 1.03

Computer Type and Operating System: Gateway 2000 4860X2/50 MSDOS

Reported By: J.V. Ramsdell

Date: $12 / 29 / 95$

Organization: PNNL

Telephone: (509) 3726316

Problem or Requested Change: If a software problem is being reported, include a description of the input data that can be used to duplicate the problem. Attach .pmf and .sdf files if possible.

If RADTRAD has been run, the names of the .PMF and .SDF files from the most recent runs should be used as defaults when RADTRAD is restarted. This would facilitate running a sequence of related scenarios.

Evaluation:

Summary: $3 \quad(1=$ Critical, $2=$ Important, 3 = Cosmetic, $4=$ Documentation, $5=$ None $)$

Evaluator: Steven Humphreys Date: 3/19/96

\section{Disposition:}

Scope: _ Current _ New

Code: $\quad$ Revise $\quad \checkmark$ Defer $\quad$ No Change Required

Documentation: _User's Guide _ Tech. Desc. _ _ No Documentation

Comments:

Minimal effort to change $<1 \mathrm{~h}$

Date: $3 / 19 / 96$ 


\section{RADTRAD $\beta$-Test Discrepancy Report}

RADTRAD Version: 1.03

Computer Type and Operating System: Gateway 2000 486DX2/50 MSDOS

Reported By: J.V. Ramsdell

Date: $\quad 12 / 29 / 95$

Organization: PNNL

Telephone: (509) 3726316

Problem or Requested Change: If a software problem is being reported, include a description of the input data that can be used to duplicate the problem. Attach .pmf and .sdf files if possible.

When a name is entered for the .PMF file, e.g. Name.PMF, the default names for the .SDF and output files should change automatically to Name.SDF and Name.TXT.

\section{Evaluation:}

The separation of the plant from the scenario information allows the user to run different scenarios for a given plant model. This capability precludes any for knowledge of what the scenario or output files might be called.

Summary: $5 \quad(1=$ Critical, $2=$ Important, $3=$ Cosmetic, $4=$ Documentation, $5=$ None $)$

Evaluator: Steven Humphreys $\quad$ Date: 3/19/96

\section{Disposition:}

$\begin{array}{rlll}\text { Scope: } & - \text { Current } & - \text { New } & \\ \text { Code: } & - \text { Revise } & - \text { Defer } & \checkmark \text { No Change Required } \\ \text { Documentation: } & - \text { User's Guide } & - \text { Tech. Desc. } & \text { - No Documentation }\end{array}$

Comments:

No correction necessary

Date: $3 / 19 / 96$ 


\section{RADTRAD $\beta$-Test Discrepancy Report}

RADTRAD Version: 1.03

Computer Type and Operating System: Gateway 2000 486DX2/50 MSDOS

Reported By: J.V. Ramsdell

Date: $\quad 12 / 29 / 95$

Organization: PNNL

Telephone: (509) 3726316

Problem or Requested Change: If a software problem is being reported, include a description of the input data that can be used to duplicate the problem. Attach .pmf and .sdf files if possible.

Once plant compartments have been defined, it should be possible to define and redefine transfer pathways without losing the plant compartment definitions. RADTRAD appears to delete all data on unused compartments when it does calculations.

\section{Evaluation:}

An error during the effective volume calculations caused the wrong compartment to be altered. This error was corrected. Since that time the effective volume subroutine has been removed altogether.

Summary: $1 \quad(1=$ Critical, $2=$ Important, $3=$ Cosmetic, $4=$ Documentation, $5=$ None $)$

Evaluator: Steven Humphreys Date: 3/19/96

\section{Disposition:}

$$
\begin{array}{lll}
\text { Scope: } & \checkmark \text { Current } & - \text { New } \\
\text { Code: } & \checkmark \text { Revise } & - \text { Defer }
\end{array}
$$

Documentation: _ User's Guide _ Tech. Desc.

Comments:

Corrected.

Date: $3 / 19 / 96$ 
RADTRAD $\beta$-Test Discrepancy Report

RADTRAD Version: 1.03

Computer Type and Operating System: Gateway 2000 486DX2/50 MSDOS

Reported By: J.V. Ramsdell

Date: $\quad 12 / 29 / 95$

Organization: PNNL

Telephone: (509) 3726316

Problem or Requested Change: If a software problem is being reported, include a description of the input data that can be used to duplicate the problem. Attach .pmf and .sdf files if possible.

When simplifying a scenario, RADTRAD doesn't delete transfer pathways correctly when the simplification is done by deleting compartments. Started with Acceptance Test Case 3 defining 4 compartments and 6 tx paths... saved .PMF file and exited. Restarted RADTRAD, recalled CASE3.PMF. Marked Air Filtration Volume unused, changed the Env. to AFV tx path to filtered path from Env to CR, marked the remaining tx paths associated with the AFV and the unfiltered path from the Env to $C R$ as unused, then saved file as CASE3A.PMF. Transfer paths in

CASE3A.PMF are not correct. Last 3 tx paths in CASE3.PMF dropped, not the correct 3. Files attached.

\section{Evaluation:}

This is a troublesome limitation of the user interface. It will be corrected for the help file version of RADTRAD.

Summary: 2 $\quad(1=$ Critical, $2=$ Important, $3=$ Cosmetic, $4=$ Documentation, $5=$ None $)$

Evaluator: Steven Humphreys Date: 3/19/96

\section{Disposition:}

\begin{tabular}{|c|c|c|c|}
\hline Scope: & $\checkmark$ Current & _New & \\
\hline Code: & $\checkmark$ Revise & _ Defer & __ No Change Required \\
\hline ntation & _User's Guide & Tech. Desc. & _ No Documentation \\
\hline
\end{tabular}

Comments:

Corrected

Date: $3 / 19 / 96$ 


\section{RADTRAD $\beta$-Test Discrepancy Report}

RADTRAD Version: 1.03

Computer Type and Operating System: Gateway 2000 486DX2/50 MSDOS

Reported By: J.V. Ramsdell

Date: $12 / 29 / 95$

Organization: PNNL

Telephone: (509) 3726316

Problem or Requested Change: If a software problem is being reported, include a description of the input data that can be used to duplicate the problem. Attach .pmf and .sdf files if possible.

Is it possible to have RADTRAD calculate the outflow from the Control Room to the Environment from the inflow to ensure an air flow balance? This is a potential source of user error, particularly because the inflow can include both real and normalized flows.

\section{Evaluation:}

Flow balancing is not required in RADTRAD.

Summary: $\underline{5} \quad(1=$ Critical, $2=$ Important, $3=$ Cosmetic, $4=$ Documentation, $5=$ None $)$

Evaluator: Steven Humphreys Date: 3/19/96

\section{Disposition:}

$\begin{array}{ccc}\text { Scope: } & \text { - Current } & \\ \text { Code: } & \text { - Revise } & - \text { Defer }\end{array}$

Documentation: _ User's Guide

Comments:

No Correction necessary.
_ Tech. Desc.
No Change Required

_ No Documentation

Date: $3 / 19 / 96$ 


\section{RADTRAD $\beta$-Test Discrepancy Report}

RADTRAD Version: 1.03

Computer Type and Operating System: Gateway 2000 486DX2/50 MSDOS

Reported By: J.V. Ramsdell

Date: $\quad 12 / 29 / 95$

Organization: PNNL

Telephone: (509) 3726316

Problem or Requested Change: If a software problem is being reported, include a description of the input data that can be used to duplicate the problem. Attach .pmf and .sdf files if possible.

When a radio button is used to select an option, the button should show the default option and it shouldn't be necessary to click on the button to select the default option (e.g. the select model buttons on the form used to edit transfer pathways in the scenario description). (This button doesn't add anything to the utility of RADTRAD does it? Perhaps the button should be deleted.)

\section{Evaluation:}

At present the buttons are necessary but there is almost certainly a better way to handle the input without requiring these buttons.

Summary: $3 \quad(1=$ Critical, $2=$ Important, $3=$ Cosmetic, $4=$ Documentation, $5=$ None $)$

Evaluator: Steven Humphreys Date: 3/29/96

Disposition:

Scope: _Current _ New

Code: $\quad$ Revise $\quad \checkmark$ Defer $\quad$ No Change Required

Documentation: _User's Guide _ _ Tech. Desc. _ No Documentation

Comments:

Moderate effort to change $<1 \mathrm{~d}$

$>1 \mathrm{~h}$

Date: $3 / 19 / 96$ 


\section{RADTRAD $\beta$-Test Discrepancy Report}

RADTRAD Version: 1.03

Computer Type and Operating System: Gateway 2000 486DX2/50 MSDOS

Reported By: J.V. Ramsdell

Date: $12 / 29 / 95$

Organization: PNNL

Telephone: (509) 3726316

Problem or Requested Change: If a software problem is being reported, include a description of the input data that can be used to duplicate the problem. Attach .pmf and sdf files if possible.

RADTRAD requires numerical input in specific form (sometimes in normal floating point form and sometimes in scientific notation). Is this necessary. The program should accept any appropriate numerical values.

\section{Evaluation:}

This problem is a function of the Hi-Screen Pro II interface software. It requires you to specify if your real values are in regular or scientific notation. You can't specify both. This problem could be corrected if we wrote our own syntax checkers. This would not be a trivial task but the resulting interface would be better.

Summary: $3 \quad(1=$ Critical, $2=$ Important, $3=$ Cosmetic, $4=$ Documentation, $5=$ None $)$

Evaluator: Steven Humphreys Date: 3/19/96

\section{Disposition:}

$$
\text { Scope: _Current } \quad \text { New }
$$

Code: _- Revise $\quad \checkmark$ Defer

Documentation: _User's Guide _ Tech. Desc. _ No Documentation
Comments:
Significant effort to change $\begin{aligned}< & 1 \text { wk } \\ & >1 \mathrm{~d}\end{aligned}$

Date: $3 / 19 / 96$ 
RADTRAD $\beta$-Test Discrepancy Report

RADTRAD Version: 1.03

Computer Type and Operating System: Gateway 2000 486DX2/50 MSDOS

Reported By: J.V. Ramsdell

Date: $\quad 12 / 29 / 95$

Organization: PNNL

Telephone: (509) 3726316

Problem or Requested Change: If a software problem is being reported, include a description of the input data that can be used to duplicate the problem. Attach .pmf and .sdf files if possible.

RADTRAD should accept input in engineering units used by NRC Staff. These units include flows in $\mathrm{ft}^{3} / \mathrm{min}$, leak rates in \%/day, and volumes in $\mathrm{ft}^{3}$. Unit conversion is a potential source of error and should be done by the program.

\section{Evaluation:}

The limitations of the High Screen Pro II interface software and screen size make this difficult to do with selectors or drop down lists. It could be done more easily by incorporating the units into the values themselves and having the syntax checker described in Report \#8 do the necessary conversion. One drawback is that the user would have to enter units for all values in the non default units.

Units recommended by the NRC will be incorporated into RADTRAD version 2.12 .

Summary: $2 \quad(1=$ Critical, $2=$ Important, $3=$ Cosmetic, $4=$ Documentation, $5=$ None $)$

Evaluator: Steven Humphreys Date: 3/19/96

\section{Disposition:}

$\begin{array}{lll}\text { Scope: } & \checkmark \text { Current } & - \text { New } \\ \text { Code: } & \checkmark \text { Revise } & - \text { Defer }\end{array}$

Documentation: _ User's Guide

Comments:

Corrected

Date: $3 / 19 / 96$
_ Defer _ No Change Required

_Tech. Desc. _ No Documentation 


\section{RADTRAD $\beta$-Test Discrepancy Report}

RADTRAD Version: 1.03

Computer Type and Operating System: Gateway 2000 486DX2/50 MSDOS

Reported By: J.V. Ramsdell

Date: $12 / 29 / 95$

Organization: PNNL

Telephone: (509) 3726316

Problem or Requested Change: If a software problem is being reported, include a description of the input data that can be used to duplicate the problem. Attach .pmf and .sdf files if possible.

There are too many data input forms... forms used to enter related information could be combined... the decay/ingrowth and iodine chemical form forms are an example...

Evaluation:

Much of the above problem can be contributed to the separation of plant and scenario information. It may be desirable to review this feature to determine whether or not it makes more sense just to use a single file per model. If so, then it would make sense to contain many of the plant and scenario screens.

Summary: $\underline{5} \quad(1=$ Critical, $2=$ Important, $3=$ Cosmetic, $4=$ Documentation, $5=$ None $)$

Evaluator: Steven Humphreys Date: 3/19/96

Disposition:

Scope: _ Current $\quad$ New

Code: _ Revise _ Defer

Documentation: _User's Guide _ _ Tech. Desc. _ No Documentation

Comments:

To make the above change would require a shift in the RADTRAD design philosophy that would in turn require extensive re-writing of the interface. This kind of change is beyond the normal scope of error correction or software maintenance and as such would require < change in the NRC requirements before it could be implemented. Since it would violate the requirement for separate plant and scenario files of information no change is presently required and in fact the codes would not meet NRC requirements if the change was made.

Date: $3 / 19 / 96$ 


\section{RADTRAD $\beta$-Test Discrepancy Report}

RADTRAD Version: 1.03

Computer Type and Operating System: Gateway 2000 486DX2/50 MSDOS

Reported By: J,V. Ramsdell

Date: $\quad 12 / 29 / 95$

Organization: PNNL

Telephone: (509) 3726316

Problem or Requested Change: If a software problem is being reported, include a description of the input data that can be used to duplicate the problem. Attach .pmf and .sdf files if possible.

Delete writing the $\mathrm{i} / \mathrm{o}$ unit status to the screen, if possible, for the RADIRAD production code.

\section{Evaluation:}

This debugging information has been removed.

Summary: $3 \quad(1=$ Critical, $2=$ Important, $3=$ Cosmetic, $4=$ Documentation, $5=$ None $)$

Evaluator: Steven Humphreys Date: 3/19/96

\section{Disposition:}

Scope: $\checkmark$ Current $\quad-$ New

Documentation: _ User's Guide

Comments:

Corrected

Date: $3 / 19 / 96$
- Defer

_ Tech. Desc.
_ No Change Required

_ No Documentation 
RADTRAD $\beta$-Test Discrepancy Report

RADTRAD Version: 1.03

Computer Type and Operating System: Gateway 2000 486DX2/50 MSDOS

Reported By: J.V. Ramsdell

Date: $12 / 29 / 95$

Organization: PNNL

Telephone: (509) 3726316

Problem or Requested Change: If a software problem is being reported, include a description of the input data that can be used to duplicate the problem. Attach .pmf and .sdf files if possible.

Selecting decay and ingrowth should be 2 options.... Decay or no decay, and if decay is selected ingrowth or no ingrowth.

Evaluation:

The user has the capability of turning on decay for one, some, or all of the compartments. Because of this feature the user might possibly proceed through the problem setup first specifying daughter ingrowth before specifying any decay or turning all decay off and then turning decay back on. Either operation would have daughtering turned off when the user wanted it on. Daughtering has no effect if there is no decay.

Summary: $5 \quad(1=$ Critical, $2=$ Important, $3=$ Cosmetic, $4=$ Documentation, $5=$ None $)$

Evaluator: Steven Humphreys

Date: $3 / 19 / 96$

Disposition:

Scope: _ Current _ New

Code: _Revise _ Defer

No Change Required

Documentation: _User's Guide_ _ Tech. Desc. _ No Documentation

Comments:

No correction necessary

Date: $3 / 19 / 96$ 


\section{RADTRAD $\beta$-Test Discrepancy Report}

RADTRAD Version: 1.03

Computer Type and Operating System: Gateway 2000 486DX2/50 MSDOS

Reported By: J.V. Ramsdell

Date: $\quad 12 / 29 / 95$

Organization: PNNL

Telephone: (509) 3726316

Problem or Requested Change: If a software problem is being reported, include a description of the input data that can be used to duplicate the problem. Attach .pmf and .sdf files if possible.

RADTRAD needs better error trapping and messages to provide users with some information on what has gone: wrong when the program fails. Before calculations are begun the program should check the input and identify errors and omissions that would cause the program to crash. This information should be provided to the user in an error message.

\section{Evaluation:}

Agreed Improvements have been made. See comments below.

Summary: 2 ( 1 = Critical, $2=$ Important, $3=$ Cosmetic, $4=$ Documentation, $5=$ None $)$

Evaluator: Steven Humphreys Date: 3/19/96

Disposition:

$$
\begin{array}{lll}
\text { Scope: } & \checkmark \text { Current } & - \text { New } \\
\text { Code: } & \checkmark \text { Revise } & \quad \text { Defer }
\end{array}
$$

Documentation: _User's Guide _ _ Tech. Desc. _ No Documentation

Comments:

Corrected. Better error handling would improve the user's interaction with the software. But, while it is important it is not critical. Almost all errors in RADTRAD now print error information to the output file so the user can determine and correct the problem causing the error in most cases.

Date: $3 / 19 / 96$ 


\section{RADTRAD $\beta$-Test Discrepancy Report}

RADTRAD Version: 1.03

Computer Type and Operating System: Gateway 2000 486DX2/50 MSDOS

Reported By: J.V. Ramsdell

Date: $\quad 12 / 29 / 95$

Organization: PNNL

Telephone: (509) 3726316

Problem or Requested Change: If a software problem is being reported, include a description of the input data that can be used to duplicate the problem. Attach .pmf and .sdf files if possible.

Occupancy factors should be input explicitly rather than requiring users to adjust X/Q or breathing rates. There isn't anything in the output that shows whether occupancy factor has been included or not.

\section{Evaluation:}

Occupying factors have been added.

Summary: $1 \quad(1=$ Critical, $2=$ Important, $3=$ Cosmetic, $4=$ Documentation, $5=$ None $)$

Evaluator: Steven Humphreys

Date: $3 / 19 / 96$

\section{Disposition:}

Scope: $\checkmark$ Current _ New

Code: $\checkmark$ Revise _ Defer _ No Change Required

Documentation: _ User's Guide _ Tech. Desc. _ No Documentation

Comments:

Corrected.

Date: $3 / 19 / 96$ 


\section{RADTRAD $\beta$-Test Discrepancy Report}

RADTRAD Version: 1.03

Computer Type and Operating System: Gateway 2000 486DX2/50 MSDOS

Reported By: J.V. Ramsdell

Date: $12 / 29 / 95$

Organization: PNNL

Telephone: (509) 3726316

Problem or Requested Change: If a software problem is being reported, include a description of the input data that can be used to duplicate the problem. Attach .pmf and .sdf files if possible.

With the TID source term it isn't normal practice for NRC to give credit for both an initial plateout of $50 \%$ of the iodine released and natural deposition. RADTRAD should provide an option to include or exclude the initial plateout.

\section{Evaluation:}

The TID source term specifies 50\% release of iodine. Reg Guides $1.5 \times 1.4$ specify $50 \%$ plateout or an effective release of $25 \%$. In cases where natural deposition in the containment is being modeled the user should use the TID_DEF.RFT file which has no plateout. Otherwise the user should use the TID_DBA.RFT which does.

Summary: 1 ( 1 = Critical, $2=$ Important, $3=$ Cosmetic, $4=$ Documentation, $5=$ None $)$

Evaluator: Steven Humphreys Date: 3/19/96

\section{Disposition:}

$\begin{array}{clll}\text { Scope: } & \checkmark \text { Current } & - \text { New } & \\ \text { Code: } & \checkmark \text { Revise } & - \text { Defer } & - \text { No Change Required } \\ \text { Documentation: } & -{ }^{\text {User's Guide }} & - \text { Tech. Desc. } & - \text { No Documentation } \\ \begin{array}{c}\text { Comments: } \\ \text { Corrected }\end{array} & & \end{array}$

Date: $\quad 3 / 19 / 96$ 


\section{RADTRAD $\beta$--Test Discrepancy Report}

RADTRAD Version: 1.03

Computer Type and Operating System: Gateway 2000 486DX2/50 MSDOS

Reported By: J.V. Ramsdell

Date: $12 / 29 / 95$

Organization: PNNL

Telephone: (509) 3726316

Problem or Requested Change: If a software problem is being reported, include a description of the input data that can be used to duplicate the problem. Attach .pmf and .sdf files if possible.

RADTRAD overwrites existing .PMF, .SDF, and .TXT files without warning. This can lead to unintentional loss of data. There should be a way to protect/unprotect these files, and there should be a warning prior to overwriting an unprotected file.

Evaluation:

This is indeed true and many users will expect and assume this kind of protection.

Summary: $3 \quad(1=$ Critical, $2=$ Important, $3=$ Cosmetic, $4=$ Documentation, $5=$ None $)$

Evaluator: Steven Humphreys Date: 3/19/96

Disposition:

Scope: _Current _ New

Code: _Revise $\quad$ Defer No Change Required

Documentation: _ User's Guide _ Tech. Desc. _ No Documentation

Comments:

Moderate effort required to change $<1 \mathrm{~d}$ $>1 \mathrm{~h}$

Date: $3 / 19 / 96$ 
RADTRAD $\beta$-Test Discrepancy Report

RADTRAD Version: 1.03

Computer Type and Operating System: Gateway 2000 486DX2/50 MSDOS

Reported By: J.V. Ramsdell

Date: $\quad 12 / 29 / 95$

Organization: PNNL

Telephone: (509) 3726316

Problem or Requested Change: If a software problem is being reported, include a description of the input data that can be used to duplicate the problem. Attach .pmf and .sdf files if possible.

Using the DOS editor from within RADTRAD causes problems... out of memory errors routinely, and occasionally it screws up the cursor when displaying the results.

\section{Evaluation:}

A new browser has been written using the Hi-Screen Pro II features.

Summary: 2 ( 1 = Critical, $2=$ Important, $3=$ Cosmetic, $4=$ Documentation, $5=$ None $)$

Evaluator: Steven Humphreys

Date: $3 / 19 / 96$

\section{Disposition:}

Scope: $\checkmark$ Current

Code: Revise

Documentation: _ User's Guide

Comments:

Corrected.

Date: $3 / 19 / 96$

- New

- Defer

- Tech. Desc.
_ No Change Required

_ No Documentation 


\section{RADTRAD $\beta$-Test Discrepancy Report}

RADTRAD Version: 1.03

Computer Type and Operating System: Gateway 2000 486DX2/50 MSDOS

Reported By: J.V. Ramsdell

Date: $\quad 12 / 29 / 95$

Organization: PNNL

Telephone: (509) 3726316

Problem or Requested Change: If a software problem is being reported, include a description of the input data that can be used to duplicate the problem. Attach .pmf and .sdf files if possible.

In one instance with bad input files, RADTRAD calculated and reported negative doses. Negative doses should not be reported.... If negative doses are calculated they should either be set to zero if they are the result of roundoff error, or the program should print an error message rather than the negative doses.

\section{Evaluation:}

This problem would be solved with better error handling in general. See report \#13.

Summary: $\underline{2} \quad(1=$ Critical, $2=$ Important, $3=$ Cosmetic, $4=$ Documentation, $5=$ None $)$

Evaluator: Steven Humphreys

Date: $3 / 19 / 96$

\section{Disposition:}

Scope: $\_$Current

_ New

Code: $\quad \checkmark$ Revise

_ Defer

_ No Change Required

Documentation:

_ User's Guide

_ Tech. Desc.

_ No Documentation

Comments:

Corrected. See Report \#13

Date: $3 / 19 / 96$ 


\section{RADTRAD -Test Discrepancy Report}

RADTRAD Version: 1.03

Computer Type and Operating System: Gateway 2000 486DX2/50 MSDOS

Reported By: J.V. Ramsdell

Date: $12 / 29 / 95$

Organization: PNNL

Telephone: (509) 3726316

Problem or Requested Change: If a software problem is being reported, include a description of the input data that can be used to duplicate the problem. Attach .pmf and .sdf files if possible.

The output is excessive for normal use... There should be a separate summary table that gives cumulative doses as a function of time. This table should also include a summary of the input data, the code name and version, and the date and time that the code was run.

\section{Evaluation:}

The output has been vastly improved since Beta 1.03 .

Summary: $2 \quad(1=$ Critical, 2 = Important, $3=$ Cosmetic, $4=$ Documentation, $5=$ None $)$

Evaluator: Steven Humphreys Date: 3/19/96

\section{Disposition:}

$\begin{array}{lll}\text { Scope: } & \checkmark \text { Current } & - \text { New } \\ \text { Code: } & \checkmark \text { Revise } & - \text { Defer }\end{array}$

Documentation: _ User's Guide

_ Tech. Desc.
_ No Change Required

_ No Documentation

Comments:

Corrected.

Date: $3 / 19 / 96$ 


\section{RADTRAD $\beta$-Test Discrepancy Report}

RADTRAD Version: $\quad 1.03$

Computer Type and Operating System: Gateway 2000 486DX2/50 MSDOS

Reported By: J.V. Ramsdell

Date: $\quad 1 / 2 / 96$

Organization: PNNL

Telephone:

(509) 3726316

Problem or Requested Change: If a software problem is being reported, include a description of the input data that can be used to duplicate the problem. Attach .pmf and .sdf files if possible.

Selection of the dose factor/nuclide data values should be part of RADTRAD. Jay Lee suggests the following names for the 3 choices:

TID-14844/FGR $11 \& 12$

NUREG/1465/FGR $11 \& 12$

NUREG/1465/ICRP 60

Evaluation:

These are now selected at program startup. Note that the inventory selected for NUREG/1465 has nothing to do with NUREG/1465. It is the MACCS Sample inventory and is therefore referred to as the MACCS inventory.

Summary: $1 \quad(1=$ Critical, $2=$ Important, $3=$ Cosmetic, $4=$ Documentation, $5=$ None $)$

Evaluator: Steven Humphreys

Date: $3 / 19 / 96$

Disposition:
Scope: $\checkmark$ Current
Code: $\quad \checkmark$ Revise
- New
- Defer

Documentation: _ User's Guide

Comments:

Corrected.

Date: $3 / 19 / 96$
_ No Change Required

_ Tech. Desc. _ _ No Documentation 


\section{RADTRAD $\beta$-Test Discrepancy Report}

RADTRAD Version: 1.03

Computer Type and Operating System: Gateway 2000 486DX2/50 MSDOS

Reported By: J.V. Ramsdell

Date: $\quad 1 / 2 / 96$

Organization: PNNL

Telephone: (509) 3726316

Problem or Requested Change: If a software problem is being reported, include a description of the input data that can be used to duplicate the problem. Attach .pmf and .sdf files if possible.

RADTRAD crashes when bwr.nif or pwr.nif file is selected after selecting TID-14844/FGR 11 \& 12 data file. There should be check for consistency in these files in RADTRAD if consistency is required by the program... The program should not crash leaving the user in the dark.

\section{Evaluation:}

I agree and the program should never crash. However, this problem isn't that disastrous. It will occur as soon as the user loads in his or her plant model file. This in itself should be a clear indication of what the problem was.

Furthermore, because it happens immediately no user data has been lost.

Summary: $3 \quad(1=$ Critical, $2=$ Important, $3=$ Cosmetic, $4=$ Documentation, $5=$ None $)$

Evaluator: Steven Humphreys

Date: $3 / 19 / 96$

\section{Disposition:}

Scope: _ Current _ New

Code: _ Revise Defer _ No Change Required

Documentation: _ User's Guide - Tech. Desc. _ No Documentation

\section{Comments:}

Moderate effort to change $<1 \mathrm{~d}$

$$
>1 \mathrm{~h}
$$

Date: $3 / 19 / 96$ 


\section{RADTRAD $\beta$-Test Discrepancy Report}

RADTRAD Version: $\quad 1.03$

Computer Type and Operating System: Gateway 2000 486DX2/50 MSDOS

Reported By: J.V. Ramsdell

Date: $\quad 1 / 2 / 96$

Organization: PNNL

Telephone: (509) 3726316

Problem or Requested Change: If a software problem is being reported, include a description of the input data that can be used to duplicate the problem. Attach .pmf and .sdf files if possible.

Default TID Inventory is selected as default when MACCS BWR/PWR / FGR 11\&12 nuclide file is selected prior to entering RADTRAD. Shouldn't default be the Default BWR or PWR? Similar comment related to the RF\&T file default.

\section{Evaluation:}

Certainly would be helpful.

Summary: $3 \quad(1=$ Critical, $2=$ Important, $3=$ Cosmetic, $4=$ Documentation, $5=$ None $)$

Evaluator: Steven Humphreys

Date: 3/19/96

\section{Disposition:}

$\begin{array}{clll}\text { Scope: } & - \text { Current } & - \text { New } & \\ \text { Code: } & - \text { Revise } & \checkmark \text { Defer } & - \text { No Change Required } \\ \text { Documentation: } & - \text { User's Guide } & - \text { Tech. Desc. } & - \text { No Documentation } \\ \text { Comments: } & & \\ \text { Moderate effort to change }<1 \mathrm{~d} & >1 \mathrm{~h} & \end{array}$

Date: $3 / 19 / 96$ 


\section{RADTRAD $\beta$-Test Discrepancy Report}

RADTRAD Version: $\quad 1.03$

Computer Type and Operating System: Gateway 2000 486DX2/50 MisDOS

Reported By: J.V. Ramsdell

Date: $\quad 1 / 2 / 96$

Organization: PNNL

Telephone: $\quad$ (509) 3726316

Problem or Requested Change: If a software problem is being reported, include a description of the input data that can be used to duplicate the problem. Attach .pmf and .sdf files if possible.

RADTRAD resets transfer rates to zero prior to calculations if a second time entry is not made for the transfer rate. Then RADTRAD crashes without giving user a clue to the problem. Second entries don't seem to be required for other parameters.

\section{Evaluation:}

All user defined inputs now accept a single input line. If the user indeed wants 0 s then he or she does need to input a second line with a non-zero time so that RADTRAD will count the first line.

Summary: 2 (1 = Critical, $2=$ Important, $3=$ Cosmetic, $4=$ Documentation, $5=$ None $)$

Evaluator: Steven Humphreys Date: 3/19/96

\section{Disposition:}

Scope:

Code:

$\checkmark$ Current

Revise

Documentation: _User's Guide

\section{Comments:}

Corrected

Date: $3 / 19 / 96$ 


\section{RADTRAD $\beta$-Test Discrepancy Report}

RADTRAD Version: $\quad 1.03$

Computer Type and Operating System: Gateway 2000 486DX2/50 MSDOS

Reported By: J.V.Ramsdell

Date: $\quad 1 / 2 / 96$

Organization: PNNL

Telephone: $\quad$ (509) 3726316

Problem or Requested Change: If a software problem is being reported, include a description of the input data that can be used to duplicate the problem. Attach .pmf and .sdf files if possible.

The .pmf and .sdf files for TEST99 were initially created to check the effects of not including a second time for the transfer rate. The were then recalled and the data were edited so that the scenario should run. However, it didn't. I have not been able to identify the source of the problem. A more slightly more complex problem (TEST99A) was entered correctly the first time and runs OK. Both sets of .PDF and .SDF files are attached.

\section{Evaluation:}

See \# 23

Summary: 2 ( 1 = Critical, $2=$ Important, $3=$ Cosmetic, $4=$ Documentation, $5=$ None $)$

Evaluator: Steven Humphreys

Date: $3 / 19 / 96$

Disposition:

Scope: $\checkmark \checkmark$ Current - New

Code: $\simeq$ Revise

- Defer

_No Change Required

Documentation: _ User's Guide

_ Tech. Desc. _ No Documentation.

Comments:

Corrected.

Date: 3/19/96 


\section{RADTRAD $\beta$-Test Discrepancy Report}

RADTRAD Version: $\quad 1.03$

Computer Type and Operating System: Gateway 2000 486DX2/50 MSDOS

Reported By: J.V. Ramsdell

Date: $\quad 1 / 2 / 96$

Organization: PNNL

Telephone: $\quad$ (509) 3726316

Problem or Requested Change: If a software problem is being reported, include a description of the input data that can be used to duplicate the problem. Attach .pmf and .sdf files if possible.

User should be warned that after shelling to DOS, the directory must be reset to the subdirectory containing RADTRAD before returning to RADTRAD. Otherwise the program doesn't know what to do and ultimately crashes. Had to reboot the computer to get it to behave properly after this crash.

\section{Evaluation:}

It is unknown at this time what causes this problem but we will try to correct it in the helpfile versions.

Summary: 2 ( 1 = Critical, $2=$ Important, $3=$ Cosmetic, $4=$ Documentation, $5=$ None $)$

Evaluator: Steven Humphreys

Date: $3 / 19 / 96$

\section{Disposition:}

Scope: _ Current

Code: _ Revise

Documentation: _ User's Guide

Comments:
_ No Change Required
_ Tech. Desc. _ No Documentation

Actual time to correct unknown but highly improbable that it would resolve more than $1 \mathrm{~d}$.

Date: $3 / 19 / 96$ 


\section{RADTRAD b-Test Discrepancy Report}

RADTRAD Version: $\quad 1.03$

Computer Type and Operating System: Gateway 2000 P5-90 MSDOS

Reported By: J.V. Ramsdell

Date: $\quad 1 / 10 / 96$

Organization: PNNL

Telephone: $\quad$ (509) 3726316

Problem or Requested Change: If a software problem is being reported, include a description of the input data that can be used to duplicate the problem. Attach .pmf and .sdf files if possible.

The plant model in TEST.PMF is not good... compartments have been dropped, therefore there are pathways for compartments that don't exist. When TEST.PMF and TEST.SDF are used for calculations, RADTRAD stops and displays the message "Compartment Unused has an invalid type of 0 ." There is no response to the OK command button, and the CANCEL button appears to cause the program to try another calculation step. Finally, after several clicks on the CANCEL button, RADTRAD appears to come to a normal completion with the output file displayed in the editor. The results are all zero, but there should be a error exit when a problem occurs rather than having the program appear to run.

\section{Evaluation:}

The test.pmt and test.sdf files are no longer valid with newer versions of RADTRAD. If a user tries to read in such a file, the program gives an error message "invalid file format," and the user is forced to either type in a new file name or escape from the screen.

Summary: $5 \quad(1=$ Critical, $2=$ Important, $3=$ Cosmetic, $4=$ Documentation, $5=$ None $)$

Evaluator: Steven Humphreys $\quad$ Date: 3/25/96

Disposition:

$\begin{array}{ccc}\text { Scope: } & \text { - Current } & - \text { New } \\ \text { Code: } & - \text { Revise } & \end{array}$

Documentation: _ User's Guide _ _ Tech. Desc. _ No Documentation

Comments:

No corrections necessary

Date: $3 / 25 / 96$ 


\section{RADTRAD b-Test Discrepancy Report}

RADTRAD Version: $\quad 1.03$

Computer Type and Operating System: Gateway 2000 P5-90 MSDOS

Reported By: J.V. Ramsdell

Date: $\quad 1 / 19 / 96$

Organization: PNNL

Telephone: $\quad$ (509) 3726316

Problem or Requested Change: If a software problem is being reported, include a description of the input data that can be used to duplicate the problem. Attach .pmf and .sdf files if possible.

Set decay on as default in all compartments.

\section{Evaluation:}

It is not clear whether or not this is desired by NRC. If it is then the change could be easily made. Any action on this discrepancy will have to be deterred until we get word from NRC or their preference.

Summary: $3 \quad(1=$ Critical, $2=$ Important, $3=$ Cosmetic, $4=$ Documentation, $5=$ None $)$

Evaluator: Steven Humphreys Date: $3 / 25 / 96$

\section{Disposition:}

$\begin{array}{rlll}\text { Scope: } & \simeq \text { Current } & - \text { New } & \\ \text { Code: } & \simeq \text { Revise } & - \text { Defer } & - \text { No Change Required } \\ \text { Documentation: } & - \text { User's Guide } & - \text { Tech. Desc. } & - \text { No Documentation }\end{array}$

Comments:

Corrected. If decay is on, it is on for all compartments.

Date: $3 / 25 / 96$ 


\section{RADTRAD b-Test Discrepancy Report}

RADTRAD Version: $\quad 1.03$

Computer Type and Operating System: Gateway 2000 P5-90 MSDOS

Reported By: J.V. Ramsdell

Date: $\quad 1 / 19 / 96$

Organization: PNNL

Telephone: $\quad$ (509) 3726316

Problem or Requested Change: If a software problem is being reported, include a description of the input data that can be used to duplicate the problem. Attach .pmf and .sdf files if possible.

Set daughter ingrowth on as default and add text box indicating that ingrowth will not take place in those compartments where decay is turned off.

\section{Evaluation:}

No changes to the daughter ingrowth default can be made until the NRC determines what they want the default to be. The additional text should be added to the screens regardless of the daughtering default.

Summary: $3 \quad(1=$ Critical, $2=$ Important, $3=$ Cosmetic, $4=$ Documentation, $5=$ None $)$

Evaluator: Steven Humphreys Date: 3/25/96

\section{Disposition:}

$\begin{array}{lll}\text { Scope: } & \checkmark \text { Current } & - \text { New } \\ \text { Code: } & \checkmark \text { Revise } & - \text { Defer }\end{array}$

_ No Change Required

Documentation: _User's Guide_ _ Tech. Desc. _ No Documentation

Comments:

Corrected. If decay and daughtering are on, then they are on for all compartments.

Date: $3 / 25 / 96$ 


\section{RADTRAD b-Test Discrepancy Report}

RADTRAD Version: $\quad 1.03$

Computer Type and Operating System: Gateway 2000 P5-90 MSDOS

Reported By: J.V. Ramsdell

Date: $\quad 1 / 19 / 96$

Organization: PNNL

Telephone:

(509) 3726316

Problem or Requested Change: If a software problem is being reported, include a description of the input data that can be used to duplicate the problem. Attach .pmf and .sdf files if possible.

Add release timing files that do not include late in-vessel and ex-vessel releases. These files should be the primary files used by RADTRAD.

\section{Evaluation:}

These files have been added. they are named as follows:

pwr_dba.rft

bwr_dba.rft

Summary: 2 ( $1=$ Critical, $2=$ Important, $3=$ Cosmetic, $4=$ Documentation, $5=$ None $)$

Evaluator: Steven Humphreys Date: 3/25/96

\section{Disposition:}

\begin{tabular}{|c|c|c|c|}
\hline Scope: & $\checkmark$ Current & - New & \\
\hline Code: & $\checkmark$ Revise & _- Defer & _ No Change Required \\
\hline Ientation: & _User's Guide & _ Tech. Desc. & _No Documentation \\
\hline
\end{tabular}

Date: $3 / 25 / 96$ 


\section{RADTRAD b-Test Discrepancy Report}

RADTRAD Version: $\quad 1.03$

Computer Type and Operating System: Gateway 2000 P5-90 MSDOS

Reported By: J.V. Ramsdell

Date: $\quad 3 / 5 / 96$

Organization: PNNL

Telephone: $\quad$ (509) 3726316

Problem or Requested Change: If a software problem is being reported, include a description of the input data that can be used to duplicate the problem. Attach .pmf and .sdf files if possible.

For dose calculations in the control room, the breathing rate and occupancy factor are combined into a single parameter. Thus, the occupancy factor is only applied to inhalation doses. It is not applied in the calculation of the immersion (deep dose). Consequently doses to control room personnel may be significantly overestimated. This will be the case whenever there is a protracted release of noble gases and when recirculation filters are used to reduce the concentration of iodines and particulates in the control room. Suggest that a default breathing rate of 3.47E-4 be used in the control room and that the occupancy factor be entered by the user.

\section{Evaluation:}

Occupancy rates as well as breathing rates for control room doses may now be entered by the user.

Summary: 1 ( $\quad(1=$ Critical, $2=$ Important, $3=$ Cosmetic, $4=$ Documentation, $5=$ None $)$

Evaluator: Steven Humphreys Date:3/25/96

Disposition:

\begin{tabular}{|c|c|c|c|}
\hline Scope: & $\checkmark$ Current & _ New & \\
\hline Code: & $\checkmark$ Revise & _ Defer & _ No Change Required \\
\hline Documentation: & _User's Guide & - Tech. Desc. & _ No Documentation \\
\hline
\end{tabular}

Date: $3 / 25 / 96$ 


\subsection{RADTRAD Acceptance Test Cases}

\subsubsection{Introduction}

The RADTRAD acceptance test plan was developed cooperatively with the U.S. Nuclear Regulatory Commission, Office of Nuclear Reactor Regulation, Division of Reactor Program Management; Sandia National Laboratories, Department of Accident Analysis and Consequence Assessment, and Pacific Northwest National Laboratory. The competing goals of validating real cases, validating ideal cases, and validating the RADTRAD code were merged through the generation of three types of acceptance cases. The first type validated the ideal cases by comparing RADTRAD solutions with analytic solutions. For this type of comparison, the quantitative acceptance criteria in the dose estimate for the environment was set at less than $1 \%$. In the 4 cases of this type, RADTRAD exceeded the performance criteria. The second type validated many of RADTRAD's features while using reactor/control configurations typical of BWRs, PWRs, and advanced PWRs. For comparison purposes, an enhanced version of the HABIT code [St95] was run and the results compared. For these sets of cases, the quantitative acceptance criteria were typically in the $10-15 \%$ range for doses in the environment and activity in the control room. In the 25 cases of this type, RADTRAD greatly exceed the performance criteria by typically comparing within $1 \%$ for doses in the control room and in the environment. The third type of validation examined the new features incorporated into the RADTRAD code, such as analytic models for spray decontamination and deposition in rooms and pipes. In these cases, there are no comparative results; hence comparison was limited to other cases where either no deposition or user-specified removal rates were used. In these 7 cases the RADTRAD code met qualitative acceptance criteria by following correct trends. This section documents the input and output for the 32 acceptance cases as well as their comparisons with each other and calculated solutions from other techniques. These test cases were developed for the purpose of testing the code and were not intended to represent real licersing situations.

\subsubsection{Acceptance Test Procedure}

As noted above, the selection of suitable acceptance test cases was a cooperative process. In general, the plan was incremental, initially defining simplified test cases that could be verified against analytic solutions, then adding complexity (typically a control room) and comparing the results against the HABIT code analysis, adding more complexity (typically a fixed input radionuclide removal rate) and comparing results with the HABIT code again, and adding yet additional complexity in the form of a model of a removal mechanism and examining the trends. HABIT is a group of codes used to evaluate control room habitability issues that was developed by PNNL. It includes an activity transport code, TACT5, which has been in general use since 1988 , as well as a control room dose evaluation code, CONHAB, that does both an atmospheric dispersion calculation and a dose calculation. The code uses explicit numerical techniques to solve the equations and link the codes together. PNNL supplied the HABIT results.

In a typical situation, for example, stepping from acceptance test case 2 to acceptance test case 3 , the acceptance test team would discuss the input modifications necessary for the case and ensure the elimination of user-induced error. They would try to estimate the expected effect of the 
modifications and perform the test cases. If the dose results did not compare to within a few percent, then the cases were recalculated with limited isotopes, either ${ }^{131} \mathrm{I}$ only or a mix of iodine, krypton, and cesium. This allowed a match of activity by volume and time for each isotope and for each chemical form (aerosol, organic, or elemental) and therefore provided a method to isolate the cause for discrepancies from expected results. Thus each case was brought into agreement with the analytic solution and with the HABIT results. After each set of cases was tested, the doses were then examined for case-to-case trends. In progressing through more complex cases, a difference in the air submersion calculation within the control room was determined to be more important than originally anticipated and corrective action was taken.

Table 3.3.1.1-1 is the original design for the acceptance plan. The sequence of acceptance tests is based on three reactors: a PWR, a BWR, and an advanced PWR. As the sequence steps from left to right, additional complexity is added. As the sequence steps from top to bottom, either a different reactor design is specified or the radionuclide release to the containment is changed. Of the 24 cases listed, only cases 17 and 18 are not reported here. These two cases were eliminated because it was concluded that they would not give significantly more information than other test cases due to the insignificant amount of pipe deposition. In addition to the resulting 22 cases, 10 perturbations were introduced to isolate particular effects, such as decay and daughtering, or a particular deposition mechanism.

The acceptance test plan was meant to assess:

- The source term models, that is, the radionuclide release fractions and timings, and the inventory, decay, and daughtering for both the TID-14844 and the NUREG-1465 models.

- The dose calculations due to cloudshine and inhalation in both the environment and in the control room.

- Nuclide transport due to forced convection in the environment.

- Pathway removal models and tables for pipes and filters.

- Compartment (containment, environment, control room) removal models and tables for sprays, recirculation filters, and natural deposition.

The rationale for the acceptance test cases for this part of the assessment of the RADTRAD code is:

- Cases 1-10 use a PWR design (block 1 of Table 3.3.1.1-1).

- Test case 1 was developed to verify that a puff release of ${ }^{131} \mathrm{I}$ would leak from the containment correctly and match the analytic solution.

- Test case 2 was developed to verify that a puff release of the entire TID source term (I, Xe, and $\mathrm{Kr}$ ) would leak from the containment correctly and match the analytic solution. 
- Test case 2 a was developed to verify that a puff release of the entire TID source term would radioactively decay correctly.

- Test case $2 b$ was developed to verify that a puff release of the entire TID source term would radioactively decay and daughter correctly.

- Test case 3 was developed to verify that a control room could be added to the calculation and give the same dose results in the environment as case 2 , and to add the control room dose calculation.

- Test case 4 was developed to verify the effect of natural deposition in the containment using the TID source term. Many believed that the $50 \%$ deposition assumption was overly optimistic.

- Test case 5 was developed to see the effect of using Henry's natural deposition correlation with the TID source term.

- Test case 6 was developed to verify that a timed release of ${ }^{131}$ I would leak from the containment correctly and match the analytic solution.

- Test case 7 was developed to verify that a timed release of the entire NUREG-1465 source term for PWRs would leak from the containment correctly and match the analytic solution.

- Test case 7a was developed to verify that a timed release of the entire NUREG-1465 source term for PWRs would radioactively decay correctly.

- Test case $7 \mathrm{~b}$ was developed to verify that a timed release of the entire NUREG-1465 source term for BWRs would leak from the containment correctly.

- Test case 7c was developed to verify that a timed release of the entire NUREG-1465 source term for BWRs would radioactively decay correctly.

- Test case 7d was developed to verify that a timed release of the entire NUREG-1465 source term for PWRs would radioactively decay correctly for $1.0 \mathrm{hr}$ before release.

- Test case 8 was developed to verify that a control room could be added to the calculation and give the same dose results in the environment as case 7 , and to add the control room dose calculation.

- Test case 9 was developed to show the effect of natural deposition in the containment using the NUREG-1465 source term.

- Test case 10 was developed to see the effect of using the Powers model for natural deposition in the containment when using the NUREG-1465 source term.

- Test case 10a was developed to see the effect of using Henry's correlation for natural deposition in the containment when using the NUREG-1465 source term. 
- Cases 11-18 use a BWR design (block 2 of Table 3.3.1.1-1)

- Test case 11 was developed to examine the BWR containment and a leak path that passed through the main steam isolation valve before going to the environment. The use of the TID ${ }^{131}$ I isotope for a puff source made the calculation easier to analyze.

- Test case 12 was developed to verify that the addition of a control room would not change the environmental doses and to add the control room dose calculation. The use of the TID ${ }^{131} I$ isotope for a puff source made the calculation easier to analyze.

- Test case 13 was developed to examine pipe deposition and to verify that the results of a more detailed model for pipe deposition could be implemented into RADTRAD. In this case the Cline model [Cl91] was chosen. The model calculated that the iodine that was released in both aerosol and elemental form was totally trapped within the pipe and condenser network; hence the RADTRAD code used large decontamination factors for these two release forms. The model also calculated that the organic iodine increased as the calculation proceeded. Thus the RADTRAD input was adjusted to yield the same results by increasing the organic fraction to the values needed to yield the final value ( $7.4 \%$ instead of $4 \%$ ), and the pipe decontamination factor table for organics had larger values at the earlier times. The use of the TID ${ }^{131} I$ isotope for a puff source made the calculation easier to analyze.

- Test case $13 \mathrm{~b}$ was developed to verify that the NUREG-1465 timed $\left({ }^{131} \mathrm{I}\right)$ source release could also be used.

- Test case 14 was developed to show the effect of using the RADTRAD pipe deposition model with the TID $\left({ }^{131} \mathrm{I}\right)$ puff source term.

- Test case $14 \mathrm{~b}$ was developed to show the effect of using the RADTRAD pipe deposition model with the NUREG-1465 $\left({ }^{131} \mathrm{I}\right)$ timed source term.

- Test case 15 was developed to examine the BWR containment and a leak path that passed through the emergency core cooling system and auxiliary building before exiting to the environment. The NUREG- $1465{ }^{131}$ I timed source term was used to make the results easier to analyze.

- Test case 16 was developed to verify that the addition of a control room would have no effect upon the environment doses and to add the control room dose calculation.

- Cases 19-24 use an advanced PWR design (block 3 of Table 3.3.1.1-1).

- Test case 19 was developed to examine an advanced PWR design. In this case containment mixing was modeled with multiple leaks to the environment. The use of the NUREG-1465 ${ }^{131} \mathrm{I}$ isotope for a timed source made the calculation easier to analyze.

- Test case 20 was developed to examine the use of the full NUREG-1465 source with the case 19 assumptions. 
- Test case 21 was developed to verify that the addition of a control room would have no effect upon the environment doses and to add the control room dose calculation.

- Test case 22 was developed to examine the effect of natural deposition in the multi-zoned containment.

- Test case 23 was developed to examine the effect of a spray removal coefficient.

- Test case 24 was developed to examine the effect of a spray removal model.

- Test case 24a was developed to examine the effect of aerosol addition to the source term on the spray removal model.

\subsubsection{Acceptance Test Results}

Tables 3.3.1.2-1 through 3.3.1.2-5 give the results for the 32 acceptance test cases compared with the HABIT results. As shown, there is less than a $1 \%$ difference in the dose calculated by the two codes both for locations in the environment and in the control room. The tables also indicate that the isotopic masses were within the test limits for those HABIT runs where activity results were available.

Table 3.3.1.2-1 delineates the PWR cases. Cases 1 through 5 use the TID source term, and cases 6 through 10A use the NUREG-1465 source term. In general, stepping away from the first row in each section increases the calculated dose as more of the source term is included. In stepping from the second to the third row, the inclusion of the control room allows a new location for a dose calculation. Note that there is no effect on the environment dose results. Stepping from. row three to four in each section, the user-specified natural deposition in the containment is added, causing the dose to go down as radionuclides are deposited within the containment. Note, however, that in progressing from case 3 to 4 the doses increase. This is because the TID source term release of $50 \%$ of the iodine inventory to the containment also assumes that $50 \%$ of the released iodine will be deposited on the surfaces. Because natural deposition was the focus of case 4 , the entire $50 \%$ was left in the containment atmosphere. Stepping from row four to row five (or six) invokes the use of the model instead of the user-supplied deposition rate. In the comparison of cases 4 and 5 , this led to an increase in dose clue to the inappropriate use of the model. These results indicate the incompatibility of using the TID source term with the new correlations because the TID assumptions on iodine distribution cause logical disconnects. In the comparison of case 9 with both 10 and 10A, where the Powers model [Po95] and the Henry correlation [Fa84] were in use, the results indicate that both methods give answers similar to an assumed deposition rate of $15 \%$ per hour. This indicates that the methods could be used to estimate deposition rates when insufficient data were available to the user.

Table 3.3.1.2-2 delineates the BWR cases. For acceptance testing, all of these cases are limited to a release of ${ }^{131} \mathrm{I}$ from the reactor to the containment. The differences between the TID and the NUREG releases are in magnitude, chemical form, and timing. Cases 11 through 14 used the TID source term to examine the case of a main steam isolation valve (MSIV) leak to the environment. These cases are modeled as a containment leak to the condenser followed by a 
pipe leak to the environment. Case 11 shows that the two codes gave similar leaks to the environment. Case 12 shows that the addition of a control room had no effect upon the environment dose results and that the dose to the control room was similar. Case 13 included the deposition specified by the Cline model [C191], which calculates total deposition of the dominant aerosol and elemental iodine forms due to the condenser's large surface area. Thus, the environmental dose is greatly reduced. However, the control room dose has increased; this is because the control filters also eliminate elemental and aerosol chemical forms. Thus, the significant component of the control room dose is the state of the organics. In the Cline model, elemental iodine is transformed into organic iodine and released; hence the organic iodine form increases in the environment and in the control room. Case 14 includes the effect of the Brockmann model for deposition of aerosols as well as the Cline correlation for organic and elemental deposition. Because the TID source term is dominated by the release of iodine in its elemental form, aerosol deposition differences were masked. As in the comparison between the Cline model and the base case, the state of the organic iodine is the controlling feature. Cases $13 \mathrm{~B}$ and 14B were introduced to examine the effect of using the NUREG-1465 source term, where the aerosols make up $95 \%$ of the released iodine, on the Cline and Brockmann deposition models. A direct comparison between the TID and NUREG-1465 results is not reasonable because the TID assumption on iodine deposition in the containment makes the released quantities dramatically different. Note that case 14B still has lower doses than case $13 \mathrm{~B}$ and the organic iodine is still controlling, thus indicating the importance of reevolution. Cases 15 and 16 examine an emergency core cooling system (ECCS) leak to an auxiliary building followed by a leak to the environment. As the table indicates, the codes calculated essentially the same answers for all these cases.

Table 3.3.1.2-3 delineates the advanced PWR cases. All of these cases use the source term of NUREG-1465. The advanced PWR required more compartments. Case 22 added a specified removal rate due to natural deposition within the containment, resulting in aerosol deposition and a reduction dose. Cases 23 and 24 examine containment sprays. Case 23 utilized a userprovided coefficient removal whereas case 24 invoked a spray model developed by Powers and Burson [Po93]. The results indicate that the spray model yields results similar to the userprovided removal rate. In case 24A, additional aerosol material was added to the release to the containment to determine the effect upon spray deposition; as the model suggests, there were negligible changes.

Table 3.3.1.2-4 delineates a series of cases used to examine radionuclide decay and daughtering. In these cases, the TID source, as shown in Section 1.4.3, was examined in cases 2, 2A, and 2B. The PWR NUREG-1465 source term (see Section 1.4.3) limited to the design basis accident assumptions was examined in cases 7 and 7A. The full BWR NUREG-1465 source term was examined in cases 7B and 7C. Both codes follow the anticipated trends, i.e., the inclusion of decay allows some of the released material to decay and be removed from the calculation and therefore reduce the total containment activity. Note in Table 3.3.1.2-4 that iodine-131 abundance has decreased, the xenon-135 has disappeared due to its short half-life, and the xenon131 has also decreased. When daughtering is also included, the quantity of xenon-131 increases due to the decay and daughtering of the iodine-131. Cases 7 and 7A show the same effect due to the inclusion of isotopic decay for the PWR source term and cases $7 \mathrm{~B}$ and $7 \mathrm{C}$ for the BWR source term. Case 7D assumes a 10-hr delay between the time the release starts and the time the 
reactor was shut down (scrammed). It shows the effect of a decay before release and should be compared with case 7A. In all these cases, the comparison with HABIT with respect to dose and activity is within $1 \%$.

Table 3.3.1.2-5 shows the results for the analytic comparison. The cases are simplistic releases from the containment to the atmosphere. The RADTRAD and HABIT codes give the same answer. In the first two cases where the TID source term is uised, the first line contains the calculated results and the second the analytic solution. For cases 6 and 7 where the NUREG1465 source term is used, the first line contains the calculated results for a design basis accident, i.e., only the first two releases of the NUREG-1465 scenario. This line should only be used to compare RADTRAD and HABIT. The following two lines give the calculated and analytic solution using the draft NUREG-1465 four-release severe accident scenario. As anticipated, the severe accident releases more than the design basis accident; hence the LPZ doses are higher (row 2 is larger than row 1). Note that the EAB time frame is only the first $2 \mathrm{hr}$ and the time frame of the design basis accident is $1.8 \mathrm{hr}$; hence the $\mathrm{EAB}$ doses (the first four columns) are very similar.

\subsubsection{Acceptance Case General Input}

All acceptance test cases estimated air submersion and inhalation doses at offsite locations. Most acceptance test cases also estimated doses in the control room. All doses are estimated using Federal Guidance Reports 11 and 12 (FGR 11 and 12) dose conversion factors (DCFs) for the following organs and pseudoorgans:

- Gonads

- Breast

- Lungs

- Red bone marrow

- Bone surface

- Thyroid

- Skin

- Effective dose equivalent

Remainder

In addition, the following derived doses are calculated:

- Whole body (effective air submersion dose)

- Thyroid (thyroid chronic inhalation dose)

- TEDE (effective air submersion dose + effective committed effective dose equivalent) 
All acceptance test cases specified the following standard breathing rates, as defined in NRC Regulatory Guides 1.3 and 1.4 for the EAB and LPZ. [Note: the table below indicates that from 0.0000 to $8.0000 \mathrm{hr}$ the breathing rate would be $0.3470 \mathrm{E}-03 \mathrm{~m}^{3} / \mathrm{s}$; from $8.0000 \mathrm{~h}$ to $24.0000 \mathrm{hr}$, at would be $0.1750 \mathrm{E}-03 \mathrm{~m}^{3} / \mathrm{s}$; and from $24.0000 \mathrm{hr}$ to the end of the test case ( 30 days), the breathing rate would be $0.2320 \mathrm{E}-03 \mathrm{~m}^{3} / \mathrm{s}$ ]:

$$
\begin{array}{ll}
0.0000 \mathrm{hr} & 0.3470 \mathrm{E}-03\left(\mathrm{~m}^{3} / \mathrm{s}\right) \\
8.0000 \mathrm{hr} & 0.1750 \mathrm{E}-03\left(\mathrm{~m}^{3} / \mathrm{s}\right) \\
24.0000 \mathrm{hr} & 0.2320 \mathrm{E}-03\left(\mathrm{~m}^{3} / \mathrm{s}\right)
\end{array}
$$

All acceptance test cases specified the breathing rate as $0.3470 \mathrm{E}-03 \mathrm{~m}^{3} / \mathrm{s}$ for the control room. All acceptance test cases use the following occupancy factors, as defined in Section 6.4 of the Murphy-Campe[Mu74] report for the control room operator:

$$
\begin{array}{ll}
0.0000 \mathrm{hr} & 1.0000 \\
24.0000 \mathrm{hr} & 0.6000 \\
96.0000 \mathrm{hr} & 0.4000
\end{array}
$$

All acceptance test cases will end at $720.0000 \mathrm{hr}$ ( 30 days).

Note: Throughout this page and the acceptance test cases, the numeric values given are presented in the same format in which they will appear on the RADTRAD user interface screens and printouts. The format should not be taken as an indicator of numeric precision or the number of available significant digits. 
Table 3.3.1.1-1 Acceptance test plan

Reactor: PWR

\begin{tabular}{|l|c|c|c|c|c|}
\hline $\begin{array}{l}\text { Scenario \& } \\
\text { Source Term }\end{array}$ & A. ${ }^{131}$ I Only & $\begin{array}{c}\text { B. Full Source } \\
\text { Term }\end{array}$ & $\begin{array}{c}\text { C. Column B } \\
\text { plus control } \\
\text { room }\end{array}$ & $\begin{array}{c}\text { D. Column C plus user- } \\
\text { specified natural } \\
\text { deposition }\end{array}$ & $\begin{array}{c}\text { E. Column C plus } \\
\text { natural deposition } \\
\text { models }\end{array}$ \\
\hline $\begin{array}{l}\text { Containment leak } \\
\text { TID-14844 }\end{array}$ & Test Case 1 & Test Case $2^{1}$ & Test Case 3 & Test Case 4 & Test Case 5 \\
\hline $\begin{array}{l}\text { Containment leak } \\
\text { NUREG-1465/PWR }\end{array}$ & Test Case 6 & Test Case $7^{1}$ & Test Case 8 & Test Case 9 & Test Case 10 \\
\hline
\end{tabular}

Reactor: BWR

\begin{tabular}{|l|c|c|c|c|c|}
\hline $\begin{array}{l}\text { Scenario \& } \\
\text { Source Term } \\
\text { I131 only }\end{array}$ & A. ${ }^{131}$ I Only & $\begin{array}{c}\text { B. Full Source } \\
\text { Term (not } \\
\text { used) }\end{array}$ & $\begin{array}{c}\text { C. Column A } \\
\text { plus control } \\
\text { room }\end{array}$ & $\begin{array}{c}\text { D. Column C plus user- } \\
\text { specified pipe } \\
\text { deposition }\end{array}$ & $\begin{array}{c}\text { E. Column C plus } \\
\text { pipe deposition } \\
\text { models }\end{array}$ \\
\hline $\begin{array}{l}\text { MSIV leak } \\
\text { TID-14844 }\end{array}$ & $\begin{array}{c}\text { Test Case } \\
11\end{array}$ & & Test Case 12 & Test Case 13 & Test Case 14 \\
\hline $\begin{array}{l}\text { ECCS leak } \\
\text { NUREG-1465/BWR }\end{array}$ & $\begin{array}{c}\text { Test Case } \\
15\end{array}$ & & Test Case 16 & Test Case 17 & Test Case $18^{2}$ \\
\hline
\end{tabular}

Reactor: Advanced PWR

\begin{tabular}{|l|c|c|c|c|c|}
\hline $\begin{array}{l}\text { Scenario \& } \\
\text { Source Term }\end{array}$ & A. ${ }^{131}$ I Only & $\begin{array}{c}\text { B. Full Source } \\
\text { Term }\end{array}$ & $\begin{array}{c}\text { C. Column B } \\
\text { plus control } \\
\text { room }\end{array}$ & $\begin{array}{c}\text { D. Column C plus user- } \\
\text { specified natural } \\
\text { deposition }\end{array}$ & $\begin{array}{c}\text { E. Column D plus } \\
\text { user sprays, and } \\
\text { spray model }\end{array}$ \\
\hline NUREG-1465/PWR & $\begin{array}{c}\text { Test Case } \\
19\end{array}$ & Test Case 20 & Test Case 21 & Test Case 22 & Test Cases 23-24 \\
\hline
\end{tabular}

$\sqrt{\text { These cases were also used to evaluate decay and daughtering capabilities }}$

${ }^{2}$ Acceptance test cases 17 and 18 have been eliminated from the acceptance matrix. 
Table 3.3.1.2-1 PWR--Control room and natural deposition tests

\begin{tabular}{|c|c|c|c|c|c|c|c|c|c|}
\hline \multirow{3}{*}{$\frac{\text { Test Case }}{\text { TID-14844 }}$} & \multirow{3}{*}{ CODE } & \multirow{2}{*}{\multicolumn{2}{|c|}{$\begin{array}{c}\begin{array}{c}\text { Exclusion } \\
\text { Area Boundary }\end{array} \\
\text { Doses (rem) }\end{array}$}} & \multirow{2}{*}{\multicolumn{2}{|c|}{$\begin{array}{c}\begin{array}{c}\text { Low Population } \\
\text { Zone }\end{array} \\
\text { Doses (rem) } \\
\end{array}$}} & \multirow{2}{*}{\multicolumn{2}{|c|}{$\frac{\text { Control Room }}{\text { Doses (rem) }}$}} & \multirow{2}{*}{\multicolumn{2}{|c|}{$\begin{array}{c}\begin{array}{c}\text { Control Room } \\
\text { Activity }\end{array} \\
\text { (curie) }\end{array}$}} \\
\hline & & & & & & & & & \\
\hline & & Thyroid & TEDE & Thyroid & TEDE & Thyroid & TEDE & 131 & ${ }^{135} \mathrm{Xe}$ \\
\hline 7 & $\begin{array}{l}\text { RADTRAD } \\
\text { HABIT }\end{array}$ & $\begin{array}{l}443 \\
443 \\
\end{array}$ & $\begin{array}{l}13.6 \\
13.6 \\
\end{array}$ & $\begin{array}{l}2992 \\
2992 \\
\end{array}$ & $\begin{array}{l}91.9 \\
91.9 \\
\end{array}$ & & & & \\
\hline 2 & $\begin{array}{c}\text { RADTRAD } \\
\text { HABIT }\end{array}$ & $\begin{array}{l}652 \\
652 \\
\end{array}$ & $\begin{array}{l}28.7 \\
28.7 \\
\end{array}$ & $\begin{array}{l}4400 \\
4400 \\
\end{array}$ & $\begin{array}{l}220 \\
220 \\
\end{array}$ & & & & \\
\hline 3 & $\begin{array}{l}\text { RADTRAD } \\
\text { HABIT }\end{array}$ & $\begin{array}{l}652 \\
653 \\
\end{array}$ & $\begin{array}{l}28.8 \\
28.8 \\
\end{array}$ & $\begin{array}{l}4400 \\
4400 \\
\end{array}$ & $\begin{array}{l}220 \\
220 \\
\end{array}$ & $\begin{array}{l}2660 \\
2650 \\
\end{array}$ & $\begin{array}{l}95.5 \\
95.1 \\
\end{array}$ & $\begin{array}{l}3.950 \mathrm{E}-03 \\
4.000 \mathrm{E}-03\end{array}$ & 0.75 \\
\hline 4 & $\begin{array}{c}\text { RADTRAD } \\
\text { HABIT }\end{array}$ & $\begin{array}{l}1130 \\
1130\end{array}$ & $\begin{array}{l}47.5 \\
47.6 \\
\end{array}$ & $\begin{array}{l}809 \\
808 \\
\end{array}$ & $\begin{array}{l}66.9 \\
66.8 \\
\end{array}$ & $\begin{array}{l}4940 \\
4920\end{array}$ & $\begin{array}{l}169 \\
168 \\
\end{array}$ & $\begin{array}{l}7.320 \mathrm{E}-03 \\
7.420 \mathrm{E}-03\end{array}$ & 0.75 \\
\hline 5 & RADTRAD & 1300 & 54.1 & 8470 & 392 & 5300 & 181 & $7.870 \mathrm{E}-03$ & .75 \\
\hline \multicolumn{10}{|c|}{ NUREG-1465/PWR } \\
\hline 6 & $\begin{array}{l}\text { RADTRAD } \\
\text { HABIT }\end{array}$ & $\begin{array}{l}354 \\
354 \\
\end{array}$ & $\begin{array}{l}10.9 \\
10.8 \\
\end{array}$ & $\begin{array}{l}4905 \\
4905 \\
\end{array}$ & $\begin{array}{l}151 \\
151 \\
\end{array}$ & & & & \\
\hline 7 & $\begin{array}{c}\text { RADTRAD } \\
\text { HABIT }\end{array}$ & $\begin{array}{l}518 \\
518 \\
\end{array}$ & $\begin{array}{l}25.3 \\
25.3 \\
\end{array}$ & $\begin{array}{l}7200 \\
7200 \\
\end{array}$ & $\begin{array}{l}382 \\
382 \\
\end{array}$ & & & & \\
\hline 8 & $\begin{array}{l}\text { RADTRAD } \\
\text { HABIT }\end{array}$ & $\begin{array}{l}518 \\
518\end{array}$ & $\begin{array}{l}25.3 \\
25.3 \\
\end{array}$ & $\begin{array}{l}7200 \\
7200 \\
\end{array}$ & $\begin{array}{l}382 \\
382 \\
\end{array}$ & $\begin{array}{l}481 \\
479 \\
\end{array}$ & $\begin{array}{l}25.7 \\
25.6 \\
\end{array}$ & $7.310 \mathrm{E}-04$ & 0.15 \\
\hline 9 & $\begin{array}{c}\text { RADTRAD } \\
\text { HABIT }\end{array}$ & $\begin{array}{l}477 \\
477\end{array}$ & $\begin{array}{l}23.4 \\
23.4\end{array}$ & $\begin{array}{l}707 \\
707\end{array}$ & $\begin{array}{l}54.4 \\
54.3\end{array}$ & $\begin{array}{l}179 \\
178\end{array}$ & $\begin{array}{l}12.7 \\
12.6\end{array}$ & $\begin{array}{l}2.520 \mathrm{E}-04 \\
2.550 \mathrm{E}-04\end{array}$ & 0.15 \\
\hline 10 & RADTRAD & 509 & 24.9 & 837 & 60.9 & 185.5 & 13 & $2.520 \mathrm{E}-04$ & 0.15 \\
\hline $10 \mathrm{~A}$ & RADTRAD & 474 & 23.2 & 690 & 53.6 & 179 & 12.7 & $2.520 \mathrm{E}-04$ & 0.15 \\
\hline
\end{tabular}


Table 3.3.1.2-2 BWR--Control room and pipe deposition tests

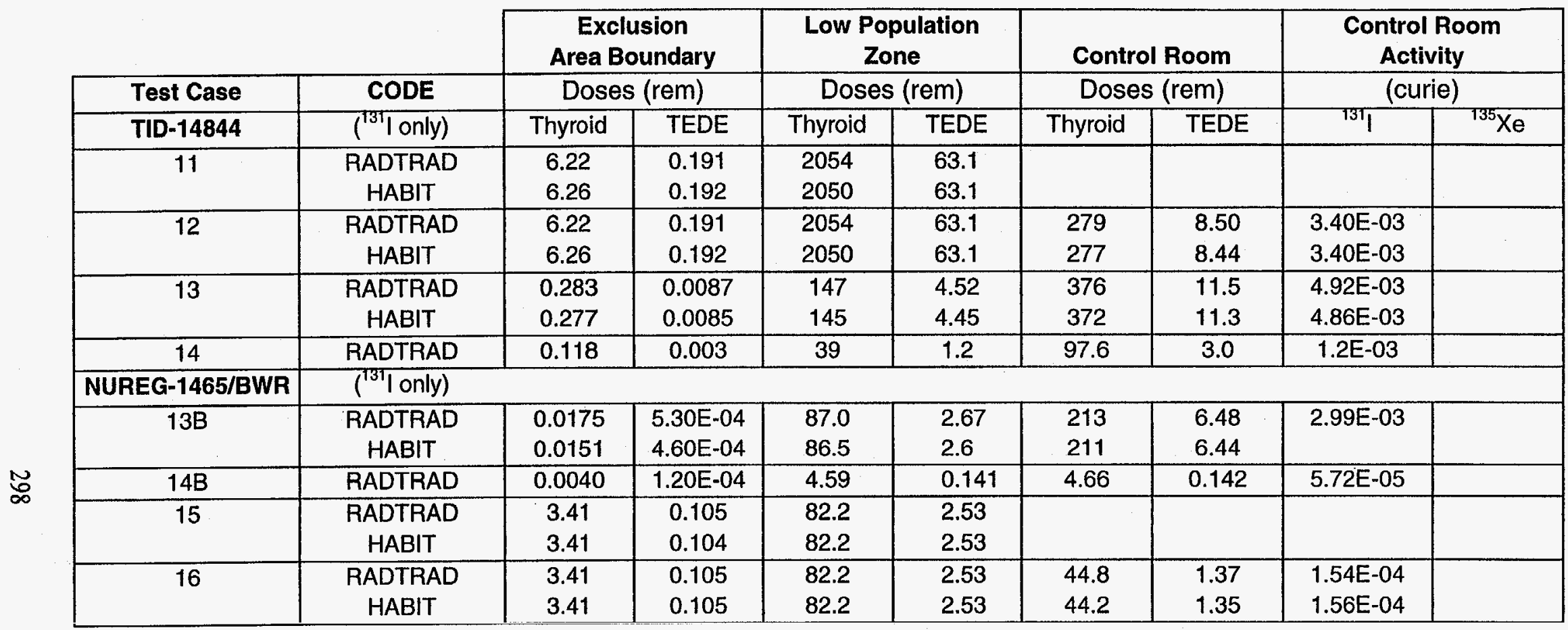


Table 3.3.1.2-3 PWR--Natural deposition and sprays tests

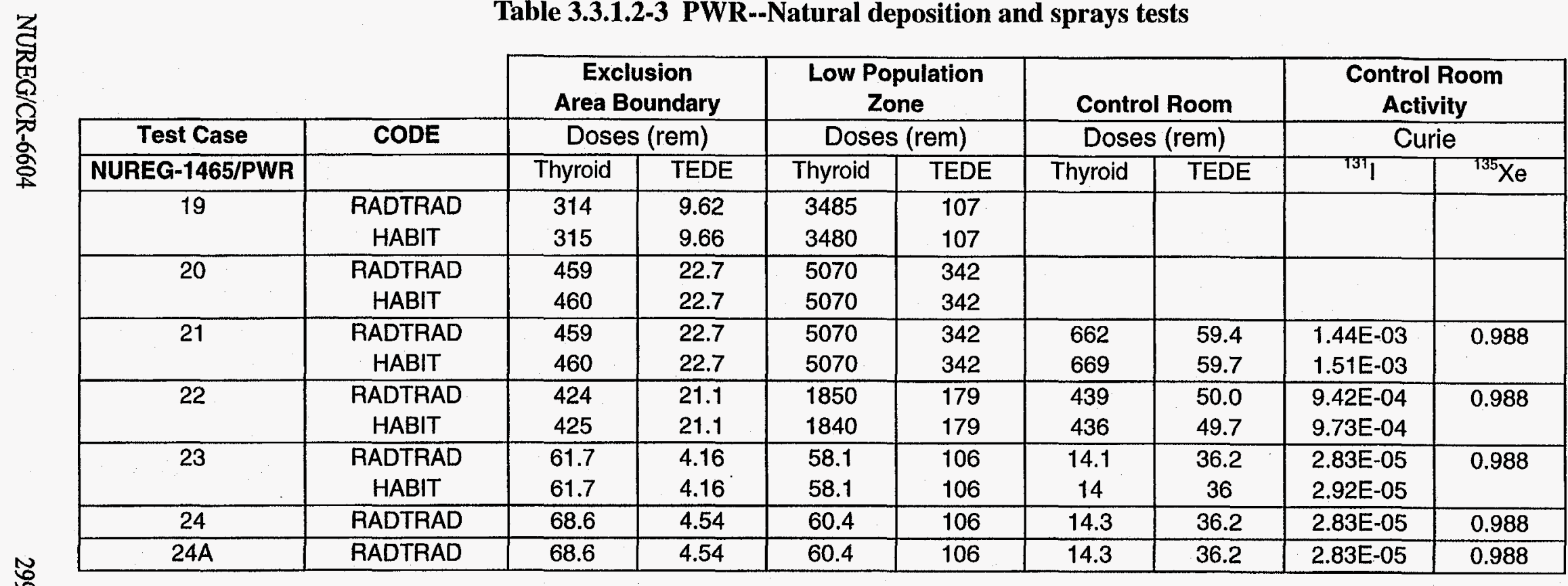


Table 3.3.1.2-4 PWR and BWR decay and daughtering tests

\begin{tabular}{|c|c|c|c|c|c|c|c|c|}
\hline & & \multirow{2}{*}{\multicolumn{2}{|c|}{$\begin{array}{c}\begin{array}{c}\text { Exclusion } \\
\text { Area Boundary }\end{array} \\
\text { Dose (rem) }\end{array}$}} & \multirow{2}{*}{\multicolumn{2}{|c|}{$\begin{array}{c}\begin{array}{c}\text { Low Population } \\
\text { Zone }\end{array} \\
\text { Dose (rem) }\end{array}$}} & \multirow{2}{*}{\multicolumn{3}{|c|}{$\frac{\text { Containment Activity }}{\text { (Curie) }}$}} \\
\hline Test Case & Code & & & & & & & \\
\hline TID-14844 & & Thyroid & TEDE & Thyroid & TEDE & $\sqrt[131]{1}$ & ${ }^{135} \mathrm{Xe}$ & ${ }^{131} \mathrm{Xe}$ \\
\hline 1 & $\begin{array}{l}\text { RADTRAD } \\
\text { HABIT }\end{array}$ & $\begin{array}{l}443 \\
443\end{array}$ & $\begin{array}{l}13.6 \\
13.6\end{array}$ & $\begin{array}{l}2992 \\
2992\end{array}$ & $\begin{array}{l}91.9 \\
91.9\end{array}$ & $\begin{array}{l}1.140 \mathrm{E}+07 \\
1.140 \mathrm{E}+07\end{array}$ & & \\
\hline 2 & $\begin{array}{c}\text { RADTRAD } \\
\text { HABIT }\end{array}$ & $\begin{array}{l}652 \\
652 \\
\end{array}$ & $\begin{array}{l}28.7 \\
28.8\end{array}$ & $\begin{array}{l}4400 \\
4400\end{array}$ & $\begin{array}{l}220 \\
220 \\
\end{array}$ & $\begin{array}{l}1.140 \mathrm{E}+07 \\
1.140 \mathrm{E}+07 \\
\end{array}$ & $\begin{array}{l}9.37 E+07 \\
9.37 E+07\end{array}$ & $\begin{array}{l}4.64 E+05 \\
4.64 E+05\end{array}$ \\
\hline $2 A$ & $\begin{array}{c}\text { RADTRAD } \\
\text { HABIT }\end{array}$ & $\begin{array}{l}640 \\
640 \\
\end{array}$ & \begin{tabular}{l|}
26.4 \\
26.2 \\
\end{tabular} & $\begin{array}{l}1625 \\
1610 \\
\end{array}$ & \begin{tabular}{l|}
53.1 \\
52.6 \\
\end{tabular} & $\begin{array}{l}8.600 E+05 \\
8.600 E+05\end{array}$ & $\begin{array}{r}0 \\
0 \\
\end{array}$ & $\begin{array}{l}8.07 E+04 \\
8.81 E+04\end{array}$ \\
\hline $2 B$ & RADTRAD & 640 & 26.4 & 1625 & 53.2 & $8.600 E+05$ & 0 & $1.07 E+05$ \\
\hline NUREG-1465/PWR & & & & & & 7311 & ${ }^{135} \mathrm{Xe}$ & ${ }^{137} \mathrm{Cs}$ \\
\hline 6 & $\begin{array}{l}\text { RADTRAD } \\
\text { HABIT }\end{array}$ & $\begin{array}{l}354 \\
354\end{array}$ & \begin{tabular}{|l|}
10.9 \\
10.8
\end{tabular} & $\begin{array}{l}4905 \\
4905\end{array}$ & $\begin{array}{l}151 \\
151\end{array}$ & $3.551 E+07$ & & \\
\hline 7 & $\begin{array}{c}\text { RADTRAD } \\
\text { HABIT }\end{array}$ & $\begin{array}{l}518 \\
518 \\
\end{array}$ & \begin{tabular}{l|}
25.3 \\
25.3 \\
\end{tabular} & $\begin{array}{l}7200 \\
7200 \\
\end{array}$ & \begin{tabular}{l|}
382 \\
382 \\
\end{tabular} & $1.89 E+07$ & $1.88 E+07$ & \\
\hline$\overline{7 A}$ & $\begin{array}{c}\text { RADTRAD } \\
\text { HABIT }\end{array}$ & $\begin{array}{l}507 \\
505 \\
\end{array}$ & \begin{tabular}{l|}
23.6 \\
23.4 \\
\end{tabular} & \begin{tabular}{l|}
2680 \\
2660 \\
\end{tabular} & $\begin{array}{l}157 \\
156 \\
\end{array}$ & $1.43 E+06$ & 0 & $1.07 \mathrm{E}+06$ \\
\hline NUREG-1465/BWR & & & & & & & & \\
\hline $7 \mathrm{~B}$ & $\begin{array}{l}\text { RADTRAD } \\
\text { HABIT }\end{array}$ & $\begin{array}{l}378 \\
378 \\
\end{array}$ & $\begin{array}{l}20.2 \\
20.2 \\
\end{array}$ & $\begin{array}{l}5530 \\
5530 \\
\end{array}$ & $\begin{array}{l}327 \\
326 \\
\end{array}$ & $1.44 \mathrm{E}+07$ & $2.40 \mathrm{E}+07$ & $1.18 \mathrm{E}+06$ \\
\hline $7 \mathrm{C}$ & $\begin{array}{l}\text { RADTRAD } \\
\text { HABIT }\end{array}$ & $\begin{array}{l}370 \\
369 \\
\end{array}$ & $\begin{array}{l}18.9 \\
18.7 \\
\end{array}$ & $\begin{array}{l}2060 \\
2040 \\
\end{array}$ & $\begin{array}{l}148 \\
147 \\
\end{array}$ & $1.09 E+06$ & 0 & $1.18 \mathrm{E}+06$ \\
\hline NUREG-1465/PWR & & & & & & & & \\
\hline $7 D$ & RADTRAD & 446 & 19.6 & 2510 & 150 & $1.38 \mathrm{E}+06$ & & \\
\hline & HABIT & 444 & 19.5 & 2500 & 150 & 1.37E+06 & 0 & \\
\hline
\end{tabular}




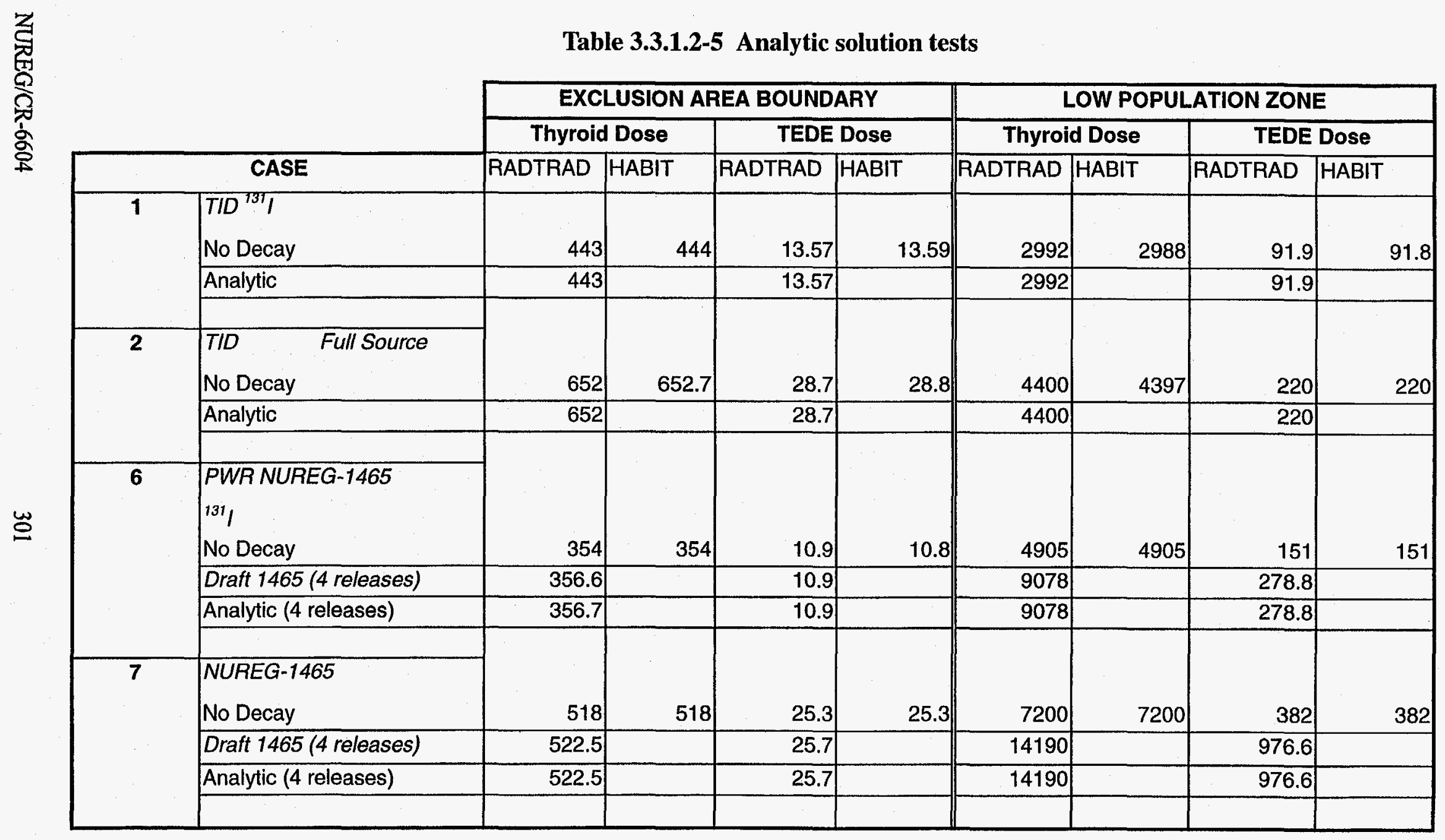




\subsubsection{Acceptance Test Case 1}

\section{Problem Description}

PWR design (see Figure 1).

Instantaneous, modified TID-14844 $\left({ }^{131}\right.$ I only) release into containment.

Release begins at reactor shutdown.

No explicit calculation of removal mechanisms in the containment, but $50 \%$ of the released iodine is deposited in accordance with TID-14844.

Containment leak to the environment.

$\mathrm{EAB}$ and $\mathrm{LPZ}$ doses calculated.

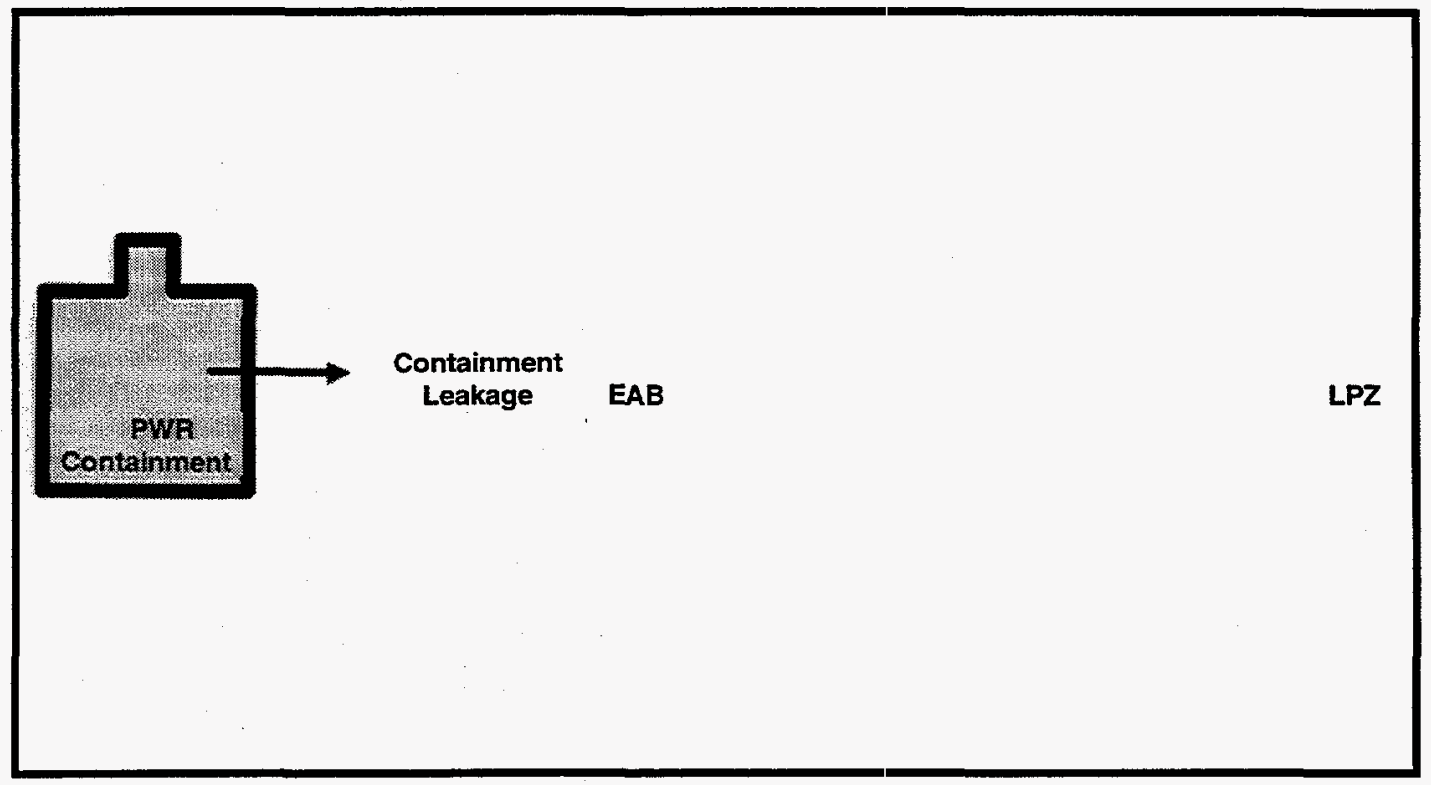

Figure 1. PWR Containment Leakage

\section{Source Term}

Release fractions and timing: TD-14844, Table IV with modifications per NRC Regulatory Guides 1.3 and 1.4 (removes mixed fission products and details iodine fractions) and further modified as described below.

Start of release:

$0.0000 \mathrm{hr}$

Iodine fractions:

Fraction of released iodine chemical form:

$\begin{array}{ll}\text { Elemental: } & 0.9100 \\ \text { Organic: } & 0.0400 \\ \text { Aerosol: } & 0.0500\end{array}$

Inventory:

TID-14844 example normalized core inventory modified as follows:

$$
{ }^{131} \text { I only }
$$

Reactor power:

1932 MWth

\section{Plant Model}

Containment volume:

$0.1730 \mathrm{E}+07 \mathrm{ft}^{3}$

Environment

Containment leakage:

Air leakage

from: Containment

to: Environment 
Scenario Description

Containment leak rate:

$0.1200 \% /$ day

Dose Parameters

EAB X/Q values:

Time (hr) X/Q $\left(\mathrm{s} / \mathrm{m}^{3}\right)$

$0.0000 \quad 0.1000 \mathrm{E}-02$

$2.0000 \quad 0.0000 \mathrm{E}+00$

LPZ X/Q values:

Time (hr) X/Q $\left(\mathrm{s} / \mathrm{m}^{3}\right)$

$0.0000 \quad 0.1350 \mathrm{E}-03$

$8.0000 \quad 0.1000 \mathrm{E}-03$

$24.0000 \quad 0.5400 \mathrm{E}-04$

$96.0000 \quad 0.2200$ E-04

\section{Success Criteria}

All of the RADTRAD-calculated doses must equal the analytically derived doses within $\pm 0.1 \%$.

\section{Acceptance Criteria}

All of the success criteria must be met or exceeded in order for the RADTRAD software to be acceptable to the NRC.

\section{Results}

\begin{tabular}{|l|l|r|c|}
\hline \multicolumn{2}{|l|}{ Test Case 1 } & RADTRAD & Analytic Solution \\
\hline EAB & Thyroid (rem) & 443 & 443 \\
\hline & TEDE (rem) & 13.57 & 13.57 \\
\hline LPZ & Thyroid (rem) & 2992 & 2992 \\
\hline & TEDE (rem) & 91.9 & 91.9 \\
\hline
\end{tabular}




\subsubsection{Acceptance Test Case 2}

\section{Problem Description}

PWR design (see Figure 1).

Instantaneous, modified TID-14844 release into containment.

Release begins at reactor shutdown.

No decay and no daughtering.

No explicit calculation of removal mechanisms in the containment, but $50 \%$ of the released iodine is deposited in accordance with TID-14844.

Containment leak to the environment.

$\mathrm{EAB}$ and LPZ doses calculated.

\section{Source Term}

Release fractions and timing: TID-14844, Table IV with modifications per NRC Regulatory Guides 1.3 and 1.4 .

Start of release:

$0.0000 \mathrm{hr}$

Iodine fractions:

Fraction of released iodine chemical form:

$\begin{array}{ll}\text { Elemental: } & 0.9100 \\ \text { Organic: } & 0.0400 \\ \text { Aerosol: } & 0.0500\end{array}$

Inventory:

TID-14844 example normalized core inventory

Reactor power:

1932 MWth

\section{Plant Model}

Containment volume: $\quad 0.1730 \mathrm{E}+07 \mathrm{ft}^{3}$

Environment

Containment leakage:

Air leakage from: Containment

to: Environment

\section{Scenario Description}

Containment leak rate:

$0.1200 \% /$ day

\section{Dose Parameters}

$\mathrm{EAB} X / \mathrm{Q}$ values:

$\begin{array}{ll}\text { Time }(\mathrm{hr}) & \mathrm{X} / \mathrm{Q}\left(\mathrm{s} / \mathrm{m}^{3}\right) \\ 0.0000 & 0.1000 \mathrm{E}-02 \\ 2.0000 & 0.0000 \mathrm{E}+00 \\ \text { Time }(\mathrm{hr}) & \mathrm{X} / \mathrm{Q}\left(\mathrm{s} / \mathrm{m}^{3}\right) \\ 0.0000 & 0.1350 \mathrm{E}-03 \\ 8.0000 & 0.1000 \mathrm{E}-03 \\ 24.0000 & 0.5400 \mathrm{E}-04 \\ 96.0000 & 0.2200 \mathrm{E}-04\end{array}$

\section{Success Criteria}

All of the RADTRAD-calculated doses must equal the analytically derived doses within $\pm 0.1 \%$.

\section{Acceptance Criteria}

All of the success criteria must be met or exceeded in order for the RADTRAD software to be acceptable to the NRC. 
Results

\begin{tabular}{|l|l|r|c|}
\hline \multicolumn{2}{|l|}{ Test Case 2 } & RADTRAD & Analytic Solution \\
\hline EAB & Thyroid (rem) & 652. & 652. \\
\hline & TEDE (rem) & 28.7 & 28.7 \\
\hline LPZ & Thyroid (rem) & 4400 & 4400 \\
\hline & TEDE (rem) & 220 & 220 \\
\hline
\end{tabular}




\subsubsection{Acceptance Test Case 2A}

\section{Problem Description}

PWR design (see Figure 1).

Instantaneous, modified TID-14844 release into containment.

Decay without daughtering included.

Release begins at reactor shutdown.

No explicit calculation of removal mechanisms in the containment, but $50 \%$ of the released iodine is deposited in accordance with TID-14844.

Containment leak to the environment.

$\mathrm{EAB}$ and $\mathrm{LPZ}$ doses calculated.

\section{Source Term}

Release fractions and timing: TID-14844, Table IV with modifications per NRC regulatory guides 1.3 and 1.4.

Start of release:

Iodine fractions:

Inventory:

Reactor power:

\section{$0.0000 \mathrm{hr}$}

Fraction of released iodine chemical form:

$\begin{array}{ll}\text { Elemental: } & 0.9100 \\ \text { Organic: } & 0.0400 \\ \text { Aerosol: } & 0.0500\end{array}$

TID-14844 example normalized core inventory

1932 MWth

$0.1730 \mathrm{E}+07 \mathrm{ft}^{3}$

Containment volume:

Environment

Containment leakage:

\section{Scenario Description}

Containment leak rate:

\section{Dose Parameters}

$\mathrm{EAB} X / Q$ values:

LPZ X/Q values:

$$
\text { Air leakage }
$$

from: Containrnent

\section{$0.1200 \% /$ day}

$$
\begin{array}{ll}
\text { Time }(\mathrm{hr}) & \mathrm{X} / \mathrm{Q}\left(\mathrm{s} / \mathrm{m}^{3}\right) \\
0.0000 & 0.1000 \mathrm{E}-02 \\
2.0000 & 0.0000 \mathrm{E}+00 \\
\text { Time }(\mathrm{hr}) & \mathrm{X} / \mathrm{Q}\left(\mathrm{s} / \mathrm{m}^{3}\right) \\
0.0000 & 0.1350 \mathrm{E}-03 \\
8.0000 & 0.1000 \mathrm{E}-03 \\
24.0000 & 0.5400 \mathrm{E}-04 \\
96.0000 & 0.2200 \mathrm{E}-04
\end{array}
$$

\section{Success Criteria}

All of the RADTRAD-calculated doses must equal the doses calculated by HABIT within $\pm 5 \%$.

\section{Acceptance Criteria}

All of the success criteria must be met or exceeded in order for the RADTRAD software to be acceptable to the NRC. 
Results

\begin{tabular}{|l|l|r|r|}
\hline \multicolumn{2}{|l|}{ Test Case 2A } & RADTRAD & HABIT \\
\hline EAB & Thyroid (rem) & 640 & 640 \\
\hline & TEDE (rem) & 26.4 & 26.17 \\
\hline LPZ & Thyroid (rem) & 1625 & 1610 \\
\hline & TEDE (rem) & 53.1 & 52.6 \\
\hline
\end{tabular}




\subsubsection{Acceptance Test Case 2B}

\section{Problem Description}

PWR design (see Figure 1).

Instantaneous, modified TID-14844 release into containment.

Decay and daughtering included.

Release begins at reactor shutdown.

No explicit calculation of removal mechanisms in the containment, but $50 \%$ of the released iodine is deposited in accordance with TID-14844.

Containment leak to the environment.

$\mathrm{EAB}$ and LPZ doses calculated.

\section{Source Term}

Release fractions and timing: TID-14844, Table IV with modifications per NRC Regulatory Guides 1.3 and 1.4 .

Start of release:

Iodine fractions:

Inventory:

Reactor power:

\section{Plant Model}

Containment volume:

Environment

Containment leakage:

\section{Scenario Description}

Containment leak rate:

\section{Dose Parameters}

EAB X/Q values:

LPZ X/Q values:
$0.0000 \mathrm{hr}$

Fraction of released iodine chemical form:

$\begin{array}{ll}\text { Elemental: } & 0.9100 \\ \text { Organic: } & 0.0400 \\ \text { Aerosol: } & 0.0500\end{array}$

TID-14844 example normalized core inventory

1932 MWth

$0.1730 \mathrm{E}+07 \mathrm{ft}^{3}$

Air leakage from: Containment to: Environment

\section{$0.1200 \% /$ day}

$\begin{array}{ll}\text { Time (hr) } & \text { X/Q }\left(\mathrm{s} / \mathrm{m}^{3}\right) \\ 0.0000 & 0.1000 \mathrm{E}-02 \\ 2.0000 & 0.0000 \mathrm{E}+00 \\ \text { Time }(\mathrm{hr}) & \mathrm{X} / \mathrm{Q}\left(\mathrm{s} / \mathrm{m}^{3}\right) \\ 0.0000 & 0.1350 \mathrm{E}-03 \\ 8.0000 & 0.1000 \mathrm{E}-03 \\ 24.0000 & 0.5400 \mathrm{E}-04 \\ 96.0000 & 0.2200 \mathrm{E}-04\end{array}$

\section{Success Criteria}

All of the RADTRAD-calculated doses must equal the doses calculated by HABIT within $\pm 5 \%$.

\section{Acceptance Criteria}

All of the success criteria must be met or exceeded in order for the RADTRAD software to be acceptable to the NRC. 


\section{Results}

\begin{tabular}{|l|l|r|r|}
\hline \multicolumn{2}{|l|}{ Test Case 2B } & RADTRAD & RADTRAD 2A \\
\hline EAB & Thyroid (rem) & 640 & 640 \\
\hline & TEDE (rem) & 26.4 & 26.4 \\
\hline LPZ & Thyroid (rem) & 1625 & 1625 \\
\hline & TEDE (rem) & 53.2 & 53.1 \\
\hline
\end{tabular}

Table 3.3.1.2-4 shows some of the isotopic changes for these two cases. 


\subsubsection{Acceptance Test Case 3}

\section{Problem Description}

PWR design (see Figure 1).

Instantaneous, modified TID-14844 release into the containment.

Release begins at reactor shutdown.

No explicit calculation of removal mechanisms in the containment, but $50 \%$ of the released iodine is deposited in accordance with NRC Regulatory Guide 1.4.

Containment leak to the environment.

Control room air filtration system (see Figure 2).

$\mathrm{EAB}, \mathrm{LPZ}$, and CR doses calculated.

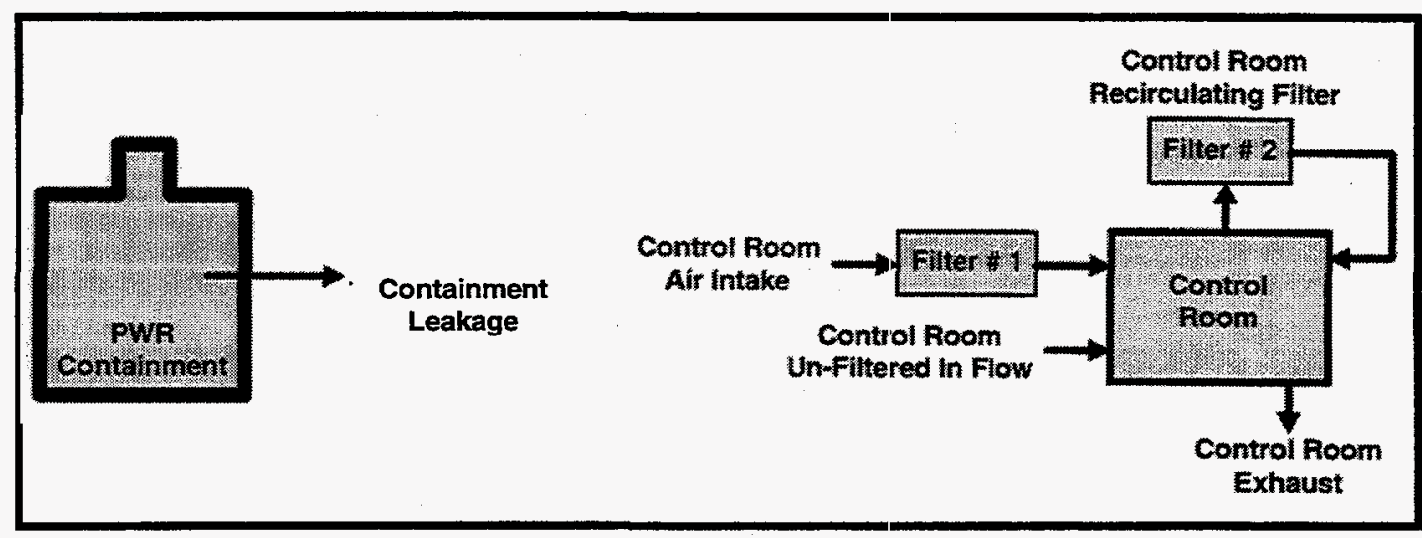

Figure 2. PWR Control Room Detail

\section{Source Term}

Release fractions and timing: TID-14844, Table IV with modifications per NRC Regulatory Guide 1.4.

Start of release:

$0.0000 \mathrm{hr}$

Iodine fractions:

Fraction of released iodine chemical form:

$\begin{array}{ll}\text { Organic } & 0.0400 \\ \text { Elemental: } & 0.9100 \\ \text { Aerosol: } & 0.0500\end{array}$

Inventory:

TID-14844 example normalized core inventory

Reactor power:

1932 MWth

\section{Plant Model}

Containment volume:

$0.1730 \mathrm{E}+07 \mathrm{ft}^{3}$

Control room volume:

$0.4238 \mathrm{E}+05 \mathrm{ft}^{3}$

Control room removal:

Recirculating filter \# 2 .

Environment

Containment leakage:

Air leakage

Filter \#1:

Filter \#2:

CR exhaust:

$\mathrm{CR}$ unfiltered inflow:
Filtered pathway

Recirculation filter

Air leakage

Air leakage from: Containment

from: Environment

from: Control room

from: Control room

from: Environment to: Environment

to: Control room

to: Control room

to: Environment

to: Control room 


\section{Scenario Description}

Containment leak rate:

$0.1200 \% /$ day

Filter \#1 flow rate:

$3000 \mathrm{cfm}$

Filter \#1 efficiencies:

Elemental iodine

$99.0000 \%$

Organic iodine

$00.0000 \%$

Aerosols

$99.0000 \%$

Filter \#2 flow rate:

$10000 \mathrm{cfm}$

Filter \#2 Efficiencies:

$\begin{array}{ll}\text { Elemental iodine } & 90.0000 \% \\ \text { Organic iodine } & 00.0000 \% \\ \text { Aerosols } & 90.0000 \%\end{array}$

CR exhaust:

$3010 \mathrm{cfm}$

$C R$ unfiltered inflow:

$10 \mathrm{cfm}$

\section{Dose Parameters}

EAB X/Q values:

$\begin{array}{ll}\text { Time }(\mathrm{hr}) & \mathrm{X} / \mathrm{Q}\left(\mathrm{s} / \mathrm{m}^{3}\right) \\ 0.0000 & 0.1000 \mathrm{E}-02 \\ 2.0000 & 0.0000 \mathrm{E}+00\end{array}$

LPZ X/Q values:

$\begin{array}{ll}\text { Time }(\mathrm{hr}) & \mathrm{X} / \mathrm{Q}\left(\mathrm{s} / \mathrm{m}^{3}\right) \\ 0.0000 & 0.1350 \mathrm{E}-03 \\ 8.0000 & 0.1000 \mathrm{E}-03 \\ 24.0000 & 0.5400 \mathrm{E}-04 \\ 96.0000 & 0.2200 \mathrm{E}-04 \\ \text { Time }(\mathrm{hr}) & \mathrm{X} / \mathrm{Q}\left(\mathrm{s}^{3} \mathrm{~m}^{3}\right) \\ 0.0000 & 0.2200 \mathrm{E}-02 \\ 2.0000 & 0.1500 \mathrm{E}-02 \\ 8.0000 & 0.1300 \mathrm{E}-02 \\ 24.0000 & 0.8400 \mathrm{E}-03 \\ 96.0000 & 0.4800 \mathrm{E}-03\end{array}$

\section{Success Criteria}

All of the RADTRAD-calculated EAB, LPZ, and CR doses must equal the doses calculated by HABIT within $\pm 5.0 \%$

\section{Acceptance Criteria}

All of the success criteria must be met or exceeded in order for the RADTRAD software to be acceptable to the NRC. 


\section{Results}

\begin{tabular}{|l|l|r|r|}
\hline \multicolumn{2}{|l|}{ Test Case 3 } & RADTRAD & HABIT \\
\hline EAB & Thyroid (rem) & 652 & 653 \\
\hline & TEDE (rem) & 28.8 & 28.8 \\
\hline LPZ & Thyroid (rem) & 4400 & 4400 \\
\hline & TEDE (rem) & 220 & 220 \\
\hline CR & Thyroid (rem) & 2660 & 2650 \\
\hline & TEDE (rem) & 95.5 & 95.1 \\
\hline
\end{tabular}




\subsubsection{Acceptance Test Case 4}

\section{Problem Description}

PWR design (see Figure 1).

Instantaneous, TID-14844 release into the containment.

Release begins at reactor shutdown.

In-containment aerosol removal via natural deposition with user-defined removal rates.

Containment leak to the environment.

Control room air filtration system (see Figure 2).

$\mathrm{EAB}, \mathrm{LPZ}$, and CR doses calculated.

\section{Source Term}

Release fractions and timing:

TID-14844, Table IV with aerosol-only modifications per NRC Regulatory Guide 1.4.

Start of release:

$0.0000 \mathrm{hr}$

Iodine fractions:

Fraction of released iodine chemical form:

$\begin{array}{ll}\text { Elemental: } & 0.9100 \\ \text { Organic: } & 0.0400 \\ \text { Aerosol: } & 0.0500\end{array}$

Inventory:

TID-14844 example normalized core inventory

Reactor power:

1932 MWth

\section{Plant Model}

Containment volume:

\section{$0.1730 \mathrm{E}+07 \mathrm{ft}^{3}$}

Containment removal:

Aerosol removal via natural deposition with user-defined removal rates.

Control room volume:

$0.4238 \mathrm{E}+05 \mathrm{ft}^{3}$

Environment

Containment leakage:

Air leakage

Filtered pathway

Filter \#1:

Filter \#2:

CR exhaust:

$\mathrm{CR}$ unfiltered inflow:

\section{Scenario Description}

Containment leak rate:

Natural deposition aerosol removal rate: elemental removal rate:

Filter \#1 flow rate:

Filter \#1 efficiencies:

Filter \#2 flow rate:

Filter \#2 efficiencies:
Recirculation filter

Air leakage

Air leakage

\section{$0.1200 \% /$ day}

$0.15 \mathrm{hr}^{-1}$

$0.15 \mathrm{hr}^{-1}$

$3000 \mathrm{cfm}$

Elemental iodine $\quad 99.0000 \%$

Organic iodine $\quad 00.0000 \%$

Aerosols

$99.0000 \%$

$10000 \mathrm{cfm}$

Elemental iodine

Organic iodine

Aerosols
from: Containment
to: Environment
from: Environment
to: Control room
from: Control room
to: Control room
from: Control room
to: Environment
from: Environment
to: Control room 
CR unfiltered inflow:

$10 \mathrm{cfm}$

CR exhaust:

$3010 \mathrm{cfm}$

\section{Dose Parameters}

$\mathrm{EAB} X / \mathrm{Q}$ values:

Time $(\mathrm{hr}) \quad \mathrm{X} / \mathrm{Q}\left(\mathrm{s} / \mathrm{m}^{3}\right)$

$$
0.0000 \quad 0.1000 \mathrm{E}-02
$$

$2.0000 \quad 0.0000 \mathrm{E}+00$

LPZ X/Q values:

Time (hr) X/Q $\left(\mathrm{s} / \mathrm{m}^{3}\right)$

$0.0000 \quad 0.1350 \mathrm{E}-03$

$8.0000 \quad 0.1000 \mathrm{E}-03$

$24.0000 \quad 0.5400 \mathrm{E}-04$

$96.0000 \quad 0.2200 \mathrm{E}-04$

$\mathrm{CR}$ air intake X/Q values: $\quad$ Time $(\mathrm{hr}) \quad \mathrm{X} / \mathrm{Q}\left(\mathrm{s} / \mathrm{m}^{3}\right)$

$\begin{array}{ll}0.0000 & 0.2200 \mathrm{E}-02 \\ 2.0000 & 0.1500 \mathrm{E}-02 \\ 8.0000 & 0.1300 \mathrm{E}-02 \\ 24.0000 & 0.8400 \mathrm{E}-03 \\ 96.0000 & 0.4800 \mathrm{E}-03\end{array}$

Success Criteria

All of the RADTRAD-calculated EAB, LPZ, and CR doses must equal the doses calculated by HABIT within $\pm 5.0 \%$.

\section{Acceptance Criteria}

All of the success criteria must be met or exceeded in order for the RADTRAD software to be acceptable to the NRC.

\section{Results}

\begin{tabular}{|l|l|r|r|}
\hline \multicolumn{2}{|l|}{ Test Case 4 } & RADTRAD & \multicolumn{1}{c|}{ HABIT } \\
\hline EAB & Thyroid (rem) & 1130 & 1130 \\
\hline & TEDE (rem) & 47.5 & 47.6 \\
\hline LPZ & Thyroid (rem) & 808 & 808 \\
\hline & TEDE & 66.9 & 66.8 \\
\hline CR & Thyroid (rem) & 4940 & 4940 \\
\hline & TEDE & 169 & 168 \\
\hline
\end{tabular}




\subsubsection{Acceptance Test Case 5}

\section{Problem Description}

PWR design (see Figure 1).

Instantaneous, TID-14844 release into the containment.

Release begins at reactor shutdown.

In-containment aerosol removal via natural deposition using Henry's correlation [Fa84]. Because this model deposits only aerosols and the TID releases elementals, the problem is duplicated as case $10 \mathrm{~A}$.

Containment leak to the environment.

Control room air filtration system (see Figure 2).

$\mathrm{EAB}, \mathrm{LPZ}$, and $\mathrm{CR}$ doses calculated.

\section{Source Term}

Release fractions and timing: TID-14844, Table IV with aerosol-only modifications per NRC Regulatory Guide 1.4.

Start of release:

$0.0000 \mathrm{hr}$

Iodine fractions:

Fraction of released iodine chemical form:

$\begin{array}{ll}\text { Elemental: } & 0.9100 \\ \text { Organic: } & 0.0400 \\ \text { Aerosol: } & 0.0500\end{array}$

Inventory:

TID-14844 example normalized core inventory

Reactor power:

1932 MWth

\section{Plant Model}

Containment volume:

\section{$0.1730 \mathrm{E}+07 \mathrm{ft}^{3}$}

Containment removal:

Aerosol removal determined by Henry's correlation for natural deposition.

Control room volume:

$0.4238 \mathrm{E}+05 \mathrm{ft}^{3}$

Environment

Containment leakage:

Air leakage

from: Containment

to: Environment

Filter \#1:

Filtered pathway

from: Environment

to: Control room

Filter \#2:

Recirculation filter

from: Control room

to: Control room

CR exhaust:

Air leakage

from: Control room

to: Environment

CR unfiltered inflow:

Air leakage

from: Environment

to: Control room

\section{Scenario Description}

Containment leak rate:

$0.1200 \% /$ day

Henry's natural deposition parameters:

$16.4 \mathrm{ft}(16.4 \mathrm{ft}=$ reference value $)$

Particle theoretical density $=1.0 \mathrm{~g} / \mathrm{cm}^{3}\left(2.27 \mathrm{~g} / \mathrm{cm}^{3}=\right.$ reference value $)$

Filter \#1 flow rate: $3000 \mathrm{cfm}$

Filter \#1 efficiencies:

Elemental iodine

Organic iodine

Aerosols

$99.0000 \%$

$00.0000 \%$

$99.0000 \%$

Filter \#2 flow rate:

$10,000 \mathrm{cfm}$ 
Filter \#2 efficiencies:

Elemental iodine

Organic iodine

Aerosols

$10 \mathrm{cfm}$

$3010 \mathrm{cfm}$

CR exhaust:

Time (hr) $\quad \mathrm{X} / \mathrm{Q}\left(\mathrm{s} / \mathrm{m}^{3}\right)$

$0.0000 \quad 0.1000 \mathrm{E}-02$

2.0000

$0.0000 \mathrm{E}+00$

LPZ X/Q values:

Time (hr) X/Q $\left(\mathrm{s} / \mathrm{m}^{3}\right)$

$0.0000 \quad 0.1350 \mathrm{E}-03$

$8.0000 \quad 0.1000 \mathrm{E}-03$

$24.0000 \quad 0.5400 \mathrm{E}-04$

$96.0000 \quad 0.2200 \mathrm{E}-04$

CR air intake X/Q values: $\quad$ Time (hr) $\mathrm{X} / \mathrm{Q}\left(\mathrm{s} / \mathrm{m}^{3}\right)$

$0.0000 \quad 0.2200 \mathrm{E}-02$

$2.0000 \quad 0.1500 \mathrm{E}-02$

$8.0000 \quad 0.1300 \mathrm{E}-02$

$24.0000 \quad 0.8400 \mathrm{E}-03$

$96.0000 \quad 0.4800 \mathrm{E}-03$

\section{Success Criteria}

Deposition rate should be found to be reasonable.

\section{Acceptance Criteria}

All of the success criteria must be met or exceeded in order for the RADTRAD software to be acceptable to the NRC. If the success criteria are not met in the RADTRAD version under test, the software will still be acceptable to the NRC. SNL will be responsible for determining the cause of the failure and correction will be expected before project closeout.

\section{Results}

\begin{tabular}{|l|l|r|r|}
\hline \multicolumn{2}{|l|}{ Test Case 5 } & RADTRAD & RADTRAI CASE 4 \\
\hline EAB & Thyroid (rem) & 1300 & 1130 \\
\hline & TEDE (rem) & 54.1 & 47.5 \\
\hline LPZ & Thyroid (rem) & 8470 & 808 \\
\hline & TEDE (rem) & 392 & 66.9 \\
\hline CR & Thyroid (rem) & 5300 & 4940 \\
\hline & TEDE (rem) & 181 & 169 \\
\hline
\end{tabular}

These calculations indicate less iodine deposition with the model, resulting in higher doses in the environment and control room. This case is an example of the code being used incorrectly. 


\subsubsection{Acceptance Test Case 6}

\section{Problem Description}

PWR design (see Figure 1).

Modified NUREG-1465 PWR ( $\left({ }^{131}\right.$ I only) release into containment.

Release begins at reactor shutdown.

No removal mechanisms.

Containment leak to the environment.

$\mathrm{EAB}$ and $\mathrm{LPZ}$ doses calculated.

\section{Source Term}

Release fractions and timing: NUREG-1465, Tables 3.12 and 3.13 with modifications as described below.

Start of release:

$0.0000 \mathrm{hr}$

Iodine fractions:

$$
1.0000
$$

Fraction of released iodine chemical form:

$\begin{array}{ll}\text { Elemental: } & 0.0485 \\ \text { Organic: } & 0.0015 \\ \text { Aerosol: } & 0.9500\end{array}$

Inventory:

Normalized MACCS sample 3412 MWth PWR core inventory modified as follows:

$$
{ }^{131} \text { I only }
$$

Reactor power: $\quad 1932$ MWth

\section{Plant Model}

Containment volume:

$$
0.1730 \mathrm{E}+07 \mathrm{ft}^{3}
$$

Environment

Containment leakage:

Air leakage

from: Containment

to: Environment

\section{Scenario Description}

Containment leak rate:

$0.1200 \% /$ day

\section{Dose Parameters}

EAB X/Q values:

$\begin{array}{ll}\text { Time (hr) } & \mathrm{X} / \mathrm{Q}\left(\mathrm{s} / \mathrm{m}^{3}\right) \\ 0.0000 & 0.1000 \mathrm{E}-02 \\ 2.0000 & 0.0000 \mathrm{E}+00\end{array}$

LPZ XJQ values:

$\begin{array}{ll}\text { Time (hr) } & X / Q\left(\mathrm{~s} / \mathrm{m}^{3}\right) \\ 0.0000 & 0.1350 \mathrm{E}-03 \\ 8.0000 & 0.1000 \mathrm{E}-03 \\ 24.0000 & 0.5400 \mathrm{E}-04 \\ 96.0000 & 0.2200 \mathrm{E}-04\end{array}$

\section{Success Criteria}

All of the RADTRAD-calculated doses must equal the analytically derived doses within $\pm 0.1 \%$.

\section{Acceptance Criteria}

All of the success criteria must be met or exceeded in order for the RADTRAD software to be acceptable to the NRC. 


\section{Results}

\begin{tabular}{|l|l|c|c|}
\hline \multicolumn{2}{|l|}{ Test Case 6 } & RADTRAD & \multicolumn{2}{c|}{ HABIT } \\
\hline EAB & Thyroid (rem) & 354 & 354 \\
\hline & TEDE (rem) & 10.9 & 10.8 \\
\hline LPZ & Thyroid (rem) & 4907 & 4905 \\
\hline & TEDE (rem) & 150.7 & 151 \\
\hline
\end{tabular}

In Table 3.3.1.2-5 this case is also compared with HABIT with decay but no in-growth included. In addition, a case using the severe accident iodine release from NUREG 1465 is compared with the analytic solution in that table. 


\subsubsection{Acceptance Test Case 7}

\section{Problem Description}

PWR design (see Figure 1).

NUREG-1465 PWR release into containment.

No decay and no daughtering.

Release begins at reactor shutdown.

No removal mechanisms.

Containment leak to the environment.

$\mathrm{EAB}$ and $\mathrm{LPZ}$ doses calculated.

\section{Source Term}

Release fractions and timing: NUREG-1465, Tables 3.12 and 3.13.

Start of release:

$0.0000 \mathrm{hr}$

Iodine fractions:

$$
1.0000
$$

Fraction of released iodine chemical form:

$\begin{array}{ll}\text { Elemental: } & 0.0485 \\ \text { Organic: } & 0.0015 \\ \text { Aerosol: } & 0.9500\end{array}$

Inventory:

Normalized MACCS sample 3412 MWth PWR core inventory

Reactor power:

1932 MWth

\section{Plant Model}

Containment volume: $\quad 0.1730 \mathrm{E}+07 \mathrm{ft}^{3}$

Environment

Containment leakage:

Air leakage from: Containment

to: Environment

\section{Scenario Description}

Containment leak rate:

$0.1200 \% /$ day

\section{Dose Parameters}

EAB X/Q values:

$\begin{array}{ll}\text { Time }(\mathrm{hr}) & \mathrm{X} / \mathrm{Q}\left(\mathrm{s} / \mathrm{m}^{3}\right) \\ 0.0000 & 0.1000 \mathrm{E}-02 \\ 2.0000 & 0.0000 \mathrm{E}+00 \\ \text { Time }(\mathrm{hr}) & \mathrm{X} / \mathrm{Q}\left(\mathrm{s} / \mathrm{m}^{3}\right) \\ 0.0000 & 0.1350 \mathrm{E}-03 \\ 8.0000 & 0.1000 \mathrm{E}-03 \\ 24.0000 & 0.5400 \mathrm{E}-04 \\ 96.0000 & 0.2200 \mathrm{E}-04\end{array}$

\section{Success Criteria}

All of the RADTRAD-calculated doses must equal the analytically derived doses within $\pm 0.1 \%$.

\section{Acceptance Criteria}

All of the success criteria must be met or exceeded in order for the RADTRAD software to be acceptable to the NRC. 


\section{Results}

\begin{tabular}{|l|l|c|c|}
\hline \multicolumn{2}{|l|}{ Test Case 7 } & \multicolumn{1}{c|}{ RADTRAD } & HABIT \\
\hline EAB & Thyroid (rem) & 518 & 518 \\
\hline & TEDE (rem) & 25.3 & 25.3 \\
\hline LPZ & Thyroid (rem) & 7200 & 7200 \\
\hline & TEDE (rem) & 382 & 382 \\
\hline
\end{tabular}

In Table 3.3.1.2-5 this case is also compared with HABIT with decay but no in-growth included. In addition, a case using the severe accident iodine release from NUREG 1465 is compared with the analytic solution in that table. 


\subsubsection{Acceptance Test Case 7A}

\section{Problem Description}

PWR design (see Figure 1).

NUREG-1465 PWR release into containment.

Decay but no daughtering.

Release begins at reactor shutdown.

No removal mechanisms.

Containment leak to the environment.

$\mathrm{EAB}$ and LPZ doses calculated.

\section{Source Term}

Release fractions and timing: NUREG-1465, Tables 3.12 and 3.13 .

Start of release:

$0.0000 \mathrm{hr}$

Iodine fractions:

Fraction of released iodine chemical form:

$\begin{array}{ll}\text { Elemental: } & 0.0485 \\ \text { Organic: } & 0.0015 \\ \text { Aerosol: } & 0.9500\end{array}$

Inventory:

Normalized MACCS sample 3412 MWth PWR core inventory

Reactor power:

1932 MWth

Plant Model

Containment volume:

$0.1730 \mathrm{E}+07 \mathrm{ft}^{3}$

Environment

Containment leakage:

Air leakage

from: Containment

to: Environment

\section{Scenario Description}

Containment leak rate:

$0.1200 \% /$ day

\section{Dose Parameters}

$\mathrm{EAB} X / \mathrm{Q}$ values:

$\begin{array}{ll}\text { Time }(\mathrm{hr}) & \mathrm{X} / \mathrm{Q}\left(\mathrm{s} / \mathrm{m}^{3}\right) \\ 0.0000 & 0.1000 \mathrm{E}-02 \\ 2.0000 & 0.0000 \mathrm{E}+00 \\ \text { Time }(\mathrm{hr}) & \mathrm{X} / \mathrm{Q}\left(\mathrm{s} / \mathrm{m}^{3}\right) \\ 0.0000 & 0.1350 \mathrm{E}-03 \\ 8.0000 & 0.1000 \mathrm{E}-03 \\ 24.0000 & 0.5400 \mathrm{E}-04 \\ 96.0000 & 0.2200 \mathrm{E}-04\end{array}$

\section{Success Criteria}

All of the RADTRAD-calculated EAB and LPZ doses must equal the doses calculated by HABIT within $\pm 5.0 \%$.

\section{Acceptance Criteria}

All of the success criteria must be met or exceeded in order for the RADTRAD software to be acceptable to the NRC. 


\section{Results}

\begin{tabular}{|l|l|r|r|}
\hline \multicolumn{2}{|l|}{ Test Case 7A } & RADTRAD & \multicolumn{1}{c|}{ HAB]T } \\
\hline EAB & Thyroid (rem) & 507 & 505 \\
\hline & TEDE (rem) & 23.6 & 23.4 \\
\hline LPZ & Thyroid (rem) & 2680 & 2660 \\
\hline & TEDE (rem) & 157 & 156 \\
\hline
\end{tabular}




\subsubsection{Acceptance Test Case 7B}

\section{Problem Description}

PWR design (see Figure 1).

NUREG-1465 BWR release into containment.

No decay and no daughtering.

Release begins at reactor shutdown.

No removal mechanisms.

Containment leak to the environment.

$\mathrm{EAB}$ and LPZ doses calculated.

\section{Source Term}

Release fractions and timing: NUREG-1465, Tables 3.12 and 3.13.

Start of release:

$0.0000 \mathrm{hr}$

Iodine fractions:

Fraction of released iodine chemical form:
Elemental: $\quad 0.0485$
Organic: $\quad 0.0015$
Aerosol: $\quad 0.9500$

Inventory:

Normalized MACCS sample 3412 MWth BWR core inventory

Reactor power: $\quad 1932$ MWth

\section{Plant Model}

Containment volume: $\quad 0.1730 \mathrm{E}+07 \mathrm{ft}^{3}$

Environment

Containment leakage:

Air leakage

from: Containment

to: Environment

\section{Scenario Description}

Containment leak rate:

$0.1200 \% /$ day

\section{Dose Parameters}

EAB X/Q values:

$\begin{array}{ll}\text { Time }(\mathrm{hr}) & \mathrm{X} / \mathrm{Q}\left(\mathrm{s} / \mathrm{m}^{3}\right) \\ 0.0000 & 0.1000 \mathrm{E}-02 \\ 2.0000 & 0.0000 \mathrm{E}+00 \\ \text { Time }(\mathrm{hr}) & \mathrm{X} / \mathrm{Q}\left(\mathrm{s} / \mathrm{m}^{3}\right) \\ 0.0000 & 0.1350 \mathrm{E}-03 \\ 8.0000 & 0.1000 \mathrm{E}-03 \\ 24.0000 & 0.5400 \mathrm{E}-04 \\ 96.0000 & 0.2200 \mathrm{E}-04\end{array}$

\section{Success Criteria}

All of the RADTRAD-calculated EAB, LPZ, and CR doses must equal the doses calculated by HABIT within $\pm 5.0 \%$.

\section{Acceptance Criteria}

All of the success criteria must be met or exceeded in order for the RADTRAD software to be acceptable to the NRC. 


\section{Results}

\begin{tabular}{|l|l|r|r|}
\hline \multicolumn{2}{|l|}{ Test Case 7B } & RADTRAD & HABIT \\
\hline EAB & Thyroid (rem) & 378 & 378 \\
\hline & TEDE (rem) & 20.2 & 20.2 \\
\hline LPZ & Thyroid (rem) & 5530 & 5530 \\
\hline & TEDE (rem) & 327 & 326 \\
\hline
\end{tabular}




\subsubsection{Acceptance Test Case 7C}

\section{Problem Description}

PWR design (see Figure 1).

NUREG-1465 BWR release into containment.

Decay but no daughtering.

Release begins at reactor shutdown.

No removal mechanisms.

Containment leak to the environment.

EAB and LPZ doses calculated.

\section{Source Term}

Release fractions and timing: NUREG-1465, Tables 3.12 and 3.13 .

Start of release:

$0.0000 \mathrm{hr}$

Iodine fractions:

Fraction of released iodine chemical form:

$\begin{array}{ll}\text { Elemental: } & 0.0485 \\ \text { Organic: } & 0.0015 \\ \text { Aerosol: } & 0.9500\end{array}$

Inventory:

Normalized MACCS sample 3412 MWth BWR core inventory

Reactor power:

1932 MWth

\section{Plant Model}

Containment volume: $\quad 0.1730 \mathrm{E}+07 \mathrm{ft}^{3}$

Environment

Containment leakage:

Air leakage

from: Containment

to: Environment

\section{Scenario Description}

Containment leak rate:

$0.1200 \% /$ day

Dose Parameters

$\mathrm{EAB} X / Q$ values:
Time (hr) X/Q $\left(\mathrm{s} / \mathrm{m}^{3}\right)$
0.0000
$0.1000 \mathrm{E}-02$
2.0000
$0.0000 \mathrm{E}+00$
Time (hr) X/Q $\left(\mathrm{s} / \mathrm{m}^{3}\right)$
$0.0000 \quad 0.1350 \mathrm{E}-03$
$8.0000 \quad 0.1000 \mathrm{E}-03$
$24.0000 \quad 0.5400 \mathrm{E}-04$
$96.0000 \quad 0.2200 \mathrm{E}-04$

LPZ X/Q values:

\section{Success Criteria}

All of the RADTRAD-calculated EAB, LPZ, and CR doses must equal the doses calculated by HABIT within $\pm 5.0 \%$.

\section{Acceptance Criteria}

All of the success criteria must be met or exceeded in order for the RADTRAD software to be acceptable to the NRC.

\section{Results}




\begin{tabular}{|l|l|r|r|}
\hline \multicolumn{2}{|l|}{ Test Case 7C } & RADTRAD & \multicolumn{1}{c|}{ HABIT } \\
\hline EAB & Thyroid (rem) & 370 & 369 \\
\hline & TEDE (rem) & 18.9 & 18.7 \\
\hline LPZ & Thyroid (rem) & 2060 & 2040 \\
\hline & TEDE (rem) & 148 & 147 \\
\hline
\end{tabular}




\subsubsection{Acceptance Test Case 7D}

\section{Problem Description}

PWR design (see Figure 1).

NUREG-1465 PWR release into containment.

Decay and no daughtering.

Release begins $10 \mathrm{hr}$ after reactor shutdown.

No removal mechanisms.

Containment leak to the environment.

$\mathrm{EAB}$ and $\mathrm{LPZ}$ doses calculated.

\section{Source Term}

Release fractions and timing: NUREG-1465, Tables 3.12 and 3.13.

Start of release:

$10.0000 \mathrm{hr}$

Iodine fractions:

Fraction of released iodine chemical form:

$\begin{array}{ll}\text { Elemental: } & 0.0485 \\ \text { Organic: } & 0.0015 \\ \text { Aerosol: } & 0.9500\end{array}$

Inventory:

Normalized MACCS sample 3412 MWth PWR core inventory

Reactor power:

1932 MWth

\section{Plant Model}

Containment volume: $\quad 0.1730 \mathrm{E}+07 \mathrm{ft}^{3}$

Environment

Containment leakage:

Air leakage

from: Containment

to: Environment

\section{Scenario Description}

Containment leak rate:

$0.1200 \% /$ day

\section{Dose Parameters}

EAB X/Q values:

$$
\begin{array}{lll}
\text { EAB X/Q values: } & \text { Time }(\mathrm{hr}) & \mathrm{X} / \mathrm{Q}\left(\mathrm{s} / \mathrm{m}^{3}\right) \\
& 0.0000 & 0.1000 \mathrm{E}-02 \\
& 2.0000 & 0.0000 \mathrm{E}+00 \\
\text { LPZ X/Q values: } & \text { Time }(\mathrm{hr}) & \mathrm{X} / \mathrm{Q}\left(\mathrm{s} / \mathrm{m}^{3}\right) \\
& 0.0000 & 0.1350 \mathrm{E}-03 \\
& 8.0000 & 0.1000 \mathrm{E}-03 \\
& 24.0000 & 0.5400 \mathrm{E}-04 \\
& 96.0000 & 0.2200 \mathrm{E}-04
\end{array}
$$

\section{Success Criteria}

All of the RADTRAD-calculated EAB, LPZ, and CR doses must equal the doses calculated by HABIT within $\pm 5.0 \%$.

\section{Acceptance Criteria}

All of the success criteria must be met or exceeded in order for the RADTRAD software to be acceptable to the NRC. 


\section{Results}

\begin{tabular}{|l|l|r|r|r|r|}
\hline \multicolumn{2}{|l|}{ RADTRAD } & \multicolumn{1}{|c|}{ Case 7 } & \multicolumn{1}{c|}{ Case 7D } & Case 7A & HABIT 7D \\
\hline EAB & Thyroid (rem) & 518 & 446 & 507 & 445 \\
\hline & TEDE (rem) & 25.3 & 19.6 & 23.4 & 19.5 \\
\hline LPZ & Thyroid (rem) & 7200 & 2510 & 2680 & 2500 \\
\hline & TEDE (rem) & 382 & 150 & 157 & 150 \\
\hline
\end{tabular}

The cases shown above are all PWRs, Note that without decay the doses are high (case 7); when decay is added, the doses fall significantly (case 7A). When the decay goes on for $10 \mathrm{hr}$ before the source term is released, the doses should be even lower, although not substantially. The comparison with the HABIT code is shown to indicate the relative accuracy. 


\subsubsection{Acceptance Test Case 8}

\section{Problem Description}

PWR design (see Figure 1).

NUREG-1465 PWR release into containment.

Release begins at reactor shutdown.

No removal mechanisms.

Containment leak to the environment.

Control room air filtration system (see Figure 2).

$\mathrm{EAB}, \mathrm{LPZ}$, and $\mathrm{CR}$ doses calculated.

\section{Source Term}

Release fractions and timing: NUREG-1465, Tables 3.12 and 3.13.

Start of release:

lodine fractions:

$$
0.0000 \mathrm{hr}
$$

$$
1.0000
$$

Fraction of released iodine chemical form:

$\begin{array}{ll}\text { Elemental: } & 0.0485 \\ \text { Organic: } & 0.0015 \\ \text { Aerosol: } & 0.9500\end{array}$

Inventory:

Normalized MACCS sample 3412 MWth PWR core inventory.

Reactor power:

1932 MWth

\section{Plant Model}

Containment volume:

Control room volume:

Control room removal:

Environment

Containment leakage:

Filter \#1:

Filter \#2:

CR exhaust:

CR unfiltered inflow:

\section{Scenario Description}

Containment leak rate:

Filter \#1 flow rate:

Filter \#1 efficiencies:

Filter \#2 flow rate:

Filter \#2 efficiencies:

CR unfiltered inflow:

CR exhaust:

$$
\begin{aligned}
& 0.1730 \mathrm{E}+07 \mathrm{ft}^{3} \\
& 0.4238 \mathrm{E}+05 \mathrm{ft}^{3}
\end{aligned}
$$

Recirculating filter \# 2
Air leakage

Filtered pathway

Recirculation filter

Air leakage

Air leakage

\section{$0.12 \% /$ day \\ $3000 \mathrm{cfm}$}

Elemental iodine $\quad 99.0000 \%$

Organic iodine $\quad 00.0000 \%$

Aerosols

$99.0000 \%$

$10000 \mathrm{cfm}$

Elemental iodine $\quad 90.0000 \%$

Organic iodine

Aerosols

$00.0000 \%$

$90.0000 \%$

$10 \mathrm{cfm}$

$3010 \mathrm{cfm}$ 


\section{Dose Parameters}

EAB X/Q values:

$$
\begin{aligned}
& \text { Time (hr) X/Q }\left(\mathrm{s} / \mathrm{m}^{3}\right) \\
& 0.0000 \quad 0.1000 \mathrm{E}-02 \\
& 2.0000 \quad 0.0000 \mathrm{E}+00 \\
& \text { LPZ X/Q values: } \quad \text { Time }(\mathrm{hr}) \quad \mathrm{X} / \mathrm{Q}\left(\mathrm{s} / \mathrm{m}^{3}\right) \\
& 0.0000 \quad 0.1350 \mathrm{E}-03 \\
& 8.0000 \quad 0.1000 \mathrm{E}-03 \\
& 24.0000 \quad 0.5400 \mathrm{E}-04 \\
& 96.0000 \quad 0.2200 \mathrm{E}-04 \\
& 0.0000 \quad 0.2200 \mathrm{E}-02 \\
& 2.0000 \quad 0.1500 \mathrm{E}-02 \\
& 8.0000 \quad 0.1300 \mathrm{E}-02 \\
& 24.0000 \quad 0.8400 \mathrm{E}-03 \\
& 96.0000 \quad 0.4800 \mathrm{E}-03
\end{aligned}
$$$$
\text { Filter \#1 X/Q values: } \quad \text { Time (hr) X/Q }\left(\mathrm{s} / \mathrm{m}^{3}\right)
$$

\section{Success Criteria}

All of the RADTRAD-calculated EAB, LPZ, and CR doses must equal the doses calculated by HABIT within $\pm 5.0 \%$.

\section{Acceptance Criteria}

All of the success criteria must be met or exceeded in order for the RADTRAD software to be acceptable to the NRC.

\section{Results}

\begin{tabular}{|l|l|c|c|}
\hline \multicolumn{2}{|l|}{ Test Case 8 } & RADTRAD & HABIT \\
\hline EAB & Thyroid (rem) & 518 & 518 \\
\hline & TEDE (rem) & 25.3 & 25.3 \\
\hline LPZ & Thyroid (rem) & 7200 & 7200 \\
\hline & TEDE (rem) & 382 & 382 \\
\hline CR & Thyroid (rem) & 481 & 479 \\
\hline & TEDE (rem) & 25.7 & 25.6 \\
\hline
\end{tabular}




\subsubsection{Acceptance Test Case 9}

\section{Problem Description}

PWR design (see Figure 1).

NUREG-1465 PWR release into containment.

Release begins at reactor shutdown.

In-containment aerosol removal via natural deposition with user-defined removal rates.

Containment leak to the environment.

Control room air filtration system (see Figure 2).

$\mathrm{EAB}, \mathrm{LPZ}$, and CR doses calculated.

\section{Source Term}

Release fractions and timing: NUREG-1465, Tables 3.12 and 3.13 .

Start of release:

$0.0000 \mathrm{hr}$

Iodine fractions:

$$
1.0000
$$

Fraction of released iodine chemical form:

$$
\begin{array}{ll}
\text { Elemental: } & 0.0485 \\
\text { Organic: } & 0.0015 \\
\text { Aerosol: } & 0.9500
\end{array}
$$

Inventory:

Normalized MACCS sample 3412 MWth PWR core inventory

Reactor power:

1932 MWth

\section{Plant Model}

Containment volume:

$0.1730 \mathrm{E}+07 \mathrm{ft}^{3}$

Containment removal:

Aerosol removal determined by user-defined rates for natural deposition.

Control room volume:

$0.4238 \mathrm{E}+05 \mathrm{ft}^{3}$

Control room removal:

Recirculating filter \# 2

Environment

Containment leakage:

Filter \#1:

Filter \#2:

CR exhaust:

CR unfiltered inflow:

\section{Scenario Description}

Containment leak rate:

Natural deposition aerosol removal rate:

Filter \#1 flow rate:

Filter \#1 efficiencies:

Filter \#2 flow rate:
Air leakage

Filtered pathway

Recirculation filter

Air leakage

Air leakage

$0.1200 \% /$ day

$0.15 \mathrm{hr}^{-1}$

$3000 \mathrm{cfm}$

Elemental iodine $\quad 99.0000 \%$

Organic iodine $\quad 00.0000 \%$

Aerosols

$99.0000 \%$

$10000 \mathrm{cfm}$
to: Environment
to: Control room
to: Control room
to: Environment
to: Control Room 
Filter \#2 efficiencies:

Elemental iodine

$90.0000 \%$

Organic iodine

$00.0000 \%$

Aerosols

$90.0000 \%$

$C R$ unfiltered inflow:

$10 \mathrm{cfm}$

CR exhaust:

$3010 \mathrm{cfm}$

\section{Dose Parameters}

EAB X/Q values:

Time (hr) X/Q $\left(\mathrm{s} / \mathrm{m}^{3}\right)$

0.0000

$0.1000 \mathrm{E}-02$

2.0000

$0.0000 \mathrm{E}+00$

LPZ X/Q values:

Time (hr) X/Q $\left(\mathrm{s} / \mathrm{m}^{3}\right)$

0.0000

$0.1350 \mathrm{E}-03$

8.0000

$0.1000 \mathrm{E}-03$

24.0000

$0.5400 \mathrm{E}-04$

96.0000

$0.2200 \mathrm{E}-04$

Filter \#1 X/Q values:

$\begin{array}{ll}\text { Time }(\mathrm{hr}) & \mathrm{X} / \mathrm{Q}\left(\mathrm{s} / \mathrm{m}^{3}\right) \\ 0.0000 & 0.2200 \mathrm{E}-02 \\ 2.0000 & 0.1500 \mathrm{E}-02 \\ 8.0000 & 0.1300 \mathrm{E}-02 \\ 24.0000 & 0.8400 \mathrm{E}-03 \\ 96.0000 & 0.4800 \mathrm{E}-03\end{array}$

\section{Success Criteria}

All of the RADTRAD-calculated EAB, LPZ, and CR doses must equal the doses calculated by HABIT within $\pm 5.0 \%$.

\section{Acceptance Criteria}

All of the success criteria must be met or exceeded in order for the RADTRAD software to be acceptable to the NRC.

\section{Results}

\begin{tabular}{|l|l|c|c|}
\hline \multicolumn{2}{|l|}{ Test Case 9 } & RADTRAD & HABIT \\
\hline EAB & Thyroid (rem) & 477 & 477 \\
\hline & TEDE (rem) & 23.4 & 23.4 \\
\hline LPZ & Thyroid (rem) & 707 & 707 \\
\hline & TEDE (rem) & 54.4 & 54.3 \\
\hline CR & Thyroid (rem) & 179 & 178 \\
\hline & TEDE (rem) & 12.7 & 12.6 \\
\hline
\end{tabular}




\subsubsection{Acceptance Test Case 10}

\section{Problem Description}

PWR design (see Figure 1).

NUREG-1465 PWR release into containment.

Release begins at reactor shutdown.

In-containment aerosol removal via natural deposition using Powers model [Po95].

Containment leak to the environment.

Control room air filtration system (see Figure 2).

$\mathrm{EAB}, \mathrm{LPZ}$, and $\mathrm{CR}$ doses calculated.

\section{Source Term}

Release fractions and timing: NUREG-1465, Tables 3.12 and 3.13.

Start of release:

$0.0000 \mathrm{~h}$

Iodine fractions:

Fraction of released iodine chemical form:

$\begin{array}{ll}\text { Elemental: } & 0.0485 \\ \text { Organic: } & 0.0015 \\ \text { Aerosol: } & 0.9500\end{array}$

Inventory:

Normalized MACCS sample 3412 MWth PWR core inventory

Reactor power:

1932 MWth

\section{Plant Model}

Containment volume:

$0.1730 \mathrm{E}+07 \mathrm{ft}^{3}$

Containment removal:

Aerosol removal determined by Powers model for natural deposition.

Control room volume:

$0.4238 \mathrm{E}+05 \mathrm{ft}^{3}$

Control room removal:

Recirculating filter \# 2

Environment

Containment leakage:

Air leakage

Filtered pathway

Recirculation filter

Air leakage

Air leakage

$0.1200 \% /$ day

Reactor type $=$ PWR design basis accident

Best-estimate solution

Filter \#1 flow rate:

Filter \#1 efficiencies:

Filter \#2 flow rate:
$3000 \mathrm{cfm}$

Elemental iodine $\quad 99.0000 \%$

Organic iodine $\quad 00.0000 \%$

Aerosols

$99.0000 \%$
$10,000 \mathrm{cfm}$ 
Filter \#2 efficiencies:

$\begin{array}{ll}\text { Elemental iodine } & 90.0000 \% \\ \text { Organic iodine } & 00.0000 \% \\ \text { Aerosols } & 90.0000 \%\end{array}$

CR unfiltered inflow:

$10 \mathrm{cfm}$

CR exhaust:

$3010 \mathrm{cfm}$

\section{Dose Parameters}

$E A B X / Q$ values:

$\begin{array}{ll}\text { Time }(\mathrm{hr}) & \mathrm{X} / \mathrm{Q}\left(\mathrm{s} / \mathrm{m}^{3}\right) \\ 0.0000 & 0.1000 \mathrm{E}-02 \\ 2.0000 & 0.0000 \mathrm{E}+00\end{array}$

LPZ X/Q values:

$\begin{array}{ll}\text { Time }(\mathrm{hr}) & \mathrm{X} / \mathrm{Q}\left(\mathrm{s} / \mathrm{m}^{3}\right) \\ 0.0000 & 0.1350 \mathrm{E}-03 \\ 8.0000 & 0.1000 \mathrm{E}-03 \\ 24.0000 & 0.5400 \mathrm{E}-04 \\ 96.0000 & 0.2200 \mathrm{E}-04\end{array}$

Filter \#1 X/Q values:

$\begin{array}{ll}\text { Time }(\mathrm{hr}) & \mathrm{X} / \mathrm{Q}\left(\mathrm{s} / \mathrm{m}^{3}\right) \\ 0.0000 & 0.2200 \mathrm{E}-02 \\ 2.0000 & 0.1500 \mathrm{E}-02 \\ 8.0000 & 0.1300 \mathrm{E}-02 \\ 24.0000 & 0.8400 \mathrm{E}-03 \\ 96.0000 & 0.4800 \mathrm{E}-03\end{array}$

\section{Success Criteria}

Deposition rate should be found to be reasonable.

\section{Acceptance Criteria}

If the success criteria not met in the RADTRAD version under test, the software will be still be acceptable to the NRC. However, SNL will be responsible for determining the cause of the failure and correction will be expected before project closeout.

\section{Results}

\begin{tabular}{|l|l|c|c|}
\hline Test Case 10 & RADTRAD & $\begin{array}{c}\text { FADTRAD } \\
\text { CASE 9 }\end{array}$ \\
\hline EAB & Thyroid (rem) & 504 & 477 \\
\hline & TEDE (rem) & 24.7 & 23.4 \\
\hline LPZ & Thyroid (rem) & 781 & 707 \\
\hline & TEDE (rem) & 58 & 54.4 \\
\hline CR & Thyroid (rem) & 183 & 179 \\
\hline & TEDE (rem) & 12.9 & 12.7 \\
\hline
\end{tabular}

The results indicate more early aerosol deposition with the Powers natural deposition model, resulting in lower doses to the environment. 


\subsubsection{Acceptance Test Case 10A}

\section{Problem Description}

PWR design (see Figure 1).

NUREG-1465 PWR release into containment.

Release begins at reactor shutdown.

In-containment aerosol removal via natural deposition using Henry's correlation [Fa84].

Containment leak to the environment.

Control room air filtration system (see Figure 2).

EAB, LPZ, and CR doses calculated.

\section{Source Term}

Release fractions and timing: NUREG-1465, Tables 3.12 and 3.13.

Start of release:

Iodine fractions:

Inventory:

Reactor power:

\section{Plant Model}

Containment volume:

Containment removal:

Control room volume:

Control room removal:

Environment

Containment leakage:

Filter \#1:

Filter \#2:

CR exhaust:

CR unfiltered inflow:

\section{Scenario Description}

Containment leak rate:

Henry's natural deposition parameters:

Filter \#1 flow rate:

Filter \#1 efficiencies:

Filter \#2 flow rate:
$0.0000 \mathrm{hr}$

1.0000

Fraction of released iodine chemical form:

$\begin{array}{ll}\text { Elemental: } & 0.0485 \\ \text { Organic: } & 0.0015 \\ \text { Aerosol: } & 0.9500\end{array}$

Normalized MACCS sample 3412 MWth PWR core inventory 1932 MWth

\section{$0.1730 \mathrm{E}+07 \mathrm{ft}^{3}$}

Aerosol removal determined by for Henry's correlation for natural deposition.

$0.4238 \mathrm{E}+05 \mathrm{ft}^{3}$

Recirculating filter \# 2.
Air leakage

Filtered pathway

Recirculation filter

Air leakage

Air leakage

$0.1200 \% /$ day
from: Containment
to: Environment
from: Environment
to: Control room
from: Control room
to: Control room
from: Control room
to: Environment
from: Environment
to: Control Room

Particle theoretical density $=1.0 \mathrm{~g} / \mathrm{cm}^{3}\left(2.27 \mathrm{~g} / \mathrm{cm}^{3}=\right.$ reference value $)$

$3000 \mathrm{cfm}$

Elemental iodine

Organic iodine

Aerosols

$99.0000 \%$

$00.0000 \%$

$99.0000 \%$

$10000 \mathrm{cfm}$ 
Filter \#2 efficiencies:

CR unfiltered inflow:

CR exhaust:

\section{Dose Parameters}

$\mathrm{EAB} X / Q$ values:

PZ X/Q values: $\begin{array}{ll}\text { Elemental iodine } & 90.0000 \% \\ \text { Organic iodine } & 00.0000 \% \\ \text { Aerosols } & 90.0000 \%\end{array}$

$10 \mathrm{cfm}$

$3010 \mathrm{cfm}$
Time (hr) X/Q $\left(\mathrm{s} / \mathrm{m}^{3}\right)$
$0.0000 \quad 0.1000 \mathrm{E}-02$
$2.0000 \quad 0.0000 \mathrm{E}+00$
Time (hr) X/Q $\left(\mathrm{s} / \mathrm{m}^{3}\right)$
$0.0000 \quad 0.1350 \mathrm{E}-03$
$8.0000 \quad 0.1000 \mathrm{E}-03$
$24.0000 \quad 0.5400 \mathrm{E}-04$
$96.0000 \quad 0.2200 \mathrm{E}-04$
Time (hr) X/Q $\left(\mathrm{s} / \mathrm{m}^{3}\right)$
$0.0000 \quad 0.2200 \mathrm{E}-02$
$2.0000 \quad 0.1500 \mathrm{E}-02$
$8.0000 \quad 0.1300 \mathrm{E}-02$
$24.0000 \quad 0.8400 \mathrm{E}-03$
$96.0000 \quad 0.4800 \mathrm{E}-03$

\section{Success Criteria}

Deposition rate should be found to be reasonable.

\section{Acceptance Criteria}

If the success criteria are not met in the RADTRAD version under test, the software will be still be acceptable to the NRC. However, SNL will be responsible for determining the cause of the failure and correction will be expected before project closeout.

\section{Results}

\begin{tabular}{|l|l|c|c|c|}
\hline Test Case 10A & $\begin{array}{c}\text { RADTRAD } \\
\text { Case 10A }\end{array}$ & $\begin{array}{c}\text { RADTRAD } \\
\text { Case 9 }\end{array}$ & \multicolumn{2}{c|}{$\begin{array}{c}\text { RADTRAD 10 } \\
\text { Case 10 }\end{array}$} \\
\hline EAB & Thyroid (rem) & 474 & 477 & 504 \\
\hline & TEDE (rem) & 23.2 & 23.4 & 24.7 \\
\hline LPZ & Thyroid (rem) & 690 & 707 & 581 \\
\hline & TEDE (rem) & 53.6 & 54.4 & 183 \\
\hline CR & Thyroid (rem) & 179 & 179 & 12.9 \\
\hline & TEDE (rem) & 12.7 & 12.7 & 58 \\
\hline
\end{tabular}

The results indicate less aerosol deposition overall with same residual aerosol at 30 days, resulting in somewhat higher doses for Henry's correlation compared with the Powers model (case 10) and the constant rate model (case 9). 


\subsubsection{Acceptance Test Case 11}

\section{Problem Description}

BWR-Mark II containment (see Figure 3).

Instantaneous, modified TID-14844 $\left({ }^{131}\right.$ I only) release into containment.

Release begins at reactor shutdown.

No explicit calculation of removal mechanisms in the containment, but $50 \%$ of the released iodine is deposited in accordance with NRC Regulatory Guide 1.3.

Containment leak into condenser via main steam line, condenser leak to the environment.

$\mathrm{EAB}$ and $\mathrm{LPZ}$ doses calculated.

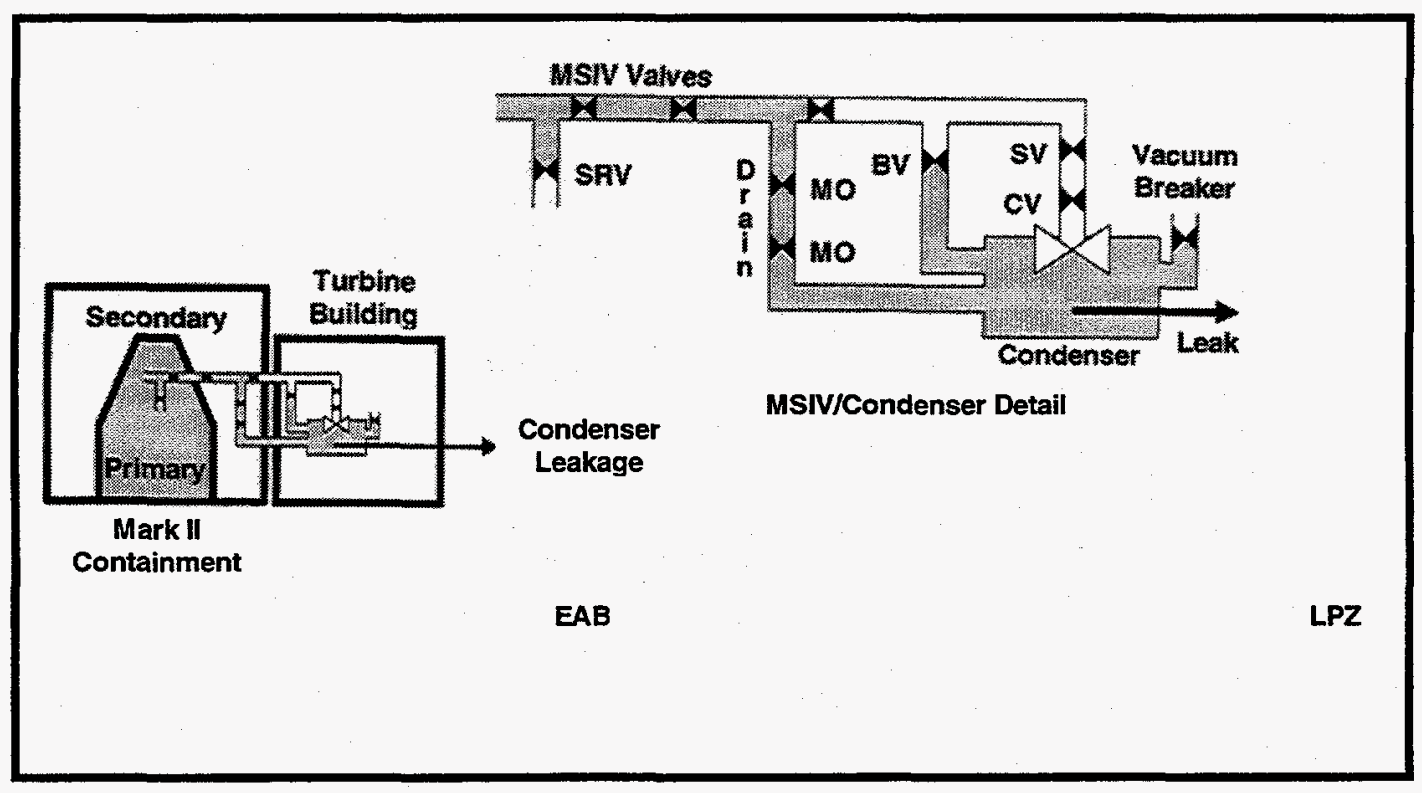

Figure 3. BWR-Mark II Containment MSIV/Condenser Leak

\section{Source Term}

Release fractions and timing: TID-14844, Table IV with modifications per NRC Regulatory Guide 1.3 and further modified as described below.

Start of release: $\quad 0.0000 \mathrm{hr}$

Iodine fractions:

Fraction of released iodine chemical form:

$\begin{array}{ll}\text { Elemental: } & 0.9100 \\ \text { Organic: } & 0.0400 \\ \text { Aerosol: } & 0.0500\end{array}$

Inventory: TID-14844 example normalized core inventory modified as follows: ${ }^{131}$ I only

Reactor power: $\quad 3441$ MWth

\section{Plant Model}

Containment volume: $\quad 0.389 \mathrm{E}+06 \mathrm{ft}^{3}$

Effective condenser volume: $\quad 0.986 \mathrm{E}+05 \mathrm{ft}^{3}$

Environment 
Containment leakage:

Condenser leakage:

Scenario Description

Containment leak rate:

Condenser leak rate:

\section{Dose Parameters}

EAB X/Q values:

$\begin{array}{lll}0.0000 & 0.1100 \mathrm{E}-02 \\ 2.0000 & 0.0000 \mathrm{E}+00 \\ & \text { Time }(\mathrm{hr}) & \mathrm{X} / \mathrm{Q}\left(\mathrm{s} / \mathrm{m}^{3}\right) \\ & 0.0000 & 0.5200 \mathrm{E}-04 \\ 8.0000 & 0.3600 \mathrm{E}-04 \\ 24.0000 & 0.1600 \mathrm{E}-04 \\ 96.0000 & 0.5300 \mathrm{E}-05\end{array}$

$$
\begin{aligned}
& 1.85 \% / \text { day } \\
& 0.7666 \mathrm{cfm}
\end{aligned}
$$

\section{Success Criteria}

$\begin{array}{lll}\text { Piping } & \text { from: Containment } & \text { to: Condenser } \\ \text { Air leakage } & \text { from: Condenser } & \text { to: Environment }\end{array}$

All of the RADTRAD-calculated EAB and LPZ doses must equal the doses calculated by HABIT within $\pm 5.0 \%$.

\section{Acceptance Criteria}

All of the success criteria must be met or exceeded in order for the RADTRAD software to be acceptable to the NRC.

\section{Results}

\begin{tabular}{|l|l|r|r|}
\hline \multicolumn{2}{|l|}{ Test Case 11 } & RADTRAD & HABIT \\
\hline EAB & Thyroid (rem) & 6.22 & 6.26 \\
\hline & TEDE (rem) & 0.191 & 0.192 \\
\hline LPZ & Thyroid (rem) & 2054 & 2050 \\
\hline & TEDE (rem) & 63.1 & 63.1 \\
\hline
\end{tabular}




\subsubsection{Acceptance Test Case 12}

\section{Problem Description}

BWR-Mark II containment (see Figure 3).

Instantaneous, modified TID-14844 ( ${ }^{131}$ I only) release into containment.

Release begins at reactor shutdown.

No explicit calculation of removal mechanisms in the containment, but $50 \%$ of the released iodine is deposited in accordance with NRC Regulatory Guide 1.3.

Containment leak into condenser via main steam line, condenser leak to the environment.

Control room air filtration system (see Figure 4).

$\mathrm{EAB}, \mathrm{LPZ}$, and $\mathrm{CR}$ doses calculated.

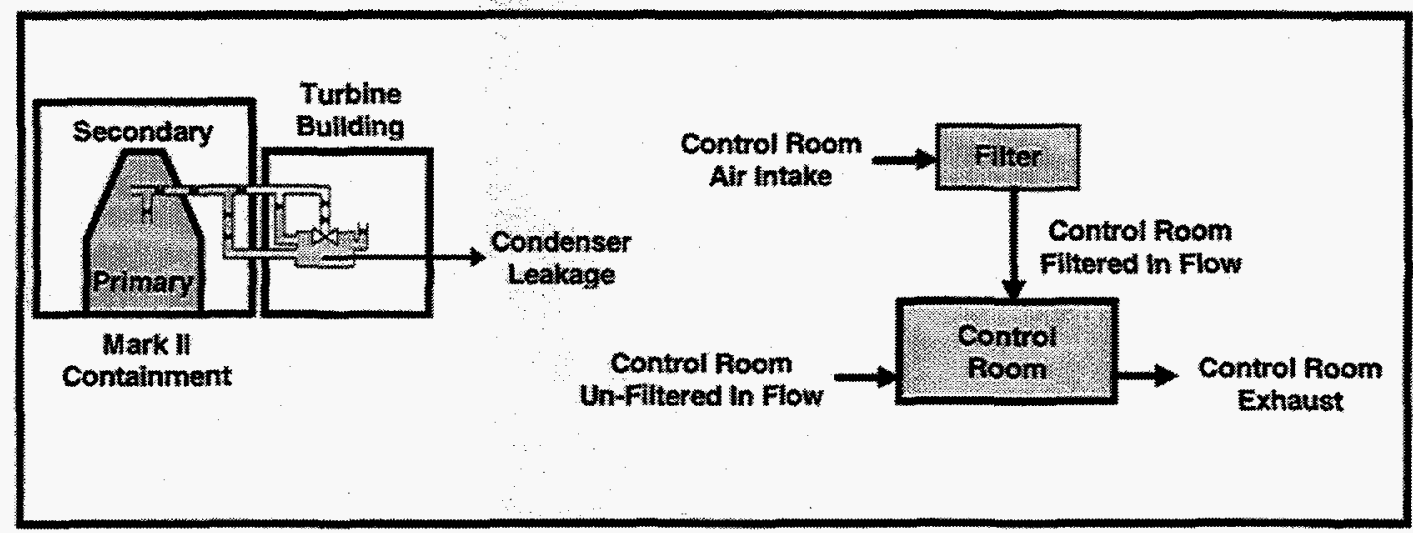

Figure 4. BWR-Mark II Containment Control Room Detail

\section{Source Term}

Release fractions and timing: TID-14844, Table IV with modifications per NRC Regulatory Guide 1.3 and further modified as described below.

Start of release: $\quad 0.0000 \mathrm{hr}$

Iodine fractions:

Fraction of released iodine chemical form:

$\begin{array}{ll}\text { Elemental: } & 0.9100 \\ \text { Organic: } & 0.0400 \\ \text { Aerosol: } & 0.0500\end{array}$

Inventory:

TID-14844 example normalized core inventory modified as follows:

${ }^{131}$ I only

Reactor power: $\quad 3441 \mathrm{MW}$ th

\section{Plant Model}

Containment volume: $\quad 0.3892 \mathrm{E}+06 \mathrm{ft}^{3}$

Effective condenser volume: $\quad 0.9860 \mathrm{E}+05 \mathrm{ft}^{3}$

Control room volume:

$0.1100 \mathrm{E}+06 \mathrm{ft}^{3}$

Environment

Containment leakage: Piping

Condenser leakage:

Air leakage

Filter:

Filtered pathway

from: Containment

from: Condenser

from: Environment to: Condenser

to: Environment

to: Control room 

CR unfiltered inflow:
Forced air
from: Environment
to: Control room
CR exhaust:
Air leakage
from: Control room
to: Environment

\section{Scenario Description}

Containment leak rate:

$1.851 \% /$ day

Condenser leak rate:

$0.7666 \mathrm{cfm}$

Filter flow rate:

$5800 \mathrm{cfm}$

Filter efficiencies:

Elemental iodine

Organic iodine

Aerosols

$99.0000 \%$

$0.0000 \%$

$10 \mathrm{cfm}$

CR exhaust:

$5810 \mathrm{cfm}$

\section{Dose Parameters}

$\mathrm{EAB} X / \mathrm{Q}$ values:

Time (hr) X/Q $\left(\mathrm{s} / \mathrm{m}^{3}\right)$

$0.0000 \quad 0.1100 \mathrm{E}-02$

$2.0000 \quad 0.0000 \mathrm{E}+00$

LPZ X/Q values:

Time (hr) $\quad \mathrm{X} / \mathrm{Q}\left(\mathrm{s} / \mathrm{m}^{3}\right)$

$0.0000 \quad 0.5200 \mathrm{E}-04$

$8.0000 \quad 0.3600 \mathrm{E}-04$

$24.0000 \quad 0.1600 \mathrm{E}-04$

$96.0000 \quad 0.5300 \mathrm{E}-05$

Filter \#1 X/Q values:

$\begin{array}{ll}\text { Time (hr) } & \text { X/Q }\left(\mathrm{s} / \mathrm{m}^{3}\right) \\ 0.0000 & 0.3320 \mathrm{E}-03 \\ 8.0000 & 0.1960 \mathrm{E}-03 \\ 24.0000 & 0.7640 \mathrm{E}-04 \\ 96.0000 & 0.2190 \mathrm{E}-04\end{array}$

\section{Success Criteria}

All of the RADTRAD-calculated EAB, LPZ, and CR doses must equal the doses calculated by HABIT within \pm $5.0 \%$.

\section{Acceptance Criteria}

All of the success criteria must be met or exceeded in order for the RADTRAD software to be acceptable to the NRC. 


\section{Results}

\begin{tabular}{|l|l|r|r|}
\hline Test Case 12 & & RADTRAD & \multicolumn{1}{|c|}{ HABIT } \\
\hline EAB & Thyroid (rem) & 6.22 & 6.26 \\
\hline & TEDE (rem) & .191 & 0.192 \\
\hline LPZ & Thyroid (rem) & 2054 & 2050 \\
\hline & TEDE (rem) & 63.1 & 63.1 \\
\hline CR & Thyroid (rem) & 279 & 277 \\
\hline & TEDE (rem) & 8.5 & 8.44 \\
\hline
\end{tabular}




\subsubsection{Acceptance Test Case 13}

\section{Problem Description}

BWR-Mark II containment (see Figure 3).

Instantaneous, modified TID-14844 ( ${ }^{131}$ I only) release into containment.

Release begins at reactor shutdown.

No explicit calculation of removal mechanisms in the containment, but $50 \%$ of the released iodine is deposited in accordance with NRC Regulatory Guide 1.3.

Pipe deposition with user-defined deposition rates as determined by Cline's model.

Containment leak into condenser via main steam line, condenser leak to the environment.

Control room air filtration system (see Figure 4).

$\mathrm{EAB}, \mathrm{LPZ}$, and CR doses calculated.

\section{Source Term}

Release fractions and timing: TID-14844, Table IV with modifications per NRC Regulatory Guides 1.3 and 1.4 and further modified as described below.

Start of release:

$0.0000 \mathrm{hr}$

Iodine fractions:

$$
1.0000
$$

Fraction of released iodine chemical form:

$\begin{array}{ll}\text { Elemental: } & 0.8760 \\ \text { Organic: } & 0.0740 \\ \text { Aerosol: } & 0.0500\end{array}$

Inventory:

TID-14844 example normalized core inventory modified as follows:

${ }^{131}$ I only

Reactor power: $\quad 3441$ MWth

Plant Model

Containment volume: $\quad 0.3892 \mathrm{E}+06 \mathrm{ft}^{3}$

Effective condenser volume: $\quad 0.9860 \mathrm{E}+05 \mathrm{ft}^{3}$

Control room volume: $\quad 0.1100 \mathrm{E}+06 \mathrm{ft}^{3}$

Environment

Containment leakage:

Piping

Condenser leakage:

Air leakage

Filter:

Filtered pathway

from: Containment

to: Condenser

$\mathrm{CR}$ unfiltered inflow:

Forced air from: Condenser

to: Environment

CR exhaust:

Air leakage

from: Environment

to: Control room

from: Environment

to: Control room

\section{Scenario Description}

Containment leak rate:

$1.85 \% /$ day

Condenser leak rate:

$0.7666 \mathrm{cfm}$

to: Environment 
Pipe deposition:

\begin{tabular}{lllll} 
Time & \multicolumn{4}{l}{ Decontamination factors } \\
Hours & Aerosiciency) & Elemental & Organic & \\
$0-40$ & 900. & 900. & 1.660 & $(40 \%)$ \\
$40-100$ & 900. & 900. & 1.428 & $(30 \%)$ \\
$100-200$ & 900. & 900. & 1.176 & $(15 \%)$ \\
$200-400$ & 900. & 900. & 1.053 & $(5 \%)$ \\
$400-$ & 900. & 900. & 1.0 & $(0 \%)$
\end{tabular}

Filter flow rate:

$5800 \mathrm{cfm}$

Filter efficiencies:

Elemental iodine Organic iodine Aerosols

$99.0000 \%$

$0.0000 \%$

$99.0000 \%$

$\mathrm{CR}$ unfiltered inflow:

$10 \mathrm{cfm}$

CR exhaust:

$5810 \mathrm{cfm}$

\section{Dose Parameters}

$\mathrm{EAB} X / \mathrm{Q}$ values:

$\begin{array}{ll}\text { Time }(\mathrm{hr}) & \mathrm{X} / \mathrm{Q}\left(\mathrm{s} / \mathrm{m}^{3}\right) \\ 0.0000 & 0.1100 \mathrm{E}-02 \\ 2.0000 & 0.0000 \mathrm{E}+00\end{array}$

LPZ X/Q values:

Time (hr) X/Q $\left(\mathrm{s} / \mathrm{m}^{3}\right)$

$0.0000 \quad 0.5200 \mathrm{E}-04$

$8.0000 \quad 0.3600 \mathrm{E}-04$

$24.0000 \quad 0.1600 \mathrm{E}-04$

$96.0000 \quad 0.5300 \mathrm{E}-05$

Filter \# $1 \mathrm{X} / \mathrm{Q}$ values:

$\begin{array}{ll}\text { Time }(\mathrm{hr}) & \mathrm{X} / \mathrm{Q}\left(\mathrm{s} / \mathrm{m}^{3}\right) \\ 0.0000 & 0.3320 \mathrm{E}-03 \\ 8.0000 & 0.1960 \mathrm{E}-03 \\ 24.0000 & 0.7640 \mathrm{E}-04 \\ 96.0000 & 0.2190 \mathrm{E}-04\end{array}$

\section{Success Criteria}

All of the RADTRAD-calculated EAB, LPZ, and CR doses must equal the doses calculated by HABIT within \pm $5.0 \%$.

\section{Acceptance Criteria}

All of the success criteria must be met or exceeded in order for the RADTRAD software to be acceptable to the NRC. 


\section{Results}

\begin{tabular}{|l|l|c|c|}
\hline \multicolumn{2}{|l|}{ Test Case 13 } & RADTRAD & HABIT \\
\hline EAB & Thyroid (rem) & 0.283 & 0.277 \\
\hline & TEDE (rem) & 0.0087 & 0.0085 \\
\hline LPZ & Thyroid (rem) & 147 & 145 \\
\hline & TEDE (rem) & 4.52 & 4.45 \\
\hline CR & Thyroid (rem) & 376 & 372 \\
\hline & TEDE (rem) & 11.5 & 11.3 \\
\hline
\end{tabular}




\subsubsection{Acceptance Test Case 13B}

\section{Problem Description}

BWR-Mark II containment (see Figure 3).

Modified NUREG-1465 BWR ( ${ }^{131}$ I only) release into containment.

Release begins at reactor shutdown.

Pipe deposition with user-defined deposition rates as determined by Cline's model [C191].

Containment leak into condenser via main steam line, condenser leak to the environment.

Control room air filtration system (see Figure 4).

$\mathrm{EAB}, \mathrm{LPZ}$, and CR doses calculated.

\section{Source Term}

Release fractions and timing: NUREG-1465 Tables 3.11 and 3.13 with modifications.

Start of release:

$0.0000 \mathrm{hr}$

Iodine fractions:

Fraction of released iodine chemical form:

$\begin{array}{ll}\text { Elemental: } & 0.0128 \\ \text { Organic: } & 0.0372 \\ \text { Aerosol: } & 0.9500\end{array}$

Inventory:

Normalized MACCS sample 3578 MWth BWR core inventory modified as follows:

${ }^{131}$ I only

Reactor power:

3441 MWth

Plant Model

Containment volume: $\quad 0.3892 \mathrm{E}+06 \mathrm{ft}^{3}$

Effective condenser volume: $\quad 0.9860 \mathrm{E}+05 \mathrm{ft}^{3}$

Control room volume: $\quad 0.1100 \mathrm{E}+06 \mathrm{ft}^{3}$

Environment

Containment leakage:

Piping

Air leakage

Condenser leakage:

Filtered pathway

to: Condenser

Filter:

Forced air

from: Environment

to: Environment

CR unfiltered inflow:

Air leakage

from: Environment

to: Control room

CR exhaust:

from: Control room

to: Control room

to: Environment

\section{Scenario Description}

Containment leak rate:

$1.851 \% /$ day

Condenser leak rate:

$0.7666 \mathrm{cfm}$ 
Pipe deposition:

\begin{tabular}{|c|c|c|c|c|}
\hline Time & \multicolumn{2}{|c|}{ Decontamination Factors } & (Efficien & \\
\hline (hr) & Aerosol & Elemental & Organic & \\
\hline $0-48$ & 900. & 900. & 5.555 & $(82 \%)$ \\
\hline $48-96$ & 900. & 900. & 2.707 & $(63 \%)$ \\
\hline $96-200$ & 900. & 900. & 1.639 & $(39 \%)$ \\
\hline $200-300$ & 900. & 900. & 1.219 & $(18 \%)$ \\
\hline 300 & 900. & 900. & 1.0417 & $(4 \%)$ \\
\hline
\end{tabular}

Filter flow rate:

$5800 \mathrm{cfm}$

Filter efficiencies:

Elemental iodine $\quad 99.0000 \%$

Organic iodine $\quad 0.0000 \%$

Aerosols

$99.0000 \%$

CR unfiltered inflow: $\quad 10 \mathrm{cfm}$

CR exhaust:

$5810 \mathrm{cfm}$

\section{Dose Parameters}

EAB X/Q values:

$\begin{array}{ll}\text { Time }(\mathrm{hr}) & \mathrm{X} / \mathrm{Q}\left(\mathrm{s} / \mathrm{m}^{3}\right) \\ 0.0000 & 0.1100 \mathrm{E}-02 \\ 2.0000 & 0.0000 \mathrm{E}+00 \\ \text { Time }(\mathrm{hr}) & \left.\mathrm{X} / \mathrm{(s} / \mathrm{m}^{3}\right) \\ 0.0000 & 0.5200 \mathrm{E}-04 \\ 8.0000 & 0.3600 \mathrm{E}-04 \\ 24.0000 & 0.1600 \mathrm{E}-04 \\ 96.0000 & 0.5300 \mathrm{E}-05 \\ \text { Time }(\mathrm{hr}) & \mathrm{X} / \mathrm{Q}\left(\mathrm{s} / \mathrm{m}^{3}\right) \\ 0.0000 & 0.3320 \mathrm{E}-03 \\ 8.0000 & 0.1960 \mathrm{E}-03 \\ 24.0000 & 0.7640 \mathrm{E}-04 \\ 96.0000 & 0.2190 \mathrm{E}-04\end{array}$

\section{Success Criteria}

All of the RADTRAD-calculated EAB, LPZ, and CR doses must equal the doses calculated by HABIT within \pm $5.0 \%$.

\section{Acceptance Criteria}

All of the success criteria must be met or exceeded in order for the RADTRAD software to be acceptable to the NRC. 
Results

\begin{tabular}{|l|l|c|c|}
\hline \multicolumn{2}{|l|}{ Test Case 13B } & RADTRAD & HABIT \\
\hline EAB & Thyroid (rem) & 0.0175 & 0.0151 \\
\hline & TEDE (rem) & 0.00040 & 0.00046 \\
\hline LPZ & Thyroid (rem) & 87.0 & 86.5 \\
\hline & TEDE (rem) & 2.67 & 2.6 \\
\hline CR & Thyroid (rem) & 213 & 211 \\
\hline & TEDE (rem) & 6.48 & 6.44 \\
\hline
\end{tabular}




\subsubsection{Acceptance Test Case 14}

\section{Problem Description}

BWR-Mark II containment (see Figure 3).

Instantaneous, modified TID-14844 ( ${ }^{131}$ I only) release into containment.

Release begins at reactor shutdown.

No explicit calculation of removal mechanisms in the containment, but $50 \%$ of the released iodine is deposited in accordance with NRC Regulatory Guide 1.3.

Pipe deposition using the Brockmann [Br91] and Bixler [Bi95] models.

Containment leak into condenser via main steam line, condenser leak to the environment.

Control room air filtration system (see Figure 4).

EAB, LPZ, and CR doses calculated.

\section{Source Term}

Release fractions and timing: TID-14844, Table IV per NRC Regulatory Guide 1.4 modifications and further modified as described below.

Start of release:

$0.0000 \mathrm{hr}$

Iodine fractions:

$$
1.0000
$$

Fraction of released iodine chemical form:

$$
\begin{array}{ll}
\text { Elemental: } & 0.9100 \\
\text { Organic: } & 0.0400 \\
\text { Aerosol: } & 0.0500
\end{array}
$$

Inventory: $\quad$ TID-14844 example normalized core inventory modified as follows:

$$
{ }^{131} \text { I only }
$$

Reactor power: $\quad 3441$ MWth

\section{Plant Model}

Containment volume:

$03892 \mathrm{E}+06 \mathrm{ft}^{3}$

Effective condenser volume:

$09860 \mathrm{E}+05 \mathrm{ft}^{3}$

Control room volume:

$01100 \mathrm{E} \div 06 \mathrm{ft}^{3}$

Environment

Containment leakage:

Piping

Condenser leakage:

Air leakage

from: Containment

to: Condenser

Filter:

Filtered pathway

from: Condenser

to: Environment

$\mathrm{CR}$ unfiltered inflow:

Forced air

from: Environment

to: Control room

CR exhaust:

Air leakage

from: Environment

to: Control room

from: Control room

to: Environment

\section{Scenario Description}

Containment leak rate:

\section{$1.851 \% /$ day}

Condenser leak rate:

$0.7666 \mathrm{cfm}$

Pipe deposition:

Pipe surface area $=500,000 \mathrm{ft}^{2}$

Pipe volume $=98,6011 \mathrm{ft}^{3}$

Pipe flow $=0.767 \mathrm{cfm}$

Pipe pressure $=1.0 \mathrm{~atm}$

Pipe temperature $=548^{\circ} \mathrm{F}$

Filter flow rate:

$5800 \mathrm{cfm}$ 
Filter efficiencies:

Elemental iodine

$99.0000 \%$

Organic iodine

$0.0000 \%$

Aerosols

$99.0000 \%$

CR unfiltered inflow:

$10 \mathrm{cfm}$

CR exhaust:

$5810 \mathrm{cfm}$

\section{Dose Parameters}

$\mathrm{EAB} X / \mathrm{Q}$ values:

Time (hr) X/Q $\left(\mathrm{s} / \mathrm{m}^{3}\right)$

$0.0000 \quad 0.1100 \mathrm{E}-02$

$2.0000 \quad 0.0000 \mathrm{E}+00$

LPZ X/Q values:

Time (hr) X/Q $\left(\mathrm{s} / \mathrm{m}^{3}\right)$

$0.0000 \quad 0.5200 \mathrm{E}-04$

$8.0000 \quad 0.3600 \mathrm{E}-04$

$24.0000 \quad 0.1600 \mathrm{E}-04$

$96.0000 \quad 0.5300 \mathrm{E}-05$

Filter \#1 X/Q values: $\quad$ Time (hr) X/Q $\left(\mathrm{s} / \mathrm{m}^{3}\right)$

$0.0000 \quad 0.3320$ E-03

$8.0000 \quad 0.1960 \mathrm{E}-03$

$24.0000 \quad 0.7640 \mathrm{E}-04$

$96.0000 \quad 0.2190 \mathrm{E}-04$

\section{Success Criteria}

Deposition rate should be found to be reasonable

\section{Acceptance Criteria}

If the success criteria are not met in the RADTRAD version under test, the software will be still be acceptable to the NRC. However, SNL will be responsible for determining the cause of the failure and correction will be expected before project closeout.

\section{Results}

\begin{tabular}{|l|l|c|c|c|}
\hline \multicolumn{2}{|l|}{} & $\begin{array}{c}\text { RADTRAD } \\
\text { Case 14 }\end{array}$ & $\begin{array}{c}\text { RADTRAD } \\
\text { Case 12 }\end{array}$ & $\begin{array}{c}\text { RADTRAD } \\
\text { Case 13 }\end{array}$ \\
\hline EAB & Thyroid (rem) & 0.118 & 6.22 & 0.283 \\
\hline & TEDE (rem) & 0.003 & 0.191 & 0.0087 \\
\hline LPZ & Thyroid (rem) & 39.00 & 2054 & 147 \\
\hline & TEDE (rem) & 1.2 & 63.1 & 4.52 \\
\hline CR & Thyroid (rem) & 97.6 & 279 & 376 \\
\hline & TEDE (rem) & 3.00 & 8.5 & 11.5 \\
\hline
\end{tabular}

The results of case 12 are dominated by the elemental iodine release. In cases 13 and 14 , where the elemental iodine is deposited on the pipe and condenser walls, the dose falls significantly. In case 13 the deposited iodine is allowed to revaporize in an organic form, which is not trapped by filters; thus the dose in 13 is higher than 14. This effect also explains why the control room dose in case 13 is higher than case 12 and indicates that revaporization is an important phenomenon. 


\subsubsection{Acceptance Test Case 14B}

\section{Problem Description}

BWR-Mark II containment (see Figure 3).

Modified NUREG-1465 BWR ( ${ }^{131} \mathrm{I}$ only) release into containment.

Release begins at reactor shutdown.

Pipe deposition using the Brockmann [Br91] and Bixler [Bi95] models.

Containment leak into condenser via main steam line, condenser leak to the environment.

Control room air filtration system (see Figure 4).

$\mathrm{EAB}, \mathrm{LPZ}$, and CR doses calculated.

\section{Source Term}

Release fractions and timing: NUREG-1465 Tables 3.11 and 3.13 with modification as described below.

Start of release:

$0.0000 \mathrm{hr}$

Iodine fractions:

$$
1.0000
$$

Fraction of released iodine chemical form:

$\begin{array}{ll}\text { Elemental: } & 0.0485 \\ \text { Organic: } & 0.0015 \\ \text { Aerosol: } & 0.9500\end{array}$

Inventory:

Normalized MACCS sample 3578 MWth BWR core inventory modified as follows:

${ }^{131}$ I only

Reactor power: $\quad 3441 \mathrm{MWth}$

\section{Plant Model}

Containment volume: $\quad 0.3892 \mathrm{E}+06 \mathrm{ft}^{3}$

Effective condenser volume: $\quad 0.9860 \mathrm{E}+05 \mathrm{ft}^{3}$

Control room volume:

$0.1100 \mathrm{E}+06 \mathrm{ft}^{3}$

Environment

Containment leakage:

Piping

Air leakage

from: Containunent

to: Condenser

Condenser leakage:

Filtered pathway

from: Condenser

to: Environment

Filter:

Forced air

from: Environment

to: Control room

$\mathrm{CR}$ unfiltered inflow:

Air leakage

from: Environment

to: Control room

CR exhaust:

from: Control room

to: Environment

\section{Scenario Description}

Containment leak rate:

$1.85 \% /$ day

Condenser leak rate:

$0.7666 \mathrm{cfm}$

Pipe deposition:

Pipe surface area $=500,000 \mathrm{ft}^{2}$

Pipe volume $=98,6011 \mathrm{ft}^{3}$

Pipe flow $=0.766 \mathrm{cfm}$

Pipe pressure $=1.0 \mathrm{~atm}$

Pipe temperature $=548^{\circ} \mathrm{F}$

Filter flow rate:

$5800 \mathrm{cfm}$ 
Filter efficiencies:

Elemental iodine $\quad 99.0000 \%$

Organic iodine $\quad 0.0000 \%$

Aerosols

$99.0000 \%$

CR unfiltered inflow: $\quad 10 \mathrm{cfm}$

CR exhaust:

$5810 \mathrm{cfm}$

\section{Dose Parameters}

$\mathrm{EAB} X / Q$ values:

$$
\begin{array}{ll}
\text { Time }(\mathrm{hr}) & \mathrm{X} / \mathrm{Q}\left(\mathrm{s} / \mathrm{m}^{3}\right) \\
0.0000 & 0.1100 \mathrm{E}-02 \\
2.0000 & 0.0000 \mathrm{E}+00
\end{array}
$$

LPZ X/Q values:

Time (hr) X/Q $\left(\mathrm{s} / \mathrm{m}^{3}\right)$

$0.0000 \quad 0.5200 \mathrm{E}-04$

$8.0000 \quad 0.3600 \mathrm{E}-04$

$24.0000 \quad 0.1600 \mathrm{E}-04$

$96.0000 \quad 0.5300 \mathrm{E}-05$

Filter \#1 X/Q values: $\quad$ Time (hr) X/Q $\left(\mathrm{s} / \mathrm{m}^{3}\right)$

$0.0000 \quad 0.3320$ E-03

$8.0000 \quad 0.1960 \mathrm{E}-03$

$24.0000 \quad 0.7640 \mathrm{E}-04$

$96.0000 \quad 0.2190 \mathrm{E}-04$

\section{Success Criteria}

The deposition rate should be found to be reasonable.

\section{Acceptance Criteria}

If the success criteria are not met in the RADTRAD version under test, the software will be still be acceptable to the NRC. However, SNL will be responsible for determining the cause of the failure and correction will be expected before project closeout. 


\section{Results}

\begin{tabular}{|c|c|c|c|}
\hline & & $\begin{array}{l}\text { RADTRAD } \\
\text { Case 13B }\end{array}$ & $\begin{array}{c}\text { RADTRAD } \\
\text { Case 14B }\end{array}$ \\
\hline \multirow[t]{2}{*}{$\overline{\mathrm{EAB}}$} & Thyroid (rem) & 0.0175 & 0.0040 \\
\hline & TEDE (rem) & 0.00040 & 0.00012 \\
\hline \multirow[t]{2}{*}{$\overline{L P Z}$} & Thyroid (rem) & 87.1 & 4.59 \\
\hline & TEDE (rem) & 2.67 & 0.141 \\
\hline \multirow[t]{2}{*}{$\mathrm{CR}$} & Thyroid (rem) & 213. & 4.66 \\
\hline & TEDE (rem) & 6.48 & 0.142 \\
\hline $\begin{array}{l}\text { Pipe and } \\
\text { condenser }\end{array}$ & $\mathrm{I}_{2}$ (atom & $0.8787 e+21$ & $0.3326 e+22$ \\
\hline Deposition & Organic (atom) & $0.2431 \mathrm{e}+21$ & $0.6713 e+20$ \\
\hline$@ 720 \mathrm{hr}$ & Aerosol $(\mathrm{kg})$ & $0.1419 \mathrm{e}-01$ & $0.1417 e-01$ \\
\hline
\end{tabular}

Case 14B removes aerosols very similarly to case 13B; however, these scenarios are depositing materials not only on the steam line walls but also within the condenser. Thus where the Cline model assumes total aerosol deposition, the Brockmann model should get very similar results, just because of the very large surface areas involved. In cases where only the pipe is involved, aerosol deposition percentages are more like $70 \%$. As can be seen from this table, the elemental $\left(\mathrm{I}_{2}\right)$ depositions are also similar, the difference being the transformation of $\mathrm{I}_{2}$ into an organic form. Likewise, the total organic deposition is different due to the revaporization in the Cline model of case 13B. The total result is that the doses for case 13B are higher than those for case 14B. 


\subsubsection{Acceptance Test Case 15}

\section{Problem Description}

BWR-Mark II containment (see Figure 5).

Modified NUREG-1465 BWR ( ${ }^{131}$ I only) release into containment.

Release begins at reactor shutdown.

Auxiliary building exhaust filter \#1.

Containment leak into auxiliary building via ECCS leak; auxiliary building leak to the environment.

EAB and LPZ doses calculated.

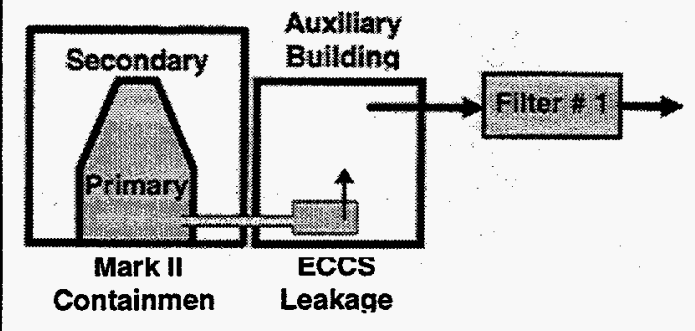

EAB

LPZ

Figure 5. BWR-Mark II Containment ECCS Leakage

\section{Source Term}

Release fractions and timing: NUREG-1465, Tables 3.11 and 3.13 with modifications as described below.

Start of release:

$0.0000 \mathrm{hr}$

Iodine fractions:

$$
1.0000
$$

Fraction of released iodine chemical form:

$\begin{array}{ll}\text { Elemental: } & 0.0485 \\ \text { Organic: } & 0.0015 \\ \text { Aerosol: } & 0.9500\end{array}$

Inventory:

Normalized MACCS sample 3578 MWth BWR core inventory modified as follows:

$$
{ }^{131} \text { I only }
$$

Reactor power: $\quad 3441$ MWth

\section{Plant Model}

Containment volume:

$0.3892 \mathrm{E}+06 \mathrm{ft}^{3}$

Auxiliary bldg. volume:

$0.1000 \mathrm{E}+07 \mathrm{ft}^{3}$

Environment

Containment leakage:

Piping

Filter \# 1:

Filtered Pathway

from: Containment

to: Auxiliary bldg.

from: Auxiliary bldg.

to: Environment 
Scenario Description

Containment leak rate:

$0.53 \% /$ day

Filter \# 1 flow rate:

$4000 \mathrm{cfm}$

Filter efficiencies:

$\begin{array}{ll}\text { Elemental iodine } & 99.0000 \% \\ \text { Organic iodine } & 0.0000 \% \\ \text { Aerosols } & 99.0000 \%\end{array}$

In this case an additional auxiliary building inlet was added to balance the exit airflow. This should cause a slightly higher dose to the auxiliary building. Based on the result, the added dose is negligible.

\section{Dose Parameters}

$\mathrm{EAB} X / \mathrm{Q}$ values:

$$
\begin{array}{ll}
\text { Time }(\mathrm{hr}) & \mathrm{X} / \mathrm{Q}\left(\mathrm{s} / \mathrm{m}^{3}\right) \\
0.0000 & 0.1100 \mathrm{E}-02 \\
2.0000 & 0.0000 \mathrm{E}+00 \\
\text { Time }(\mathrm{hr}) & \mathrm{X} / \mathrm{Q}\left(\mathrm{s} / \mathrm{m}^{3}\right) \\
0.0000 & 0.5200 \mathrm{E}-04 \\
8.0000 & 0.3600 \mathrm{E}-04 \\
24.0000 & 0.1600 \mathrm{E}-04 \\
96.0000 & 0.5300 \mathrm{E}-05
\end{array}
$$

LPZ X/Q values: $\quad$ Time (hr) $\quad \mathrm{X} / \mathrm{Q}\left(\mathrm{s} / \mathrm{m}^{3}\right)$

\section{Success Criteria}

All of the RADTRAD-calculated EAB and LPZ doses must equal the doses calculated by HABIT within $\pm 5.0 \%$.

\section{Acceptance Criteria}

All of the success criteria must be met or exceeded in order for the RADTRAD software to be acceptable to the NRC.

\section{Results}

\begin{tabular}{|l|l|c|c|}
\hline \multicolumn{2}{|l|}{ Test Case 15 } & RADTRAD & \multicolumn{1}{c|}{ HABIT } \\
\hline EAB & Thyroid (rem) & 3.41 & 3.41 \\
\hline & TEDE (rem) & 0.105 & 0.104 \\
\hline LPZ & Thyroid (rem) & 82.2 & 82.2 \\
\hline & TEDE (rem) & 2.53 & 2.53 \\
\hline
\end{tabular}




\subsubsection{Acceptance Test Case 16}

\section{Problem Description}

BWR-Mark II containment (see Figure 5).

Modified NUREG-1465 BWR $\left({ }^{131}\right.$ I only) release into containment.

Release begins at reactor shutdown.

Auxiliary building exhaust filter \#1.

Containment leak into auxiliary building via ECCS leak; auxiliary building leak to the environment.

Control room air filtration system (see Figure 6).

$\mathrm{EAB}, \mathrm{LPZ}$, and CR doses calculated.

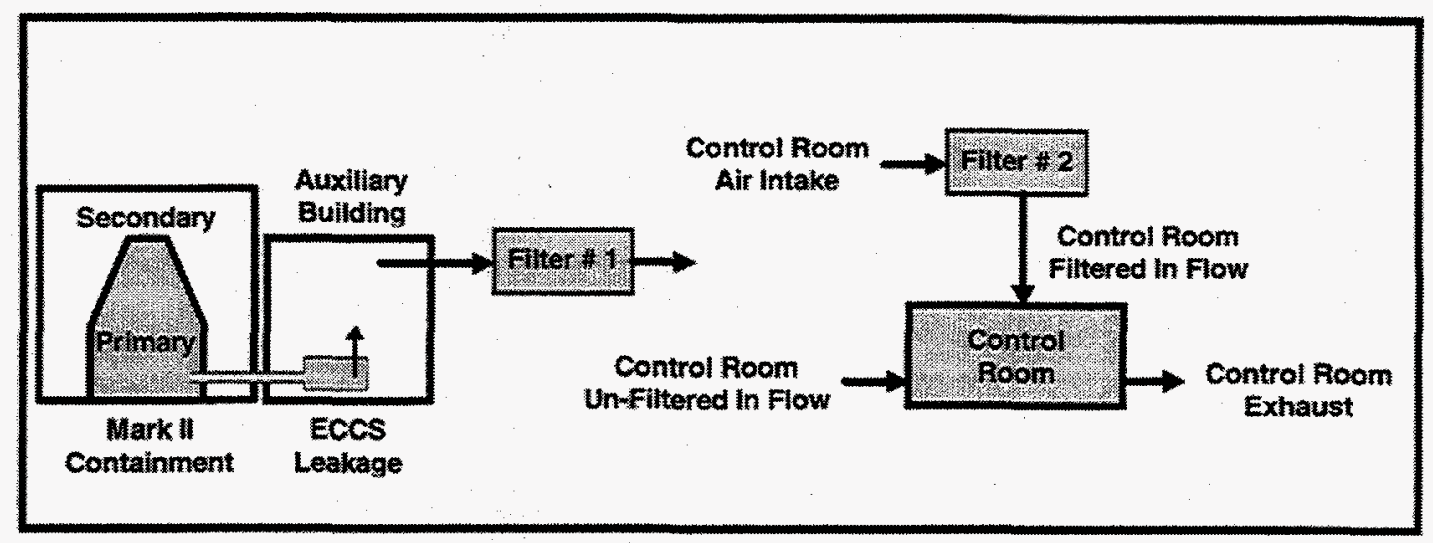

Figure 6. BWR-Mark II Containment Control Room Detail

\section{Source Term}

Release fractions and timing: NUREG-1465, Tables 3.11 and 3.13 with modifications as described below.

Start of release:

$0.0000 \mathrm{hr}$

Iodine fractions:

$$
1.0000
$$

Fraction of released iodine chemical form:

$$
\begin{array}{ll}
\text { Elemental: } & 0.0485 \\
\text { Organic: } & 0.0015 \\
\text { Aerosol: } & 0.9500
\end{array}
$$

Inventory:

Normalized MACCS sample 3578 MWth BWR core inventory modified as follows:

$$
{ }^{131} \text { I only }
$$

Reactor power:

$$
3441 \text { MWth }
$$

\section{Plant Model}

Containment volume: $\quad 0.3892 \mathrm{E}+06 \mathrm{ft}^{3}$

Auxiliary building volume: $\quad 0.1000 \mathrm{E}+07 \mathrm{ft}^{3}$

Control room volume:

$$
0.1100 \mathrm{E}+06 \mathrm{ft}^{3}
$$

Environment

Containment leakage:

\section{Piping}

Filtered pathway

Filtered pathway from: Containment

to: Auxiliary bldg.

from: Auxiliary bldg.

from: Environment to: Environment

to: Control room 
CR unfiltered inflow:

CR leakage:

\section{Scenario Description}

Containment leak rate:

Filter \#1 flow rate:

Filter \#1 efficiencies:

Filter \#2 flow rate:

Filter \#2 efficiencies:

CR unfiltered inflow:

CR exhaust:

\section{Dose Parameters}

EAB X/Q values:

LPZ X/Q values:

Filter \#2 X/Q values:
Forced air
from: Environment
to: Control room
Air leakage
from: Control room
to: Environment

$0.5300 \% /$ day

$4000 \mathrm{cfm}$

$\begin{array}{ll}\text { Elemental iodine } & 99.0000 \% \\ \text { Organic iodine } & 0.0000 \% \\ \text { Aerosols } & 99.0000 \%\end{array}$

$5800 \mathrm{cfm}$

$\begin{array}{ll}\text { Elemental iodine } & 99.0000 \% \\ \text { Organic iodine } & 0.0000 \% \\ \text { Aerosols } & 99.0000 \%\end{array}$

\section{$10 \mathrm{cfm}$}

$5810 \mathrm{cfm}$

Time (hr) X/Q $\left(\mathrm{s} / \mathrm{m}^{3}\right)$

$0.0000 \quad 0.1100 \mathrm{E}-02$

$2.0000 \quad 0.0000 \mathrm{E}+00$

$\begin{array}{ll}\text { Time }(\mathrm{hr}) & \mathrm{X} / \mathrm{Q}\left(\mathrm{s} / \mathrm{m}^{3}\right) \\ 0.0000 & 0.5200 \mathrm{E}-04 \\ 8.0000 & 0.3600 \mathrm{E}-04 \\ 24.0000 & 0.1600 \mathrm{E}-04 \\ 96.0000 & 0.5300 \mathrm{E}-05\end{array}$

\section{Success Criteria}

All of the RADTRAD-calculated EAB, LPZ, and CR doses must equal the doses calculated by HABIT within $\pm 5.0 \%$.

\section{Acceptance Criteria}

All of the success criteria must be met or exceeded in order for the RADTRAD software to be acceptable to the NRC. 


\section{Results}

\begin{tabular}{|l|l|c|c|}
\hline \multicolumn{2}{|l|}{ Test Case 16 } & RADTRAD & HABIT \\
\hline EAB & Thyroid (rem) & 3.41 & 3.41 \\
\hline & TEDE (rem) & .105 & .105 \\
\hline LPZ & Thyroid (rem) & 82.2 & 82.2 \\
\hline & TEDE (rem) & 2.53 & 2.53 \\
\hline CR & Thyroid (rem) & 44.8 & 44.2 \\
\hline & TEDE (rem & 1.37 & 1.35 \\
\hline
\end{tabular}




\subsubsection{Acceptance Test Case 17}

\section{Problem Description}

Acceptance test deleted per NRC's request.

\subsubsection{Acceptance Test Case 18}

\section{Problem Description}

Acceptance test deleted per NRC's request. 


\subsubsection{Acceptance Test Case 19}

\section{Problem Description}

Advanced PWR design (see Figure 7).

Modified NUREG-1465 PWR ( ${ }^{131}$ I only) release into sprayed ${ }^{1}$ containment region.

Release begins at reactor shutdown.

Containment annulus exhaust filter.

Sprayed containment region mixes with unsprayed region; both leak to the environment and annulus;

annulus also exhausts to the environment.

$\mathrm{EAB}$ and LPZ doses calculated.

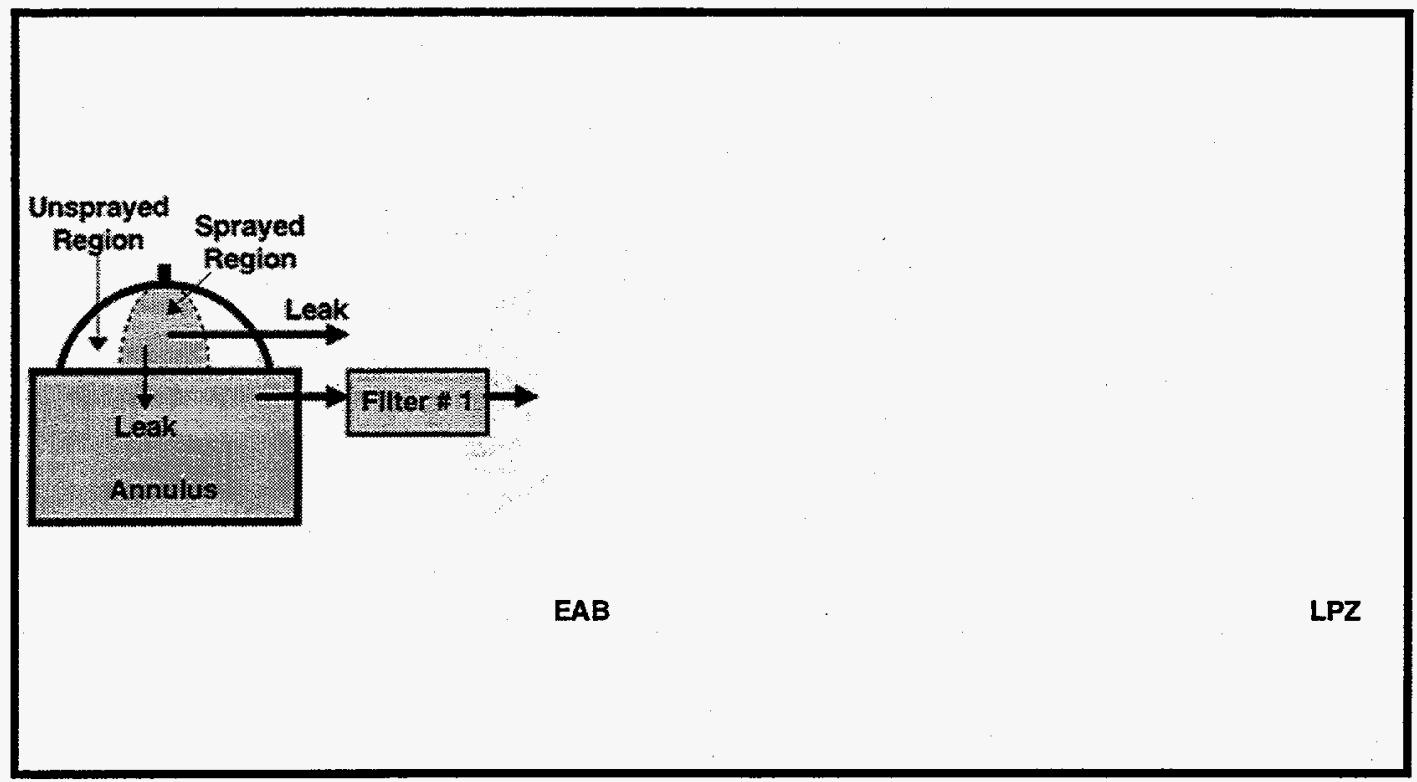

Figure 7. Advanced PWR Containment Leakage

\section{Source Term}

Release fractions and timing: NUREG-1465, Tables 3.12 and 3.13 with modifications as described below.

Start of release:

Iodine fractions:

Inventory:

Reactor power:

$$
0.0000 \mathrm{hr}
$$

Fraction of released iodine chemical form:

$\begin{array}{ll}\text { Elemental: } & 0.0485 \\ \text { Organic: } & 0.0015 \\ \text { Aerosol: } & 0.9500\end{array}$

Normalized MACCS sample 3412 MWth PWR core inventory modified as follows:

$$
{ }^{131} \text { I only. }
$$

3992 MWth

\footnotetext{
${ }^{1}$ For this and the next several test cases, the (un)sprayed containment region or (un)sprayed containment only refers to the containment region that can or cannot be sprayed. It does not indicate that sprays are being modeled. The sprays will be modeled in test cases 23 and 24 .
} 


\section{Plant Model}

Sprayed containment volume: $\quad 0.2740 \mathrm{E}+07 \mathrm{ft}^{3}$

Unsprayed containment volume: $\quad 0.6011 \mathrm{E}+06 \mathrm{ft}^{3}$

Annulus volume: $\quad 0.6162 \mathrm{E}+06 \mathrm{ft}^{3}$

Environment

Sprayed containment leakage:
Bypass:
Air leakage
from: Sprayed
to: Environment
Annulus:
Air leakage
from: Sprayed
to: Annulus

Unsprayed containment leakage:

Bypass:

Annulus:

Air leakage

Air leakage

Containment mixing:

Sprayed:

Unsprayed:

Filter \# 1:

\section{Scenario Description}

Containment mixing:

$\begin{array}{ll} & 0.000 \\ & 0.1667 \\ & 0.500 \\ & 1.175 \\ & 1.800 \\ & \text { Time (hr) } \\ & 0.0000 \\ \text { Sprayed zone leak rate: } & 24.0000 \\ & \text { Time (hr) } \\ \text { Unsprayed zone leak rate: } & 0.0000 \\ & 24.0000 \\ & \text { Time (hr) } \\ \text { Annulus exhaust: } & 0.0000 \\ & 0.0278 \\ & 0.2778\end{array}$

Filter \#1 efficiencies:

$\begin{array}{ll}\text { Elemental iodine } & 0.0000 \% \\ \text { Organic iodine } & 0.0000 \% \\ \text { Aerosols } & 99.0000 \%\end{array}$

from: Unsprayed

from: Unsprayed

from: Sprayed

from: Unsprayed

from: Annulus

Sprayed to unsprayed (\%/day)

$0.1053 \mathrm{E}+04$

$0.4056 \mathrm{E}+04$

$0.4425 \mathrm{E}+04$

$0.2951 \mathrm{E}+04$

$0.1053 \mathrm{E}+04$

Bypass rate (\%/day)

$0.5000 \mathrm{E}-01$

$0.2500 \mathrm{E}-01$

Bypass rate (\%/day)

$0.5000 \mathrm{E}-01$

$0.2500 \mathrm{E}-01$

Filter \#1 Flow rate

$0 \mathrm{cfm}$

$14000 \mathrm{cfm}$

$1000 \mathrm{cfm}$

$99.0000 \%$

\section{Dose Parameters}

$\mathrm{EAB} X / \mathrm{Q}$ values:

$\begin{array}{ll}\text { Time (hr) } & \mathrm{X} / \mathrm{Q}\left(\mathrm{s} / \mathrm{m}^{3}\right) \\ 0.0000 & 0.1000 \mathrm{E}-02 \\ 2.0000 & 0.0000 \mathrm{E}+00\end{array}$

to: Unsprayed

to: Sprayed

to: Environment

Unsprayed to sprayed (\%/day)

$0.4800 \mathrm{E}+04$

$0.1845 \mathrm{E}+04$

$0.2016 \mathrm{E}+05$

$0.1344 \mathrm{E}+05$

$0.4800 \mathrm{E}+04$

Annulus rate (\%/day)

0.4500

0.2250

Annulus rate (\%/day)

0.4500

0.2250 
LPZ X/Q values:
Time (hr)
$\mathrm{X} / \mathrm{Q}\left(\mathrm{s} / \mathrm{m}^{3}\right)$
0.0000
0.1350E-03
8.0000
$0.1000 \mathrm{E}-03$
24.0000
$0.5400 \mathrm{E}-04$
96.0000
$0.2200 \mathrm{E}-04$

\section{Success Criteria}

All of the RADTRAD-calculated EAB and LPZ doses must equal the doses calculated by HABIT within $\pm 10.0 \%$.

\section{Acceptance Criteria}

All of the success criteria must be met or exceeded in order for the RADTRAD software to be acceptable to the NRC.

\section{Results}

\begin{tabular}{|l|l|r|r|}
\hline \multicolumn{2}{|l|}{ Test Case 19 } & RADTRAD & \multicolumn{1}{c|}{ HABIT } \\
\hline EAB & Thyroid (rem) & 314 & 315 \\
\hline & TEDE $(\mathrm{rem})$ & 9.62 & 9.66 \\
\hline LPZ & Thyroid (rem) & 3485 & 3480 \\
\hline & TEDE $(\mathrm{rem}$ & 107 & 107 \\
\hline
\end{tabular}




\subsubsection{Acceptance Test Case 20}

\section{Problem Description}

Advanced PWR design (see Figure 7).

NUREG-1465 PWR release into sprayed ${ }^{2}$ containment region.

Release begins at reactor shutdown.

Containment annulus exhaust filter.

Sprayed containment region mixes with unsprayed region; both leak to the environment and annulus; annulus also exhausts to the environment.

$\mathrm{EAB}$ and $\mathrm{LPZ}$ doses calculated.

\section{Source Term}

Release fractions and timing: NUREG-1465, Tables 3.12 and 3.13.

Start of release:

$0.0000 \mathrm{hr}$

Iodine fractions:

$$
1.0000
$$

Fraction of released iodine chemical form:

$\begin{array}{ll}\text { Elemental: } & 0.0485 \\ \text { Organic: } & 0.0015 \\ \text { Aerosol: } & 0.9500\end{array}$

Inventory:

Normalized MACCS sample 3412 MWth PWR core inventory.

Reactor power:

3992 MWth

\section{Plant Model}

Sprayed

$$
\text { containment volume: } \quad 0.2740 \mathrm{E}+07 \mathrm{ft}^{3}
$$

Unsprayed

$$
\text { containment volume: } \quad 00.6011 \mathrm{E}+06 \mathrm{ft}^{3}
$$

Annulus volume: $\quad 00.6162 \mathrm{E}+06 \mathrm{ft}^{3}$

Environment

Sprayed containment leakage:

Bypass:
Annulus:

Air leakage

Air leakage

Unsprayed containment leakage:

Bypass:

Annulus:

Air leakage

Air leakage

Containment mixing:

Sprayed:

Unsprayed:

Filter \# 1:

$\begin{array}{ll}\text { Air Leakage } & \text { from: Sprayed } \\ \text { Air Leakage } & \text { from: Unsprayed }\end{array}$

Filtered Pathway

from: Annulus from: Sprayed
from: Sprayed

to: Environment

to: Annulus

from: Unsprayed

from: Unsprayed

to: Environment

to: Annulus

to: Unsprayed

to: Sprayed

to: Environment

\footnotetext{
${ }^{2}$ For this and the next several test cases, the (un)sprayed containment region or (un)sprayed containment only refers to the containment region that can or cannot be sprayed. It does not inclicate that sprays are being modeled. The sprays will be modeled in test cases 23 and 24 .
} 
Scenario Description

Containment mixing:

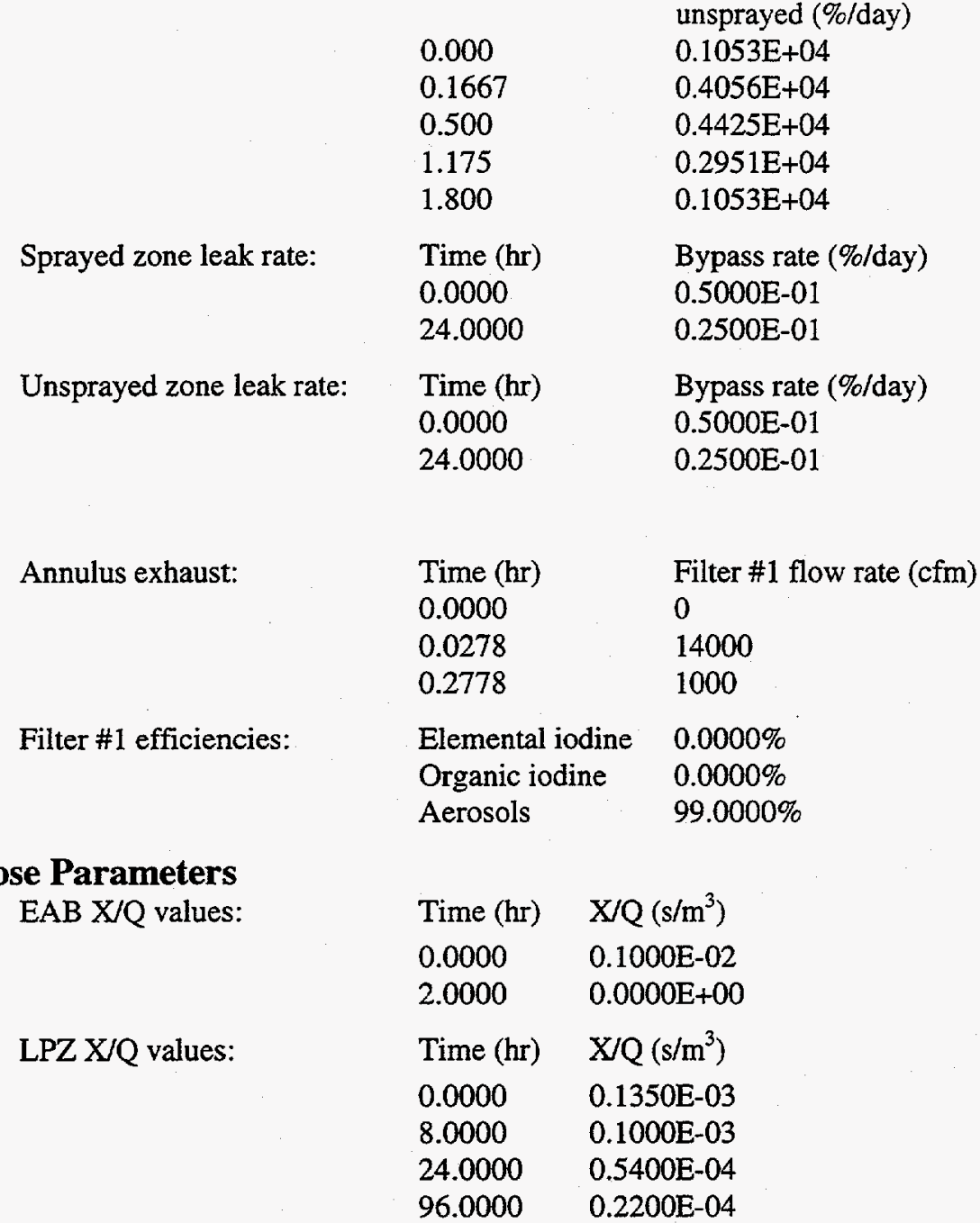

Unsprayed

to sprayed (\%/day)

$0.4800 \mathrm{E}+04$

$0.1845 \mathrm{E}+05$

$0.2016 \mathrm{E}+05$

$0.1344 \mathrm{E}+05$

$0.4800 \mathrm{E}+04$

Annulus rate (\%/day)

0.4500

0.2250

Annulus rate (\%/day)

0.4500

0.2250

\section{Success Criteria}

All of the RADTRAD-calculated EAB and LPZ doses must equal the doses calculated by HABIT within $\pm 10.0 \%$.

\section{Acceptance Criteria}

All of the success criteria must be met or exceeded in order for the RADTRAD software to be acceptable to the NRC.

\section{Results}

\begin{tabular}{|l|l|r|r|}
\hline \multicolumn{2}{|l|}{ Test Case 20 } & RADTRAD & \multicolumn{1}{c|}{ HABIT } \\
\hline EAB & Thyroid (rem) & 459 & 460 \\
\hline & TEDE (rem) & 22.7 & 22.7 \\
\hline LPZ & Thyroid (rem) & 5070 & 5070 \\
\hline & TEDE (rem) & 342 & 342 \\
\hline
\end{tabular}




\subsubsection{Acceptance Test Case 21}

\section{Problem Description}

Advanced PWR design (see Figure 7).

NUREG-1465 PWR release into sprayed ${ }^{3}$ containment region.

Release begins at reactor shutdown.

Containment annulus exhaust filter.

Sprayed containment region mixes with unsprayed region; both leak to the environment and annulus; annulus also exhausts to the environment.

Control room air filtration system (see Figure 8).

$\mathrm{EAB}, \mathrm{LPZ}$, and CR doses calculated.

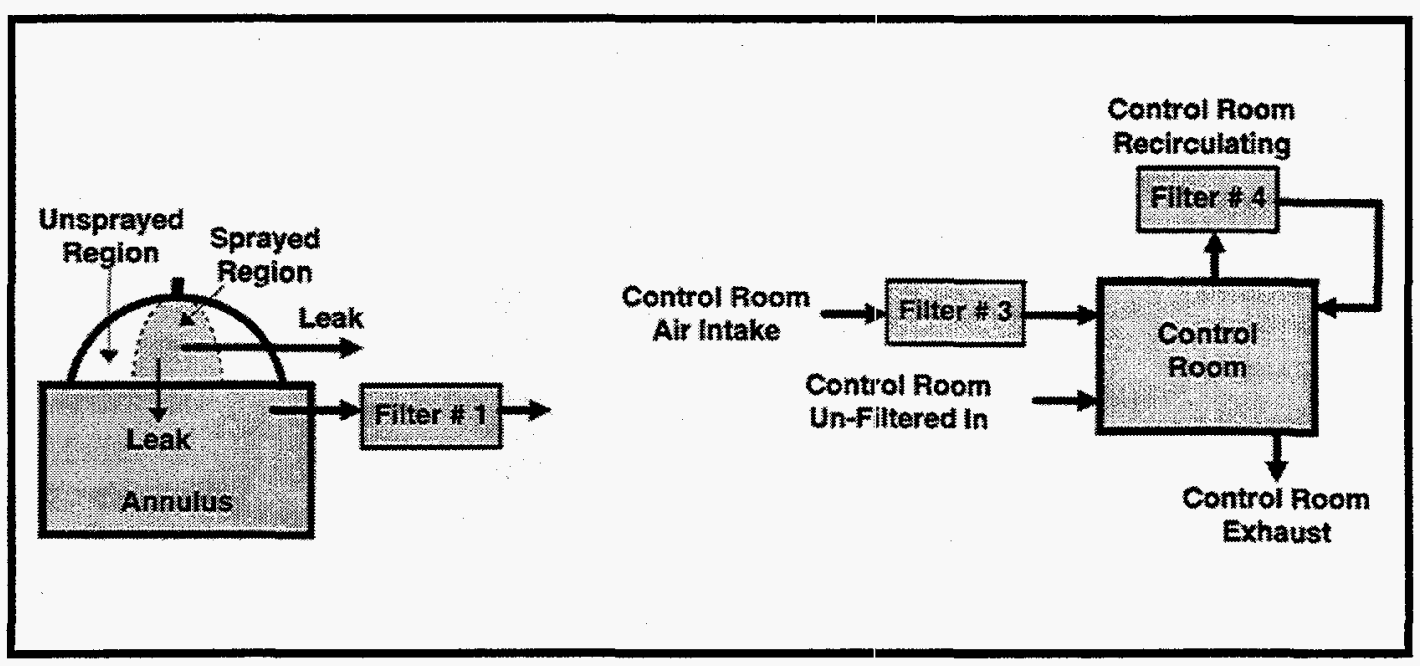

Figure 8. Advanced PWR Control Room Detail

\section{Source Term}

Release fractions and timing: NUREG-1465, Tables 3.12 and 3.13 .

Start of release:

$0.0000 \mathrm{hr}$

Iodine fractions:

Fraction of released iodine chemical form:

$\begin{array}{ll}\text { Elemental: } & 0.0485 \\ \text { Organic: } & 0.0015 \\ \text { Aerosol: } & 0.9500\end{array}$

Inventory:

Normalized MACCS sample 3412 MWth PWR core inventory.

Reactor power:

3992 MWth

\section{Plant Model}

Sprayed

containment volume: $\quad 0.2740 \mathrm{E}+07 \mathrm{ft}^{3}$

Unsprayed
containment volume:
$0.6011 \mathrm{E}+06 \mathrm{ft}^{3}$

\footnotetext{
${ }^{3}$ For this and the next several test cases, the (un)sprayed containment region or (un)sprayed containment only refers to the containment region that can or cannot be sprayed. It does not indicate that sprays are being modeled. The sprays will be modeled in test cases 23 and 24 .
} 
Annulus volume:

$0.6169 \mathrm{E}+06 \mathrm{ft}^{3}$

Control room volume:

$0.673 \mathrm{E}+05 \mathrm{ft}^{3}$

Environment

Sprayed containment leakage:

$\begin{array}{ll}\text { Bypass: } & \text { Air leakage } \\ \text { Annulus: } & \text { Air leakage }\end{array}$

Unsprayed containment leakage:

Bypass:

Annulus:

Containment mixing:

Sprayed:

Unsprayed:

Filter \#1:

Filter \#2:

Filter \#3:

CR exhaust:

$\mathrm{CR}$ unfiltered inflow:

\section{Scenario Description}

Containment mixing:

$\begin{array}{ll} & 0.000 \\ & 0.1667 \\ & 0.500 \\ & 1.175 \\ & 1.800 \\ & \text { Time (hr) } \\ \text { Sprayed zone leak rate: } & 0.0000 \\ & 24.0000 \\ & \text { Time (hr) } \\ \text { Unsprayed zone leak rate: } & 0.0000 \\ & 24.0000\end{array}$

Annulus exhaust:

Filter \#1 efficiencies:
Filter \#2 flow rate:
Filter \#3 efficiencies:

Time (hr)

0.0000

0.0278

0.2778

Aerosols

$2000 \mathrm{cfm}$
Air leakage

Air leakage

Air leakage

Air leakage

Filtered pathway

Filtered pathway

Recirculation filter

Air leakage

Air leakage from: Sprayed
from: Sprayed

from: Unsprayed

from: Unsprayed

from: Sprayed

from: Unsprayed

from: Annulus

from: Environment

from: Control room

from: Control room

from: Environment to: Environment

to: Annulus

to: Environment

to: Annulus

to: Unsprayed

to: Sprayed

to: Environment

to: Control room

to: Control room

to: Environment

to: Control room

Unsprayed

Sprayed to unsprayed (\%/day)

to sprayed (\%/day)

$0.1053 \mathrm{E}+04 \quad 0.4800 \mathrm{E}+04$

$0.4056 \mathrm{E}+04 \quad 0.1845 \mathrm{E}+05$

$0.4425 \mathrm{E}+04 \quad 0.2016 \mathrm{E}+05$

$0.2951 \mathrm{E}+04$

$0.1344 \mathrm{E}+05$

$0.1053 \mathrm{E}+04$

$0.4800 \mathrm{E}+04$

Bypass rate (\%/day)

$0.5000 \mathrm{E}-01$

Annulus rate (\%/day)

$0.2500 \mathrm{E}-01$

0.4500

0.2250

Bypass rate (\%/day)

Annulus rate (\%/day)

$0.5000 \mathrm{E}-01 \quad 0.4500$

$0.2500 \mathrm{E}-01 \quad 0.2250$

Filter \#1 Flow rate (cfm)

0

14000

1000

Elemental iodine

Organic iodine

$0.0000 \%$

$0.0000 \%$

$99.0000 \%$

Elemental iodine

Organic iodine

Aerosols

$95.0000 \%$

$95.0000 \%$

$99.0000 \%$ 
Filter \#4 flow rate:

$4000 \mathrm{cfm}$

Filter \#4 efficiencies:

Elemental iodine

Organic iodine

Aerosols

$2010 \mathrm{cfm}$

CR exhaust:

CR unfiltered inflow:

\section{Dose Parameters}

$E A B X / Q$ values:

LPZ X/Q values:

Filter \#2 X/Q values:

$$
10 \mathrm{cfm}
$$

$95.0000 \%$

$95.0000 \%$

$99.0000 \%$

$\begin{array}{ll}\text { Time }(\mathrm{hr}) & \mathrm{X} / \mathrm{Q}\left(\mathrm{s} / \mathrm{m}^{3}\right) \\ 0.0000 & 0.1000 \mathrm{E}-02 \\ 2.0000 & 0.0000 \mathrm{E}+00 \\ \text { Time }(\mathrm{hr}) & \mathrm{X} / \mathrm{Q}\left(\mathrm{s} / \mathrm{m}^{3}\right) \\ 0.0000 & 0.1350 \mathrm{E}-03 \\ 8.0000 & 0.1000 \mathrm{E}-03 \\ 24.0000 & 0.5400 \mathrm{E}-04 \\ 96.0000 & 0.2200 \mathrm{E}-04 \\ & \\ \text { Time }(\mathrm{hr}) & \mathrm{X} / \mathrm{Q}\left(\mathrm{s} / \mathrm{m}^{3}\right) \\ 0.0000 & 0.2200 \mathrm{E}-02 \\ 2.0000 & 0.1500 \mathrm{E}-02 \\ 8.0000 & 0.1300 \mathrm{E}-02 \\ 24.0000 & 0.8400 \mathrm{E}-03 \\ 96.0000 & 0.4800 \mathrm{E}-03\end{array}$

\section{Success Criteria}

All of the RADTRAD-calculated EAB, LPZ, and CR doses must equal the doses calculated by HABIT within $\pm 10.0 \%$.

\section{Acceptance Criteria}

All of the success criteria must be met or exceeded in order for the RADTRAD software to be acceptable to the NRC.

\section{Results}

\begin{tabular}{|l|l|r|r|}
\hline \multicolumn{2}{|l|}{ Test Case 21 } & RADTRAD & \multicolumn{1}{c|}{ HABIT } \\
\hline EAB & Thyroid (rem) & 459 & 460 \\
\hline & TEDE (rem) & 22.7 & 22.7 \\
\hline LPZ & Thyroid (rem) & 5070 & 5070 \\
\hline & TEDE (rem) & 342 & 342 \\
\hline CR & Thyroid (rem) & 662 & 669 \\
\hline & TEDE (rem) & 59.4 & 59.7 \\
\hline
\end{tabular}




\subsubsection{Acceptance Test Case 22}

\section{Problem Description}

Advanced PWR design (see Figure 7).

NUREG-1465 PWR release into sprayed ${ }^{4}$ containment region.

Release begins at reactor shutdown.

Containment annulus exhaust filter.

Sprayed containment region mixes with unsprayed region, both leak to the environment and annulus, annulus also exhausts to the environment.

Control room air filtration system (see Figure 8).

User-defined natural deposition in sprayed and unsprayed containment regions.

\section{Source Term}

Release fractions and timing: NUREG-1465, Tables 3.12 and 3.13 .

Start of release:

$0.0000 \mathrm{hr}$

Iodine fractions:

$$
1.0000
$$

Fraction of released iodine chemical form:

$\begin{array}{ll}\text { Elemental: } & 0.0485 \\ \text { Organic: } & 0.0015 \\ \text { Aerosol: } & 0.9500\end{array}$

Inventory:

Normalized MACCS sample 3412 MWth PWR core inventory.

Reactor power:

3992 MWth

\section{Plant Model}

Sprayed

$$
\text { containment volume: } \quad 0.2740 \mathrm{E}+07 \mathrm{ft}^{3}
$$

Unsprayed

$$
\text { containment volume: } \quad 0.6011 \mathrm{E}+06 \mathrm{ft}^{3}
$$

Annulus volume:

$$
0.6162 \mathrm{E}+06 \mathrm{ft}^{3}
$$

Control room volume:

$$
0.6731 \mathrm{E}+05 \mathrm{ft}^{3}
$$

Environment

Sprayed containment leakage:
Bypass:
Air leakage
from: Sprayed
from: Sprayed
to: Environment
Annulus:
Air leakage
to: Annulus

Unsprayed containment leakage:

Bypass:

Annulus:

Air leakage

Air leakage

Containment mixing:

Sprayed:

Unsprayed:

\begin{abstract}
Air Leakage
Air Leakage
\end{abstract}

from: Unsprayed

from: Unsprayed

to: Environment

to: Annulus

from: Sprayed

from: Unsprayed to: Unsprayed

to: Sprayed

\footnotetext{
${ }^{4}$ For this and the next several test cases, the (un)sprayed containment region or (un)sprayed containment only refers to the containment region that can or cannot be sprayed. It does not indicate that sprays are being modeled. The sprays will be modeled in test cases 23 and 24 .
} 
Filter \#1:

Filter \#2:

Filter \#3:

CR exhaust:

CR unfiltered inflow:

\section{Scenario Description}

Containment mixing:

\begin{tabular}{|c|c|c|}
\hline & 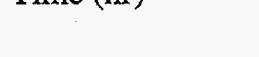 & unsprayed (\%/day) \\
\hline & 0.000 & $0.1053 E+04$ \\
\hline & 0.1667 & $0.4056 \mathrm{E}+04$ \\
\hline & 0.500 & $0.4425 \mathrm{E}+04$ \\
\hline & 1.175 & $0.2951 E+04$ \\
\hline & 1.800 & $0.1053 \mathrm{E}+04$ \\
\hline Sprayed zone leak rate: & Time (hr) & Bypass rate (\%/day) \\
\hline & 0.0000 & $0.5000 \mathrm{E}-01$ \\
\hline & 24.0000 & $0.2500 \mathrm{E}-01$ \\
\hline Unsprayed zone leak rate: & Time (hr) & Bypass rate (\%/day) \\
\hline & 0.0000 & $0.5000 \mathrm{E}-01$ \\
\hline & 24.0000 & $0.2500 \mathrm{E}-01$ \\
\hline Natural deposition & & \\
\hline aerosol removal rate: & $0.15 / \mathrm{hr}$ & \\
\hline Annulus exhaust: & Time (hr) & Filter \#1 flow rate \\
\hline & 0.0000 & $0 \mathrm{cfm}$ \\
\hline & 0.0278 & $14000 \mathrm{cfm}$ \\
\hline & 0.2778 & $1000 \mathrm{cfm}$ \\
\hline Filter \#1 efficiencies: & Elemental iodine & $0.0000 \%$ \\
\hline & Organic iodine & $0.0000 \%$ \\
\hline & Aerosols & $99.0000 \%$ \\
\hline Filter \#2 flow rate: & $2000 \mathrm{cfm}$ & \\
\hline Filter \#2 efficiencies: & Elemental iodine & $95.0000 \%$ \\
\hline & Organic iodine & $95.0000 \%$ \\
\hline & Aerosols & $99.0000 \%$ \\
\hline Filter \#3 flow rate: & $4000 \mathrm{cfm}$ & \\
\hline Filter \#3 efficiencies: & Elemental iodine & $95.0000 \%$ \\
\hline & Organic iodine & $95.0000 \%$ \\
\hline & Aerosols & $99.0000 \%$ \\
\hline CR exhaust: & $2010 \mathrm{cfm}$ & \\
\hline CR unfiltered inflow: & $10 \mathrm{cfm}$ & \\
\hline se Parameters & & \\
\hline EAB X/Q values: & Time (hr) & $\left.\mathrm{s} / \mathrm{m}^{3}\right)$ \\
\hline & $\begin{array}{l}0.0000 \\
2.0000\end{array}$ & OE-02 \\
\hline
\end{tabular}

\begin{tabular}{|c|c|}
\hline Filtered pathway & from: Annulus \\
\hline Filtered pathway & from: Environment \\
\hline Recirculation Filter & from: Control Room \\
\hline Air leakage & from: Control room \\
\hline Air leakage & from: Environment \\
\hline
\end{tabular}

to: Environment

to: Control Room

to: Control Room

to: Environment

to: Control Room
Unsprayed to sprayed (\%/day)

$0.4800 \mathrm{E}+04$

$0.1845 \mathrm{E}+05$ day

$0.2016 \mathrm{E}+05$

$0.1344 \mathrm{E}+05$

$0.4800 \mathrm{E}+04$

Annulus rate (\%/day)

0.4500

0.2250

Annulus rate (\%/day)

0.4500

0.2250 
LPZ X/Q values:

Time (hr) X/Q $\left(\mathrm{s} / \mathrm{m}^{3}\right)$

$0.0000 \quad 0.1350 \mathrm{E}-03$

$8.0000 \quad 0.1000 \mathrm{E}-03$

$24.0000 \quad 0.5400 \mathrm{E}-04$

$96.0000 \quad 0.2200 \mathrm{E}-04$

Filter \#2 X/Q values:

Time (hr) X/Q $\left(\mathrm{s} / \mathrm{m}^{3}\right)$

$0.0000 \quad 0.2200 \mathrm{E}-02$

$2.0000 \quad 0.1500 \mathrm{E}-02$

$8.0000 \quad 0.1300 \mathrm{E}-02$

$24.0000 \quad 0.8400 \mathrm{E}-03$

$96.0000 \quad 0.4800 \mathrm{E}-03$

\section{Success Criteria}

All of the RADTRAD-calculated EAB, LPZ, and CR doses must equal the doses calculated by HABIT within $\pm 10.0 \%$.

\section{Acceptance Criteria}

All of the success criteria must be met or exceeded in order for the RADTRAD software to be acceptable to the NRC.

\section{Results}

\begin{tabular}{|l|l|r|r|}
\hline \multicolumn{2}{|l|}{ Test Case 22 } & RADTRAD & \multicolumn{1}{c|}{ HABIT } \\
\hline EAB & Thyroid (rem) & 424 & 425 \\
\hline & TEDE (rem) & 21.1 & 21.1 \\
\hline LPZ & Thyroid (rem) & 1845 & 1840 \\
\hline & TEDE (rem) & 179 & 179 \\
\hline CR & Thyroid (rem) & 439 & 436 \\
\hline & TEDE (rem) & 50.0 & 49.7 \\
\hline
\end{tabular}




\subsubsection{Acceptance Test Case 23}

\section{Problem Description}

Advanced PWR design (see Figure 7).

NUREG-1465 PWR release into sprayed containment region.

Release begins at reactor shutdown.

Containment annulus exhaust filter.

Sprayed containment region mixes with unsprayed region; both leak to the environment and annulus; annulus also exhausts to the environment.

Control room air filtration system (see Figure 8).

User-defined natural deposition in unsprayed containment region.

User-defined spray removal coefficients.

\section{Source Term}

Release fractions and timing: NUREG-1465, Tables 3.12 and 3.13.

Start of release:

$0.0000 \mathrm{hr}$

Iodine fractions:

$$
1.0000
$$

Fraction of released iodine chemical form:

$\begin{array}{ll}\text { Elemental: } & 0.0485 \\ \text { Organic: } & 0.0015 \\ \text { Aerosol: } & 0.9500\end{array}$

Inventory:

Normalized MACCS sample 3412 MWth PWR core inventory.

Reactor power:

3992 MWth

\section{Plant Model}

Sprayed

containment volume: $\quad 0.2740 \mathrm{E}+07 \mathrm{ft}^{3}$

Unsprayed

containment volume: $\quad 0.6011 \mathrm{E}+06 \mathrm{ft}^{3}$

Annulus volume: $\quad 0.6162 \mathrm{E}+06 \mathrm{ft}^{3}$

Control room volume: $\quad 0.6731 \mathrm{E}+05 \mathrm{ft}^{3}$

Environment

Sprayed containment leakage:
Bypass:
Air leakage
Annulus:
Air leakage
from: Sprayed
from: Sprayed
to: Environment
to: Annulus

Unsprayed containment leakage:
Bypass:
Annulus:
Air leakage
Air leakage

\section{Containment Mixing:}

Sprayed:

Unsprayed:

Filter \#1:

Filter \#2:

\begin{abstract}
Air leakage
Air leakage
\end{abstract}

Filtered pathway

Filtered pathway from: Unsprayed

from: Unsprayed

to: Environment

to: Annulus

from: Sprayed

from: Unsprayed

to: Unsprayed

to: Sprayed

from: Annulus

to: Environment

from: Environment

to: Control room 
Filter \#3:

CR exhaust:

CR unfiltered inflow:

\section{Scenario Description}

Containment mixing:

\begin{tabular}{|c|c|c|c|}
\hline \multirow[t]{6}{*}{ Containment mixing: } & Time (hr) & $\begin{array}{l}\text { Sprayed to } \\
\text { unsprayed (\%/day) }\end{array}$ & $\begin{array}{l}\text { Unsprayed } \\
\text { to sprayed (\%/day) }\end{array}$ \\
\hline & 0.000 & $0.1053 E+04$ & $0.4800 \mathrm{E}+04$ \\
\hline & 0.1667 & $0.4056 \mathrm{E}+04$ & $0.1845 \mathrm{E}+05$ \\
\hline & 0.500 & $0.4425 E+04$ & $0.2016 \mathrm{E}+05$ \\
\hline & 1.175 & $0.2951 E+04$ & $0.1344 E+05$ \\
\hline & 1.800 & $0.1053 E+04$ & $0.4800 \mathrm{E}+04$ \\
\hline \multirow[t]{3}{*}{ Sprayed zone leak rate: } & Time (hr) & Bypass rate (\%/day) & Annulus rate (\%/day) \\
\hline & 0.0000 & $0.5000 \mathrm{E}-01$ & 0.4500 \\
\hline & 24.0000 & $0.2500 \mathrm{E}-01$ & 0.2250 \\
\hline \multirow[t]{3}{*}{ Unsprayed zone leak rate: } & Time (hr) & Bypass rate (\%/day) & Annulus rate (\%/day) \\
\hline & 0.0000 & $0.5000 \mathrm{E}-01$ & 0.4500 \\
\hline & 24.0000 & $0.2500 \mathrm{E}-01$ & 0.2250 \\
\hline \multicolumn{4}{|l|}{ Natural deposition } \\
\hline aerosol removal rate: & $0.15 / \mathrm{hr}$ & & \\
\hline \multirow[t]{8}{*}{ Spray removal coefficients: } & Time (hr) & \multicolumn{2}{|c|}{ Removal coefficient for aerosols and elementals $\left(\mathrm{hr}^{-1}\right)$} \\
\hline & 0.0000 & \multirow{5}{*}{\multicolumn{2}{|c|}{$\begin{array}{l}1.5 \\
12 \\
5 \\
2 \\
0.4\end{array}$}} \\
\hline & 0.50 & & \\
\hline & 1.8 & & \\
\hline & 2.0 & & \\
\hline & 8.0 & & \\
\hline & Time (hr) & \multirow{2}{*}{\multicolumn{2}{|c|}{$\begin{array}{l}\text { Removal coefficients for organics } \\
0.00\end{array}$}} \\
\hline & 0.0000 & & \\
\hline \multirow[t]{4}{*}{ Annulus exhaust: } & Time (hr) & \multicolumn{2}{|c|}{ Filter \#1 flow rate (cfm) } \\
\hline & 0.0000 & \multicolumn{2}{|c|}{0} \\
\hline & 0.0278 & \multicolumn{2}{|l|}{14000} \\
\hline & 0.2778 & \multicolumn{2}{|l|}{1000} \\
\hline \multirow[t]{3}{*}{ Filter \#1 efficiencies: } & Elemental iodine & \multirow{3}{*}{\multicolumn{2}{|c|}{$\begin{array}{l}0.0000 \% \\
0.0000 \% \\
99.0000 \%\end{array}$}} \\
\hline & Organic iodine & & \\
\hline & Aerosols & & \\
\hline Filter \#2 flow rate: & $2000 \mathrm{cfm}$ & & \\
\hline \multirow[t]{3}{*}{ Filter \#2 efficiencies: } & Elemental iodine & \multirow{3}{*}{\multicolumn{2}{|c|}{$\begin{array}{l}95.0000 \% \\
95.0000 \% \\
99.0000 \%\end{array}$}} \\
\hline & Organic iodine & & \\
\hline & Aerosols & & \\
\hline Filter \#4 flow rate: & $4000 \mathrm{cfm}$ & & \\
\hline \multirow{3}{*}{ Filter \#4 efficiencies: } & Elemental iodine & \multicolumn{2}{|l|}{$95.0000 \%$} \\
\hline & Organic iodine & \multicolumn{2}{|l|}{$95.0000 \%$} \\
\hline & Aerosols & \multicolumn{2}{|l|}{$99.0000 \%$} \\
\hline CR exhaust: & $2010 \mathrm{cfm}$ & & \\
\hline CR unfiltered inflow: & $10 \mathrm{cfm}$ & & \\
\hline
\end{tabular}

Recirculation filter from: Control Room to: Control room

Air leakage

from: Control room

to: Environment

from: Environment

to: Control room

Air leakage

$$
\text { from: Environment }
$$

\author{
to: Control room
}




\section{Dose Parameters}

EAB X/Q values:

$$
\begin{array}{ll}
\text { Time (hr) } & \text { X/Q }\left(\mathrm{s} / \mathrm{m}^{3}\right) \\
0.0000 & 0.1000 \mathrm{E}-02 \\
2.0000 & 0.0000 \mathrm{E}+00
\end{array}
$$

$0.0000 \quad 0.1000 \mathrm{E}-02$

LPZ X/Q values:

Time (hr) X/Q $\left(\mathrm{s} / \mathrm{m}^{3}\right)$

$0.0000 \quad 0.1350 \mathrm{E}-03$

$8.0000 \quad 0.1000 \mathrm{E}-03$

$24.0000 \quad 0.5400 \mathrm{E}-04$

$96.0000 \quad 0.2200 \mathrm{E}-04$

Filter \#2 X/Q values:

$\begin{array}{ll}\text { Time (hr) } & \text { X/Q }\left(\mathrm{s} / \mathrm{m}^{3}\right) \\ 0.0000 & 0.2200 \mathrm{E}-02 \\ 2.0000 & 0.1500 \mathrm{E}-02 \\ 8.0000 & 0.1300 \mathrm{E}-02 \\ 24.0000 & 0.8400 \mathrm{E}-03 \\ 96.0000 & 0.4800 \mathrm{E}-03\end{array}$

\section{Success Criteria}

All of the RADTRAD-calculated EAB, LPZ, and CR doses must equal the doses calculated by HABIT within $\pm 10.0 \%$

\section{Acceptance Criteria}

All of the success criteria must be met or exceeded in order for the RADTRAD software to be acceptable to the NRC.

\section{Results}

\begin{tabular}{|l|l|c|c|}
\hline \multicolumn{2}{|l|}{ Test Case 23 } & RADTRAD & HABIT \\
\hline EAB & Thyroid (rem) & 61.7 & 61.7 \\
\hline & TEDE (rem) & 4.16 & 4.16 \\
\hline LPZ & Thyroid (rem) & 58.1 & 58.1 \\
\hline & TEDE (rem) & 106 & 106 \\
\hline CR & Thyroid (rem) & 14.1 & 14 \\
\hline & TEDE (rem) & 36.2 & 36 \\
\hline
\end{tabular}




\subsubsection{Acceptance Test Case 24}

\section{Problem Description}

Advanced PWR design (see Figure 7).

NUREG-1465 PWR release into sprayed containment region.

Release begins at reactor shutdown.

Containment annulus exhaust filter.

Sprayed containment region mixes with unsprayed region; both leak to the environment and annulus; annulus also exhausts to the environment.

Control room air filtration system (see Figure 8).

User-defined natural deposition in unsprayed containment region.

Spray removal coefficients determined from Powers model [Po93].

\section{Source Term}

Release fractions and timing: NUREG-1465, Tables 3.12 and 3.13 .

Start of release:

$0.0000 \mathrm{hr}$

Iodine fractions:

Fraction of released iodine chemical form:

$\begin{array}{ll}\text { Elemental: } & 0.0485 \\ \text { Organic: } & 0.0015 \\ \text { Aerosol: } & 0.9500\end{array}$

Inventory:

Normalized MACCS sample 3412 MWth PWR core inventory.

Reactor power:

3992 MWth

\section{Plant Model}

Sprayed

containment volume: $\quad 0.2740 \mathrm{E}+07 \mathrm{ft}^{3}$

Unsprayed containment volume: $\quad 0.6011 \mathrm{E}+06 \mathrm{ft}^{3}$

Annulus volume: $\quad 0.6162 \mathrm{E}+06 \mathrm{ft}^{3}$

Control room volume: $\quad 0.6731 \mathrm{E}+05 \mathrm{ft}^{3}$

Environment

Sprayed containment leakage:
Bypass:
Air leakage
Annulus:
Air leakage
from: Sprayed
to: Environment
from: Sprayed
to: Annulus

Unsprayed containment leakage:

Bypass:

Annulus:

Air leakage

Air leakage

Containment Mixing:

Sprayed:

Unsprayed:

Filter \#1:

Filter \#2:
Air leakage

Air leakage

Filtered pathway

Filtered pathway from: Unsprayed

from: Unsprayed

from: Sprayed

from: Unsprayed

from: Annulus

from: Environment to: Environment

to: Annulus

to: Unsprayed

to: Sprayed

to: Environment

to: Control room 
Filter \#3:

CR exhaust:

CR unfiltered inflow:

\section{Scenario Description}

Containment mixing:

$\begin{array}{ll} & 0.000 \\ & 0.1667 \\ & 0.500 \\ & 1.175 \\ & 1.800 \\ & \text { Time (hr) } \\ & 0.0000 \\ \text { Sprayed zone leak rate: } & 24.0000 \\ & \text { Time (hr) } \\ & 0.0000 \\ \text { Unsprayed zone leak rate: } & 24.0000 \\ & \\ \text { Natural deposition } & 0.1500 \mathrm{hr}^{-1} \\ \text { aerosol removal rate: } & \\ \text { Powers' spray removal: } & 85 \mathrm{ft} \\ \text { Nozzle height: } & 0.3230 \mathrm{E}+05 \mathrm{ft}^{2} \\ \text { Mean cross section: } & 1.0 \\ \text { Spray volume fraction: } & 50 \% \\ \text { Confidence level: } & \text { Time } \\ \text { Water flow } & 0.0 \\ & 3.0\end{array}$

Spray removal coefficients: Time (hr)

Annulus exhaust:

Filter \#1 efficiencies:

Filter \#2 flow rate:

Filter \#2 efficiencies:

Aerosols
Recirculation filter

Air leakage

Air leakage

Time (hr)

Sprayed to
unsprayed

$0.1053 \mathrm{E}+04$

$0.4056 \mathrm{E}+04$

$0.4425 \mathrm{E}+04$

$0.2951 \mathrm{E}+04$

$0.1053 \mathrm{E}+04$

Bypass rate (\%/day)

$0.5000 \mathrm{E}-01$

$0.2500 \mathrm{E}-01$

Bypass rate (\%/day)

$0.5000 \mathrm{E}-01$

$0.2500 \mathrm{E}-01$ to: Control room

to: Environment

to: Control room
Unsprayed

to sprayed (\%/day)

$0.4800 \mathrm{E}+04$

$0.1845 E+05$

$0.2016 \mathrm{E}+05$

$0.1344 \mathrm{E}+05$

$0.4800 \mathrm{E}+04$

Annulus rate (\%/day)

0.4500

0.2250

Annulus rate (\%/day)

0.4500

0.2250

0.0000

0.50

1.8

2.0

8.0

Time (hr) 0.0000

Time (hr)
0.0000
0.0278
0.2778

Flow rate/coverage area (FLUX)

$0.3937 \mathrm{E}-01 \mathrm{ctm} / \mathrm{ft}^{2}$

$0.0 \mathrm{cfm} / \mathrm{ft}^{2}$

Removal coefficient for elementals $\left(\mathrm{hr}^{-1}\right)$

1.5

12

5

2

0.4

Removal coefficients for organics 0.00

Filter \#1 flow rate

$0 \mathrm{cfm}$

$1400 \mathrm{cfm}$

$1000 \mathrm{cfm}$

Elemental iodine $\quad 0.0000 \%$

Organic iodine $\quad 0.0000 \%$

$99.0000 \%$

$2000 \mathrm{cfm}$

Elemental iodine $\quad 95.0000 \%$

Organic iodine $\quad 95.0000 \%$

Aerosols

$99.0000 \%$ 
Filter \#3 flow rate:

Filter \#3 efficiencies:

CR exhaust:

CR unfiltered inflow:

\section{Dose Parameters}

$\mathrm{EAB} X / \mathrm{Q}$ values:

LPZ X/Q values:

Filter \#2 X/Q values:
$4000 \mathrm{cfm}$

Elemental iodine $\quad 95.0000 \%$

Organic iodine $\quad 95.0000 \%$

Aerosols

\section{$2010 \mathrm{cfm}$}

$10 \mathrm{cfm}$

$$
\begin{array}{ll}
\text { Time }(\mathrm{hr}) & \mathrm{X} / \mathrm{Q}\left(\mathrm{s} / \mathrm{m}^{3}\right) \\
0.0000 & 0.1000 \mathrm{E}-02 \\
2.0000 & 0.0000 \mathrm{E}+0 \\
\text { Time }(\mathrm{hr}) & \mathrm{X} / \mathrm{Q}\left(\mathrm{s} / \mathrm{m}^{3}\right) \\
0.0000 & 0.1350 \mathrm{E}-03 \\
8.0000 & 0.1000 \mathrm{E}-03 \\
24.0000 & 0.5400 \mathrm{E}-04 \\
96.0000 & 0.2200 \mathrm{E}-04 \\
\text { Time }(\mathrm{hr}) & \mathrm{X} / \mathrm{Q}\left(\mathrm{s} / \mathrm{m}^{3}\right) \\
0.0000 & 0.2200 \mathrm{E}-02 \\
2.0000 & 0.1500 \mathrm{E}-02 \\
8.0000 & 0.1300 \mathrm{E}-02 \\
24.0000 & 0.8400 \mathrm{E}-03 \\
96.0000 & 0.4800 \mathrm{E}-03
\end{array}
$$

\section{Success Criteria}

The deposition rate should be found to be reasonable.

\section{Acceptance Criteria}

If the success criteria are not met in the RADTRAD version under test, the software will be still be acceptable to the NRC. However, SNL will be responsible for determining the cause of the failure and correction will be expected before project closeout.

\section{Results}

\begin{tabular}{|l|l|r|r|}
\hline \multicolumn{2}{|l|}{ Test Case 24 } & RADTRAD & \multicolumn{2}{c|}{$\begin{array}{c}\text { RADTRAD } \\
\text { Case 23 }\end{array}$} \\
\hline EAB & Thyroid (rem) & 68.6 & 61.7 \\
\hline & TEDE (rem) & 4.54 & 4.16 \\
\hline LPZ & Thyroid (rem) & 60.4 & 58.1 \\
\hline & TEDE (rem) & 106 & 106 \\
\hline CR & Thyroid (rem) & 14.3 & 14 \\
\hline & TEDE (rem) & 36.2 & 36 \\
\hline
\end{tabular}

The results are similar to those for the user-supplied spray decontamination coefficient input. This indicates that the model has the correct trends. 


\subsubsection{Acceptance Test Case 24A}

\section{Problem Description}

Advanced PWR design (see Figure 7).

NUREG-1465 PWR release into sprayed containment region.

NUREG-1465 DRAFT PWR aerosol release into sprayed containment region.

Release begins at reactor shutdown.

Containment annulus exhaust filter.

Sprayed containment region mixes with unsprayed region; both leak to the environment and annulus; annulus also exhausts to the environment.

Control room air filtration system (see Figure 8).

User-defined natural deposition in unsprayed containment region.

Spray removal coefficients determined from Powers model [PO93].

\section{Source Term}

Release fractions and timing: NUREG-1465, Tables 3.12 and 3.13.

Start of release:

$0.0000 \mathrm{hr}$

Iodine fractions:

Fraction of released iodine chemical form:
Elemental: $\quad 0.0485$
Organic: $\quad 0.0015$
Aerosol: $\quad 0.9500$

Inventory:

Normalized MACCS sample 3412 MWth PWR core inventory.

Reactor power:

3992 MWth

\section{Plant Model}

Sprayed

containment volume: $\quad 0.2740 \mathrm{E}+07 \mathrm{ft}^{3}$

Unsprayed containment volume: $\quad 0.6011 \mathrm{E}+06 \mathrm{ft}^{3}$

Annulus volume: $\quad 0.6162 \mathrm{E}+06 \mathrm{ft}^{3}$

Control room volume: $\quad 0.6731 \mathrm{E}+05 \mathrm{ft}^{3}$

Environment

Sprayed containment leakage:

$\begin{array}{llll}\text { Bypass: } & \text { Air leakage } & \text { from: Sprayed } & \text { to: Environment } \\ \text { Annulus: } & \text { Air leakage } & \text { from: Sprayed } & \text { to: Annulus }\end{array}$

Unsprayed containment leakage:

Bypass: $\quad$ Air leakage from: Unsprayed

Annulus:

Air leakage

Containment mixing:

Sprayed:

Unsprayed:

Filter \#1:

Filter \#3:
Air leakage

Air leakage

Filtered pathway

Filtered pathway from: Unsprayed

to: Environment

to: Annulus

from: Sprayed

from: Unsprayed

to: Unsprayed

to: Sprayed

from: Annulus

to: Environment

from: Environment

to: Control room 
Filter \#4:

CR exhaust:

CR unfiltered inflow:

Scenario Description

Containment mixing:

$\begin{array}{ll} & 0.000 \\ & 0.1667 \\ & 0.500 \\ & 1.175 \\ & 1.800 \\ \text { Sprayed zone leak rate: } & \text { Time (hr) } \\ & 0.0000 \\ & 24.0000 \\ \text { Unsprayed zone leak rate: } & \text { Time (hr) } \\ & 0.0000 \\ & 24.0000\end{array}$

Natural deposition aerosol removal rate:

$0.1500 \mathrm{hr}^{-1}$

Powers' spray removal:

Nozzle height:

Mean cross section:

Spray volume fraction:

Confidence level:

Water Flow$$
\text { ater Flow }
$$

Spray removal coefficients:

$85 \mathrm{ft}$
$03230 \mathrm{E}+05 \mathrm{ft}^{2}$
1.0
$50 \%$

Time

0.0
3.0
0.0000

0.50

1.8

2.0

8.0

Time (hr)

0.0000

Annulus exhaust:

Filter \#1 efficiencies:

Filter \#3 flow rate:

0.0000
0.0278
0.2778

Elemental iodine

Organic iodine

Aerosols

$2000 \mathrm{cfm}$ from: Control room from: Control room

from: Environment to: Control room

to: Environment

to: Control room
Sprayed to unsprayed (\%/day)

$0.1053 \mathrm{E}+04$

$0.4056 \mathrm{E}+04$

$0.4425 \mathrm{E}+04$

$0.2951 \mathrm{E}+04$

$0.1053 E+04$

Bypass rate (\%/day)

$0.5000 \mathrm{E}-01$

$0.2500 \mathrm{E}-01$

Bypass rate (\%/day)

$0.5000 \mathrm{E}-01$

$0.2500 \mathrm{E}-01$
Unsprayed

to sprayed (\%/day)

$0.4800 \mathrm{E}+04$

$0.1845 E+04$

$0.2016 \mathrm{E}+05$

$0.1344 \mathrm{E}+05$

$0.4800 E+04$

Annulus rate (\%/day)

0.4500

0.2250

Annulus rate (\%/day)

0.4500

0.2250

Filter \#1 flow rate

$0 \mathrm{cfm}$

$14000 \mathrm{cfm}$

$1000 \mathrm{cfm}$

Flow rate/coverage area (flux)

$0.3937 \mathrm{E}-01 \mathrm{cfm} / \mathrm{ft}^{2}$

$0.0 \mathrm{cfm} / / \mathrm{ft}^{2}$

Removal coefficient for elementals $\left(\mathrm{hr}^{-1}\right)$

1.5

12

5

2

0.4

Removal coefficients for organics

0.00

$0.0000 \%$

$0.0000 \%$

$99.0000 \%$ 
Filter \#3 efficiencies:

Filter \#4 flow rate:

Filter \#4 efficiencies:

CR exhaust:

CR unfiltered inflow:

\section{Dose Parameters}

$\mathrm{EAB} X / \mathrm{Q}$ values:

LPZ X/Q values:

Filter \#3 X/Q values:

$\begin{array}{ll}\text { Elemental iodine } & 95.0000 \% \\ \text { Organic iodine } & 95.0000 \% \\ \text { Aerosols } & 99.0000 \%\end{array}$

$0.1888 \mathrm{E}+01 \mathrm{~m}^{3} / \mathrm{s}$

Elemental Iodine $95.0000 \%$

Organic lodine $\quad 95.0000 \%$

Aerosols

$2010 \mathrm{cfm}$

$10 \mathrm{cfm}$

$\begin{array}{lll}\text { EAB X/Q values: } & \text { Time }(\mathrm{hr}) & \mathrm{X} / \mathrm{Q}\left(\mathrm{s} / \mathrm{m}^{3}\right) \\ & 0.0000 & 0.1000 \mathrm{E}-02 \\ & 2.0000 & 0.0000 \mathrm{E}+00 \\ \text { LPZ X/Q values: } & \text { Time }(\mathrm{hr}) & \mathrm{X} / \mathrm{Q}\left(\mathrm{s} / \mathrm{m}^{3}\right) \\ & 0.0000 & 0.1350 \mathrm{E}-03 \\ & 8.0000 & 0.1000 \mathrm{E}-03 \\ & 24.0000 & 0.5400 \mathrm{E}-04 \\ & 96.0000 & 0.2200 \mathrm{E}-04 \\ \text { Filter \#3 X/Q values: } & \text { Time (hr) } & \text { X/Q }\left(\mathrm{s} / \mathrm{m}^{3}\right) \\ & 0.0000 & 0.2200 \mathrm{E}-02 \\ & 2.0000 & 0.1500 \mathrm{E}-02 \\ & 8.0000 & 0.1300 \mathrm{E}-02 \\ & 24.0000 & 0.8400 \mathrm{E}-03 \\ & 96.0000 & 0.4800 \mathrm{E}-03\end{array}$

\section{Success Criteria}

The deposition rate should be found to be reasonable.

\section{Acceptance Criteria}

All of the success criteria must be met or exceeded in order for the RADTRAD software to be acceptable to the NRC. 


\section{Results}

\begin{tabular}{|l|l|r|r|}
\hline \multicolumn{2}{|l|}{} & \multicolumn{1}{c|}{$\begin{array}{c}\text { RADTRAD } \\
\text { Case 24 }\end{array}$} & \multicolumn{2}{c|}{\begin{tabular}{c} 
Case 24A \\
\hline EAB
\end{tabular}} & Thyroid (rem) & 68.6 & 68.6 \\
\hline & TEDE (rem) & 4.54 & 4.54 \\
\hline LPZ & Thyroid (rem) & 60.4 & 60.4 \\
\hline & TEDE (rem) & 106 & 106 \\
\hline CR & Thyroid (rem) & 14.3 & 14.3 \\
\hline & TEDE (rem & 36.2 & 36.2 \\
\hline
\end{tabular}

In the Powers spray model, the decontamination factors were derived in terms of mass fractions. The addition of aerosol to the mix will have no effect on dose due to this first minor assumption. 


\subsection{Configuration Control}

One purpose of configuration control is to maintain a standard, recognized version of the code. Code assessment efforts are hindered when a code is undergoing continuous development. The code must be frozen, documented, and distributed to code users so that all users have the same code, and assessment results and user experience can be exchanged knowing that results are interrelated and are not specific to a unique version of the code. Freezing of the code is defined as the avoidance of changes in models and correlations to improve the code's predictive capability. Changes to the frozen code are allowed to correct errors, for improved input/output, and for user conveniences. The intent is to freeze the code for a sufficient time to establish a considered view of its capabilities and accuracy.

If an error is discovered by a user, the information (see Section 1.7) should be sent (via fax or e-mail) to the developers. The problem should be described and suggestions for possible fixes included. The problem will be reviewed to ensure that it is indeed an error and that the problem is relevant to conditions that might be expected to occur in a nuclear power plant. Error corrections will typically be released semiannually.

The use of the code assessment and applications is expected to reveal deficiencies that need to be addressed through code development. The information gathered during the period when the code is kept frozen will be considered continuously in preparing new versions of the code. A priority based implementation system will be used for all code revisions. User requirements to resolve safety and regulatory needs are the foremost determinant of priority.

\subsection{References}

[Bi95] N.E. Bixler, "Simple Model of Pipe Decontamination for RADTRAD," Internal Sandia National Laboratories Memorandum to D. Monroe, August 29, 1995.

[Br91] J.E. Brockmann, "Sampling and Transport of Aerosols," in "Aerosol Measurement: Principles, Techniques, and Applications," K. Wilke and P.A. Baron, Eds., Van Nostrand Reinhold, New York, 1991.

[Cl91] J.E. Cline, "MSIV Leakage Iodine Transport Analysis," NRC-03-87-029, Nuclear Regulatory Commission, Washington, DC, 1991.

[Di62] J.J. DiNunno, R.E. Baker, F.D. Anderson, and R.L. Waterfield, "Calculation of Distance Factors for Power and Test Reactor Sites," TD-14844, U.S. Atomic Energy Agency, Washington, DC, 1962.

[Fa84] Fauske and Associates, Inc., FAI Aerosol Correlation, An Industry Degraded Core Rulemaking (IDCOR) Program Report, Bethesda, MD, 1984. 
Federal Guidance Report No. 11, "Limiting Values of Radionuclide Intake and Air Concentration and Dose Conversion Factors for Inhalation, Submersion, and Ingestion," USEPA, EPA 520/188-020 U.S. Environmental Protection Agency, Washington, DC, 1988.

Federal Guidance Report No. 12, "External Exposure to Radionuclides in Air, Water and Soil," EPA 420-r-93-081 U.S. Environmental Protection Agency, 1993.

[Mu74] K.G. Murphy and K.M. Campe, "Nuclear Power Plant Control Room Ventilation System Design for Meeting General Design Criterion 19," in Proceedings of the $13^{\text {th }}$ AEC Air Cleaning Conference, CONF-740807, U.S. Atomic Energy Commission, Washington, DC.

[Po92] D.A. Powers and J.L. Sprung, A Simplified Model of Aerosol Scrubbing by a Water Pool Overlying Core Debris Interacting with Concrete, NUREG/CR-5901, SAND92-1422, Sandia National Laboratories, Albuquerque, NM, 1992.

[Po93] D.A. Powers and S.B. Burson, A Simplified Model of Aerosol Removal by Containment Sprays, NUREG/CR-5966, SAND92-2689, Sandia National Laboratories, Albuquerque, NM, 1993.

[Po95] D.A. Powers, K.E. Washington, S.B. Burson, and J.L. Sprung, "A Simplified Model of Aerosol Removal by Natural Process in Reactor Containments," NUREG-6189, SAND94-0407, Sandia National Laboratories, Albuquerque, NM, 1995.

[So95] L. Soffer, S.B. Burson, C.M. Ferrell, R.Y. Lee, and J.N. Ridgely, "Accident Source Terms for Light-Water Nuclear Power Plants," NUREG-1465, Nuclear Regulatory Commission, Washington, DC, 1995.

[St 95] S.A. Stage, "Computer Codes for Evaluation of Control Room Habitability (HABIT)," Draft, NUREG/CR-6210 (PNL-10496), Pacific Northwest National Laboratory, Richland, WA, 1995.

USNRC [74] "Assumptions Used for Evaluating the Potential Radiological Consequences of a Loss of Coolant Accident for Boiling Water Reactors," Regulatory Guide 1.3, Revision 2, U.S. Nuclear Regulatory Commission, Washington, DC, June 1974.

USNRC [74] "Assumptions Used for Evaluating the Potential Radiological Consequences of a Loss of Coolant Accident for Pressurized Water Reactors," Regulatory Guide 1.4, Revision 2, U.S. Nuclear Regulatory Commission, Washington, DC, June 1974. 



\section{RADTRAD Programmer's Guide}

The first two parts of this document have dealt with the RADTRAD from the user's perspective. They have provided the information needed to install and run the RADTRAD program and to understand and apply the results for the program. The last two parts deal specifically with the RADTRAD computer code. This material is intended for individuals who may be called upon to revise the code or place the code in a different shell. Chapter 3 deals with quality assurance (i.e., does the code solve the problem correctly?), while this section details the actual code and its logic.

\subsection{General Computational Approach}

RADTRAD is implemented as a modular code. It includes two distinct components. The first is written using Fortran and the Hi-Screen Pro II user-interface tool. It provides the graphical user interface (GUI) used for data input and permits RADTRAD to run one scenario after another without the user having to restart the program. Its first call is the INTROMENU, from which the user decides by the choice of screen where to go.

The other component is written in Fortran. It performs RADTRAD's computational tasks. This part of the document deals primarily with the second component. The computational part of the RADTRAD code consists of a main program (RADCALC) to control the program execution and 70 subroutines and functions to handle the data and perform the model calculations. Each program unit is coded using a structured approach.

\subsubsection{Code Languages}

The computational portion of RADTRAD is written using a subset of the ANSI Fortran-90 programming language.

\subsubsection{Coding Standards}

The following coding standards are followed in all RADTRAD Fortran program units.

The code for each program unit includes two blocks:

- definition block - the definition block includes the program unit name, history, description, and relationship to other units and INCLUDE files. It may also contain references, algorithm descriptions, and variable definitions.

- code block - code and comments.

The code block in each Fortran program unit begins with the type, and the dimensions of all variables are defined in type statements. 
The primary method of passing variables between major program units is named "common blocks." Common blocks contain related information, and the block name indicates the general nature of the information. The common blocks are defined in code segments contained in INCLUDE files. The INCLUDE files also contain parameter statements, type definitions, and dimensions associated with the variables defined in the file.

Argument lists are used to pass variables to subroutines and functions if use of a formal argument list facilitates program development, verification, and maintenance. Variables are passed to functions via argument lists.

Structured programming techniques (IF ... THEN, ELSE IF... THEN, DO WHILE) have been used, and the unnecessary use of statement numbers has been avoided. END DO statements have been used to decrease the number of statement numbers.

The RADTRAD code aborts execution if an abnormal condition is encountered during execution of the code. Traps have been provided for abnormal conditions related to file operations, data input, and data output. In general, the error messages are written to the console, to the log file, and to other output files to describe the abnormal condition that is causing the code execution to be aborted.

The use of system-dependent calls has been limited to two places: (1) the numerical solution section where a call to ZPORC is used to return the zeros of a polynomial; and (2) the system subroutines, where there is a call to get the date and time of program execution from the system clock.

\subsubsection{Data Input}

RADTRAD is usually run through the interactive screen mode, but it may also be run in a batch mode. User interactions and data input are via data input files. The names of the run specification files (plant model file and scenario description file) are entered as command line arguments at the time code execution is initiated. The output file name is the third command line argument (XXX.OUT).

\subsubsection{Interactive Input}

When RADTRAD is run interactively via the GUI, then the user has the option of either entering the plant model and scenario description via the GUI, or specifying the file names of the data input files.

\subsection{RADTRAD Code structure}

\subsubsection{RADTRAD Logic Flow}

The RADionuclide Transport, Removal, and Dose code (RADTRAD) calculates doses to receptors, with the locations defined by the user. Locations may be at offsite locations, for 
example, the exclusion area boundary or the low population zone, and in the control room. A general logic flow chart, with subroutine names given in parentheses, is given below. A detailed flow charge indicating all subprogram relationships is shown in Section 4.2.1.1.

Screen input (INTRO.IMG)

Initialization for RADTRAD [INITALL]

For each time interval [AUTODT]

RADTRAD assumes a single-source compartment. Default source terms are for BWRs and PWRs, described in NUREG-1465 or in TID-14844 [SOURCE].

Material is transported through the containment building, as well as through other buildings and the environment.

Credit is taken for removal mechanisms. Containment sprays may remove aerosols, elemental iodine, and organic iodine. The flow between buildings or rooms may be through HEPA filters or a suppression pool. Leakage to the environment may occur [COEFFS].

Evaluate matrix-inverse in Laplace space by transport group [INVMAT].

Calculate separately the contribution factors from each compartment's nuclide inventory, source term, and the initial values and rates for the transport groups to each compartment's inventory at the end of the time step [COENUM, ZERODN, RESADER].

Update the nuclide inventories in each compartment based upon transport results [XNUPDT].

Calculate doses at the specified locations [DOSES].

Calculate the decay and ingrowth of nuclides in the compartment atmosphere [DKNGRO].

Edit results [PRINTOUT].

Prepare for another time step [AUTODT].

\subsubsection{RADTRAD Flowchart}

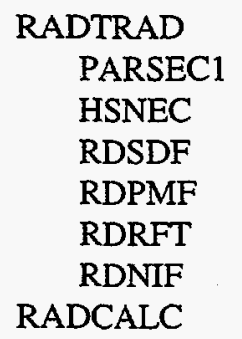




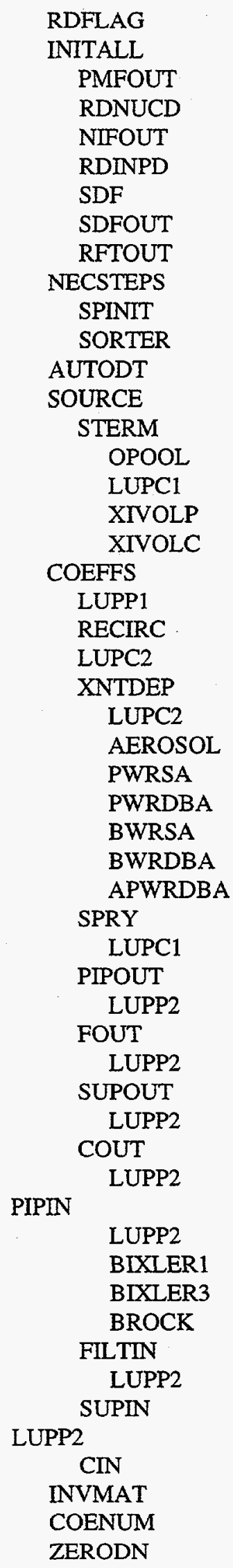


DZPORC

RADCALC (continued)

\author{
NDPEXC \\ RESADER \\ EQUAL \\ SUMPWR \\ COENUM \\ ZERODN \\ DZPORC \\ NDPEXC \\ RESADER \\ EQUAL \\ SUMPWR \\ COENUM \\ ZERODN \\ DZPORC \\ NDPEXC \\ RESADER \\ EQUAL \\ SUMPWR \\ XNUPDT \\ DOSES \\ LUPL2 \\ DKNGRO \\ PRINTOUT \\ AUTODT \\ ZRINV \\ TSHIFT
}

\title{
4.2.1.2 Input Screen Subroutines
}

Screen names and numbers and the subroutines that create them are listed below. Note that only those screens that are currently active have subroutines. As additional models and options are included, additional screens will be written.

Screen

Title

Welcome

Main Menu

Plant Model

Load Plant Model

Nuclide Inventory

Select Compartment

Compartment Description

Select Transfer Pathways

Transfer Pathway Description

Select Compartment

Review Plant Model

Save Plant Model
Screen

Number

0000

1000

1100

1200

1300

1310

1400

1410

1411

1500

1600
Subroutine

Name

INTROMENU

MENU_PM

FILE_PM

INVEN_PM

COMPT_PM

CINFO_PM

TRANR_PM

PINFO_PM

SELECT_C

VIEWFILE

SAVE_PM 
Release Fractions and Timing

Aerosol Removal by Sprays

User-specified

AESP3_SD

Removal Coefficient

$2311 \mathrm{~T}$

AESP1_SD

Powers Model

Elemental Iodine Removal by Sprays

User-specified

$$
\text { Removal Coefficient }
$$

EISPR_SD

Organic Iodine Removal by Sprays

User-specified

Removal Coefficient 2313A

Sump - Iodine volatilization (no user input)

OISPR_SD

Recirculating Filters

Filters-Recirculating Air

Natural Deposition

Aerosol Removal

User-specified

Henry Model

$2341 \mathrm{M}$

Powers Model

2341M2

Elemental Iodine Removal

User-specified

Decontamination due to Overlying Pool

Initial Water Volume

Aerosol Removal due to Overlying Pool User-specified Aerosoll DF

Powers Aerosol DF Model

Elemental Iodine DF

User-specified

Organic Iodine DF

User-specified

Select Transfer Pathway

Transfer Pathways

\section{Piping}

User-specified Removal Coefficient

Brockmann Model
$2342 \mathrm{~T}$

2350

2351

2352

$2352 \mathrm{~T}$

$2352 \mathrm{M}$

$2353 \mathrm{~T}$

$2354 \mathrm{~T}$

2400

2410

$2410 \mathrm{~T}$

$2410 \mathrm{M}$
FULTR_SD

ND3_SD

NDA32_SD

NDAE_SD

NDEP_SD

NDPC_SD

NDEI_SP

OPOOL_SD

VOLUM_SD

AEOP3_SD

AEOP2_SD

AEOP1_SD

OPEI_SD

OPOI_SD

PATH_SD

PIPEM_SD

PIPEV_SD

PIPEB_SD 
Screen

Title
Screen

Number

Subroutine

Name

Filters

User-Specified Filter

Parameters

$2420 \mathrm{~T}$

$2420 \mathrm{~T} 2$

2430

TPFIL_SD

Air Intake X/Qs

Suppression Pool

User-Specified Decontamination

Factors for Aerosol Removal

User-Specified Decontamination

Factors for Elemental Iodine

Removal

User-Specified Decontamination

Factors for Organic Iodine

Removal

Air Leakage

Dose Calculation Input

User-specified

$2431 \mathrm{~T}$

LN_EV_SD

TPSP_SD

PAESP_SP

$2432 \mathrm{~T}$

PEISP_SD

Select Dose Conversion Factors

Create/Modify Dose Location Selection

Dose Location

Location Compartment

Control Room

Environment

$2433 \mathrm{~T}$

POISP_SD

$2440 t$

PCONV_SD

2510

DOSECON

2520

DOSE_SD

$2520 \mathrm{~A}$

LNDOS_SD

2521

SELEC2_C

2522

LN_CR_SD

2523

LN_E_SD

Review Scenario Description

2600

Save Scenario Description

2700

VIEWFILE

SAVE_SD

Calculate Results

3000

SIMULATE

4000

RESULTS

5000

QUIT

\subsubsection{Program Module Description}

AEROSOL. This subroutine receives the decontamination coefficient as a function of reactor type (PWR or BWR) and release scenario (design basis accident or severe accident) in units of $\mathrm{hr}^{-1}$ and converts it to $\mathrm{s}^{-1}$. The correlations used are discussed in Section 2.2.2.1.2. It is called by the routine controlling natural deposition, XNTDEP.

APWRDBA. This subroutine calculates the decontamination coefficient for natural deposition using the Powers model for a design basis accident in an AP-600 reactor. It is called by the main routine controlling aerosol deposition, AEROSOL.

AUTODT. This subroutine determines the next time step based on user-specified events and a manual time step control called by the drive routine, RADCALC. 
BIXLER1. This subroutine calculates the decontamination coefficient for elemental iodine in a pipe as discussed in Section 2.2.6.2. It assumes that revaporization is negligible. It is called by the routine controlling pipe deposition, PIPINB.

BIXLER3. This subroutine calculates the decontamination coefficient for organic iodine in a pipe as discussed in Section 2.2.6.3. It assumes that revaporization is negligible. It is called by the routine controlling pipe deposition, PIPINB.

BROCK. This subroutine calculates the transport efficiency of aerosols for piping pathways as discussed in Section 2.2.6.1. The subroutine assumes continuous flow from the pipeway inlet. It is based upon a lumped-node model description. Subroutine BROCK includes the following effects: (1) gravitational settling, for low-flow-rate conditions; (2) turbulent inertial deposition, along straight pipe sections; (3) Brownian diffusion, along straight sections of pipe; and (4) inertial deposition in bends. It is called by the routine controlling pipe deposition, PIPINB.

BWRDBA. This subroutine calculates the decontamination coefficient for natural deposition using the Powers model for a design basis accident in a boiling water reactor. It is called by the main routine for aerosol deposition, AEROSOL.

BWRSA. This subroutine calculates the decontamination coefficient for natural deposition using the Powers model for a severe accident in a boiling water reactor. It is called by the main routine for aerosol deposition, AEROSOL.

CIN. This subroutine calculates the normalized flow into the compartment due to convection (air leakage). The user specifies the compartment volume and the volumetric flow rate between the compartments. These values are returned as removal coefficients $\left(\mathrm{s}^{-1}\right)$ for the transport groups (1) nobles, (2) elemental iodine, (3) organic iodine, and (4) aerosols. This routine is typically used in determining leak rates from containment. It is called by the coefficient driver routine, COEFFS.

COEFFS. This subroutine combines the coefficients for the individual removal and transport mechanisms to obtain the system matrix for the differential equation governing nuclide removal and transport.

For each of the four transport groups, there will be obtained a set of coefficients, $C_{i j}$, with the indices $i$ and $j$ designating compartment numbers. The value of $C_{i j}$ is positive when it corresponds to a loss in the content of the $i^{\text {th }}$ compartment. It is negative when it corresponds to a gain in the content of the $i^{\text {th }}$ compartment. This loss or gain is proportional to the content of the $j^{\text {th }}$ compartment. At present, all losses occur on the diagonal of the system matrix and all gains occur off the diagonal. This is because there are no removal mechanisms that can affect removal in another compartment.

COENUM evaluates the numerator of the transformed equation in Laplace space. One such numerator exists for each compartment location. It is used in combination with ZERODN and RESADER to evaluate the transport of radionuclides. It is called by the main driver routine, 
RADCALC, three times to calculate each compartment's nuclide contribution to every other compartment.

COUT calculates the flow out of a compartment by way of a pathway as a result of convection (also referred to as air leakage) for each transport group. It is called by the main driver routine, RADCALC, three times to calculate each compartment's nuclide contribution to every other compartment.

DKNGRO calculates the radioactive decay and daughter in-growth for all nuclides. It is called from the main driver routine, RADCALC.

DOSES. Environment and control room doses are calculated for receptors located at userdefined locations [e.g., exclusion area boundary (EAB), low population zone (LPZ), control room operator]. The user supplies the $\mathrm{X} / \mathrm{Q}$ s and breathing rates as a function of time for each location. The user also supplies the occupancy factors as a function of time for the control room. It is called from the main driver routine, RADCALC.

DZPORC returns the double precision or the complex zeros of a given polynomial with double precision coefficients. The required inputs are NDEG, the degree of the polynomial, and COEFF, the vector of the double-precision coefficients (with the zeroth power occurring first). The output is ZERO, the vector of double precision or complex roots (whose number is NDEG).

EQUAL. This subroutine returns 1 if the two arguments are equal or nearly equal to each other and a 0 if they are not. The degree of equality is determined by the values of ABS_EPSILON and REL_EPSILON, which are defined in icodelconstant.h. It is called from RESADER.

FILTIN. This subroutine calculates the flow rate and removal coefficient called from RESADER for filtering incoming air. The coefficient is calculated for each transport group for the given time step and is stored in the array FILFIN(4). The user must specify the volume of the "from" compartment, the flow through the filter, and the efficiency of the filter for the various groups. The routine is called by the coefficient evaluation routine, COEFFS, and is discussed in Section 2.2.7.

FOUT. This subroutine calculates the flow rate for filtered air. The user must specify the volume of the "from" compartment and the flow through the filter for the various groups. The routine is called by the coefficient evaluation routine, COEFFS.

INITALL sets input for the RADTRAD program reads. It calls routines that read the nuclide inventory and dose files discussed in Section 1.4.3.3 and that edit the plant model file information and the scenario description file. It is called up from the main drive routine RADCALC.

INTRO.IMG generates the "Welcome to RADTRAD" initial input screen, which then calls the main menu screen (screen number 0000), where the user begins the input process.

INVMAT. This subroutine determines the inverse of the Laplace system matrix (the resolvent matrix) using an algorithm called Leverrier's algorithm (also called Fadeeva's method). If $A$ is 
the system matrix, $I$ is the identity matrix, and $s$ is the Laplace transform variable, then the resolvent matrix can be defined as:

$$
(s I-A)^{-1}
$$

It is called from the main drive routine, RADCALC.

LUPC1 is a lookup table function for one-dimensional arrays; for example, the overlying pool tables.

LUPC2 is a lookup table function for two-dimensional arrays; for example, the filter and spray tables.

LUPL2 is a lookup table function for two-dimensional arrays; for example, the control room occupancy factor and breathing rates.

LUPP1 is a lookup table function for one-dimensional arrays; for example, the X/Q for the control room inlet.

LUPP2 is a lookup table function for one-dimensional, double-precision arrays; for example, the piping deposition tables.

NECSTEPS. This subroutine determines the minimum set of time steps that are necessary to capture all of the events and other system changes that are included in the user input. It is called from the main driver routine RADCALC.

NIFOUT outputs the nuclide inventory file data if the detailed output control variable (LEVELNIFOUT) is set greater than 1 in FLAG.SET.

OPOOL. For each of the four transport groups, this subroutine calculates decontamination factors resulting from an overlying pool. Both user-provided and model-developed decontamination factors are implemented in this subroutine. The model is discussed in Section 2.2.3. The effects considered in this subroutine only occur during the ex-vessel portion of the release.

PIPIN. This subroutine calculates the removal rate for material flowing into a compartment by way of a pipeway, as discussed in Section 2.2.6. The user may specify the flow rate for iodine and aerosol transport groups. In addition, the user may specify the DFs for these same transport groups or request that they be obtained using the Brockmann/Bixler algorithms. They are called from the coefficient evaluation routine, COEFFS.

PIPOUT. This subroutine calculates the normalized flow out of a given compartment by way of piping for all four transport groups. It is called from the coefficient evaluation routine, COEFFS.

PMFOUT. This subroutine outputs plant model file information if the detailed output control variable (LEVEL_PMFOUT) is set greater than 0 in FLAG.SET. 
PRINTOUT. This routine handles all user-requested RADTRAD output. All other output from all other routines should be used for debugging purposes only. This debug output will be interspersed among the normal descriptive and dose output, but should be completely turned off for most runs. Compartment detailed output is stored and printed after the dose output. The detailed output contains information about nuclide and transport group inventories for each compartment for which detailed output is requested.

The descriptive information describes the software, model, and scenario used to obtain the resulting output.

The dose information is the organ, whole-body, and TEDE doses in sieverts at the end of each user-defined time step. Both accumulated doses and those acquired during the time step are given.

If requested, nuclide inventories are given in atoms, kilograms, curies, and becquerels at the end of each user time step. Transport group inventories are given in atoms and kilograms.

PWRDBA. This subroutine calculates the decontamination coefficient for natural deposition using the Powers model for a design basis accident in a pressurized water reactor. It is called by the aerosol deposition main routine, AEROSOL.

PWRSA. This subroutine calculates the decontamination coefficient for natural deposition using the Powers model for a severe accident in a pressurized water reactor. It is called by the main aerosol deposition routine, AEROSOL.

RADCALC. This is the main driver routine for the calculational aspects of RADTRAD. It calculates doses to receptors having locations defined by the user. Locations may be at the site boundary, in the low population zone, and in the control room.

RDFLAG reads detailed-output control flags from FLAG.SET for subroutines.

RDINPD reads dose information from MCODEDOSEDATA.INP. It is called from the input control routine, INITALL.

RDNUCD reads nuclides, atomic weights, half-lives, and half-life units of measurement information from MCODELNUC.NIS. It is called from the input control routine, INITALL.

RECIRC. This subroutine calculates the removal coefficient for the recirculating filters in a compartment. The subroutine loops over all transport groups. The user provides the filter efficiency as a function of transport group, flow rates, and volume information. The routine is called from COEFFS and is discussed in Section 2.2.7.

RESADER. This subroutine calculates the amount of each transport group in each compartment using the method of residues, which is the process of inverting the Laplace transform obtained in solving the first-order differential equations describing transport and removal within the system. A sequence of eleven code blocks (designated as RESADER0, RESADER $1, \ldots, R E S A D E R X)$ is utilized to process the denominator roots of the transform. The first block of code, RESADER0, 
is used to determine the level of multiplicity, or repetitions, in the denominator. The other blocks of code are used for each level of multiplicity; thus a multiplicity level of 3 would use the RESADER3 block of code. When there is more than one zero in the denominator, an analytical solution of the derivative is determined in this routine. The technique is discussed in Section 2.4.2.

RFTOUT. This is a subroutine to produce output concerning the release fractions and timings when the detailed output control variable (LEVEL_RFTOUT) is set greater than 1 in FLAG.SET.

SDF. This is a subroutine that can be used to override scenario input.

SDFOUT. This is a subroutine to output a data set in 'SDF.FOR' concerning the scenario descriptions when the detailed output control variable (LEVEL_SDFOUT) is set greater than 1 in FLAG.SET.

SORTER. This subroutine does a selection sort on the NVAL elements of the double-precision array VALS. It returns the sorted elements in the same array. These elements are time values in RADTRAD, but any set of double-precision numbers can be sorted in the same way.

SOURCE. This subroutine controls the calculation of source material in each compartment. Source material arises from core heatup and degradation determined by the user's choice of inventory and release fractions. It is called from the main driver RADCALC and is discussed in Section 1.4.3.

SPINIT is the Powers spray model initialization routine. It is used to estimate the internal time steps need for stability of the model due to the intense removal of aerosols.

SPRY. This subroutine determines the removal coefficients for all four transport groups due to the action of sprays. It is assumed that no removal effect occurs for noble gases. For the other three transport groups, the removal coefficient can be specified by the user. For the aerosol transport group, the removal coefficient can also be calculated using the Powers mechanistic model. This is discussed in Section 2.2.1.

STERM. This subroutine calculates the release rate of material from the core inventory to the source term compartment. It also accounts for the effects of decay.

SUMPWR is the sum of the inverse numerator powers, less the sum of the inverse denominator powers. Each numerator power is of the form $(s-z n)^{* *}(-n)$, where $s$ is equal to the given denominator zero for which the residues are being determined. If $s$ happens to equal $z n$, then the term is excluded from the sum. In this case a numerator multiplicity is said to occur, and the SUMPWR will apply to the reduced function. Each denominator power is of the form $(s-z d)^{* *}(-n)$ and is multiplied by its denominator multiplicity. When $s$ happens to equal $z d$, then the term is excluded.

SUPIN. This subroutine calculates the removal coefficient for material flowing into a compartment through a suppression pool. The user must specify DFs for elemental iodine, organic iodine, and aerosols. This is discussed in Section 2.2.5. 
SUPOUT. This subroutine calculates the normalized flow out of a given compartment by way of a suppression pool. The values obtained are returned in the array SUPFL(4).

TSHIFT shifts results and updates old values to new values at the end of a time step.

XNTDEP. This subroutine calculates the removal coefficient for natural deposition. This removal mechanism includes many phenomena, such as gravitational settling, impaction, and diffusion. The subroutine considers all four transport groups. However, natural deposition is considered applicable only to elemental iodine and aerosols. Noble gases and organic iodine are not affected because they are nonreactive.

The removal coefficients for elemental iodine and aerosols can be specified by the user. In the case of elemental iodine removal, the user should include the effects of reemission of iodine, because this effect is not modeled explicitly. The removal coefficients can also be determined from either Henry's correlation or the Powers model as discussed in Section 2.2.2.

XNUPDT updates the nuclide inventory. It updates inventories using previous compartment inventories and the inventory and source term contribution factors. It only deals with nuclides transported in the atmosphere. Nuclides in other compartment sublocations are not transported. The only source term rate per nuclide is the primary source term from the source term compartment.

ZERODN for each transport group, subroutine ZERODN, determines the zeros of the scalar denominator and the zeros of the vector numerator.

ZRINV is the routine used to zero the inventories in a particular type of compartment. This routine was written for general use, but currently is used only for environment compartments (compartment type $=3$ ). The current use is to zero environment inventories for plume simulations.

\subsubsection{Diagnostic Control}

To obtain additional output for a particular subroutine, a level-of-detail system is used. In this system, increasing the flag value from 0 to 5 will increase the information flow. It is recommended that the user start with values like 1 or 2 and trace through the logic to localize the problem. Below is the standard level of detail flags that exist in file FLAG.DAT.

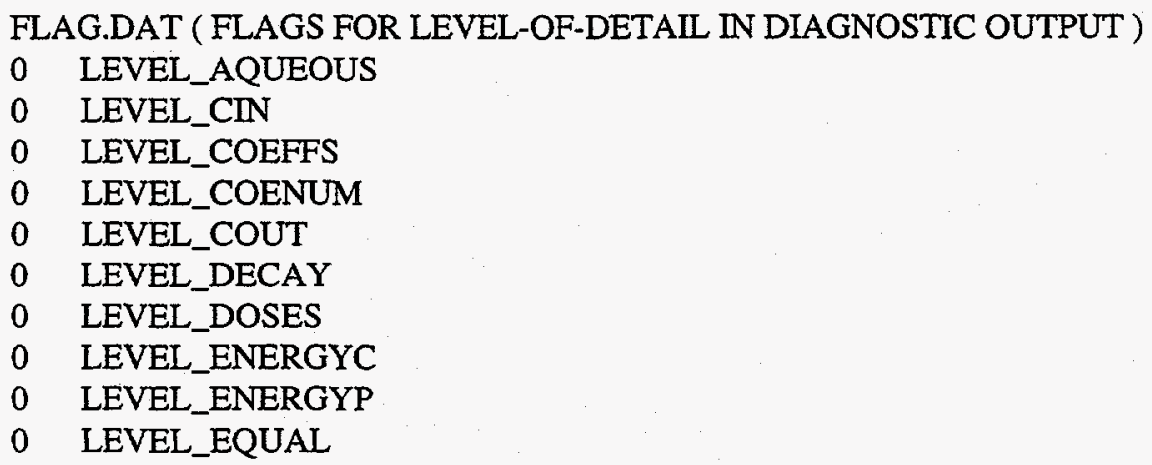




$\begin{array}{ll}0 & \text { LEVEL_EXTEDE } \\ 0 & \text { LEVEL_FILTIN } \\ 0 & \text { LEVEL_FOUT } \\ 0 & \text { LEVEL_INITALL } \\ 0 & \text { LEVEL_INVMAT } \\ 0 & \text { LEVEL_LUPC1 } \\ 0 & \text { LEVEL_LUPC2 } \\ 0 & \text { LEVEL_LUPC3 } \\ 0 & \text { LEVEL_LUPL2 } \\ 0 & \text { LEVEL_LUPP1 } \\ 0 & \text { LEVEL_LUPP2 } \\ 0 & \text { LEVEL_MASSTR } \\ 0 & \text { LEVEL_NECSTEPS } \\ 0 & \text { LEVEL_NIFOUT } \\ 0 & \text { LEVEL_NUCLIDE } \\ 0 & \text { LEVEL_OPFLAG } \\ 0 & \text { LEVEL_OPOOL } \\ 0 & \text { LEVEL_OUTPUT } \\ 0 & \text { LEVEL_PIPIN } \\ 0 & \text { LEVEL_PIPOUT } \\ 0 & \text { LEVEL_PMF } \\ 0 & \text { LEVEL_PMFOUT } \\ 0 & \text { LEVEL_PRINTOUT } \\ 0 & \text { LEVEL_RADCALC } \\ 0 & \text { LEVEL_RDBWR } \\ 0 & \text { LEVEL_RDFLAG } \\ 0 & \text { LEVEL_RDINPD } \\ 0 & \text { LEVEL_RD_NIF } \\ 0 & \text { LEVEL_RDNUCD } \\ 0 & \text { LEVEL_RDPMF } \\ 0 & \text { LEVEL_RDPWR } \\ 0 & \text { LEVEL_RECIRC } \\ 0 & \text { LEVEL_RESIDUE } \\ 0 & \text { LEVEL_RFTBWR } \\ 0 & \text { LEVEL_RFTOUT } \\ 0 & \text { LEVEL_RFTPWR } \\ 0 & \text { LEVEL_SDF } \\ 0 & \text { LEVEL_SSDFOUT } \\ 0 & \text { LEVEL_SORTER } \\ 0 & \text { LEVEL_SOURCE } \\ 0 & \text { LEVEL_SPRY } \\ 0 & \text { LEVEL_STERM } \\ 0 & \text { LEVEL_SUPIN } \\ 0 & \text { LEVEL_SUPOUT } \\ 0 & \text { LEVEL_TSHIFT } \\ 0 & \text { LEVEL_XIVOLC } \\ 0 & \text { LEVEL_XIVOLP } \\ 0 & \text { LEVEL_XNTDEP } \\ & \text { LEVEL__ZNURORT } \\ & \end{array}$




\subsection{RADTRAD Executable and Source Code Listing}

The RADTRAD executable and source code listing is available in electronic format on 3.5-inch high-density IBM PC-compatible floppy disks. Either is available by request from:

Sandia National Laboratories

Accident and Consequence Analysis Department

P.O. Box 5800

Albuquerque, NM 87185-0748

(505) 844-3321 (fax) 
2. TITLE AND SUBTITLE

(See instructions on the reverse)

NUREG/CR-6604

SAND98-0272

RADTRAD: A Simplified Model for RADionuclide Transport and

Removal And Dose Estimation

\section{AUTHORIS)}

S.L. Humphreys, SNL; T.J. Heames, ITSC; L.A. Miller, SNL;

D.K. Monroe, SNL

\begin{tabular}{|c|c|}
\hline DATE & PUBLISKED \\
\hline MONTH & YEAE \\
\hline April & 1998 \\
\hline \multicolumn{2}{|c|}{ 4. FIN OR GRANT NUMBER } \\
\hline \multicolumn{2}{|c|}{ 6. TYPE OF REPORT } \\
\hline \multicolumn{2}{|c|}{ Technical } \\
\hline 7. PERIODCO & Inclusive Dates. \\
\hline
\end{tabular}

8. PERFORMING ORGANIZATION - NAME AND ADDRESS (II NRC, provide Division, Office or Region, U.S. Nuclear Regularany Commiscion, and mailing address: if contractor, providir name and mailing eddress.)

Sandia National Laboratories ITSC

P.O. Box 5800, MS0748 8015 Mountain Road Place, NE

Albuquerque, NM 87185-0748

Albuquerque, NM 87110

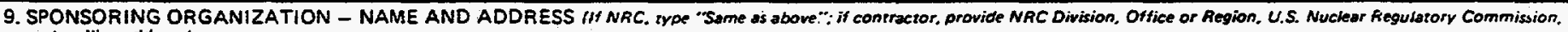
and mailing adoress.)

Division of Reactor Program Management

Office of Nuclear Reactor Regulation

U.S. Nuclear Regulatory Commission

Washington, DC 20555-0001

10. SUPPLEMENTARY NOTES

Jay Y. Lee, NRC Project Manager

11. ABSTRACT 1200 wores or less)

This report documents the RADTRAD computer code developed for the U.S. Nuclear

Regulatory Comission, Office of Nuclear Reactor Regulation to estimate transport

and removal of radionuclides and dose at selected receptors. The document includes

a users' guide to the code, a description of the technical basis for the code, the quality assurance and code acceptance testing documentation, and a programmers' guide. The RADTRAD code can be used to estimate the containment release using either the TID-14844 or NUREG-1465 source terms, and assumptions, or a user-specified table. In addition, the code can account for the reduction in the quantity of radioactive material due to containment sprays, natural deposition, filters, and other natural and engineered safety features. The RADTRAD code uses a combination of tables and/or numerical models of source term reduction phenomena to determine the time dependent dose at user specified locations for a given accident scenario. The code also provides the inventory, decay chain, and dose conversion factors needed for the dose calculation. The RADTRAD code can be used for occupational radiation exposure assessments, typically in the control room, for site boundary dose estimates, and for dose attenuation estimates due to facility or accident sequence modifications.

12. KEY WORDS/DESCR!PTORS (List words or phroses that will assist researchers in locating she reporr. I

nuclear power reactor accident, design basis accident, severe accident, source terms, removal mechanisms, environment dose, control room dose

\begin{tabular}{l} 
13. AVAILABILITY STATEMENT \\
unlimited \\
\hline 14. SECURITY CLASSIFICATION \\
This Pagel \\
unclassified \\
iThis ReponI \\
unclassified \\
15. NUMBER OF PAGES \\
\hline 16. PRICE
\end{tabular}

NRC FORM 335 (2-89) 